\title{
Metal-Free Synthesis of Indolopyrans and 2,3-Dihydrofurans Based on Tandem Oxidative Cycloaddition
}

\author{
Subin Choi ${ }^{a}$, Hyeonji Oh ${ }^{a}$, Jeongwoo Sim ${ }^{a}$, Eunsoo $\mathrm{Yu}^{a}$, Seunghoon Shin ${ }^{b}$ and Cheol-Min \\ Park $^{* a}$ \\ ${ }^{a}$ Department of Chemistry, UNIST (Ulsan National Institute of Science \& Technology), Ulsan 44919, Korea. \\ ${ }^{b}$ Department of Chemistry, Hanyang University, Seoul 04763, Korea. \\ correspondence to: Cheol-Min Park, cmpark@unist.ac.kr
}

\section{TABLE OF CONTENTS}

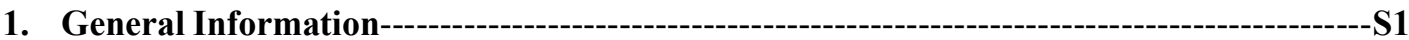

2. Optimization of the reaction conditions for Indolopyrans---

3. Preparation and characterization of substrates

3.1 Preparation and characterization of activated methylene compounds-----------------------S3

3.2 Preparation and characterization of substituted indoles---

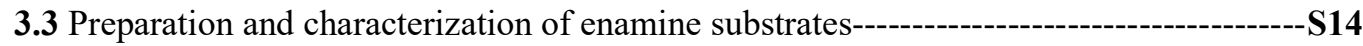

4. General procedure and characterization of pyrans---S16

5. General procedure and characterization of 2,3-dihydrofurans---_-_-S32

6. Synthetic applications

6.1 Sequential one-pot indolomalonate synthesis/pyran synthesis procedure-------------S36

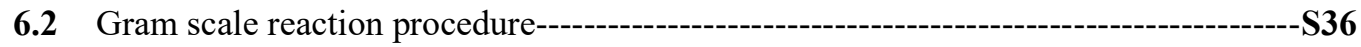

6.3 Synthesis of 7- and 8-membered rings--

6.4 Synthesis of cyclobutane derivatives--

7. Mechanistic studies

7.1 Identification of intermediates along the reaction pathway--------------------S40

7.2 Probing the presence of radical species derived from the reactants-----------------------S43

7.3 Procedures for identification of intermediates along the reaction pathway--------------S43

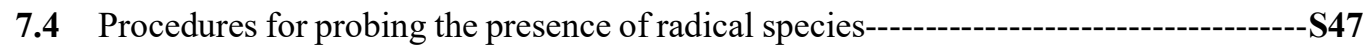

7.5 Identification of indolomalonate radical species by HRMS ------------------S50

7.6 Effect of reaction concentration supporting the cage-collapse cross-coupling ----------S51

7.7 Effect of radical scavenger----

7.8 Gibbs free energy for the SET between electron donors and electron acceptors--------S53

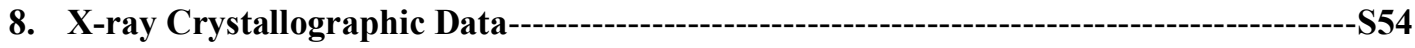

9. Computational details and references----

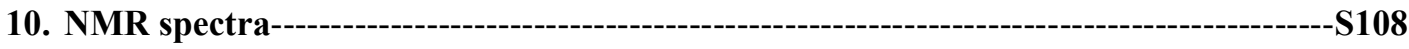

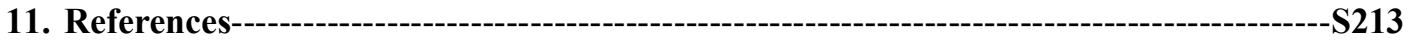




\section{General Information}

All reactions were conducted under a nitrogen atmosphere with oven-dried glassware and standard Schlenk or vacuum line techniques. Oil baths were used as a heat source for a reaction. All solutions were handled under nitrogen and transferred via syringe. Anhydrous solvents were purchased and stored over activated 4 A molecular sieves. Unless otherwise stated, reagents were commercially available and used as purchased without further purification. Chemicals were purchased from Sigma-Aldrich, Acros, Alfa Aesar or TCI. Progress of reactions was monitored by thin-layer chromatography using Merck 60 F254 precoated silica gel plate and visualized by shortwave ultraviolet light as well as by treatment with basic solution of potassium permanganate or acidic solution of ceric molybdate. Flash chromatography was performed with Silica Flash P60 silica gel ( $230-400$ mesh). ${ }^{1} \mathrm{H}$ and ${ }^{13} \mathrm{C}$ NMR spectra were obtained using an Agilent 400-MR DD2 Fourier-transform NMR spectrometer at 400 and $100 \mathrm{MHz}$, respectively. Chemical shifts were reported in units of parts per million (ppm) downfield from tetramethylsilane (TMS), and all coupling constants were reported in hertz. The residual solvent signals were taken as the reference $\left(\mathrm{CDCl}_{3}, 7.26 \mathrm{ppm}\right.$ or DMSO_$d_{6}, 2.50 \mathrm{ppm}$ for ${ }^{1} \mathrm{H}$ NMR spectra and $\mathrm{CDCl}_{3}, 77.0 \mathrm{ppm}$ or DMSO $\_d_{6}, 39.52 \mathrm{ppm}$ for ${ }^{13} \mathrm{C}$ NMR spectra). The signals observed are described as: $\mathrm{s}$ (singlet), $\mathrm{d}$ (doublet), $\mathrm{t}$ (triplet), q (quartet), m (multiplets). Mass analysis were carried out using Advion Expression CMS mass spectrometer and LC-ELSD analysis was carried out using Agilent 1260 Infinity ELSD coupled with Agilent 1220 HPLC. High resolution mass analysis was performed with JOEL AccuTOF 4G+ DART-HRMS and on Bruker, 1200 Series \& HCT Basic System. IR spectra were recorded on a Spectrum Two FT-IR Spectrometer. Melting point were measured with SRS MPA120 EZ-Melt Melting Point Apparatus. 


\section{Optimization of the reaction conditions for indolopyran ${ }^{a}$}

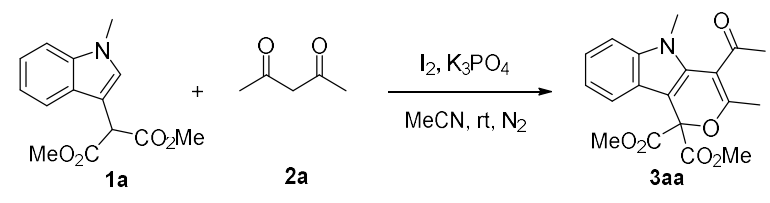

\begin{tabular}{|c|c|c|c|c|c|c|}
\hline Entry & $\begin{array}{c}2 a \\
\text { (equiv) }\end{array}$ & Oxidant (equiv) & Base (equiv) & Solvent (M) & $\begin{array}{c}\text { Time } \\
\text { (h) }\end{array}$ & NMR Yield \\
\hline 1 & 2 & $\mathrm{BrCCl}_{3}(7)$ & $\mathrm{K}_{3} \mathrm{PO}_{4}(7)$ & $\operatorname{MeCN}(0.1)$ & 1 & $70 \%$ \\
\hline 2 & 2 & DDQ (7) & $\mathrm{K}_{3} \mathrm{PO}_{4}(7)$ & $\operatorname{MeCN}(0.1)$ & $1 \mathrm{~d}$ & NR \\
\hline 3 & 2 & PIFA (7) & $\mathrm{K}_{3} \mathrm{PO}_{4}(7)$ & $\mathrm{MeCN}(0.1)$ & $1 \mathrm{~d}$ & NR \\
\hline 4 & 2 & $\mathrm{O}_{2}(7)$ & $\mathrm{K}_{3} \mathrm{PO}_{4}(7)$ & $\mathrm{MeCN}(0.1)$ & 30 & $\begin{array}{c}\text { Hydroxylated } \\
\text { product }(7 \mathbf{n}): \\
54 \%\end{array}$ \\
\hline 5 & 2 & $\mathrm{CBr}_{4}(7)$ & $\mathrm{K}_{3} \mathrm{PO}_{4}(7)$ & $\operatorname{MeCN}(0.1)$ & $1 \mathrm{~d}$ & $76 \%$ \\
\hline 6 & 1.1 & $I_{2}(2.2)$ & $\mathrm{K}_{3} \mathrm{PO}_{4}(5)$ & $\operatorname{MeCN}(0.1)$ & $20 \mathrm{~min}$ & $>99 \%$ \\
\hline 7 & 1.1 & $\mathrm{I}_{2}(2.2)$ & $\mathrm{KH}_{2} \mathrm{PO}_{4}(5)$ & $\operatorname{MeCN}(0.1)$ & $1 \mathrm{~d}$ & NR \\
\hline 8 & 1.1 & $\mathrm{I}_{2}(2.2)$ & $\mathrm{K}_{2} \mathrm{HPO}_{4}(5)$ & $\operatorname{MeCN}(0.1)$ & 9 & $10 \%$ \\
\hline 9 & 1.1 & $\mathrm{I}_{2}(2.2)$ & $\mathrm{Na}_{3} \mathrm{PO}_{4}(5)$ & $\operatorname{MeCN}(0.1)$ & 13 & $67 \%$ \\
\hline 10 & 1.1 & $\mathrm{I}_{2}(2.2)$ & TMEDA (5) & $\mathrm{MeCN}(0.1)$ & $25 \mathrm{~min}$ & $77 \%$ \\
\hline 11 & 1.1 & $\mathrm{I}_{2}(2.2)$ & DIPEA (5) & $\operatorname{MeCN}(0.1)$ & $1 \mathrm{~d}$ & NR \\
\hline 12 & 1.1 & $\mathrm{I}_{2}(2.2)$ & $\mathrm{K}_{3} \mathrm{PO}_{4}(5)$ & DMF $(0.1)$ & 3.5 & $74 \%$ \\
\hline 13, dark & 1.1 & $\mathrm{I}_{2}(2.2)$ & $\mathrm{K}_{3} \mathrm{PO}_{4}(5)$ & $\operatorname{MeCN}(0.1)$ & $20 \mathrm{~min}$ & $92 \%$ \\
\hline $14^{b},-10^{\circ} \mathrm{C}$ & 1.1 & $\mathrm{I}_{2}(2.2)$ & $\mathrm{K}_{3} \mathrm{PO}_{4}(5)$ & $\operatorname{MeCN}(0.1)$ & 1 & $87 \%$ \\
\hline $15^{c}$ & 1.1 & $\mathrm{I}_{2}(2.2)$ & $\mathrm{K}_{3} \mathrm{PO}_{4}(5)$ & $\mathrm{MeCN}(0.01)$ & 1 & $81 \%$ \\
\hline
\end{tabular}

${ }^{a}$ Unless noted otherwise, the reaction was carried out with $0.05 \mathrm{mmol}$ scale in $0.5 \mathrm{~mL}$ solvent. The yield was determined by ${ }^{1} \mathrm{H}$ NMR analysis $\left(\mathrm{CDCl}_{3}\right)$ of the crude reaction mixture using trichloroethylene as the internal standard. DDQ $=$ 2,3-Dichloro-5,6-dicyano-p-benzoquinone, PIFA $=$ (Bis(trifluoroacetoxy)iodo)benzene, TMEDA $=$ Tetramethylethylenediamine, DIPEA $=\mathrm{N}, \mathrm{N}$-Diisopropylethylamine. ${ }^{b}$ Low temperature does not affect the reaction. ${ }^{c}$ Dilution does not affect the in-cage reaction. 


\section{Preparation and characterization of substrates}

\subsection{Preparation and characterization of activated methylene compounds}

\section{1,3-Di-p-tolylpropane-1,3-dione (2c)}<smiles>Cc1ccc(C(=O)CC(=O)c2ccc(C)cc2)cc1</smiles>

It was prepared according to reported procedure. ${ }^{1}$ hexane:EA $=4: 1,201.8$ mg, $0.8 \mathrm{mmol}, 80 \%$ yield, white solid; ${ }^{1} \mathrm{H}$ NMR (400 MHz, $\left.\mathrm{CDCl}_{3}\right) \delta=$ $16.96(\mathrm{~s}, 1 \mathrm{H}), 7.91-7.87(\mathrm{~m}, 4 \mathrm{H}), 7.31-7.27(\mathrm{~m}, 4 \mathrm{H}), 6.81(\mathrm{~s}, 1 \mathrm{H})$,

$2.43(\mathrm{~s}, 6 \mathrm{H})$.

\section{1,3-Bis(4-methoxyphenyl)propane-1,3-dione (2d)}

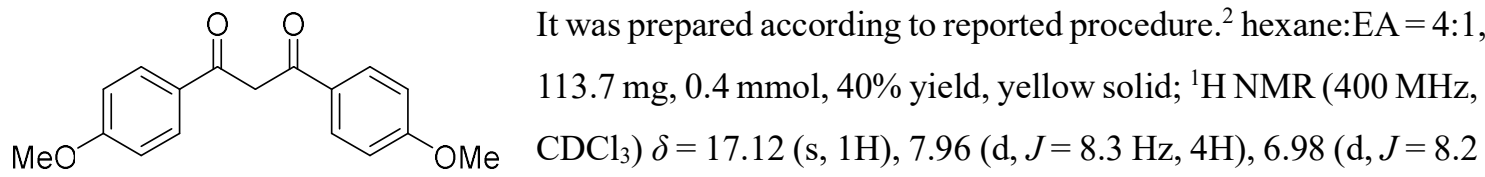

$\mathrm{Hz}, 4 \mathrm{H}), 6.73$ (s, 1H), 3.88 (s, 6H).

\section{1,3-Bis(4-nitrophenyl)propane-1,3-dione (2e)}<smiles>O=C(CC(=O)c1ccc([N+](=O)[O-])cc1)c1ccc([N+](=O)[O-])cc1</smiles>

4-Nitrobenzoyl chloride was prepared according to reported procedure using 4-nitrobenzoic acid. ${ }^{3}$ The resulting acyl chloride was obtained in almost quantitative yield and used for the next step without further purification. To a solution of ketone $\left(1 \mathrm{mmol}, 1\right.$ equiv) in THF $(0.4 \mathrm{M})$ at $-78{ }^{\circ} \mathrm{C}$ was added LiHMDS ( 3 equiv) and stirred for $1 \mathrm{hr}$. The 4-nitrobenzoyl chloride (1 equiv) was added dropwise and stirred for hours at room temperature. After the completion of reaction, $\mathrm{pH}$ of solution was adjusted to 7.0 by saturated $\mathrm{NH}_{4} \mathrm{Cl}$. The solution was diluted with ethyl acetate, washed with brine and dried over $\mathrm{NaSO}_{4}$. The solution was concentrated in vacuo and crude material was purified by flash chromatography. toluene:diethyl ether $=95: 5,141.4 \mathrm{mg}, 0.45 \mathrm{mmol}, 45 \%$ yield, yellow solid; ${ }^{1} \mathrm{H}$ NMR $\left(400 \mathrm{MHz}, \mathrm{CDCl}_{3}\right) \delta=16.43(\mathrm{~s}, 1 \mathrm{H}), 8.40-8.34(\mathrm{~m}, 4 \mathrm{H}), 8.19-8.14(\mathrm{~m}, 4 \mathrm{H}), 6.93(\mathrm{~s}, 1 \mathrm{H})$; The compound was identified by spectral comparison with literature data. ${ }^{4}$

1-(4-Methoxyphenyl)-3-(4-nitrophenyl)propane-1,3-dione (2f) 
<smiles>COc1ccc(C(=O)CC(=O)c2ccc([N+](=O)[O-])cc2)cc1</smiles>

4-Methoxybenzoyl chloride was prepared according to reported procedure using 4-methoxybenzoic acid. ${ }^{3}$ The resulting acyl chloride was obtained in almost quantitative yield and used for the next step without further purification. To a solution of 4nitroacetophenone $\left(0.3 \mathrm{mmol}, 1\right.$ equiv) in THF $(0.4 \mathrm{M})$ at $-78^{\circ} \mathrm{C}$ was added LiHMDS ( 3 equiv) and the resulting solution was stirred for $1 \mathrm{hr}$. The 4-metnoxybenzoyl chloride (1 equiv) was added dropwise and the solution was warmed to room temperature and stirred. Once the reaction was determined to be completed by TLC, $\mathrm{NH}_{4} \mathrm{Cl}$ (saturated solution in water) was added, the $\mathrm{pH}$ adjusted to 7.0. The organic and aqueous layers of the liquid phase were separated and the aqueous layer then extracted with ethyl acetate. The combined organic layers were washed with brine, dried over $\mathrm{NaSO}_{4}$ and then concentrated in vacuo. The product was purified through the flash chromatography. hexane:EA ; 4:1, $83.4 \mathrm{mg}, 0.28$ mmol, 93\% yield; ${ }^{1} \mathrm{H}$ NMR (400 MHz, $\left.\mathrm{CDCl}_{3}\right) \delta=16.81(\mathrm{~s}, 1 \mathrm{H}), 8.35-8.30(\mathrm{~m}, 2 \mathrm{H}), 8.13-8.09$ (m, $2 \mathrm{H}), 8.03-7.98(\mathrm{~m}, 2 \mathrm{H}), 7.00(\mathrm{~d}, \mathrm{~J}=8.9,2 \mathrm{H}), 6.83(\mathrm{~s}, 1 \mathrm{H}), 3.90(\mathrm{~s}, 3 \mathrm{H}) ;{ }^{13} \mathrm{C} \mathrm{NMR}\left(100 \mathrm{MHz}, \mathrm{CDCl}_{3}\right)$ $\delta=187.8,179.9,163.8,149.7,141.1,129.7,127.8,127.8,123.8,114.2,93.6,55.6$; HRMS (DART) $\mathrm{m} / \mathrm{z}:[\mathrm{M}+\mathrm{H}]^{+}$Calcd. for $\mathrm{C}_{16} \mathrm{H}_{13} \mathrm{NO}_{5}$ 300.0866; Found 300.0866

\section{3-Oxo-N-phenylbutanamide (2k)}<smiles>CC(=O)CC(=O)Nc1ccccc1</smiles>

It was prepared according to reported procedure using aniline $(0.4 \mathrm{mmol}){ }^{5}$ hexane:acetone $=3: 2,46.8 \mathrm{mg}, 0.26 \mathrm{mmol}, 66 \%$ yield, yellow solid; ${ }^{1} \mathrm{H} \mathrm{NMR}$ $\left(400 \mathrm{MHz}, \mathrm{CDCl}_{3}\right) \delta=9.12(\mathrm{~s}, 1 \mathrm{H}), 7.57-7.52(\mathrm{~m}, 2 \mathrm{H}), 7.32(\mathrm{tt}, \mathrm{J}=7.4,0.9,2 \mathrm{H})$, $7.12(\mathrm{t}, \mathrm{J}=7.3,1 \mathrm{H}), 3.58(\mathrm{~s}, 2 \mathrm{H}), 2.33-2.31(\mathrm{~m}, 3 \mathrm{H})$; The compound was identified by spectral comparison with literature data. ${ }^{6}$

\section{3-Oxo-3-phenylpropanenitrile (2I)}

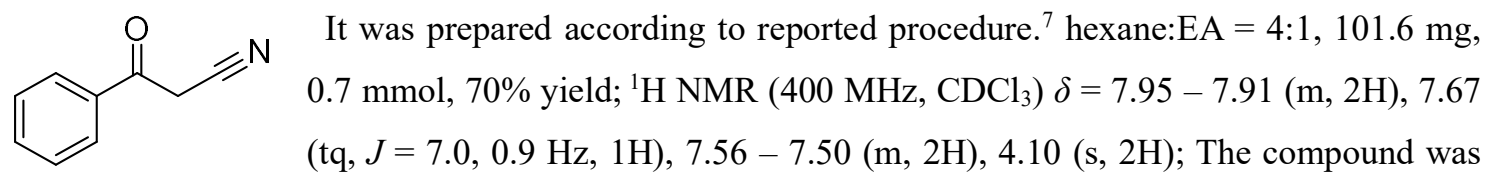
identified by spectral comparison with literature data. ${ }^{8}$

\section{1-Phenyl-2-(phenylsulfonyl)ethan-1-one (2m)}


<smiles>O=C(CS(=O)(=O)c1ccccc1)c1ccccc1</smiles>
It was prepared according to reported procedure using 2-chloro-1-phenylethan1-one $(0.5 \mathrm{mmol}) .{ }^{9}$ hexane:EA $=4: 1,83 \mathrm{mg}, 0.34 \mathrm{mmol}, 64 \%$ yield, white solid; ${ }^{1} \mathrm{H}$ NMR (400 MHz, $\mathrm{CDCl}_{3}$ ) $\delta=7.92$ (ddd, J=16.1, 8.4, 1.2, 4H), $7.69-7.59$ $(\mathrm{m}, 2 \mathrm{H}), 7.55(\mathrm{t}, \mathrm{J}=7.7,2 \mathrm{H}), 7.48(\mathrm{t}, \mathrm{J}=7.7,2 \mathrm{H}), 4.74(\mathrm{~s}, 2 \mathrm{H})$; The compound was identified by spectral comparison with literature data. ${ }^{9}$

\section{Methyl (E)-3-oxo-5-phenylpent-4-enoate (2o)}<smiles>COC(=O)CC(=O)/C=C/c1ccccc1</smiles>

It was prepared according to reported procedure. ${ }^{10} \mathrm{CH}_{2} \mathrm{Cl}_{2}: \mathrm{MeOH}=19: 1$, $121 \mathrm{mg}, 0.6 \mathrm{mmol}, 60 \%$ yield, white solid; ${ }^{1} \mathrm{H} \mathrm{NMR}\left(400 \mathrm{MHz}, \mathrm{CDCl}_{3}\right.$ ) $\delta=11.9(\mathrm{~s}, 1 \mathrm{H}), 7.5(\mathrm{~d}, J=7.6 \mathrm{~Hz}, 2 \mathrm{H}), 7.4(\mathrm{~d}, J=16.1 \mathrm{~Hz}, 1 \mathrm{H}), 7.3(\mathrm{dd}, J=$ 10.0, $7.4 \mathrm{~Hz}, 3 \mathrm{H}), 6.4$ (dd, $J=15.9,1.6 \mathrm{~Hz}, 1 \mathrm{H}), 5.2$ (s, 1H), 3.8 (d, $J=1.3 \mathrm{~Hz}$,

$3 \mathrm{H})$. The compound was identified by spectral comparison with literature data. ${ }^{10}$

\section{(1E,6E)-1,7-Diphenylhepta-1,6-diene-3,5-dione (2p)}<smiles>O=C(/C=C/c1ccccc1)/C=C/c1ccccc1</smiles>

It was prepared according to reported procedure using benzaldehyde $(3 \mathrm{mmol}) .{ }^{11}$ hexane:toluene $=1: 1,2.4 \mathrm{~g}, 9 \mathrm{mmol}$, $45 \%$ yield, yellow solid; ${ }^{1} \mathrm{H}$ NMR $\left(400 \mathrm{MHz}, \mathrm{CDCl}_{3}\right) \delta=15.89$

(s, 1H), $7.68(\mathrm{~d}, J=15.9 \mathrm{~Hz}, 2 \mathrm{H}), 7.59-7.55(\mathrm{~m}, 4 \mathrm{H}), 7.45-7.35(\mathrm{~m}, 6 \mathrm{H}), 6.64(\mathrm{~d}, J=15.9 \mathrm{~Hz}, 2 \mathrm{H})$, $5.86(\mathrm{~s}, 1 \mathrm{H})$; The compound was identified by spectral comparison with literature data. ${ }^{12}$

\section{General procedure (A) for dehydration of alcohol compound}

The 2q-2t were prepared according to reported procedure using pentane-2,4-dione. ${ }^{13}$ The corresponding alcohol was heated at reflux in a mixture of Dioxane and $1 \mathrm{~N} \mathrm{HCl}(4: 1)[0.2 \mathrm{M}]$. Once the reaction was determined to be complete by TLC, the reaction mixture was diluted with ethyl acetate washed with water. $\mathrm{NaHCO}_{3}$ and brine and dried over anhydrous $\mathrm{Na}_{2} \mathrm{SO}_{4}$. The solvent was evaporated and the residue was purified by column chromatography.

\section{(1E,4Z)-5-Hydroxy-1-phenylhexa-1,4-dien-3-one (2q)}

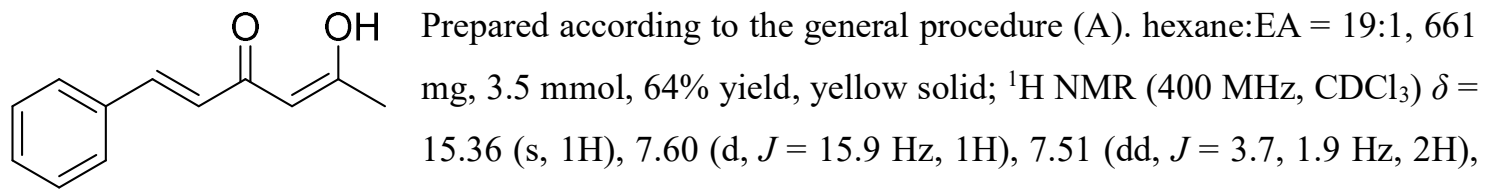


$7.43-7.34(\mathrm{~m}, 3 \mathrm{H}), 6.47(\mathrm{~d}, J=15.9 \mathrm{~Hz}, 1 \mathrm{H}), 5.65(\mathrm{~s}, 1 \mathrm{H}), 2.17(\mathrm{~s}, 3 \mathrm{H})$; The compound was identified by spectral comparison with literature data. ${ }^{14}$

\section{(1E,4Z)-5-Hydroxy-1-(4-methoxyphenyl)hexa-1,4-dien-3-one (2r)}<smiles>COc1ccc(/C=C/C(=O)/C=C(\C)O)cc1</smiles>

Prepared according to the general procedure (A). hexane:EA $=19: 1$, $661 \mathrm{mg}, 3.5 \mathrm{mmol}, 71 \%$ yield, yellow solid; ${ }^{1} \mathrm{H}$ NMR $(600 \mathrm{MHz}$, $\left.\mathrm{CDCl}_{3}\right) \delta=7.56(\mathrm{~d}, J=15.8 \mathrm{~Hz}, 1 \mathrm{H}), 7.51-7.44(\mathrm{~m}, 2 \mathrm{H}), 6.92-$ $6.87(\mathrm{~m}, 2 \mathrm{H}), 6.34(\mathrm{~d}, J=15.8 \mathrm{~Hz}, 1 \mathrm{H}) ;{ }^{13} \mathrm{C} \mathrm{NMR}\left(100 \mathrm{MHz}, \mathrm{CDCl}_{3}\right) \delta=197.1,177.9,161.2,139.6$, $129.6,127.7,120.3,114.4,100.8,55.3,26.8$; The compound was identified by spectral comparison with literature data. ${ }^{15}$

\section{(1E,4Z)-5-Hydroxy-1-(4-nitrophenyl)hexa-1,4-dien-3-one (2s)}

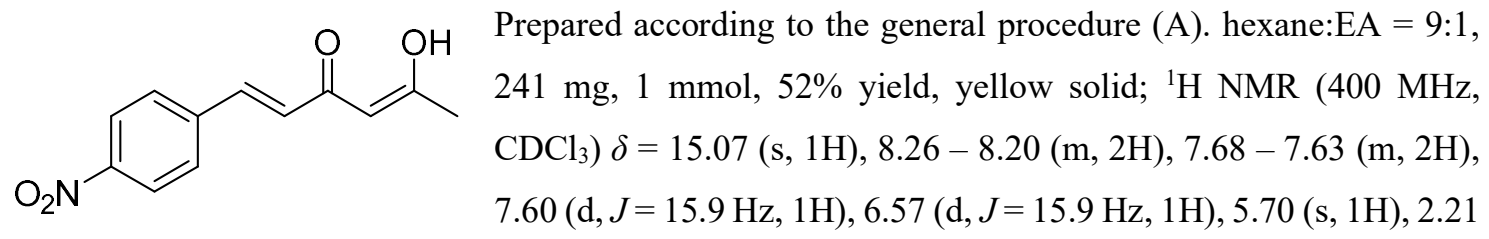

(s, 3H). ${ }^{13} \mathrm{C} \mathrm{NMR}\left(100 \mathrm{MHz}, \mathrm{CDCl}_{3}\right) \delta=199.8,174.0,148.1,141.3,136.4,128.3,126.8,124.2,102.5$, 27.7; HRMS (DART) m/z: [M+H] ${ }^{+}$Calcd. for $\mathrm{C}_{12} \mathrm{H}_{11} \mathrm{NO}_{4} 234.0761$; Found 234.0760

\section{(1E,4Z)-1-(4-Chlorophenyl)-5-hydroxyhexa-1,4-dien-3-one (2t)}

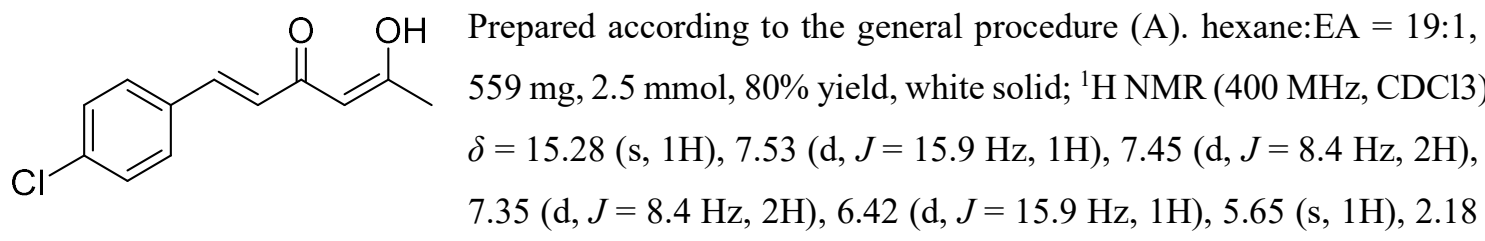

(s, 3H); ${ }^{13} \mathrm{C}$ NMR $\left(100 \mathrm{MHz}, \mathrm{CDCl}_{3}\right) \delta 198.4,176.1,138.2,135.7,133.6,129.1,129.0,123.2,101.5$, 27.2; HRMS (DART) $\mathrm{m} / \mathrm{z}$ : $[\mathrm{M}+\mathrm{H}]^{+}$Calcd. for $\mathrm{C}_{12} \mathrm{H}_{11} \mathrm{ClO}_{2} 223.0520$; Found 223.0520

\section{1-(Phenylsulfonyl)propan-2-one (2u)}<smiles>CC(=O)CS(=O)(=O)c1ccccc1</smiles>

It was prepared according to reported procedure using sodium benzenesulfinate $(1 \mathrm{mmol}) .{ }^{16}$ hexane:acetone $=4: 1,158.6 \mathrm{mg}, 0.81 \mathrm{mmol}, 81 \%$ yield, white solid; ${ }^{1} \mathrm{H} \mathrm{NMR}\left(400 \mathrm{MHz}, \mathrm{CDCl}_{3}\right) \delta=7.90(\mathrm{~d}, \mathrm{~J}=8.1 \mathrm{~Hz}, 2 \mathrm{H}), 7.70(\mathrm{t}, \mathrm{J}=7.1 \mathrm{~Hz}, 1 \mathrm{H})$, $7.59(\mathrm{t}, \mathrm{J}=7.7 \mathrm{~Hz}, 2 \mathrm{H}), 4.16(\mathrm{~s}, 2 \mathrm{H}), 2.42(\mathrm{~s}, 3 \mathrm{H})$; The compound was identified by spectral comparison with literature data. ${ }^{17}$ 


\subsection{Preparation and characterization of substituted indoles}

\section{General procedure (B)}

Copper (II) or Rh (II) catalyst (x mol \%) was added to a solution of indole (x equiv) and diketone (x equiv) dissolved in solvent (x M) under $\mathrm{N}_{2}$ at ambient temperature. After the completion of reaction, the reaction mixture was filtered through a celite pad and concentrated in vacuo. The crude material was purified by flash chromatography.

\section{Dimethyl 2-(1-methyl-1H-indol-3-yl)malonate (1a)}

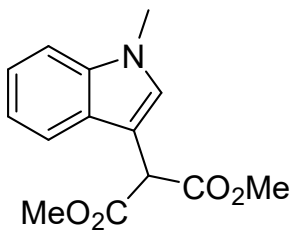

Prepared according to the general procedure (B) using $\mathrm{Cu}(\mathrm{hfacac})_{2}(2 \mathrm{~mol} \%)$, dimethyl 2-diazomalonate (1.2 equiv), 1-methyl-1 $H$-indole ( $1 \mathrm{mmol}, 1$ equiv) in $\operatorname{DCE}(0.1 \mathrm{M})$ at $60{ }^{\circ} \mathrm{C}$, hexane:EA $=7: 3,248.2 \mathrm{mg}, 0.95 \mathrm{mmol}, 95 \%$ yield, white solid; ${ }^{1} \mathrm{H}$ NMR $\left(400 \mathrm{MHz}, \mathrm{CDCl}_{3}\right) \delta=7.62(\mathrm{~d}, J=8.0 \mathrm{~Hz}, 1 \mathrm{H}), 7.33-7.27(\mathrm{~m}$, 2H), $7.23(\mathrm{~d}, J=8.1 \mathrm{~Hz}, 1 \mathrm{H}), 7.15(\mathrm{t}, J=7.4 \mathrm{~Hz}, 1 \mathrm{H}), 4.97$ (s, 1H), 3.79 (s, 3H), 3.76 (s, 6H); The compound was identified by spectral comparison with literature data. ${ }^{18}$

\section{Dimethyl 2-(5-methoxy-1-methyl-1 $H$-indol-3-yl)malonate (1b)}

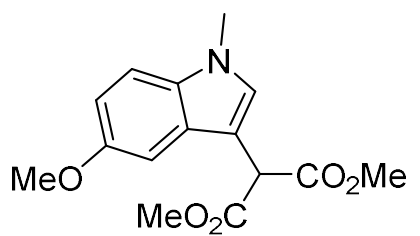

Prepared according to the general procedure (B) using $\mathrm{Cu}(\mathrm{hfacac})_{2}(2$ mol \%), dimethyl 2-diazomalonate (1.2 equiv), 5-methoxy-1-methyl$1 H$-indole $\left(0.19 \mathrm{mmol}, 1\right.$ equiv) in $\mathrm{DCE}(0.1 \mathrm{M})$ at $35^{\circ} \mathrm{C}$, hexane:EA $=$ 4:1, $30.7 \mathrm{mg}, 0.1 \mathrm{mmol}, 55 \%$ yield, yellow solid; ${ }^{1} \mathrm{H}$ NMR $(400 \mathrm{MHz}$, $\left.\mathrm{CDCl}_{3}\right) \delta=7.24(\mathrm{~s}, 1 \mathrm{H}), 7.19(\mathrm{~d}, J=8.9 \mathrm{~Hz}, 1 \mathrm{H}), 7.06(\mathrm{~d}, J=2.4 \mathrm{~Hz}, 1 \mathrm{H}), 6.90(\mathrm{dd}, J=8.9,2.4 \mathrm{~Hz}$, $1 \mathrm{H}), 4.92(\mathrm{~s}, 1 \mathrm{H}), 3.86(\mathrm{~s}, 3 \mathrm{H}), 3.77(\mathrm{~s}, 6 \mathrm{H}), 3.75(\mathrm{~s}, 3 \mathrm{H})$; The compound was identified by spectral comparison with literature data. ${ }^{19}$

\section{Dimethyl 2-(5-((tert-butoxycarbonyl)amino)-1-methyl-1 $H$-indol-3-yl)malonate (1c)}<smiles>CC(=O)OC(C)(C)C(=O)Nc1ccc2c(c1)c(C(C)=O)cn2C</smiles>

Prepared according to the general procedure (B) using $\mathrm{Cu}(\mathrm{hfacac})_{2}(5$ mol \%), methyl dimethyl 2-diazomalonate (1.5 equiv), tert-butyl (1methyl-1H-indol-5-yl)carbamate (1.5 mmol, 1 equiv) in DCE $(0.2 \mathrm{M})$ at $40{ }^{\circ} \mathrm{C}$. hexane: $\mathrm{EA}=7: 3,381.7 \mathrm{mg}, 1.0 \mathrm{mmol}, 68 \%$ yield, white solid;

${ }^{1} \mathrm{H}$ NMR $\left(400 \mathrm{MHz}, \mathrm{CDCl}_{3}\right) \delta=7.57(\mathrm{~s}, 1 \mathrm{H}), 7.23(\mathrm{~d}, \mathrm{~J}=13.5,3 \mathrm{H}), 6.48(\mathrm{~s}, 1 \mathrm{H}), 4.93(\mathrm{~s}, 1 \mathrm{H}), 3.76(\mathrm{~s}$, $6 \mathrm{H}), 3.75(\mathrm{~s}, 3 \mathrm{H}), 1.53(\mathrm{~s}, 9 \mathrm{H}) ;{ }^{13} \mathrm{C} \mathrm{NMR}\left(100 \mathrm{MHz}, \mathrm{CDCl}_{3}\right) \delta=169.1,153.5,133.6,131.1,129.3$, 
127.1 115.5, 109.6, 109.1 105.3, 80.0, 52.8, 49.0, 33.0, 28.4; HRMS (DART) m/z: [M+H] ${ }^{+}$Calcd. for $\mathrm{C}_{19} \mathrm{H}_{24} \mathrm{~N}_{2} \mathrm{O}_{6} 377.1707$; Found 377.1703

\section{Dimethyl 2-(1-methyl-5-nitro-1H-indol-3-yl)malonate (1d)}

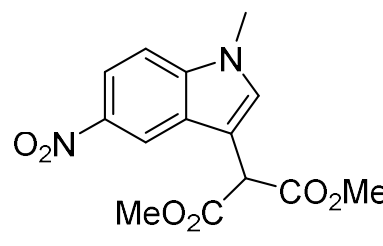

Prepared according to the general procedure (B) using $\mathrm{Cu}(\mathrm{acac})_{2}$ (3 mol \%), methyl dimethyl 2-diazomalonate (1.5 equiv), 1-methyl-5-nitro$1 H$-indole $\left(0.4 \mathrm{mmol}, 1\right.$ equiv) in benzene $(0.2 \mathrm{M})$ at $90{ }^{\circ} \mathrm{C}$. hexane:EA =

7:3, $91.1 \mathrm{mg}, 0.3 \mathrm{mmol}, 75 \%$ yield, brown solid; ${ }^{1} \mathrm{H}$ NMR $(400 \mathrm{MHz}$, $\left.\mathrm{CDCl}_{3}\right) \delta=8.61(\mathrm{~d}, \mathrm{~J}=2.1,1 \mathrm{H}), 8.15(\mathrm{dd}, \mathrm{J}=9.1,1.9,1 \mathrm{H}), 7.46(\mathrm{~s}, 1 \mathrm{H}), 7.34(\mathrm{~d}, \mathrm{~J}=9.0,1 \mathrm{H}), 4.98(\mathrm{~s}, 1 \mathrm{H})$, $3.85(\mathrm{~s}, 3 \mathrm{H}), 3.80(\mathrm{~s}, 6 \mathrm{H})$; The compound was identified by spectral comparison with literature data. ${ }^{19}$

\section{Dimethyl 2-(5-cyano-1-methyl-1H-indol-3-yl)malonate (1e)}

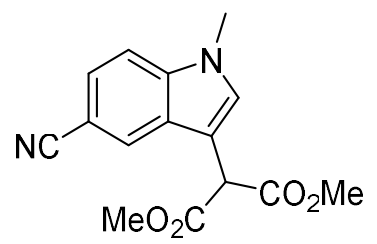

Prepared according to the general procedure (B) using $\mathrm{Cu}(\mathrm{acac})_{2}(5 \mathrm{~mol} \%)$, methyl dimethyl 2-diazomalonate (1.5 equiv), 1-methyl-1 $H$-indole-5carbonitrile ( $0.5 \mathrm{mmol}, 1$ equiv) in $\mathrm{DCE}(0.2 \mathrm{M})$ at $90^{\circ} \mathrm{C}$. hexane:EA=7:3, $50.0 \mathrm{mg}, 0.175 \mathrm{mmol}, 35 \%$ yield, white solid; ${ }^{1} \mathrm{H} \mathrm{NMR}\left(400 \mathrm{MHz}, \mathrm{CDCl}_{3}\right)$ $\delta=8.00(\mathrm{dd}, \mathrm{J}=1.5,0.7,1 \mathrm{H}), 7.47(\mathrm{dd}, \mathrm{J}=8.6,1.5,1 \mathrm{H}), 7.41(\mathrm{~s}, 1 \mathrm{H}), 7.36(\mathrm{dd}, \mathrm{J}=8.6,0.7,1 \mathrm{H}), 4.92(\mathrm{~d}$, $\mathrm{J}=0.6,1 \mathrm{H}), 3.82(\mathrm{~s}, 3 \mathrm{H}), 3.78(\mathrm{~s}, 6 \mathrm{H}) ;{ }^{13} \mathrm{C} \mathrm{NMR}\left(100 \mathrm{MHz}, \mathrm{CDCl}_{3}\right) \delta 168.4,138.3,131.0,126.7,125.1$, 124.9, 120.6, 110.4, 106.9, 102.9, 53.0, 49.0, 33.2; HRMS (DART) m/z: $[\mathrm{M}+\mathrm{H}]^{+}$Calcd. for $\mathrm{C}_{15} \mathrm{H}_{14} \mathrm{~N}_{2} \mathrm{O}_{4}$ 287.1026; Found 287.1027

\section{Dimethyl 2-(5-(methoxycarbonyl)-1-methyl-1H-indol-3-yl)malonate (1f)}

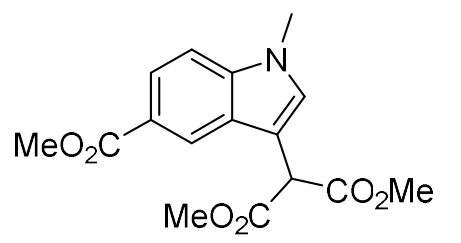

Prepared according to the general procedure (B) using $\mathrm{Cu}(\mathrm{acac})_{2}$ (5 mol \%), methyl dimethyl 2-diazomalonate (1.5 equiv), methyl 1methyl-1H-indole-5-carboxylate $(0.3 \mathrm{mmol}, 1$ equiv) in DCE $(0.2 \mathrm{M})$ at $80{ }^{\circ} \mathrm{C}$. hexane:EA $=7: 3,49.8 \mathrm{mg}, 0.16 \mathrm{mmol}, 52 \%$ yield, white solid;

${ }^{1} \mathrm{H}$ NMR (400 MHz, $\left.\mathrm{CDCl}_{3}\right) \delta=8.37(\mathrm{~d}, \mathrm{~J}=1.5,1 \mathrm{H}), 7.95(\mathrm{dd}, \mathrm{J}=8.7,1.6,1 \mathrm{H}), 7.36(\mathrm{~s}, 1 \mathrm{H}), 7.31(\mathrm{~d}$, $\mathrm{J}=8.7,1 \mathrm{H}), 5.01(\mathrm{~s}, 1 \mathrm{H}), 3.93(\mathrm{~s}, 3 \mathrm{H}), 3.81(\mathrm{~s}, 3 \mathrm{H}), 3.78(\mathrm{~s}, 6 \mathrm{H}) ;{ }^{13} \mathrm{C} \mathrm{NMR}\left(100 \mathrm{MHz}, \mathrm{CDCl}_{3}\right) \delta=168.8$, 168.0, 139.1, 130.1, 126.6, 123.4, 121.8, 121.8, 109.2, 107.3, 52.9, 51.9, 48.8, 33.1; HRMS (DART) $\mathrm{m} / \mathrm{z}:[\mathrm{M}+\mathrm{H}]^{+}$Calcd. For $\mathrm{C}_{16} \mathrm{H}_{17} \mathrm{NO}_{6} 320.1129$; Found 320.1122 


\section{Dimethyl 2-(4-(methoxycarbonyl)-1-methyl-1H-indol-3-yl)malonate (1g)}<smiles>COCc1cccc2c1c(C(COC)C(C)=O)cn2C</smiles>

Prepared according to the general procedure (B) using $\mathrm{Cu}(\mathrm{hfacac})_{2}(2$ mol \%), dimethyl 2-diazomalonate (1.2 equiv), methyl 1-methyl- $1 \mathrm{H}$ indole-4-carboxylate $\left(0.3 \mathrm{mmol}, 1\right.$ equiv) in DCE $(0.1 \mathrm{M})$ at $35{ }^{\circ} \mathrm{C}$, hexane:EA $=7: 3,80.7 \mathrm{mg}, 0.24 \mathrm{mmol}, 84 \%$ yield, white solid; ${ }^{1} \mathrm{H}$ NMR $\left(400 \mathrm{MHz}, \mathrm{CDCl}_{3}\right) \delta=7.77(\mathrm{dd}, J=7.5,1.0 \mathrm{~Hz}, 1 \mathrm{H}), 7.50(\mathrm{dd}, J=8.2,1.0$ $\mathrm{Hz}, 1 \mathrm{H}), 7.34(\mathrm{~d}, J=0.6 \mathrm{~Hz}, 1 \mathrm{H}), 7.24(\mathrm{dd}, J=8.2,7.5 \mathrm{~Hz}, 1 \mathrm{H}), 5.85(\mathrm{~s}, 1 \mathrm{H}), 3.92(\mathrm{~s}, 3 \mathrm{H}), 3.81(\mathrm{~s}, 3 \mathrm{H})$, $3.77(\mathrm{~s}, 6 \mathrm{H}) ;{ }^{13} \mathrm{C} \mathrm{NMR}\left(100 \mathrm{MHz}, \mathrm{CDCl}_{3}\right) \delta=170.1,168.5,137.9,131.2,125.0,123.8,123.3,120.7$, 114.1, 106.5, 52.6, 52.1, 50.3, 33.1; HRMS (DART) m/z: $[\mathrm{M}+\mathrm{H}]^{+}$Calcd. For $\mathrm{C}_{16} \mathrm{H}_{17} \mathrm{NO}_{6} 320.1129$; Found 320.1128

\section{Dimethyl 2-(5-bromo-1-methyl-1H-indol-3-yl)malonate (1h)}<smiles>CC(=O)C(C(C)=O)c1cn(C)c2ccc(Br)cc12</smiles>

Prepared according to the general procedure (B) using $\mathrm{Cu}(\mathrm{acac})_{2}(2 \mathrm{~mol} \%)$, dimethyl 2-diazomalonate ( $0.24 \mathrm{mmol}, 1$ equiv), 5-bromo-1-methyl-1 $H$ indole (1.5 equiv) in benzene $(0.25 \mathrm{M})$ at $85^{\circ} \mathrm{C}$, hexane:EA $=4: 1,59.6 \mathrm{mg}$,

$0.18 \mathrm{mmol}, 73 \%$ yield, white solid; ${ }^{1} \mathrm{H}$ NMR $\left(400 \mathrm{MHz}, \mathrm{CDCl}_{3}\right) \delta=7.74$ (d, $J=1.9 \mathrm{~Hz}, 1 \mathrm{H}), 7.31$ (dd, $J=8.7,1.9 \mathrm{~Hz}, 1 \mathrm{H}), 7.28(\mathrm{~s}, 1 \mathrm{H}), 7.17(\mathrm{~d}, J=8.7 \mathrm{~Hz}, 1 \mathrm{H}), 4.88(\mathrm{~s}, 1 \mathrm{H})$, $3.77(\mathrm{~s}, 6 \mathrm{H}), 3.76(\mathrm{~s}, 3 \mathrm{H})$; The compound was identified by spectral comparison with literature data. ${ }^{19}$

\section{Dimethyl 2-(1H-indol-3-yl)malonate (1i)}

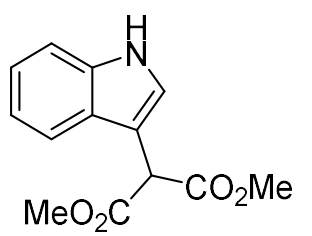

Prepared according to the general procedure (B) using $\mathrm{Cu}(\mathrm{acac})_{2}(2 \mathrm{~mol} \%)$, dimethyl 2-diazomalonate (1.2 equiv), $1 H$-indole ( $0.2 \mathrm{mmol}, 1$ equiv) in DCE $(0.1 \mathrm{M})$ at $60^{\circ} \mathrm{C}$, hexane:EA $=4: 1,34.6 \mathrm{mg}, 0.14 \mathrm{mmol}, 70 \%$ yield, white solid; ${ }^{1} \mathrm{H}$ NMR (400 MHz, $\left.\mathrm{CDCl}_{3}\right) \delta=8.30(\mathrm{~s}, 1 \mathrm{H}), 7.65(\mathrm{dd}, J=7.8,1.4 \mathrm{~Hz}, 1 \mathrm{H})$, $7.32(\mathrm{t}, J=2.6 \mathrm{~Hz}, 2 \mathrm{H}), 7.18(\mathrm{dtd}, J=20.4,7.1,1.1 \mathrm{~Hz}, 2 \mathrm{H}), 4.99$ (s, 1H), 3.77 (s, 6H); The compound was identified by spectral comparison with literature data. ${ }^{19}$

\section{Dimethyl 2-(1-benzyl-1H-indol-3-yl)malonate (1j)}

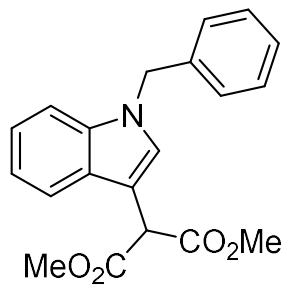

Prepared according to the general procedure (B) using $\mathrm{Cu}(\mathrm{acac})_{2}(2 \mathrm{~mol} \%)$, dimethyl 2-diazomalonate (1.2 equiv), 1-benzyl- $1 H$-indole ( $0.2 \mathrm{mmol}, 1$ equiv) in DCE $(0.1 \mathrm{M})$ at $60^{\circ} \mathrm{C}$, hexane:EA $=4: 1,47.2 \mathrm{mg}, 0.14 \mathrm{mmol}, 70 \%$ yield, white solid; ${ }^{1} \mathrm{H}$ NMR (400 MHz, $\left.\mathrm{CDCl}_{3}\right) \delta=7.74-7.67(\mathrm{~m}, 1 \mathrm{H}), 7.42-7.38(\mathrm{~m}, 1 \mathrm{H})$, 
$7.31(\mathrm{td}, J=9.1,8.5,3.9 \mathrm{~Hz}, 4 \mathrm{H}), 7.19(\mathrm{dt}, J=19.8,7.2 \mathrm{~Hz}, 4 \mathrm{H}), 5.30(\mathrm{~s}, 2 \mathrm{H}), 5.03(\mathrm{~s}, 1 \mathrm{H}), 3.79$ (s, $6 \mathrm{H})$; The compound was identified by spectral comparison with literature data. ${ }^{19}$

\section{Dimethyl 2-(1-(p-tolyl)-1H-indol-3-yl)malonate (1 k)}

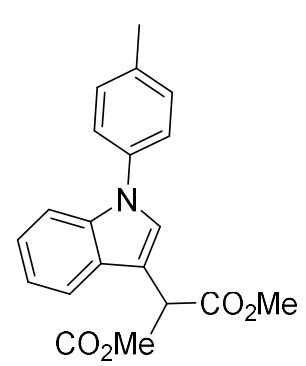

Prepared according to the general procedure (B) using $\mathrm{Cu}(\mathrm{acac})_{2}(2 \mathrm{~mol} \%)$, dimethyl 2-diazomalonate (1.2 equiv), 1-( $p$-tolyl)- $1 H$-indole ( 1 mmol, 1 equiv) in DCE $(0.1 \mathrm{M})$ at $60{ }^{\circ} \mathrm{C}$, hexane: $\mathrm{EA}=4: 1,269.9 \mathrm{mg}, 0.8 \mathrm{mmol}, 80 \%$ yield, white solid; ${ }^{1} \mathrm{H}$ NMR (400 MHz, $\left.\mathrm{CDCl}_{3}\right) \delta=7.72-7.68(\mathrm{~m}, 1 \mathrm{H}), 7.54(\mathrm{~s}, 1 \mathrm{H})$, $7.53-7.50(\mathrm{~m}, 1 \mathrm{H}), 7.42-7.38(\mathrm{~m}, 2 \mathrm{H}), 7.33-7.29(\mathrm{~m}, 2 \mathrm{H}), 7.22(\mathrm{dtd}, J=$ 14.1, 7.0, $1.3 \mathrm{~Hz}, 2 \mathrm{H}), 5.04$ (s, 1H), 3.79 (s, 6H), 2.44 (s, 3H); ${ }^{13} \mathrm{C}$ NMR (100 $\left.\mathrm{MHz}, \mathrm{CDCl}_{3}\right) \delta=168.9,136.8,136.6,136.0,130.1,127.9,127.7,124.4,122.7,120.5,119.3,110.8$, 107.8, 52.8, 49.3, 21.1; HRMS (DART) m/z: $[\mathrm{M}+\mathrm{H}]^{+}$Calcd. For $\mathrm{C}_{20} \mathrm{H}_{19} \mathrm{NO}_{4} 338.1387$; Found 338.1387

\section{Dimethyl 2-(1-acetyl-1H-indol-3-yl)malonate (11)}

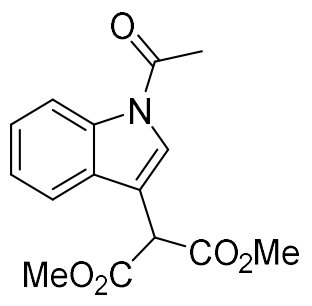

Prepared according to the general procedure (B) using $\mathrm{Cu}(\mathrm{hfacac})_{2}(5 \mathrm{~mol} \%$ ), methyl dimethyl 2 -diazomalonate (1.5 equiv), 1-(1H-indol-1-yl)ethan-1-one (2 mmol, 1 equiv) in benzotrifluoride $(0.1 \mathrm{M})$ at $90^{\circ} \mathrm{C}$. hexane:EA $=7: 3,312.4 \mathrm{mg}$, $1.1 \mathrm{mmol}, 54 \%$ yield, pink solid; ${ }^{1} \mathrm{H}$ NMR $\left(400 \mathrm{MHz}, \mathrm{CDCl}_{3}\right) \delta=8.46(\mathrm{~d}, \mathrm{~J}=8.2$, 1H), $7.71(\mathrm{~s}, 1 \mathrm{H}), 7.57(\mathrm{~d}, \mathrm{~J}=7.7,1 \mathrm{H}), 7.41-7.34(\mathrm{~m}, 1 \mathrm{H}), 7.31(\mathrm{t}, \mathrm{J}=7.0,1 \mathrm{H})$, $4.93(\mathrm{~s}, 1 \mathrm{H}), 3.80(\mathrm{~s}, 6 \mathrm{H}), 2.66$ (s, 3H); ${ }^{13} \mathrm{C} \mathrm{NMR}\left(100 \mathrm{MHz}, \mathrm{CDCl}_{3}\right) \delta=168.6,168.0,135.5,129.1$, 125.6, 125.5, 125.0, 125.0, 123.8, 119.0, 119.0, 116.7, 113.6, 53.1, 48.8, 23.9, 23.9; HRMS (DART) m/z: $[\mathrm{M}+\mathrm{H}]^{+}$Calcd. For $\mathrm{C}_{15} \mathrm{H}_{15} \mathrm{NO}_{5} 290.1023$; Found 290.1025

\section{Methyl 2-(1-methyl-1 $H$-indol-3-yl)-3-oxobutanoate (1m)}

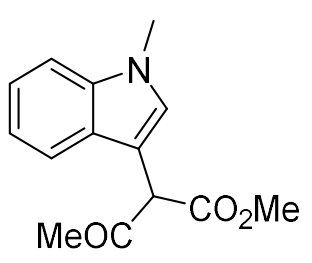

Prepared according to the general procedure (B) using $\mathrm{Cu}(\mathrm{hfacac})_{2}(6 \mathrm{~mol} \%)$, methyl 2-diazo-3-oxobutanoate (1.1 equiv), 1-methyl-1 $H$-indole ( $1.5 \mathrm{mmol}, 1$ equiv) in DCE $(0.1 \mathrm{M})$ at $50{ }^{\circ} \mathrm{C}$. hexane:EA $=4: 1,184.0 \mathrm{mg}, 0.75 \mathrm{mmol}, 50 \%$ yield, brown solid; ${ }^{1} \mathrm{H}$ NMR (400 MHz, $\left.\mathrm{CDCl}_{3}\right) \delta=13.27$ (s, $\left.1 \mathrm{H}\right), 7.58$ (d, $J=8.0$, $1 \mathrm{H}), 7.39$ (d, $J=7.9,1 \mathrm{H}), 7.34$ (d, $J=8.2,2 \mathrm{H}), 7.29$ (s, 1H), 7.24 (d, $J=8.7,2 \mathrm{H})$, 7.15 (dt, J=15.3, 7.4, 2H), 6.90 (s, 1H), 4.99 (s, 1H), 3.81 (s, 3H), 3.81 (s, 3H), 3.78 (s, 3H), 3.67 (s, $3 \mathrm{H}), 2.21(\mathrm{~s}, 3 \mathrm{H}), 1.91$ (s, 3H); ${ }^{13} \mathrm{C}$ NMR $\left(100 \mathrm{MHz}, \mathrm{CDCl}_{3}\right) \delta=202.2,175.6,173.9,169.7,136.9$, 136.6, 129.0, 128.7, 128.4, 127.0, 122.1, 121.6, 119.8, 119.6, 119.3, 118.8, 109.6, 109.3, 108.3, 105.7, 
95.0, 57.1, 52.5, 51.8, 32.9, 32.8, 28.2, 20.1; HRMS (DART) m/z: $[\mathrm{M}+\mathrm{H}]^{+}$Calcd. For $\mathrm{C}_{14} \mathrm{H}_{15} \mathrm{NO}_{3}$ 246.1125; Found 246.1138

\section{3-(1-Methyl-1H-indol-3-yl)pentane-2,4-dione (1n)}

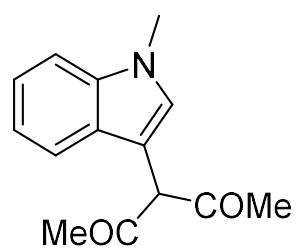

Prepared according to the general procedure (B) using $\mathrm{Cu}(\mathrm{hfacac})_{2}(6 \mathrm{~mol} \%), 3-$ diazopentane-2,4-dione (1.5 equiv), 1-methyl- $1 H$-indole ( $2 \mathrm{mmol}, 1$ equiv) in DCE $(0.1 \mathrm{M})$ at $70{ }^{\circ} \mathrm{C}$. hexane:EA $=19: 1,229.3 \mathrm{mg}, 1 \mathrm{mmol}, 50 \%$ yield, brown $7.28(\mathrm{t}, J=7.7,1 \mathrm{H}), 7.16(\mathrm{t}, J=7.4,1 \mathrm{H}), 6.91(\mathrm{~s}, 1 \mathrm{H}), 3.84(\mathrm{~s}, 3 \mathrm{H}), 1.94(\mathrm{~s}, 6 \mathrm{H}) ;{ }^{13} \mathrm{C}$ NMR $(100 \mathrm{MHz}$, $\left.\mathrm{CDCl}_{3}\right) \delta=192.8,136.9,128.8,128.3,121.9,119.6,119.3,110.3,109.4,105.5,32.8,24.0$; HRMS (DART) m/z: $[\mathrm{M}+\mathrm{H}]^{+}$Calcd. For $\mathrm{C}_{14} \mathrm{H}_{15} \mathrm{NO}_{2} 230.1176$; Found 230.1177

\section{Dimethyl (1-(1-methyl-1H-indol-3-yl)-2-oxopropyl)phosphonate (10)}

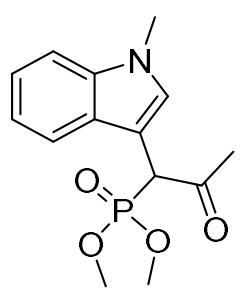

Prepared according to the general procedure (B) using $\mathrm{Cu}(\mathrm{acac})_{2}$ (2 mol \%), dimethyl (1-diazo-2-oxopropyl)phosphonate (3.2 equiv), 1-methyl-1 $H$-indole $(0.5 \mathrm{mmol}, 1$ equiv) in DCE $(0.1 \mathrm{M})$ at $80{ }^{\circ} \mathrm{C}, \mathrm{CH}_{2} \mathrm{Cl}_{2}: \mathrm{EA}=4: 1,35 \mathrm{mg}, 0.12 \mathrm{mmol}, 24 \%$ yield, brown gum; ${ }^{1} \mathrm{H}$ NMR $\left(400 \mathrm{MHz}, \mathrm{CDCl}_{3}\right) \delta=7.56-7.54(\mathrm{~m}, 1 \mathrm{H}), 7.44(\mathrm{~d}, J=2.6$ $\mathrm{Hz}, 1 \mathrm{H}), 7.32(\mathrm{~d}, J=8.2 \mathrm{~Hz}, 1 \mathrm{H}), 7.28-7.22(\mathrm{~m}, 1 \mathrm{H}), 7.15$ (ddd, $J=8.0,7.0,1.1$ $\mathrm{Hz}, 1 \mathrm{H}), 4.70$ (d, $J=23.9 \mathrm{~Hz}, 1 \mathrm{H}), 3.80$ (s, 3H), 3.76 (d, $J=11.0 \mathrm{~Hz}, 3 \mathrm{H}), 3.64$ (d, $J=10.9 \mathrm{~Hz}, 3 \mathrm{H})$, $2.29(\mathrm{~s}, 3 \mathrm{H}) ;{ }^{13} \mathrm{C} \mathrm{NMR}\left(100 \mathrm{MHz}, \mathrm{CDCl}_{3}\right) \delta=202.0-201.9(\mathrm{~m}), 136.6,129.1$ (d, J = 5.9 Hz), $127.3(\mathrm{~d}$, $\mathrm{J}=8.7 \mathrm{~Hz}), 122.1,119.7,118.2,109.5,103.1(\mathrm{~d}, \mathrm{~J}=8.5 \mathrm{~Hz}), 53.8(\mathrm{~d}, \mathrm{~J}=6.8 \mathrm{~Hz}), 53.3$ (d, J = 7.1 Hz), 51.0, 49.6, 33.0, 29.6 (d, J = 2.6 Hz); HRMS (DART) m/z: $[\mathrm{M}+\mathrm{H}]^{+}$Calcd. For $\mathrm{C}_{14} \mathrm{H}_{18} \mathrm{NO}_{4} \mathrm{P} 296.1046$; Found 296.1046

\section{Methyl 2-(1-methyl-1H-indol-2-yl)-3-oxobutanoate (1p)}

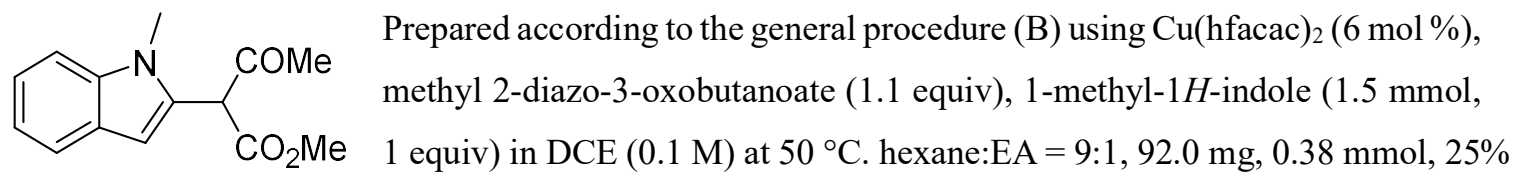
yield, white solid; ${ }^{1} \mathrm{H}$ NMR (400 MHz, $\left.\mathrm{CDCl}_{3}\right) \delta=7.79(\mathrm{dd}, J=7.9,1.4 \mathrm{~Hz}, 1 \mathrm{H}), 7.40(\mathrm{~m}, 2 \mathrm{H}), 7.21$ (m, 1H), $7.16(\mathrm{dd}, J=8.2,1.7 \mathrm{~Hz}, 2 \mathrm{H}), 6.99(\mathrm{~m}, 2 \mathrm{H}), 6.66$ (ddd, $J=7.9,7.1,1.7 \mathrm{~Hz}, 1 \mathrm{H}), 5.84(\mathrm{bs}, 1 \mathrm{H})$; ${ }^{13} \mathrm{C}$ NMR $\left(100 \mathrm{MHz}, \mathrm{CDCl}_{3}\right) \delta=178.1,172.7,137.1,133.6,127.7,121.4,120.5,119.4,109.4,103.3$, 94.8, 52.0, 29.8, 20.1; HRMS (DART) m/z: $[\mathrm{M}+\mathrm{H}]^{+}$Calcd. For $\mathrm{C}_{14} \mathrm{H}_{15} \mathrm{NO}_{3} 246.1125$; Found 246.1123 


\section{Dimethyl 2-(1-methyl-4-oxo-4,5,6,7-tetrahydro-1H-indol-2-yl)malonate (1q)}

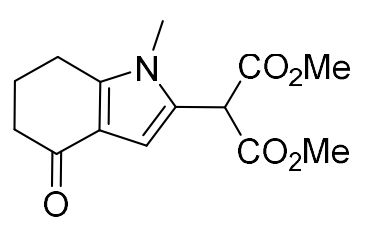

Prepared according to the general procedure (B) using $\mathrm{Rh}_{2}(\mathrm{OAc})_{4}(2 \mathrm{~mol} \%)$, dimethyl 2-diazomalonate (1.2 equiv), 1-methyl-1,5,6,7-tetrahydro- $4 H$ indol-4-one ( $0.2 \mathrm{mmol}, 1$ equiv) in DCE ( $0.2 \mathrm{M})$ at rt, hexane:EA = 3:7, 51.2 mg, $0.18 \mathrm{mmol}$, black solid, 92\% yield ; ${ }^{1} \mathrm{H}$ NMR (400 MHz, $\left.\mathrm{CDCl}_{3}\right) \delta=$ $6.57(\mathrm{~s}, 1 \mathrm{H}), 4.74(\mathrm{~s}, 1 \mathrm{H}), 3.80(\mathrm{~s}, 6 \mathrm{H}), 3.48(\mathrm{~s}, 3 \mathrm{H}), 2.74(\mathrm{t}, J=6.2 \mathrm{~Hz}, 2 \mathrm{H}), 2.49-2.43(\mathrm{~m}, 2 \mathrm{H}), 2.17$ $(\mathrm{p}, J=6.3 \mathrm{~Hz}, 2 \mathrm{H}) ;{ }^{13} \mathrm{C} \mathrm{NMR}\left(100 \mathrm{MHz}, \mathrm{CDCl}_{3}\right) \delta=193.7,167.3,145.2,125.2,120.0,107.1,53.2$, 50.5, 37.6, 31.3, 23.3, 22.0; HRMS (DART) m/z: $[\mathrm{M}+\mathrm{H}]^{+}$Calcd. For $\mathrm{C}_{14} \mathrm{H}_{17} \mathrm{NO}_{5}$ 280.1179; Found 280.1179

\section{Dimethyl 2-(benzofuran-3-yl)malonate (1r)}

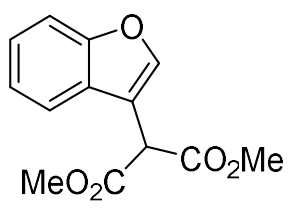

3-Bromobenzofuran (1"r) was prepared according to reported procedure using benzofuran. ${ }^{20}$ The resulting $\mathbf{1}^{\prime \prime} \mathbf{r}$ was obtained in almost quantitative yield and used for the next step without further purification. According to the reported procedure, $\mathbf{1 r}$ was prepared using $\mathbf{1}{ }^{\prime \prime} \mathbf{r}(0.4 \mathrm{mmol}){ }^{21}$ toluene:diethyl ether $=19: 1$, $33.8 \mathrm{mg}, 0.136 \mathrm{mmol}, 0.34 \%$ yield, white solid; ${ }^{1} \mathrm{H}$ NMR (400 MHz, CDCl3) $\delta=7.82(\mathrm{~s}, 1 \mathrm{H}), 7.63-$ $7.60(\mathrm{~m}, 1 \mathrm{H}), 7.49(\mathrm{~d}, \mathrm{~J}=8.0,1 \mathrm{H}), 7.32(\mathrm{td}, \mathrm{J}=8.2,7.8,1.4,1 \mathrm{H}), 7.27(\mathrm{dd}, \mathrm{J}=7.6,1.1,1 \mathrm{H}), 4.86(\mathrm{~s}, 1 \mathrm{H})$, $3.79(\mathrm{~s}, 6 \mathrm{H})$; The ${ }^{13} \mathrm{C}$ NMR data for this compound match the literature data. ${ }^{22}$

\section{Dimethyl 2-(benzo[b]thiophen-3-yl)malonate (1s)}

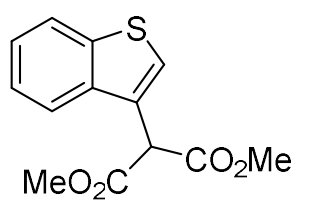

It was prepared according to reported procedure using 3bromobenzo[b]thiophene $(0.2 \mathrm{mmol}){ }^{21}$ hexane:EA $=4: 1,34.3 \mathrm{mg}, 0.13 \mathrm{mmol}$, $57 \%$ yield, yellow solid; ${ }^{1} \mathrm{H}$ NMR (400 MHz, $\left.\mathrm{CDCl}_{3}\right) \delta=7.87$ (dd, J=7.2, 1.3, 1H), 7.77 (dd, J=7.2, 1.4, 1H), $7.66(\mathrm{~s}, 1 \mathrm{H}), 7.44-7.34(\mathrm{~m}, 2 \mathrm{H}), 5.10(\mathrm{~s}, 1 \mathrm{H})$, 3.78 (s, 6H); ${ }^{13} \mathrm{C} \mathrm{NMR}\left(100 \mathrm{MHz}, \mathrm{CDCl}_{3}\right) \delta=167.9,140.0,137.8,126.4,126.3,124.6,124.4,122.9$, 121.5, 53.0, 51.3; HRMS (DART) m/z: $[\mathrm{M}+\mathrm{H}]^{+}$Calcd. For $\mathrm{C}_{13} \mathrm{H}_{12} \mathrm{O}_{4} \mathrm{~S} 265.0529$; Found 265.0529

\section{Dimethyl 2-(1-allyl-1H-indol-3-yl)malonate (1t)}

Prepared according to the general procedure (B) using $\mathrm{Cu}(\mathrm{hfacac})_{2}$ ( 3 mol \%), methyl dimethyl 2- 


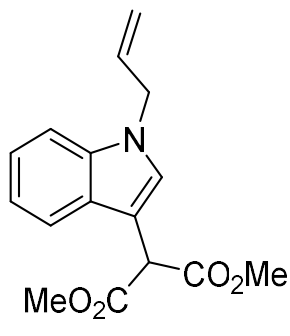

diazomalonate ( 1.5 equiv), 1 -allyl- $1 H$-indole $(0.2 \mathrm{mmol}, 1$ equiv) in DCE $(0.1$ M) at $90{ }^{\circ} \mathrm{C}$. hexane:EA = 4:1, $40.2 \mathrm{mg}, 0.14 \mathrm{mmol}, 70 \%$ yield, brown oil; ${ }^{1} \mathrm{H}$ $\operatorname{NMR}\left(400 \mathrm{MHz}, \mathrm{CDCl}_{3}\right) \delta 7.63(\mathrm{~d}, \mathrm{~J}=7.9,1 \mathrm{H}), 7.31(\mathrm{~d}, \mathrm{~J}=8.4,2 \mathrm{H}), 7.21$ (t, J=7.4, $1 \mathrm{H}), 7.14(\mathrm{t}, \mathrm{J}=7.4,1 \mathrm{H}), 5.99$ (ddt, $\mathrm{J}=15.8,10.6,5.6,1 \mathrm{H}), 5.23-5.19(\mathrm{~m}, 1 \mathrm{H})$, $5.17-5.10(\mathrm{~m}, 1 \mathrm{H}), 4.97(\mathrm{~s}, 1 \mathrm{H}), 4.70(\mathrm{dt}, \mathrm{J}=5.6,1.6,2 \mathrm{H}), 3.75(\mathrm{~s}, 6 \mathrm{H}) ;{ }^{13} \mathrm{C} \mathrm{NMR}$ $\left(100 \mathrm{MHz}, \mathrm{CDCl}_{3}\right) \delta 169.0,136.1,133.1,127.6,127.2,122.0,119.8,119.1,117.7$,

109.8, 106.0, 52.8, 49.2, 49.0; HRMS (DART) m/z: $[\mathrm{M}+\mathrm{H}]^{+}$Calcd. For $\mathrm{C}_{16} \mathrm{H}_{17} \mathrm{NO}_{4}$ 288.1230; Found 288.1227

\section{Dimethyl 2-(1-(but-3-en-1-yl)-1H-indol-3-yl)malonate (1u)}

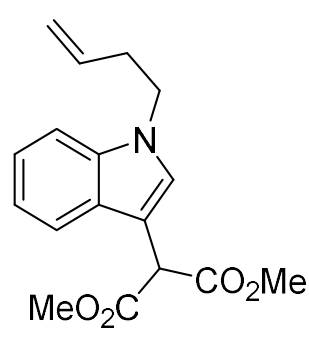

Prepared according to the general procedure (B) using $\mathrm{Cu}(\mathrm{hfacac})_{2}(3 \mathrm{~mol} \%)$, methyl dimethyl 2 - diazomalonate (2 equiv), 1-(but-3-en-1-yl)-1 $H$-indole ( 0.5 mmol, 1 equiv $)$ in DCE $(0.1 \mathrm{M})$ at $90{ }^{\circ} \mathrm{C}$. hexane:toluene $=1: 1,57.3 \mathrm{mg}, 0.19$ mmol, 38\% yield, brown oil; ${ }^{1} \mathrm{H}$ NMR (400 MHz, $\left.\mathrm{CDCl}_{3}\right) \delta=7.63(\mathrm{~d}, J=8.7$ $\mathrm{Hz}, 1 \mathrm{H}), 7.34$ (d, $J=7.5 \mathrm{~Hz}, 2 \mathrm{H}), 7.23$ (t, $J=7.6 \mathrm{~Hz}, 1 \mathrm{H}), 7.19-7.10$ (m, $1 \mathrm{H}), 5.87-5.72(\mathrm{~m}, 1 \mathrm{H}), 5.14-5.03(\mathrm{~m}, 2 \mathrm{H}), 4.97(\mathrm{~s}, 1 \mathrm{H}), 4.21-4.12(\mathrm{~m}$, 2H), 3.76 (s, 6H), $2.65-2.53(\mathrm{~m}, 2 \mathrm{H}) ;{ }^{13} \mathrm{C} \mathrm{NMR}\left(100 \mathrm{MHz}, \mathrm{CDCl}_{3}\right) \delta=169.0,135.9,134.4,127.5$, 127.1, 121.9, 119.6, 119.1, 117.5, 109.6, 105.7, 52.7, 49.2, 46.1, 34.5; HRMS (DART) m/z: $[\mathrm{M}+\mathrm{H}]^{+}$ Calcd. For $\mathrm{C}_{17} \mathrm{H}_{19} \mathrm{NO}_{4}$ 302.1387; Found 302.1386 


\subsection{Preparation and characterization of enamine substrates}

General procedure $(C)$

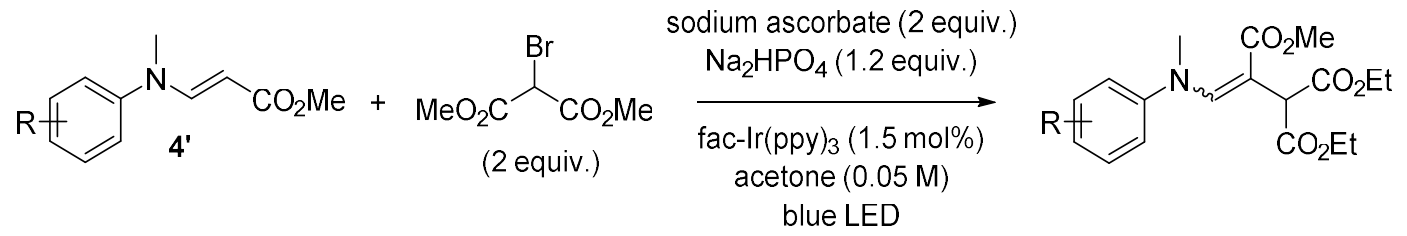

To an oven-dried $4 \mathrm{~mL}$ vial equipped with a stir bar was added $f a c-\operatorname{Ir}(\mathrm{ppy})_{3}(1.5 \mathrm{~mol} \%)$, sodium ascorbate ( 2 equiv), anhydrous sodium phosphate dibasic (powder) (1.2 equiv) and $4^{\prime 23}$ (1 equiv). Anhydrous degassed acetone $(0.05 \mathrm{M})$ and diethyl 2-bromomalonate (2 equiv) were added under nitrogen atmosphere. The solution was irradiated by a $12 \mathrm{~W}$ blue LED lamp $\left(5 \mathrm{~cm}\right.$ away, $25{ }^{\circ} \mathrm{C}$ maintained with a cooling fan). After the completion of the reaction, the solution was filtered on celite and concentrated under reduced pressure. The residue was purified by flash chromatograph.

\section{1,1-Diethyl 2-methyl 3-(methyl(phenyl)amino)prop-2-ene-1,1,2-tricarboxylate (4a)}<smiles></smiles>

Prepared according to the general procedure (C) using $\mathbf{4}^{\prime} \mathbf{a}(1 \mathrm{mmol})$. hexane:acetone $=4: 1,199.1 \mathrm{mg}, 0.57 \mathrm{mmol}, 57 \%$ yield, yellow oil; ${ }^{1} \mathrm{H}$ $\operatorname{NMR}\left(400 \mathrm{MHz}, \mathrm{CDCl}_{3}\right) \delta=7.76(\mathrm{~s}, 1 \mathrm{H}), 7.37-7.32(\mathrm{~m}, 2 \mathrm{H}), 7.21-7.16$ (m, 1H), $7.14-7.11(\mathrm{~m}, 2 \mathrm{H}), 4.18-4.10(\mathrm{~m}, 4 \mathrm{H}), 4.09$ (d, J = 0.6 Hz, 1H), $3.70(\mathrm{~s}, 3 \mathrm{H}), 3.39(\mathrm{~s}, 3 \mathrm{H})$, $1.22(\mathrm{t}, \mathrm{J}=7.1 \mathrm{~Hz}, 6 \mathrm{H}) ;{ }^{13} \mathrm{C} \mathrm{NMR}\left(100 \mathrm{MHz}, \mathrm{CDCl}_{3}\right) \delta=168.9,168.6,148.3,146.1,129.7,125.6$, 123.0, 97.9, 61.3, 51.4, 49.4, 43.8, 14.0; HRMS (DART) m/z: $[\mathrm{M}+\mathrm{H}]^{+}$Calcd. for $\mathrm{C}_{18} \mathrm{H}_{24} \mathrm{NO}_{6} 350.1598$; Found 350.1599

\section{1,1-Diethyl 2-methyl 3-(methyl(m-tolyl)amino)prop-2-ene-1,1,2-tricarboxylate (4b)}

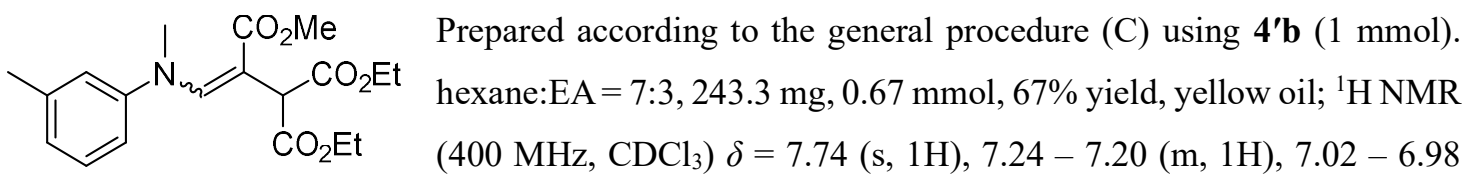
(m, 1H), $6.94-6.90(\mathrm{~m}, 2 \mathrm{H}), 4.17-4.07(\mathrm{~m}, 4 \mathrm{H}), 4.06(\mathrm{~s}, 1 \mathrm{H}), 3.69(\mathrm{~s}, 3 \mathrm{H}), 3.36(\mathrm{~s}, 3 \mathrm{H}), 2.33(\mathrm{~s}, 3 \mathrm{H})$, $1.21(\mathrm{t}, \mathrm{J}=7.1 \mathrm{~Hz}, 6 \mathrm{H}) ;{ }^{13} \mathrm{C}$ NMR $(100 \mathrm{MHz}, \mathrm{CDCl} 3) \delta=169.0,168.6,148.4,145.9,139.8,129.5$, 126.6, 123.9, 120.2, 97.4, 61.2, 51.3, 49.3, 44.1, 21.4, 14.0; HRMS (DART) m/z: [M+H] ${ }^{+}$Calcd. for $\mathrm{C}_{19} \mathrm{H}_{26} \mathrm{NO}_{6} 364.1755$; Found 364.1754 
1,1-Diethyl 2-methyl 3-(methyl(p-tolyl)amino)prop-2-ene-1,1,2-tricarboxylate (4c)

Prepared according to the general procedure (C) using 4'c (1 mmol).
hexane:EA = 7:3,178.0 mg, 0.49 mmol, $49 \%$ yield, yellow oil; ${ }^{1} \mathrm{H}$

$7.02(\mathrm{~d}, \mathrm{~J}=8.4 \mathrm{~Hz}, 2 \mathrm{H}), 4.16-4.06(\mathrm{~m}, 4 \mathrm{H}), 4.01(\mathrm{~s}, 1 \mathrm{H}), 3.69(\mathrm{~s}, 3 \mathrm{H}), 3.36(\mathrm{~s}, 3 \mathrm{H}), 2.34(\mathrm{~s}, 3 \mathrm{H}), 1.21$

$(\mathrm{t}, \mathrm{J}=7.1 \mathrm{~Hz}, 6 \mathrm{H}) ;{ }^{13} \mathrm{C} \mathrm{NMR}\left(100 \mathrm{MHz}, \mathrm{CDCl}_{3}\right) \delta=169.1,168.7,148.6,143.3,135.9,130.3,123.6$, 96.4, 61.2, 51.3, 49.1, 44.7, 20.9, 14.0; HRMS (DART) m/z: $[\mathrm{M}+\mathrm{H}]^{+}$Calcd. for $\mathrm{C}_{19} \mathrm{H}_{26} \mathrm{NO}_{6} 364.1755$;

Found 364.1753

1,1-Diethyl 2-methyl 3-((4-methoxyphenyl)(methyl)amino)prop-2-ene-1,1,2-tricarboxylate (4d)

Prepared according to the general procedure (C) using 4'd (2 mmol).
hexane:EA=1:1, $555 \mathrm{mg}, 1.46 \mathrm{mmol}, 73 \%$ yield, yellow oil; ${ }^{1} \mathrm{H}$ $6.86(\mathrm{~d}, \mathrm{~J}=8.9 \mathrm{~Hz}, 2 \mathrm{H}), 4.10$ (qq, J = 10.7, $7.1 \mathrm{~Hz}, 4 \mathrm{H}), 3.93$ (s, 1H), 3.80 (s, 3H), 3.68 (s, 3H), 3.34 $(\mathrm{s}, 3 \mathrm{H}), 1.20(\mathrm{t}, \mathrm{J}=7.1 \mathrm{~Hz}, 6 \mathrm{H}) ;{ }^{13} \mathrm{C} \mathrm{NMR}\left(100 \mathrm{MHz}, \mathrm{CDCl}_{3}\right) \delta=169.2,168.7,158.0,149.0,138.5$, 125.9, 115.0, 95.3, 61.1, 55.4, 51.2, 48.7, 45.8, 14.0; HRMS (DART) m/z: $[\mathrm{M}+\mathrm{H}]^{+}$Calcd. for $\mathrm{C}_{19} \mathrm{H}_{26} \mathrm{NO}_{7} 380.1704$; Found 380.1701

\section{1,1-Diethyl 2-methyl 3-((4-bromophenyl)(methyl)amino)prop-2-ene-1,1,2-tricarboxylate (4e)}

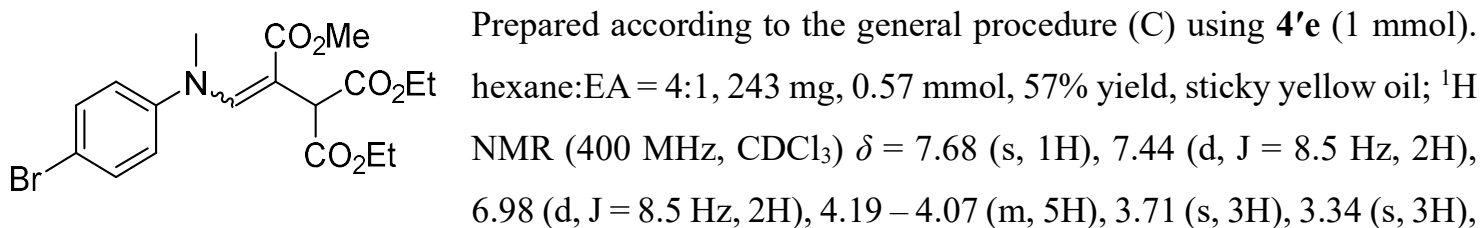
$1.22\left(\mathrm{t}, \mathrm{J}=7.1 \mathrm{~Hz}, 6 \mathrm{H} ;{ }^{13} \mathrm{C} \mathrm{NMR}\left(100 \mathrm{MHz}, \mathrm{CDCl}_{3}\right) \delta=168.6,168.3,147.8,145.2,132.7,124.0,118.2\right.$, 100.0, 61.5, 51.5, 49.7, 43.4, 14.0; HRMS (DART) m/z: $[\mathrm{M}+\mathrm{H}]^{+}$Calcd. for $\mathrm{C}_{18} \mathrm{H}_{23} \mathrm{BrNO}_{6} 428.0703$; Found 428.0700

\section{1,1-Diethyl 2-methyl 3-((4-(methoxycarbonyl)phenyl)(methyl)amino)prop-2-ene-1,1,2- tricarboxylate (4f)}


<smiles></smiles>

Prepared according to the general procedure (C) using $\mathbf{4}^{\prime} \mathbf{f}$ ( $1 \mathrm{mmol}$, 1 equiv). hexane:acetone $=4: 1,244.5 \mathrm{mg}, 0.6 \mathrm{mmol}, 60 \%$ yield, yellow oil; ${ }^{1} \mathrm{H} \mathrm{NMR}\left(400 \mathrm{MHz}, \mathrm{CDCl}_{3}\right) \delta=8.00(\mathrm{~d}, \mathrm{~J}=8.7 \mathrm{~Hz}, 2 \mathrm{H})$, $7.76(\mathrm{~s}, 1 \mathrm{H}), 7.07$ (d, J = 8.7 Hz, 2H), 4.33 (s, 1H), 4.16 (dddd, $\mathrm{J}=$ 17.9, 10.8, 7.1, $3.7 \mathrm{~Hz}, 4 \mathrm{H}), 3.91(\mathrm{~s}, 3 \mathrm{H}), 3.76(\mathrm{~s}, 3 \mathrm{H}), 3.40(\mathrm{~s}, 3 \mathrm{H}), 1.22(\mathrm{t}, \mathrm{J}=7.1 \mathrm{~Hz}, 6 \mathrm{H}) ;{ }^{13} \mathrm{C} \mathrm{NMR}$ $\left(100 \mathrm{MHz}, \mathrm{CDCl}_{3}\right) \delta=168.1,168.1,166.4,149.9,146.8,131.1,124.9,105.2,61.6,52.0,51.8,50.3$, 41.3, 13.9; HRMS (DART) m/z: [M+H] ${ }^{+}$Calcd. for $\mathrm{C}_{20} \mathrm{H}_{26} \mathrm{NO}_{8} 408.1653$; Found 408.1654

\section{1,1-Diethyl 2-methyl 3-(methyl(4-(trifluoromethyl)phenyl)amino)prop-2-ene-1,1,2-tricarboxylate} $(4 \mathrm{~g})$

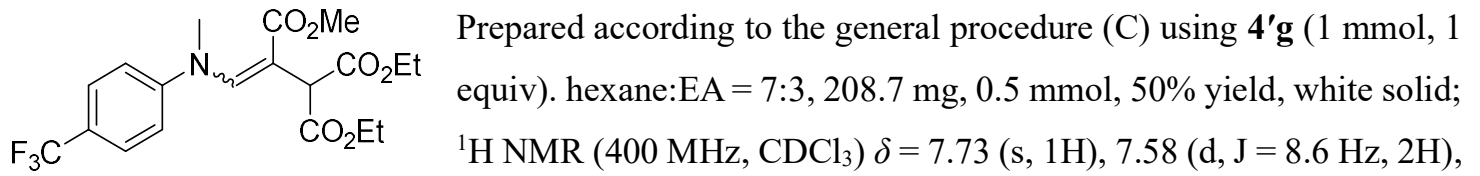
$7.13(\mathrm{~d}, \mathrm{~J}=8.5 \mathrm{~Hz}, 2 \mathrm{H}), 4.28$ (s, 1H), $4.23-4.09$ (m, 4H), 3.75 (s, 3H), 3.39 (s, 3H), 1.23 (t, J = 7.1 $\mathrm{Hz}, 6 \mathrm{H}) ;{ }^{13} \mathrm{C} \mathrm{NMR}\left(100 \mathrm{MHz}, \mathrm{CDCl}_{3}\right) \delta=168.1,168.0,149.1,146.9,128.1,126.8,126.7,126.7,126.6$, $126.1,125.8,125.5,125.4,125.1,122.7,120.0,119.9$, 104.6, 61.6, 51.7, 50.2, 41.7, 13.9; HRMS (DART) m/z: $[\mathrm{M}+\mathrm{H}]^{+}$Calcd. for $\mathrm{C}_{19} \mathrm{H}_{23} \mathrm{~F}_{3} \mathrm{NO}_{6} 418.1472$; Found 418.1471

\section{General Procedure (D) and characterization of pyrans}

To a suspension of carbonyl compound $2(0.11 \mathrm{mmol}, 1.1$ equiv $)$ and $\mathrm{K}_{3} \mathrm{PO}_{4}(0.5 \mathrm{mmol}, 5.0$ equiv $)$ in anhydrous acetonitrile $(1 \mathrm{~mL}, 0.1 \mathrm{M})$ at room temperature was added indole substrate $\mathbf{1}^{19}(0.1 \mathrm{mmol}$, 1.0 equiv) dropwise under a nitrogen atmosphere. $I_{2}(0.22 \mathrm{mmol}, 2.2$ equiv) was added under nitrogen and the reaction was stirred at room temperature. The reaction mixture was monitored by TLC until indole was consumed, opened to air, quenched with 2-3 drops of $1 \mathrm{~N} \mathrm{Na}_{2} \mathrm{~S}_{2} \mathrm{O}_{3}$ solution, diluted with ethyl acetate, and filtered over a pad of celite. The pad was rinsed with an additional ethyl acetate, and the combined solutions were concentrated in vacuo. The crude material was purified by column chromatography to give the product.

(1 was prepared according to reported procedure.)

\section{Dimethyl 4-acetyl-3,5-dimethylpyrano[4,3-b]indole-1,1(5H)-dicarboxylate (3aa)}




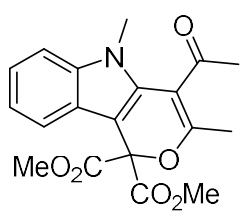

Prepared according to the general procedure (D). hexane:EA = 7:3, $32.8 \mathrm{mg}, 0.092$ mmol, 92\% yield, yellow solid, m.p. $105-110{ }^{\circ} \mathrm{C} ;{ }^{1} \mathrm{H}$ NMR $\left(400 \mathrm{MHz}, \mathrm{CDCl}_{3}\right) \delta=$ $7.46(\mathrm{~d}, J=7.9 \mathrm{~Hz}, 1 \mathrm{H}), 7.29$ (d, $J=8.1 \mathrm{~Hz}, 1 \mathrm{H}), 7.25-7.20(\mathrm{~m}, 1 \mathrm{H}), 7.18-7.13$ (m, 1H), $3.84(\mathrm{~s}, 6 \mathrm{H}), 3.57$ (s, 3H), $2.42(\mathrm{~s}, 3 \mathrm{H}), 2.28(\mathrm{~s}, 3 \mathrm{H}) ;{ }^{13} \mathrm{C} \mathrm{NMR}(100 \mathrm{MHz}$, $\left.\mathrm{CDCl}_{3}\right) \delta=198.5,167.3,157.2,139.0,131.1,123.8,122.1,121.1,119.0,111.3,109.8,99.9,84.8,53.4$, 32.1, 31.7, 18.2; IR (film): 2955, 1748, 1683, 1589, 1273, 1225, 1201, 744, $743 \mathrm{~cm}^{-1}$; HRMS (DART) $\mathrm{m} / \mathrm{z}:[\mathrm{M}+\mathrm{Na}]^{+} \mathrm{Calcd}$. for $\mathrm{C}_{19} \mathrm{H}_{19} \mathrm{NNaO}_{6} 380.1105$; Found 380.1105

\section{Dimethyl 4-acetyl-8-methoxy-3,5-dimethylpyrano[4,3-b]indole-1,1(5H)-dicarboxylate (3ba)}

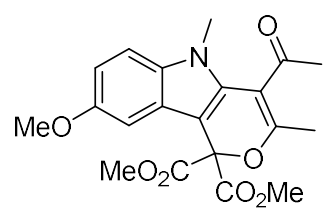

Prepared according to the general procedure (D). hexane:EA $=7: 3,28.6 \mathrm{mg}$, $0.074 \mathrm{mmol}, 74 \%$ yield, yellow solid, m.p. $100-105^{\circ} \mathrm{C} ;{ }^{1} \mathrm{H}$ NMR $(400 \mathrm{MHz}$, $\left.\mathrm{CDCl}_{3}\right) \delta=8.42(\mathrm{dd}, J=2.3,0.5 \mathrm{~Hz}, 1 \mathrm{H}), 8.11(\mathrm{ddd}, J=9.1,2.2,0.7 \mathrm{~Hz}, 1 \mathrm{H})$,

$7.32(\mathrm{dd}, J=9.1,0.5 \mathrm{~Hz}, 1 \mathrm{H}), 3.88(\mathrm{~s}, 6 \mathrm{H}), 3.62(\mathrm{~s}, 3 \mathrm{H}), 2.47(\mathrm{~s}, 3 \mathrm{H}), 2.31(\mathrm{~s}$, $3 \mathrm{H}) ;{ }^{13} \mathrm{C} \mathrm{NMR}\left(100 \mathrm{MHz}, \mathrm{CDCl}_{3}\right) \delta=198.6,167.4,156.9,155.0,134.4,131.6,124.3,111.8,111.4$, 110.4, 101.3, 99.5, 84.7, 55.9, 53.4, 32.2, 31.7, 18.1; IR (film): 2955, 1747, 1680, 1607, 1256, 1233, 1190, 828, 791, $754 \mathrm{~cm}^{-1}$; HRMS (DART) m/z: [M+Na $]^{+}$Calcd. for $\mathrm{C}_{20} \mathrm{H}_{21} \mathrm{NNaO}_{7}$ 410.1210; Found 410.1210

\section{Dimethyl 4-acetyl-8-((tert-butoxycarbonyl)amino)-3,5-dimethylpyrano[4,3-b]indole-1,1(5H)- dicarboxylate (3ca)}

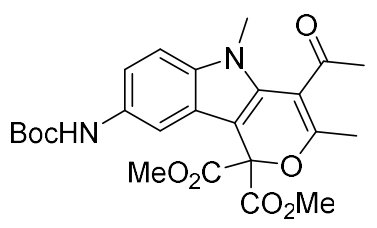

Prepared according to the general procedure (D). hexane:EA $=7: 3,43 \mathrm{mg}$, $0.091 \mathrm{mmol}, 91 \%$ yield, yellow solid, m.p. $190-195^{\circ} \mathrm{C}$; ${ }^{1} \mathrm{H}$ NMR $(400 \mathrm{MHz}$, $\left.\mathrm{CDCl}_{3}\right) \delta=7.34-7.28(\mathrm{~m}, 2 \mathrm{H}), 7.17(\mathrm{~d}, J=8.8 \mathrm{~Hz}, 1 \mathrm{H}), 6.53(\mathrm{~s}, 1 \mathrm{H}), 3.85$ $(\mathrm{s}, 6 \mathrm{H}), 3.52(\mathrm{~s}, 3 \mathrm{H}), 2.41(\mathrm{~s}, 3 \mathrm{H}), 2.26(\mathrm{~s}, 3 \mathrm{H}) ;{ }^{13} \mathrm{C} \mathrm{NMR}\left(100 \mathrm{MHz}, \mathrm{CDCl}_{3}\right)$ $\delta=198.6,167.4,156.9,155.0,134.4,131.6,124.3,111.8,111.4,110.4,101.3,99.5,84.7,55.9,53.4$, 32.2, 31.7, 18.1; IR (film): 3376, 2960, 1740, 1672, 1582, 1455, 1265, 1232, 1161, 805, $770 \mathrm{~cm}^{-1}$; HRMS (DART) m/z: [M+Na $]^{+}$Calcd. for $\mathrm{C}_{24} \mathrm{H}_{28} \mathrm{~N}_{2} \mathrm{NaO}_{8} 495.1738$; Found 495.1739

\section{Dimethyl 4-acetyl-3,5-dimethyl-8-nitropyrano[4,3-b] indole-1,1(5H)-dicarboxylate (3da)}

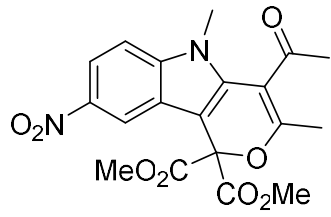

Prepared according to the general procedure (D). toluene:diethyl ether $=9: 1$, $27.3 \mathrm{mg}, 0.068 \mathrm{mmol}, 68 \%$ yield, yellow solid, m.p. $190-195{ }^{\circ} \mathrm{C} ;{ }^{1} \mathrm{H}$ NMR $\left(400 \mathrm{MHz}, \mathrm{CDCl}_{3}\right) \delta=7.17(\mathrm{dd}, J=8.9,0.6 \mathrm{~Hz}, 1 \mathrm{H}), 6.91(\mathrm{~d}, J=2.3 \mathrm{~Hz}, 1 \mathrm{H})$, 
$6.87(\mathrm{dd}, J=8.8,2.5 \mathrm{~Hz}, 1 \mathrm{H}), 3.85(\mathrm{~s}, 6 \mathrm{H}), 3.84(\mathrm{~s}, 3 \mathrm{H}), 3.54(\mathrm{~s}, 3 \mathrm{H}), 2.42(\mathrm{~s}, 3 \mathrm{H}), 2.26(\mathrm{~s}, 3 \mathrm{H}) ;{ }^{13} \mathrm{C}$ $\operatorname{NMR}\left(100 \mathrm{MHz}, \mathrm{CDCl}_{3}\right) \delta=198.6,167.4,156.9,155.0,134.4,131.6,124.3,111.8,111.4,110.4,101.3$, 99.5, 84.7, 55.9, 53.4, 32.2, 31.7, 18.1; IR (film): 2961, 1747, 1679, 1600, 1514, 1337, 1254, 1200812 , $750,735 \mathrm{~cm}^{-1}$; HRMS (DART) m/z: [M+Na] $]^{+}$Calcd. for $\mathrm{C}_{19} \mathrm{H}_{18} \mathrm{~N}_{2} \mathrm{NaO}_{8} 425.0955$; Found 425.0955

\section{Dimethyl 4-acetyl-8-cyano-3,5-dimethylpyrano[4,3-b]indole-1,1(5H)-dicarboxylate (3ea)}

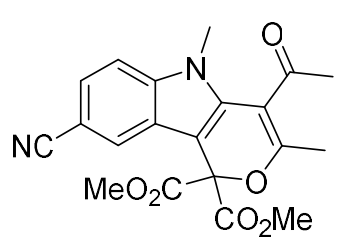

Prepared according to the general procedure (D). hexane:EA $=7: 3,27.1 \mathrm{mg}$, $0.071 \mathrm{mmol}, 71 \%$ yield, white solid, m.p. $160-165{ }^{\circ} \mathrm{C}$; ${ }^{1} \mathrm{H}$ NMR $(400 \mathrm{MHz}$, $\left.\mathrm{CDCl}_{3}\right) \delta=7.83(\mathrm{~d}, J=1.5 \mathrm{~Hz}, 1 \mathrm{H}), 7.46-7.43(\mathrm{~m}, 1 \mathrm{H}), 7.34(\mathrm{dd}, J=8.6$, $0.7 \mathrm{~Hz}, 1 \mathrm{H}), 3.87$ (s, 6H), 3.59 (s, 3H), 2.46 (s, 3H), 2.30 (s, 3H); ${ }^{13} \mathrm{C}$ NMR $\left(100 \mathrm{MHz}, \mathrm{CDCl}_{3}\right) \delta=198.1,166.6,158.6,140.4,133.5,124.8,124.8,123.7,120.4,110.7,110.6,104.3$, 100.1, 84.3, 53.7, 32.5, 32.0, 18.5; IR (film): 2962, 2220, 1744, 1700, 1619, 1272, 1212, 1142, 850, $779,760 \mathrm{~cm}^{-1}$; HRMS (DART) m/z: [M+Na] $]^{+}$Calcd. for $\mathrm{C}_{20} \mathrm{H}_{18} \mathrm{~N}_{2} \mathrm{NaO}_{6} 405.1057$; Found 405.1057

\section{Trimethyl 4-acetyl-3,5-dimethylpyrano[4,3-b]indole-1,1,8(5H)-tricarboxylate (3fa)}

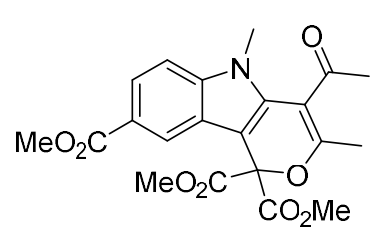

Prepared according to the general procedure (D). hexane:EA $=3: 2,29 \mathrm{mg}$, 0.07 mmol, 70\% yield, yellow solid, m.p. $190-195{ }^{\circ} \mathrm{C} ;{ }^{1} \mathrm{H}$ NMR $(400 \mathrm{MHz}$, DMSO- $\left.d_{6}\right) \delta=8.01(\mathrm{~d}, J=1.6 \mathrm{~Hz}, 1 \mathrm{H}), 7.80(\mathrm{dd}, J=8.7,1.7 \mathrm{~Hz}, 1 \mathrm{H}), 7.59$ (d, $J=8.7 \mathrm{~Hz}, 1 \mathrm{H}), 3.86(\mathrm{~s}, 3 \mathrm{H}), 3.81(\mathrm{~s}, 6 \mathrm{H}), 3.58(\mathrm{~s}, 3 \mathrm{H}), 2.49$ (s, 3H), $2.21(\mathrm{~s}, 3 \mathrm{H}) ;{ }^{13} \mathrm{C}$ NMR $\left(100 \mathrm{MHz}, \mathrm{DMSO}-d_{6}\right) \delta=199.1,167.3,167.2,156.9,141.2,132.9,123.1,122.9$, 122.6, 121.0, 111.6, 111.1, 100.4, 84.4, 54.1, 52.4, 32.8, 32.6, 18.6; IR (film): 2954, 1742, 1667, 1593, 1264, 1151, 810, 764, $743 \mathrm{~cm}^{-1}$; HRMS (DART) m/z: $[\mathrm{M}+\mathrm{Na}]^{+}$Calcd. for $\mathrm{C}_{21} \mathrm{H}_{21} \mathrm{NNaO}_{8} 438.1159$; Found 438.1159

\section{Trimethyl 4-acetyl-3,5-dimethylpyrano[4,3-b]indole-1,1,9(5H)-tricarboxylate (3ga)}

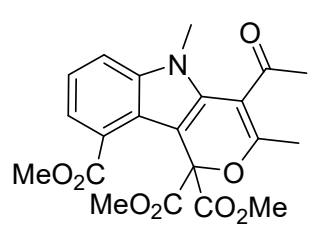

Prepared according to the general procedure (D). hexane:EA $=7: 3,41.1 \mathrm{mg}$, 0.099 mmol, $99 \%$ yield, yellow solid, m.p. $180-185{ }^{\circ} \mathrm{C}$; ${ }^{1} \mathrm{H}$ NMR $(400 \mathrm{MHz}$, $\left.\mathrm{CDCl}_{3}\right) \delta=7.85(\mathrm{dd}, J=7.5,1.1 \mathrm{~Hz}, 1 \mathrm{H}), 7.49(\mathrm{dd}, J=8.3,1.1 \mathrm{~Hz}, 1 \mathrm{H}), 7.25-$ $7.21(\mathrm{~m}, 1 \mathrm{H}), 3.82(\mathrm{~s}, 3 \mathrm{H}), 3.81(\mathrm{~s}, 6 \mathrm{H}), 3.59(\mathrm{~s}, 3 \mathrm{H}), 2.37(\mathrm{~s}, 3 \mathrm{H}), 2.31(\mathrm{~s}, 3 \mathrm{H})$;

${ }^{13} \mathrm{C}$ NMR $\left(100 \mathrm{MHz}, \mathrm{CDCl}_{3}\right) \delta=197.3,168.2,167.1,161.1,140.1,134.6,124.9,122.6,122.2,121.0$, 114.3, 111.7, 102.4, 86.5, 53.4, 51.9, 32.4, 31.4, 18.3; IR (film): 2953, 1741, 1680, 1578, 1283, 1263 , 1209, 1189, 793, 750, $739 \mathrm{~cm}^{-1}$; HRMS (DART) m/z: [M+Na ${ }^{+}$Calcd. for $\mathrm{C}_{21} \mathrm{H}_{21} \mathrm{NNaO}_{8} 438.1159$; 


\section{Dimethyl 4-acetyl-8-bromo-3,5-dimethylpyrano[4,3-b]indole-1,1(5H)-dicarboxylate (3ha)}

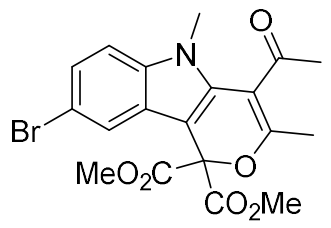

Prepared according to the general procedure (D). hexane:EA $=4: 1,34 \mathrm{mg}$, $0.078 \mathrm{mmol}, 78 \%$ yield, brown solid, m.p. $110-115{ }^{\circ} \mathrm{C} ;{ }^{1} \mathrm{H}$ NMR $(400 \mathrm{MHz}$, $\left.\mathrm{CDCl}_{3}\right) \delta=7.57(\mathrm{dd}, J=1.9,0.5 \mathrm{~Hz}, 1 \mathrm{H}), 7.28(\mathrm{dd}, J=8.7,1.9 \mathrm{~Hz}, 1 \mathrm{H}), 7.14$ (dd, $J=8.7,0.5 \mathrm{~Hz}, 1 \mathrm{H}), 3.85(\mathrm{~s}, 6 \mathrm{H}), 3.53(\mathrm{~s}, 3 \mathrm{H}), 2.42$ (s, 3H), 2.27 (s, 3H);

${ }^{13} \mathrm{C}$ NMR $\left(100 \mathrm{MHz}, \mathrm{CDCl}_{3}\right) \delta=198.3,167.0,157.9,137.7,132.3,125.3,124.9,121.7,114.5,111.1$, 111.0, 99.2, 84.5, 53.5, 32.3, 31.9, 18.3; IR (film): 2954, 1738, 1693, 1607, 1304, 1196, 1143, 773, 748, $639 \mathrm{~cm}^{-1}$; HRMS (DART) m/z: $[\mathrm{M}+\mathrm{Na}]^{+}$Calcd. for $\mathrm{C}_{19} \mathrm{H}_{18} \mathrm{BrNNaO}_{6} 458.0210$; Found 458.0210

\section{Dimethyl 4-acetyl-3-methylpyrano[4,3-b]indole-1,1(5H)-dicarboxylate (3ia)}

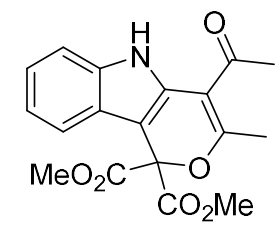

Prepared according to the general procedure (D). hexane:EA =7:3, 26.1 mg, 0.076 mmol, 76\% yield, yellow solid, m.p. $175-180{ }^{\circ} \mathrm{C} ;{ }^{1} \mathrm{H}$ NMR $\left(400 \mathrm{MHz}, \mathrm{CDCl}_{3}\right) \delta$ $=10.17(\mathrm{~s}, 1 \mathrm{H}), 7.49(\mathrm{dq}, J=7.7,0.9 \mathrm{~Hz}, 1 \mathrm{H}), 7.36(\mathrm{dt}, J=8.2,0.9 \mathrm{~Hz}, 1 \mathrm{H}), 7.25$ $-7.07(\mathrm{~m}, 2 \mathrm{H}), 3.83(\mathrm{~s}, 6 \mathrm{H}), 2.57(\mathrm{~s}, 3 \mathrm{H}), 2.52(\mathrm{~s}, 3 \mathrm{H}) ;{ }^{13} \mathrm{C} \mathrm{NMR}(100 \mathrm{MHz}$, $\left.\mathrm{CDCl}_{3}\right) \delta=197.2,167.0,164.7,136.2,129.4,123.6,121.9,120.6,119.3,111.4,110.0,96.6,85.2,53.3$, 32.2, 21.9; IR (film): 3403, 2962, 1751, 1641, 1273, 1201, 1167, 749, $731 \mathrm{~cm}^{-1}$; HRMS (DART) m/z: $[\mathrm{M}+\mathrm{Na}]^{+}$Calcd. for $\mathrm{C}_{18} \mathrm{H}_{17} \mathrm{NNaO}_{6} 366.0948$; Found 366.0948

\section{Dimethyl 4-acetyl-5-benzyl-3-methylpyrano[4,3-b]indole-1,1(5H)-dicarboxylate (3ja)}

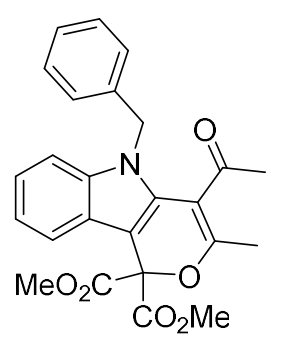

Prepared according to the general procedure (D). toluene:diethyl ether $=19: 1,20.4$ mg, $0.047 \mathrm{mmol}, 47 \%$ yield, yellow solid, m.p. $160-165{ }^{\circ} \mathrm{C}$; ${ }^{1} \mathrm{H}$ NMR $(400 \mathrm{MHz}$, $\left.\mathrm{CDCl}_{3}\right) \delta=7.51-7.48(\mathrm{~m}, 1 \mathrm{H}), 7.25(\mathrm{~d}, J=1.7 \mathrm{~Hz}, 1 \mathrm{H}), 7.24-7.18(\mathrm{~m}, 3 \mathrm{H}), 7.16$ $-7.13(\mathrm{~m}, 2 \mathrm{H}), 6.96-6.92(\mathrm{~m}, 2 \mathrm{H}), 5.21(\mathrm{~s}, 2 \mathrm{H}), 3.86(\mathrm{~s}, 6 \mathrm{H}), 2.21(\mathrm{~s}, 3 \mathrm{H}), 2.08$ (s, 3H); ${ }^{13} \mathrm{C} \mathrm{NMR}\left(100 \mathrm{MHz}, \mathrm{CDCl}_{3}\right) \delta=199.2,167.4,155.9,138.8,136.6,130.6$, $128.8,127.5,126.2,124.1,122.2,121.2,119.2,111.7,110.5,100.6,84.7,53.4,48.4,31.9,18.5$; IR (film): 2952, 1736, 1680, 1597, 1247, 1190, 1167, 795, $740 \mathrm{~cm}^{-1}$; HRMS (DART) m/z: $[\mathrm{M}+\mathrm{H}]^{+} \mathrm{Calcd}$. for $\mathrm{C}_{25} \mathrm{H}_{24} \mathrm{NO}_{6} 434.1598$; Found 434.1598 
Dimethyl 4-acetyl-3-methyl-5-(p-tolyl)pyrano[4,3-b]indole-1,1(5H)-dicarboxylate (3ka)

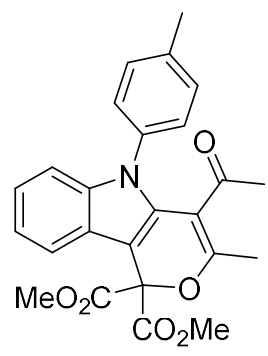

Prepared according to the general procedure (D). hexane:EA $=4: 1,22.5 \mathrm{mg}, 0.052$ mmol, 52\% yield, orange solid, m.p. $160-165{ }^{\circ} \mathrm{C} ;{ }^{1} \mathrm{H} \mathrm{NMR}\left(400 \mathrm{MHz}, \mathrm{CDCl}_{3}\right) \delta=$ $7.55-7.51(\mathrm{~m}, 1 \mathrm{H}), 7.34-7.27(\mathrm{~m}, 3 \mathrm{H}), 7.23-7.14(\mathrm{~m}, 4 \mathrm{H}), 3.88(\mathrm{~s}, 6 \mathrm{H}), 2.42$ (s, 3H), $2.26(\mathrm{~s}, 3 \mathrm{H}), 1.65(\mathrm{~s}, 3 \mathrm{H}) ;{ }^{13} \mathrm{C} \mathrm{NMR}\left(100 \mathrm{MHz}, \mathrm{CDCl}_{3}\right) \delta=197.9,167.4$, 157.6, 138.7, 138.2, 134.5, 130.5, 130.3, 127.1, 124.0, 122.5, 121.7, 119.1, 111.3, 110.7, 101.5, 84.6, 53.5, 30.8, 21.2, 18.1; IR (film): 2955, 1752, 1686, 1604, 1289,

1211, 1164, 830, 782, $744 \mathrm{~cm}^{-1}$; HRMS (DART) m/z: [M+Na $]^{+}$Calcd. for $\mathrm{C}_{25} \mathrm{H}_{24} \mathrm{NO}_{6} 456.1418$; Found 456.1418

Dimethyl 4,5-diacetyl-3-methylpyrano[4,3-b]indole-1,1(5H)-dicarboxylate (3la)

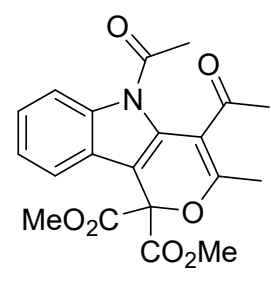

Prepared according to the general procedure (D). hexane:EA $=7: 3,11.6 \mathrm{mg}, 0.03$ mmo, 30\% yield, white solid, m.p. $140-145{ }^{\circ} \mathrm{C} ;{ }^{1} \mathrm{H}$ NMR (400 MHz, $\left.\mathrm{CDCl}_{3}\right) \delta$ $7.74-7.71(\mathrm{~m}, 1 \mathrm{H}), 7.47-7.43(\mathrm{~m}, 1 \mathrm{H}), 7.35-7.27(\mathrm{~m}, 2 \mathrm{H}), 3.85(\mathrm{~s}, 6 \mathrm{H}), 2.73$ (s, 3H), 2.38 (s, 3H), 2.27 (s, 3H); ${ }^{13} \mathrm{C}$ NMR (100 MHz, $\left.\mathrm{CDCl}_{3}\right) \delta$ 195.5, 170.1, $166.7,158.0,136.5,131.4,126.3,124.6,124.0,119.9,114.4,113.3,109.3,83.7$,

53.6, 30.8, 27.0, 18.7; IR (film): 2924, 1746, 1687, 1625, 1290, 1218, 1160, 793, $740 \mathrm{~cm}^{-1}$; HRMS (DART) m/z: [M+Na] $]^{+}$Calcd. for $\mathrm{C}_{20} \mathrm{H}_{19} \mathrm{NNaO}_{7} 408.1054$; Found 408.1054

\section{Methyl 1,4-diacetyl-3,5-dimethyl-1,5-dihydropyrano[4,3-b]indole-1-carboxylate (3ma)}

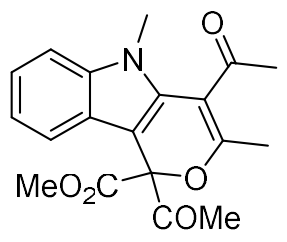

Prepared according to the general procedure (D). hexane:EA $=4: 1,22.9 \mathrm{mg}, 0.067$ mmol, 67\% yield, white solid, m.p. $120-125^{\circ} \mathrm{C} ;{ }^{1} \mathrm{H}$ NMR (400 MHz, $\left.\mathrm{CDCl}_{3}\right) \delta=$ $7.43(\mathrm{dt}, J=7.9,1.1 \mathrm{~Hz}, 1 \mathrm{H}), 7.33-7.29(\mathrm{~m}, 1 \mathrm{H}), 7.26-7.21(\mathrm{~m}, 1 \mathrm{H}), 7.19-$ $7.14(\mathrm{~m}, 1 \mathrm{H}), 3.82(\mathrm{~s}, 3 \mathrm{H}), 3.60(\mathrm{~s}, 3 \mathrm{H}), 2.42(\mathrm{~s}, 3 \mathrm{H}), 2.31(\mathrm{~s}, 3 \mathrm{H}), 2.30(\mathrm{~s}, 3 \mathrm{H})$;

${ }^{13} \mathrm{C}$ NMR $\left(100 \mathrm{MHz}, \mathrm{CDCl}_{3}\right) \delta=199.8,198.5,168.4,158.0,139.0,131.4,123.5,122.2,121.3,118.5$, 111.6, 109.9, 99.7, 89.0, 53.1, 32.1, 31.7, 25.5, 18.2; IR (film): 2954, 1741, 1722, 1659, 1589, 1246, 1198, 1142, 797, $759 \mathrm{~cm}^{-1}$; HRMS (DART) m/z: $[\mathrm{M}+\mathrm{Na}]^{+}$Calcd. for $\mathrm{C}_{19} \mathrm{H}_{19} \mathrm{NNaO}_{5} 364.1155$; Found 364.1155

\section{1,1',1"-(3,5-Dimethyl-1,5-dihydropyrano[4,3-b]indole-1,1,4-triyl)tris(ethan-1-one) (3na)}




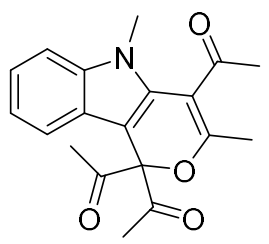

Prepared according to the general procedure (D). hexane:EA = 4:1, $23.4 \mathrm{mg}, 0.072$ mmol, $72 \%$ yield; Yellow oil; ${ }^{1} \mathrm{H}$ NMR $\left(400 \mathrm{MHz}, \mathrm{CDCl}_{3}\right) \delta=7.48$ (ddd, $J=7.9$, 1.3, $0.8 \mathrm{~Hz}, 1 \mathrm{H}), 7.32(\mathrm{dt}, J=8.2,1.0 \mathrm{~Hz}, 1 \mathrm{H}), 7.24(\mathrm{dd}, J=8.2,1.3 \mathrm{~Hz}, 1 \mathrm{H}), 7.18$ (ddd, $J=8.1,7.0,1.2 \mathrm{~Hz}, 1 \mathrm{H}), 3.60(\mathrm{~s}, 3 \mathrm{H}), 2.38(\mathrm{~s}, 3 \mathrm{H}), 2.34$ (s, 3H), 2.32 (s, 6H); ${ }^{13} \mathrm{C} \mathrm{NMR}\left(100 \mathrm{MHz}, \mathrm{CDCl}_{3}\right) \delta=201.9,198.2,159.5,139.1,132.1,123.3,122.2,121.5,118.1,111.5$, 110.0, 99.4, 93.6, 32.1 , 31.6, 26.5, 18.3; IR (film): 2974, 2866, 1719, 1689, 1587, 1290, 1194, $745 \mathrm{~cm}^{-}$ 1; HRMS (DART) m/z: [M+Na] $]^{+}$Calcd. for $\mathrm{C}_{19} \mathrm{H}_{19} \mathrm{NNaO}_{4}$ 348.1206; Found 348.1206

\section{Dimethyl (1,4-diacetyl-3,5-dimethyl-1,5-dihydropyrano[4,3-b]indol-1-yl)phosphonate (3oa)}

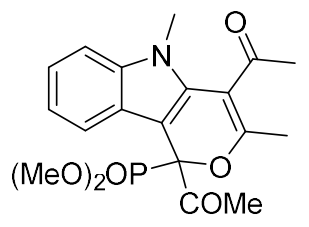

Prepared according to the general procedure (D). hexane:EA = 9:1, 34.0 mg, $0.087 \mathrm{mmol}, 87 \%$ yield; Brown oil; ${ }^{1} \mathrm{H}$ NMR $\left(400 \mathrm{MHz}, \mathrm{CDCl}_{3}\right) \delta 7.85$ (dq, $J=$ $7.8,0.9 \mathrm{~Hz}, 1 \mathrm{H}), 7.29$ (d, $J=1.3 \mathrm{~Hz}, 1 \mathrm{H}), 7.20$ (dddt, $J=19.3,8.0,6.9,1.0 \mathrm{~Hz}$, 2H), $3.81(\mathrm{~d}, J=10.7 \mathrm{~Hz}, 3 \mathrm{H}), 3.77(\mathrm{~d}, J=10.7 \mathrm{~Hz}, 3 \mathrm{H}), 3.58(\mathrm{~s}, 3 \mathrm{H}), 2.41(\mathrm{~s}$, $3 \mathrm{H}), 2.33$ (s, 3H), 2.26 (s, 3H); ${ }^{13} \mathrm{C} \mathrm{NMR}\left(100 \mathrm{MHz}, \mathrm{CDCl}_{3}\right) \delta 200.9,200.8,198.8,158.1,158.1,139.0$, $132.0,131.9,123.5,123.5,122.2$, 121.5, 119.9, 111.4, 109.6, 97.4, 90.4, 88.7, 54.5, 54.5, 54.3, 54.2, 32.1, 31.4, 26.1, 26.0, 18.1; IR (film): 2957, 2854, 1720, 1690, 1595, 1256, 1180, 1032, $747 \mathrm{~cm}^{-1}$; HRMS (DART) m/z: [M+Na] ${ }^{+}$Calcd. for $\mathrm{C}_{19} \mathrm{H}_{22} \mathrm{NNaO}_{6} \mathrm{P} 414.1077$; Found 414.1077

\section{Methyl 1,1-diacetyl-3,5-dimethyl-1,5-dihydropyrano[4,3-b]indole-4-carboxylate (3pa)}

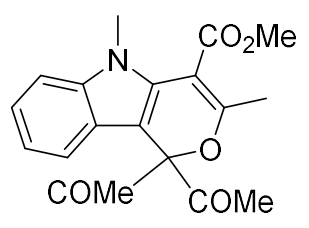

Prepared according to the general procedure (D). hexane:EA =4:1, $5.8 \mathrm{mg}, 0.017$ mmol, $17 \%$ yield, white solid, m.p. $95-100{ }^{\circ} \mathrm{C} ;{ }^{1} \mathrm{H}$ NMR $\left(400 \mathrm{MHz}, \mathrm{CDCl}_{3}\right) \delta=$ 7.45 (ddd, $J=8.0,1.3,0.7 \mathrm{~Hz}, 1 \mathrm{H}$ ), 7.32 (dt, $J=8.4,1.0 \mathrm{~Hz}, 1 \mathrm{H}$ ), 7.23 (ddd, $J$ $=8.3,7.1,1.2 \mathrm{~Hz}, 1 \mathrm{H}), 7.16(\mathrm{ddd}, J=8.1,7.0,1.1 \mathrm{~Hz}, 1 \mathrm{H}), 3.85(\mathrm{~s}, 3 \mathrm{H}), 3.60(\mathrm{~s}$, 3H), $2.48(\mathrm{~s}, 3 \mathrm{H}), 2.31(\mathrm{~s}, 6 \mathrm{H}) ;{ }^{13} \mathrm{C} \mathrm{NMR}\left(100 \mathrm{MHz}, \mathrm{CDCl}_{3}\right) \delta=201.9,166.0,163.8,138.9,131.5$, 122.9, 122.0, 121.2, 117.9, 109.8, 102.2, 99.0, 93.6, 51.8, 32.0, 26.6, 19.0; IR (film): 2949, 1714, 1597, 1296, 1188, 1159, 754, $744 \mathrm{~cm}^{-1}$; HRMS (DART) m/z: $[\mathrm{M}+\mathrm{Na}]^{+}$Calcd. for $\mathrm{C}_{19} \mathrm{H}_{19} \mathrm{NNaO}_{7} 396.1054$; Found 396.1054

\section{Dimethyl}

4-acetyl-3,9-dimethyl-5-oxo-6,7,8,9-tetrahydropyrano[3,4-b]indole-1,1(5H)dicarboxylate (3qa) 


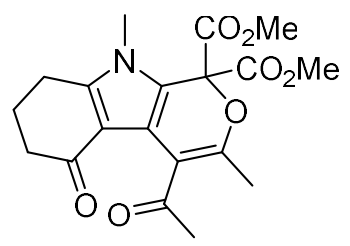

Prepared according to the general procedure (D). hexane:EA $=1: 9,12.0 \mathrm{mg}$, $0.032 \mathrm{mmol}, 32 \%$ yield, white solid, m.p. $190-195{ }^{\circ} \mathrm{C}$; ${ }^{1} \mathrm{H}$ NMR $(400 \mathrm{MHz}$, $\left.\mathrm{CDCl}_{3}\right) \delta=3.89(\mathrm{~s}, 6 \mathrm{H}), 3.48(\mathrm{~s}, 3 \mathrm{H}), 2.75(\mathrm{t}, J=6.2 \mathrm{~Hz}, 2 \mathrm{H}), 2.44-2.39(\mathrm{~m}$, $2 \mathrm{H}), 2.25(\mathrm{~s}, 3 \mathrm{H}), 2.20-2.13(\mathrm{~m}, 2 \mathrm{H}), 2.12(\mathrm{~s}, 3 \mathrm{H}) ;{ }^{13} \mathrm{C} \mathrm{NMR}(100 \mathrm{MHz}$, $\left.\mathrm{CDCl}_{3}\right) \delta=199.4,192.7,167.2,149.7,145.9,128.4,118.9,114.6,114.1$, 112.5, 53.9, 37.8, 31.8, 30.9, 22.8, 22.1, 17.0; IR (film): 2924, 2854, 1761, 1747, 1690, 1654, 1478, 1240, $1099 \mathrm{~cm}^{-1}$; HRMS (DART) m/z: [M+Na] ${ }^{+}$Calcd. for $\mathrm{C}_{19} \mathrm{H}_{21} \mathrm{NNaO}_{7} 398.1210$; Found 398.1210

\section{Dimethyl 4-acetyl-3-methyl-1 $H$-pyrano[4,3-b]benzofuran-1,1-dicarboxylate (3ra)}

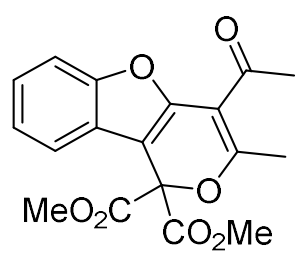

Prepared according to the general procedure (D). hexane:EA $=4: 1,10.3 \mathrm{mg}, 0.03$ mmol, 30\% yield, yellow solid, m.p. $125-130{ }^{\circ} \mathrm{C} ;{ }^{1} \mathrm{H} \mathrm{NMR}\left(400 \mathrm{MHz}, \mathrm{CDCl}_{3}\right) \delta$ $=7.55-7.50(\mathrm{~m}, 1 \mathrm{H}), 7.48(\mathrm{ddd}, \mathrm{J}=5.9,3.3,0.7 \mathrm{~Hz}, 1 \mathrm{H}), 7.29-7.25(\mathrm{~m}, 2 \mathrm{H})$, $3.86(\mathrm{~s}, 6 \mathrm{H}), 2.65$ (s, 3H), 2.47 (s, 3H); ${ }^{13} \mathrm{C}$ NMR $\left(100 \mathrm{MHz}, \mathrm{CDCl}_{3}\right) \delta=194.4$, $166.2,165.4,154.8,147.9,124.7,124.1,123.9,120.3,111.3,107.8,100.5,84.5$,

53.6, 32.4, 20.0; IR (film): 2960, 1745, 1674, 1613, 1602, 1451, 1235, 1163, 748, $734 \mathrm{~cm}^{-1}$; HRMS (DART) $\mathrm{m} / \mathrm{z}:[\mathrm{M}+\mathrm{Na}]^{+}$Calcd. for $\mathrm{C}_{18} \mathrm{H}_{16} \mathrm{NaO}_{7} 367.0788$; Found 367.0793

\section{Dimethyl 4-benzoyl-5-methyl-3-phenylpyrano[4,3-b] indole-1,1(5H)-dicarboxylate (3ab)}

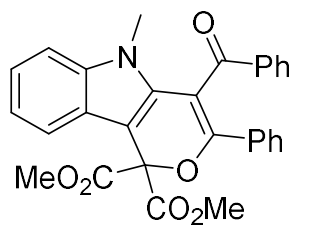

Prepared according to the general procedure (D). hexane:EA $=7: 3,35.6 \mathrm{mg}$, $0.074 \mathrm{mmol}, 74 \%$ yield, yellow solid, m.p. $185-190{ }^{\circ} \mathrm{C}$; ${ }^{1} \mathrm{H}$ NMR $(400 \mathrm{MHz}$, $\left.\mathrm{CDCl}_{3}\right) \delta=7.86(\mathrm{dd}, J=8.2,1.0 \mathrm{~Hz}, 2 \mathrm{H}), 7.78-7.74(\mathrm{~m}, 2 \mathrm{H}), 7.57-7.53(\mathrm{~m}$, 1H), $7.45-7.40(\mathrm{~m}, 1 \mathrm{H}), 7.31-7.26(\mathrm{~m}, 3 \mathrm{H}), 7.25(\mathrm{~d}, J=3.6 \mathrm{~Hz}, 2 \mathrm{H}), 7.21$ (dd, $J=4.9,1.2 \mathrm{~Hz}, 2 \mathrm{H}), 7.17(\mathrm{~d}, J=8.0 \mathrm{~Hz}, 1 \mathrm{H}), 3.91(\mathrm{~s}, 6 \mathrm{H}), 3.47(\mathrm{~s}, 3 \mathrm{H}) ;{ }^{13} \mathrm{C} \mathrm{NMR}\left(100 \mathrm{MHz}, \mathrm{CDCl}_{3}\right)$ $\delta=194.1,167.8,152.9,138.9,137.6,133.7,133.1,131.2,130.0,129.8,129.6,128.6,127.9,123.5$, 122.2, 120.9, 119.5, 109.5, 108.2, 99.2, 53.4, 31.7; IR (film): 2955, 1764, 1668, 1613, 1591, 1274, 1241, 1218, 1142, 775, $743 \mathrm{~cm}^{-1}$; HRMS (DART) m/z: $[\mathrm{M}+\mathrm{Na}]^{+}$Calcd. for $\mathrm{C}_{29} \mathrm{H}_{23} \mathrm{NNaO}_{6}$ 504.1418; Found 504.1418

\section{Dimethyl 5-methyl-4-(4-methylbenzoyl)-3-(p-tolyl)pyrano[4,3-b]indole-1,1(5H)-dicarboxylate (3ac)}




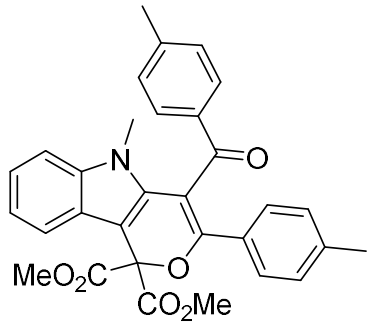

Prepared according to the general procedure (D). hexane:EA $=4: 1,38.2 \mathrm{mg}$, $0.075 \mathrm{mmol}, 75 \%$ yield, yellow solid, m.p. $95-100{ }^{\circ} \mathrm{C} ;{ }^{1} \mathrm{H}$ NMR $(400 \mathrm{MHz}$, $\left.\mathrm{CDCl}_{3}\right) \delta=7.76(\mathrm{~d}, J=8.3 \mathrm{~Hz}, 2 \mathrm{H}), 7.68(\mathrm{~d}, J=8.1 \mathrm{~Hz}, 2 \mathrm{H}), 7.55-7.52$ (m, 1H), $7.21-7.13(\mathrm{~m}, 3 \mathrm{H}), 7.08$ (td, $J=8.3,0.7 \mathrm{~Hz}, 4 \mathrm{H}), 3.90(\mathrm{~s}, 6 \mathrm{H})$, $3.46(\mathrm{~s}, 3 \mathrm{H}), 2.30(\mathrm{~s}, 3 \mathrm{H}), 2.27$ (s, 3H); ${ }^{13} \mathrm{C} \mathrm{NMR}\left(100 \mathrm{MHz}, \mathrm{CDCl}_{3}\right) \delta=$ 193.8, 167.9, 152.5, 144.7, 140.1, 138.9, 135.2, 131.5, 130.3, 129.8, 129.7, 129.4, 128.6, 123.5, 122.0, 120.8, 119.4, 109.4, 107.8, 99.0, 84.9, 53.3, 31.5, 21.7, 21.4; IR (film): 2952, $2863,1745,1661,1602,1569,1435,1273,1247,1222,1115,826,788,742 \mathrm{~cm}^{-1}$; HRMS (DART) m/z: $[\mathrm{M}+\mathrm{Na}]^{+}$Calcd. for $\mathrm{C}_{31} \mathrm{H}_{27} \mathrm{NNaO}_{6} 532.1731$; Found 532.1731

\section{Dimethyl 4-(4-methoxybenzoyl)-3-(4-methoxyphenyl)-5-methylpyrano[4,3-b]indole-1,1(5H)- dicarboxylate (3ad)}

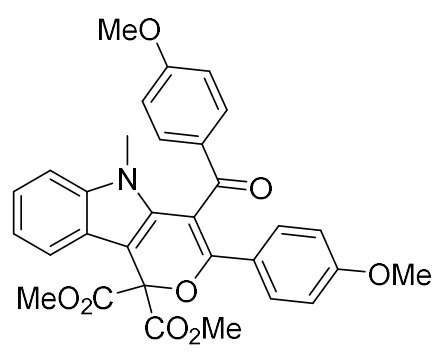

Prepared according to the general procedure (D). hexane:EA $=4: 1,35.2$ $\mathrm{mg}, 0.065 \mathrm{mmol}, 65 \%$ yield, yellow solid, m.p. $150-155{ }^{\circ} \mathrm{C}$; ${ }^{1} \mathrm{H}$ NMR $\left(400 \mathrm{MHz}, \mathrm{CDCl}_{3}\right) \delta=7.87-7.83(\mathrm{~m}, 2 \mathrm{H}), 7.80-7.75(\mathrm{~m}, 2 \mathrm{H}), 7.53$ $(\mathrm{dd}, J=7.1,1.5 \mathrm{~Hz}, 1 \mathrm{H}), 7.23-7.18(\mathrm{~m}, 2 \mathrm{H}), 7.18-7.13(\mathrm{~m}, 1 \mathrm{H})$, $6.81-6.75(\mathrm{~m}, 4 \mathrm{H}), 3.90(\mathrm{~s}, 6 \mathrm{H}), 3.78(\mathrm{~s}, 3 \mathrm{H}), 3.76(\mathrm{~s}, 3 \mathrm{H}), 3.49(\mathrm{~s}$, $3 \mathrm{H}) ;{ }^{13} \mathrm{C} \mathrm{NMR}\left(100 \mathrm{MHz}, \mathrm{CDCl}_{3}\right) \delta=192.8,168.0,164.0,160.8,152.1$, $138.9,132.1,131.6,131.5,130.8,125.7,123.5,121.9,120.8,119.3,113.9,113.3,109.4,107.2,98.8$, 55.4, 55.2, 53.3, 31.5; IR (film): 2959, 1745, 1659, 1594, 1572, 1451, 1298, 1253, 1223, 1129, 779, 740 $\mathrm{cm}^{-1}$; HRMS (DART) m/z: [M+Na] ${ }^{+}$Calcd. for $\mathrm{C}_{31} \mathrm{H}_{27} \mathrm{NNaO}_{8}$ 564.1629; Found 564.1629

Dimethyl dicarboxylate (3ae)

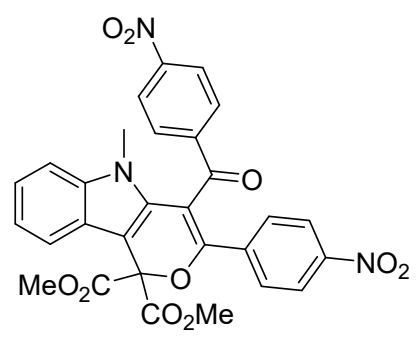

5-methyl-4-(4-nitrobenzoyl)-3-(4-nitrophenyl)pyrano[4,3-b]indole-1,1(5H)-

Prepared according to the general procedure (D). hexane:EA $=4: 1,34.3$ $\mathrm{mg}, 0.06 \mathrm{mmol}, 60 \%$ yield, red solid, m.p. $235-240{ }^{\circ} \mathrm{C} ;{ }^{1} \mathrm{H}$ NMR $(400$ $\left.\mathrm{MHz}, \mathrm{CDCl}_{3}\right) \delta=8.15(\mathrm{dd}, J=8.9,6.9 \mathrm{~Hz}, 4 \mathrm{H}), 8.00(\mathrm{dd}, J=11.7,8.9$ $\mathrm{Hz}, 4 \mathrm{H}), 7.57$ (d, J=7.9 Hz, 1H), $7.26-7.18(\mathrm{~m}, 3 \mathrm{H}), 3.94$ (s, 6H), 3.47 $(\mathrm{s}, 3 \mathrm{H}) ;{ }^{13} \mathrm{C} \mathrm{NMR}\left(100 \mathrm{MHz}, \mathrm{CDCl}_{3}\right) \delta=191.8,167.3,150.9,150.7$, $148.5,141.3,139.3,138.5,131.0,130.6,129.6,124.1,123.3,123.2$, 789, $749 \mathrm{~cm}^{-1}$; HRMS (DART) m/z: [M+Na $]^{+}$Calcd. for $\mathrm{C}_{29} \mathrm{H}_{21} \mathrm{~N}_{3} \mathrm{NaO}_{10}$ 594.1119; Found 594.1119 
dicarboxylate (3af)

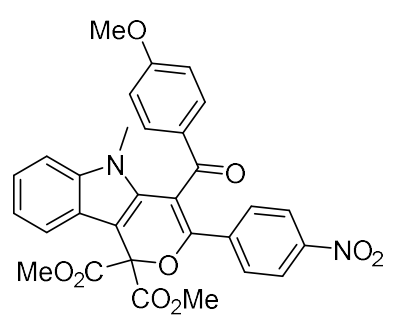

Prepared according to the general procedure (D). hexane:EA $=4: 1,35.0$ $\mathrm{mg}, 0.063 \mathrm{mmol}, 63 \%$ yield (total $95 \%$ ), orange solid, m.p. $170-175{ }^{\circ} \mathrm{C}$; ${ }^{1} \mathrm{H}$ NMR $\left(400 \mathrm{MHz}, \mathrm{CDCl}_{3}\right) \delta=8.10(\mathrm{~d}, J=8.9 \mathrm{~Hz}, 2 \mathrm{H}), 7.98(\mathrm{~d}, J=9.1$ $\mathrm{Hz}, 2 \mathrm{H}), 7.84(\mathrm{~d}, J=8.9 \mathrm{~Hz}, 2 \mathrm{H}), 7.56(\mathrm{dd}, J=7.9,1.1 \mathrm{~Hz}, 1 \mathrm{H}), 7.25-$ $7.16(\mathrm{~m}, 3 \mathrm{H}), 6.78(\mathrm{~d}, J=9.0 \mathrm{~Hz}, 2 \mathrm{H}), 3.91(\mathrm{~s}, 6 \mathrm{H}), 3.79(\mathrm{~s}, 3 \mathrm{H}), 3.48(\mathrm{~s}$, $3 \mathrm{H}) ;{ }^{13} \mathrm{C} \mathrm{NMR}\left(100 \mathrm{MHz}, \mathrm{CDCl}_{3}\right) \delta=192.1,167.6,164.6,148.6,148.1$, 139.3, 139.2, 132.1, 130.4, 130.3, 130.1, 123.3, 123.1, 122.8, 121.2, 119.9, 114.3, 110.7, 109.6, 100.2, 85.1, 55.5, 53.5, 31.5; IR (film): 2923, 1744, 1673, 1592, 1519, 1459, 1342, 1255, 1224, 1172, 803, 747, $725 \mathrm{~cm}^{-1}$; HRMS (DART) m/z: [M+Na] ${ }^{+}$Calcd. for $\mathrm{C}_{30} \mathrm{H}_{24} \mathrm{~N}_{2} \mathrm{NaO}_{9}$ 579.1374; Found 579.1374

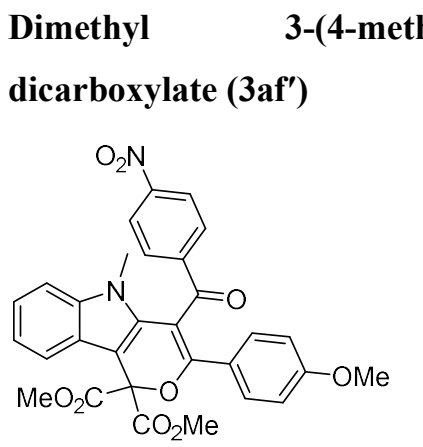

Prepared according to the general procedure (D). hexane:EA $=4: 1,17.8$ $\mathrm{mg}, 0.032 \mathrm{mmol}, 32 \%$ yield (total $95 \%$ ), orange solid, m.p. $155-160{ }^{\circ} \mathrm{C}$; ${ }^{1} \mathrm{H} \mathrm{NMR}\left(400 \mathrm{MHz}, \mathrm{CDCl}_{3}\right) \delta=8.12-8.06(\mathrm{~m}, 2 \mathrm{H}), 8.00-7.95(\mathrm{~m}, 2 \mathrm{H})$, $7.71-7.65(\mathrm{~m}, 2 \mathrm{H}), 7.52(\mathrm{dd}, J=7.6,1.3 \mathrm{~Hz}, 1 \mathrm{H}), 7.24-7.14(\mathrm{~m}, 3 \mathrm{H})$, $6.79-6.74(\mathrm{~m}, 2 \mathrm{H}), 3.91(\mathrm{~s}, 6 \mathrm{H}), 3.74(\mathrm{~s}, 3 \mathrm{H}), 3.56(\mathrm{~s}, 3 \mathrm{H}) ;{ }^{13} \mathrm{C} \mathrm{NMR}$ $\left(100 \mathrm{MHz}, \mathrm{CDCl}_{3}\right) \delta=192.3,167.6,161.5,155.5,150.1,142.6,139.1,132.0,130.8,130.5,125.2$, 123.7, 123.3, 122.4, 121.1, 119.4, 113.5, 109.6, 106.8, 99.5, 55.3, 53.5, 32.1; IR (film): 2919, 2850, 1666, 1602, 1507, 1463, 1342, 1250, 1175, 844, 742, $703 \mathrm{~cm}^{-1}$; HRMS (DART) m/z: [M+Na] ${ }^{+}$Calcd. for $\mathrm{C}_{30} \mathrm{H}_{24} \mathrm{~N}_{2} \mathrm{NaO}_{9} 579.1374$; Found 579.1374

\section{Dimethyl}

3,3,11-trimethyl-1-oxo-2,3,4,11-tetrahydrochromeno[4,3-b]indole-6,6(1H)dicarboxylate (3ag)

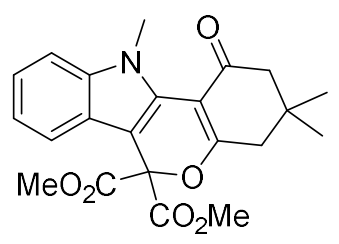

Prepared according to the general procedure (D). hexane:EA $=4: 1,31.0 \mathrm{mg}$, $0.078 \mathrm{mmol}, 78 \%$ yield, orange solid, m.p. $145-150{ }^{\circ} \mathrm{C} ;{ }^{1} \mathrm{H}$ NMR $(400 \mathrm{MHz}$, $\left.\mathrm{CDCl}_{3}\right) \delta=7.39(\mathrm{~d}, J=7.9 \mathrm{~Hz}, 1 \mathrm{H}), 7.34(\mathrm{~d}, J=8.2 \mathrm{~Hz}, 1 \mathrm{H}), 7.21(\mathrm{ddd}, J=$ 8.2, 7.0, $1.2 \mathrm{~Hz}, 1 \mathrm{H}), 7.16-7.10(\mathrm{~m}, 1 \mathrm{H}), 3.84(\mathrm{~s}, 3 \mathrm{H}), 3.83(\mathrm{~s}, 6 \mathrm{H}), 2.68(\mathrm{~s}$, 2H), 2.45 (s, 2H), $1.18(\mathrm{~s}, 6 \mathrm{H}) ;{ }^{13} \mathrm{C} \mathrm{NMR}\left(100 \mathrm{MHz}, \mathrm{CDCl}_{3}\right) \delta=193.6,171.3,166.9,139.5,129.3$, 123.4, 122.0, 120.7, 118.6, 110.2, 109.6, 100.2, 85.4, 53.5, 51.7, 42.6, 34.4, 31.6, 28.3; IR (film): 2956, 1751, 1666, 1588, 1467, 1369, 1285, 1246, 1087, $742 \mathrm{~cm}^{-1}$; HRMS (DART) m/z: [M+Na] $]^{+}$Calcd. for 
$\mathrm{C}_{22} \mathrm{H}_{23} \mathrm{NNaO}_{6}$ 420.1418; Found 420.1415

\section{Dimethyl 4-benzoyl-3,5-dimethylpyrano[4,3-b]indole-1,1(5H)-dicarboxylate (3ah)}

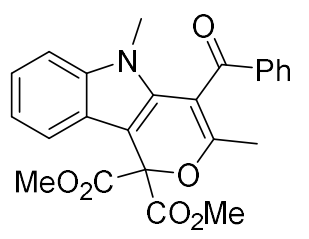

Prepared according to the general procedure (D). hexane:EA = 9:1, $31.4 \mathrm{mg}$, 0.075 mmol, $75 \%$ yield, yellow solid, m.p. $175-180{ }^{\circ} \mathrm{C} ;{ }^{1} \mathrm{H}$ NMR $(400 \mathrm{MHz}$, $\left.\mathrm{CDCl}_{3}\right) \delta=7.89(\mathrm{dd}, J=8.1,1.4 \mathrm{~Hz}, 2 \mathrm{H}), 7.57(\mathrm{t}, J=7.4 \mathrm{~Hz}, 1 \mathrm{H}), 7.52-7.48$ (m, 1H), $7.42(\mathrm{t}, J=7.7 \mathrm{~Hz}, 2 \mathrm{H}), 7.19-7.11(\mathrm{~m}, 3 \mathrm{H}), 3.88(\mathrm{~s}, 6 \mathrm{H}), 3.22(\mathrm{~s}, 3 \mathrm{H})$, 2.13 (s, 3H); ${ }^{13} \mathrm{C}$ NMR $\left(100 \mathrm{MHz}, \mathrm{CDCl}_{3}\right) \delta=193.5,167.6,156.6,138.4,138.1,133.8,131.6,129.4$, 129.0, 123.7, 121.8, 120.8, 119.2, 109.4, 108.1, 98.2, 84.9, 53.4, 31.6, 18.4; IR (film): 2952, 1757, 1744, 1664, 1623, 1594, 1448, 1274, 1240, 754, $699 \mathrm{~cm}^{-1}$; HRMS (DART) m/z: [M+Na ${ }^{+}$Calcd. for $\mathrm{C}_{24} \mathrm{H}_{21} \mathrm{NNaO}_{6} 442.1261$; Found 442.1261

\section{Trimethyl 3,5-dimethylpyrano[4,3-b]indole-1,1,4(5H)-tricarboxylate (3ai)}

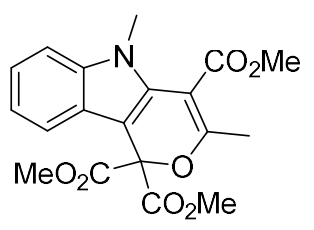

Prepared according to the general procedure (D). hexane:EA $=9: 1,31.0 \mathrm{mg}$, $0.083 \mathrm{mmol}, 83 \%$ yield, yellow solid, m.p. $145-150{ }^{\circ} \mathrm{C} ;{ }^{1} \mathrm{H}$ NMR $(400 \mathrm{MHz}$, $\left.\mathrm{CDCl}_{3}\right) \delta=7.44-7.40(\mathrm{~m}, 1 \mathrm{H}), 7.28(\mathrm{dt}, J=8.3,1.0 \mathrm{~Hz}, 1 \mathrm{H}), 7.20$ (ddd, $J=$ 8.3, 7.0, $1.3 \mathrm{~Hz}, 1 \mathrm{H}), 7.13(\mathrm{ddd}, J=8.0,7.0,1.1 \mathrm{~Hz}, 1 \mathrm{H}), 3.86(\mathrm{~s}, 3 \mathrm{H}), 3.83(\mathrm{~s}$, $6 \mathrm{H}), 3.57$ (s, 3H), 2.42 (s, 3H); ${ }^{13} \mathrm{C}$ NMR (100 MHz, $\left.\mathrm{CDCl}_{3}\right) \delta=167.3,166.1,161.4,138.8,130.4$, 123.4, 121.9, 120.8, 118.8, 109.6, 102.2, 99.6, 85.0, 53.4, 51.9, 31.9, 18.8; IR (film): 2954, 1759, 1748, 1709, 1614, 1593, 1429, 1301, 1204, 750, $741 \mathrm{~cm}^{-1}$; HRMS (DART) m/z: [M+Na] $]^{+}$Calcd. for $\mathrm{C}_{19} \mathrm{H}_{19} \mathrm{NNaO}_{7} 396.1054$; Found 396.1054

\section{4-Ethyl 1,1-dimethyl 5-methyl-3-phenylpyrano[4,3-b]indole-1,1,4(5H)-tricarboxylate (3aj)}

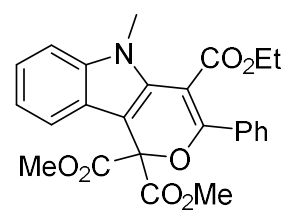

Prepared according to the general procedure (D). hexane:EA $=4: 1,37.3 \mathrm{mg}$, $0.083 \mathrm{mmol}, 83 \%$ yield, yellow solid, m.p. $135-140{ }^{\circ} \mathrm{C}$; ${ }^{1} \mathrm{H}$ NMR $(400 \mathrm{MHz}$, $\left.\mathrm{CDCl}_{3}\right) \delta=7.86-7.83(\mathrm{~m}, 2 \mathrm{H}), 7.50-7.47(\mathrm{~m}, 1 \mathrm{H}), 7.45-7.40(\mathrm{~m}, 3 \mathrm{H}), 7.32$ (d, $J=8.2 \mathrm{~Hz}, 1 \mathrm{H}), 7.23$ (ddd, $J=8.3,7.1,1.2 \mathrm{~Hz}, 1 \mathrm{H}), 7.18-7.14(\mathrm{~m}, 1 \mathrm{H}), 4.11$ $(\mathrm{q}, J=7.2 \mathrm{~Hz}, 2 \mathrm{H}), 3.85(\mathrm{~s}, 6 \mathrm{H}), 3.72(\mathrm{~s}, 3 \mathrm{H}), 1.06(\mathrm{t}, J=7.1 \mathrm{~Hz}, 3 \mathrm{H}) ;{ }^{13} \mathrm{C}$ NMR $\left(100 \mathrm{MHz}, \mathrm{CDCl}_{3}\right) \delta$ $=167.5,165.9,156.6,139.0,133.4,130.2,130.2,129.7,127.8,123.4,122.2,120.9,119.2,109.6,103.3$, 99.7, 85.1, 61.5, 53.4, 31.6, 13.6; IR (film): 2954, 1761, 1743, 1706, 1612, 1591, 1448, 1277, 1233, 1215, 744, $710 \mathrm{~cm}^{-1}$; HRMS (DART) m/z: [M+Na] $]^{+}$Calcd. for $\mathrm{C}_{25} \mathrm{H}_{23} \mathrm{NNaO}_{7}$ 472.1367; Found 472.1367 


\section{Dimethyl 3,5-dimethyl-4-(phenylcarbamoyl)pyrano[4,3-b]indole-1,1(5H)-dicarboxylate (3ak)}

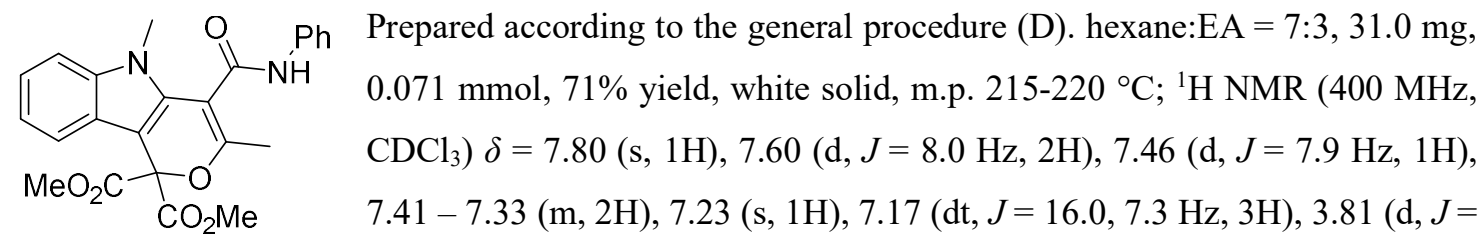

$5.9 \mathrm{~Hz}, 6 \mathrm{H}), 3.65(\mathrm{~d}, J=2.3 \mathrm{~Hz}, 3 \mathrm{H}), 2.29(\mathrm{~d}, J=1.8 \mathrm{~Hz}, 3 \mathrm{H}) ;{ }^{13} \mathrm{C} \mathrm{NMR}\left(100 \mathrm{MHz}, \mathrm{CDCl}_{3}\right) \delta=167.6$, $163.8,154.9,138.6,137.5,129.8,129.3,124.9,123.8,122.0,121.0,119.7,119.2$, 109.5, 105.5, 98.3, 84.8, 53.3, 31.0, 18.0IR (film): 3241, 2952, 1749, 1644, 1596, 1546, 1443, 1276, 1242, 1221, 1143, 758, 732, $691 \mathrm{~cm}^{-1}$; HRMS (DART) m/z: [M+Na] Calcd. for $\mathrm{C}_{24} \mathrm{H}_{22} \mathrm{~N}_{2} \mathrm{NaO}_{6} 457.1370$; Found 457.1371

\section{Dimethyl 4-cyano-5-methyl-3-phenylpyrano[4,3-b]indole-1,1(5H)-dicarboxylate (3al)}

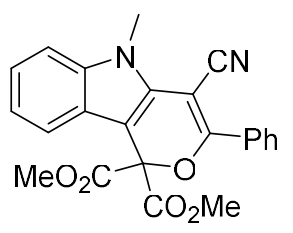

Prepared according to the general procedure (D). hexane:EA $=9: 1,37.0 \mathrm{mg}, 0.092$ mmol, $92 \%$ yield, yellow solid, m.p. $170-175{ }^{\circ} \mathrm{C} ;{ }^{1} \mathrm{H}$ NMR (400 MHz, $\left.\mathrm{CDCl}_{3}\right) \delta$ $=8.14-8.10(\mathrm{~m}, 2 \mathrm{H}), 7.57-7.50(\mathrm{~m}, 4 \mathrm{H}), 7.36(\mathrm{~d}, J=8.2 \mathrm{~Hz}, 1 \mathrm{H}), 7.28(\mathrm{ddd}, J$ $=8.3,6.9,1.2 \mathrm{~Hz}, 1 \mathrm{H}), 7.20(\mathrm{ddd}, J=8.0,7.0,1.0 \mathrm{~Hz}, 1 \mathrm{H}), 4.05(\mathrm{~s}, 3 \mathrm{H}), 3.87(\mathrm{~d}$, $J=0.9 \mathrm{~Hz}, 6 \mathrm{H}) ;{ }^{13} \mathrm{C} \mathrm{NMR}\left(100 \mathrm{MHz}, \mathrm{CDCl}_{3}\right) \delta=166.7,164.7,139.0,132.1,131.1,129.5,128.6,126.8$, 123.1, 122.9, 121.4, 119.4, 116.7, 109.7, 99.2, 85.6, 81.1, 53.7, 30.6; IR (film): 2215, 1749, 1588, 1567, $1275,1244,1223,1154,740,701 \mathrm{~cm}^{-1}$; HRMS (DART) m/z: $[\mathrm{M}+\mathrm{H}]^{+}$Calcd. for $\mathrm{C}_{23} \mathrm{H}_{19} \mathrm{~N}_{2} \mathrm{O}_{5} 403.1288$; Found 403.1288

\section{Dimethyl 5-methyl-3-phenyl-4-(phenylsulfonyl)pyrano[4,3-b]indole-1,1(5H)-dicarboxylate (3am)}

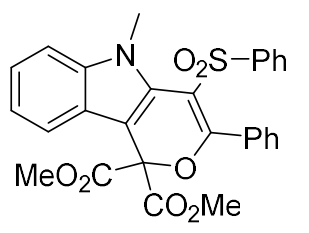

Prepared according to the general procedure (D). hexane:EA $=1: 1,50.7 \mathrm{mg}$, $0.098 \mathrm{mmol}, 98 \%$ yield, yellow solid, m.p. $225-230{ }^{\circ} \mathrm{C}$; ${ }^{1} \mathrm{H}$ NMR $(400 \mathrm{MHz}$, $\left.\mathrm{CDCl}_{3}\right) \delta=7.65(\mathrm{~d}, J=7.3 \mathrm{~Hz}, 2 \mathrm{H}), 7.45-7.35(\mathrm{~m}, 3 \mathrm{H}), 7.33-7.27(\mathrm{~m}, 4 \mathrm{H})$, $7.20(\mathrm{q}, J=8.0 \mathrm{~Hz}, 3 \mathrm{H}), 7.10$ (dd, $J=8.4,7.3 \mathrm{~Hz}, 2 \mathrm{H}), 4.15$ (s, 3H), 3.87 (s, 6H);

${ }^{13} \mathrm{C}$ NMR $\left(100 \mathrm{MHz}, \mathrm{CDCl}_{3}\right) \delta=167.1,162.0,141.6,140.0,132.4,132.2,131.9,131.5,129.9,127.9$, 127.8, 127.7, 122.9, 122.6, 121.0, 118.4, 116.1, 110.5, 102.3, 85.2, 53.6, 34.7; IR (film): 2952, 1755, 1558, 1446, 1307, 1273, 1250, 1160, 747, 716, $686 \mathrm{~cm}^{-1}$; HRMS (DART) m/z: $[\mathrm{M}+\mathrm{H}]^{+}$Calcd. for $\mathrm{C}_{23} \mathrm{H}_{22} \mathrm{NO}_{7} \mathrm{~S} 456.1111$; Found 456.1111 
Dimethyl 4-(dimethoxyphosphoryl)-3,5-dimethylpyrano[4,3-b]indole-1,1(5H)-dicarboxylate (3an)

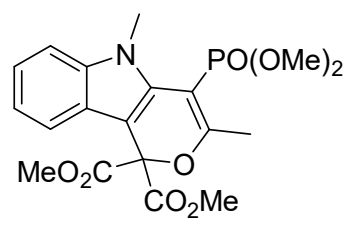

Prepared according to the general procedure (D). hexane:EA $=3: 7,37.0 \mathrm{mg}$, $0.088 \mathrm{mmol}, 88 \%$ yield, yellow solid, m.p. $145-150{ }^{\circ} \mathrm{C} ;{ }^{1} \mathrm{H}$ NMR $(400 \mathrm{MHz}$, $\left.\mathrm{CDCl}_{3}\right) \delta=7.36(\mathrm{dt}, J=8.0,1.0 \mathrm{~Hz}, 1 \mathrm{H}), 7.31(\mathrm{dt}, J=8.3,0.9 \mathrm{~Hz}, 1 \mathrm{H}), 7.20$ (ddd, $J=8.2,7.0,1.2 \mathrm{~Hz}, 1 \mathrm{H}), 7.12(\mathrm{ddd}, J=8.0,7.0,1.1 \mathrm{~Hz}, 1 \mathrm{H}), 3.86$ (s,

$3 \mathrm{H}), 3.83(\mathrm{~s}, 6 \mathrm{H}), 3.77(\mathrm{~s}, 3 \mathrm{H}), 3.74(\mathrm{~s}, 3 \mathrm{H}), 2.45(\mathrm{~d}, J=2.3 \mathrm{~Hz}, 3 \mathrm{H}) ;{ }^{13} \mathrm{C} \mathrm{NMR}\left(100 \mathrm{MHz}, \mathrm{CDCl}_{3}\right) \delta=$ 167.7, 167.5, 167.1, 139.5, 131.6, 131.6, 123.0, 121.9, 120.8, 118.3, 110.1, 100.5, 100.4, 96.1, 94.0, 84.7, 53.5, 52.6, 52.5, 33.0, 21.1, 21.1; IR (film): 2953, 1748, 1608, 1572, 1464, 1284, 1243, 1049, 1025, $756 \mathrm{~cm}^{-1}$; HRMS (DART) m/z: $[\mathrm{M}+\mathrm{H}]^{+}$Calcd. for $\mathrm{C}_{19} \mathrm{H}_{23} \mathrm{NO}_{8} \mathrm{P} 424.1156$; Found 424.1156

\section{Trimethyl (E)-3-styrylpyrano[4,3-b]indole-1,1,4(5H)-tricarboxylate (3io)}

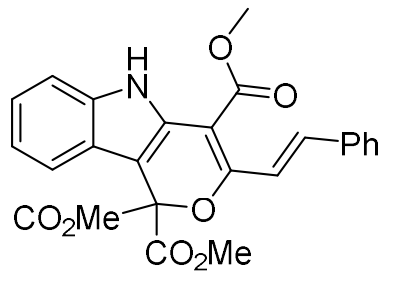

$\left(100 \mathrm{MHz}, \mathrm{CDCl}_{3}\right) \delta=167.3,165.9,158.3,138.7,136.7,136.3,129.4,129.3,128.8,128.0,124.2$, 122.5, 120.9, 119.7, 119.5, 111.3, 99.7, 99.3, 84.5, 53.4, 52.2; IR (film): 3399, 2955, 1739, 1715, 1616, 1311, 1250, 1227, 1172, 978, 765, $744 \mathrm{~cm}^{-1}$; HRMS (DART) m/z: $[\mathrm{M}+\mathrm{H}]^{+}$Calcd. for $\mathrm{C}_{25} \mathrm{H}_{22} \mathrm{NO}_{7}$ 448.1391; Found 448.1391

\section{Dimethyl 4-cinnamoyl-5-methyl-3-((E)-styryl)pyrano[4,3-b]indole-1,1(5H)-dicarboxylate (3ap)}

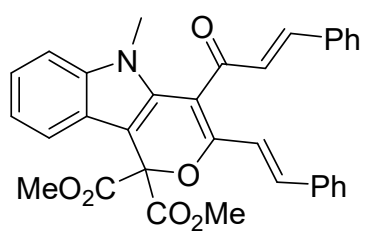

Prepared according to the general procedure (D). hexane:EA $=4: 1,37.3 \mathrm{mg}$, $0.07 \mathrm{mmol}, 70 \%$ yield, red solid, m.p. $95-100{ }^{\circ} \mathrm{C} ;{ }^{1} \mathrm{H}$ NMR $(400 \mathrm{MHz}$, $\left.\mathrm{CDCl}_{3}\right) \delta=7.64(\mathrm{~d}, J=16.1 \mathrm{~Hz}, 1 \mathrm{H}), 7.57-7.48(\mathrm{~m}, 6 \mathrm{H}), 7.41-7.27(\mathrm{~m}$, $6 \mathrm{H}), 7.24(\mathrm{dd}, J=6.6,1.3 \mathrm{~Hz}, 1 \mathrm{H}), 7.18(\mathrm{~s}, 1 \mathrm{H}), 7.13(\mathrm{~d}, J=15.6 \mathrm{~Hz}, 1 \mathrm{H})$, $7.00(\mathrm{~d}, J=16.1 \mathrm{~Hz}, 1 \mathrm{H}), 3.91(\mathrm{~s}, 6 \mathrm{H}), 3.59$ (s, 3H); ${ }^{13} \mathrm{C} \mathrm{NMR}\left(100 \mathrm{MHz}, \mathrm{CDCl}_{3}\right) \delta=192.2,167.8$, 151.6, 147.0, 139.3, 136.3, 136.0, 134.1, 131.7, 131.1, 129.0, 128.8, 128.7, 128.6, 127.8, 127.6, 123.9, 122.5, 121.1, 119.2, 118.2, 110.5, 109.7, 101.7, 84.4, 53.5, 31.9; IR (film): 2921, 1745, 1594, 1574, 1447, 1315, 1247, 1194, 911, 747, $688 \mathrm{~cm}^{-1}$; HRMS (DART) m/z: $[\mathrm{M}+\mathrm{H}]^{+}$Calcd. for $\mathrm{C}_{33} \mathrm{H}_{27} \mathrm{NNaO}_{6}$ 556.1731; Found 556.1730 


\section{Dimethyl 4-cinnamoyl-3-((E)-styryl)pyrano[4,3-b]indole-1,1(5H)-dicarboxylate (3ip)}

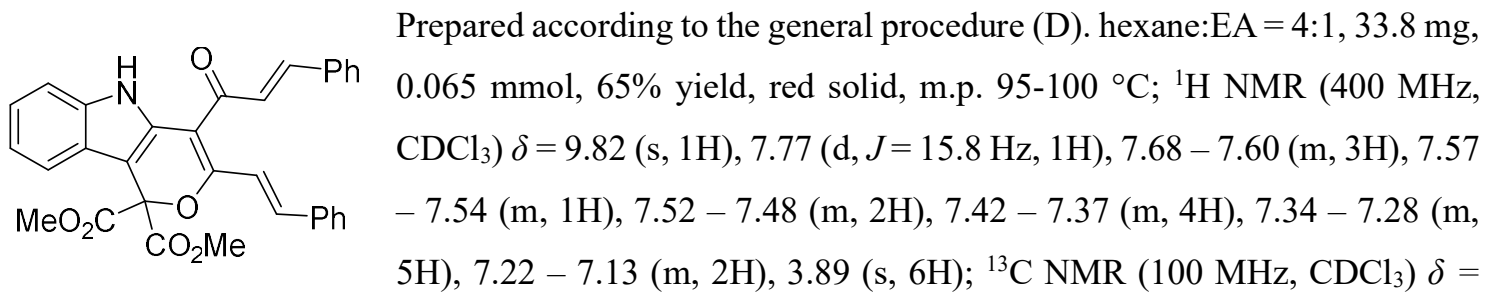

189.7, 167.3, 157.0, 143.5, 138.2, 137.0, 135.7, 134.5, 130.8, 130.0, 129.6, 129.1, 128.9, 128.4, 127.7, 127.0, 124.2, 122.4, 120.8, 119.3, 119.1, 111.5, 110.5, 99.4, 53.5; IR (film): 3394, 2953, 1767, 1644, 1583, 1448, 1342, 1265, 1200, 971, 727, $679 \mathrm{~cm}^{-1}$; HRMS (DART) m/z: $[\mathrm{M}+\mathrm{H}]^{+}$Calcd. for $\mathrm{C}_{32} \mathrm{H}_{25} \mathrm{NNaO}_{6}$ 542.1574; Found 542.1574

\section{Dimethyl (E)-4-acetyl-5-methyl-3-styrylpyrano[4,3-b]indole-1,1(5H)-dicarboxylate (3aq)}

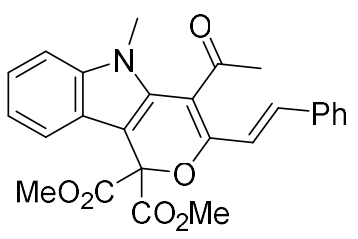

Prepared according to the general procedure (D). hexane:EA $=4: 1,17.8 \mathrm{mg}$, $0.04 \mathrm{mmol}, 40 \%$ yield ( $85 \%$ total yield), red solid, m.p. $125-130{ }^{\circ} \mathrm{C} ;{ }^{1} \mathrm{H}$ NMR $\left(400 \mathrm{MHz}, \mathrm{CDCl}_{3}\right) \delta=7.58-7.49(\mathrm{~m}, 4 \mathrm{H}), 7.38-7.30(\mathrm{~m}, 4 \mathrm{H}), 7.28-7.23$

(m, 2H), $7.20-7.16(\mathrm{~m}, 2 \mathrm{H}), 3.87(\mathrm{~s}, 6 \mathrm{H}), 3.63(\mathrm{~s}, 3 \mathrm{H}), 2.47(\mathrm{~s}, 3 \mathrm{H}) ;{ }^{13} \mathrm{C}$ $\operatorname{NMR}\left(100 \mathrm{MHz}, \mathrm{CDCl}_{3}\right) \delta=199.0,167.6,151.3,139.6,137.2,136.2,131.6,129.0,128.7,127.7,123.9$, 122.6, 121.3, 119.1, 117.7, 112.0, 109.8, 102.6, 84.3, 53.5, 32.2, 32.0; IR (film): 2923, 1744, 1688, 1615, 1435, 1245, 759, 746, $690 \mathrm{~cm}^{-1}$; HRMS (DART) m/z: $[\mathrm{M}+\mathrm{H}]^{+}$Calcd. for $\mathrm{C}_{26} \mathrm{H}_{23} \mathrm{NNaO}_{6} 468.1418$; Found 468.1418

\section{Dimethyl 4-cinnamoyl-3,5-dimethylpyrano[4,3-b]indole-1,1(5H)-dicarboxylate (3aq')}

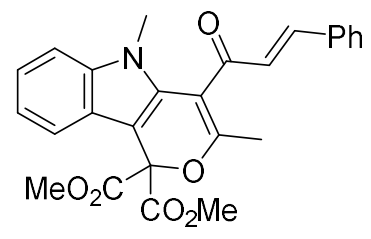

Prepared according to the general procedure (D). hexane:EA $=4: 1,20.0 \mathrm{mg}$, $0.045 \mathrm{mmol}, 45 \%$ yield (85\% total yield), red solid, m.p. $125-130{ }^{\circ} \mathrm{C} ;{ }^{1} \mathrm{H}$ NMR $\left(400 \mathrm{MHz}, \mathrm{CDCl}_{3}\right) \delta=7.63(\mathrm{~d}, J=16.1 \mathrm{~Hz}, 1 \mathrm{H}), 7.51(\mathrm{td}, J=6.1$, $5.1,1.3 \mathrm{~Hz}, 3 \mathrm{H}), 7.42-7.37$ (m, 3H), $7.25-7.14(\mathrm{~m}, 3 \mathrm{H}), 6.95$ (dd, $J=$ 16.1, $0.9 \mathrm{~Hz}, 1 \mathrm{H}), 3.88$ (d, $J=0.6 \mathrm{~Hz}, 6 \mathrm{H}), 3.54$ (s, 3H), 2.25 (s, 3H); ${ }^{13} \mathrm{C}$ NMR $\left(100 \mathrm{MHz}, \mathrm{CDCl}_{3}\right) \delta$ $=192.3,167.6,156.5,146.3,138.7,134.1,131.2,131.1,129.0,128.6,127.7,123.8,121.9,120.9,119.1$, 109.6, 109.0, 98.9, 84.9, 53.4, 31.9, 18.4; IR (film): 2950, 1748, 1621, 1448, 1276, 1247, 1200, 766, 743, $727 \mathrm{~cm}^{-1}$; HRMS (DART) m/z: [M+H] $]^{+}$Calcd. for $\mathrm{C}_{26} \mathrm{H}_{23} \mathrm{NNaO}_{6} 468.1418$; Found 468.1418

Dimethyl (E)-4-acetyl-3-styrylpyrano[4,3-b]indole-1,1(5H)-dicarboxylate (3iq) and Dimethyl 4- 
cinnamoyl-3-methylpyrano $[4,3-b]$ indole-1,1(5H)-dicarboxylate (3iq')

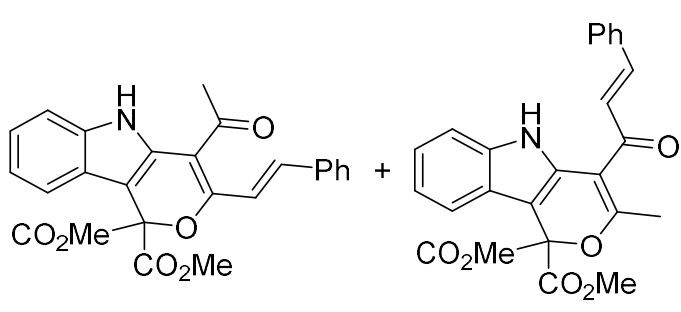

Prepared according to the general procedure (D). hexane:EA $=4: 1,34.5 \mathrm{mg}, 0.08 \mathrm{mmol}, 80 \%$ total yield, red solid, m.p. $125-130{ }^{\circ} \mathrm{C} ;{ }^{1} \mathrm{H}$ NMR $(400 \mathrm{MHz}$, $\left.\mathrm{CDCl}_{3}\right) \delta=10.08(\mathrm{~s}, 1 \mathrm{H}), 9.77(\mathrm{~s}, 0.7 \mathrm{H}), 7.71(\mathrm{~d}, J=$ $5.8 \mathrm{~Hz}, 0.8 \mathrm{H}), 7.67(\mathrm{~d}, J=6.2 \mathrm{~Hz}, 1 \mathrm{H}), 7.58(\mathrm{dd}, J=$ $8.3,6.3 \mathrm{~Hz}, 3.5 \mathrm{H}), 7.52(\mathrm{t}, J=6.6 \mathrm{~Hz}, 1.7 \mathrm{H}), 7.43-$ $7.35(\mathrm{~m}, 7.3 \mathrm{H}), 7.30(\mathrm{~s}, 1 \mathrm{H}), 7.20-7.07(\mathrm{~m}, 4.6 \mathrm{H}), 3.86(\mathrm{~s}, 6 \mathrm{H}), 3.85(\mathrm{~s}, 5 \mathrm{H}), 2.65(\mathrm{~s}, 3 \mathrm{H}), 2.50(\mathrm{~s}, 2 \mathrm{H})$; ${ }^{13} \mathrm{C}$ NMR $\left(100 \mathrm{MHz}, \mathrm{CDCl}_{3}\right) \delta=197.4,190.3,167.3,167.0,162.7,158.1,143.7,139.7,136.9,136.4$, $135.7,134.6,130.7,129.8,129.8,129.0,129.0,128.4,127.9,126.4,124.1,123.8,122.4,121.9,120.8$, 120.7, 119.3, 119.3, 118.9, 111.5, 111.5, 110.4, 53.4, 53.4, 32.6, 21.3; IR (film): 3385, 2900, 2850, 1743, 1644, 1605, 1576, 1449, 1318, 1281, 1257, 1162, 967, 763, 738, $715 \mathrm{~cm}^{-1}$; HRMS (DART) m/z: $[\mathrm{M}+\mathrm{Na}]^{+}$Calcd. for $\mathrm{C}_{25} \mathrm{H}_{21} \mathrm{NNaO}_{6} 454.1261$; Found 454.1261

Dimethyl (E)-4-acetyl-3-(4-methoxystyryl)pyrano[4,3-b]indole-1,1(5H)-dicarboxylate (3ir) and Dimethyl (E)-4-(3-(4-methoxyphenyl)acryloyl)-3-methylpyrano[4,3-b]indole-1,1(5H)dicarboxylate (3ir')
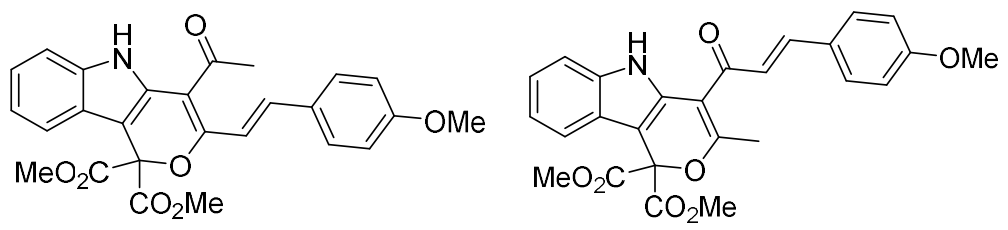

Prepared according to the
general procedure (D).
hexane:EA $=4: 1,28.6 \mathrm{mg}$,
$0.062 \mathrm{mmol}, 62 \%$ total yield,

red solid, m.p. $155-160{ }^{\circ} \mathrm{C}$; ${ }^{1} \mathrm{H}$ NMR $\left(400 \mathrm{MHz}, \mathrm{CDCl}_{3}\right) \delta=10.11(\mathrm{~s}, 1.2 \mathrm{H}), 9.73(\mathrm{~s}, 0.9 \mathrm{H}), 7.66(\mathrm{~d}, J$ $=15.4 \mathrm{~Hz}, 2.2 \mathrm{H}), 7.54(\mathrm{t}, J=8.7 \mathrm{~Hz}, 7 \mathrm{H}), 7.36(\mathrm{~d}, J=1.1 \mathrm{~Hz}, 2.5 \mathrm{H}), 7.20-7.10(\mathrm{~m}, 7.3 \mathrm{H}), 7.00-6.90$ $(\mathrm{m}, 5.7 \mathrm{H}), 3.86(\mathrm{~s}, 9 \mathrm{H}), 3.85(\mathrm{~s}, 12 \mathrm{H}), 2.64(\mathrm{~s}, 4 \mathrm{H}), 2.49(\mathrm{~s}, 3 \mathrm{H}) ;{ }^{13} \mathrm{C} \mathrm{NMR}\left(100 \mathrm{MHz}, \mathrm{CDCl}_{3}\right) \delta=197.4$, 190.4, 167.4, 167.1, 162.1, 161.9, 161.1, 158.9, 143.7, 139.6, 136.8, 136.4, 130.3, 130.2, 129.8, 129.5, $128.5,127.3,124.1,124.1,123.8,122.2,121.9,120.7,120.7,119.3,119.2,116.7,114.5,114.5,111.5$, 109.9, 109.7, 98.9, 55.5, 55.4, 53.4, 53.4, 32.6, 21.2; IR (film): 3426, 2838, 1637, 1597, 1431, 1314, 1245, 1173, 985, 834, $755 \mathrm{~cm}^{-1}$; HRMS (DART) m/z: $[\mathrm{M}+\mathrm{H}]^{+}$Calcd. for $\mathrm{C}_{26} \mathrm{H}_{23} \mathrm{NO}_{7}$ 461.1547; Found 461.1547

Dimethyl (E)-4-acetyl-3-(4-nitrostyryl)pyrano[4,3-b]indole-1,1(5H)-dicarboxylate $\quad(3 i s)$ and Dimethyl (E)-3-methyl-4-(3-(4-nitrophenyl)acryloyl)pyrano[4,3-b]indole-1,1(5H)-dicarboxylate (3is') 

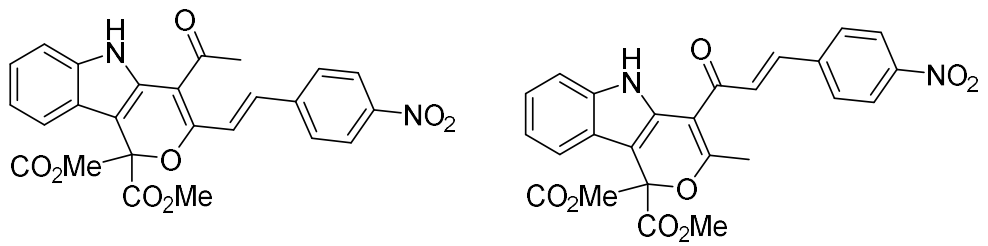

Prepared according to the
general procedure
hexane:EA $=7: 3,26.2 \mathrm{mg}$,
$0.055 \mathrm{mmol}, 55 \%$ total yield, red solid, m.p. $165-170{ }^{\circ} \mathrm{C} ;{ }^{1} \mathrm{H}$ NMR $\left(400 \mathrm{MHz}, \mathrm{CDCl}_{3}\right) \delta=9.95$ (s, $\left.1 \mathrm{H}\right), 9.78(\mathrm{~s}, 0.5 \mathrm{H}), 8.25(\mathrm{dd}, J=$ $12.9,8.8 \mathrm{~Hz}, 3 \mathrm{H}), 7.75-7.63(\mathrm{~m}, 5 \mathrm{H}), 7.56-7.49(\mathrm{~m}, 1.5 \mathrm{H}), 7.43-7.33(\mathrm{~m}, 3 \mathrm{H}), 7.23-7.11(\mathrm{~m}, 4 \mathrm{H})$, $3.88(\mathrm{~s}, 6 \mathrm{H}), 3.86(\mathrm{~s}, 3 \mathrm{H}), 2.67(\mathrm{~s}, 3 \mathrm{H}), 2.53(\mathrm{~s}, \mathrm{H}) ;{ }^{13} \mathrm{C} \mathrm{NMR}\left(100 \mathrm{MHz}, \mathrm{CDCl}_{3}\right) \delta=197.2,189.4$, 167.2 , 166.9, 163.8, 156.0, 148.6, 147.9, 141.9, 140.7, 140.1, 137.2, 136.4, 136.0, 129.9, 129.2, 129.1, $128.9,128.2,124.3,124.2,123.9,123.7,123.0,122.6,122.2,121.0,120.9,119.6,119.4,112.1,111.6$, 111.5, 110.0, 100.3, 97.1, 85.4, 84.6, 53.6, 53.5, 32.7, 21.6; IR (film): 3388, 2850, 1742, 1634, 1596, 1519, 1339, 1281, 1170, 965, 836, 784, $750 \mathrm{~cm}^{-1}$; HRMS (DART) m/z: $[\mathrm{M}+\mathrm{Na}]^{+}$Calcd. for $\mathrm{C}_{25} \mathrm{H}_{20} \mathrm{~N}_{2} \mathrm{NaO}_{8} 499.1112$; Found 499.1112

Dimethyl (E)-4-acetyl-3-(4-chlorostyryl)pyrano[4,3-b]indole-1,1(5H)-dicarboxylate $(3 i t)$ and Dimethyl (E)-4-(3-(4-chlorophenyl)acryloyl)-3-methylpyrano[4,3-b] indole-1,1(5H)-dicarboxylate $\left(3 i^{\prime}\right)$
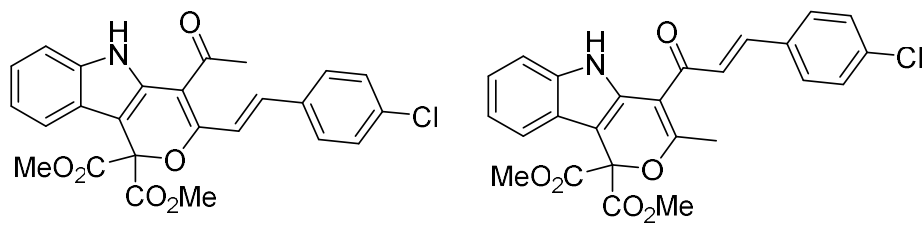

Prepared according to the general procedure (D). hexane:EA $=4: 1$, $32.6 \mathrm{mg}, 0.07 \mathrm{mmol}, 70 \%$ total yield, red solid, m.p. $125-130{ }^{\circ} \mathrm{C} ;{ }^{1} \mathrm{H}$ NMR

$\left(400 \mathrm{MHz}, \mathrm{CDCl}_{3}\right) \delta=10.04(\mathrm{~s}, 1 \mathrm{H}), 9.75(\mathrm{~s}, 0.8 \mathrm{H}), 7.64(\mathrm{~d}, J=5.6 \mathrm{~Hz}, 0.8 \mathrm{H}), 7.60(\mathrm{~d}, J=6.0 \mathrm{~Hz}$, 1H), $7.54-7.47(\mathrm{~m}, 5.6 \mathrm{H}), 7.40-7.35(\mathrm{~m}, 5.7 \mathrm{H}), 7.22(\mathrm{~s}, 0.7 \mathrm{H}), 7.21-7.10(\mathrm{~m}, 4 \mathrm{H}), 7.06(\mathrm{~d}, J=15.8$ $\mathrm{Hz}, 1 \mathrm{H}), 3.86(\mathrm{~s}, 6 \mathrm{H}), 3.85$ (s, 5H), $2.64(\mathrm{~s}, 3 \mathrm{H}), 2.50$ (s, 2H); ${ }^{13} \mathrm{C} \mathrm{NMR}\left(100 \mathrm{MHz}, \mathrm{CDCl}_{3}\right) \delta=197.3$, 190.0, 167.2, 167.0, 157.5, 142.1, 138.0, 137.0, 136.6, 136.4, 135.6, 134.3, 133.0, 129.7, 129.5, 129.4, $129.3,129.2,128.9,126.7,123.8,122.5,122.0,120.8,120.7,119.4,119.3,119.3,111.5,111.5,110.7$, 99.4, 85.3, 53.4, 53.4, 32.6, 21.3; IR (film): 3389, 2920, 1748, 1607, 1566, 1490, 1318, 1254, 1168, 815, $744 \mathrm{~cm}^{-1}$; HRMS (DART) m/z: [M+Na] ${ }^{+}$Calcd. for $\mathrm{C}_{25} \mathrm{H}_{20} \mathrm{ClNNaO}_{6} 488.0871$; Found 488.0871

\section{Dimethyl (E)-4-acetyl-5-allyl-3-styrylpyrano[4,3-b]indole-1,1(5H)-dicarboxylate (3tq)}

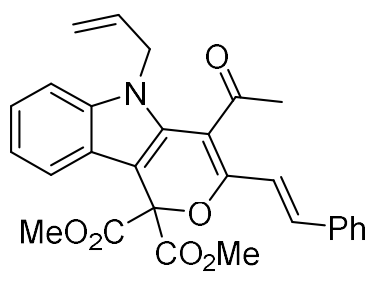

Prepared according to the general procedure (D). toluene:diethyl ether $=$ 19:1, $14.1 \mathrm{mg}, 0.03 \mathrm{mmol}, 30 \%$ yield (75\% total yield), red solid, m.p. $65-$ $70{ }^{\circ} \mathrm{C} ;{ }^{1} \mathrm{H}$ NMR $\left(400 \mathrm{MHz}, \mathrm{CDCl}_{3}\right) \delta=7.56-7.49(\mathrm{~m}, 4 \mathrm{H}), 7.38-7.30$ $(\mathrm{m}, 4 \mathrm{H}), 7.24-7.15(\mathrm{~m}, 2 \mathrm{H}), 7.11(\mathrm{~d}, J=15.6 \mathrm{~Hz}, 1 \mathrm{H}), 5.92-5.81(\mathrm{~m}$, 
1H), $5.20(\mathrm{dq}, J=10.6,1.5 \mathrm{~Hz}, 1 \mathrm{H}), 5.03(\mathrm{dq}, J=17.1,1.3 \mathrm{~Hz}, 1 \mathrm{H}), 4.61(\mathrm{dt}, J=4.9,1.8 \mathrm{~Hz}, 2 \mathrm{H}), 3.88$ (s, 6H), $2.46(\mathrm{~s}, 3 \mathrm{H}) ;{ }^{13} \mathrm{C} \mathrm{NMR}\left(100 \mathrm{MHz}, \mathrm{CDCl}_{3}\right) \delta=199.4,167.6,150.6,139.2,136.9,136.2,132.4$, 131.0, 129.0, 128.7, 127.7, 124.33, 122.6, 121.4, 119.2, 117.7, 117.7, 112.4, 110.7, 103.3, 84.2, 53.4, 47.6, 32.7; IR (film): 2920, 2850, 1745, 1598, 1458, 1314, 1247, 1193, 979, 743, $692 \mathrm{~cm}^{-1}$; HRMS (DART) $\mathrm{m} / \mathrm{z}$ : $[\mathrm{M}+\mathrm{H}]^{+}$Calcd. for $\mathrm{C}_{28} \mathrm{H}_{25} \mathrm{NO}_{6} 472.1755$; Found 472.1755

\section{Dimethyl 5-allyl-4-cinnamoyl-3-methylpyrano[4,3-b]indole-1,1(5H)-dicarboxylate (3tq')}

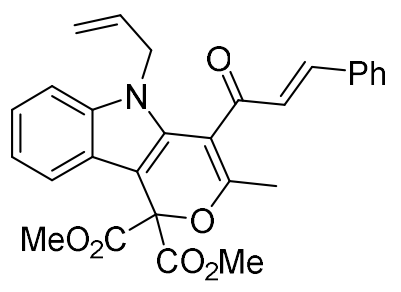

Prepared according to the general procedure (D). toluene:diethyl ether $=$ 19:1, $21.2 \mathrm{mg}, 0.045 \mathrm{mmol}, 45 \%$ yield (75\% total yield), red solid m.p. $65-70{ }^{\circ} \mathrm{C} ;{ }^{1} \mathrm{H}$ NMR $\left(400 \mathrm{MHz}, \mathrm{CDCl}_{3}\right) \delta=7.58(\mathrm{~d}, J=16.2 \mathrm{~Hz}, 1 \mathrm{H}), 7.52$ -7.46 (m, 3H), 7.39 (ddd, $J=5.2,4.3,2.3 \mathrm{~Hz}, 3 \mathrm{H}), 7.25-7.22(\mathrm{~m}, 1 \mathrm{H})$, $7.18-7.13(\mathrm{~m}, 2 \mathrm{H}), 6.88(\mathrm{~d}, J=16.1 \mathrm{~Hz}, 1 \mathrm{H}), 5.73(\mathrm{ddt}, J=17.2,10.2$, $4.9 \mathrm{~Hz}, 1 \mathrm{H}), 5.04(\mathrm{dq}, J=10.2,1.4 \mathrm{~Hz}, 1 \mathrm{H}), 4.89$ (dq, $J=17.3,1.6 \mathrm{~Hz}, 1 \mathrm{H}), 4.58(\mathrm{dt}, J=4.9,1.8 \mathrm{~Hz}$, 2H), $3.88(\mathrm{~s}, 6 \mathrm{H}), 2.21$ (s, 3H); ${ }^{13} \mathrm{C} \mathrm{NMR}\left(100 \mathrm{MHz}, \mathrm{CDCl}_{3}\right) \delta=192.5,167.6,155.8,146.7,138.4$, 134.1, 132.7, 131.0, 130.7, 129.0, 128.7, 128.6, 127.9, 124.1, 121.9, 121.0, 119.1, 117.2, 110.4, 108.9, 99.5, 84.8, 53.4, 47.2, 18.4; IR (film): 2920, 2850, 1745, 1598, 1458, 1314, 1247, 1193, 979, 743, 692 $\mathrm{cm}^{-1}$; HRMS (DART) m/z: [M+H] $]^{+}$Calcd. for $\mathrm{C}_{28} \mathrm{H}_{25} \mathrm{NO}_{6} 472.1755$; Found 472.1755

Dimethyl (E)-4-acetyl-5-(but-3-en-1-yl)-3-styrylpyrano[4,3-b]indole-1,1(5H)-dicarboxylate (3uq) and Dimethyl 5-(but-3-en-1-yl)-4-cinnamoyl-3-methylpyrano[4,3-b]indole-1,1(5H)-dicarboxylate (3uq')

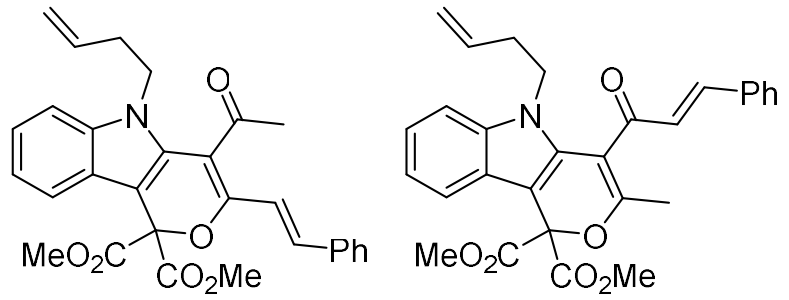

Prepared according to the general procedure (D). hexane:acetone $=4: 1,42.2 \mathrm{mg}, 0.087$ mmol, $87 \%$ yield (regioisomers mixture), yellow solid, m.p. $65-70{ }^{\circ} \mathrm{C} ;{ }^{1} \mathrm{H}$ NMR $(400$ $\left.\mathrm{MHz}, \mathrm{CDCl}_{3}\right) \delta=7.61(\mathrm{~d}, J=16.1 \mathrm{~Hz}, 1 \mathrm{H})$, $7.56-7.46$ (m, 6H), $7.43-7.26(\mathrm{~m}, 9 \mathrm{H}), 7.25-7.08(\mathrm{~m}, 5 \mathrm{H}), 6.93(\mathrm{~d}, J=16.1 \mathrm{~Hz}, 1 \mathrm{H}), 5.69-5.57$ $(\mathrm{m}, 2 \mathrm{H}), 5.03-4.97(\mathrm{~m}, 2 \mathrm{H}), 4.96(\mathrm{t}, J=1.2 \mathrm{~Hz}, 1 \mathrm{H}), 4.06-4.01(\mathrm{~m}, 1 \mathrm{H}), 4.00-3.94(\mathrm{~m}, 2 \mathrm{H}), 3.88$ (s, 6H), $3.87(\mathrm{~s}, 3 \mathrm{H}), 2.48(\mathrm{~s}, 1 \mathrm{H}), 2.40-2.31(\mathrm{~m}, 3 \mathrm{H}), 2.19(\mathrm{~s}, 3 \mathrm{H}) ;{ }^{13} \mathrm{C} \mathrm{NMR}\left(100 \mathrm{MHz}, \mathrm{CDCl}_{3}\right) \delta=$ 199.3, 192.8, 167.6, 167.6, 155.2, 150.3, 146.9, 138.7, 137.9, 136.8, 136.2, 134.1, 133.8, 133.4, 131.1, $130.4,129.0,128.9,128.7,127.7,127.7,124.5,124.1,122.5,121.8,121.2,120.9,119.4,119.2,117.9$, 117.6, 117.4, 110.3, 109.9, 108.8, 99.3, 84.2, 53.4, 53.4, 44.6, 44.2, 34.0, 33.8, 32.5, 18.3; IR (film): 2952, 1745, 1614, 1448, 1433, 1313, 1246, 1089, 920, 742, 691 $\mathrm{cm}^{-1}$; HRMS (DART) m/z: $[\mathrm{M}+\mathrm{H}]^{+}$ Calcd. for $\mathrm{C}_{29} \mathrm{H}_{28} \mathrm{NO}_{6} 486.1911$; Found 486.1911 


\section{General Procedure (E) and characterization of 2,3-dihydrofurans}

To a suspension of carbonyl compound ( $0.11 \mathrm{mmol}, 1.1$ equiv) and $\mathrm{K}_{3} \mathrm{PO}_{4}(0.3 \mathrm{mmol}, 3.0$ equiv) in anhydrous acetonitrile at room temperature was added $\mathrm{I}_{2}(0.11 \mathrm{mmol}, 1.1$ equiv) under a nitrogen atmosphere. Then a solution of enamine $(0.1 \mathrm{mmol}, 1.0$ equiv) in anhydrous acetonitrile was added under nitrogen and the reaction was stirred at room temperature. The reaction mixture was monitored by TLC until enamine was consumed, opened to air, quenched with 2-3 drops of $1 \mathrm{~N} \mathrm{Na}_{2} \mathrm{~S}_{2} \mathrm{O}_{3}$ solution, diluted with dichloromethane, and filtered over a pad of celite. The pad was rinsed with an additional dichloromethane, and the combined solutions were concentrated in vacuo. The crude material was purified by column chromatography to give the product.

\section{Diethyl 2-((2R,3S)-4-acetyl-2-(methoxycarbonyl)-5-methyl-3-(methyl(phenyl)amino)-2,3-} dihydrofuran-2-yl)malonate (5aa)

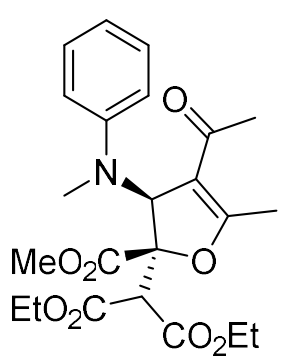

Prepared according to the general procedure $(\mathrm{E})$. hexane:EA $=7: 3,33.6 \mathrm{mg}$, $0.075 \mathrm{mmol}, 75 \%$ yield; Yellow oil; ${ }^{1} \mathrm{H}$ NMR $\left(400 \mathrm{MHz}, \mathrm{CDCl}_{3}\right) \delta=7.29-7.23$ $(\mathrm{m}, 2 \mathrm{H}), 6.88(\mathrm{~d}, \mathrm{~J}=8.3 \mathrm{~Hz}, 2 \mathrm{H}), 6.81(\mathrm{t}, \mathrm{J}=7.3 \mathrm{~Hz}, 1 \mathrm{H}), 5.75(\mathrm{~s}, 1 \mathrm{H}), 4.35-$ $4.30(\mathrm{~m}, 1 \mathrm{H}), 4.25(\mathrm{~s}, 1 \mathrm{H}), 4.23-4.10(\mathrm{~m}, 3 \mathrm{H}), 3.55(\mathrm{~s}, 3 \mathrm{H}), 2.64(\mathrm{~s}, 3 \mathrm{H}), 2.39$ (s, 3H), $1.85(\mathrm{~s}, 3 \mathrm{H}), 1.28(\mathrm{~d}, \mathrm{~J}=7.1 \mathrm{~Hz}, 3 \mathrm{H}), 1.23(\mathrm{~d}, \mathrm{~J}=7.2 \mathrm{~Hz}, 3 \mathrm{H}) ;{ }^{13} \mathrm{C}$ NMR $\left(100 \mathrm{MHz}, \mathrm{CDCl}_{3}\right) \delta=194.7,170.5,167.6,166.0,165.9,149.3,129.4,118.6$, $113.5,111.5,91.2,69.2,62.5,62.4,57.0,52.7,32.7,29.4,15.1,14.1,14.0 ;$ IR (film): 2980, 1728, 1678, 1598, 1434, 1311, 1204, 750, $693 \mathrm{~cm}^{-1}$; HRMS (DART) m/z: $[\mathrm{M}+\mathrm{H}]^{+} \mathrm{Calcd}$. for $\mathrm{C}_{23} \mathrm{H}_{30} \mathrm{NO}_{8} 448.1966$; Found 448.1978

\section{Diethyl 2-((2R,3S)-4-acetyl-2-(methoxycarbonyl)-5-methyl-3-(methyl(m-tolyl)amino)-2,3-} dihydrofuran-2-yl)malonate (5ba)

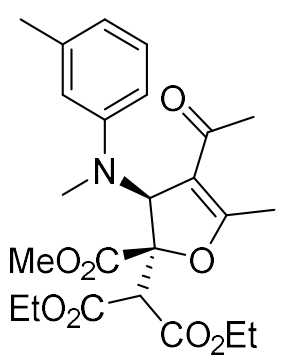

Prepared according to the general procedure (E). hexane:EA = 7:3, $34.1 \mathrm{mg}, 0.074$ mmol, 74\% yield; Colorless oil; ${ }^{1} \mathrm{H}$ NMR (400 MHz, $\left.\mathrm{CDCl}_{3}\right) \delta=7.18-7.10(\mathrm{~m}$, $1 \mathrm{H}), 6.73-6.61(\mathrm{~m}, 3 \mathrm{H}), 5.73(\mathrm{~s}, 1 \mathrm{H}), 4.35-4.11(\mathrm{~m}, 5 \mathrm{H}), 3.56(\mathrm{~s}, 3 \mathrm{H}), 2.62(\mathrm{~s}$, $3 \mathrm{H}), 2.38(\mathrm{~s}, 3 \mathrm{H}), 2.33(\mathrm{~s}, 3 \mathrm{H}), 1.84(\mathrm{~s}, 3 \mathrm{H}), 1.29(\mathrm{t}, \mathrm{J}=7.1 \mathrm{~Hz}, 3 \mathrm{H}), 1.24(\mathrm{t}, \mathrm{J}=$ $7.1 \mathrm{~Hz}, 3 \mathrm{H}) ;{ }^{13} \mathrm{C} \mathrm{NMR}\left(100 \mathrm{MHz}, \mathrm{CDCl}_{3}\right) \delta=194.6,170.2,167.5,165.8,165.8$, 149.3, 138.8, 129.0, 119.4, 114.0, 111.4, 110.7, 91.1, 69.0, 62.3, 62.3, 56.8, 52.6, 32.6, 29.4, 21.9, 15.0, 13.9, 13.9; IR (film): 2981, 1728, 1678, 1600, 1495, 1304, 1218, 937, $628 \mathrm{~cm}^{-1}$; 
HRMS (DART) m/z: [M+H] $]^{+}$Calcd. for $\mathrm{C}_{24} \mathrm{H}_{32} \mathrm{NO}_{8}$ 462.2122; Found 462.2131

Diethyl 2-((2R,3S)-4-acetyl-2-(methoxycarbonyl)-5-methyl-3-(methyl(p-tolyl)amino)-2,3dihydrofuran-2-yl)malonate (5ca)

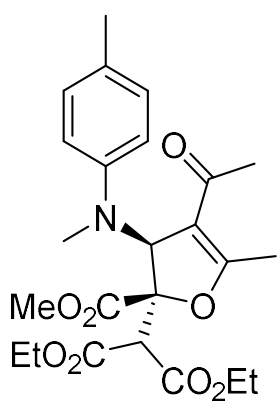

Prepared according to the general procedure (E). hexane:EA $=7: 3,24.4 \mathrm{mg}, 53 \%$ yield; Colorless oil; ${ }^{1} \mathrm{H}$ NMR (400 MHz, $\left.\mathrm{CDCl}_{3}\right) \delta=7.07$ (d, J = 8.4 Hz, 2H), $6.78(\mathrm{~d}, \mathrm{~J}=8.5 \mathrm{~Hz}, 2 \mathrm{H}), 5.68(\mathrm{~s}, 1 \mathrm{H}), 4.32(\mathrm{dq}, \mathrm{J}=10.6,7.3 \mathrm{~Hz}, 2 \mathrm{H}), 4.25(\mathrm{~s}$, $1 \mathrm{H}), 4.18-4.10(\mathrm{~m}, 2 \mathrm{H}), 3.58(\mathrm{~s}, 3 \mathrm{H}), 2.61(\mathrm{~s}, 3 \mathrm{H}), 2.38(\mathrm{~s}, 3 \mathrm{H}), 2.26(\mathrm{~s}, 3 \mathrm{H})$, $1.84(\mathrm{~s}, 3 \mathrm{H}), 1.28(\mathrm{~d}, \mathrm{~J}=7.1 \mathrm{~Hz}, 3 \mathrm{H}), 1.23(\mathrm{t}, \mathrm{J}=7.1 \mathrm{~Hz}, 3 \mathrm{H}) ;{ }^{13} \mathrm{C} \mathrm{NMR}(100$ $\left.\mathrm{MHz}, \mathrm{CDCl}_{3}\right) \delta=194.6,170.2,167.6,165.8,165.8,147.2,129.7,127.6,113.4$, $111.4,91.2,69.5,62.3,62.3,56.8,52.6,32.6,29.4,20.3,14.9,13.9,13.9$; IR (film): 2980, 1728, 1678, 1607, 1434, 1305, 1230, 1202, 806, $631 \mathrm{~cm}^{-1}$; HRMS (DART) m/z: $[\mathrm{M}+\mathrm{H}]^{+}$ Calcd. for $\mathrm{C}_{24} \mathrm{H}_{32} \mathrm{NO}_{8} 462.2122$; Found 462.2092

Diethyl 2-((2R,3S)-4-acetyl-2-(methoxycarbonyl)-3-((4-methoxyphenyl)(methyl)amino)-5methyl-2,3-dihydrofuran-2-yl)malonate (5da)

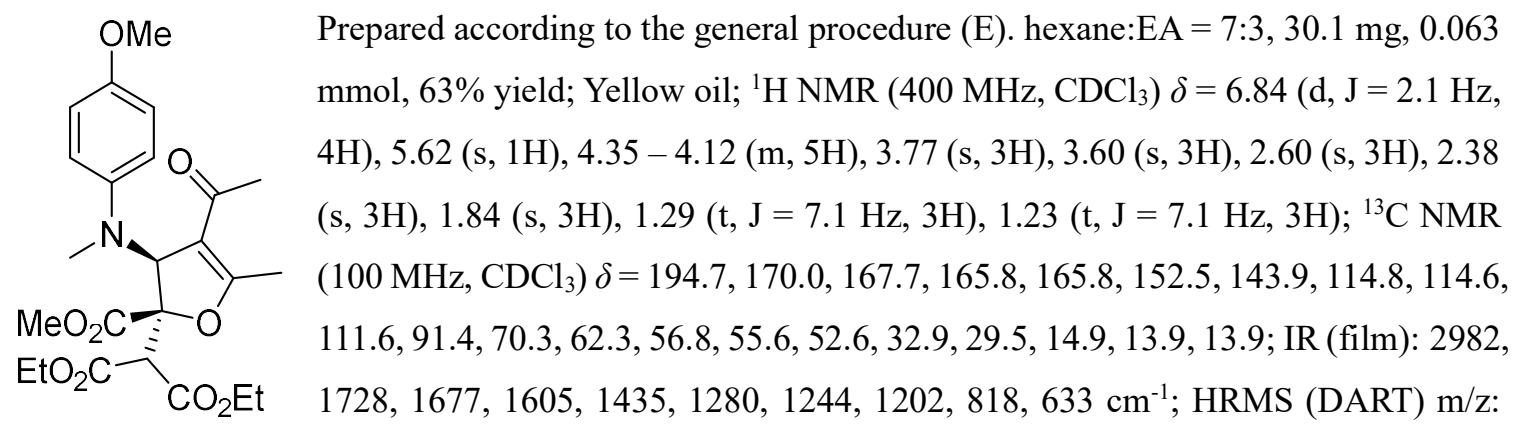

$[\mathrm{M}+\mathrm{H}]^{+}$Calcd. for $\mathrm{C}_{24} \mathrm{H}_{32} \mathrm{NO}_{9} 478.2072$; Found 478.2090

Diethyl 2-((2R,3S)-4-acetyl-3-((4-bromophenyl)(methyl)amino)-2-(methoxycarbonyl)-5-methyl-<smiles>CCOC(=O)C(C(C)=O)[C@@H]1OC(C)=C(C(C)=O)[C@@H]1N(C)c1ccc(Br)cc1</smiles2,3-dihydrofuran-2-yl)malonate (5ea)

Prepared according to the general procedure (E). $76 \%$ yield; Yellow oil; ${ }^{1} \mathrm{H}$ NMR $\left(400 \mathrm{MHz}, \mathrm{CDCl}_{3}\right) \delta=7.33(\mathrm{~d}, \mathrm{~J}=9.2 \mathrm{~Hz}, 2 \mathrm{H}), 6.75(\mathrm{~d}, \mathrm{~J}=9.1 \mathrm{~Hz}, 2 \mathrm{H}), 5.70(\mathrm{~s}$, 1H), $4.32-4.12$ (m, 5H), 3.57 (s, 3H), 2.59 (s, 3H), 2.38 (s, 3H), $1.86(\mathrm{~s}, 3 \mathrm{H}), 1.29$ $(\mathrm{t}, \mathrm{J}=7.1 \mathrm{~Hz}, 3 \mathrm{H}), 1.23(\mathrm{t}, \mathrm{J}=7.1 \mathrm{~Hz}, 3 \mathrm{H}) ;{ }^{13} \mathrm{C} \mathrm{NMR}\left(100 \mathrm{MHz}, \mathrm{CDCl}_{3}\right) \delta=194.1$, $170.3,167.4,165.7,165.7,148.2,132.0,115.0,111.3,110.5,91.0,68.8,62.4,62.4$, 56.7, 52.7, 32.7, 29.3, 15.0, 13.9, 13.9; IR (film): 2979, 1728, 1679, 1608, 1492, 
1435, 1308, 1203, 812, 629, $517 \mathrm{~cm}^{-1}$; HRMS (DART) m/z: $[\mathrm{M}+\mathrm{H}]^{+}$Calcd. for $\mathrm{C}_{23} \mathrm{H}_{29} \mathrm{BrNO}_{8}$ 526.1071; Found 526.1065

Diethyl

2-((2R,3S)-4-acetyl-2-(methoxycarbonyl)-3-((4-

(methoxycarbonyl)phenyl)(methyl)amino)-5-methyl-2,3-dihydrofuran-2-yl)malonate (5fa)

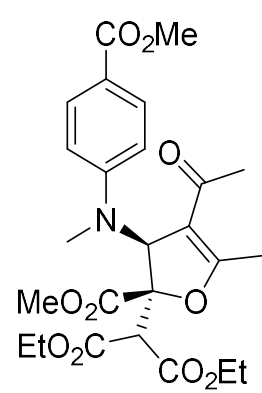

Prepared according to the general procedure $(\mathrm{E})$. hexane:EA $=3: 2,38.9 \mathrm{mg}$, $0.077 \mathrm{mmol}, 77 \%$ yield; Yellow oil; ${ }^{1} \mathrm{H}$ NMR $\left(400 \mathrm{MHz}, \mathrm{CDCl}_{3}\right) \delta=7.95(\mathrm{~d}, \mathrm{~J}=$ $8.9 \mathrm{~Hz}, 2 \mathrm{H}), 6.89(\mathrm{~d}, \mathrm{~J}=8.9 \mathrm{~Hz}, 2 \mathrm{H}), 5.91(\mathrm{~s}, 1 \mathrm{H}), 4.37-4.21(\mathrm{~m}, 3 \mathrm{H}), 4.19$ (d, $\mathrm{J}=2.3 \mathrm{~Hz}, 1 \mathrm{H}), 4.19-4.09(\mathrm{~m}, 2 \mathrm{H}), 3.87(\mathrm{~s}, 3 \mathrm{H}), 3.51(\mathrm{~s}, 3 \mathrm{H}), 2.69(\mathrm{~s}, 3 \mathrm{H}), 2.40$ (s, 3H), $2.05(\mathrm{~s}, 1 \mathrm{H}), 1.88(\mathrm{~s}, 3 \mathrm{H}), 1.30(\mathrm{t}, \mathrm{J}=7.2 \mathrm{~Hz}, 3 \mathrm{H}), 1.25$ (d, J = $7.1 \mathrm{~Hz}$, $3 \mathrm{H}) ;{ }^{13} \mathrm{C} \mathrm{NMR}\left(100 \mathrm{MHz}, \mathrm{CDCl}_{3}\right) \delta=193.9,170.7,167.1,167.0,165.6,165.6$, $152.3,131.3,119.6,112.1,111.1,90.8,67.7,62.5,62.4,56.7,52.6,51.6,32.7$, 29.1, 15.0, 13.9, 13.9; IR (film): 2977, 1770, 1710, 1680, 1602, 1434, 1279, 1188, 1114, 832, $630 \mathrm{~cm}^{-}$ ${ }^{1}$; HRMS (DART) m/z: $[\mathrm{M}+\mathrm{H}]^{+}$Calcd. for $\mathrm{C}_{25} \mathrm{H}_{32} \mathrm{NO}_{10} 506.2021$; Found 506.2190

Diethyl

2-((2R,3S)-4-acetyl-2-(methoxycarbonyl)-5-methyl-3-(methyl(4-

(trifluoromethyl)phenyl)amino)-2,3-dihydrofuran-2-yl)malonate (5ga)

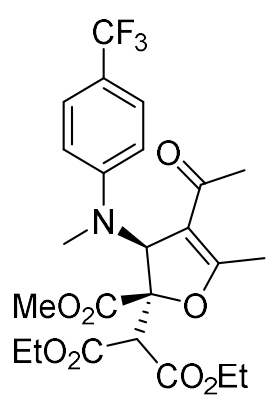

Prepared according to the general procedure (E). hexane:EA $=7: 3,40.7 \mathrm{mg}, 0.079$ mmol, 79\% yield; Colorless oil; ${ }^{1} \mathrm{H}$ NMR $\left(400 \mathrm{MHz}, \mathrm{CDCl}_{3}\right) \delta=7.50(\mathrm{~d}, \mathrm{~J}=8.5$ $\mathrm{Hz}, 2 \mathrm{H}), 6.93$ (d, J = 8.8 Hz, 2H), $5.86(\mathrm{~s}, 1 \mathrm{H}), 4.35-4.19$ (m, 3H), $4.18(\mathrm{~s}, 1 \mathrm{H})$, $4.17-4.11(\mathrm{~m}, 1 \mathrm{H}), 3.56(\mathrm{~s}, 3 \mathrm{H}), 2.67(\mathrm{~s}, 3 \mathrm{H}), 2.40(\mathrm{~d}, \mathrm{~J}=1.0 \mathrm{~Hz}, 3 \mathrm{H}), 1.89$ (s, $3 \mathrm{H}), 1.30(\mathrm{t}, \mathrm{J}=7.1 \mathrm{~Hz}, 3 \mathrm{H}), 1.26(\mathrm{~d}, \mathrm{~J}=7.1 \mathrm{~Hz}, 3 \mathrm{H}) ;{ }^{13} \mathrm{C} \mathrm{NMR}\left(100 \mathrm{MHz}, \mathrm{CDCl}_{3}\right)$ $\delta=193.8,170.5,167.3,165.7,165.6,151.3,126.6,126.6,126.5,126.5,126.1$, $123.4,120.5,120.1,119.8,119.5,117.1,112.5,111.3,90.9,68.1,62.5,62.4,56.7$, 52.7, 32.7, 29.1, 15.0, 13.9, 13.9; IR (film): 2983, 1729, 1680, 1612, 1436, 1318, 1253, 1200, 1153, 821, $628 \mathrm{~cm}^{-1}$; HRMS (DART) m/z: [M+H] ${ }^{+}$Calcd. for $\mathrm{C}_{24} \mathrm{H}_{29} \mathrm{~F}_{3} \mathrm{NO}_{8}$ 515.1840; Found 516.1842

Dimethyl (2R,3S)-2-(1,3-diethoxy-1,3-dioxopropan-2-yl)-5-methyl-3-(methyl(phenyl)amino)-2,3-<smiles>CCOC(=O)C(CC)[C@H]1OC(C)=C(C(C)=O)[C@H]1N(C)c1ccccc1</smilesdihydrofuran-2,4-dicarboxylate (5ai)

Prepared according to the general procedure (E). hexane:EA $=4: 1,38.0 \mathrm{mg}$, $0.082 \mathrm{mmol}, 82 \%$ yield; Colorless oil; ${ }^{1} \mathrm{H}$ NMR (400 MHz, $\left.\mathrm{CDCl}_{3}\right) \delta=7.23$ (t, $\mathrm{J}=7.6 \mathrm{~Hz}, 2 \mathrm{H}), 6.82(\mathrm{dd}, \mathrm{J}=14.5,7.8 \mathrm{~Hz}, 3 \mathrm{H}), 5.56(\mathrm{~s}, 1 \mathrm{H}), 4.33-4.11(\mathrm{~m}$, $5 \mathrm{H}), 3.59(\mathrm{~s}, 3 \mathrm{H}), 3.50(\mathrm{~s}, 3 \mathrm{H}), 2.59(\mathrm{~s}, 3 \mathrm{H}), 2.36(\mathrm{~s}, 3 \mathrm{H}), 1.28(\mathrm{t}, \mathrm{J}=7.1 \mathrm{~Hz}$, $3 \mathrm{H}), 1.21(\mathrm{t}, \mathrm{J}=7.1 \mathrm{~Hz}, 3 \mathrm{H}) ;{ }^{13} \mathrm{C} \mathrm{NMR}\left(100 \mathrm{MHz}, \mathrm{CDCl}_{3}\right) \delta=170.5,167.7$, $165.9,165.8,165.0,150.5,128.7,118.4,114.4,102.9,91.2,70.0,62.2,62.2,56.8,52.6,51.1,32.7,14.3$, 
13.9, 13.8; IR (film): 2952, 1731, 1705, 1654, 1597, 1434, 1311, 1191, 1146, 750, $693 \mathrm{~cm}^{-1}$; HRMS (DART) $\mathrm{m} / \mathrm{z}$ : $[\mathrm{M}+\mathrm{H}]^{+}$Calcd. for $\mathrm{C}_{23} \mathrm{H}_{30} \mathrm{NO}_{9} 464.1915$; Found 464.1915

Diethyl 2-((2R,3R)-2-(methoxycarbonyl)-5-methyl-3-(methyl(phenyl)amino)-4-(phenylsulfonyl)2,3-dihydrofuran-2-yl)malonate (5au)

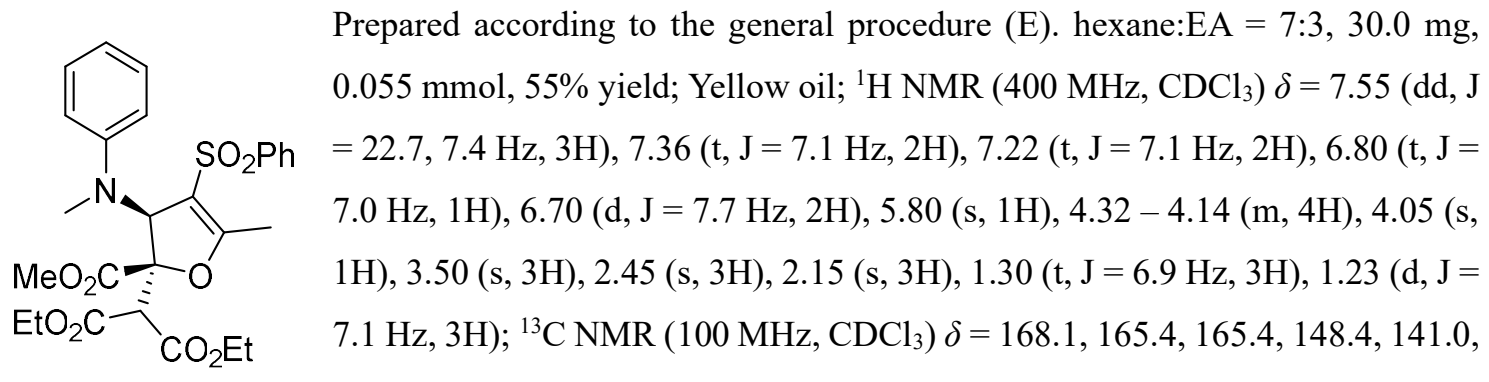
133.1, 129.0, 128.9, 127.6, 118.4, 113.7, 111.7, 90.7, 68.2, 62.5, 62.4, 56.7, 52.6, 31.9, 14.0, 13.9, 13.8; IR (film): 2981, 1729, 1637, 1597, 1446, 1318, 1255, 1204, 1157, 750, $688 \mathrm{~cm}^{-1}$; HRMS (DART) m/z: $[\mathrm{M}+\mathrm{H}]^{+}$Calcd. for $\mathrm{C}_{27} \mathrm{H}_{32} \mathrm{NO}_{9} \mathrm{~S} 546.1792$; Found 546.1800

Diethyl

2-((2R,3R)-4-(dimethoxyphosphoryl)-2-(methoxycarbonyl)-5-methyl-3(methyl(phenyl)amino)-2,3-dihydrofuran-2-yl)malonate (5an)

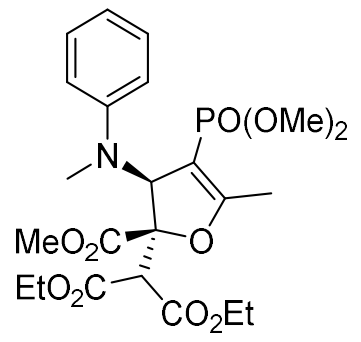

Prepared according to the general procedure (E). 100\% EA, $27.2 \mathrm{mg}, 0.053$ mmol, 53\% yield; Yellow oil; ${ }^{1} \mathrm{H}$ NMR (400 MHz, $\left.\mathrm{CDCl}_{3}\right) \delta=7.22(\mathrm{t}, \mathrm{J}=$ $7.9 \mathrm{~Hz}, 2 \mathrm{H}), 6.86(\mathrm{~d}, \mathrm{~J}=8.2 \mathrm{~Hz}, 2 \mathrm{H}), 6.76(\mathrm{t}, \mathrm{J}=7.3 \mathrm{~Hz}, 1 \mathrm{H}), 5.77(\mathrm{~d}, \mathrm{~J}=$ $2.9 \mathrm{~Hz}, 1 \mathrm{H}), 4.38-4.19(\mathrm{~m}, 4 \mathrm{H}), 4.06(\mathrm{~s}, 1 \mathrm{H}), 3.60(\mathrm{~d}, \mathrm{~J}=11.2 \mathrm{~Hz}, 3 \mathrm{H})$, $3.58(\mathrm{~s}, 3 \mathrm{H}), 3.52(\mathrm{~d}, \mathrm{~J}=11.2 \mathrm{~Hz}, 3 \mathrm{H}), 2.67(\mathrm{~s}, 3 \mathrm{H}), 2.29-2.26(\mathrm{~m}, 3 \mathrm{H})$, $1.29(\mathrm{td}, \mathrm{J}=7.1,1.4 \mathrm{~Hz}, 6 \mathrm{H}) ;{ }^{13} \mathrm{C} \mathrm{NMR}\left(100 \mathrm{MHz}, \mathrm{CDCl}_{3}\right) \delta=170.5,170.2$,

$167.5,165.8,165.8,149.6,128.8,118.1,114.0,97.6,95.4,91.4,91.2,70.3,70.2,62.3,62.2,56.6,52.5$, 52.4, 52.3, 52.3, 52.2, 32.7, 14.2, 13.9, 13.9; IR (film): 2953, 1729, 1644, 1597, 1434, 1311, 1245, 1203 , 1022, 750, $693 \mathrm{~cm}^{-1}$; HRMS (DART) m/z: $[\mathrm{M}+\mathrm{H}]^{+}$Calcd. for $\mathrm{C}_{23} \mathrm{H}_{33} \mathrm{NO}_{10} \mathrm{P} 514.1837$; Found 514.1841

\section{Synthesis applications}

\subsection{Sequential one-pot indolomalonate synthesis/pyran synthesis procedure}

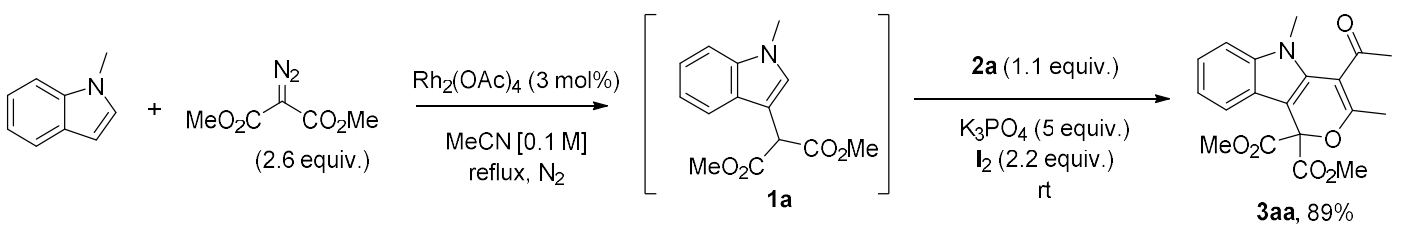


An oven-dried Schlenk flask equipped with a stir bar was sealed with a rubber septum and was connected to a Schlenk line, evacuated, and refilled with nitrogen (repeated three times). To a solution of $\mathrm{N}$-methyl indole $\left(0.1 \mathrm{mmol}, 1.0\right.$ equiv) and $\mathrm{Rh}_{2}(\mathrm{OAc})_{4}(0.003 \mathrm{mmol}, 3 \mathrm{~mol} \%)$ in anhydrous acetonitrile $(0.5 \mathrm{~mL})$ at room temperature was added a solution of diazo malonate $(0.22 \mathrm{mmol}, 2.2$ equiv) in anhydrous acetonitrile $(0.5 \mathrm{~mL})$ dropwise under a nitrogen atmosphere. The reaction mixture was stirred under $\mathrm{N}_{2}$ at reflux. After completion of the reaction, $\mathrm{K}_{3} \mathrm{PO}_{4}$ was added to the Schlenk flask. Next, carbonyl compound (2a, $0.11 \mathrm{mmol}, 1.1$ equiv) and $\mathrm{I}_{2}(0.22 \mathrm{mmol}, 2.2$ equiv) were added and the reaction mixture was stirred at room temperature. The reaction mixture was monitored by TLC until indole was consumed, opened to air, quenched with 2-3 drops of $1 \mathrm{~N} \mathrm{Na}_{2} \mathrm{~S}_{2} \mathrm{O}_{3}$ solution, diluted with ethyl acetate, and filtered over a pad of celite. The pad was rinsed with an additional ethyl acetate, and the combined solutions were concentrated in vacuo. The crude material was purified by column chromatography (hexane:EA $=7: 3)$ to give the product 3aa as a yellow solid $(31.8 \mathrm{mg}, 0.089 \mathrm{mmol}$, $89 \%$ yield).

\subsection{Gram scale reaction procedure}
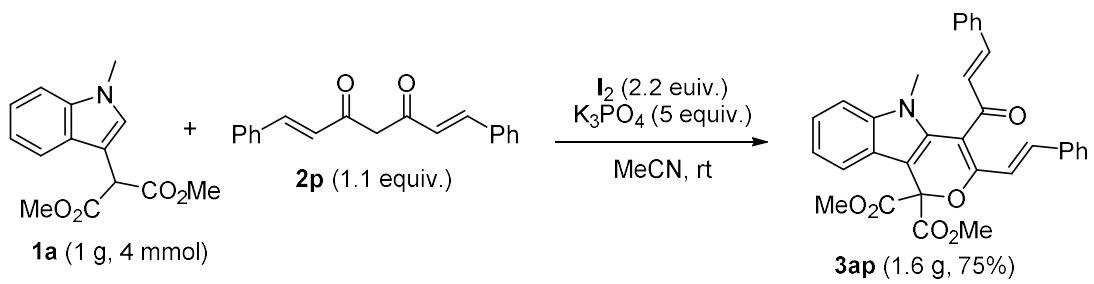

An oven-dried $100 \mathrm{~mL}$ Schlenk tube equipped with a stir bar was sealed with a rubber septum and was connected to a Schlenk line, evacuated, and refilled with nitrogen (repeated three times). To a solution of N-methyl indolomalonate (1a, $1.05 \mathrm{~g}, 4.0 \mathrm{mmol})$ and $\mathrm{K}_{3} \mathrm{PO}_{4}(4.25 \mathrm{~g}, 5.0 \mathrm{mmol})$ in anhydrous acetonitrile $(30 \mathrm{~mL})$ at room temperature was added a solution of activated methylene compound $(\mathbf{2} \mathbf{p}$, $1.22 \mathrm{~g}, 4.4 \mathrm{mmol})$ in anhydrous acetonitrile $(10 \mathrm{~mL})$ dropwise under a nitrogen atmosphere. $\mathrm{I}_{2}(2.23 \mathrm{~g}$, $8.8 \mathrm{mmol}$ ) was added under nitrogen and the reaction was stirred at room temperature. After completion of the reaction, the solvent was evaporated and $1 \mathrm{~N} \mathrm{Na}_{2} \mathrm{~S}_{2} \mathrm{O}_{3}$ solution was added, and the mixture was extracted with ethyl acetate three times. The combined organic solvent was dried over anhydrous $\mathrm{Na}_{2} \mathrm{SO}_{4}$, and concentrated in vacuo. The crude material was purified by flash chromatography (hexane:EA $=7: 3$ ) to give the product $3 \mathrm{ap}$ as a yellow solid (1.6 g, $3.0 \mathrm{mmol}, 75 \%$ yield).

\subsection{General procedure (A) for the metathesis reactions (6a and $6 \mathrm{~b})$}




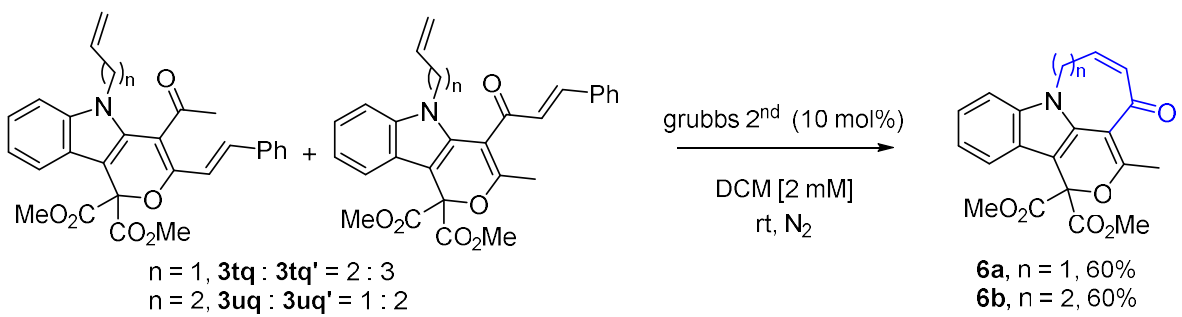

To a solution of dihyropyran substrate (regioisomers mixture, $0.1 \mathrm{mmol}, 1.0$ equiv) in anhydrous DCM $(50 \mathrm{~mL}, 2 \mathrm{mM})$ at room temperature was added grubbs $2^{\text {nd }}$ generation catalyst $(10 \mathrm{~mol} \%)$ under a nitrogen atmosphere. The reaction mixture was stirred at room temperature and monitored by TLC until dihyropyran was consumed, opened to air, diluted with ethyl acetate, and filtered over a pad of $\mathrm{SiO}_{2}$. The pad was rinsed with an additional ethyl acetate, and the combined solutions were concentrated in vacuo. The crude material was purified by column chromatography on silica gel to give the product.

\section{Dimethyl 3-methyl-4-oxo-4,7-dihydro-1H-2-oxa-7a-azacyclohepta[jk]fluorene-1,1-dicarboxylate} (6a)<smiles>CCOC(C)(C)C1(C(C)=O)OC(C)=C2C(=O)C=CCn3c2c1c1ccccc13</smiles>

Prepared according to the general procedure (A) using regioisomers mixture (3tq and 3tq'). toluene:diethyl ether $=19: 1,22.0 \mathrm{mg}, 0.06 \mathrm{mmol}, 60 \%$ yield, yellow solid, m.p. $175-180{ }^{\circ} \mathrm{C} ;{ }^{1} \mathrm{H}$ NMR $\left(400 \mathrm{MHz}, \mathrm{CDCl}_{3}\right) \delta=7.47(\mathrm{~d}, J=7.8$ $\mathrm{Hz}, 1 \mathrm{H}), 7.31(\mathrm{~d}, J=8.1 \mathrm{~Hz}, 1 \mathrm{H}), 7.28-7.12(\mathrm{~m}, 2 \mathrm{H}), 6.54(\mathrm{dt}, J=11.7,5.6$ $\mathrm{Hz}, 1 \mathrm{H}), 6.35(\mathrm{~d}, J=12.4 \mathrm{~Hz}, 1 \mathrm{H}), 4.79(\mathrm{~d}, J=5.5 \mathrm{~Hz}, 2 \mathrm{H}), 3.84(\mathrm{~s}, 6 \mathrm{H}), 2.62$ $(\mathrm{s}, 3 \mathrm{H}) ;{ }^{13} \mathrm{C} \mathrm{NMR}\left(100 \mathrm{MHz}, \mathrm{CDCl}_{3}\right) \delta=186.9,169.0,166.9,137.7,136.2,132.1,129.7,124.2,122.0$, 121.3, 119.2, 109.3, 108.9, 98.1, 84.8, 53.5, 43.1, 21.9; IR (film): 2954, 1745, 1670, 1632, 1557, 1287 , 1224, 1212, 735, $708 \mathrm{~cm}^{-1}$; HRMS (DART) m/z: $[\mathrm{M}+\mathrm{H}]^{+}$Calcd. for $\mathrm{C}_{20} \mathrm{H}_{18} \mathrm{NO}_{6}$ 368.1129; Found 368.1130

Dimethyl

(Z)-3-methyl-4-oxo-7,8-dihydro-1 $H, 4 H-2-o x a-8 a-a z a c y c l o o c t a[j k]$ fluorene-1,1dicarboxylate (6b)

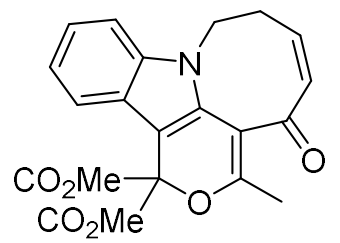

Prepared according to the general procedure (A) using regioisomers mixture (3uq and 3uq'). toluene:diethyl ether $=19: 1,23.0 \mathrm{mg}, 0.06 \mathrm{mmol}, 60 \%$ yield, yellow solid, m.p. $190-195{ }^{\circ} \mathrm{C} ;{ }^{1} \mathrm{H}$ NMR $\left(400 \mathrm{MHz}, \mathrm{CDCl}_{3}\right) \delta=7.55-7.48$ (m, 1H), $7.30(\mathrm{dd}, J=8.0,1.2 \mathrm{~Hz}, 1 \mathrm{H}), 7.26-7.12(\mathrm{~m}, 2 \mathrm{H}), 5.98$ (dt, $J=13.3$, $2.2 \mathrm{~Hz}, 1 \mathrm{H}), 5.74(\mathrm{dt}, J=13.4,3.8 \mathrm{~Hz}, 1 \mathrm{H}), 4.20(\mathrm{dd}, J=6.2,4.6 \mathrm{~Hz}, 2 \mathrm{H}), 3.84(\mathrm{~s}, 6 \mathrm{H}), 2.73-2.65(\mathrm{~m}$, 2H), 2.37 (s, 3H); ${ }^{13} \mathrm{C}$ NMR (100 MHz, $\left.\mathrm{CDCl}_{3}\right) \delta=194.4,167.1,161.1,136.9,130.7,130.0,129.5$, 124.0, 121.9, 121.0, 119.5, 109.4, 108.9, 97.3, 85.1, 53.4, 40.5, 31.7, 18.4; IR (film): 2956, 1747, 1671, 
1614, 1597, 1275, 1213, 785, $745 \mathrm{~cm}^{-1}$; HRMS (DART) m/z: $[\mathrm{M}+\mathrm{H}]^{+}$Calcd. for $\mathrm{C}_{21} \mathrm{H}_{20} \mathrm{NO}_{6} 382.1285$;

Found 382.1288

\subsection{General procedure $(B)$ for the synthesis of cyclobutane derivatives $(7 a-7 c)$}

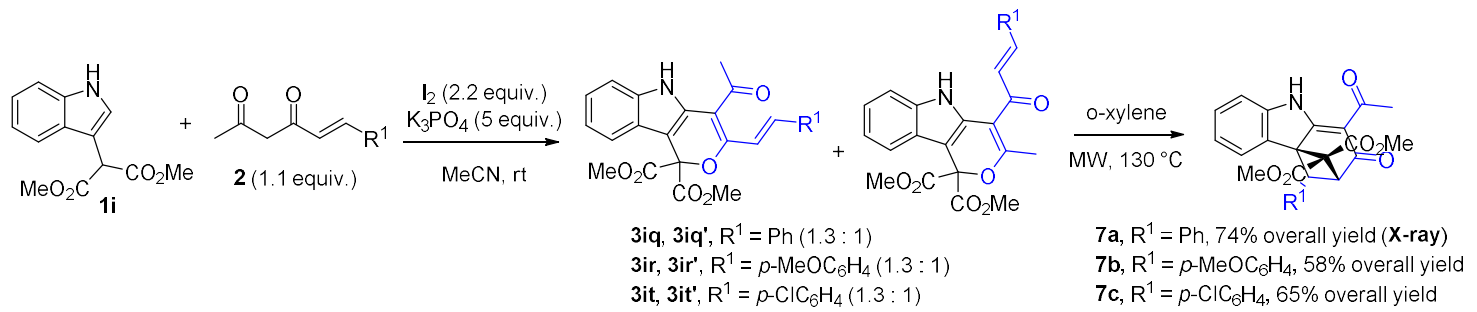

To a solution of indole $1 \mathbf{i}(0.1 \mathrm{mmol}, 1.0$ equiv $)$ and $\mathrm{K}_{3} \mathrm{PO}_{4}(0.5 \mathrm{mmol}, 5.0$ equiv) in anhydrous acetonitrile $(1 \mathrm{~mL}, 0.1 \mathrm{M})$ at room temperature was added carbonyl compound $\mathbf{2}(0.11 \mathrm{mmol}, 1.1$ equiv) dropwise under a nitrogen atmosphere. $\mathrm{I}_{2}(0.22 \mathrm{mmol}, 2.2$ equiv) was added under nitrogen and the reaction was stirred at room temperature. The reaction mixture was monitored by TLC until indole was consumed, opened to air, quenched with 2-3 drops of $1 \mathrm{~N} \mathrm{Na}_{2} \mathrm{~S}_{2} \mathrm{O}_{3}$ solution, diluted with ethyl acetate, and filtered over a pad of celite. The pad was rinsed with an additional ethyl acetate, and the combined solutions were concentrated in vacuo. Without further purification, the crude mixture was used directly in the next step. The pyran and o-xylene $(1 \mathrm{~mL})$ were mixed in the microwave instrument vial. The vial was flushed with nitrogen and irradiated with stirring at $130{ }^{\circ} \mathrm{C}$ for $0.5 \mathrm{~h}$. Upon completion of the reaction, the crude material was purified by column chromatography on silica gel to give the product.

Dimethyl $\quad(3 R, 4 a R, 10 R)-1-a c e t y l-2-o x o-10-p h e n y l-2,9-d i h y d r o-3,4 a-m e t h a n o c a r b a z o l e-4,4(3 H)$ dicarboxylate (7a)

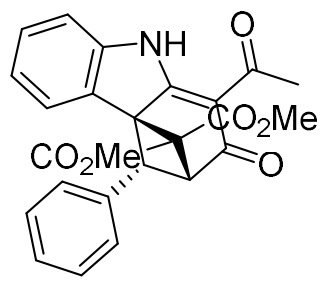

Prepared according to the general procedure (B) using 2q. hexane:EA $=7: 3$, $31.9 \mathrm{mg}, 0.074 \mathrm{mmol}, 74 \%$ overall yield, yellow solid, m.p. $180-185{ }^{\circ} \mathrm{C} ;{ }^{1} \mathrm{H}$ $\operatorname{NMR}\left(400 \mathrm{MHz}, \mathrm{CDCl}_{3}\right) \delta=11.18(\mathrm{~s}, 1 \mathrm{H}), 7.60(\mathrm{~d}, J=7.5 \mathrm{~Hz}, 1 \mathrm{H}), 7.46(\mathrm{t}, J$ $=7.7 \mathrm{~Hz}, 1 \mathrm{H}), 7.26-7.17(\mathrm{~m}, 2 \mathrm{H}), 7.10-7.03(\mathrm{~m}, 3 \mathrm{H}), 6.61-6.56(\mathrm{~m}, 2 \mathrm{H})$, $5.12(\mathrm{~d}, J=6.1 \mathrm{~Hz}, 1 \mathrm{H}), 4.10(\mathrm{~d}, J=6.2 \mathrm{~Hz}, 1 \mathrm{H}), 3.90$ (s, 3H), $3.56(\mathrm{~s}, 3 \mathrm{H})$, $2.36(\mathrm{~s}, 3 \mathrm{H}) ;{ }^{13} \mathrm{C} \mathrm{NMR}\left(100 \mathrm{MHz}, \mathrm{CDCl}_{3}\right) \delta=197.6,190.5,174.1,167.1,166.7,145.3,136.3,130.3$, 128.5, 127.8, 127.0, 125.8, 125.2, 124.4, 112.3, 103.9, 68.1, 62.4, 55.6, 53.5, 53.3, 52.9, 30.3; IR (film): 3294, 2922, 2852, 1751, 1715, 1668, 1634, 1448, 1280, 1199, 736, $692 \mathrm{~cm}^{-1}$; HRMS (DART) m/z:

$[\mathrm{M}+\mathrm{Na}]^{+}$Calcd. for $\mathrm{C}_{25} \mathrm{H}_{21} \mathrm{NNaO}_{6} 454.1267$; Found 454.1261 


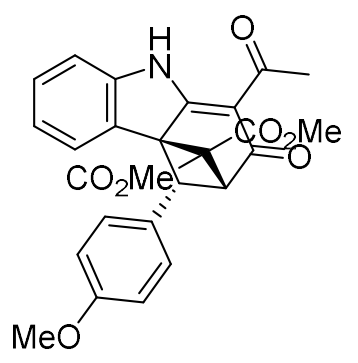

Prepared according to the general procedure (B) using 2r. hexane:EA $=1: 1$, $26.7 \mathrm{mg}, 0.058 \mathrm{mmol}, 58 \%$ overall yield, yellow solid, m.p. $185-190{ }^{\circ} \mathrm{C} ;{ }^{1} \mathrm{H}$ NMR (400 MHz, $\left.\mathrm{CDCl}_{3}\right) \delta=11.19(\mathrm{~s}, 1 \mathrm{H}), 7.58(\mathrm{~d}, J=7.5 \mathrm{~Hz}, 1 \mathrm{H}), 7.44(\mathrm{t}$, $J=7.7 \mathrm{~Hz}, 1 \mathrm{H}), 7.23$ (d, $J=7.6 \mathrm{~Hz}, 1 \mathrm{H}), 7.17$ (d, $J=7.9 \mathrm{~Hz}, 1 \mathrm{H}), 6.61-$ $6.56(\mathrm{~m}, 2 \mathrm{H}), 6.53-6.47(\mathrm{~m}, 2 \mathrm{H}), 5.04(\mathrm{~d}, J=6.1 \mathrm{~Hz}, 1 \mathrm{H}), 4.06$ (d, $J=6.1$ $\mathrm{Hz}, 1 \mathrm{H}), 3.89$ (s, 3H), 3.66 (s, 3H), 3.55 (s, 3H), 2.38 (s, 3H); ${ }^{13} \mathrm{C}$ NMR (100 $\left.\mathrm{MHz}, \mathrm{CDCl}_{3}\right) \delta=197.6,190.7,174.2,167.1,166.7,158.3,145.3,130.2,128.2,127.8,126.9,125.2$, 124.4, 113.9, 112.3, 104.0, 68.2, 62.4, 55.6, 55.1, 53.5, 52.9, 52.9, 30.4; IR (film): 3276, 2957, 1726, $1638,1615,1462,1277,1234,1188,846,748 \mathrm{~cm}^{-1}$; HRMS (DART) m/z: $[\mathrm{M}+\mathrm{H}]^{+}$Calcd. for $\mathrm{C}_{26} \mathrm{H}_{23} \mathrm{NO}_{7}$ 461.1475; Found 462.1547

Dimethyl (3R,4aR,10R)-1-acetyl-10-(4-chlorophenyl)-2-oxo-2,9-dihydro-3,4a-methanocarbazole4,4(3H)-dicarboxylate $(7 \mathrm{c})$

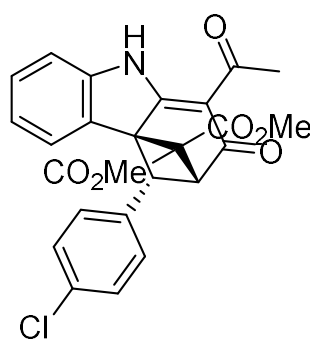

Prepared according to the general procedure (B) using 2t. hexane:EA $=7: 3$, $30.2 \mathrm{mg}, 0.065 \mathrm{mmol}, 65 \%$ overall yield, yellow solid, m.p. $200-205{ }^{\circ} \mathrm{C} ;{ }^{1} \mathrm{H}$ $\operatorname{NMR}\left(400 \mathrm{MHz}, \mathrm{CDCl}_{3}\right) \delta=11.21(\mathrm{~s}, 1 \mathrm{H}), 7.60(\mathrm{~d}, J=7.5 \mathrm{~Hz}, 1 \mathrm{H}), 7.46$ (t, $J$ $=7.8 \mathrm{~Hz}, 1 \mathrm{H}), 7.29-7.25(\mathrm{~m}, 1 \mathrm{H}), 7.20(\mathrm{~d}, J=7.9 \mathrm{~Hz}, 1 \mathrm{H}), 7.04(\mathrm{~d}, J=7.8$ $\mathrm{Hz}, 2 \mathrm{H}), 6.52(\mathrm{~d}, J=8.2 \mathrm{~Hz}, 2 \mathrm{H}), 5.08(\mathrm{~d}, J=6.1 \mathrm{~Hz}, 1 \mathrm{H}), 4.07$ (d, $J=6.1 \mathrm{~Hz}$, $1 \mathrm{H}), 3.91(\mathrm{~s}, 3 \mathrm{H}), 3.57(\mathrm{~s}, 3 \mathrm{H}), 2.39(\mathrm{~s}, 3 \mathrm{H}) ;{ }^{13} \mathrm{C} \mathrm{NMR}\left(100 \mathrm{MHz}, \mathrm{CDCl}_{3}\right) \delta=$ $197.5,190.3,173.9,167.0,166.6,145.3,134.9,132.9,130.5,128.7,127.5,127.2,125.2,124.5,112.5$, 103.8, 68.1, 62.3, 55.5, 53.6, 52.9, 52.7, 30.4; IR (film): 3254, 1743, 1732, 1675, 1613, 1461, 1273, 1198, 842, 789, $752 \mathrm{~cm}^{-1}$; HRMS (DART) m/z: $[\mathrm{M}+\mathrm{H}]^{+}$Calcd. for $\mathrm{C}_{25} \mathrm{H}_{20} \mathrm{ClNO}_{6}$ 465.0979; Found 466.1052 


\section{Mechanistic studies}

\subsection{Identification of intermediates along the reaction pathway.}

Exp. 1. Identification of intermediate 3'aa by employing 1.1 equivalents of $\mathrm{I}_{2}$.

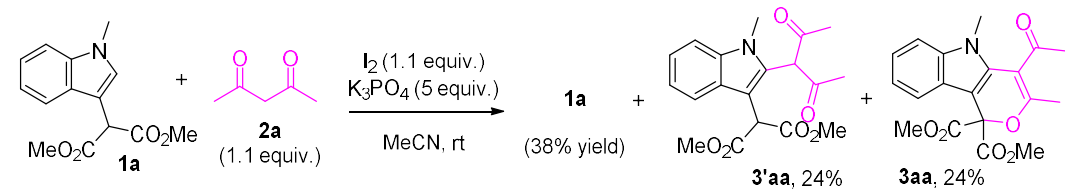

Exp. 2. Confirmation of $\mathbf{3}^{\prime} \mathbf{a a}$ as an intermediate by subjecting $\mathbf{3}^{\prime} \mathbf{a a}$ to the reaction conditions.

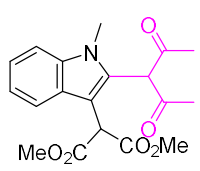

3'aa

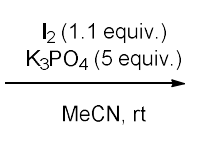

CN, it

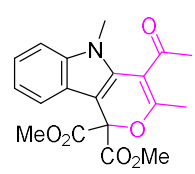

3aa, $97 \%$

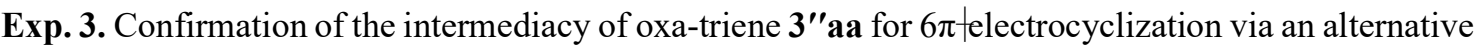
oxidation method.
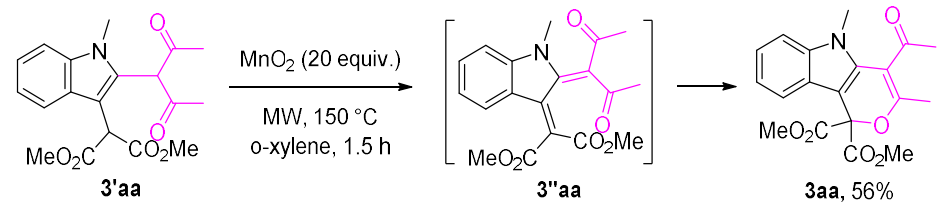

Exp. 4. Confirmation of the intermediacy of iododiketone 8a by reaction with $1 \mathbf{a}$ in the absence of $\mathrm{I}_{2}$ to afford a mixture of $\mathbf{3}$ 'ab and $\mathbf{3 a b}$.
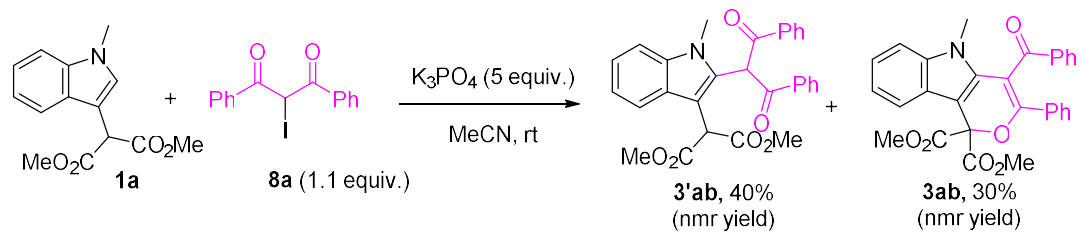

- The in situ formation of molecular iodine during the reaction is responsible for the formation of pyran 3ab, which was confirmed by NMR study (Exp. 5).

Exp. 5. NMR experiment to identify in situ formation of molecular iodine.

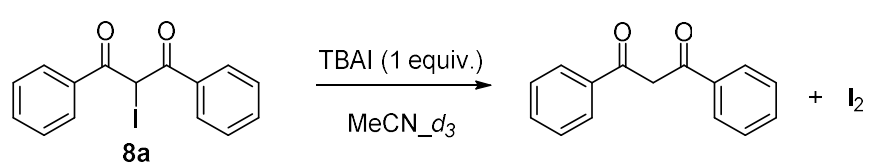


(a)

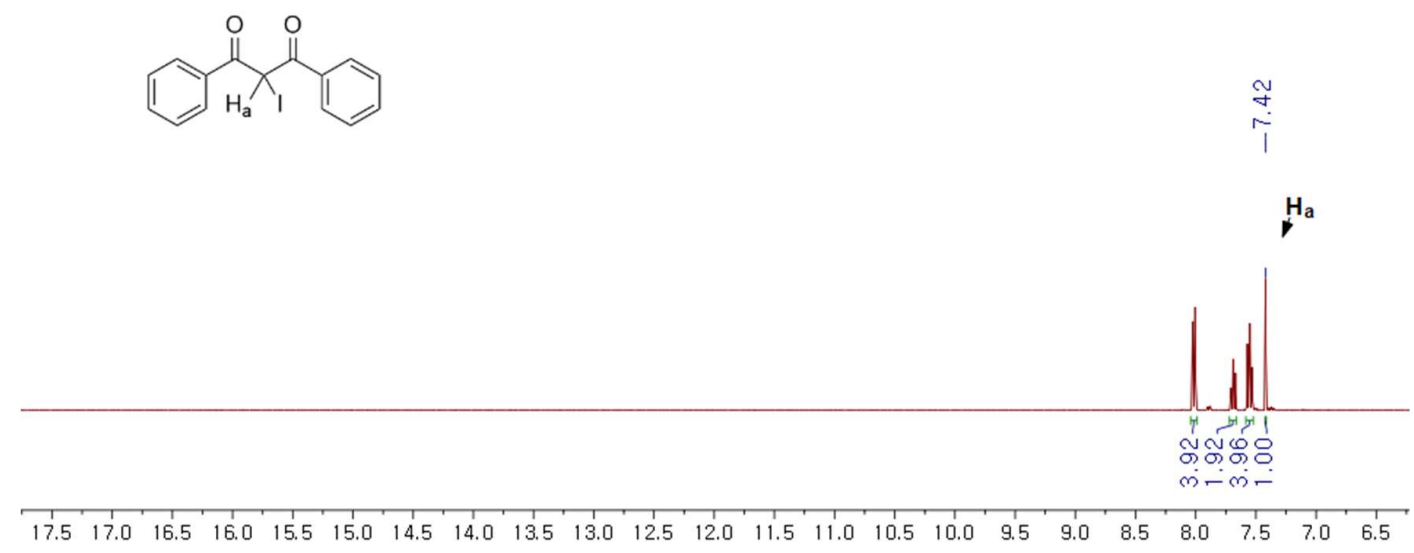

(b)

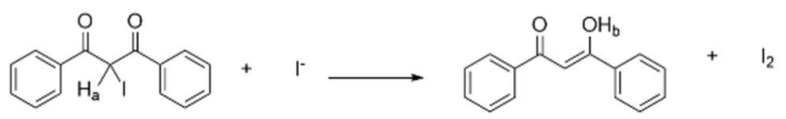

$\stackrel{\frac{1}{\Gamma}}{\stackrel{0}{r}}$

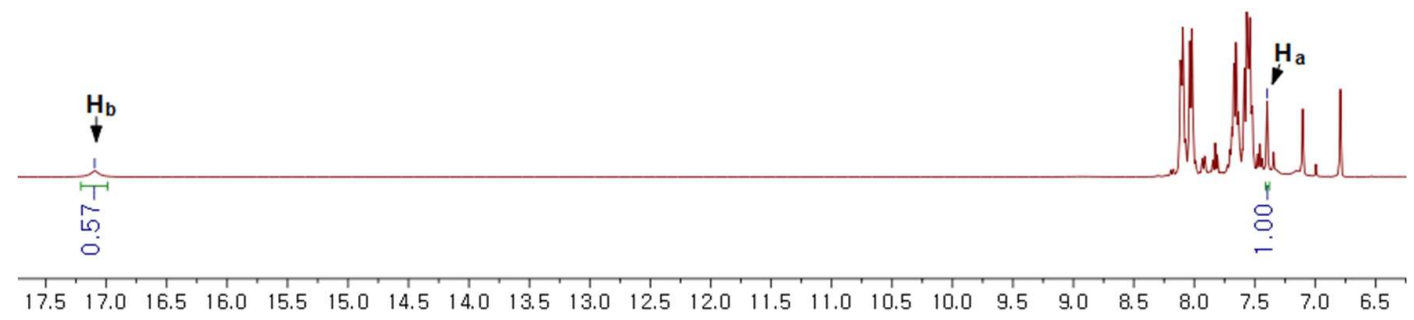

Supplementary Figure 1. (a) ${ }^{1} \mathrm{H}$ NMR (400 MHz, $\mathrm{MeCN}-d_{3}$ ) spectra of 2-iodo-1,3-diphenylpropane-1,3-dione (8a) (b) ${ }^{1} \mathrm{H}$ NMR (400 MHz, MeCN-d $d_{3}$ ) spectra of reaction mixture (Exp. 5) after $10 \mathrm{~h}$.

\section{2-Iodo-1,3-diphenylpropane-1,3-dione (8a)}<smiles>O=C(c1ccccc1)C(I)C(=O)c1ccccc1</smiles>

It was prepared according to reported procedure. ${ }^{24}$ hexane:EA $=4: 1,315.1 \mathrm{mg}$, $0.9 \mathrm{mmol}, 90 \%$ yield; ${ }^{1} \mathrm{H}$ NMR $\left(400 \mathrm{MHz}, \mathrm{CDCl}_{3}\right) \delta=8.02-7.96(\mathrm{~m}, 4 \mathrm{H}), 7.60(\mathrm{t}, J$ $=7.4 \mathrm{~Hz}, 2 \mathrm{H}), 7.47(\mathrm{t}, J=7.7 \mathrm{~Hz}, 4 \mathrm{H}), 6.94(\mathrm{~s}, 1 \mathrm{H})$. 
Exp. 6. Reaction of iododiketone 8a with $1 \mathrm{a}$ in the presence of 1.1 equivalents of $I_{2}$ provides complete conversion to 3ab.
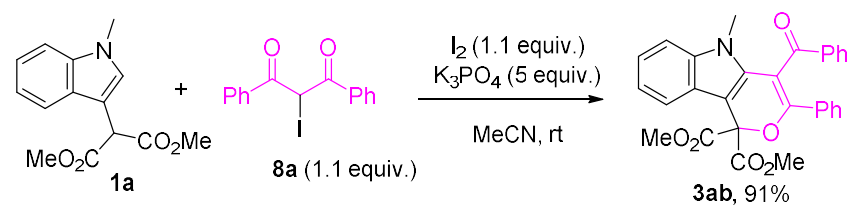

Exp. 7 and 8. Comparison of the reaction sequences confirms that the reaction is initiated by the iodination of the AMCs.

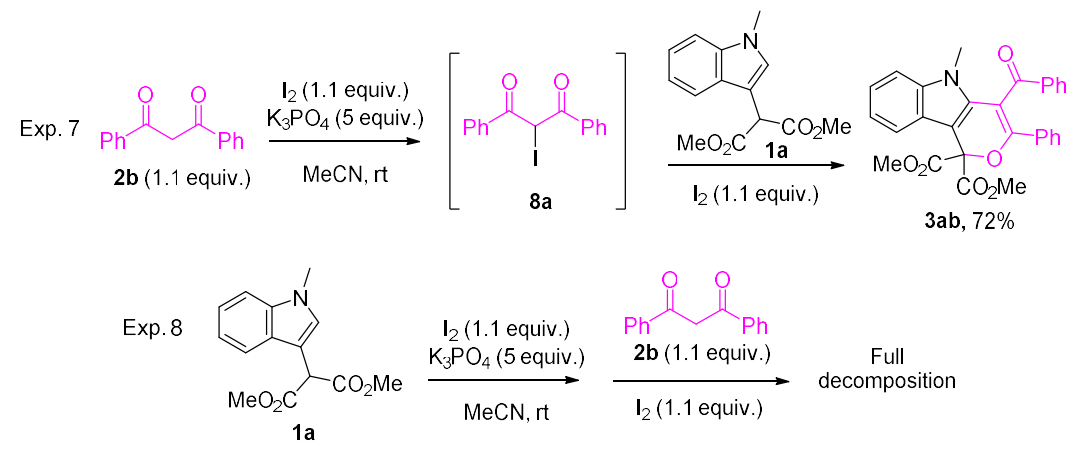

- Diketone $\mathbf{2 b}$ was first treated with iodine and a base, followed by the addition of indolomalonate $\mathbf{1 a}$ along with an equivalent addition of iodine (Exp. 7). 


\subsection{Probing the presence of radical species derived from the reactants}

(i) $\overbrace{\mathrm{Ph}}^{\mathrm{OII}} \frac{\mathrm{TBAl}(1.1 \text { equiv.) }}{\mathrm{MeCN} \text { rt air }}$

(ii)

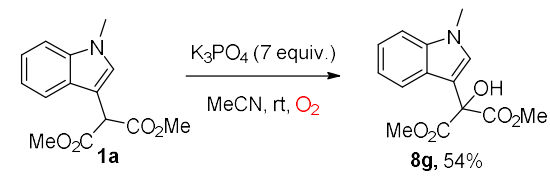

(iii)
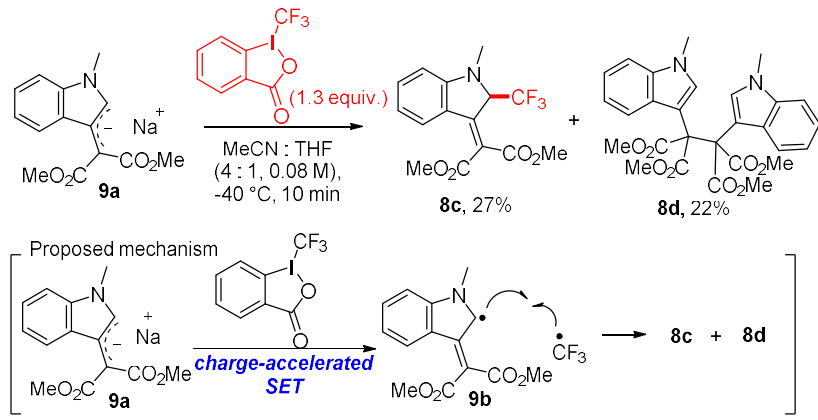

(i) - The ability of iododicarbonyl compounds to form radical species has been confirmed by the formation of tricarbonyl compound $\mathbf{8 b}$ when iododiketone $\mathbf{8 a}$ was treated with n-tetrabutylammonium iodide, which may arise from the corresponding peroxy intermediate.

(ii) - The capability of indolomalonate to form a radical species has been confirmed by the formation of hydroxylation product $\mathbf{8 g}$ when indolomalonate 1a was treated with a base under an oxygen atmosphere.

(iii) - The formation of a radical species on the indolomalonate was evidenced by reacting indolomalonate anion 9a with Togni reagent II, a well known electron acceptor. The reaction was completed at $-40{ }^{\circ} \mathrm{C}$ in 10 min to produce trifluoromethylated product $8 \mathbf{c}$ and dimer $8 \mathbf{d}$ in $27 \%$ and $22 \%$, respectively, both of which strongly suggests the presence of indolomalonate radical $\mathbf{9 b}$.

\subsection{Procedure for identification of intermediates along the reaction pathway}

\section{3-1. (=Exp. 1)}

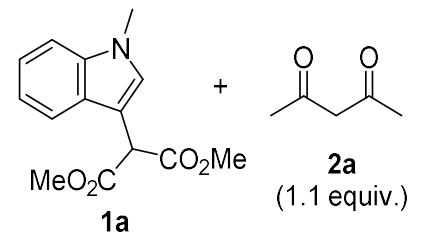

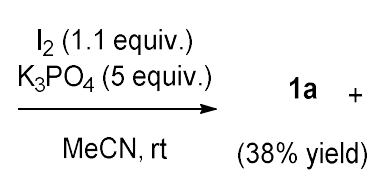

$1 a$

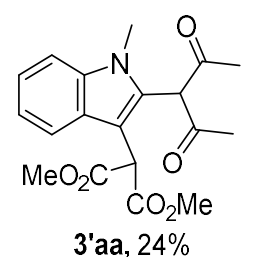

3'aa, $24 \%$

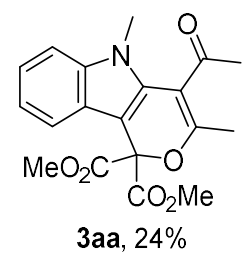

3aa, 24\%

To a solution of indolomalonate $1 \mathrm{a}\left(0.1 \mathrm{mmol}, 1.0\right.$ equiv) and $\mathrm{K}_{3} \mathrm{PO}_{4}(0.5 \mathrm{mmol}, 5.0$ equiv $)$ in anhydrous acetonitrile $(1 \mathrm{~mL}, 0.1 \mathrm{M})$ at room temperature was added acetylacetone $2 \mathrm{a}(0.11 \mathrm{mmol}, 1.1$ equiv) 
dropwise under a nitrogen atmosphere. $\mathrm{I}_{2}(0.11 \mathrm{mmol}, 1.1$ equiv) was added under nitrogen and the reaction was stirred at room temperature. The reaction mixture was monitored by TLC until indole was consumed, opened to air, quenched with 2-3 drops of $1 \mathrm{~N} \mathrm{Na}_{2} \mathrm{~S}_{2} \mathrm{O}_{3}$ solution, diluted with ethyl acetate, and filtered over a pad of celite. The pad was rinsed with an additional ethyl acetate, and the combined solutions were concentrated in vacuo. The crude material was purified by column chromatography (toluene:diethyl ether $=19: 1)$ to afford the $\mathbf{1 a}$ as a white solid $(9.9 \mathrm{mg}, 0.038 \mathrm{mmol}, 38 \%$ recovered yield), 3' aa as a yellow solid ( $8.6 \mathrm{mg}, 0.024 \mathrm{mmol}, 24 \%$ yield), and 3aa as a yellow solid (8.6 mg, $0.024 \mathrm{mmol}, 25 \%$ yield).

\section{Dimethyl 2-(2-(2,4-dioxopentan-3-yl)-1-methyl-1H-indol-3-yl)malonate (3'aa)}<smiles>CC(=O)c1c(C(C(C)=O)C(C)=O)c2ccccc2n1C</smiles>

toluene:diethyl ether $=19: 1$, yellow solid, m.p. $155-160{ }^{\circ} \mathrm{C} ;{ }^{1} \mathrm{H}$ NMR $(400$ $\left.\mathrm{MHz}, \mathrm{CDCl}_{3}\right) \delta=16.97(\mathrm{~s}, 1 \mathrm{H}), 7.74(\mathrm{~d}, J=8.0 \mathrm{~Hz}, 1 \mathrm{H}), 7.36(\mathrm{~d}, J=8.2 \mathrm{~Hz}$, $1 \mathrm{H}), 7.32-7.26(\mathrm{~m}, 1 \mathrm{H}), 7.20-7.15(\mathrm{~m}, 1 \mathrm{H}), 4.73(\mathrm{~s}, 1 \mathrm{H}), 3.72(\mathrm{~s}, 6 \mathrm{H}), 3.60$ $(\mathrm{s}, 3 \mathrm{H}), 1.87(\mathrm{~s}, 6 \mathrm{H}) ;{ }^{13} \mathrm{C} \mathrm{NMR}\left(100 \mathrm{MHz}, \mathrm{CDCl}_{3}\right) \delta=193.8,168.7,137.1$, 134.2, 126.1, 122.5, 120.6, 120.3, 109.6, 107.1, 102.5, 52.6, 49.9, 29.9, 23.7; IR (film): 2954, 2923, 1738, 1599, 1294, 1153, $745 \mathrm{~cm}^{-1}$; HRMS (DART) m/z: [M+H] $]^{+}$Calcd. for $\mathrm{C}_{19} \mathrm{H}_{22} \mathrm{NO}_{6} 360.1442$; Found 360.1411

\section{3-2. (=Exp. 2)}

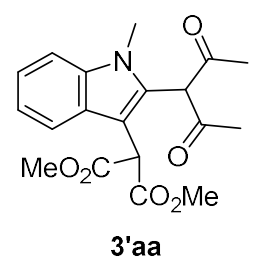

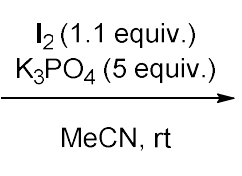

3'aa

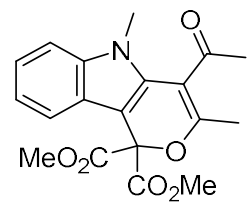

3aa, $97 \%$

To a solution of $\mathrm{C} 2$-intermediate $\mathbf{3}$ 'aa $\left(0.1 \mathrm{mmol}, 1.0\right.$ equiv) and $\mathrm{K}_{3} \mathrm{PO}_{4}(0.5 \mathrm{mmol}, 5.0$ equiv) in anhydrous acetonitrile $(1 \mathrm{~mL}, 0.1 \mathrm{M})$ at room temperature was added $\mathrm{I}_{2}(0.11 \mathrm{mmol}, 1.1$ equiv) under a nitrogen atmosphere and the reaction was stirred at room temperature. The reaction mixture was monitored by TLC until indole was consumed, opened to air, quenched with 2-3 drops of $1 \mathrm{~N} \mathrm{Na}_{2} \mathrm{~S}_{2} \mathrm{O}_{3}$ solution, diluted with ethyl acetate, and filtered over a pad of celite. The pad was rinsed with an additional ethyl acetate, and the combined solutions were concentrated in vacuo. The crude material was purified by column chromatography (hexane:EA $=5: 1$ ) to give the product 3aa as a yellow solid (34.6 mg, $0.097 \mathrm{mmol}$, 97\% yield). 


\section{3-3. (=Exp. 3)}

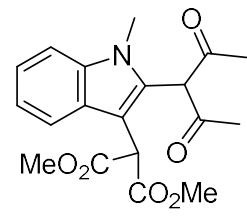

3'aa

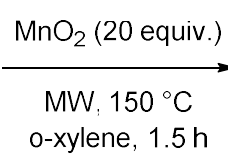

o-xylene, $1.5 \mathrm{~h}$

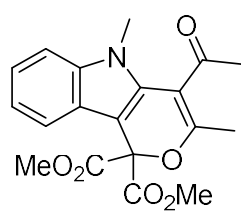

3aa, $56 \%$

The C2-intermediate 3'aa (0.1 mmol), $\mathrm{MnO}_{2}(2 \mathrm{mmol}, 20$ equiv) and dried o-xylene ( $1 \mathrm{~mL}, 0.1 \mathrm{M})$ were mixed in the microwave instrument vial. The vial was flushed with nitrogen and irradiated with stirring at $150{ }^{\circ} \mathrm{C}$ for $1.5 \mathrm{~h}$. Upon completion of the reaction, the crude material was purified by column chromatography (hexane:EA $=5: 1)$ on silica gel to give the product 3aa as a yellow solid $(20.0 \mathrm{mg}$, $0.056 \mathrm{mmol}, 56 \%$ yield).

\section{3-4. (=Exp. 4)}<smiles>CC(=O)C(C(C)=O)c1cn(C)c2ccccc12</smiles>

$1 \mathrm{a}$<smiles>O=C(C(I)c1ccccc1)C(I)c1ccccc1</smiles>

(1.1 eq.)

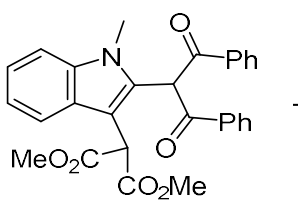

3 'ab, $40 \%$

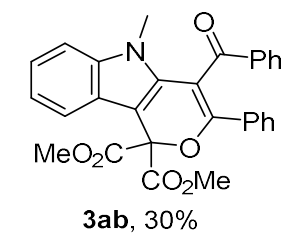

$3 \mathrm{ab}, 30 \%$

To a solution of $\mathrm{C} 2$-intermediate $1 \mathrm{a}\left(0.1 \mathrm{mmol}, 1.0\right.$ equiv) and $\mathrm{K}_{3} \mathrm{PO}_{4}(0.5 \mathrm{mmol}, 5.0$ equiv) in anhydrous acetonitrile $(1 \mathrm{~mL}, 0.1 \mathrm{M})$ at room temperature was added iododicarbonyl $\mathbf{8 a}(0.11 \mathrm{mmol}$, 1.1 equiv) under a nitrogen atmosphere and the reaction was stirred at room temperature. The reaction mixture was monitored by TLC until indole was consumed, opened to air, quenched with 2-3 drops of $1 \mathrm{~N} \mathrm{Na} \mathrm{S}_{2} \mathrm{O}_{3}$ solution, diluted with ethyl acetate, and filtered over a pad of celite. The pad was rinsed with an additional ethyl acetate, and the combined solutions were concentrated in vacuo. The yield was determined by ${ }^{1} \mathrm{H}$ NMR analysis $\left(\mathrm{CDCl}_{3}\right)$ of the crude reaction mixture.

\section{3-5. (=Exp. 5)}<smiles>O=C(c1ccccc1)C(O)C(=O)c1ccccc1</smiles>

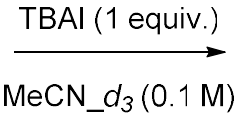<smiles>O=C(CC(=O)c1ccccc1)c1ccccc1</smiles>

The iododicarbonyl $8 \mathrm{a}(0.05 \mathrm{mmol}, 1.0$ equiv), tetrabutylammonium iodide (TBAI, $0.05 \mathrm{mmol}, 1.0$ equiv) and MeCN_d $d_{3}(0.5 \mathrm{~mL}, 0.1 \mathrm{M})$ were mixed in the nmr tube. After $10 \mathrm{~h}$, the reaction mixture was directly determined by ${ }^{1} \mathrm{H}$ NMR. 
7.3-6. (=Exp. 6)

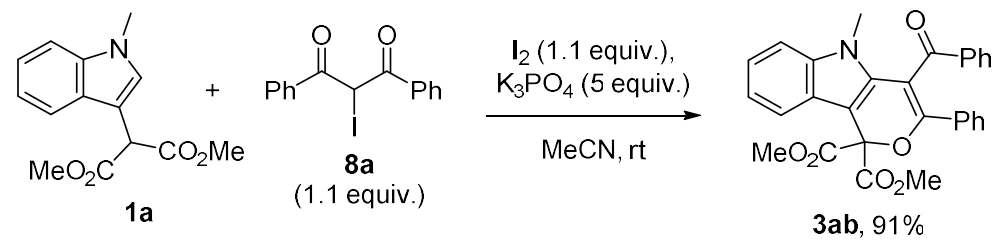

To a solution of indolomalonate $1 \mathrm{a}\left(0.1 \mathrm{mmol}, 1.0\right.$ equiv) and $\mathrm{K}_{3} \mathrm{PO}_{4}(0.5 \mathrm{mmol}, 5.0$ equiv $)$ in anhydrous acetonitrile $(1 \mathrm{~mL}, 0.1 \mathrm{M})$ at room temperature was added iododicarbonyl $\mathbf{8 a}(0.11 \mathrm{mmol}, 1.1$ equiv) dropwise under a nitrogen atmosphere. $\mathrm{I}_{2}(0.11 \mathrm{mmol}, 1.1$ equiv) was added under nitrogen and the reaction was stirred at room temperature. The reaction mixture was monitored by TLC until indole was consumed, opened to air, quenched with 2-3 drops of $1 \mathrm{~N} \mathrm{Na}_{2} \mathrm{~S}_{2} \mathrm{O}_{3}$ solution, diluted with ethyl acetate, and filtered over a pad of celite. The pad was rinsed with an additional ethyl acetate, and the combined solutions were concentrated in vacuo. The crude material was purified by column chromatography (hexane:EA $=5: 1$ ) to give the product 3ab as a yellow solid (43.8 $\mathrm{mg}, 0.091 \mathrm{mmol}, 91 \%$ yield).

\section{3-7. (=Exp. 7)}

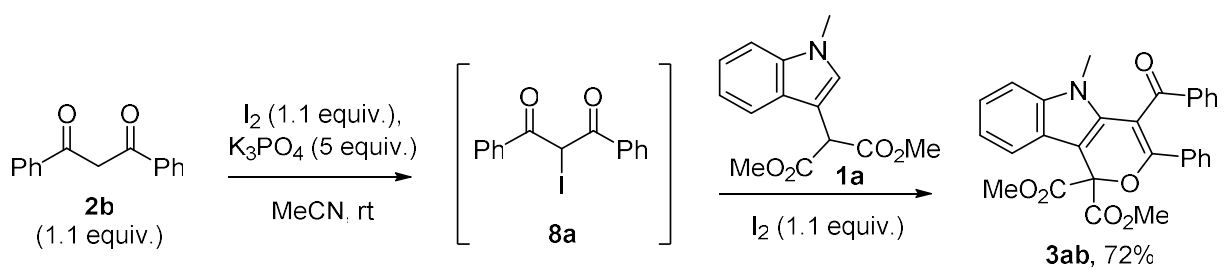

To a solution of activated methylene compound $\mathbf{2 b}\left(0.11 \mathrm{mmol}, 1.1\right.$ equiv) and $\mathrm{K}_{3} \mathrm{PO}_{4}(0.5 \mathrm{mmol}, 5.0$ equiv) in anhydrous acetonitrile $(1 \mathrm{~mL}, 0.1 \mathrm{M})$ at room temperature was added $\mathrm{I}_{2}(0.11 \mathrm{mmol}, 1.1$ equiv) dropwise under a nitrogen atmosphere and the reaction was stirred at room temperature. The reaction mixture was monitored by TLC until activated methylene compound $\mathbf{2 b}$ was almost consumed (about $10 \mathrm{~min})$, indolomalonate $1 \mathrm{a}\left(0.1 \mathrm{mmol}, 1\right.$ equiv) and $\mathrm{I}_{2}(0.11 \mathrm{mmol}, 1.1$ equiv) were added under nitrogen and the reaction was stirred at room temperature. The reaction mixture was monitored by TLC until indole was consumed, opened to air, quenched with 2-3 drops of $1 \mathrm{~N} \mathrm{Na}_{2} \mathrm{~S}_{2} \mathrm{O}_{3}$ solution, diluted with ethyl acetate, and filtered over a pad of celite. The pad was rinsed with an additional ethyl acetate, and the combined solutions were concentrated in vacuo. The crude material was purified by column chromatography (hexane:EA $=5: 1)$ to give the product $\mathbf{3 a b}$ as a yellow solid $(34.7 \mathrm{mg}, 0.072 \mathrm{mmol}$, $72 \%$ yield). 
7.3-8. (=Exp. 8)

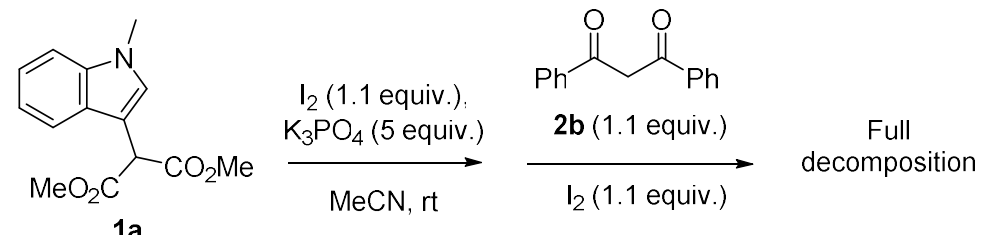

$1 \mathrm{a}$

To a solution of indolomalonate $1 \mathrm{a}\left(0.1 \mathrm{mmol}, 1\right.$ equiv) and $\mathrm{K}_{3} \mathrm{PO}_{4}(0.5 \mathrm{mmol}, 5.0$ equiv) in anhydrous acetonitrile $(1 \mathrm{~mL}, 0.1 \mathrm{M})$ at room temperature was added $\mathrm{I}_{2}(0.11 \mathrm{mmol}, 1.1$ equiv $)$ dropwise under a nitrogen atmosphere and the reaction was stirred at room temperature. The reaction mixture was monitored by TLC until indolomalonate 1a was almost consumed (about $10 \mathrm{~min}$ ), activated methylene compound $\mathbf{2 b}$ ( $0.11 \mathrm{mmol}, 1.1$ equiv) and $\mathrm{I}_{2}(0.11 \mathrm{mmol}, 1.1$ equiv) were added under nitrogen and the reaction was stirred at room temperature.

\subsection{Procedures for probing the presence of radical species}

\section{4-1. Reaction with iododicarbonyl}

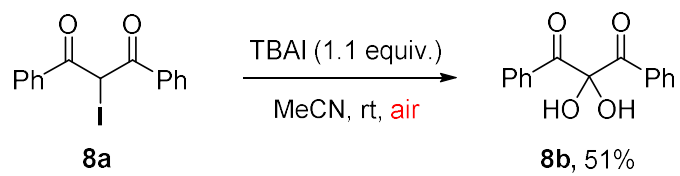

A solution of $8 \mathbf{a}(0.1 \mathrm{mmol})$ and TBAI (Tetrabutylammonium iodide) (1.1 equiv) in acetonitrile (1.0 $\mathrm{mL}, 0.1 \mathrm{M}$ ) was stirred at room temperature for $0.5 \mathrm{~h}$. After complete disappearance of $\mathbf{8 a}$, as judged by TLC analysis, the reaction was quenched by addition of $1 \mathrm{~N}$ thiosulfate solution, and the reaction mixture was extracted twice with ethyl acetate. The combined organic phase was washed with brine, dried over magnesium sulfate and concentrated under reduced pressure. Purification of the crude material by column chromatography (hexane:EA = 3:2) to afford the $\mathbf{8 b}$ as a white solid (13 $\mathrm{mg}, 0.051 \mathrm{mmol}, 51 \%$ yield).

\section{2,2-Dihydroxy-1,3-diphenylpropane-1,3-dione (8b)}<smiles>O=C(c1ccccc1)C(O)(O)C(=O)c1ccccc1</smiles>

White solid, $51 \%$ yield; ${ }^{1} \mathrm{H}$ NMR $\left(400 \mathrm{MHz}, \mathrm{CDCl}_{3}\right) \delta=7.94(\mathrm{dd}, J=8.5,1.3 \mathrm{~Hz}$, $4 \mathrm{H}), 7.54-7.50(\mathrm{~m}, 2 \mathrm{H}), 7.39-7.34(\mathrm{~m}, 4 \mathrm{H}), 5.89$ (s, 2H). The compound was identified by spectral comparison with literature data. ${ }^{25}$ 
7.4-2. Reaction with indolomalonate 1a (The capability of indolomaonate to form a radical species)

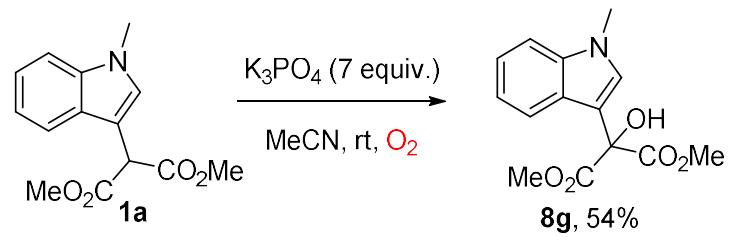

A solution of $1 \mathrm{a}(0.1 \mathrm{mmol})$ and $\mathrm{K}_{3} \mathrm{PO}_{4}(7$ equiv) in acetonitrile $(1.0 \mathrm{~mL}, 0.1 \mathrm{M})$ was stirred at room temperature with $\mathrm{O}_{2}$ balloon for overnight. After complete disappearance of 1a, as judged by TLC analysis, the reaction was quenched by addition of saturated $\mathrm{NH}_{4} \mathrm{Cl}$ solution, and the reaction mixture was extracted twice with ethyl acetate. The combined organic phase was washed with brine, dried over magnesium sulfate and concentrated under reduced pressure. Purification of the crude material by column chromatography (hexane:EA = 1:1) to afford the $8 \mathrm{~g}$ as a white solid (15.0 $\mathrm{mg}, 0.054 \mathrm{mmol}, 54 \%$ yield).

\section{Dimethyl 2-hydroxy-2-(1-methyl-1 $H$-indol-3-yl)malonate (8g)}

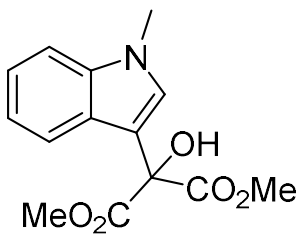

White solid, 54\% yield; m.p. 75-80 ${ }^{\circ} \mathrm{C} ;{ }^{1} \mathrm{H}$ NMR $\left(400 \mathrm{MHz}, \mathrm{CDCl}_{3}\right) \delta=7.67$ (d, $J=8.1 \mathrm{~Hz}, 1 \mathrm{H}), 7.36(\mathrm{~s}, 1 \mathrm{H}), 7.31(\mathrm{~d}, J=8.2 \mathrm{~Hz}, 1 \mathrm{H}), 7.23(\mathrm{~d}, J=7.9 \mathrm{~Hz}, 1 \mathrm{H})$, $7.13(\mathrm{t}, J=7.5 \mathrm{~Hz}, 1 \mathrm{H}), 4.29(\mathrm{~s}, 1 \mathrm{H}), 3.84(\mathrm{~s}, 6 \mathrm{H}), 3.78(\mathrm{~s}, 3 \mathrm{H}) ;{ }^{13} \mathrm{C}$ NMR $(100$ 53.6, 32.9; IR (film): 3481, 2953, 1732, 1475, 1434, 1334, 1231, 1201, 1103, $741 \mathrm{~cm}^{-1}$; HRMS (DART) $\mathrm{m} / \mathrm{z}:[\mathrm{M}+\mathrm{H}]^{+}$Calcd. for $\mathrm{C}_{14} \mathrm{H}_{16} \mathrm{NO}_{5} 278.103$; Found 278.1023

\section{4-3. Preparation of anion salt 9a.}
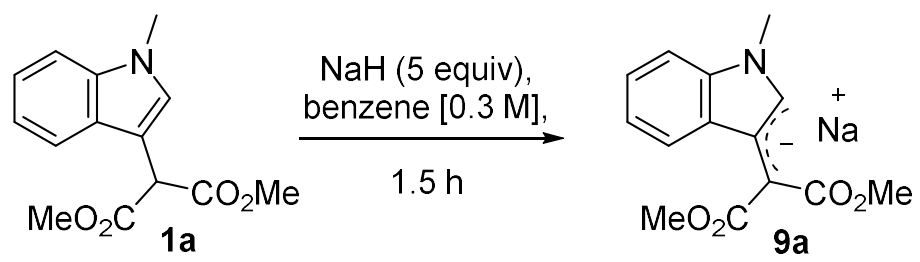

The mixture of sodium hydride $(1 \mathrm{mmol})$ in dried benzene $(0.6 \mathrm{~mL})$ was stirred for $5 \mathrm{~min}$ at room temperature. After centrifuging, the liquid component of mixture at the top was removed (repeated three times). A mixture of dimethyl 2-(1-methyl-1H-indol-3-yl)malonate $1 \mathbf{a}(0.2 \mathrm{mmol})$ in dried benzene $(0.6$ $\mathrm{mL}$ ) was added. Then the mixture was stirred for $1.5 \mathrm{~h}$. After centrifuging, the liquid component of mixture at the top was transferred, concentrated and dried under vacuum. 
1,3-Dimethoxy-2-(1-methyl-1H-indol-3-yl)-1,3-dioxopropan-2-ide (9a)<smiles>CC(=O)C(C(C)=O)C1CN(C)c2ccccc21</smiles>

${ }^{1} \mathrm{H}$ NMR $\left(400 \mathrm{MHz}\right.$, DMSO- $\left.d_{6}\right) \delta=7.24(\mathrm{dd}, J=8.1,2.3 \mathrm{~Hz}, 1 \mathrm{H}), 7.17(\mathrm{dd}, J=$ 8.0, $3.8 \mathrm{~Hz}, 1 \mathrm{H}), 6.98$ (ddt, $J=8.0,3.4,1.8 \mathrm{~Hz}, 1 \mathrm{H}), 6.85$ (td, $J=7.4,3.0 \mathrm{~Hz}$, $1 \mathrm{H}), 6.73(\mathrm{~s}, 1 \mathrm{H}), 3.69(\mathrm{~s}, 3 \mathrm{H}), 3.24(\mathrm{~s}, 6 \mathrm{H})$.

\section{4-4. Trapping with Togni's reagent}

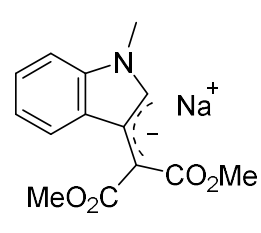

$9 a$

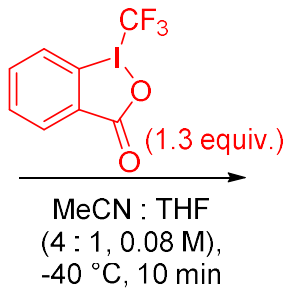

$-40^{\circ} \mathrm{C}, 10 \mathrm{~min}$

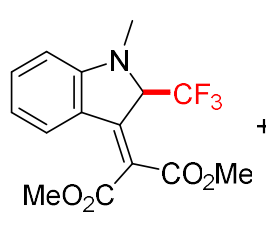

$8 c, 27 \%$

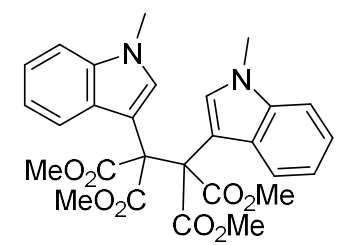

$8 d, 22 \%$

A solution of $9 \mathrm{a}(0.1 \mathrm{mmol})$ and Togni reagent $(5$ equiv $)$ in acetonitrile $(1.0 \mathrm{~mL}, 0.1 \mathrm{M})$ was stirred at $40{ }^{\circ} \mathrm{C}$ under $\mathrm{N}_{2}$ atmosphere for $10 \mathrm{~min}$. After complete disappearance of 1a (protonated 9a), as judged by TLC analysis, the reaction was quenched by addition of $1 \mathrm{~N} \mathrm{NH}_{4} \mathrm{Cl}$ solution, and the reaction mixture was extracted twice with ethyl acetate. The combined organic phase was washed with brine, dried over magnesium sulfate and concentrated under reduced pressure. Purification of the crude material by column chromatography (hexane:EA $=9: 1$ to $1: 1$ ) to afford the $\mathbf{8 c}$ as an orange solid (8.9 $\mathrm{mg}, 0.027$ mmol, $27 \%$ yield) and $\mathbf{8 d}$ as a yellow solid (5.7 $\mathrm{mg}, 0.011 \mathrm{mmol}, 22 \%$ yield).

\section{Dimethyl 2-(1-methyl-2-(trifluoromethyl)indolin-3-ylidene)malonate (8c)}<smiles>CC(=O)C(C(C)=O)=C1c2ccccc2N(C)C1C(F)(F)F</smiles>

Orange solid, $27 \%$ yield; m.p. $105-110{ }^{\circ} \mathrm{C} ;{ }^{1} \mathrm{H} \mathrm{NMR}\left(400 \mathrm{MHz}, \mathrm{CDCl}_{3}\right) \delta=7.33$ (ddd, $J=8.3,7.3,1.2 \mathrm{~Hz}, 1 \mathrm{H}), 7.26-7.23(\mathrm{~m}, 1 \mathrm{H}), 6.71(\mathrm{ddd}, J=8.1,7.3,1.0$ $\mathrm{Hz}, 1 \mathrm{H}), 6.63(\mathrm{~d}, J=8.2 \mathrm{~Hz}, 1 \mathrm{H}), 5.79(\mathrm{q}, J=6.0 \mathrm{~Hz}, 1 \mathrm{H}), 3.93(\mathrm{~s}, 3 \mathrm{H}), 3.82(\mathrm{~s}$, $3 \mathrm{H}), 3.09(\mathrm{~s}, 3 \mathrm{H}) ;{ }^{13} \mathrm{C} \mathrm{NMR}\left(100 \mathrm{MHz}, \mathrm{CDCl}_{3}\right) \delta=166.4,164.5,155.9,147.6$, 134.5, 124.8, 124.2 (d, $\left.J=287.1 \mathrm{~Hz}, \mathrm{CF}_{3}\right), 121.6,118.7,118.2,108.2,67.0$ (q, $\left.J=29.4 \mathrm{~Hz}, \mathrm{CH}\right), 52.9$, 52.4, 33.2; IR (film): 2956, 1729, 1712, 1605, 1437, 1253, 1156, 1117, $747 \mathrm{~cm}^{-1} ;$ HRMS (DART) m/z: $[\mathrm{M}+\mathrm{Na}]^{+}$Calcd. for $\mathrm{C}_{15} \mathrm{H}_{14} \mathrm{~F}_{3} \mathrm{NNaO}_{4} 352.0767$; Found 352.0767 
Tetramethyl 1,2-bis(1-methyl-1 $H$-indol-3-yl)ethane-1,1,2,2-tetracarboxylate (8d)<smiles>COC(=O)C(C)(C(C)=O)C(C)(C)c1cn(C)c2ccccc12</smiles>

Yellow solid, 22\% yield; m.p. $80-85{ }^{\circ} \mathrm{C} ;{ }^{1} \mathrm{H}$ NMR (400 MHz, $\left.\mathrm{CDCl}_{3}\right) \delta=7.17$ $(\mathrm{d}, J=8.0 \mathrm{~Hz}, 1 \mathrm{H}), 7.07$ (t, $J=7.5 \mathrm{~Hz}, 1 \mathrm{H}), 6.77(\mathrm{t}, J=7.7 \mathrm{~Hz}, 1 \mathrm{H}), 6.71(\mathrm{~s}$, $1 \mathrm{H}), 6.53(\mathrm{~s}, 1 \mathrm{H}), 3.78(\mathrm{~s}, 6 \mathrm{H}), 3.43(\mathrm{~s}, 3 \mathrm{H}) ;{ }^{13} \mathrm{C} \mathrm{NMR}\left(100 \mathrm{MHz}, \mathrm{CDCl}_{3}\right) \delta$ $=169.8,136.5,133.2,127.6,122.2,120.6,118.5,108.9,105.6,65.3,53.0$, 32.5; IR (film): 2950, 1726, 1431, 1246, 1201, $737 \mathrm{~cm}^{-1}$; HRMS (DART) m/z: $[\mathrm{M}+\mathrm{Na}]^{+}$Calcd. for $\mathrm{C}_{28} \mathrm{H}_{28} \mathrm{~N}_{2} \mathrm{NaO}_{8}$ 543.1738; Found 543.1738

\subsection{Identification of indolomalonate radical species 9d by HRMS}

(i) To investigate the plausible radical species, indolomalonate $\mathbf{1 a}(0.01 \mathrm{mmol}, 1$ equiv) and TMEDA ( $0.05 \mathrm{mmol}, 5$ equiv) were treated with iododiketone $\mathbf{8 a}(0.011 \mathrm{mmol}, 1.1$ equiv) in $1 \mathrm{~mL}$ of acetonitrile in a $4 \mathrm{~mL}$ vial. After that, the vial with the reaction mixture was stirred for $10 \mathrm{~min}$. An aliquot of this solution was directly injected into the ESI source.

(ii) As a control experiment, indolomalonate $1 \mathrm{a}(0.01 \mathrm{mmol}, 1$ equiv) and TMEDA( $0.05 \mathrm{mmol}, 5$ equiv) were treated with iododiketone $8 \mathrm{a}(0.011 \mathrm{mmol}, 1.1$ equiv) in $1 \mathrm{~mL}$ of acetonitrile in a $4 \mathrm{~mL}$ vial. After that, the vial with the reaction mixture was stirred for $10 \mathrm{~min}$. An aliquot of this solution was directly injected into the ESI source.
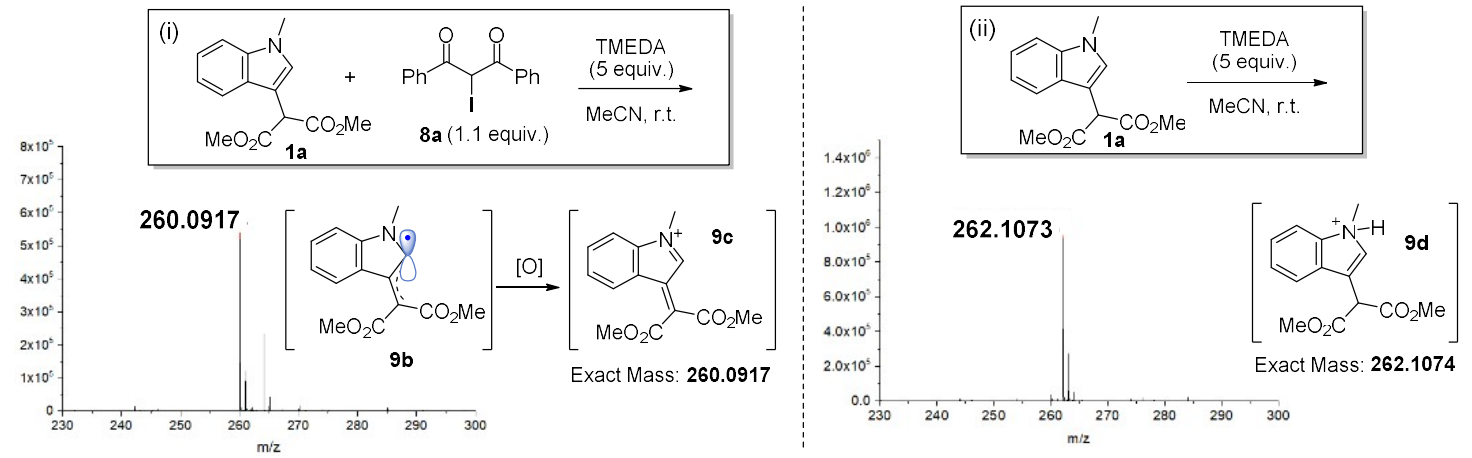


\subsection{Effect of reaction concentration supporting the cage-collapse cross-coupling}

- Dilution does not affect the radical-radical cross-coupling supporting that the coupling occurs via cage-collapse.

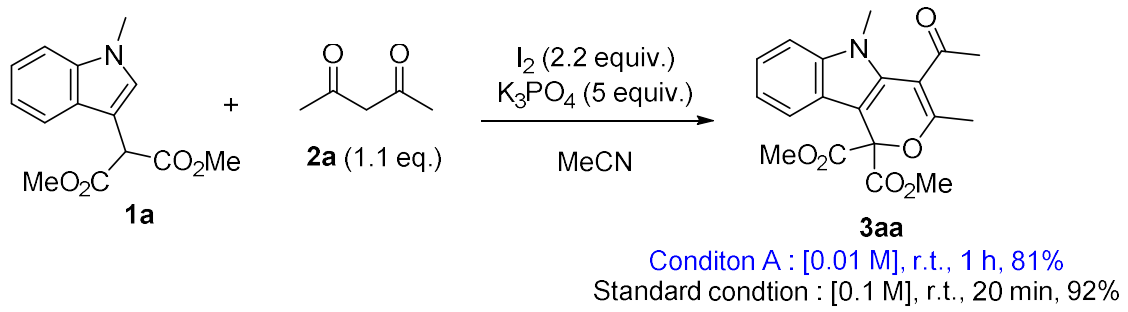

(Condition A) To a solution of indole $1 \mathrm{a}\left(0.1 \mathrm{mmol}, 1.0\right.$ equiv) and $\mathrm{K}_{3} \mathrm{PO}_{4}(0.5 \mathrm{mmol}, 5.0$ equiv) in anhydrous acetonitrile $(10 \mathrm{~mL}, 0.01 \mathrm{M})$ at room temperature was added carbonyl compound $2 \mathrm{a}(0.11$ mmol, 1.1 equiv) dropwise under a nitrogen atmosphere. $\mathrm{I}_{2}$ ( $0.22 \mathrm{mmol}, 2.2$ equiv) was added under nitrogen and the reaction was stirred at room temperature. The reaction mixture was monitored by TLC until indole was consumed, opened to air, quenched with 2-3 drops of $1 \mathrm{~N} \mathrm{Na}_{2} \mathrm{~S}_{2} \mathrm{O}_{3}$ solution, diluted with ethyl acetate, and filtered over a pad of celite. The pad was rinsed with an additional ethyl acetate, and the combined solutions were concentrated in vacuo. The crude material was purified by column chromatography (hexane:EA $=5: 1)$ to give the product 3aa as a yellow solid $(28.9 \mathrm{mg}, 0.081 \mathrm{mmol}$, $81 \%$ yield).

\subsection{Effect of radical scavenger (to support the cage-collapse cross-coupling)}

- TEMPO did not interfere cage-collapse radical-radical cross-coupling.

- The different ratio between $\mathbf{3}^{\prime} \mathbf{a b}$ and 3ab in the two reactions are due to the quenching of molecular iodine formed in situ by TEMPO resulting in interruption of further oxidation of $\mathbf{3}^{\prime} \mathbf{a b}$ to $\mathbf{3} \mathbf{a b}$.
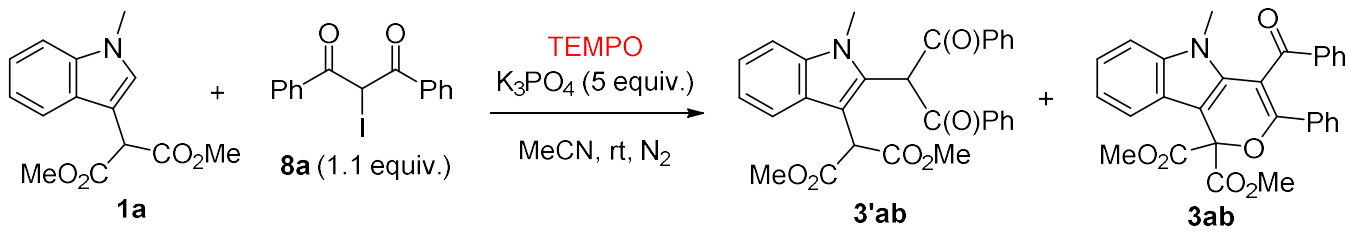

with TEMPO (5 equiv.) : $\mathbf{3}$ 'ab (63\%) $\mathbf{3} \mathbf{a b}(10 \%)$ without TEMPO : 3 'ab (40\%), 3ab (30\%)

(with TEMPO) To a solution of indole $1 \mathrm{a}\left(0.1 \mathrm{mmol}, 1.0\right.$ equiv), $\mathrm{K}_{3} \mathrm{PO}_{4}(0.5 \mathrm{mmol}, 5.0$ equiv) and TEMPO ( $0.5 \mathrm{mmol}, 5.0$ equiv) in anhydrous acetonitrile $(1 \mathrm{~mL}, 0.1 \mathrm{M})$ at room temperature was added iodocarbonyl compound $8 \mathbf{8}(0.11 \mathrm{mmol}, 1.1$ equiv) dropwise under a nitrogen atmosphere and the reaction was stirred at room temperature. The reaction mixture was monitored by TLC until indole was 
consumed, opened to air, quenched with 2-3 drops of $1 \mathrm{~N} \mathrm{Na}_{2} \mathrm{~S}_{2} \mathrm{O}_{3}$ solution, diluted with ethyl acetate, and filtered over a pad of celite. The pad was rinsed with an additional ethyl acetate, and the combined solutions were concentrated in vacuo. The crude material was purified by column chromatography (toluene:diethyl ether $=19: 1)$ to give the $\mathbf{3} \mathbf{\prime} \mathbf{a b}$ as a yellow solid (30.4 $\mathrm{mg}, 0.063 \mathrm{mmol}, 63 \%$ yield) and 3ab as a yellow solid ( $4.8 \mathrm{mg}, 0.01 \mathrm{mmol}, 10 \%$ yield).

(without TEMPO) To a solution of indole $1 \mathrm{a}\left(0.1 \mathrm{mmol}, 1.0\right.$ equiv) and $\mathrm{K}_{3} \mathrm{PO}_{4}(0.5 \mathrm{mmol}, 5.0$ equiv) in anhydrous acetonitrile $(1 \mathrm{~mL}, 0.1 \mathrm{M})$ at room temperature was added iodocarbonyl compound $\mathbf{8 a}$ ( $0.11 \mathrm{mmol}, 1.1$ equiv) dropwise under a nitrogen atmosphere and the reaction was stirred at room temperature. The reaction mixture was monitored by TLC until indole was consumed, opened to air, quenched with 2-3 drops of $1 \mathrm{~N} \mathrm{Na}_{2} \mathrm{~S}_{2} \mathrm{O}_{3}$ solution, diluted with ethyl acetate, and filtered over a pad of celite. The pad was rinsed with an additional ethyl acetate, and the combined solutions were concentrated in vacuo. The crude material was purified by column chromatography (toluene:diethyl ether $=19: 1)$ to give the $\mathbf{3}^{\prime} \mathbf{a b}$ as a yellow solid (19.3 $\mathrm{mg}, 0.04 \mathrm{mmol}, 40 \%$ yield) and $\mathbf{3 a b}$ as a yellow solid (14.4 mg, $0.03 \mathrm{mmol}, 30 \%$ yield).

Dimethyl 2-(2-(1,3-dioxo-1,3-diphenylpropan-2-yl)-1-methyl-1H-indol-3-yl)malonate (3'ab)

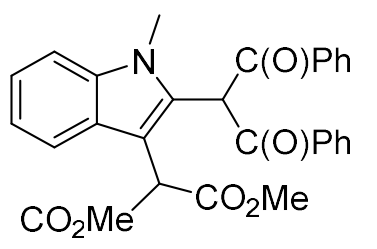

Yellow solid, m.p. $200-205{ }^{\circ} \mathrm{C} ;{ }^{1} \mathrm{H}$ NMR $\left(400 \mathrm{MHz}, \mathrm{CDCl}_{3}\right) \delta=18.73$ (s, $1 \mathrm{H}), 7.64-7.60(\mathrm{~m}, 1 \mathrm{H}), 7.33-7.28(\mathrm{~m}, 2 \mathrm{H}), 7.26-7.19(\mathrm{~m}, 6 \mathrm{H}), 7.15-$ $7.11(\mathrm{~m}, 5 \mathrm{H}), 4.68(\mathrm{~s}, 1 \mathrm{H}), 3.43(\mathrm{~s}, 6 \mathrm{H}), 3.28(\mathrm{~s}, 3 \mathrm{H}) ;{ }^{13} \mathrm{C} \mathrm{NMR}(100 \mathrm{MHz}$, $\left.\mathrm{CDCl}_{3}\right) \delta=190.0,168.3,136.7,136.0,134.2,131.4,128.3,128.1,126.3$, 122.2, 121.2, 120.2, 109.4, 107.3, 100.2, 52.4, 49.8, 30.1; IR (film): 2947, 1726, 1597, 1318, 1237, 1150, 747, $696 \mathrm{~cm}^{-1}$; HRMS (DART) m/z: $[\mathrm{M}+\mathrm{Na}]^{+}$Calcd. for $\mathrm{C}_{29} \mathrm{H}_{25} \mathrm{NNaO}_{6}$ 506.1574; Found 506.1574

- TEMPO did not interfere cage-collapse radical-radical cross-coupling<smiles>O=C(C(Br)c1ccccc1)C(Br)c1ccccc1</smiles>

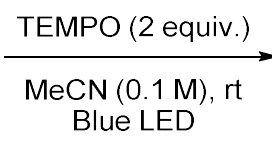

$8 a$

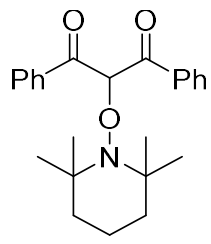

8e, $91 \%$ 
To an oven dried Schlenk flask equipped with a stir bar was added $\mathbf{8 a}(0.05 \mathrm{mmol}, 1$ equiv $)$ and TEMPO ( $0.1 \mathrm{mmol}, 2$ equiv). Required amounts of anhydrous acetonitrile $(0.5 \mathrm{~mL})$ was added under $\mathrm{N}_{2}$ atmosphere. The reaction mixture was irradiated with a blue LED lamp $\left(5 \mathrm{~cm}\right.$ away, $25{ }^{\circ} \mathrm{C}$ maintained with a cooling fan) for $6 \mathrm{~h}$. After evaporating the solvent under reduced pressure, the crude material was purified by column chromatography (hexane:EA $=9: 1$ ) to give the product $\mathbf{8 e}$ as a white solid (17.3 mg, $0.046 \mathrm{mmol}, 91 \%$ yield).

\section{1,3-Diphenyl-2-((2,2,6,6-tetramethylpiperidin-1-yl)oxy)propane-1,3-dione (8e)}<smiles>CC1(C)CCCC(C)(C)N1OC(C(=O)c1ccccc1)C(=O)c1ccccc1</smiles>

White solid, ${ }^{1} \mathrm{H}$ NMR $\left(400 \mathrm{MHz}, \mathrm{CDCl}_{3}\right) \delta=8.19(\mathrm{~d}, J=7.8 \mathrm{~Hz}, 4 \mathrm{H}), 7.53(\mathrm{~d}, J=$ $7.4 \mathrm{~Hz}, 2 \mathrm{H}), 7.44(\mathrm{t}, J=7.6 \mathrm{~Hz}, 4 \mathrm{H}), 6.29(\mathrm{~s}, 1 \mathrm{H}), 1.61-1.50(\mathrm{~m}, 1 \mathrm{H}), 1.47-1.40$ $(\mathrm{m}, 4 \mathrm{H}), 1.32-1.25(\mathrm{~m}, 1 \mathrm{H}), 1.13(\mathrm{~s}, 6 \mathrm{H}), 0.95(\mathrm{~s}, 6 \mathrm{H})$. The compound was identified by spectral comparison with literature data. ${ }^{25}$

\subsection{Gibbs free energy for the SET between electron donors and electron acceptors}

(i)
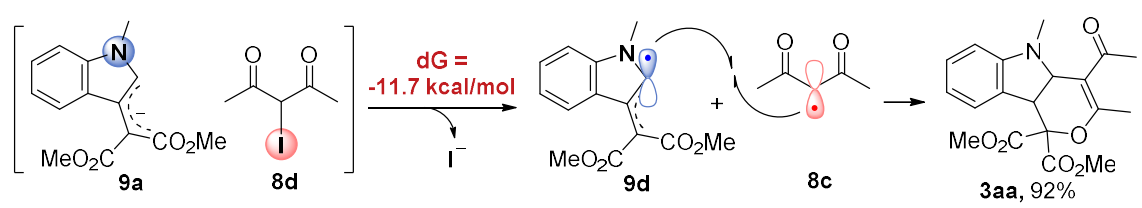

(ii)
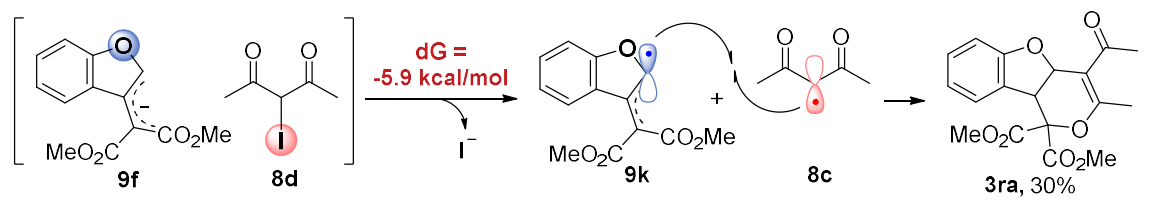

(iii)

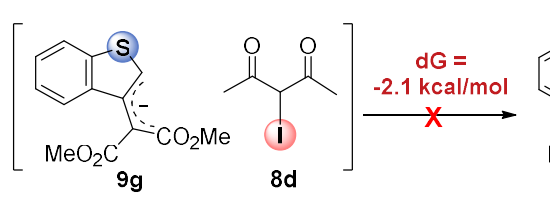

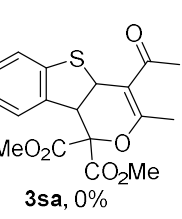

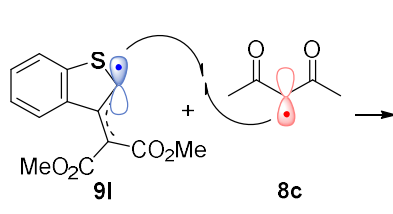

Supplementary Figure 2. Gibbs free energy changes were calculated at the B3LYP-D3/6-31+G(d,p)/LANL2DZdp level in MeCN (SMD).

DFT calculations support the crucial role of the nitrogen-conjugated unsaturation for the reaction. The stability of the radical species resulting from SET accounts for the efficiency of the reaction, in which the formation of $9 \mathbf{d}$ is highly favorable as opposed to those for $9 \mathbf{k}$ and 91 . The Gibbs free energies calculated by DFT are consistent with the experimental results (3ra and 3sa). 


\section{X-ray Crystallographic Data}

Single Crystal X-ray Diffraction Data for the pyran 3da $(\mathrm{CCDC}=1881731$, Thermal ellipsoids were drawn at the $50 \%$ probability levels). The crystals of 3da were obtained by dissolving 3da in hexane:diethyl ether (1:1) followed by slow evaporation of diethyl ether.
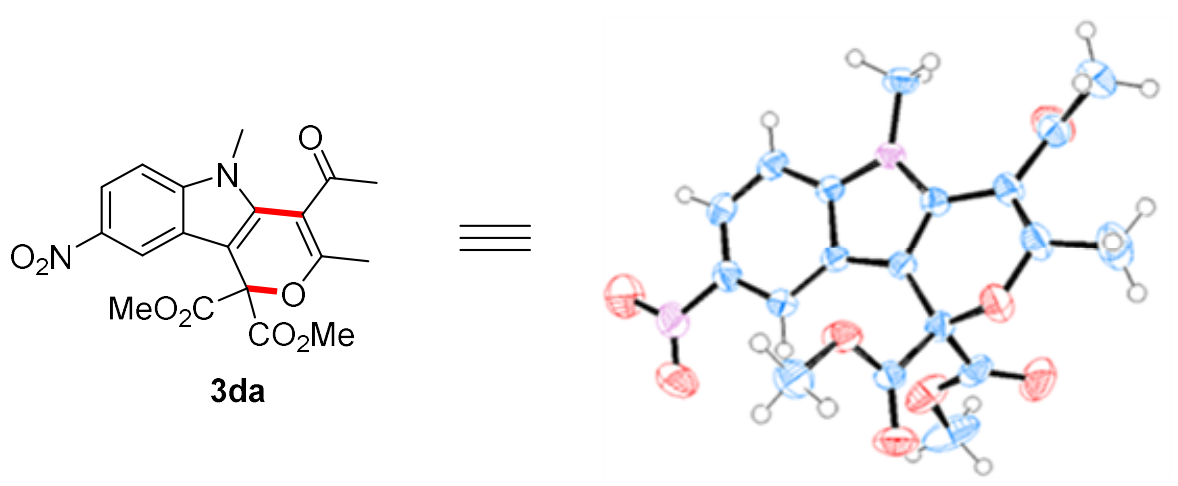

Table 1. Crystal data and structure refinement for $\mathbf{3 d a}$.

Identification code

Empirical formula

Formula weight

Temperature

Wavelength

Crystal system

Space group

Unit cell dimensions

Volume

Z

Density (calculated)

Absorption coefficient

$\mathrm{F}(000)$

Crystal size

Theta range for data collection

Index ranges

Reflections collected

Independent reflections

Completeness to theta $=25.242^{\circ}$

Absorption correction
$3 \mathrm{da}$

C19 H18 N2 O8

402.35

223(2) K

$0.71073 \AA$

Triclinic

P-1

$a=7.6454(13) \AA$ $\alpha=80.687(5)^{\circ}$.

$\mathrm{b}=10.2360(16) \AA$

$\beta=80.271(5)^{\circ}$.

$\mathrm{c}=13.1012(18) \AA$

$\gamma=69.660(5)^{\circ}$.

2

$1.419 \mathrm{Mg} / \mathrm{m}^{3}$

$0.112 \mathrm{~mm}^{-1}$

420

$0.210 \times 0.150 \times 0.070 \mathrm{~mm}^{3}$

2.799 to $28.586^{\circ}$.

$-10<=\mathrm{h}<=10,-13<=\mathrm{k}<=13,-17<=\mathrm{l}<=17$

31195

$4768[\mathrm{R}($ int $)=0.0662]$

$99.7 \%$

Semi-empirical from equivalents 
Max. and min. transmission

Refinement method

Data / restraints / parameters

Goodness-of-fit on $\mathrm{F}^{2}$

Final $R$ indices $[\mathrm{I}>2 \operatorname{sigma}(\mathrm{I})]$

$\mathrm{R}$ indices (all data)

Extinction coefficient

Largest diff. peak and hole
0.7457 and 0.6875

Full-matrix least-squares on $\mathrm{F}^{2}$

4768 / 0 / 267

1.022

$\mathrm{R} 1=0.0569, \mathrm{wR} 2=0.1197$

$\mathrm{R} 1=0.0961, \mathrm{wR} 2=0.1392$

$\mathrm{n} / \mathrm{a}$

0.779 and -0.202 e. $\AA^{-3}$

Table 2. Atomic coordinates ( $\left.\times 10^{4}\right)$ and equivalent isotropic displacement parameters $\left(\AA^{2} \times 10^{3}\right)$ for 3da. U(eq) is defined as one third of the trace of the orthogonalized $U^{i j}$ tensor.

\begin{tabular}{|c|c|c|c|c|}
\hline & $\mathrm{x}$ & $\mathrm{y}$ & $\mathrm{z}$ & $\mathrm{U}(\mathrm{eq})$ \\
\hline $\mathrm{N}(1)$ & $7666(2)$ & 4921(2) & $4058(1)$ & $30(1)$ \\
\hline $\mathrm{C}(1)$ & $6474(2)$ & $6044(2)$ & $4554(1)$ & $27(1)$ \\
\hline$C(2)$ & $6263(3)$ & $6273(2)$ & $5594(2)$ & $34(1)$ \\
\hline$C(3)$ & 4990(3) & $7512(2)$ & $5889(2)$ & $38(1)$ \\
\hline$C(4)$ & $3955(3)$ & $8490(2)$ & $5159(2)$ & $36(1)$ \\
\hline$C(5)$ & $4110(3)$ & $8286(2)$ & $4128(2)$ & $32(1)$ \\
\hline$C(6)$ & $5383(2)$ & $7026(2)$ & $3819(1)$ & $28(1)$ \\
\hline$C(7)$ & $5942(2)$ & $6414(2)$ & $2867(1)$ & $27(1)$ \\
\hline$C(8)$ & $5297(3)$ & $6882(2)$ & $1816(1)$ & $31(1)$ \\
\hline $\mathrm{O}(1)$ & $5755(2)$ & $5710(2)$ & $1234(1)$ & $43(1)$ \\
\hline $\mathrm{C}(9)$ & $7400(3)$ & $4610(2)$ & $1327(2)$ & $40(1)$ \\
\hline$C(10)$ & $8259(3)$ & $4275(2)$ & 2197(2) & $34(1)$ \\
\hline$C(11)$ & $7345(2)$ & $5166(2)$ & $3025(1)$ & $28(1)$ \\
\hline$C(12)$ & $8867(3)$ & $3644(2)$ & $4587(2)$ & $38(1)$ \\
\hline $\mathrm{N}(2)$ & 2602(3) & $9790(2)$ & $5504(2)$ & $50(1)$ \\
\hline $\mathrm{O}(2)$ & $2377(4)$ & $9942(2)$ & $6424(2)$ & $91(1)$ \\
\hline $\mathrm{O}(3)$ & 1741(3) & $10675(2)$ & $4872(2)$ & $74(1)$ \\
\hline$C(13)$ & $3148(3)$ & $7507(2)$ & $1827(1)$ & $30(1)$ \\
\hline $\mathrm{O}(4)$ & $2395(2)$ & $8462(2)$ & $1228(1)$ & $54(1)$ \\
\hline $\mathrm{O}(5)$ & 2292(2) & $6794(2)$ & $2535(1)$ & $40(1)$ \\
\hline$C(14)$ & $289(3)$ & $7168(3)$ & $2528(2)$ & $46(1)$ \\
\hline$C(15)$ & $6198(3)$ & $7942(3)$ & $1195(2)$ & $41(1)$ \\
\hline $\mathrm{O}(6)$ & $7239(3)$ & $\begin{array}{r}7736(2) \\
\text { S55 }\end{array}$ & $410(1)$ & $70(1)$ \\
\hline
\end{tabular}




\begin{tabular}{lrrrr}
$\mathrm{O}(7)$ & $5713(3)$ & $9064(2)$ & $1682(1)$ & $57(1)$ \\
$\mathrm{C}(16)$ & $6462(5)$ & $10176(3)$ & $1196(2)$ & $78(1)$ \\
$\mathrm{C}(17)$ & $7977(4)$ & $3927(3)$ & $348(2)$ & $66(1)$ \\
$\mathrm{C}(18)$ & $10203(3)$ & $3239(2)$ & $2246(2)$ & $41(1)$ \\
$\mathrm{O}(8)$ & $11383(2)$ & $3616(2)$ & $2500(2)$ & $60(1)$ \\
$\mathrm{C}(19)$ & $10644(4)$ & $1761(3)$ & $2034(2)$ & $62(1)$ \\
\hline
\end{tabular}

Table 3. Bond lengths $[\AA]$ and angles $\left[^{\circ}\right]$ for $\mathbf{3 d a}$.

\begin{tabular}{ll}
\hline $\mathrm{N}(1)-\mathrm{C}(1)$ & $1.374(2)$ \\
$\mathrm{N}(1)-\mathrm{C}(11)$ & $1.384(2)$ \\
$\mathrm{N}(1)-\mathrm{C}(12)$ & $1.455(2)$ \\
$\mathrm{C}(1)-\mathrm{C}(2)$ & $1.392(3)$ \\
$\mathrm{C}(1)-\mathrm{C}(6)$ & $1.418(2)$ \\
$\mathrm{C}(2)-\mathrm{C}(3)$ & $1.371(3)$ \\
$\mathrm{C}(2)-\mathrm{H}(2)$ & 0.9400 \\
$\mathrm{C}(3)-\mathrm{C}(4)$ & $1.392(3)$ \\
$\mathrm{C}(3)-\mathrm{H}(3)$ & 0.9400 \\
$\mathrm{C}(4)-\mathrm{C}(5)$ & $1.379(3)$ \\
$\mathrm{C}(4)-\mathrm{N}(2)$ & $1.457(3)$ \\
$\mathrm{C}(5)-\mathrm{C}(6)$ & $1.392(3)$ \\
$\mathrm{C}(5)-\mathrm{H}(5)$ & 0.9400 \\
$\mathrm{C}(6)-\mathrm{C}(7)$ & $1.420(3)$ \\
$\mathrm{C}(7)-\mathrm{C}(11)$ & $1.365(3)$ \\
$\mathrm{C}(7)-\mathrm{C}(8)$ & $1.491(2)$ \\
$\mathrm{C}(8)-\mathrm{O}(1)$ & $1.434(2)$ \\
$\mathrm{C}(8)-\mathrm{C}(15)$ & $1.535(3)$ \\
$\mathrm{C}(8)-\mathrm{C}(13)$ & $1.540(3)$ \\
$\mathrm{O}(1)-\mathrm{C}(9)$ & $1.374(2)$ \\
$\mathrm{C}(9)-\mathrm{C}(10)$ & $1.348(3)$ \\
$\mathrm{C}(9)-\mathrm{C}(17)$ & $1.487(3)$ \\
$\mathrm{C}(10)-\mathrm{C}(11)$ & $1.455(3)$ \\
$\mathrm{C}(10)-\mathrm{C}(18)$ & $1.501(3)$ \\
$\mathrm{C}(12)-\mathrm{H}(12 \mathrm{~A})$ & 0.9700 \\
$\mathrm{C}(12)-\mathrm{H}(12 \mathrm{~B})$ & 0.9700 \\
$\mathrm{C}(12)-\mathrm{H}(12 \mathrm{C})$ & 0.9700 \\
& \\
& \\
&
\end{tabular}




\begin{tabular}{|c|c|}
\hline $\mathrm{N}(2)-\mathrm{O}(3)$ & $1.211(3)$ \\
\hline $\mathrm{N}(2)-\mathrm{O}(2)$ & $1.216(3)$ \\
\hline $\mathrm{C}(13)-\mathrm{O}(4)$ & $1.190(2)$ \\
\hline $\mathrm{C}(13)-\mathrm{O}(5)$ & $1.315(2)$ \\
\hline $\mathrm{O}(5)-\mathrm{C}(14)$ & $1.445(2)$ \\
\hline $\mathrm{C}(14)-\mathrm{H}(14 \mathrm{~A})$ & 0.9700 \\
\hline $\mathrm{C}(14)-\mathrm{H}(14 \mathrm{~B})$ & 0.9700 \\
\hline $\mathrm{C}(14)-\mathrm{H}(14 \mathrm{C})$ & 0.9700 \\
\hline $\mathrm{C}(15)-\mathrm{O}(6)$ & $1.188(3)$ \\
\hline $\mathrm{C}(15)-\mathrm{O}(7)$ & $1.314(3)$ \\
\hline $\mathrm{O}(7)-\mathrm{C}(16)$ & $1.457(3)$ \\
\hline $\mathrm{C}(16)-\mathrm{H}(16 \mathrm{~A})$ & 0.9700 \\
\hline $\mathrm{C}(16)-\mathrm{H}(16 \mathrm{~B})$ & 0.9700 \\
\hline $\mathrm{C}(16)-\mathrm{H}(16 \mathrm{C})$ & 0.9700 \\
\hline $\mathrm{C}(17)-\mathrm{H}(17 \mathrm{~A})$ & 0.9700 \\
\hline $\mathrm{C}(17)-\mathrm{H}(17 \mathrm{~B})$ & 0.9700 \\
\hline $\mathrm{C}(17)-\mathrm{H}(17 \mathrm{C})$ & 0.9700 \\
\hline $\mathrm{C}(18)-\mathrm{O}(8)$ & $1.208(3)$ \\
\hline$C(18)-C(19)$ & $1.493(3)$ \\
\hline $\mathrm{C}(19)-\mathrm{H}(19 \mathrm{~A})$ & 0.9700 \\
\hline $\mathrm{C}(19)-\mathrm{H}(19 \mathrm{~B})$ & 0.9700 \\
\hline $\mathrm{C}(19)-\mathrm{H}(19 \mathrm{C})$ & 0.9700 \\
\hline $\mathrm{C}(1)-\mathrm{N}(1)-\mathrm{C}(11)$ & $108.05(14)$ \\
\hline $\mathrm{C}(1)-\mathrm{N}(1)-\mathrm{C}(12)$ & $124.18(16)$ \\
\hline $\mathrm{C}(11)-\mathrm{N}(1)-\mathrm{C}(12)$ & $127.37(16)$ \\
\hline $\mathrm{N}(1)-\mathrm{C}(1)-\mathrm{C}(2)$ & $129.38(17)$ \\
\hline $\mathrm{N}(1)-\mathrm{C}(1)-\mathrm{C}(6)$ & $108.60(16)$ \\
\hline $\mathrm{C}(2)-\mathrm{C}(1)-\mathrm{C}(6)$ & $122.02(17)$ \\
\hline $\mathrm{C}(3)-\mathrm{C}(2)-\mathrm{C}(1)$ & $117.83(18)$ \\
\hline $\mathrm{C}(3)-\mathrm{C}(2)-\mathrm{H}(2)$ & 121.1 \\
\hline $\mathrm{C}(1)-\mathrm{C}(2)-\mathrm{H}(2)$ & 121.1 \\
\hline$C(2)-C(3)-C(4)$ & $119.99(18)$ \\
\hline $\mathrm{C}(2)-\mathrm{C}(3)-\mathrm{H}(3)$ & 120.0 \\
\hline $\mathrm{C}(4)-\mathrm{C}(3)-\mathrm{H}(3)$ & 120.0 \\
\hline $\mathrm{C}(5)-\mathrm{C}(4)-\mathrm{C}(3)$ & 123.61(19) \\
\hline $\mathrm{C}(5)-\mathrm{C}(4)-\mathrm{N}(2)$ & 117.92(19) \\
\hline $\mathrm{C}(3)-\mathrm{C}(4)-\mathrm{N}(2)$ & $118.46(19)$ \\
\hline
\end{tabular}

$118.46(19)$ 


\begin{tabular}{|c|c|}
\hline$C(4)-C(5)-C(6)$ & $117.07(18)$ \\
\hline $\mathrm{C}(4)-\mathrm{C}(5)-\mathrm{H}(5)$ & 121.5 \\
\hline $\mathrm{C}(6)-\mathrm{C}(5)-\mathrm{H}(5)$ & 121.5 \\
\hline$C(5)-C(6)-C(1)$ & $119.43(17)$ \\
\hline$C(5)-C(6)-C(7)$ & $134.80(17)$ \\
\hline$C(1)-C(6)-C(7)$ & $105.76(16)$ \\
\hline$C(11)-C(7)-C(6)$ & $108.13(16)$ \\
\hline $\mathrm{C}(11)-\mathrm{C}(7)-\mathrm{C}(8)$ & $119.28(17)$ \\
\hline$C(6)-C(7)-C(8)$ & $132.58(16)$ \\
\hline $\mathrm{O}(1)-\mathrm{C}(8)-\mathrm{C}(7)$ & $110.73(15)$ \\
\hline $\mathrm{O}(1)-\mathrm{C}(8)-\mathrm{C}(15)$ & $108.74(16)$ \\
\hline$C(7)-C(8)-C(15)$ & $111.29(16)$ \\
\hline $\mathrm{O}(1)-\mathrm{C}(8)-\mathrm{C}(13)$ & $102.07(14)$ \\
\hline $\mathrm{C}(7)-\mathrm{C}(8)-\mathrm{C}(13)$ & $114.49(15)$ \\
\hline$C(15)-C(8)-C(13)$ & $109.03(16)$ \\
\hline $\mathrm{C}(9)-\mathrm{O}(1)-\mathrm{C}(8)$ & $120.44(15)$ \\
\hline $\mathrm{C}(10)-\mathrm{C}(9)-\mathrm{O}(1)$ & $122.15(18)$ \\
\hline$C(10)-C(9)-C(17)$ & $128.9(2)$ \\
\hline $\mathrm{O}(1)-\mathrm{C}(9)-\mathrm{C}(17)$ & $108.94(18)$ \\
\hline $\mathrm{C}(9)-\mathrm{C}(10)-\mathrm{C}(11)$ & $115.68(17)$ \\
\hline $\mathrm{C}(9)-\mathrm{C}(10)-\mathrm{C}(18)$ & $122.51(18)$ \\
\hline $\mathrm{C}(11)-\mathrm{C}(10)-\mathrm{C}(18)$ & $120.86(17)$ \\
\hline $\mathrm{C}(7)-\mathrm{C}(11)-\mathrm{N}(1)$ & $109.40(16)$ \\
\hline$C(7)-C(11)-C(10)$ & $122.29(17)$ \\
\hline $\mathrm{N}(1)-\mathrm{C}(11)-\mathrm{C}(10)$ & $128.30(17)$ \\
\hline $\mathrm{N}(1)-\mathrm{C}(12)-\mathrm{H}(12 \mathrm{~A})$ & 109.5 \\
\hline $\mathrm{N}(1)-\mathrm{C}(12)-\mathrm{H}(12 \mathrm{~B})$ & 109.5 \\
\hline $\mathrm{H}(12 \mathrm{~A})-\mathrm{C}(12)-\mathrm{H}(12 \mathrm{~B})$ & 109.5 \\
\hline $\mathrm{N}(1)-\mathrm{C}(12)-\mathrm{H}(12 \mathrm{C})$ & 109.5 \\
\hline $\mathrm{H}(12 \mathrm{~A})-\mathrm{C}(12)-\mathrm{H}(12 \mathrm{C})$ & 109.5 \\
\hline $\mathrm{H}(12 \mathrm{~B})-\mathrm{C}(12)-\mathrm{H}(12 \mathrm{C})$ & 109.5 \\
\hline $\mathrm{O}(3)-\mathrm{N}(2)-\mathrm{O}(2)$ & $122.4(2)$ \\
\hline $\mathrm{O}(3)-\mathrm{N}(2)-\mathrm{C}(4)$ & $119.33(19)$ \\
\hline $\mathrm{O}(2)-\mathrm{N}(2)-\mathrm{C}(4)$ & $118.3(2)$ \\
\hline $\mathrm{O}(4)-\mathrm{C}(13)-\mathrm{O}(5)$ & $125.31(18)$ \\
\hline $\mathrm{O}(4)-\mathrm{C}(13)-\mathrm{C}(8)$ & $123.45(18)$ \\
\hline $\mathrm{O}(5)-\mathrm{C}(13)-\mathrm{C}(8)$ & $111.11(15)$ \\
\hline $\mathrm{C}(13)-\mathrm{O}(5)-\mathrm{C}(14)$ & $116.10(16)$ \\
\hline
\end{tabular}




\begin{tabular}{|c|c|}
\hline $\mathrm{O}(5)-\mathrm{C}(14)-\mathrm{H}(14 \mathrm{~A})$ & 109.5 \\
\hline $\mathrm{O}(5)-\mathrm{C}(14)-\mathrm{H}(14 \mathrm{~B})$ & 109.5 \\
\hline $\mathrm{H}(14 \mathrm{~A})-\mathrm{C}(14)-\mathrm{H}(14 \mathrm{~B})$ & 109.5 \\
\hline $\mathrm{O}(5)-\mathrm{C}(14)-\mathrm{H}(14 \mathrm{C})$ & 109.5 \\
\hline $\mathrm{H}(14 \mathrm{~A})-\mathrm{C}(14)-\mathrm{H}(14 \mathrm{C})$ & 109.5 \\
\hline $\mathrm{H}(14 \mathrm{~B})-\mathrm{C}(14)-\mathrm{H}(14 \mathrm{C})$ & 109.5 \\
\hline $\mathrm{O}(6)-\mathrm{C}(15)-\mathrm{O}(7)$ & $125.7(2)$ \\
\hline $\mathrm{O}(6)-\mathrm{C}(15)-\mathrm{C}(8)$ & $124.1(2)$ \\
\hline $\mathrm{O}(7)-\mathrm{C}(15)-\mathrm{C}(8)$ & $110.11(17)$ \\
\hline $\mathrm{C}(15)-\mathrm{O}(7)-\mathrm{C}(16)$ & $117.1(2)$ \\
\hline $\mathrm{O}(7)-\mathrm{C}(16)-\mathrm{H}(16 \mathrm{~A})$ & 109.5 \\
\hline $\mathrm{O}(7)-\mathrm{C}(16)-\mathrm{H}(16 \mathrm{~B})$ & 109.5 \\
\hline $\mathrm{H}(16 \mathrm{~A})-\mathrm{C}(16)-\mathrm{H}(16 \mathrm{~B})$ & 109.5 \\
\hline $\mathrm{O}(7)-\mathrm{C}(16)-\mathrm{H}(16 \mathrm{C})$ & 109.5 \\
\hline $\mathrm{H}(16 \mathrm{~A})-\mathrm{C}(16)-\mathrm{H}(16 \mathrm{C})$ & 109.5 \\
\hline $\mathrm{H}(16 \mathrm{~B})-\mathrm{C}(16)-\mathrm{H}(16 \mathrm{C})$ & 109.5 \\
\hline $\mathrm{C}(9)-\mathrm{C}(17)-\mathrm{H}(17 \mathrm{~A})$ & 109.5 \\
\hline C(9)-C(17)-H(17B) & 109.5 \\
\hline $\mathrm{H}(17 \mathrm{~A})-\mathrm{C}(17)-\mathrm{H}(17 \mathrm{~B})$ & 109.5 \\
\hline C(9)-C(17)-H(17C) & 109.5 \\
\hline $\mathrm{H}(17 \mathrm{~A})-\mathrm{C}(17)-\mathrm{H}(17 \mathrm{C})$ & 109.5 \\
\hline $\mathrm{H}(17 \mathrm{~B})-\mathrm{C}(17)-\mathrm{H}(17 \mathrm{C})$ & 109.5 \\
\hline $\mathrm{O}(8)-\mathrm{C}(18)-\mathrm{C}(19)$ & $120.8(2)$ \\
\hline $\mathrm{O}(8)-\mathrm{C}(18)-\mathrm{C}(10)$ & $118.3(2)$ \\
\hline$C(19)-C(18)-C(10)$ & $120.9(2)$ \\
\hline $\mathrm{C}(18)-\mathrm{C}(19)-\mathrm{H}(19 \mathrm{~A})$ & 109.5 \\
\hline C(18)-C(19)-H(19B) & 109.5 \\
\hline H(19A)-C(19)-H(19B) & 109.5 \\
\hline $\mathrm{C}(18)-\mathrm{C}(19)-\mathrm{H}(19 \mathrm{C})$ & 109.5 \\
\hline $\mathrm{H}(19 \mathrm{~A})-\mathrm{C}(19)-\mathrm{H}(19 \mathrm{C})$ & 109.5 \\
\hline H(19B)-C(19)-H(19C) & 109.5 \\
\hline
\end{tabular}

Symmetry transformations used to generate equivalent atoms:

Table 4. Anisotropic displacement parameters $\left(\AA^{2} \times 10^{3}\right)$ for 3da. The anisotropic displacement factor exponent takes the form: $-2 \pi^{2}\left[\mathrm{~h}^{2} \mathrm{a}^{* 2} \mathrm{U}^{11}+\ldots+2 \mathrm{hk} \mathrm{a*} \mathrm{b}^{*} \mathrm{U}^{12}\right]$ 
$\begin{array}{llllll}\mathrm{U}^{11} & \mathrm{U}^{22} & \mathrm{U}^{33} & \mathrm{U}^{23} & \mathrm{U}^{13} & \mathrm{U}^{12}\end{array}$

\begin{tabular}{lllllll}
\hline $\mathrm{N}(1)$ & $26(1)$ & $30(1)$ & $30(1)$ & $1(1)$ & $-7(1)$ & $-7(1)$ \\
$\mathrm{C}(1)$ & $24(1)$ & $31(1)$ & $28(1)$ & $0(1)$ & $-4(1)$ & $-12(1)$ \\
$\mathrm{C}(2)$ & $34(1)$ & $41(1)$ & $28(1)$ & $2(1)$ & $-7(1)$ & $-16(1)$ \\
$\mathrm{C}(3)$ & $44(1)$ & $46(1)$ & $27(1)$ & $-7(1)$ & $-2(1)$ & $-18(1)$ \\
$\mathrm{C}(4)$ & $36(1)$ & $34(1)$ & $38(1)$ & $-8(1)$ & $1(1)$ & $-12(1)$ \\
$\mathrm{C}(5)$ & $31(1)$ & $31(1)$ & $32(1)$ & $-2(1)$ & $-5(1)$ & $-8(1)$ \\
$\mathrm{C}(6)$ & $25(1)$ & $30(1)$ & $28(1)$ & $0(1)$ & $-5(1)$ & $-10(1)$ \\
$\mathrm{C}(7)$ & $23(1)$ & $31(1)$ & $27(1)$ & $-1(1)$ & $-4(1)$ & $-9(1)$ \\
$\mathrm{C}(8)$ & $27(1)$ & $39(1)$ & $26(1)$ & $-4(1)$ & $-5(1)$ & $-9(1)$ \\
$\mathrm{O}(1)$ & $32(1)$ & $55(1)$ & $39(1)$ & $-20(1)$ & $-12(1)$ & $-1(1)$ \\
$\mathrm{C}(9)$ & $28(1)$ & $47(1)$ & $44(1)$ & $-18(1)$ & $-3(1)$ & $-6(1)$ \\
$\mathrm{C}(10)$ & $27(1)$ & $35(1)$ & $38(1)$ & $-9(1)$ & $-3(1)$ & $-7(1)$ \\
$\mathrm{C}(11)$ & $23(1)$ & $31(1)$ & $32(1)$ & $-3(1)$ & $-5(1)$ & $-10(1)$ \\
$\mathrm{C}(12)$ & $33(1)$ & $34(1)$ & $42(1)$ & $6(1)$ & $-10(1)$ & $-7(1)$ \\
$\mathrm{N}(2)$ & $56(1)$ & $41(1)$ & $48(1)$ & $-13(1)$ & $4(1)$ & $-10(1)$ \\
$\mathrm{O}(2)$ & $123(2)$ & $71(1)$ & $50(1)$ & $-31(1)$ & $6(1)$ & $8(1)$ \\
$\mathrm{O}(3)$ & $84(1)$ & $45(1)$ & $68(1)$ & $-12(1)$ & $-9(1)$ & $14(1)$ \\
$\mathrm{C}(13)$ & $29(1)$ & $33(1)$ & $28(1)$ & $-4(1)$ & $-7(1)$ & $-8(1)$ \\
$\mathrm{O}(4)$ & $38(1)$ & $56(1)$ & $57(1)$ & $22(1)$ & $-14(1)$ & $-9(1)$ \\
$\mathrm{O}(5)$ & $26(1)$ & $48(1)$ & $45(1)$ & $11(1)$ & $-11(1)$ & $-14(1)$ \\
$\mathrm{C}(14)$ & $26(1)$ & $58(1)$ & $52(1)$ & $-2(1)$ & $-8(1)$ & $-13(1)$ \\
$\mathrm{C}(15)$ & $32(1)$ & $64(2)$ & $28(1)$ & $9(1)$ & $-10(1)$ & $-17(1)$ \\
$\mathrm{O}(6)$ & $53(1)$ & $95(2)$ & $47(1)$ & $6(1)$ & $14(1)$ & $-23(1)$ \\
$\mathrm{O}(7)$ & $83(1)$ & $61(1)$ & $40(1)$ & $9(1)$ & $-11(1)$ & $-47(1)$ \\
$\mathrm{C}(16)$ & $106(2)$ & $82(2)$ & $68(2)$ & $39(2)$ & $-39(2)$ & $-68(2)$ \\
$\mathrm{C}(17)$ & $54(2)$ & $82(2)$ & $54(2)$ & $-38(2)$ & $-15(1)$ & $5(1)$ \\
$\mathrm{C}(18)$ & $34(1)$ & $43(1)$ & $38(1)$ & $-5(1)$ & $-2(1)$ & $-4(1)$ \\
$\mathrm{O}(8)$ & $33(1)$ & $64(1)$ & $79(1)$ & $-11(1)$ & $-16(1)$ & $-6(1)$ \\
$\mathrm{C}(19)$ & $65(2)$ & $41(1)$ & $63(2)$ & $-12(1)$ & $-2(1)$ & $2(1)$ \\
\hline & & & & & & \\
\hline
\end{tabular}

Table 5. Hydrogen coordinates $\left(\mathrm{x} 10^{4}\right)$ and isotropic displacement parameters $\left(\AA^{2} \times 10^{3}\right)$ for $\mathbf{3 d a}$.

\begin{tabular}{ccccc}
\hline $\mathrm{x}$ & $\mathrm{y}$ & $\mathrm{z}$ & $\mathrm{U}(\mathrm{eq})$ \\
& $\mathrm{S} 60$
\end{tabular}




$\begin{array}{lrrrr}\mathrm{H}(2) & 6970 & 5600 & 6077 & 41 \\ \mathrm{H}(3) & 4816 & 7701 & 6585 & 46 \\ \mathrm{H}(5) & 3389 & 8968 & 3655 & 38 \\ \mathrm{H}(12 \mathrm{~A}) & 10104 & 3715 & 4569 & 57 \\ \mathrm{H}(12 \mathrm{~B}) & 8974 & 2848 & 4238 & 57 \\ \mathrm{H}(12 \mathrm{C}) & 8323 & 3517 & 5305 & 57 \\ \mathrm{H}(14 \mathrm{~A}) & -325 & 8130 & 2682 & 69 \\ \mathrm{H}(14 \mathrm{~B}) & -190 & 6550 & 3051 & 69 \\ \mathrm{H}(14 \mathrm{C}) & 37 & 7076 & 1847 & 69 \\ \mathrm{H}(16 \mathrm{~A}) & 7784 & 9891 & 1279 & 117 \\ \mathrm{H}(16 \mathrm{~B}) & 5784 & 11024 & 1526 & 117 \\ \mathrm{H}(16 \mathrm{C}) & 6316 & 10355 & 460 & 117 \\ \mathrm{H}(17 \mathrm{~A}) & 9232 & 3250 & 362 & 99 \\ \mathrm{H}(17 \mathrm{~B}) & 7971 & 4633 & -243 & 99 \\ \mathrm{H}(17 \mathrm{C}) & 7104 & 3454 & 288 & 99 \\ \mathrm{H}(19 \mathrm{~A}) & 11429 & 1612 & 1371 & 92 \\ \mathrm{H}(19 \mathrm{~B}) & 9486 & 1585 & 2010 & 92 \\ \mathrm{H}(19 \mathrm{C}) & 11306 & 1126 & 2585 & 92 \\ & & & & \end{array}$

Table 6. Torsion angles $\left[^{\circ}\right]$ for $\mathbf{3 d a}$.

\begin{tabular}{|c|c|}
\hline $\mathrm{C}(11)-\mathrm{N}(1)-\mathrm{C}(1)-\mathrm{C}(2)$ & $179.34(19)$ \\
\hline $\mathrm{C}(12)-\mathrm{N}(1)-\mathrm{C}(1)-\mathrm{C}(2)$ & $6.2(3)$ \\
\hline $\mathrm{C}(11)-\mathrm{N}(1)-\mathrm{C}(1)-\mathrm{C}(6)$ & $-0.5(2)$ \\
\hline $\mathrm{C}(12)-\mathrm{N}(1)-\mathrm{C}(1)-\mathrm{C}(6)$ & $-173.62(17)$ \\
\hline $\mathrm{N}(1)-\mathrm{C}(1)-\mathrm{C}(2)-\mathrm{C}(3)$ & $178.38(19)$ \\
\hline $\mathrm{C}(6)-\mathrm{C}(1)-\mathrm{C}(2)-\mathrm{C}(3)$ & $-1.9(3)$ \\
\hline $\mathrm{C}(1)-\mathrm{C}(2)-\mathrm{C}(3)-\mathrm{C}(4)$ & $0.2(3)$ \\
\hline $\mathrm{C}(2)-\mathrm{C}(3)-\mathrm{C}(4)-\mathrm{C}(5)$ & $0.8(3)$ \\
\hline $\mathrm{C}(2)-\mathrm{C}(3)-\mathrm{C}(4)-\mathrm{N}(2)$ & $179.43(19)$ \\
\hline $\mathrm{C}(3)-\mathrm{C}(4)-\mathrm{C}(5)-\mathrm{C}(6)$ & $-0.1(3)$ \\
\hline $\mathrm{N}(2)-\mathrm{C}(4)-\mathrm{C}(5)-\mathrm{C}(6)$ & $-178.74(17)$ \\
\hline $\mathrm{C}(4)-\mathrm{C}(5)-\mathrm{C}(6)-\mathrm{C}(1)$ & $-1.5(3)$ \\
\hline$C(4)-C(5)-C(6)-C(7)$ & $179.3(2)$ \\
\hline $\mathrm{N}(1)-\mathrm{C}(1)-\mathrm{C}(6)-\mathrm{C}(5)$ & $-177.63(16)$ \\
\hline
\end{tabular}




\begin{tabular}{|c|c|}
\hline$C(2)-C(1)-C(6)-C(5)$ & $2.6(3)$ \\
\hline $\mathrm{N}(1)-\mathrm{C}(1)-\mathrm{C}(6)-\mathrm{C}(7)$ & $1.8(2)$ \\
\hline$C(2)-C(1)-C(6)-C(7)$ & $-178.02(17)$ \\
\hline$C(5)-C(6)-C(7)-C(11)$ & $176.8(2)$ \\
\hline$C(1)-C(6)-C(7)-C(11)$ & $-2.5(2)$ \\
\hline$C(5)-C(6)-C(7)-C(8)$ & $-3.0(4)$ \\
\hline $\mathrm{C}(1)-\mathrm{C}(6)-\mathrm{C}(7)-\mathrm{C}(8)$ & $177.69(19)$ \\
\hline $\mathrm{C}(11)-\mathrm{C}(7)-\mathrm{C}(8)-\mathrm{O}(1)$ & $22.2(2)$ \\
\hline $\mathrm{C}(6)-\mathrm{C}(7)-\mathrm{C}(8)-\mathrm{O}(1)$ & $-158.02(19)$ \\
\hline$C(11)-C(7)-C(8)-C(15)$ & $-98.9(2)$ \\
\hline$C(6)-C(7)-C(8)-C(15)$ & $80.9(3)$ \\
\hline$C(11)-C(7)-C(8)-C(13)$ & $136.88(18)$ \\
\hline$C(6)-C(7)-C(8)-C(13)$ & $-43.3(3)$ \\
\hline $\mathrm{C}(7)-\mathrm{C}(8)-\mathrm{O}(1)-\mathrm{C}(9)$ & $-36.0(2)$ \\
\hline $\mathrm{C}(15)-\mathrm{C}(8)-\mathrm{O}(1)-\mathrm{C}(9)$ & $86.6(2)$ \\
\hline $\mathrm{C}(13)-\mathrm{C}(8)-\mathrm{O}(1)-\mathrm{C}(9)$ & $-158.31(17)$ \\
\hline $\mathrm{C}(8)-\mathrm{O}(1)-\mathrm{C}(9)-\mathrm{C}(10)$ & $26.7(3)$ \\
\hline $\mathrm{C}(8)-\mathrm{O}(1)-\mathrm{C}(9)-\mathrm{C}(17)$ & $-153.2(2)$ \\
\hline $\mathrm{O}(1)-\mathrm{C}(9)-\mathrm{C}(10)-\mathrm{C}(11)$ & $-0.7(3)$ \\
\hline$C(17)-C(9)-C(10)-C(11)$ & $179.2(2)$ \\
\hline $\mathrm{O}(1)-\mathrm{C}(9)-\mathrm{C}(10)-\mathrm{C}(18)$ & $-169.6(2)$ \\
\hline$C(17)-C(9)-C(10)-C(18)$ & $10.4(4)$ \\
\hline$C(6)-C(7)-C(11)-N(1)$ & $2.3(2)$ \\
\hline $\mathrm{C}(8)-\mathrm{C}(7)-\mathrm{C}(11)-\mathrm{N}(1)$ & $-177.87(16)$ \\
\hline$C(6)-C(7)-C(11)-C(10)$ & $-179.00(17)$ \\
\hline$C(8)-C(7)-C(11)-C(10)$ & $0.9(3)$ \\
\hline $\mathrm{C}(1)-\mathrm{N}(1)-\mathrm{C}(11)-\mathrm{C}(7)$ & $-1.1(2)$ \\
\hline$C(12)-N(1)-C(11)-C(7)$ & $171.75(18)$ \\
\hline$C(1)-N(1)-C(11)-C(10)$ & $-179.77(18)$ \\
\hline $\mathrm{C}(12)-\mathrm{N}(1)-\mathrm{C}(11)-\mathrm{C}(10)$ & $-6.9(3)$ \\
\hline$C(9)-C(10)-C(11)-C(7)$ & $-12.8(3)$ \\
\hline$C(18)-C(10)-C(11)-C(7)$ & $156.26(19)$ \\
\hline $\mathrm{C}(9)-\mathrm{C}(10)-\mathrm{C}(11)-\mathrm{N}(1)$ & $165.65(19)$ \\
\hline $\mathrm{C}(18)-\mathrm{C}(10)-\mathrm{C}(11)-\mathrm{N}(1)$ & $-25.3(3)$ \\
\hline $\mathrm{C}(5)-\mathrm{C}(4)-\mathrm{N}(2)-\mathrm{O}(3)$ & $-4.1(3)$ \\
\hline $\mathrm{C}(3)-\mathrm{C}(4)-\mathrm{N}(2)-\mathrm{O}(3)$ & $177.2(2)$ \\
\hline $\mathrm{C}(5)-\mathrm{C}(4)-\mathrm{N}(2)-\mathrm{O}(2)$ & $175.8(2)$ \\
\hline $\mathrm{C}(3)-\mathrm{C}(4)-\mathrm{N}(2)-\mathrm{O}(2)$ & $-3.0(3)$ \\
\hline
\end{tabular}




\begin{tabular}{lc}
$\mathrm{O}(1)-\mathrm{C}(8)-\mathrm{C}(13)-\mathrm{O}(4)$ & $-96.6(2)$ \\
$\mathrm{C}(7)-\mathrm{C}(8)-\mathrm{C}(13)-\mathrm{O}(4)$ & $143.7(2)$ \\
$\mathrm{C}(15)-\mathrm{C}(8)-\mathrm{C}(13)-\mathrm{O}(4)$ & $18.3(3)$ \\
$\mathrm{O}(1)-\mathrm{C}(8)-\mathrm{C}(13)-\mathrm{O}(5)$ & $79.46(18)$ \\
$\mathrm{C}(7)-\mathrm{C}(8)-\mathrm{C}(13)-\mathrm{O}(5)$ & $-40.2(2)$ \\
$\mathrm{C}(15)-\mathrm{C}(8)-\mathrm{C}(13)-\mathrm{O}(5)$ & $-165.61(16)$ \\
$\mathrm{O}(4)-\mathrm{C}(13)-\mathrm{O}(5)-\mathrm{C}(14)$ & $3.8(3)$ \\
$\mathrm{C}(8)-\mathrm{C}(13)-\mathrm{O}(5)-\mathrm{C}(14)$ & $-172.22(17)$ \\
$\mathrm{O}(1)-\mathrm{C}(8)-\mathrm{C}(15)-\mathrm{O}(6)$ & $-6.3(3)$ \\
$\mathrm{C}(7)-\mathrm{C}(8)-\mathrm{C}(15)-\mathrm{O}(6)$ & $116.0(2)$ \\
$\mathrm{C}(13)-\mathrm{C}(8)-\mathrm{C}(15)-\mathrm{O}(6)$ & $-116.8(2)$ \\
$\mathrm{O}(1)-\mathrm{C}(8)-\mathrm{C}(15)-\mathrm{O}(7)$ & $176.43(15)$ \\
$\mathrm{C}(7)-\mathrm{C}(8)-\mathrm{C}(15)-\mathrm{O}(7)$ & $-61.3(2)$ \\
$\mathrm{C}(13)-\mathrm{C}(8)-\mathrm{C}(15)-\mathrm{O}(7)$ & $65.9(2)$ \\
$\mathrm{O}(6)-\mathrm{C}(15)-\mathrm{O}(7)-\mathrm{C}(16)$ & $2.4(3)$ \\
$\mathrm{C}(8)-\mathrm{C}(15)-\mathrm{O}(7)-\mathrm{C}(16)$ & $179.69(19)$ \\
$\mathrm{C}(9)-\mathrm{C}(10)-\mathrm{C}(18)-\mathrm{O}(8)$ & $123.6(3)$ \\
$\mathrm{C}(11)-\mathrm{C}(10)-\mathrm{C}(18)-\mathrm{O}(8)$ & $-44.7(3)$ \\
$\mathrm{C}(9)-\mathrm{C}(10)-\mathrm{C}(18)-\mathrm{C}(19)$ & $-59.2(3)$ \\
$\mathrm{C}(11)-\mathrm{C}(10)-\mathrm{C}(18)-\mathrm{C}(19)$ & $132.5(2)$ \\
& \\
\hline
\end{tabular}

Symmetry transformations used to generate equivalent atoms: 
Single Crystal X-ray Diffraction Data for the pyran 3af $(\mathrm{CCDC}=1881736$, Thermal ellipsoids were drawn at the $50 \%$ probability levels). The crystals of 3af were obtained by dissolving 3af in hexane:diethyl ether (1:1) followed by slow evaporation of diethyl ether.
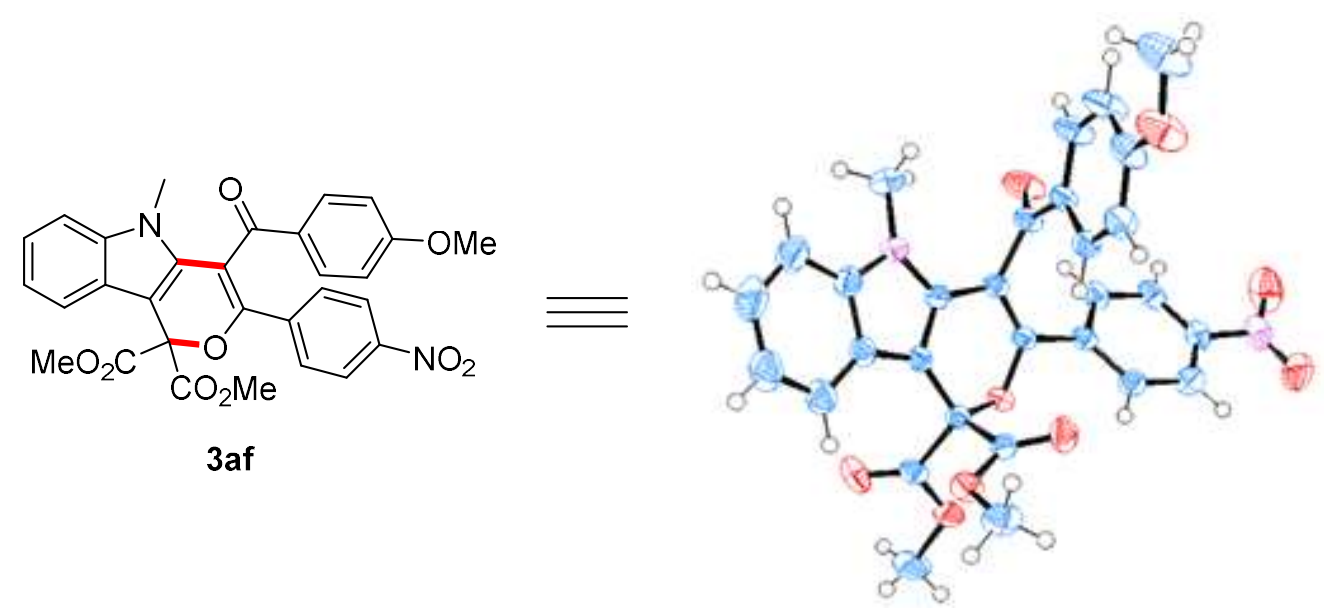

Table 1. Crystal data and structure refinement for $\mathbf{3 a f}$.

Identification code

Empirical formula

Formula weight

Temperature

Wavelength

Crystal system

Space group

Unit cell dimensions

Volume

Z

Density (calculated)

Absorption coefficient

$\mathrm{F}(000)$

Crystal size

Theta range for data collection

Index ranges

Reflections collected

Independent reflections

Completeness to theta $=25.242^{\circ}$

Absorption correction
3 af

C30 H24 N2 O9

556.51

223(2) K

$0.71073 \AA$

Monoclinic

$\mathrm{P} 21 / \mathrm{c}$

$\mathrm{a}=10.9386(8) \AA$

$\alpha=90^{\circ}$.

$\mathrm{b}=15.4387(12) \AA$

$\beta=104.272(3)^{\circ}$.

$\mathrm{c}=16.1311(12) \AA$

$\gamma=90^{\circ}$.

4

$1.400 \mathrm{Mg} / \mathrm{m}^{3}$

$0.105 \mathrm{~mm}^{-1}$

1160

$0.230 \times 0.200 \times 0.100 \mathrm{~mm}^{3}$

2.331 to $28.372^{\circ}$.

$-14<=\mathrm{h}<=14,-20<=\mathrm{k}<=20,-21<=\mathrm{l}<=21$

86051

$6606[\mathrm{R}(\mathrm{int})=0.0950]$

$99.9 \%$

Semi-empirical from equivalents 
Max. and min. transmission

Refinement method

Data / restraints / parameters

Goodness-of-fit on $\mathrm{F}^{2}$

Final $R$ indices $[\mathrm{I}>2 \operatorname{sigma}(\mathrm{I})]$

$\mathrm{R}$ indices (all data)

Extinction coefficient

Largest diff. peak and hole
0.7457 and 0.7103

Full-matrix least-squares on $\mathrm{F}^{2}$

$6606 / 0$ / 374

1.029

$\mathrm{R} 1=0.0528, w \mathrm{R} 2=0.1016$

$\mathrm{R} 1=0.0956, \mathrm{wR} 2=0.1197$

$\mathrm{n} / \mathrm{a}$

0.275 and -0.255 e. $\AA^{-3}$

Table 2. Atomic coordinates $\left(\mathrm{x} 10^{4}\right)$ and equivalent isotropic displacement parameters $\left(\AA^{2} \times 10^{3}\right)$ for 3 af. $\mathrm{U}(\mathrm{eq})$ is defined as one third of the trace of the orthogonalized $\mathrm{U}^{\mathrm{ij}}$ tensor.

\begin{tabular}{|c|c|c|c|c|}
\hline & $\mathrm{x}$ & $\mathrm{y}$ & $\mathrm{z}$ & $\mathrm{U}(\mathrm{eq})$ \\
\hline $\mathrm{N}(1)$ & $630(1)$ & $-1595(1)$ & $1468(1)$ & $35(1)$ \\
\hline $\mathrm{C}(1)$ & $1798(2)$ & $-1976(1)$ & $1776(1)$ & $36(1)$ \\
\hline $\mathrm{C}(2)$ & $2085(2)$ & $-2838(1)$ & $1978(2)$ & $49(1)$ \\
\hline$C(3)$ & $3328(3)$ & $-3050(2)$ & $2307(2)$ & $60(1)$ \\
\hline $\mathrm{C}(4)$ & $4270(2)$ & $-2425(2)$ & $2426(2)$ & $55(1)$ \\
\hline $\mathrm{C}(5)$ & $3997(2)$ & $-1575(1)$ & $2215(1)$ & $43(1)$ \\
\hline$C(6)$ & $2732(2)$ & $-1331(1)$ & $1880(1)$ & $32(1)$ \\
\hline$C(7)$ & $2084(2)$ & $-541(1)$ & 1611(1) & $28(1)$ \\
\hline $\mathrm{C}(8)$ & $2516(2)$ & $374(1)$ & $1566(1)$ & $27(1)$ \\
\hline $\mathrm{O}(1)$ & $1523(1)$ & $924(1)$ & 1093(1) & $29(1)$ \\
\hline $\mathrm{C}(9)$ & $281(2)$ & $747(1)$ & $1060(1)$ & $26(1)$ \\
\hline$C(10)$ & $-132(2)$ & $-50(1)$ & $1172(1)$ & $27(1)$ \\
\hline $\mathrm{C}(11)$ & $818(2)$ & $-718(1)$ & $1387(1)$ & $28(1)$ \\
\hline$C(12)$ & $-524(2)$ & $-2085(2)$ & $1134(2)$ & $51(1)$ \\
\hline$C(13)$ & $2984(2)$ & $758(1)$ & $2471(1)$ & $29(1)$ \\
\hline $\mathrm{O}(2)$ & $2366(1)$ & $1214(1)$ & $2806(1)$ & $43(1)$ \\
\hline $\mathrm{O}(3)$ & $4146(1)$ & $485(1)$ & $2812(1)$ & $40(1)$ \\
\hline$C(14)$ & $4709(2)$ & $733(2)$ & $3689(1)$ & $48(1)$ \\
\hline$C(15)$ & $3596(2)$ & $442(1)$ & $1106(1)$ & $32(1)$ \\
\hline $\mathrm{O}(4)$ & $4177(1)$ & $-163(1)$ & $953(1)$ & $48(1)$ \\
\hline $\mathrm{O}(5)$ & 3799(1) & $1259(1)$ & $943(1)$ & $47(1)$ \\
\hline$C(16)$ & $4779(2)$ & $1394(2)$ & $491(2)$ & $61(1)$ \\
\hline $\mathrm{C}(17)$ & $-483(2)$ & $\begin{array}{r}1545(1) \\
\text { S65 }\end{array}$ & $940(1)$ & $28(1)$ \\
\hline
\end{tabular}




\begin{tabular}{lrrrr}
$\mathrm{C}(18)$ & $-1669(2)$ & $1578(1)$ & $369(1)$ & $34(1)$ \\
$\mathrm{C}(19)$ & $-2401(2)$ & $2312(1)$ & $310(1)$ & $37(1)$ \\
$\mathrm{C}(20)$ & $-1948(2)$ & $3003(1)$ & $830(1)$ & $35(1)$ \\
$\mathrm{C}(21)$ & $-762(2)$ & $3001(1)$ & $1382(1)$ & $39(1)$ \\
$\mathrm{C}(22)$ & $-24(2)$ & $2273(1)$ & $1424(1)$ & $34(1)$ \\
$\mathrm{N}(2)$ & $-2750(2)$ & $3766(1)$ & $815(1)$ & $46(1)$ \\
$\mathrm{O}(6)$ & $-3849(2)$ & $3722(1)$ & $399(1)$ & $70(1)$ \\
$\mathrm{O}(7)$ & $-2300(2)$ & $4400(1)$ & $1235(1)$ & $65(1)$ \\
$\mathrm{C}(23)$ & $-1440(2)$ & $-215(1)$ & $1286(1)$ & $31(1)$ \\
$\mathrm{O}(8)$ & $-2337(1)$ & $-311(1)$ & $682(1)$ & $53(1)$ \\
$\mathrm{C}(24)$ & $-1553(2)$ & $-302(1)$ & $2177(1)$ & $31(1)$ \\
$\mathrm{C}(25)$ & $-2570(2)$ & $-748(2)$ & $2338(1)$ & $46(1)$ \\
$\mathrm{C}(26)$ & $-2672(2)$ & $-888(2)$ & $3162(1)$ & $53(1)$ \\
$\mathrm{C}(27)$ & $-1767(2)$ & $-555(2)$ & $3837(1)$ & $44(1)$ \\
$\mathrm{C}(28)$ & $-774(2)$ & $-75(1)$ & $3690(1)$ & $41(1)$ \\
$\mathrm{C}(29)$ & $-656(2)$ & $37(1)$ & $2867(1)$ & $33(1)$ \\
$\mathrm{O}(9)$ & $-1765(2)$ & $-676(1)$ & $4674(1)$ & $67(1)$ \\
$\mathrm{C}(30)$ & $-2673(2)$ & $-1248(2)$ & $4863(2)$ & $73(1)$ \\
& & & & \\
\hline
\end{tabular}

Table 3. Bond lengths $[\AA]$ and angles $\left[{ }^{\circ}\right]$ for 3af.

\begin{tabular}{ll}
\hline $\mathrm{N}(1)-\mathrm{C}(11)$ & $1.380(2)$ \\
$\mathrm{N}(1)-\mathrm{C}(1)$ & $1.382(2)$ \\
$\mathrm{N}(1)-\mathrm{C}(12)$ & $1.457(2)$ \\
$\mathrm{C}(1)-\mathrm{C}(2)$ & $1.388(3)$ \\
$\mathrm{C}(1)-\mathrm{C}(6)$ & $1.407(3)$ \\
$\mathrm{C}(2)-\mathrm{C}(3)$ & $1.372(3)$ \\
$\mathrm{C}(2)-\mathrm{H}(2)$ & 0.9400 \\
$\mathrm{C}(3)-\mathrm{C}(4)$ & $1.391(4)$ \\
$\mathrm{C}(3)-\mathrm{H}(3)$ & 0.9400 \\
$\mathrm{C}(4)-\mathrm{C}(5)$ & $1.370(3)$ \\
$\mathrm{C}(4)-\mathrm{H}(4)$ & 0.9400 \\
$\mathrm{C}(5)-\mathrm{C}(6)$ & $1.406(3)$ \\
$\mathrm{C}(5)-\mathrm{H}(5)$ & 0.9400 \\
$\mathrm{C}(6)-\mathrm{C}(7)$ & $1.425(3)$ \\
$\mathrm{C}(7)-\mathrm{C}(11)$ & $1.369(2)$
\end{tabular}




\begin{tabular}{|c|c|}
\hline $\mathrm{C}(7)-\mathrm{C}(8)$ & $1.498(3)$ \\
\hline $\mathrm{C}(8)-\mathrm{O}(1)$ & $1.439(2)$ \\
\hline$C(8)-C(13)$ & $1.543(2)$ \\
\hline$C(8)-C(15)$ & $1.544(2)$ \\
\hline $\mathrm{O}(1)-\mathrm{C}(9)$ & $1.373(2)$ \\
\hline$C(9)-C(10)$ & $1.338(2)$ \\
\hline $\mathrm{C}(9)-\mathrm{C}(17)$ & $1.475(2)$ \\
\hline $\mathrm{C}(10)-\mathrm{C}(11)$ & $1.445(2)$ \\
\hline$C(10)-C(23)$ & $1.509(2)$ \\
\hline $\mathrm{C}(12)-\mathrm{H}(12 \mathrm{~A})$ & 0.9700 \\
\hline $\mathrm{C}(12)-\mathrm{H}(12 \mathrm{~B})$ & 0.9700 \\
\hline $\mathrm{C}(12)-\mathrm{H}(12 \mathrm{C})$ & 0.9700 \\
\hline $\mathrm{C}(13)-\mathrm{O}(2)$ & $1.193(2)$ \\
\hline $\mathrm{C}(13)-\mathrm{O}(3)$ & $1.323(2)$ \\
\hline $\mathrm{O}(3)-\mathrm{C}(14)$ & $1.448(2)$ \\
\hline $\mathrm{C}(14)-\mathrm{H}(14 \mathrm{~A})$ & 0.9700 \\
\hline $\mathrm{C}(14)-\mathrm{H}(14 \mathrm{~B})$ & 0.9700 \\
\hline $\mathrm{C}(14)-\mathrm{H}(14 \mathrm{C})$ & 0.9700 \\
\hline $\mathrm{C}(15)-\mathrm{O}(4)$ & $1.189(2)$ \\
\hline $\mathrm{C}(15)-\mathrm{O}(5)$ & $1.319(2)$ \\
\hline $\mathrm{O}(5)-\mathrm{C}(16)$ & $1.453(2)$ \\
\hline $\mathrm{C}(16)-\mathrm{H}(16 \mathrm{~A})$ & 0.9700 \\
\hline $\mathrm{C}(16)-\mathrm{H}(16 \mathrm{~B})$ & 0.9700 \\
\hline$C(16)-H(16 C)$ & 0.9700 \\
\hline $\mathrm{C}(17)-\mathrm{C}(22)$ & $1.390(3)$ \\
\hline $\mathrm{C}(17)-\mathrm{C}(18)$ & $1.393(2)$ \\
\hline $\mathrm{C}(18)-\mathrm{C}(19)$ & $1.378(3)$ \\
\hline $\mathrm{C}(18)-\mathrm{H}(18)$ & 0.9400 \\
\hline$C(19)-C(20)$ & $1.371(3)$ \\
\hline $\mathrm{C}(19)-\mathrm{H}(19)$ & 0.9400 \\
\hline$C(20)-C(21)$ & $1.380(3)$ \\
\hline $\mathrm{C}(20)-\mathrm{N}(2)$ & $1.465(3)$ \\
\hline$C(21)-C(22)$ & $1.376(3)$ \\
\hline $\mathrm{C}(21)-\mathrm{H}(21)$ & 0.9400 \\
\hline $\mathrm{C}(22)-\mathrm{H}(22)$ & 0.9400 \\
\hline $\mathrm{N}(2)-\mathrm{O}(7)$ & $1.222(2)$ \\
\hline $\mathrm{N}(2)-\mathrm{O}(6)$ & $1.225(2)$ \\
\hline $\mathrm{C}(23)-\mathrm{O}(8)$ & $1.210(2)$ \\
\hline
\end{tabular}




\begin{tabular}{|c|c|}
\hline$C(23)-C(24)$ & $1.479(3)$ \\
\hline$C(24)-C(25)$ & $1.386(3)$ \\
\hline C(24)-C(29) & $1.392(3)$ \\
\hline$C(25)-C(26)$ & $1.378(3)$ \\
\hline $\mathrm{C}(25)-\mathrm{H}(25)$ & 0.9400 \\
\hline$C(26)-C(27)$ & $1.379(3)$ \\
\hline $\mathrm{C}(26)-\mathrm{H}(26)$ & 0.9400 \\
\hline $\mathrm{C}(27)-\mathrm{O}(9)$ & $1.362(2)$ \\
\hline $\mathrm{C}(27)-\mathrm{C}(28)$ & $1.383(3)$ \\
\hline $\mathrm{C}(28)-\mathrm{C}(29)$ & $1.376(3)$ \\
\hline $\mathrm{C}(28)-\mathrm{H}(28)$ & 0.9400 \\
\hline $\mathrm{C}(29)-\mathrm{H}(29)$ & 0.9400 \\
\hline $\mathrm{O}(9)-\mathrm{C}(30)$ & $1.417(3)$ \\
\hline $\mathrm{C}(30)-\mathrm{H}(30 \mathrm{~A})$ & 0.9700 \\
\hline $\mathrm{C}(30)-\mathrm{H}(30 \mathrm{~B})$ & 0.9700 \\
\hline $\mathrm{C}(30)-\mathrm{H}(30 \mathrm{C})$ & 0.9700 \\
\hline $\mathrm{C}(11)-\mathrm{N}(1)-\mathrm{C}(1)$ & $107.81(15)$ \\
\hline $\mathrm{C}(11)-\mathrm{N}(1)-\mathrm{C}(12)$ & $127.62(17)$ \\
\hline $\mathrm{C}(1)-\mathrm{N}(1)-\mathrm{C}(12)$ & $123.44(17)$ \\
\hline $\mathrm{N}(1)-\mathrm{C}(1)-\mathrm{C}(2)$ & $128.93(19)$ \\
\hline $\mathrm{N}(1)-\mathrm{C}(1)-\mathrm{C}(6)$ & $108.73(16)$ \\
\hline$C(2)-C(1)-C(6)$ & $122.34(19)$ \\
\hline $\mathrm{C}(3)-\mathrm{C}(2)-\mathrm{C}(1)$ & $117.7(2)$ \\
\hline $\mathrm{C}(3)-\mathrm{C}(2)-\mathrm{H}(2)$ & 121.2 \\
\hline $\mathrm{C}(1)-\mathrm{C}(2)-\mathrm{H}(2)$ & 121.2 \\
\hline$C(2)-C(3)-C(4)$ & $121.2(2)$ \\
\hline $\mathrm{C}(2)-\mathrm{C}(3)-\mathrm{H}(3)$ & 119.4 \\
\hline $\mathrm{C}(4)-\mathrm{C}(3)-\mathrm{H}(3)$ & 119.4 \\
\hline$C(5)-C(4)-C(3)$ & $121.4(2)$ \\
\hline $\mathrm{C}(5)-\mathrm{C}(4)-\mathrm{H}(4)$ & 119.3 \\
\hline $\mathrm{C}(3)-\mathrm{C}(4)-\mathrm{H}(4)$ & 119.3 \\
\hline$C(4)-C(5)-C(6)$ & 119.1(2) \\
\hline $\mathrm{C}(4)-\mathrm{C}(5)-\mathrm{H}(5)$ & 120.5 \\
\hline $\mathrm{C}(6)-\mathrm{C}(5)-\mathrm{H}(5)$ & 120.5 \\
\hline$C(5)-C(6)-C(1)$ & $118.24(18)$ \\
\hline$C(5)-C(6)-C(7)$ & $135.68(18)$ \\
\hline$C(1)-C(6)-C(7)$ & $106.07(16)$ \\
\hline
\end{tabular}




\begin{tabular}{|c|c|}
\hline$C(11)-C(7)-C(6)$ & $107.76(16)$ \\
\hline$C(11)-C(7)-C(8)$ & $119.12(16)$ \\
\hline $\mathrm{C}(6)-\mathrm{C}(7)-\mathrm{C}(8)$ & $133.09(16)$ \\
\hline $\mathrm{O}(1)-\mathrm{C}(8)-\mathrm{C}(7)$ & $112.04(13)$ \\
\hline $\mathrm{O}(1)-\mathrm{C}(8)-\mathrm{C}(13)$ & $107.81(14)$ \\
\hline $\mathrm{C}(7)-\mathrm{C}(8)-\mathrm{C}(13)$ & $110.56(14)$ \\
\hline $\mathrm{O}(1)-\mathrm{C}(8)-\mathrm{C}(15)$ & $105.82(14)$ \\
\hline$C(7)-C(8)-C(15)$ & $112.05(15)$ \\
\hline $\mathrm{C}(13)-\mathrm{C}(8)-\mathrm{C}(15)$ & $108.33(13)$ \\
\hline $\mathrm{C}(9)-\mathrm{O}(1)-\mathrm{C}(8)$ & $120.92(13)$ \\
\hline $\mathrm{C}(10)-\mathrm{C}(9)-\mathrm{O}(1)$ & $122.73(16)$ \\
\hline$C(10)-C(9)-C(17)$ & $125.88(15)$ \\
\hline $\mathrm{O}(1)-\mathrm{C}(9)-\mathrm{C}(17)$ & $111.29(15)$ \\
\hline $\mathrm{C}(9)-\mathrm{C}(10)-\mathrm{C}(11)$ & $116.23(15)$ \\
\hline$C(9)-C(10)-C(23)$ & $122.29(16)$ \\
\hline$C(11)-C(10)-C(23)$ & $119.87(16)$ \\
\hline $\mathrm{C}(7)-\mathrm{C}(11)-\mathrm{N}(1)$ & $109.58(16)$ \\
\hline$C(7)-C(11)-C(10)$ & $122.79(16)$ \\
\hline $\mathrm{N}(1)-\mathrm{C}(11)-\mathrm{C}(10)$ & $127.44(16)$ \\
\hline $\mathrm{N}(1)-\mathrm{C}(12)-\mathrm{H}(12 \mathrm{~A})$ & 109.5 \\
\hline $\mathrm{N}(1)-\mathrm{C}(12)-\mathrm{H}(12 \mathrm{~B})$ & 109.5 \\
\hline $\mathrm{H}(12 \mathrm{~A})-\mathrm{C}(12)-\mathrm{H}(12 \mathrm{~B})$ & 109.5 \\
\hline $\mathrm{N}(1)-\mathrm{C}(12)-\mathrm{H}(12 \mathrm{C})$ & 109.5 \\
\hline $\mathrm{H}(12 \mathrm{~A})-\mathrm{C}(12)-\mathrm{H}(12 \mathrm{C})$ & 109.5 \\
\hline $\mathrm{H}(12 \mathrm{~B})-\mathrm{C}(12)-\mathrm{H}(12 \mathrm{C})$ & 109.5 \\
\hline $\mathrm{O}(2)-\mathrm{C}(13)-\mathrm{O}(3)$ & $126.38(17)$ \\
\hline $\mathrm{O}(2)-\mathrm{C}(13)-\mathrm{C}(8)$ & $124.26(16)$ \\
\hline $\mathrm{O}(3)-\mathrm{C}(13)-\mathrm{C}(8)$ & $109.34(15)$ \\
\hline $\mathrm{C}(13)-\mathrm{O}(3)-\mathrm{C}(14)$ & $117.08(15)$ \\
\hline $\mathrm{O}(3)-\mathrm{C}(14)-\mathrm{H}(14 \mathrm{~A})$ & 109.5 \\
\hline $\mathrm{O}(3)-\mathrm{C}(14)-\mathrm{H}(14 \mathrm{~B})$ & 109.5 \\
\hline $\mathrm{H}(14 \mathrm{~A})-\mathrm{C}(14)-\mathrm{H}(14 \mathrm{~B})$ & 109.5 \\
\hline $\mathrm{O}(3)-\mathrm{C}(14)-\mathrm{H}(14 \mathrm{C})$ & 109.5 \\
\hline $\mathrm{H}(14 \mathrm{~A})-\mathrm{C}(14)-\mathrm{H}(14 \mathrm{C})$ & 109.5 \\
\hline $\mathrm{H}(14 \mathrm{~B})-\mathrm{C}(14)-\mathrm{H}(14 \mathrm{C})$ & 109.5 \\
\hline $\mathrm{O}(4)-\mathrm{C}(15)-\mathrm{O}(5)$ & $125.71(18)$ \\
\hline $\mathrm{O}(4)-\mathrm{C}(15)-\mathrm{C}(8)$ & $123.94(18)$ \\
\hline $\mathrm{O}(5)-\mathrm{C}(15)-\mathrm{C}(8)$ & $110.30(16)$ \\
\hline
\end{tabular}

$110.30(16)$ 


\begin{tabular}{|c|c|}
\hline$C(15)-O(5)-C(16)$ & $114.80(17)$ \\
\hline $\mathrm{O}(5)-\mathrm{C}(16)-\mathrm{H}(16 \mathrm{~A})$ & 109.5 \\
\hline $\mathrm{O}(5)-\mathrm{C}(16)-\mathrm{H}(16 \mathrm{~B})$ & 109.5 \\
\hline $\mathrm{H}(16 \mathrm{~A})-\mathrm{C}(16)-\mathrm{H}(16 \mathrm{~B})$ & 109.5 \\
\hline $\mathrm{O}(5)-\mathrm{C}(16)-\mathrm{H}(16 \mathrm{C})$ & 109.5 \\
\hline $\mathrm{H}(16 \mathrm{~A})-\mathrm{C}(16)-\mathrm{H}(16 \mathrm{C})$ & 109.5 \\
\hline $\mathrm{H}(16 \mathrm{~B})-\mathrm{C}(16)-\mathrm{H}(16 \mathrm{C})$ & 109.5 \\
\hline $\mathrm{C}(22)-\mathrm{C}(17)-\mathrm{C}(18)$ & $119.39(17)$ \\
\hline$C(22)-C(17)-C(9)$ & $119.11(15)$ \\
\hline $\mathrm{C}(18)-\mathrm{C}(17)-\mathrm{C}(9)$ & $121.47(16)$ \\
\hline$C(19)-C(18)-C(17)$ & $120.36(18)$ \\
\hline $\mathrm{C}(19)-\mathrm{C}(18)-\mathrm{H}(18)$ & 119.8 \\
\hline $\mathrm{C}(17)-\mathrm{C}(18)-\mathrm{H}(18)$ & 119.8 \\
\hline $\mathrm{C}(20)-\mathrm{C}(19)-\mathrm{C}(18)$ & $118.81(17)$ \\
\hline $\mathrm{C}(20)-\mathrm{C}(19)-\mathrm{H}(19)$ & 120.6 \\
\hline $\mathrm{C}(18)-\mathrm{C}(19)-\mathrm{H}(19)$ & 120.6 \\
\hline$C(19)-C(20)-C(21)$ & $122.15(18)$ \\
\hline $\mathrm{C}(19)-\mathrm{C}(20)-\mathrm{N}(2)$ & $119.38(17)$ \\
\hline $\mathrm{C}(21)-\mathrm{C}(20)-\mathrm{N}(2)$ & $118.46(18)$ \\
\hline$C(22)-C(21)-C(20)$ & $118.77(18)$ \\
\hline $\mathrm{C}(22)-\mathrm{C}(21)-\mathrm{H}(21)$ & 120.6 \\
\hline $\mathrm{C}(20)-\mathrm{C}(21)-\mathrm{H}(21)$ & 120.6 \\
\hline$C(21)-C(22)-C(17)$ & $120.37(17)$ \\
\hline $\mathrm{C}(21)-\mathrm{C}(22)-\mathrm{H}(22)$ & 119.8 \\
\hline $\mathrm{C}(17)-\mathrm{C}(22)-\mathrm{H}(22)$ & 119.8 \\
\hline $\mathrm{O}(7)-\mathrm{N}(2)-\mathrm{O}(6)$ & 123.67(19) \\
\hline $\mathrm{O}(7)-\mathrm{N}(2)-\mathrm{C}(20)$ & $118.43(18)$ \\
\hline $\mathrm{O}(6)-\mathrm{N}(2)-\mathrm{C}(20)$ & $117.87(19)$ \\
\hline $\mathrm{O}(8)-\mathrm{C}(23)-\mathrm{C}(24)$ & $121.75(17)$ \\
\hline $\mathrm{O}(8)-\mathrm{C}(23)-\mathrm{C}(10)$ & $121.87(16)$ \\
\hline$C(24)-C(23)-C(10)$ & $116.29(14)$ \\
\hline$C(25)-C(24)-C(29)$ & $118.55(17)$ \\
\hline$C(25)-C(24)-C(23)$ & $119.36(16)$ \\
\hline$C(29)-C(24)-C(23)$ & $122.08(16)$ \\
\hline$C(26)-C(25)-C(24)$ & $121.16(19)$ \\
\hline $\mathrm{C}(26)-\mathrm{C}(25)-\mathrm{H}(25)$ & 119.4 \\
\hline $\mathrm{C}(24)-\mathrm{C}(25)-\mathrm{H}(25)$ & 119.4 \\
\hline$C(25)-C(26)-C(27)$ & $119.4(2)$ \\
\hline
\end{tabular}




$\begin{array}{ll}\mathrm{C}(25)-\mathrm{C}(26)-\mathrm{H}(26) & 120.3 \\ \mathrm{C}(27)-\mathrm{C}(26)-\mathrm{H}(26) & 120.3 \\ \mathrm{O}(9)-\mathrm{C}(27)-\mathrm{C}(26) & 123.9(2) \\ \mathrm{O}(9)-\mathrm{C}(27)-\mathrm{C}(28) & 115.79(19) \\ \mathrm{C}(26)-\mathrm{C}(27)-\mathrm{C}(28) & 120.31(18) \\ \mathrm{C}(29)-\mathrm{C}(28)-\mathrm{C}(27) & 119.86(18) \\ \mathrm{C}(29)-\mathrm{C}(28)-\mathrm{H}(28) & 120.1 \\ \mathrm{C}(27)-\mathrm{C}(28)-\mathrm{H}(28) & 120.1 \\ \mathrm{C}(28)-\mathrm{C}(29)-\mathrm{C}(24) & 120.59(18) \\ \mathrm{C}(28)-\mathrm{C}(29)-\mathrm{H}(29) & 119.7 \\ \mathrm{C}(24)-\mathrm{C}(29)-\mathrm{H}(29) & 119.7 \\ \mathrm{C}(27)-\mathrm{O}(9)-\mathrm{C}(30) & 117.96(19) \\ \mathrm{O}(9)-\mathrm{C}(30)-\mathrm{H}(30 \mathrm{~A}) & 109.5 \\ \mathrm{O}(9)-\mathrm{C}(30)-\mathrm{H}(30 \mathrm{~B}) & 109.5 \\ \mathrm{H}(30 \mathrm{~A})-\mathrm{C}(30)-\mathrm{H}(30 \mathrm{~B}) & 109.5 \\ \mathrm{O}(9)-\mathrm{C}(30)-\mathrm{H}(30 \mathrm{C}) & 109.5 \\ \mathrm{H}(30 \mathrm{~A})-\mathrm{C}(30)-\mathrm{H}(30 \mathrm{C}) & 109.5 \\ \mathrm{H}(30 \mathrm{~B})-\mathrm{C}(30)-\mathrm{H}(30 \mathrm{C}) & 109.5 \\ \end{array}$

Symmetry transformations used to generate equivalent atoms:

Table 4. Anisotropic displacement parameters $\left(\AA^{2} \times 10^{3}\right)$ for 3af. The anisotropic displacement factor exponent takes the form: $\quad-2 \pi^{2}\left[\mathrm{~h}^{2} \mathrm{a}^{* 2} \mathrm{U}^{11}+\ldots+2 \mathrm{hk} \mathrm{a} \mathrm{b}^{*} \mathrm{U}^{12}\right]$

\begin{tabular}{lllllll}
\hline & $\mathrm{U}^{11}$ & $\mathrm{U}^{22}$ & $\mathrm{U}^{33}$ & $\mathrm{U}^{23}$ & $\mathrm{U}^{13}$ & $\mathrm{U}^{12}$ \\
\hline $\mathrm{N}(1)$ & $32(1)$ & $32(1)$ & $40(1)$ & $-6(1)$ & $10(1)$ & $-6(1)$ \\
$\mathrm{C}(1)$ & $41(1)$ & $32(1)$ & $37(1)$ & $-4(1)$ & $13(1)$ & $1(1)$ \\
$\mathrm{C}(2)$ & $60(1)$ & $34(1)$ & $58(1)$ & $0(1)$ & $22(1)$ & $0(1)$ \\
$\mathrm{C}(3)$ & $72(2)$ & $38(1)$ & $70(2)$ & $6(1)$ & $20(1)$ & $17(1)$ \\
$\mathrm{C}(4)$ & $50(1)$ & $50(1)$ & $62(2)$ & $3(1)$ & $6(1)$ & $18(1)$ \\
$\mathrm{C}(5)$ & $36(1)$ & $42(1)$ & $47(1)$ & $-2(1)$ & $5(1)$ & $8(1)$ \\
$\mathrm{C}(6)$ & $34(1)$ & $35(1)$ & $28(1)$ & $-5(1)$ & $8(1)$ & $2(1)$ \\
$\mathrm{C}(7)$ & $25(1)$ & $32(1)$ & $26(1)$ & $-6(1)$ & $7(1)$ & $0(1)$ \\
$\mathrm{C}(8)$ & $20(1)$ & $33(1)$ & $28(1)$ & $-4(1)$ & $6(1)$ & $-1(1)$ \\
$\mathrm{O}(1)$ & $21(1)$ & $34(1)$ & $33(1)$ & $1(1)$ & $8(1)$ & $-1(1)$ \\
$\mathrm{C}(9)$ & $22(1)$ & $35(1)$ & $22(1)$ & $-5(1)$ & $6(1)$ & $-2(1)$ \\
& & & & $\mathrm{S} 71$ & &
\end{tabular}




\begin{tabular}{llllccc}
$\mathrm{C}(10)$ & $22(1)$ & $36(1)$ & $23(1)$ & $-4(1)$ & $5(1)$ & $-3(1)$ \\
$\mathrm{C}(11)$ & $28(1)$ & $31(1)$ & $27(1)$ & $-6(1)$ & $9(1)$ & $-4(1)$ \\
$\mathrm{C}(12)$ & $43(1)$ & $44(1)$ & $64(2)$ & $-9(1)$ & $12(1)$ & $-17(1)$ \\
$\mathrm{C}(13)$ & $30(1)$ & $29(1)$ & $29(1)$ & $-3(1)$ & $9(1)$ & $-5(1)$ \\
$\mathrm{O}(2)$ & $47(1)$ & $48(1)$ & $35(1)$ & $-11(1)$ & $12(1)$ & $6(1)$ \\
$\mathrm{O}(3)$ & $29(1)$ & $57(1)$ & $30(1)$ & $-11(1)$ & $1(1)$ & $-3(1)$ \\
$\mathrm{C}(14)$ & $41(1)$ & $64(2)$ & $32(1)$ & $-7(1)$ & $-1(1)$ & $-11(1)$ \\
$\mathrm{C}(15)$ & $22(1)$ & $46(1)$ & $25(1)$ & $-5(1)$ & $3(1)$ & $-4(1)$ \\
$\mathrm{O}(4)$ & $40(1)$ & $60(1)$ & $49(1)$ & $6(1)$ & $23(1)$ & $16(1)$ \\
$\mathrm{O}(5)$ & $43(1)$ & $47(1)$ & $60(1)$ & $-14(1)$ & $31(1)$ & $-17(1)$ \\
$\mathrm{C}(16)$ & $52(1)$ & $73(2)$ & $73(2)$ & $-10(1)$ & $41(1)$ & $-23(1)$ \\
$\mathrm{C}(17)$ & $26(1)$ & $36(1)$ & $24(1)$ & $0(1)$ & $10(1)$ & $0(1)$ \\
$\mathrm{C}(18)$ & $32(1)$ & $44(1)$ & $26(1)$ & $-5(1)$ & $7(1)$ & $2(1)$ \\
$\mathrm{C}(19)$ & $26(1)$ & $53(1)$ & $30(1)$ & $3(1)$ & $5(1)$ & $7(1)$ \\
$\mathrm{C}(20)$ & $38(1)$ & $36(1)$ & $33(1)$ & $5(1)$ & $13(1)$ & $9(1)$ \\
$\mathrm{C}(21)$ & $41(1)$ & $34(1)$ & $40(1)$ & $-2(1)$ & $7(1)$ & $-1(1)$ \\
$\mathrm{C}(22)$ & $28(1)$ & $35(1)$ & $38(1)$ & $0(1)$ & $3(1)$ & $0(1)$ \\
$\mathrm{N}(2)$ & $48(1)$ & $50(1)$ & $42(1)$ & $7(1)$ & $12(1)$ & $16(1)$ \\
$\mathrm{O}(6)$ & $56(1)$ & $80(1)$ & $63(1)$ & $-5(1)$ & $-8(1)$ & $34(1)$ \\
$\mathrm{O}(7)$ & $63(1)$ & $42(1)$ & $90(1)$ & $-9(1)$ & $17(1)$ & $12(1)$ \\
$\mathrm{C}(23)$ & $24(1)$ & $40(1)$ & $29(1)$ & $-3(1)$ & $5(1)$ & $-4(1)$ \\
$\mathrm{O}(8)$ & $30(1)$ & $94(1)$ & $32(1)$ & $1(1)$ & $1(1)$ & $-19(1)$ \\
$\mathrm{C}(24)$ & $22(1)$ & $40(1)$ & $30(1)$ & $-1(1)$ & $7(1)$ & $1(1)$ \\
$\mathrm{C}(25)$ & $27(1)$ & $76(2)$ & $34(1)$ & $1(1)$ & $4(1)$ & $-12(1)$ \\
$\mathrm{C}(26)$ & $32(1)$ & $90(2)$ & $38(1)$ & $7(1)$ & $12(1)$ & $-14(1)$ \\
$\mathrm{C}(27)$ & $37(1)$ & $68(2)$ & $30(1)$ & $4(1)$ & $13(1)$ & $5(1)$ \\
$\mathrm{C}(28)$ & $36(1)$ & $56(1)$ & $30(1)$ & $-6(1)$ & $5(1)$ & $-1(1)$ \\
$\mathrm{C}(29)$ & $28(1)$ & $37(1)$ & $34(1)$ & $-3(1)$ & $8(1)$ & $0(1)$ \\
$\mathrm{O}(9)$ & $54(1)$ & $118(2)$ & $32(1)$ & $5(1)$ & $16(1)$ & $-11(1)$ \\
$\mathrm{C}(30)$ & $56(2)$ & $121(3)$ & $46(1)$ & $27(2)$ & $24(1)$ & $0(2)$ \\
& & & & & & \\
\hline & & & & & & \\
& & & & & \\
& & & & & \\
\end{tabular}

Table 5. Hydrogen coordinates $\left(x 10^{4}\right)$ and isotropic displacement parameters $\left(\AA^{2} \times 10^{3}\right)$ for $\mathbf{3 a f}$.

\begin{tabular}{llllll}
\hline$x$ & y & z & U(eq) \\
\hline
\end{tabular}




\begin{tabular}{|c|c|c|c|c|}
\hline $\mathrm{H}(2)$ & 1449 & -3262 & 1892 & 59 \\
\hline $\mathrm{H}(3)$ & 3546 & -3629 & 2455 & 72 \\
\hline $\mathrm{H}(4)$ & 5113 & -2589 & 2656 & 66 \\
\hline $\mathrm{H}(5)$ & 4645 & -1161 & 2292 & 51 \\
\hline $\mathrm{H}(12 \mathrm{~A})$ & -319 & -2632 & 905 & 76 \\
\hline $\mathrm{H}(12 \mathrm{~B})$ & -1082 & -1756 & 683 & 76 \\
\hline $\mathrm{H}(12 \mathrm{C})$ & -938 & -2194 & 1590 & 76 \\
\hline $\mathrm{H}(14 \mathrm{~A})$ & 4608 & 1352 & 3754 & 71 \\
\hline $\mathrm{H}(14 \mathrm{~B})$ & 5600 & 590 & 3833 & 71 \\
\hline $\mathrm{H}(14 \mathrm{C})$ & 4297 & 424 & 4068 & 71 \\
\hline $\mathrm{H}(16 \mathrm{~A})$ & 5561 & 1139 & 815 & 92 \\
\hline $\mathrm{H}(16 \mathrm{~B})$ & 4899 & 2010 & 424 & 92 \\
\hline $\mathrm{H}(16 \mathrm{C})$ & 4531 & 1124 & -69 & 92 \\
\hline $\mathrm{H}(18)$ & -1971 & 1096 & 23 & 41 \\
\hline $\mathrm{H}(19)$ & -3196 & 2339 & -79 & 44 \\
\hline $\mathrm{H}(21)$ & -465 & 3487 & 1722 & 47 \\
\hline $\mathrm{H}(22)$ & 797 & 2268 & 1782 & 41 \\
\hline $\mathrm{H}(25)$ & -3201 & -959 & 1876 & 56 \\
\hline $\mathrm{H}(26)$ & -3353 & -1207 & 3262 & 64 \\
\hline $\mathrm{H}(28)$ & -181 & 175 & 4150 & 49 \\
\hline $\mathrm{H}(29)$ & 37 & 344 & 2770 & 39 \\
\hline $\mathrm{H}(30 \mathrm{~A})$ & -3512 & -1014 & 4633 & 109 \\
\hline $\mathrm{H}(30 \mathrm{~B})$ & -2524 & -1311 & 5478 & 109 \\
\hline $\mathrm{H}(30 \mathrm{C})$ & -2605 & -1809 & 4608 & 109 \\
\hline
\end{tabular}


Table 6. Torsion angles $\left[{ }^{\circ}\right]$ for $\mathbf{3 a f}$.

\begin{tabular}{|c|c|}
\hline $\mathrm{C}(11)-\mathrm{N}(1)-\mathrm{C}(1)-\mathrm{C}(2)$ & $178.4(2)$ \\
\hline $\mathrm{C}(12)-\mathrm{N}(1)-\mathrm{C}(1)-\mathrm{C}(2)$ & $-12.9(3)$ \\
\hline $\mathrm{C}(11)-\mathrm{N}(1)-\mathrm{C}(1)-\mathrm{C}(6)$ & $-0.7(2)$ \\
\hline$C(12)-N(1)-C(1)-C(6)$ & 167.91(18) \\
\hline $\mathrm{N}(1)-\mathrm{C}(1)-\mathrm{C}(2)-\mathrm{C}(3)$ & $-177.6(2)$ \\
\hline$C(6)-C(1)-C(2)-C(3)$ & $1.5(3)$ \\
\hline$C(1)-C(2)-C(3)-C(4)$ & $-0.7(4)$ \\
\hline $\mathrm{C}(2)-\mathrm{C}(3)-\mathrm{C}(4)-\mathrm{C}(5)$ & $-0.4(4)$ \\
\hline$C(3)-C(4)-C(5)-C(6)$ & $0.8(4)$ \\
\hline$C(4)-C(5)-C(6)-C(1)$ & $0.0(3)$ \\
\hline$C(4)-C(5)-C(6)-C(7)$ & $178.3(2)$ \\
\hline $\mathrm{N}(1)-\mathrm{C}(1)-\mathrm{C}(6)-\mathrm{C}(5)$ & $178.08(17)$ \\
\hline$C(2)-C(1)-C(6)-C(5)$ & $-1.2(3)$ \\
\hline $\mathrm{N}(1)-\mathrm{C}(1)-\mathrm{C}(6)-\mathrm{C}(7)$ & $-0.7(2)$ \\
\hline $\mathrm{C}(2)-\mathrm{C}(1)-\mathrm{C}(6)-\mathrm{C}(7)$ & $-179.94(19)$ \\
\hline$C(5)-C(6)-C(7)-C(11)$ & $-176.6(2)$ \\
\hline$C(1)-C(6)-C(7)-C(11)$ & $1.9(2)$ \\
\hline$C(5)-C(6)-C(7)-C(8)$ & $1.1(4)$ \\
\hline $\mathrm{C}(1)-\mathrm{C}(6)-\mathrm{C}(7)-\mathrm{C}(8)$ & $179.58(18)$ \\
\hline $\mathrm{C}(11)-\mathrm{C}(7)-\mathrm{C}(8)-\mathrm{O}(1)$ & $-13.7(2)$ \\
\hline $\mathrm{C}(6)-\mathrm{C}(7)-\mathrm{C}(8)-\mathrm{O}(1)$ & $168.80(17)$ \\
\hline $\mathrm{C}(11)-\mathrm{C}(7)-\mathrm{C}(8)-\mathrm{C}(13)$ & $106.56(18)$ \\
\hline$C(6)-C(7)-C(8)-C(13)$ & $-70.9(2)$ \\
\hline $\mathrm{C}(11)-\mathrm{C}(7)-\mathrm{C}(8)-\mathrm{C}(15)$ & $-132.50(16)$ \\
\hline$C(6)-C(7)-C(8)-C(15)$ & $50.0(3)$ \\
\hline $\mathrm{C}(7)-\mathrm{C}(8)-\mathrm{O}(1)-\mathrm{C}(9)$ & $28.4(2)$ \\
\hline $\mathrm{C}(13)-\mathrm{C}(8)-\mathrm{O}(1)-\mathrm{C}(9)$ & $-93.48(17)$ \\
\hline $\mathrm{C}(15)-\mathrm{C}(8)-\mathrm{O}(1)-\mathrm{C}(9)$ & $150.78(14)$ \\
\hline $\mathrm{C}(8)-\mathrm{O}(1)-\mathrm{C}(9)-\mathrm{C}(10)$ & $-24.7(2)$ \\
\hline $\mathrm{C}(8)-\mathrm{O}(1)-\mathrm{C}(9)-\mathrm{C}(17)$ & $151.84(14)$ \\
\hline $\mathrm{O}(1)-\mathrm{C}(9)-\mathrm{C}(10)-\mathrm{C}(11)$ & $3.8(2)$ \\
\hline $\mathrm{C}(17)-\mathrm{C}(9)-\mathrm{C}(10)-\mathrm{C}(11)$ & $-172.30(15)$ \\
\hline $\mathrm{O}(1)-\mathrm{C}(9)-\mathrm{C}(10)-\mathrm{C}(23)$ & $169.23(15)$ \\
\hline $\mathrm{C}(17)-\mathrm{C}(9)-\mathrm{C}(10)-\mathrm{C}(23)$ & $-6.8(3)$ \\
\hline $\mathrm{C}(6)-\mathrm{C}(7)-\mathrm{C}(11)-\mathrm{N}(1)$ & $-2.4(2)$ \\
\hline $\mathrm{C}(8)-\mathrm{C}(7)-\mathrm{C}(11)-\mathrm{N}(1)$ & $179.51(15)$ \\
\hline
\end{tabular}




\begin{tabular}{|c|c|}
\hline$C(6)-C(7)-C(11)-C(10)$ & $172.94(16)$ \\
\hline$C(8)-C(7)-C(11)-C(10)$ & $-5.1(3)$ \\
\hline $\mathrm{C}(1)-\mathrm{N}(1)-\mathrm{C}(11)-\mathrm{C}(7)$ & $2.0(2)$ \\
\hline $\mathrm{C}(12)-\mathrm{N}(1)-\mathrm{C}(11)-\mathrm{C}(7)$ & $-166.05(19)$ \\
\hline $\mathrm{C}(1)-\mathrm{N}(1)-\mathrm{C}(11)-\mathrm{C}(10)$ & $-173.11(17)$ \\
\hline $\mathrm{C}(12)-\mathrm{N}(1)-\mathrm{C}(11)-\mathrm{C}(10)$ & $18.9(3)$ \\
\hline$C(9)-C(10)-C(11)-C(7)$ & $11.1(3)$ \\
\hline $\mathrm{C}(23)-\mathrm{C}(10)-\mathrm{C}(11)-\mathrm{C}(7)$ & $-154.78(17)$ \\
\hline $\mathrm{C}(9)-\mathrm{C}(10)-\mathrm{C}(11)-\mathrm{N}(1)$ & $-174.43(17)$ \\
\hline $\mathrm{C}(23)-\mathrm{C}(10)-\mathrm{C}(11)-\mathrm{N}(1)$ & $19.7(3)$ \\
\hline $\mathrm{O}(1)-\mathrm{C}(8)-\mathrm{C}(13)-\mathrm{O}(2)$ & $24.2(2)$ \\
\hline $\mathrm{C}(7)-\mathrm{C}(8)-\mathrm{C}(13)-\mathrm{O}(2)$ & $-98.6(2)$ \\
\hline $\mathrm{C}(15)-\mathrm{C}(8)-\mathrm{C}(13)-\mathrm{O}(2)$ & $138.28(19)$ \\
\hline $\mathrm{O}(1)-\mathrm{C}(8)-\mathrm{C}(13)-\mathrm{O}(3)$ & $-157.24(14)$ \\
\hline $\mathrm{C}(7)-\mathrm{C}(8)-\mathrm{C}(13)-\mathrm{O}(3)$ & $79.98(18)$ \\
\hline $\mathrm{C}(15)-\mathrm{C}(8)-\mathrm{C}(13)-\mathrm{O}(3)$ & $-43.2(2)$ \\
\hline $\mathrm{O}(2)-\mathrm{C}(13)-\mathrm{O}(3)-\mathrm{C}(14)$ & $2.7(3)$ \\
\hline $\mathrm{C}(8)-\mathrm{C}(13)-\mathrm{O}(3)-\mathrm{C}(14)$ & $-175.80(16)$ \\
\hline $\mathrm{O}(1)-\mathrm{C}(8)-\mathrm{C}(15)-\mathrm{O}(4)$ & $-135.79(18)$ \\
\hline$C(7)-C(8)-C(15)-O(4)$ & $-13.4(2)$ \\
\hline $\mathrm{C}(13)-\mathrm{C}(8)-\mathrm{C}(15)-\mathrm{O}(4)$ & $108.8(2)$ \\
\hline $\mathrm{O}(1)-\mathrm{C}(8)-\mathrm{C}(15)-\mathrm{O}(5)$ & $46.47(18)$ \\
\hline $\mathrm{C}(7)-\mathrm{C}(8)-\mathrm{C}(15)-\mathrm{O}(5)$ & $168.85(15)$ \\
\hline $\mathrm{C}(13)-\mathrm{C}(8)-\mathrm{C}(15)-\mathrm{O}(5)$ & $-68.93(18)$ \\
\hline $\mathrm{O}(4)-\mathrm{C}(15)-\mathrm{O}(5)-\mathrm{C}(16)$ & $3.8(3)$ \\
\hline $\mathrm{C}(8)-\mathrm{C}(15)-\mathrm{O}(5)-\mathrm{C}(16)$ & $-178.53(16)$ \\
\hline$C(10)-C(9)-C(17)-C(22)$ & $131.49(19)$ \\
\hline $\mathrm{O}(1)-\mathrm{C}(9)-\mathrm{C}(17)-\mathrm{C}(22)$ & $-44.9(2)$ \\
\hline $\mathrm{C}(10)-\mathrm{C}(9)-\mathrm{C}(17)-\mathrm{C}(18)$ & $-46.7(3)$ \\
\hline $\mathrm{O}(1)-\mathrm{C}(9)-\mathrm{C}(17)-\mathrm{C}(18)$ & $136.82(17)$ \\
\hline$C(22)-C(17)-C(18)-C(19)$ & $-2.6(3)$ \\
\hline $\mathrm{C}(9)-\mathrm{C}(17)-\mathrm{C}(18)-\mathrm{C}(19)$ & $175.63(17)$ \\
\hline $\mathrm{C}(17)-\mathrm{C}(18)-\mathrm{C}(19)-\mathrm{C}(20)$ & $-0.8(3)$ \\
\hline$C(18)-C(19)-C(20)-C(21)$ & $2.9(3)$ \\
\hline $\mathrm{C}(18)-\mathrm{C}(19)-\mathrm{C}(20)-\mathrm{N}(2)$ & $-175.92(17)$ \\
\hline$C(19)-C(20)-C(21)-C(22)$ & $-1.4(3)$ \\
\hline $\mathrm{N}(2)-\mathrm{C}(20)-\mathrm{C}(21)-\mathrm{C}(22)$ & $177.39(18)$ \\
\hline$C(20)-C(21)-C(22)-C(17)$ & $-2.1(3)$ \\
\hline
\end{tabular}




\begin{tabular}{|c|c|}
\hline $\mathrm{C}(18)-\mathrm{C}(17)-\mathrm{C}(22)-\mathrm{C}(21)$ & $4.1(3)$ \\
\hline $\mathrm{C}(9)-\mathrm{C}(17)-\mathrm{C}(22)-\mathrm{C}(21)$ & $-174.19(17)$ \\
\hline $\mathrm{C}(19)-\mathrm{C}(20)-\mathrm{N}(2)-\mathrm{O}(7)$ & $-174.82(19)$ \\
\hline $\mathrm{C}(21)-\mathrm{C}(20)-\mathrm{N}(2)-\mathrm{O}(7)$ & $6.4(3)$ \\
\hline $\mathrm{C}(19)-\mathrm{C}(20)-\mathrm{N}(2)-\mathrm{O}(6)$ & $7.2(3)$ \\
\hline $\mathrm{C}(21)-\mathrm{C}(20)-\mathrm{N}(2)-\mathrm{O}(6)$ & $-171.6(2)$ \\
\hline $\mathrm{C}(9)-\mathrm{C}(10)-\mathrm{C}(23)-\mathrm{O}(8)$ & $86.4(2)$ \\
\hline $\mathrm{C}(11)-\mathrm{C}(10)-\mathrm{C}(23)-\mathrm{O}(8)$ & $-108.6(2)$ \\
\hline $\mathrm{C}(9)-\mathrm{C}(10)-\mathrm{C}(23)-\mathrm{C}(24)$ & $-96.9(2)$ \\
\hline $\mathrm{C}(11)-\mathrm{C}(10)-\mathrm{C}(23)-\mathrm{C}(24)$ & $68.1(2)$ \\
\hline $\mathrm{O}(8)-\mathrm{C}(23)-\mathrm{C}(24)-\mathrm{C}(25)$ & $19.8(3)$ \\
\hline $\mathrm{C}(10)-\mathrm{C}(23)-\mathrm{C}(24)-\mathrm{C}(25)$ & $-156.89(19)$ \\
\hline $\mathrm{O}(8)-\mathrm{C}(23)-\mathrm{C}(24)-\mathrm{C}(29)$ & $-161.4(2)$ \\
\hline$C(10)-C(23)-C(24)-C(29)$ & $21.9(3)$ \\
\hline $\mathrm{C}(29)-\mathrm{C}(24)-\mathrm{C}(25)-\mathrm{C}(26)$ & $-2.6(3)$ \\
\hline $\mathrm{C}(23)-\mathrm{C}(24)-\mathrm{C}(25)-\mathrm{C}(26)$ & $176.2(2)$ \\
\hline$C(24)-C(25)-C(26)-C(27)$ & $2.0(4)$ \\
\hline$C(25)-C(26)-C(27)-O(9)$ & $-178.2(2)$ \\
\hline $\mathrm{C}(25)-\mathrm{C}(26)-\mathrm{C}(27)-\mathrm{C}(28)$ & $0.9(4)$ \\
\hline $\mathrm{O}(9)-\mathrm{C}(27)-\mathrm{C}(28)-\mathrm{C}(29)$ & $176.1(2)$ \\
\hline$C(26)-C(27)-C(28)-C(29)$ & $-3.1(3)$ \\
\hline$C(27)-C(28)-C(29)-C(24)$ & $2.4(3)$ \\
\hline $\mathrm{C}(25)-\mathrm{C}(24)-\mathrm{C}(29)-\mathrm{C}(28)$ & $0.4(3)$ \\
\hline$C(23)-C(24)-C(29)-C(28)$ & $-178.37(19)$ \\
\hline $\mathrm{C}(26)-\mathrm{C}(27)-\mathrm{O}(9)-\mathrm{C}(30)$ & $6.5(4)$ \\
\hline $\mathrm{C}(28)-\mathrm{C}(27)-\mathrm{O}(9)-\mathrm{C}(30)$ & $-172.6(2)$ \\
\hline
\end{tabular}

Symmetry transformations used to generate equivalent atoms: 
Single Crystal X-ray Diffraction Data for the pyran 7a $(\mathrm{CCDC}=1881740$, Thermal ellipsoids were drawn at the $50 \%$ probability levels). The crystals of $7 \mathbf{a}$ were obtained by dissolving $7 \mathbf{a}$ in diethyl ether followed by slow evaporation of diethyl ether.

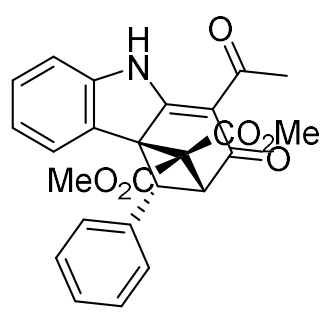

$7 a$

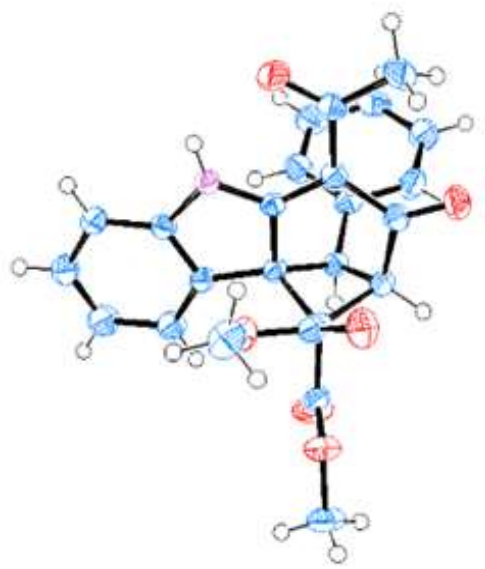

Table 1. Crystal data and structure refinement for 7a.

Empirical formula

Formula weight

Temperature

Wavelength

Crystal system

Space group

Unit cell dimensions

Volume

Z

Density (calculated)

Absorption coefficient

$\mathrm{F}(000)$

Crystal size

Theta range for data collection

Index ranges

Reflections collected

Independent reflections

Completeness to theta $=25.242^{\circ}$

Absorption correction

Max. and min. transmission
C25 H21 N O6

431.43

223(2) K

$0.71073 \AA$

Monoclinic

$\mathrm{C} 2 / \mathrm{c}$

$\mathrm{a}=32.1915(15) \AA$

$\alpha=90^{\circ}$.

$\mathrm{b}=8.2241(3) \AA$

$\beta=113.5420(15)^{\circ}$

$\mathrm{c}=17.0529(8) \AA$

$\gamma=90^{\circ}$.
8

$1.385 \mathrm{Mg} / \mathrm{m}^{3}$

$0.100 \mathrm{~mm}^{-1}$

1808

$0.240 \times 0.140 \times 0.100 \mathrm{~mm}^{3}$

2.413 to $28.338^{\circ}$.

$-42<=\mathrm{h}<=42,-10<=\mathrm{k}<=10,-22<=1<=22$

65527

$5147[\mathrm{R}(\mathrm{int})=0.0555]$

$100.0 \%$

Semi-empirical from equivalents

0.7457 and 0.7209 
Refinement method

Data / restraints / parameters

Goodness-of-fit on $\mathrm{F}^{2}$

Final $\mathrm{R}$ indices $[\mathrm{I}>2 \operatorname{sigma}(\mathrm{I})]$

$\mathrm{R}$ indices (all data)

Extinction coefficient

Largest diff. peak and hole
Full-matrix least-squares on $\mathrm{F}^{2}$

5147 / 0 / 292

1.201

$\mathrm{R} 1=0.0459, \mathrm{wR} 2=0.1479$

$\mathrm{R} 1=0.0681, \mathrm{wR} 2=0.1656$

$\mathrm{n} / \mathrm{a}$

0.372 and -0.220 e. $\AA^{-3}$

Table 2. Atomic coordinates $\left(\times 10^{4}\right)$ and equivalent isotropic displacement parameters $\left(\AA^{2} \times 10^{3}\right)$ for 7 a. U(eq) is defined as one third of the trace of the orthogonalized $U^{i j}$ tensor.

\begin{tabular}{|c|c|c|c|c|}
\hline & $\mathrm{x}$ & $\mathrm{y}$ & $\mathrm{z}$ & $\mathrm{U}(\mathrm{eq})$ \\
\hline $\mathrm{N}(1)$ & $1148(1)$ & $10147(1)$ & 2051(1) & $26(1)$ \\
\hline $\mathrm{C}(1)$ & 1271(1) & $8931(2)$ & $1671(1)$ & $23(1)$ \\
\hline$C(2)$ & $1168(1)$ & $7328(2)$ & 1996(1) & $23(1)$ \\
\hline$C(3)$ & $963(1)$ & $7853(2)$ & $2600(1)$ & $25(1)$ \\
\hline$C(4)$ & $787(1)$ & $7004(2)$ & $3095(1)$ & $35(1)$ \\
\hline$C(5)$ & $618(1)$ & $7863(2)$ & $3605(1)$ & $45(1)$ \\
\hline$C(6)$ & $625(1)$ & $9546(2)$ & $3620(1)$ & $44(1)$ \\
\hline$C(7)$ & $801(1)$ & $10429(2)$ & $3127(1)$ & $35(1)$ \\
\hline $\mathrm{C}(8)$ & $964(1)$ & $9558(2)$ & $2622(1)$ & $26(1)$ \\
\hline$C(9)$ & $1466(1)$ & $8980(2)$ & $1079(1)$ & $25(1)$ \\
\hline$C(10)$ & $1570(1)$ & $7378(2)$ & $831(1)$ & $26(1)$ \\
\hline $\mathrm{C}(11)$ & $1448(1)$ & $5944(2)$ & $1269(1)$ & $26(1)$ \\
\hline$C(12)$ & $1614(1)$ & $6291(2)$ & $2249(1)$ & $24(1)$ \\
\hline$C(13)$ & $1546(1)$ & $10550(2)$ & $759(1)$ & $29(1)$ \\
\hline$C(14)$ & 1761(1) & $10620(2)$ & $125(1)$ & $39(1)$ \\
\hline $\mathrm{O}(1)$ & $1437(1)$ & $11815(1)$ & 1013(1) & $40(1)$ \\
\hline $\mathrm{O}(2)$ & $1736(1)$ & $7116(1)$ & $314(1)$ & $40(1)$ \\
\hline$C(15)$ & $2066(1)$ & $7176(2)$ & $2670(1)$ & $26(1)$ \\
\hline $\mathrm{O}(3)$ & $2369(1)$ & $7071(2)$ & $2438(1)$ & $40(1)$ \\
\hline $\mathrm{O}(4)$ & 2073(1) & $8075(1)$ & $3325(1)$ & $32(1)$ \\
\hline$C(16)$ & 2491(1) & $8961(2)$ & $3770(1)$ & $42(1)$ \\
\hline$C(17)$ & $1620(1)$ & $4732(2)$ & $2746(1)$ & $27(1)$ \\
\hline $\mathrm{O}(5)$ & $1298(1)$ & $3890(1)$ & 2641(1) & $43(1)$ \\
\hline $\mathrm{O}(6)$ & $2035(1)$ & $\begin{array}{r}4408(1) \\
\mathrm{S} 78\end{array}$ & $3297(1)$ & $36(1)$ \\
\hline
\end{tabular}




\begin{tabular}{lrrrr}
$\mathrm{C}(18)$ & $2093(1)$ & $2903(2)$ & $3766(1)$ & $44(1)$ \\
$\mathrm{C}(19)$ & $956(1)$ & $6158(2)$ & $1213(1)$ & $25(1)$ \\
$\mathrm{C}(20)$ & $579(1)$ & $6676(2)$ & $393(1)$ & $27(1)$ \\
$\mathrm{C}(21)$ & $221(1)$ & $7614(2)$ & $391(1)$ & $37(1)$ \\
$\mathrm{C}(22)$ & $-150(1)$ & $7907(2)$ & $-370(1)$ & $43(1)$ \\
$\mathrm{C}(23)$ & $-163(1)$ & $7281(2)$ & $-1126(1)$ & $40(1)$ \\
$\mathrm{C}(24)$ & $195(1)$ & $6375(2)$ & $-1134(1)$ & $44(1)$ \\
$\mathrm{C}(25)$ & $568(1)$ & $6081(2)$ & $-379(1)$ & $39(1)$ \\
\hline
\end{tabular}

Table 3. Bond lengths $[\AA]$ and angles $\left[{ }^{\circ}\right]$ for $7 \mathbf{a}$.

\begin{tabular}{ll}
\hline $\mathrm{N}(1)-\mathrm{C}(1)$ & $1.3352(17)$ \\
$\mathrm{N}(1)-\mathrm{C}(8)$ & $1.4116(18)$ \\
$\mathrm{N}(1)-\mathrm{H}(1 \mathrm{~N})$ & 0.8700 \\
$\mathrm{C}(1)-\mathrm{C}(9)$ & $1.385(2)$ \\
$\mathrm{C}(1)-\mathrm{C}(2)$ & $1.5174(18)$ \\
$\mathrm{C}(2)-\mathrm{C}(3)$ & $1.4928(19)$ \\
$\mathrm{C}(2)-\mathrm{C}(19)$ & $1.5646(19)$ \\
$\mathrm{C}(2)-\mathrm{C}(12)$ & $1.5739(19)$ \\
$\mathrm{C}(3)-\mathrm{C}(4)$ & $1.379(2)$ \\
$\mathrm{C}(3)-\mathrm{C}(8)$ & $1.402(2)$ \\
$\mathrm{C}(4)-\mathrm{C}(5)$ & $1.389(2)$ \\
$\mathrm{C}(4)-\mathrm{H}(4)$ & 0.9400 \\
$\mathrm{C}(5)-\mathrm{C}(6)$ & $1.384(3)$ \\
$\mathrm{C}(5)-\mathrm{H}(5)$ & 0.9400 \\
$\mathrm{C}(6)-\mathrm{C}(7)$ & $1.391(2)$ \\
$\mathrm{C}(6)-\mathrm{H}(6)$ & 0.9400 \\
$\mathrm{C}(7)-\mathrm{C}(8)$ & $1.375(2)$ \\
$\mathrm{C}(7)-\mathrm{H}(7)$ & 0.9400 \\
$\mathrm{C}(9)-\mathrm{C}(10)$ & $1.4636(19)$ \\
$\mathrm{C}(9)-\mathrm{C}(13)$ & $1.4641(19)$ \\
$\mathrm{C}(10)-\mathrm{O}(2)$ & $1.2193(17)$ \\
$\mathrm{C}(10)-\mathrm{C}(11)$ & $1.530(2)$ \\
$\mathrm{C}(11)-\mathrm{C}(19)$ & $1.558(2)$ \\
$\mathrm{C}(11)-\mathrm{C}(12)$ & $1.563(2)$ \\
$\mathrm{C}(11)-\mathrm{H}(11)$ & 0.9900 \\
& \\
& \\
& \\
& \\
& \\
&
\end{tabular}




\begin{tabular}{|c|c|}
\hline$C(12)-C(15)$ & $1.5254(19)$ \\
\hline$C(12)-C(17)$ & $1.5326(18)$ \\
\hline $\mathrm{C}(13)-\mathrm{O}(1) \# 1$ & $1.2288(19)$ \\
\hline $\mathrm{C}(13)-\mathrm{O}(1)$ & $1.2288(19)$ \\
\hline $\mathrm{C}(13)-\mathrm{C}(14)$ & $1.501(2)$ \\
\hline $\mathrm{C}(14)-\mathrm{H}(14 \mathrm{~A})$ & 0.9700 \\
\hline $\mathrm{C}(14)-\mathrm{H}(14 \mathrm{~B})$ & 0.9700 \\
\hline $\mathrm{C}(14)-\mathrm{H}(14 \mathrm{C})$ & 0.9700 \\
\hline $\mathrm{O}(1)-\mathrm{O}(1) \# 1$ & $0.000(3)$ \\
\hline $\mathrm{C}(15)-\mathrm{O}(3)$ & $1.1929(18)$ \\
\hline $\mathrm{C}(15)-\mathrm{O}(4)$ & $1.3338(18)$ \\
\hline $\mathrm{O}(4)-\mathrm{C}(16)$ & $1.4494(19)$ \\
\hline $\mathrm{C}(16)-\mathrm{H}(16 \mathrm{~A})$ & 0.9700 \\
\hline $\mathrm{C}(16)-\mathrm{H}(16 \mathrm{~B})$ & 0.9700 \\
\hline $\mathrm{C}(16)-\mathrm{H}(16 \mathrm{C})$ & 0.9700 \\
\hline $\mathrm{C}(17)-\mathrm{O}(5)$ & $1.1995(18)$ \\
\hline $\mathrm{C}(17)-\mathrm{O}(6)$ & $1.3170(18)$ \\
\hline $\mathrm{O}(6)-\mathrm{C}(18)$ & $1.4447(18)$ \\
\hline $\mathrm{C}(18)-\mathrm{H}(18 \mathrm{~A})$ & 0.9700 \\
\hline $\mathrm{C}(18)-\mathrm{H}(18 \mathrm{~B})$ & 0.9700 \\
\hline $\mathrm{C}(18)-\mathrm{H}(18 \mathrm{C})$ & 0.9700 \\
\hline$C(19)-C(20)$ & $1.500(2)$ \\
\hline $\mathrm{C}(19)-\mathrm{H}(19)$ & 0.9900 \\
\hline $\mathrm{C}(20)-\mathrm{C}(21)$ & $1.386(2)$ \\
\hline$C(20)-C(25)$ & $1.390(2)$ \\
\hline $\mathrm{C}(21)-\mathrm{C}(22)$ & $1.389(2)$ \\
\hline $\mathrm{C}(21)-\mathrm{H}(21)$ & 0.9400 \\
\hline $\mathrm{C}(22)-\mathrm{C}(23)$ & $1.373(3)$ \\
\hline $\mathrm{C}(22)-\mathrm{H}(22)$ & 0.9400 \\
\hline $\mathrm{C}(23)-\mathrm{C}(24)$ & $1.376(3)$ \\
\hline $\mathrm{C}(23)-\mathrm{H}(23)$ & 0.9400 \\
\hline $\mathrm{C}(24)-\mathrm{C}(25)$ & $1.386(2)$ \\
\hline $\mathrm{C}(24)-\mathrm{H}(24)$ & 0.9400 \\
\hline $\mathrm{C}(25)-\mathrm{H}(25)$ & 0.9400 \\
\hline $\mathrm{C}(1)-\mathrm{N}(1)-\mathrm{C}(8)$ & $111.43(11)$ \\
\hline $\mathrm{C}(1)-\mathrm{N}(1)-\mathrm{H}(1 \mathrm{~N})$ & 124.3 \\
\hline $\mathrm{C}(8)-\mathrm{N}(1)-\mathrm{H}(1 \mathrm{~N})$ & 124.3 \\
\hline
\end{tabular}




\begin{tabular}{|c|c|}
\hline $\mathrm{N}(1)-\mathrm{C}(1)-\mathrm{C}(9)$ & $129.82(12)$ \\
\hline $\mathrm{N}(1)-\mathrm{C}(1)-\mathrm{C}(2)$ & $108.85(12)$ \\
\hline$C(9)-C(1)-C(2)$ & $121.32(12)$ \\
\hline$C(3)-C(2)-C(1)$ & $102.80(11)$ \\
\hline$C(3)-C(2)-C(19)$ & $127.11(12)$ \\
\hline$C(1)-C(2)-C(19)$ & $107.84(11)$ \\
\hline $\mathrm{C}(3)-\mathrm{C}(2)-\mathrm{C}(12)$ & $125.13(12)$ \\
\hline$C(1)-C(2)-C(12)$ & $105.28(11)$ \\
\hline$C(19)-C(2)-C(12)$ & $86.60(10)$ \\
\hline$C(4)-C(3)-C(8)$ & $119.14(13)$ \\
\hline$C(4)-C(3)-C(2)$ & $132.74(13)$ \\
\hline$C(8)-C(3)-C(2)$ & $108.11(12)$ \\
\hline$C(3)-C(4)-C(5)$ & $119.02(15)$ \\
\hline $\mathrm{C}(3)-\mathrm{C}(4)-\mathrm{H}(4)$ & 120.5 \\
\hline $\mathrm{C}(5)-\mathrm{C}(4)-\mathrm{H}(4)$ & 120.5 \\
\hline$C(6)-C(5)-C(4)$ & $120.79(16)$ \\
\hline $\mathrm{C}(6)-\mathrm{C}(5)-\mathrm{H}(5)$ & 119.6 \\
\hline $\mathrm{C}(4)-\mathrm{C}(5)-\mathrm{H}(5)$ & 119.6 \\
\hline$C(5)-C(6)-C(7)$ & $121.25(16)$ \\
\hline $\mathrm{C}(5)-\mathrm{C}(6)-\mathrm{H}(6)$ & 119.4 \\
\hline $\mathrm{C}(7)-\mathrm{C}(6)-\mathrm{H}(6)$ & 119.4 \\
\hline$C(8)-C(7)-C(6)$ & $117.12(15)$ \\
\hline $\mathrm{C}(8)-\mathrm{C}(7)-\mathrm{H}(7)$ & 121.4 \\
\hline $\mathrm{C}(6)-\mathrm{C}(7)-\mathrm{H}(7)$ & 121.4 \\
\hline$C(7)-C(8)-C(3)$ & $122.66(13)$ \\
\hline $\mathrm{C}(7)-\mathrm{C}(8)-\mathrm{N}(1)$ & $128.55(13)$ \\
\hline $\mathrm{C}(3)-\mathrm{C}(8)-\mathrm{N}(1)$ & $108.78(12)$ \\
\hline $\mathrm{C}(1)-\mathrm{C}(9)-\mathrm{C}(10)$ & $114.06(12)$ \\
\hline$C(1)-C(9)-C(13)$ & $119.69(13)$ \\
\hline $\mathrm{C}(10)-\mathrm{C}(9)-\mathrm{C}(13)$ & $126.25(13)$ \\
\hline $\mathrm{O}(2)-\mathrm{C}(10)-\mathrm{C}(9)$ & $125.89(13)$ \\
\hline $\mathrm{O}(2)-\mathrm{C}(10)-\mathrm{C}(11)$ & $119.34(13)$ \\
\hline$C(9)-C(10)-C(11)$ & $114.77(12)$ \\
\hline$C(10)-C(11)-C(19)$ & $109.66(11)$ \\
\hline$C(10)-C(11)-C(12)$ & $109.66(11)$ \\
\hline $\mathrm{C}(19)-\mathrm{C}(11)-\mathrm{C}(12)$ & $87.19(10)$ \\
\hline $\mathrm{C}(10)-\mathrm{C}(11)-\mathrm{H}(11)$ & 115.6 \\
\hline $\mathrm{C}(19)-\mathrm{C}(11)-\mathrm{H}(11)$ & 115.6 \\
\hline
\end{tabular}




\begin{tabular}{|c|c|}
\hline $\mathrm{C}(12)-\mathrm{C}(11)-\mathrm{H}(11)$ & 115.6 \\
\hline$C(15)-C(12)-C(17)$ & $109.83(11)$ \\
\hline $\mathrm{C}(15)-\mathrm{C}(12)-\mathrm{C}(11)$ & $116.41(12)$ \\
\hline $\mathrm{C}(17)-\mathrm{C}(12)-\mathrm{C}(11)$ & $111.25(11)$ \\
\hline $\mathrm{C}(15)-\mathrm{C}(12)-\mathrm{C}(2)$ & $117.64(11)$ \\
\hline$C(17)-C(12)-C(2)$ & $114.42(11)$ \\
\hline $\mathrm{C}(11)-\mathrm{C}(12)-\mathrm{C}(2)$ & $85.47(10)$ \\
\hline $\mathrm{O}(1) \# 1-\mathrm{C}(13)-\mathrm{O}(1)$ & $0.00(11)$ \\
\hline $\mathrm{O}(1) \# 1-\mathrm{C}(13)-\mathrm{C}(9)$ & $119.90(14)$ \\
\hline $\mathrm{O}(1)-\mathrm{C}(13)-\mathrm{C}(9)$ & $119.90(14)$ \\
\hline $\mathrm{O}(1) \# 1-\mathrm{C}(13)-\mathrm{C}(14)$ & $119.93(14)$ \\
\hline $\mathrm{O}(1)-\mathrm{C}(13)-\mathrm{C}(14)$ & $119.93(14)$ \\
\hline $\mathrm{C}(9)-\mathrm{C}(13)-\mathrm{C}(14)$ & $120.17(14)$ \\
\hline $\mathrm{C}(13)-\mathrm{C}(14)-\mathrm{H}(14 \mathrm{~A})$ & 109.5 \\
\hline $\mathrm{C}(13)-\mathrm{C}(14)-\mathrm{H}(14 \mathrm{~B})$ & 109.5 \\
\hline $\mathrm{H}(14 \mathrm{~A})-\mathrm{C}(14)-\mathrm{H}(14 \mathrm{~B})$ & 109.5 \\
\hline $\mathrm{C}(13)-\mathrm{C}(14)-\mathrm{H}(14 \mathrm{C})$ & 109.5 \\
\hline $\mathrm{H}(14 \mathrm{~A})-\mathrm{C}(14)-\mathrm{H}(14 \mathrm{C})$ & 109.5 \\
\hline $\mathrm{H}(14 \mathrm{~B})-\mathrm{C}(14)-\mathrm{H}(14 \mathrm{C})$ & 109.5 \\
\hline $\mathrm{O}(1) \# 1-\mathrm{O}(1)-\mathrm{C}(13)$ & $0(10)$ \\
\hline $\mathrm{O}(3)-\mathrm{C}(15)-\mathrm{O}(4)$ & $125.18(14)$ \\
\hline $\mathrm{O}(3)-\mathrm{C}(15)-\mathrm{C}(12)$ & $124.21(14)$ \\
\hline $\mathrm{O}(4)-\mathrm{C}(15)-\mathrm{C}(12)$ & $110.61(12)$ \\
\hline $\mathrm{C}(15)-\mathrm{O}(4)-\mathrm{C}(16)$ & $114.68(12)$ \\
\hline $\mathrm{O}(4)-\mathrm{C}(16)-\mathrm{H}(16 \mathrm{~A})$ & 109.5 \\
\hline $\mathrm{O}(4)-\mathrm{C}(16)-\mathrm{H}(16 \mathrm{~B})$ & 109.5 \\
\hline $\mathrm{H}(16 \mathrm{~A})-\mathrm{C}(16)-\mathrm{H}(16 \mathrm{~B})$ & 109.5 \\
\hline $\mathrm{O}(4)-\mathrm{C}(16)-\mathrm{H}(16 \mathrm{C})$ & 109.5 \\
\hline $\mathrm{H}(16 \mathrm{~A})-\mathrm{C}(16)-\mathrm{H}(16 \mathrm{C})$ & 109.5 \\
\hline $\mathrm{H}(16 \mathrm{~B})-\mathrm{C}(16)-\mathrm{H}(16 \mathrm{C})$ & 109.5 \\
\hline $\mathrm{O}(5)-\mathrm{C}(17)-\mathrm{O}(6)$ & $124.06(13)$ \\
\hline $\mathrm{O}(5)-\mathrm{C}(17)-\mathrm{C}(12)$ & $125.46(13)$ \\
\hline $\mathrm{O}(6)-\mathrm{C}(17)-\mathrm{C}(12)$ & $110.45(12)$ \\
\hline $\mathrm{C}(17)-\mathrm{O}(6)-\mathrm{C}(18)$ & $115.98(12)$ \\
\hline $\mathrm{O}(6)-\mathrm{C}(18)-\mathrm{H}(18 \mathrm{~A})$ & 109.5 \\
\hline $\mathrm{O}(6)-\mathrm{C}(18)-\mathrm{H}(18 \mathrm{~B})$ & 109.5 \\
\hline $\mathrm{H}(18 \mathrm{~A})-\mathrm{C}(18)-\mathrm{H}(18 \mathrm{~B})$ & 109.5 \\
\hline $\mathrm{O}(6)-\mathrm{C}(18)-\mathrm{H}(18 \mathrm{C})$ & 109.5 \\
\hline
\end{tabular}




$\begin{array}{ll}\mathrm{H}(18 \mathrm{~A})-\mathrm{C}(18)-\mathrm{H}(18 \mathrm{C}) & 109.5 \\ \mathrm{H}(18 \mathrm{~B})-\mathrm{C}(18)-\mathrm{H}(18 \mathrm{C}) & 109.5 \\ \mathrm{C}(20)-\mathrm{C}(19)-\mathrm{C}(11) & 120.85(12) \\ \mathrm{C}(20)-\mathrm{C}(19)-\mathrm{C}(2) & 122.43(11) \\ \mathrm{C}(11)-\mathrm{C}(19)-\mathrm{C}(2) & 85.96(10) \\ \mathrm{C}(20)-\mathrm{C}(19)-\mathrm{H}(19) & 108.5 \\ \mathrm{C}(11)-\mathrm{C}(19)-\mathrm{H}(19) & 108.5 \\ \mathrm{C}(2)-\mathrm{C}(19)-\mathrm{H}(19) & 108.5 \\ \mathrm{C}(21)-\mathrm{C}(20)-\mathrm{C}(25) & 119.00(15) \\ \mathrm{C}(21)-\mathrm{C}(20)-\mathrm{C}(19) & 121.49(14) \\ \mathrm{C}(25)-\mathrm{C}(20)-\mathrm{C}(19) & 119.24(13) \\ \mathrm{C}(20)-\mathrm{C}(21)-\mathrm{C}(22) & 120.19(16) \\ \mathrm{C}(20)-\mathrm{C}(21)-\mathrm{H}(21) & 119.9 \\ \mathrm{C}(22)-\mathrm{C}(21)-\mathrm{H}(21) & 119.9 \\ \mathrm{C}(23)-\mathrm{C}(22)-\mathrm{C}(21) & 120.32(16) \\ \mathrm{C}(23)-\mathrm{C}(22)-\mathrm{H}(22) & 119.8 \\ \mathrm{C}(21)-\mathrm{C}(22)-\mathrm{H}(22) & 119.8 \\ \mathrm{C}(22)-\mathrm{C}(23)-\mathrm{C}(24) & 119.97(16) \\ \mathrm{C}(22)-\mathrm{C}(23)-\mathrm{H}(23) & 120.0 \\ \mathrm{C}(24)-\mathrm{C}(23)-\mathrm{H}(23) & 120.0 \\ \mathrm{C}(23)-\mathrm{C}(24)-\mathrm{C}(25) & 120.20(17) \\ \mathrm{C}(23)-\mathrm{C}(24)-\mathrm{H}(24) & 119.9 \\ \mathrm{C}(25)-\mathrm{C}(24)-\mathrm{H}(24) & 119.9 \\ \mathrm{C}(24)-\mathrm{C}(25)-\mathrm{C}(20) & 120.28(16) \\ \mathrm{C}(24)-\mathrm{C}(25)-\mathrm{H}(25) & \\ \mathrm{C}(20)-\mathrm{C}(25)-\mathrm{H}(25) & 19.9 \\ & \end{array}$

Symmetry transformations used to generate equivalent atoms:

$\# 1 \mathrm{x}, \mathrm{y}, \mathrm{z}$

Table 4. Anisotropic displacement parameters $\left(\AA^{2} \times 10^{3}\right)$ for 7a. The anisotropic displacement factor exponent takes the form: $-2 \pi^{2}\left[h^{2} a^{* 2} U^{11}+\ldots+2 h k a^{*} b^{*} U^{12}\right]$

\begin{tabular}{llllrrr}
\hline & $\mathrm{U}^{11}$ & $\mathrm{U}^{22}$ & $\mathrm{U}^{33}$ & $\mathrm{U}^{23}$ & $\mathrm{U}^{13}$ & $\mathrm{U}^{12}$ \\
\hline $\mathrm{N}(1)$ & $30(1)$ & $21(1)$ & $29(1)$ & $3(1)$ & $15(1)$ & $2(1)$ \\
$\mathrm{C}(1)$ & $21(1)$ & $22(1)$ & $25(1)$ & $2(1)$ & $9(1)$ & $1(1)$
\end{tabular}




\begin{tabular}{|c|c|c|c|c|c|c|}
\hline$C(2)$ & $22(1)$ & $21(1)$ & $27(1)$ & $2(1)$ & $12(1)$ & $1(1)$ \\
\hline $\mathrm{C}(3)$ & $22(1)$ & $27(1)$ & $29(1)$ & $3(1)$ & $12(1)$ & $2(1)$ \\
\hline $\mathrm{C}(4)$ & $38(1)$ & $31(1)$ & $45(1)$ & $8(1)$ & $26(1)$ & $2(1)$ \\
\hline $\mathrm{C}(5)$ & $54(1)$ & $46(1)$ & $55(1)$ & 14(1) & $41(1)$ & $9(1)$ \\
\hline$C(6)$ & $56(1)$ & $45(1)$ & $46(1)$ & $8(1)$ & $36(1)$ & $16(1)$ \\
\hline$C(7)$ & $45(1)$ & $30(1)$ & $38(1)$ & $4(1)$ & $23(1)$ & $10(1)$ \\
\hline $\mathrm{C}(8)$ & $27(1)$ & $28(1)$ & $27(1)$ & $4(1)$ & $13(1)$ & $4(1)$ \\
\hline $\mathrm{C}(9)$ & $25(1)$ & $25(1)$ & $28(1)$ & $2(1)$ & $14(1)$ & $-2(1)$ \\
\hline $\mathrm{C}(10)$ & $25(1)$ & $30(1)$ & $27(1)$ & $0(1)$ & $13(1)$ & $-2(1)$ \\
\hline $\mathrm{C}(11)$ & $26(1)$ & $22(1)$ & $30(1)$ & $0(1)$ & $13(1)$ & $1(1)$ \\
\hline $\mathrm{C}(12)$ & $24(1)$ & 21(1) & $31(1)$ & $2(1)$ & $14(1)$ & $1(1)$ \\
\hline$C(13)$ & $30(1)$ & $28(1)$ & $30(1)$ & $3(1)$ & $12(1)$ & $-3(1)$ \\
\hline $\mathrm{C}(14)$ & $49(1)$ & $37(1)$ & $40(1)$ & $6(1)$ & $26(1)$ & $-5(1)$ \\
\hline $\mathrm{O}(1)$ & $54(1)$ & $25(1)$ & $50(1)$ & $5(1)$ & $31(1)$ & $-1(1)$ \\
\hline $\mathrm{O}(2)$ & $51(1)$ & $38(1)$ & $43(1)$ & $-1(1)$ & $33(1)$ & $1(1)$ \\
\hline$C(15)$ & $24(1)$ & $24(1)$ & $31(1)$ & $4(1)$ & $12(1)$ & $2(1)$ \\
\hline $\mathrm{O}(3)$ & $28(1)$ & $51(1)$ & $47(1)$ & $-5(1)$ & $21(1)$ & $-4(1)$ \\
\hline $\mathrm{O}(4)$ & $28(1)$ & $31(1)$ & $39(1)$ & $-6(1)$ & $14(1)$ & $-4(1)$ \\
\hline$C(16)$ & $35(1)$ & $33(1)$ & $52(1)$ & $-7(1)$ & $11(1)$ & $-8(1)$ \\
\hline $\mathrm{C}(17)$ & $29(1)$ & $23(1)$ & $31(1)$ & $2(1)$ & $14(1)$ & $3(1)$ \\
\hline $\mathrm{O}(5)$ & $33(1)$ & $36(1)$ & $54(1)$ & $17(1)$ & $12(1)$ & $-5(1)$ \\
\hline $\mathrm{O}(6)$ & $29(1)$ & $34(1)$ & $41(1)$ & $15(1)$ & $11(1)$ & $4(1)$ \\
\hline$C(18)$ & $42(1)$ & $40(1)$ & $50(1)$ & $23(1)$ & $18(1)$ & $10(1)$ \\
\hline $\mathrm{C}(19)$ & $25(1)$ & $21(1)$ & $30(1)$ & $1(1)$ & $12(1)$ & $-1(1)$ \\
\hline$C(20)$ & $25(1)$ & $23(1)$ & $32(1)$ & $2(1)$ & $12(1)$ & $-3(1)$ \\
\hline$C(21)$ & $32(1)$ & $44(1)$ & $35(1)$ & $2(1)$ & $15(1)$ & $5(1)$ \\
\hline$C(22)$ & $31(1)$ & $51(1)$ & $46(1)$ & $9(1)$ & $14(1)$ & $10(1)$ \\
\hline$C(23)$ & $33(1)$ & $40(1)$ & $37(1)$ & $10(1)$ & $4(1)$ & $0(1)$ \\
\hline $\mathrm{C}(24)$ & $49(1)$ & $44(1)$ & $32(1)$ & $-5(1)$ & $9(1)$ & $4(1)$ \\
\hline$C(25)$ & $40(1)$ & $35(1)$ & $38(1)$ & $-6(1)$ & $12(1)$ & $7(1)$ \\
\hline
\end{tabular}


Table 5. Hydrogen coordinates ( x $\left.10^{4}\right)$ and isotropic displacement parameters $\left(\AA^{2} \times 10^{3}\right)$ for $7 \mathbf{a}$.

\begin{tabular}{|c|c|c|c|c|}
\hline & $\mathrm{x}$ & $\mathrm{y}$ & $\mathrm{z}$ & $\mathrm{U}(\mathrm{eq})$ \\
\hline $\mathrm{H}(1 \mathrm{~N})$ & 1177 & 11172 & 1958 & 31 \\
\hline $\mathrm{H}(4)$ & 782 & 5862 & 3087 & 42 \\
\hline $\mathrm{H}(5)$ & 497 & 7295 & 3944 & 54 \\
\hline $\mathrm{H}(6)$ & 509 & 10103 & 3970 & 53 \\
\hline $\mathrm{H}(7)$ & 808 & 11572 & 3139 & 43 \\
\hline $\mathrm{H}(11)$ & 1522 & 4853 & 1115 & 31 \\
\hline $\mathrm{H}(14 \mathrm{~A})$ & 1726 & 11704 & -117 & 59 \\
\hline $\mathrm{H}(14 \mathrm{~B})$ & 1616 & 9837 & -328 & 59 \\
\hline $\mathrm{H}(14 \mathrm{C})$ & 2081 & 10363 & 411 & 59 \\
\hline $\mathrm{H}(16 \mathrm{~A})$ & 2746 & 8225 & 3910 & 63 \\
\hline $\mathrm{H}(16 \mathrm{~B})$ & 2489 & 9425 & 4292 & 63 \\
\hline $\mathrm{H}(16 \mathrm{C})$ & 2517 & 9825 & 3405 & 63 \\
\hline $\mathrm{H}(18 \mathrm{~A})$ & 1947 & 2987 & 4163 & 66 \\
\hline $\mathrm{H}(18 \mathrm{~B})$ & 2414 & 2692 & 4080 & 66 \\
\hline $\mathrm{H}(18 \mathrm{C})$ & 1958 & 2019 & 3369 & 66 \\
\hline $\mathrm{H}(19)$ & 864 & 5141 & 1410 & 30 \\
\hline $\mathrm{H}(21)$ & 229 & 8052 & 906 & 44 \\
\hline $\mathrm{H}(22)$ & -393 & 8537 & -368 & 51 \\
\hline $\mathrm{H}(23)$ & -416 & 7472 & -1639 & 48 \\
\hline $\mathrm{H}(24)$ & 186 & 5954 & -1652 & 53 \\
\hline $\mathrm{H}(25)$ & 813 & 5477 & -388 & 46 \\
\hline
\end{tabular}

Table 6. Torsion angles $\left[{ }^{\circ}\right]$ for $\mathbf{7 a}$.

\begin{tabular}{|c|c|}
\hline $\mathrm{C}(8)-\mathrm{N}(1)-\mathrm{C}(1)-\mathrm{C}(9)$ & $-179.54(14)$ \\
\hline $\mathrm{C}(8)-\mathrm{N}(1)-\mathrm{C}(1)-\mathrm{C}(2)$ & $-0.14(16)$ \\
\hline $\mathrm{N}(1)-\mathrm{C}(1)-\mathrm{C}(2)-\mathrm{C}(3)$ & $1.01(15)$ \\
\hline $\mathrm{C}(9)-\mathrm{C}(1)-\mathrm{C}(2)-\mathrm{C}(3)$ & $-179.53(13)$ \\
\hline $\mathrm{N}(1)-\mathrm{C}(1)-\mathrm{C}(2)-\mathrm{C}(19)$ & $137.32(12)$ \\
\hline $\mathrm{C}(9)-\mathrm{C}(1)-\mathrm{C}(2)-\mathrm{C}(19)$ & $-43.22(17)$ \\
\hline $\mathrm{N}(1)-\mathrm{C}(1)-\mathrm{C}(2)-\mathrm{C}(12)$ & $-131.35(12)$ \\
\hline
\end{tabular}




\begin{tabular}{|c|c|}
\hline$C(9)-C(1)-C(2)-C(12)$ & $48.11(17)$ \\
\hline $\mathrm{C}(1)-\mathrm{C}(2)-\mathrm{C}(3)-\mathrm{C}(4)$ & $178.45(16)$ \\
\hline$C(19)-C(2)-C(3)-C(4)$ & $54.0(2)$ \\
\hline $\mathrm{C}(12)-\mathrm{C}(2)-\mathrm{C}(3)-\mathrm{C}(4)$ & $-62.2(2)$ \\
\hline $\mathrm{C}(1)-\mathrm{C}(2)-\mathrm{C}(3)-\mathrm{C}(8)$ & $-1.50(14)$ \\
\hline$C(19)-C(2)-C(3)-C(8)$ & $-125.96(14)$ \\
\hline $\mathrm{C}(12)-\mathrm{C}(2)-\mathrm{C}(3)-\mathrm{C}(8)$ & $117.85(14)$ \\
\hline$C(8)-C(3)-C(4)-C(5)$ & $-0.3(2)$ \\
\hline $\mathrm{C}(2)-\mathrm{C}(3)-\mathrm{C}(4)-\mathrm{C}(5)$ & $179.73(16)$ \\
\hline $\mathrm{C}(3)-\mathrm{C}(4)-\mathrm{C}(5)-\mathrm{C}(6)$ & $-0.1(3)$ \\
\hline $\mathrm{C}(4)-\mathrm{C}(5)-\mathrm{C}(6)-\mathrm{C}(7)$ & $0.0(3)$ \\
\hline$C(5)-C(6)-C(7)-C(8)$ & $0.4(3)$ \\
\hline $\mathrm{C}(6)-\mathrm{C}(7)-\mathrm{C}(8)-\mathrm{C}(3)$ & $-0.8(2)$ \\
\hline $\mathrm{C}(6)-\mathrm{C}(7)-\mathrm{C}(8)-\mathrm{N}(1)$ & $178.27(15)$ \\
\hline $\mathrm{C}(4)-\mathrm{C}(3)-\mathrm{C}(8)-\mathrm{C}(7)$ & $0.8(2)$ \\
\hline $\mathrm{C}(2)-\mathrm{C}(3)-\mathrm{C}(8)-\mathrm{C}(7)$ & $-179.23(14)$ \\
\hline $\mathrm{C}(4)-\mathrm{C}(3)-\mathrm{C}(8)-\mathrm{N}(1)$ & $-178.46(13)$ \\
\hline $\mathrm{C}(2)-\mathrm{C}(3)-\mathrm{C}(8)-\mathrm{N}(1)$ & $1.50(15)$ \\
\hline $\mathrm{C}(1)-\mathrm{N}(1)-\mathrm{C}(8)-\mathrm{C}(7)$ & $179.93(15)$ \\
\hline $\mathrm{C}(1)-\mathrm{N}(1)-\mathrm{C}(8)-\mathrm{C}(3)$ & $-0.86(16)$ \\
\hline N(1)-C(1)-C(9)-C(10) & $177.66(14)$ \\
\hline$C(2)-C(1)-C(9)-C(10)$ & $-1.7(2)$ \\
\hline $\mathrm{N}(1)-\mathrm{C}(1)-\mathrm{C}(9)-\mathrm{C}(13)$ & $-2.7(2)$ \\
\hline $\mathrm{C}(2)-\mathrm{C}(1)-\mathrm{C}(9)-\mathrm{C}(13)$ & $178.00(12)$ \\
\hline $\mathrm{C}(1)-\mathrm{C}(9)-\mathrm{C}(10)-\mathrm{O}(2)$ & 178.97(14) \\
\hline $\mathrm{C}(13)-\mathrm{C}(9)-\mathrm{C}(10)-\mathrm{O}(2)$ & $-0.7(3)$ \\
\hline$C(1)-C(9)-C(10)-C(11)$ & $-0.16(19)$ \\
\hline $\mathrm{C}(13)-\mathrm{C}(9)-\mathrm{C}(10)-\mathrm{C}(11)$ & $-179.82(13)$ \\
\hline $\mathrm{O}(2)-\mathrm{C}(10)-\mathrm{C}(11)-\mathrm{C}(19)$ & $-131.07(14)$ \\
\hline $\mathrm{C}(9)-\mathrm{C}(10)-\mathrm{C}(11)-\mathrm{C}(19)$ & $48.12(16)$ \\
\hline $\mathrm{O}(2)-\mathrm{C}(10)-\mathrm{C}(11)-\mathrm{C}(12)$ & $134.78(14)$ \\
\hline $\mathrm{C}(9)-\mathrm{C}(10)-\mathrm{C}(11)-\mathrm{C}(12)$ & $-46.03(16)$ \\
\hline $\mathrm{C}(10)-\mathrm{C}(11)-\mathrm{C}(12)-\mathrm{C}(15)$ & $-37.29(16)$ \\
\hline $\mathrm{C}(19)-\mathrm{C}(11)-\mathrm{C}(12)-\mathrm{C}(15)$ & $-147.18(11)$ \\
\hline $\mathrm{C}(10)-\mathrm{C}(11)-\mathrm{C}(12)-\mathrm{C}(17)$ & $-164.12(11)$ \\
\hline $\mathrm{C}(19)-\mathrm{C}(11)-\mathrm{C}(12)-\mathrm{C}(17)$ & $85.98(12)$ \\
\hline $\mathrm{C}(10)-\mathrm{C}(11)-\mathrm{C}(12)-\mathrm{C}(2)$ & $81.41(12)$ \\
\hline$C(19)-C(11)-C(12)-C(2)$ & $-28.48(9)$ \\
\hline
\end{tabular}




\begin{tabular}{|c|c|}
\hline$C(3)-C(2)-C(12)-C(15)$ & $-79.90(17)$ \\
\hline$C(1)-C(2)-C(12)-C(15)$ & $38.33(16)$ \\
\hline$C(19)-C(2)-C(12)-C(15)$ & $145.90(12)$ \\
\hline$C(3)-C(2)-C(12)-C(17)$ & $51.27(18)$ \\
\hline $\mathrm{C}(1)-\mathrm{C}(2)-\mathrm{C}(12)-\mathrm{C}(17)$ & $169.50(11)$ \\
\hline $\mathrm{C}(19)-\mathrm{C}(2)-\mathrm{C}(12)-\mathrm{C}(17)$ & $-82.93(12)$ \\
\hline$C(3)-C(2)-C(12)-C(11)$ & $162.58(13)$ \\
\hline$C(1)-C(2)-C(12)-C(11)$ & $-79.20(11)$ \\
\hline$C(19)-C(2)-C(12)-C(11)$ & $28.37(9)$ \\
\hline $\mathrm{C}(1)-\mathrm{C}(9)-\mathrm{C}(13)-\mathrm{O}(1) \# 1$ & $-0.4(2)$ \\
\hline $\mathrm{C}(10)-\mathrm{C}(9)-\mathrm{C}(13)-\mathrm{O}(1) \# 1$ & $179.23(14)$ \\
\hline $\mathrm{C}(1)-\mathrm{C}(9)-\mathrm{C}(13)-\mathrm{O}(1)$ & $-0.4(2)$ \\
\hline $\mathrm{C}(10)-\mathrm{C}(9)-\mathrm{C}(13)-\mathrm{O}(1)$ & $179.23(14)$ \\
\hline $\mathrm{C}(1)-\mathrm{C}(9)-\mathrm{C}(13)-\mathrm{C}(14)$ & $179.30(14)$ \\
\hline $\mathrm{C}(10)-\mathrm{C}(9)-\mathrm{C}(13)-\mathrm{C}(14)$ & $-1.1(2)$ \\
\hline $\mathrm{C}(9)-\mathrm{C}(13)-\mathrm{O}(1)-\mathrm{O}(1) \# 1$ & $0.00(19)$ \\
\hline $\mathrm{C}(14)-\mathrm{C}(13)-\mathrm{O}(1)-\mathrm{O}(1) \# 1$ & $0.0(2)$ \\
\hline $\mathrm{C}(17)-\mathrm{C}(12)-\mathrm{C}(15)-\mathrm{O}(3)$ & $97.44(16)$ \\
\hline $\mathrm{C}(11)-\mathrm{C}(12)-\mathrm{C}(15)-\mathrm{O}(3)$ & $-30.1(2)$ \\
\hline $\mathrm{C}(2)-\mathrm{C}(12)-\mathrm{C}(15)-\mathrm{O}(3)$ & $-129.33(15)$ \\
\hline $\mathrm{C}(17)-\mathrm{C}(12)-\mathrm{C}(15)-\mathrm{O}(4)$ & $-82.69(14)$ \\
\hline $\mathrm{C}(11)-\mathrm{C}(12)-\mathrm{C}(15)-\mathrm{O}(4)$ & $149.78(12)$ \\
\hline $\mathrm{C}(2)-\mathrm{C}(12)-\mathrm{C}(15)-\mathrm{O}(4)$ & $50.54(16)$ \\
\hline $\mathrm{O}(3)-\mathrm{C}(15)-\mathrm{O}(4)-\mathrm{C}(16)$ & $-0.4(2)$ \\
\hline $\mathrm{C}(12)-\mathrm{C}(15)-\mathrm{O}(4)-\mathrm{C}(16)$ & $179.77(12)$ \\
\hline $\mathrm{C}(15)-\mathrm{C}(12)-\mathrm{C}(17)-\mathrm{O}(5)$ & $169.88(15)$ \\
\hline $\mathrm{C}(11)-\mathrm{C}(12)-\mathrm{C}(17)-\mathrm{O}(5)$ & $-59.76(19)$ \\
\hline $\mathrm{C}(2)-\mathrm{C}(12)-\mathrm{C}(17)-\mathrm{O}(5)$ & $35.0(2)$ \\
\hline $\mathrm{C}(15)-\mathrm{C}(12)-\mathrm{C}(17)-\mathrm{O}(6)$ & $-11.99(16)$ \\
\hline $\mathrm{C}(11)-\mathrm{C}(12)-\mathrm{C}(17)-\mathrm{O}(6)$ & $118.38(13)$ \\
\hline $\mathrm{C}(2)-\mathrm{C}(12)-\mathrm{C}(17)-\mathrm{O}(6)$ & $-146.84(12)$ \\
\hline $\mathrm{O}(5)-\mathrm{C}(17)-\mathrm{O}(6)-\mathrm{C}(18)$ & $2.4(2)$ \\
\hline $\mathrm{C}(12)-\mathrm{C}(17)-\mathrm{O}(6)-\mathrm{C}(18)$ & $-175.74(13)$ \\
\hline $\mathrm{C}(10)-\mathrm{C}(11)-\mathrm{C}(19)-\mathrm{C}(20)$ & $44.50(16)$ \\
\hline $\mathrm{C}(12)-\mathrm{C}(11)-\mathrm{C}(19)-\mathrm{C}(20)$ & $154.39(12)$ \\
\hline$C(10)-C(11)-C(19)-C(2)$ & $-81.24(12)$ \\
\hline $\mathrm{C}(12)-\mathrm{C}(11)-\mathrm{C}(19)-\mathrm{C}(2)$ & $28.65(9)$ \\
\hline$C(3)-C(2)-C(19)-C(20)$ & $74.52(18)$ \\
\hline
\end{tabular}




$\begin{array}{lc}\mathrm{C}(1)-\mathrm{C}(2)-\mathrm{C}(19)-\mathrm{C}(20) & -47.84(17) \\ \mathrm{C}(12)-\mathrm{C}(2)-\mathrm{C}(19)-\mathrm{C}(20) & -152.80(13) \\ \mathrm{C}(3)-\mathrm{C}(2)-\mathrm{C}(19)-\mathrm{C}(11) & -161.13(13) \\ \mathrm{C}(1)-\mathrm{C}(2)-\mathrm{C}(19)-\mathrm{C}(11) & 76.51(11) \\ \mathrm{C}(12)-\mathrm{C}(2)-\mathrm{C}(19)-\mathrm{C}(11) & -28.46(9) \\ \mathrm{C}(11)-\mathrm{C}(19)-\mathrm{C}(20)-\mathrm{C}(21) & -147.81(14) \\ \mathrm{C}(2)-\mathrm{C}(19)-\mathrm{C}(20)-\mathrm{C}(21) & -41.4(2) \\ \mathrm{C}(11)-\mathrm{C}(19)-\mathrm{C}(20)-\mathrm{C}(25) & 38.16(19) \\ \mathrm{C}(2)-\mathrm{C}(19)-\mathrm{C}(20)-\mathrm{C}(25) & 144.56(14) \\ \mathrm{C}(25)-\mathrm{C}(20)-\mathrm{C}(21)-\mathrm{C}(22) & 2.0(2) \\ \mathrm{C}(19)-\mathrm{C}(20)-\mathrm{C}(21)-\mathrm{C}(22) & -172.05(15) \\ \mathrm{C}(20)-\mathrm{C}(21)-\mathrm{C}(22)-\mathrm{C}(23) & -0.5(3) \\ \mathrm{C}(21)-\mathrm{C}(22)-\mathrm{C}(23)-\mathrm{C}(24) & -0.8(3) \\ \mathrm{C}(22)-\mathrm{C}(23)-\mathrm{C}(24)-\mathrm{C}(25) & 0.5(3) \\ \mathrm{C}(23)-\mathrm{C}(24)-\mathrm{C}(25)-\mathrm{C}(20) & 1.1(3) \\ \mathrm{C}(21)-\mathrm{C}(20)-\mathrm{C}(25)-\mathrm{C}(24) & -2.3(2) \\ \mathrm{C}(19)-\mathrm{C}(20)-\mathrm{C}(25)-\mathrm{C}(24) & 171.89(15)\end{array}$

Symmetry transformations used to generate equivalent atoms:

\#1 x,y,z

Table 7. Hydrogen bonds for $7 \mathbf{a}\left[\AA\right.$ and $\left.^{\circ}\right]$.

\begin{tabular}{lcccc}
\hline D-H...A & d(D-H) & d(H...A & $d(D \ldots A)$ & $<$ (DHA) \\
\hline $\mathrm{N}(1)-\mathrm{H}(1 \mathrm{~N}) \ldots \mathrm{O}(1) \# 1$ & 0.87 & 2.16 & $2.6838(15)$ & 118.4 \\
\hline
\end{tabular}

Symmetry transformations used to generate equivalent atoms:

$\# 1 \mathrm{x}, \mathrm{y}, \mathrm{z}$ 


\section{Computational details}

All DFT calculations were carried out using the B3LYP hybrid functional with Grimme's D3 dispersion corrections. ${ }^{26}$ Geometry optimization was performed with the $6-31+\mathrm{g}(\mathrm{d}, \mathrm{p})$ basis set for $\mathrm{H}, \mathrm{C}, \mathrm{N}, \mathrm{O}, \mathrm{S}$ atoms ${ }^{27}$ and the LANL2DZdp basis set/ECP for I, ${ }^{28}$ which were obtained from EMSL Basis Set Exchange. ${ }^{29}$ Frequency calculations were performed for every optimized geometry with the same level of theory to obtain vibrational frequencies and thermochemical data at $298.15 \mathrm{~K}$. The SMD solvation model with the solvent of acetonitrile $(\varepsilon$ $=35.688$ ) was used for all calculations. ${ }^{30}$ The transition states were identified by having one imaginary frequency, and intrinsic reaction coordinate (IRC) calculations were performed to connect transition states with corresponding intermediates. ${ }^{31}$ Each intermediate was verified as minima by having no imaginary frequency, and the geometries of intermediates with possibility of multiple conformations were optimized with several different starting geometries to find the lowest energy conformation. Relaxed scan of $\mathbf{3}^{\prime} \mathbf{a a}$ was investigated with UB3LYP, where the keyword guess $=($ mix,always $)$ was used. All calculations were performed using the Gaussian 09 software (Rev D.01). ${ }^{32}$

\subsection{DFT studies of proposed reaction mechanisms}

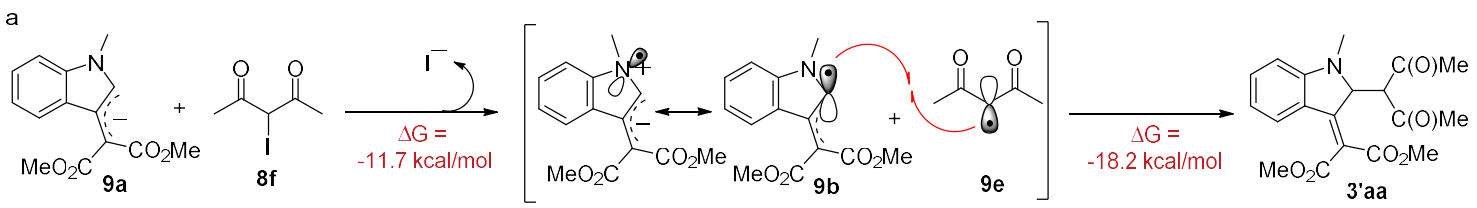

b
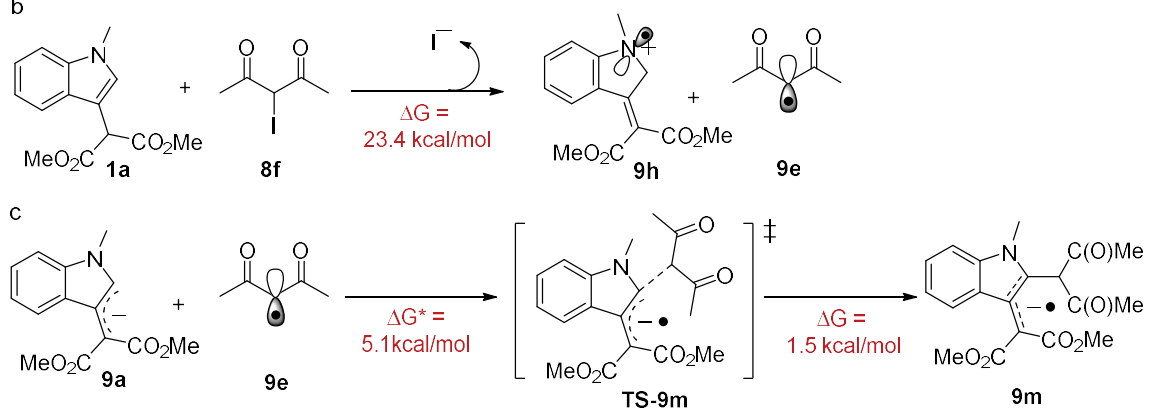

Supplementary Figure 3. Gibbs free energy profile or radical-radical cross-coupling 
9.2 Tables of energies in hartree

$\begin{array}{cccc}\text { Geometry } & \mathbf{E} & \mathbf{H} 298 & \mathbf{G}_{298} \\ \mathbf{9 f} & -878.324451 & -878.090242 & -878.151791 \\ \mathbf{9 k} & -878.176738 & -877.941351 & -878.004873 \\ \mathbf{9 g} & -1201.302775 & -1201.071272 & -1201.135670 \\ \mathbf{9 l} & -1201.152250 & -1200.919415 & -1200.982789 \\ \mathbf{9 a} & -897.787915 & -897.511718 & -897.579469 \\ \mathbf{9 b} & -897.652243 & -897.374279 & -897.441860 \\ \mathbf{9 e} & -356.625892 & -356.503690 & -356.552074 \\ \mathbf{8 f} & -345.189702 & -345.072013 & -345.115178 \\ \mathbf{1 a} & -898.284441 & -897.993853 & -898.061231 \\ \mathbf{9 h} & -898.089245 & -897.798505 & -897.867695 \\ \mathbf{3} \mathbf{a} & -1242.903398 & -1242.501981 & -1242.585976 \\ \text { TS-9m } & -1,242.997376 & -1242.600919 & -1242.686466 \\ \mathbf{9 m} & -1,243.004654 & -1242.606979 & -1242.692319\end{array}$




\subsection{Relaxed scan of 9e after SET}

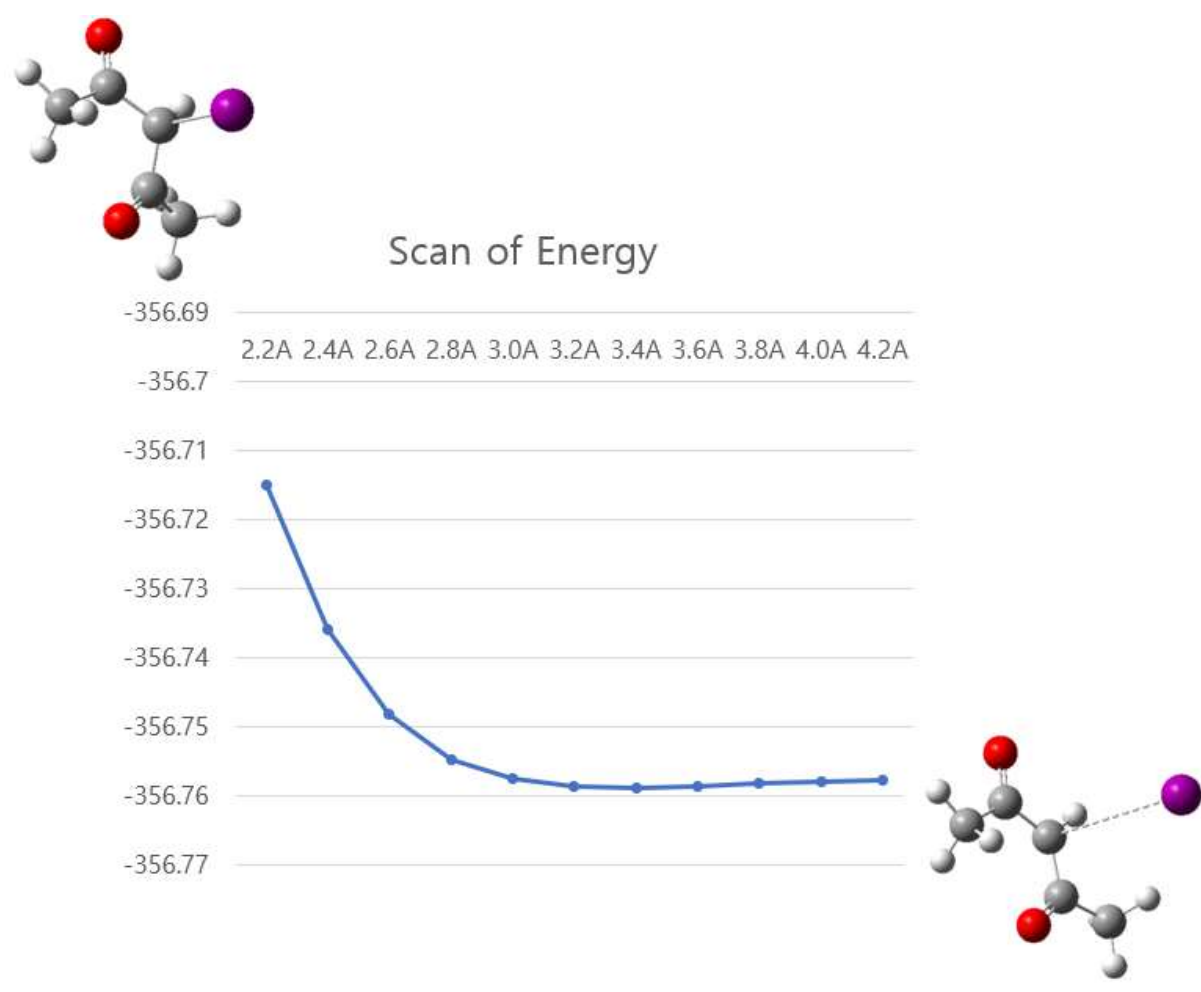

\subsection{Relaxed scan of 3'aa}

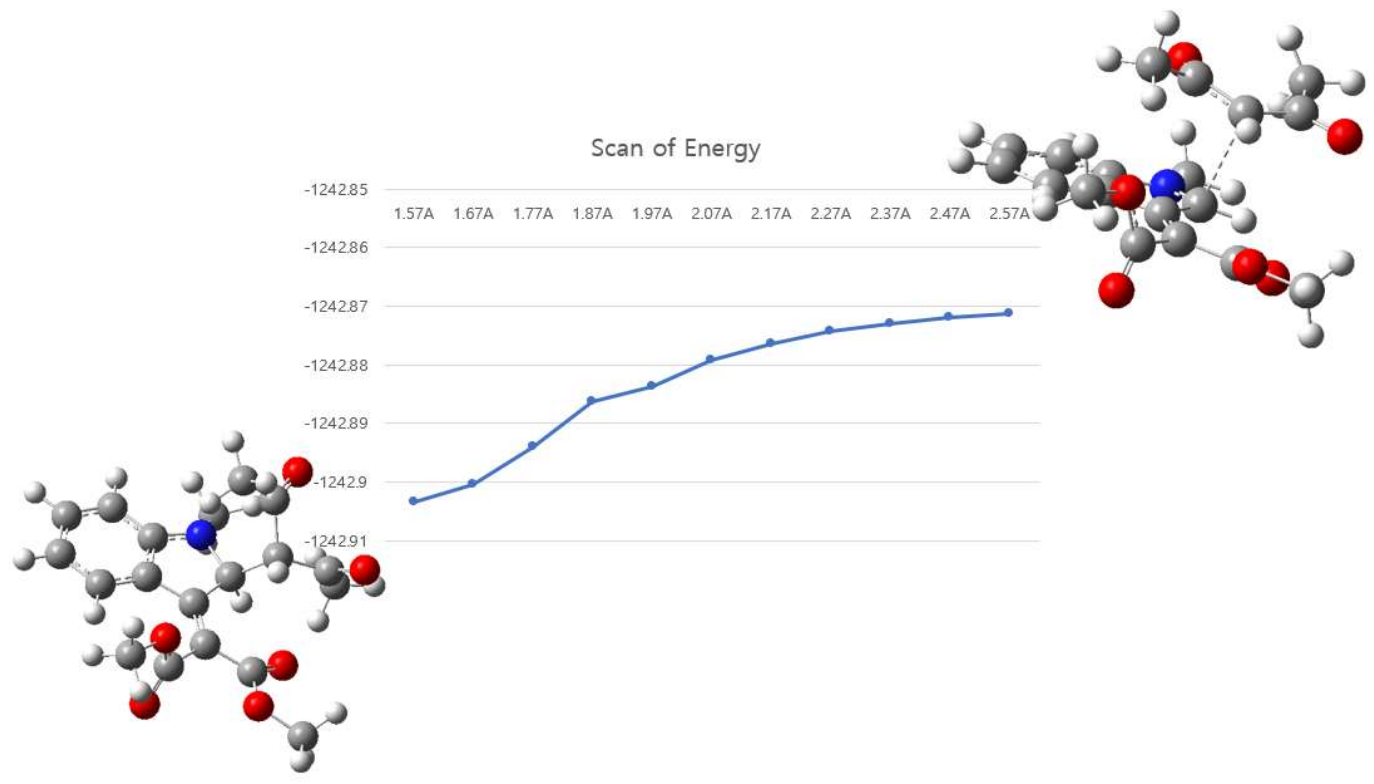

According to the scan of $\mathbf{3}$ 'aa, there is no activation barrier for the radical-radical cross-coupling between $\mathbf{9 b}$ and $8 \mathbf{8}$. 
9.5 Structures and coordinates of optimized geometries

9f

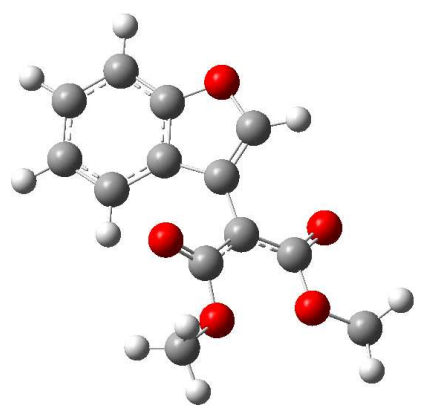

$\begin{array}{llll}\text { C } & -4.313420 & 0.610767 & 0.728295\end{array}$

$\begin{array}{llll}\text { C } & -3.264513 & 1.460255 & 1.141959\end{array}$

$\begin{array}{llll}\text { C } & -1.934328 & 1.177958 & 0.824747\end{array}$

$\begin{array}{llll}\text { C } & -1.649424 & 0.019827 & 0.081911\end{array}$

$\begin{array}{llll}\text { C } & -2.718010 & -0.812433 & -0.301487\end{array}$

$\begin{array}{llll}\text { C } & -4.053081 & -0.551341 & -0.005239\end{array}$

$\begin{array}{lllll}\text { C } & & -0.430511 & -0.616183 & -0.407434\end{array}$

$\begin{array}{llll}\text { C } & -0.866304 & -1.752679 & -1.025254\end{array}$

$\begin{array}{llll}\text { C } & 0.961079 & -0.168111 & -0.191550\end{array}$

$\begin{array}{llll}\text { C } & 1.217977 & 1.218718 & -0.472784\end{array}$

$\begin{array}{llll}\mathrm{O} & 0.418074 & 1.979810 & -1.050530\end{array}$

$\begin{array}{llll}\mathrm{O} & 2.427025 & 1.718208 & -0.052068\end{array}$

$\begin{array}{llll}\text { C } & 2.686951 & 3.087483 & -0.382103\end{array}$

$\begin{array}{llll}\text { C } & 1.907496 & -1.164077 & 0.232943\end{array}$

$\begin{array}{llll}\mathrm{O} & 1.643348 & -2.365119 & 0.435798\end{array}$

$\begin{array}{llll}\text { O } & 3.202529 & -0.732261 & 0.404121\end{array}$

$\begin{array}{llll}\text { C } & 4.138418 & -1.722142 & 0.845579\end{array}$

$\begin{array}{llll}\mathrm{H} & -5.339038 & 0.860312 & 0.986011\end{array}$

$\begin{array}{llll}\mathrm{H} & -3.500729 & 2.352915 & 1.715396\end{array}$

$\begin{array}{llll}\mathrm{H} & -1.132917 & 1.840696 & 1.133274\end{array}$

$\mathrm{H} \quad-\quad-4.846139 \quad-1.221049 \quad-0.324188$

$\begin{array}{llll}\mathrm{H} & -0.336805 & -2.560779 & -1.506267\end{array}$

$\begin{array}{llll}\mathrm{H} & 3.689657 & 3.294128 & -0.000444\end{array}$

$\begin{array}{llll}\mathrm{H} & 2.662665 & 3.252239 & -1.464770\end{array}$

$\begin{array}{llll}\mathrm{H} & 1.965225 & 3.759986 & 0.093853\end{array}$

$\begin{array}{llll}\mathrm{H} & 5.094060 & -1.199734 & 0.934625\end{array}$

$\begin{array}{llll}\mathrm{H} & 3.854053 & -2.138785 & 1.817893\end{array}$

$\begin{array}{llll}\mathrm{H} & 4.231429 & -2.541273 & 0.124243\end{array}$

$\begin{array}{llll}\mathrm{O} & -2.245407 & -1.904002 & -0.978211\end{array}$ 


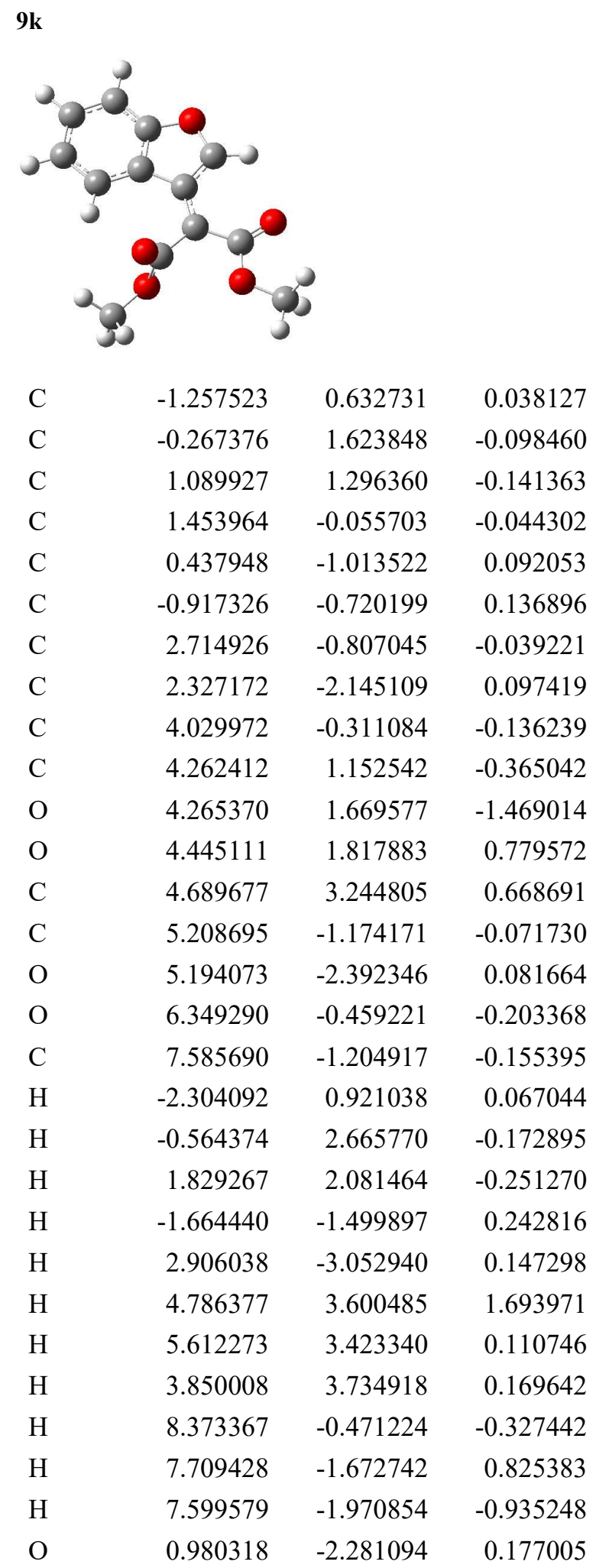




\begin{tabular}{|c|c|c|c|}
\hline \\
\hline & 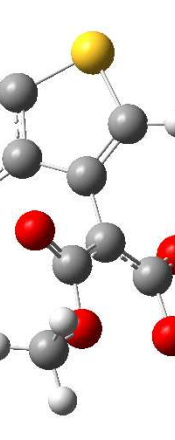 & & \\
\hline $\mathrm{C}$ & -3.838660 & 1.269744 & 1.264416 \\
\hline $\mathrm{C}$ & -2.585026 & 1.811454 & 1.618657 \\
\hline $\mathrm{C}$ & -1.408524 & 1.283091 & 1.095410 \\
\hline $\mathrm{C}$ & -1.463939 & 0.194207 & 0.203887 \\
\hline $\mathrm{C}$ & -2.732937 & -0.339644 & -0.132734 \\
\hline $\mathrm{C}$ & -3.922709 & 0.188876 & 0.387543 \\
\hline $\mathrm{C}$ & -0.358204 & -0.505350 & -0.437932 \\
\hline $\mathrm{C}$ & -0.817989 & -1.522530 & -1.223259 \\
\hline $\mathrm{C}$ & 1.076085 & -0.178476 & -0.222635 \\
\hline $\mathrm{C}$ & 1.478364 & 1.117917 & -0.684042 \\
\hline $\mathrm{O}$ & 0.744161 & 1.886801 & -1.336792 \\
\hline $\mathrm{O}$ & 2.751885 & 1.524041 & -0.360724 \\
\hline $\mathrm{C}$ & 3.135581 & 2.817550 & -0.842444 \\
\hline $\mathrm{C}$ & 1.865409 & -1.174736 & 0.443185 \\
\hline $\mathrm{O}$ & 1.430257 & -2.254641 & 0.888843 \\
\hline $\mathrm{O}$ & 3.206217 & -0.892079 & 0.576796 \\
\hline $\mathrm{C}$ & 3.986162 & -1.876807 & 1.264697 \\
\hline $\mathrm{H}$ & -4.748245 & 1.695549 & 1.679333 \\
\hline $\mathrm{H}$ & -2.540389 & 2.651912 & 2.306342 \\
\hline $\mathrm{H}$ & -0.442691 & 1.702021 & 1.362461 \\
\hline $\mathrm{H}$ & -4.886660 & -0.233961 & 0.117720 \\
\hline $\mathrm{H}$ & -0.222104 & -2.213883 & -1.806832 \\
\hline $\mathrm{H}$ & 4.165120 & 2.961101 & -0.505854 \\
\hline $\mathrm{H}$ & 3.094443 & 2.868176 & -1.935848 \\
\hline $\mathrm{H}$ & 2.499253 & 3.606518 & -0.427291 \\
\hline $\mathrm{H}$ & 5.003616 & -1.479037 & 1.289188 \\
\hline $\mathrm{H}$ & 3.626231 & -2.033018 & 2.287366 \\
\hline $\mathrm{H}$ & 3.978792 & -2.836584 & 0.736752 \\
\hline S & -2.566826 & -1.707096 & -1.228551 \\
\hline
\end{tabular}




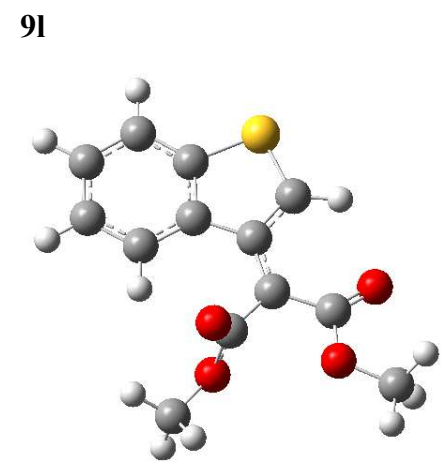

$\begin{array}{lrrr}\mathrm{C} & 4.069724 & 1.316696 & -0.005281 \\ \mathrm{C} & 2.913893 & 2.114293 & 0.017622 \\ \mathrm{C} & 1.643997 & 1.539707 & 0.029157 \\ \mathrm{C} & 1.502750 & 0.139098 & 0.020355 \\ \mathrm{C} & 2.685688 & -0.638160 & -0.014173 \\ \mathrm{C} & 3.963909 & -0.073212 & -0.024904 \\ \mathrm{C} & 0.288792 & -0.701076 & 0.017076 \\ \mathrm{C} & 0.637115 & -2.060129 & -0.036236 \\ \mathrm{C} & -1.049310 & -0.243350 & 0.067383 \\ \mathrm{C} & -1.372134 & 1.196481 & 0.338243 \\ \mathrm{O} & -1.372882 & 1.689011 & 1.453389 \\ \mathrm{O} & -1.653379 & 1.869438 & -0.782710 \\ \mathrm{C} & -2.012679 & 3.267038 & -0.627373 \\ \mathrm{C} & -2.214759 & -1.127072 & -0.055890 \\ \mathrm{O} & -2.198806 & -2.327324 & -0.311836 \\ \mathrm{O} & -3.363054 & -0.440755 & 0.150780 \\ \mathrm{C} & -4.591742 & -1.193397 & 0.052479 \\ \mathrm{H} & 5.051192 & 1.781454 & -0.012534 \\ \mathrm{H} & 3.006258 & 3.196256 & 0.025044 \\ \mathrm{H} & 0.783413 & 2.194900 & 0.041809 \\ \mathrm{H} & 4.850167 & -0.700074 & -0.051075 \\ \mathrm{H} & -0.053184 & -2.890632 & -0.047840 \\ \mathrm{H} & -2.175712 & 3.636716 & -1.639238 \\ \mathrm{H} & -2.926260 & 3.354103 & -0.033742 \\ \mathrm{H} & -1.200277 & 3.816164 & -0.144562 \\ \mathrm{H} & -5.386801 & -0.477577 & 0.261745 \\ \mathrm{H} & -4.706252 & -1.606522 & -0.953514 \\ \mathrm{H} & -4.602947 & -2.001523 & 0.788748 \\ \mathrm{~S} & 2.329619 & -2.365483 & -0.061411\end{array}$




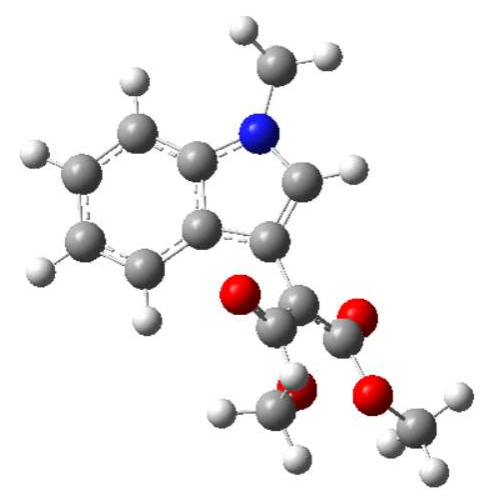

$\begin{array}{lrrr}\mathrm{C} & -3.849406 & 1.346275 & 1.267040 \\ \mathrm{C} & -2.628056 & 1.912353 & 1.704536 \\ \mathrm{C} & -1.405280 & 1.430384 & 1.237903 \\ \mathrm{C} & -1.391305 & 0.361729 & 0.322915 \\ \mathrm{C} & -2.635795 & -0.192418 & -0.106648 \\ \mathrm{C} & -3.869702 & 0.286557 & 0.358868 \\ \mathrm{C} & -0.335714 & -0.367296 & -0.341509 \\ \mathrm{C} & -0.977767 & -1.305866 & -1.122060 \\ \mathrm{~N} & -2.356294 & -1.211292 & -0.990942 \\ \mathrm{C} & -3.338067 & -2.084240 & -1.610092 \\ \mathrm{C} & 1.128293 & -0.162780 & -0.189378 \\ \mathrm{C} & 1.639871 & 1.066979 & -0.721154 \\ \mathrm{O} & 0.969123 & 1.889217 & -1.378641 \\ \mathrm{O} & 2.964953 & 1.348446 & -0.464840 \\ \mathrm{C} & 3.468458 & 2.560740 & -1.034849 \\ \mathrm{C} & 1.847120 & -1.190876 & 0.506643 \\ \mathrm{O} & 1.331466 & -2.203332 & 1.023821 \\ \mathrm{O} & 3.215550 & -1.035536 & 0.583152 \\ \mathrm{C} & 3.923300 & -2.058233 & 1.291016 \\ \mathrm{H} & -4.788114 & 1.741905 & 1.646391 \\ \mathrm{H} & -2.648483 & 2.735812 & 2.414195 \\ \mathrm{H} & -0.468926 & 1.869148 & 1.574122 \\ \mathrm{H} & -4.806601 & -0.150294 & 0.024558 \\ \mathrm{H} & -0.554402 & -2.063590 & -1.768910 \\ \mathrm{H} & -4.106980 & -1.494643 & -2.120266 \\ \mathrm{H} & -2.836653 & -2.716771 & -2.345012 \\ \mathrm{H} & -3.823087 & -2.724266 & -0.863273 \\ \mathrm{H} & 4.522944 & 2.601344 & -0.750473\end{array}$




$\begin{array}{lrrr}\mathrm{H} & 3.381837 & 2.559231 & -2.127065 \\ \mathrm{H} & 2.946997 & 3.440517 & -0.642173 \\ \mathrm{H} & 4.974270 & -1.759452 & 1.262956 \\ \mathrm{H} & 3.590249 & -2.133313 & 2.332012 \\ \mathrm{H} & 3.806569 & -3.036801 & 0.812217\end{array}$

9b

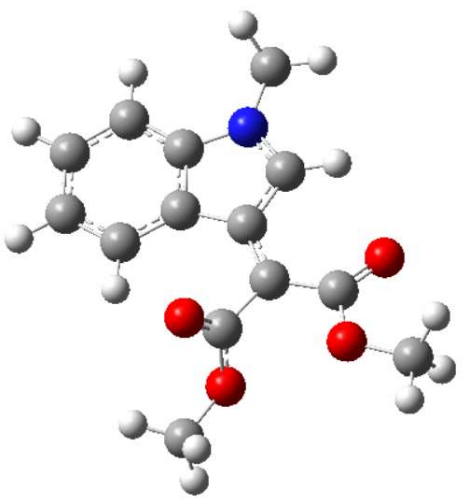

$\begin{array}{lrrr}\mathrm{C} & 4.007357 & 1.537256 & -0.018129 \\ \mathrm{C} & 2.858710 & 2.351122 & 0.024569 \\ \mathrm{C} & 1.577190 & 1.795814 & 0.053683 \\ \mathrm{C} & 1.438658 & 0.398660 & 0.038570 \\ \mathrm{C} & 2.612920 & -0.396352 & -0.012887 \\ \mathrm{C} & 3.899671 & 0.145137 & -0.039351 \\ \mathrm{C} & 0.301671 & -0.526601 & 0.039465 \\ \mathrm{C} & 0.878823 & -1.818765 & -0.016781 \\ \mathrm{~N} & 2.230780 & -1.735591 & -0.041894 \\ \mathrm{C} & 3.153001 & -2.859342 & -0.124375 \\ \mathrm{C} & -1.077900 & -0.227930 & 0.070653 \\ \mathrm{C} & -1.541000 & 1.175664 & 0.297733 \\ \mathrm{O} & -1.412975 & 1.778689 & 1.352543 \\ \mathrm{O} & -2.107238 & 1.708130 & -0.795295 \\ \mathrm{C} & -2.661578 & 3.039131 & -0.654555 \\ \mathrm{C} & -2.108510 & -1.253238 & -0.032017 \\ \mathrm{O} & -1.926687 & -2.444126 & -0.286558 \\ \mathrm{O} & -3.345543 & -0.746061 & 0.214510 \\ \mathrm{C} & -4.449755 & -1.667318 & 0.119536 \\ \mathrm{H} & 4.991196 & 1.997265 & -0.037286 \\ \mathrm{H} & 2.970304 & 3.431569 & 0.036649\end{array}$




$\begin{array}{lrrr}\mathrm{H} & 0.713635 & 2.449139 & 0.092631 \\ \mathrm{H} & 4.778614 & -0.490565 & -0.077627 \\ \mathrm{H} & 0.382380 & -2.775551 & -0.037879 \\ \mathrm{H} & 3.862475 & -2.821366 & 0.707286 \\ \mathrm{H} & 2.583739 & -3.788054 & -0.073209 \\ \mathrm{H} & 3.706077 & -2.822551 & -1.068213 \\ \mathrm{H} & -3.077148 & 3.284955 & -1.631662 \\ \mathrm{H} & -3.446197 & 3.040615 & 0.106811 \\ \mathrm{H} & -1.878366 & 3.751011 & -0.381990 \\ \mathrm{H} & -5.340661 & -1.073511 & 0.327140 \\ \mathrm{H} & -4.507446 & -2.097248 & -0.884635 \\ \mathrm{H} & -4.350070 & -2.469047 & 0.856964\end{array}$

$9 e$

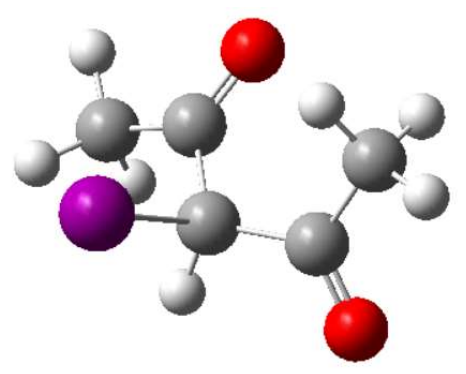

$\begin{array}{lrrr}\mathrm{C} & -0.936114 & 2.664957 & 0.586237 \\ \mathrm{C} & -1.244339 & 1.359441 & -0.098670 \\ \mathrm{C} & -0.681563 & 0.109334 & 0.583785 \\ \mathrm{C} & -1.426981 & -1.203716 & 0.349372 \\ \mathrm{C} & -1.745285 & -1.662633 & -1.048623 \\ \mathrm{O} & -1.937744 & 1.285410 & -1.102092 \\ \mathrm{O} & -1.769513 & -1.847087 & 1.333323 \\ \mathrm{I} & 1.407978 & -0.132348 & -0.073524 \\ \mathrm{H} & -1.553960 & 2.728384 & 1.491915 \\ \mathrm{H} & 0.110201 & 2.709660 & 0.902958 \\ \mathrm{H} & -1.173853 & 3.502914 & -0.071845 \\ \mathrm{H} & -2.738160 & -1.277365 & -1.308704 \\ \mathrm{H} & -1.038598 & -1.279365 & -1.785927 \\ \mathrm{H} & -1.775579 & -2.754929 & -1.071956 \\ \mathrm{H} & -0.589140 & 0.274282 & 1.657859\end{array}$




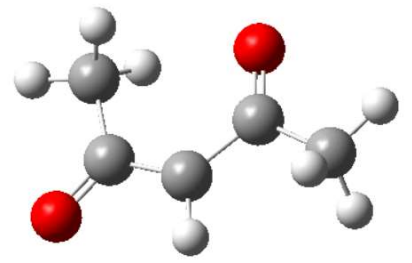

$\begin{array}{lrrr}\mathrm{C} & -1.716160 & 1.182612 & 0.000225 \\ \mathrm{C} & -1.348762 & -0.277187 & 0.000068 \\ \mathrm{C} & 0.046684 & -0.722683 & 0.000000 \\ \mathrm{C} & 1.250407 & 0.112990 & -0.000158 \\ \mathrm{C} & 2.571227 & -0.617809 & 0.000188 \\ \mathrm{O} & -2.224381 & -1.158921 & 0.000087 \\ \mathrm{O} & 1.187509 & 1.349871 & -0.000533 \\ \mathrm{H} & -1.292141 & 1.681738 & -0.876885 \\ \mathrm{H} & -2.804040 & 1.279962 & 0.000611 \\ \mathrm{H} & -1.291479 & 1.681784 & 0.876983 \\ \mathrm{H} & 0.181881 & -1.801570 & 0.000058 \\ \mathrm{H} & 2.641692 & -1.267023 & -0.881213 \\ \mathrm{H} & 3.397080 & 0.096251 & -0.000067 \\ \mathrm{H} & 2.641612 & -1.266278 & 0.882145\end{array}$




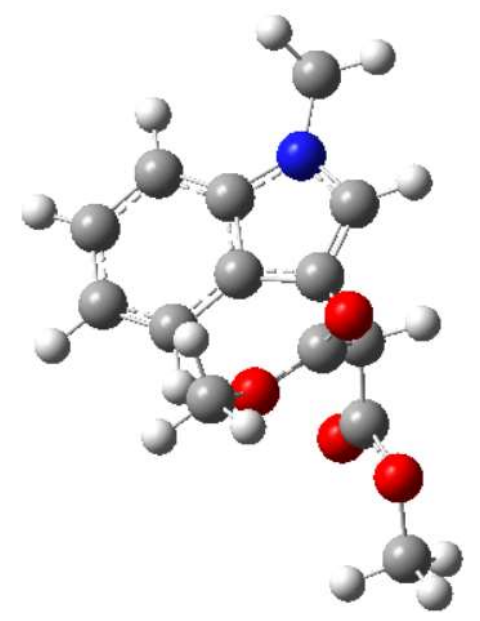

$\begin{array}{lrrr}\mathrm{C} & 3.275656 & 2.095242 & -0.698534 \\ \mathrm{C} & 1.931215 & 2.522922 & -0.805064 \\ \mathrm{C} & 0.873478 & 1.648119 & -0.567537 \\ \mathrm{C} & 1.155974 & 0.314504 & -0.212866 \\ \mathrm{C} & 2.519436 & -0.095187 & -0.114617 \\ \mathrm{C} & 3.587754 & 0.781692 & -0.352949 \\ \mathrm{C} & 0.358646 & -0.848792 & 0.089607 \\ \mathrm{C} & 1.244910 & -1.873017 & 0.353928 \\ \mathrm{~N} & 2.542322 & -1.431817 & 0.231868 \\ \mathrm{C} & 3.743667 & -2.225387 & 0.440128 \\ \mathrm{C} & -1.135939 & -0.981973 & 0.188188 \\ \mathrm{C} & -1.659321 & -0.207466 & 1.402117 \\ \mathrm{O} & -1.877418 & -0.710540 & 2.489476 \\ \mathrm{O} & -1.793311 & 1.098343 & 1.136401 \\ \mathrm{C} & -2.206678 & 1.950731 & 2.232507 \\ \mathrm{C} & -1.897216 & -0.599772 & -1.084506 \\ \mathrm{O} & -1.397840 & -0.289390 & -2.147890 \\ \mathrm{H} & -3.219844 & -0.698906 & -0.875768 \\ \mathrm{H} & -4.081343 & -0.372909 & -1.993486 \\ \mathrm{H} & 4.078316 & 2.802032 & -0.891073 \\ \mathrm{H} & 1.722411 & 3.553509 & -1.079909 \\ \mathrm{H} & -0.153616 & 1.985292 & -0.652170 \\ \mathrm{H} & 4.617822 & 0.447115 & -0.273527 \\ \mathrm{H} & 1.039772 & -2.899931 & 0.627231 \\ \mathrm{H} & -351346 & -1.793323 & 1.241834 \\ \mathrm{H} & -3.239174 & 0.720389\end{array}$




$\begin{array}{lrrr}\mathrm{H} & 4.340985 & -2.265616 & -0.476718 \\ \mathrm{H} & -2.265739 & 2.954244 & 1.811579 \\ \mathrm{H} & -3.182800 & 1.635702 & 2.609664 \\ \mathrm{H} & -1.464955 & 1.915857 & 3.034475 \\ \mathrm{H} & -5.097851 & -0.502152 & -1.622146 \\ \mathrm{H} & -3.917696 & 0.661534 & -2.306349 \\ \mathrm{H} & -3.890211 & -1.050186 & -2.829813 \\ \mathrm{H} & -1.381686 & -2.030483 & 0.384202\end{array}$

9h

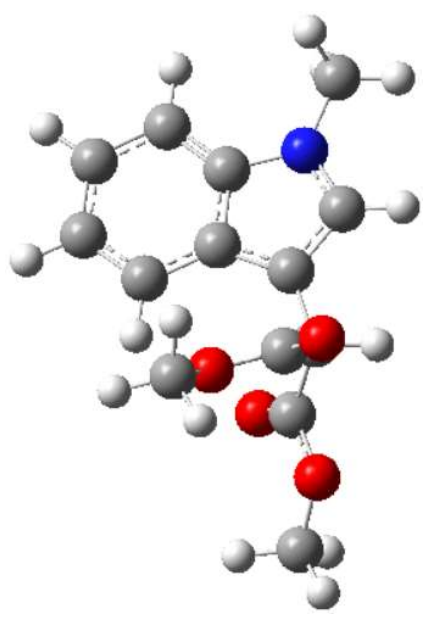

$\begin{array}{lrrr}\mathrm{C} & 3.275656 & 2.095242 & -0.698534 \\ \mathrm{C} & 1.931215 & 2.522922 & -0.805064 \\ \mathrm{C} & 0.873478 & 1.648119 & -0.567537 \\ \mathrm{C} & 1.155974 & 0.314504 & -0.212866 \\ \mathrm{C} & 2.519436 & -0.095187 & -0.114617 \\ \mathrm{C} & 3.587754 & 0.781692 & -0.352949 \\ \mathrm{C} & 0.358646 & -0.848792 & 0.089607 \\ \mathrm{C} & 1.244910 & -1.873017 & 0.353928 \\ \mathrm{~N} & 2.542322 & -1.431817 & 0.231868 \\ \mathrm{C} & 3.743667 & -2.225387 & 0.440128 \\ \mathrm{C} & -1.135939 & -0.981973 & 0.188188 \\ \mathrm{C} & -1.659321 & -0.207466 & 1.402117 \\ \mathrm{O} & -1.877418 & -0.710540 & 2.489476 \\ \mathrm{O} & -1.793311 & 1.098343 & 1.136401 \\ \mathrm{C} & -2.206678 & 1.950731 & 2.232507 \\ \mathrm{C} & -1.897216 & -0.599772 & -1.084506\end{array}$




$\begin{array}{lrrr}\mathrm{O} & -1.397840 & -0.289390 & -2.147890 \\ \mathrm{O} & -3.219844 & -0.698906 & -0.875768 \\ \mathrm{C} & -4.081343 & -0.372909 & -1.993486 \\ \mathrm{H} & 4.078316 & 2.802032 & -0.891073 \\ \mathrm{H} & 1.722411 & 3.553509 & -1.079909 \\ \mathrm{H} & -0.153616 & 1.985292 & -0.652170 \\ \mathrm{H} & 4.617822 & 0.447115 & -0.273527 \\ \mathrm{H} & 1.039772 & -2.899931 & 0.627231 \\ \mathrm{H} & 4.351346 & -1.793323 & 1.241834 \\ \mathrm{H} & 3.453512 & -3.239174 & 0.720389 \\ \mathrm{H} & 4.340985 & -2.265616 & -0.476718 \\ \mathrm{H} & -2.265739 & 2.954244 & 1.811579 \\ \mathrm{H} & -3.182800 & 1.635702 & 2.609664 \\ \mathrm{H} & -1.464955 & 1.915857 & 3.034475 \\ \mathrm{H} & -5.097851 & -0.502152 & -1.622146 \\ \mathrm{H} & -3.917696 & 0.661534 & -2.306349 \\ \mathrm{H} & -3.890211 & -1.050186 & -2.829813 \\ \mathrm{H} & -1.381686 & -2.030483 & 0.384202\end{array}$

3'aa

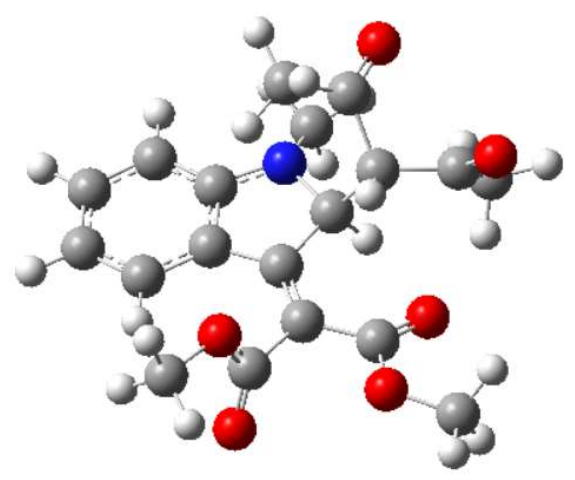

$\begin{array}{lrrc}\mathrm{C} & 1.777393 & 0.848383 & 2.524894 \\ \mathrm{C} & 2.526221 & 0.063996 & 1.480616 \\ \mathrm{C} & 1.676494 & -0.838988 & 0.559000 \\ \mathrm{C} & 2.497819 & -2.073196 & 0.158320 \\ \mathrm{C} & 3.155853 & -2.163033 & -1.191298 \\ \mathrm{O} & 3.746509 & 0.085544 & 1.397449 \\ \mathrm{O} & 2.615417 & -2.966247 & 0.988017 \\ \mathrm{C} & -0.332261 & 4.512919 & -0.498856 \\ \mathrm{C} & -1.602010 & 3.934546 & -0.292023 \\ & & & \mathrm{~S} 102\end{array}$




\begin{tabular}{|c|c|c|c|}
\hline $\mathrm{C}$ & -1.737779 & 2.551912 & -0.229010 \\
\hline $\mathrm{C}$ & -0.597580 & 1.736399 & -0.361620 \\
\hline $\mathrm{C}$ & 0.678327 & 2.334638 & -0.552034 \\
\hline $\mathrm{C}$ & 0.812082 & 3.733066 & -0.637564 \\
\hline $\mathrm{C}$ & -0.427945 & 0.296824 & -0.416850 \\
\hline $\mathrm{C}$ & 1.069487 & 0.040021 & -0.594198 \\
\hline $\mathrm{N}$ & 1.670931 & 1.388578 & -0.621876 \\
\hline $\mathrm{C}$ & 2.910647 & 1.605242 & -1.355609 \\
\hline $\mathrm{C}$ & -1.378103 & -0.683750 & -0.336837 \\
\hline $\mathrm{C}$ & -2.813075 & -0.360706 & -0.042316 \\
\hline $\mathrm{O}$ & -3.709402 & -0.365365 & -0.868780 \\
\hline $\mathrm{O}$ & -2.995867 & -0.056600 & 1.252499 \\
\hline $\mathrm{C}$ & -4.342935 & 0.304784 & 1.647136 \\
\hline $\mathrm{C}$ & -1.079347 & -2.096823 & -0.606881 \\
\hline $\mathrm{O}$ & -0.033837 & -2.528762 & -1.083386 \\
\hline $\mathrm{O}$ & -2.111586 & -2.898899 & -0.270883 \\
\hline $\mathrm{C}$ & -1.942575 & -4.310386 & -0.530500 \\
\hline $\mathrm{H}$ & 0.814582 & 1.214057 & 2.158633 \\
\hline $\mathrm{H}$ & 1.565855 & 0.164191 & 3.358474 \\
\hline $\mathrm{H}$ & 2.388628 & 1.676828 & 2.889732 \\
\hline $\mathrm{H}$ & 2.389578 & -2.310732 & -1.960060 \\
\hline $\mathrm{H}$ & 3.690277 & -1.240571 & -1.434892 \\
\hline $\mathrm{H}$ & 3.845502 & -3.009441 & -1.205363 \\
\hline $\mathrm{H}$ & -0.242941 & 5.594529 & -0.554794 \\
\hline $\mathrm{H}$ & -2.476388 & 4.569942 & -0.191977 \\
\hline $\mathrm{H}$ & -2.723588 & 2.125533 & -0.091160 \\
\hline $\mathrm{H}$ & 1.779526 & 4.195958 & -0.797945 \\
\hline $\mathrm{H}$ & 2.761846 & 1.488655 & -2.438702 \\
\hline $\mathrm{H}$ & 3.289412 & 2.607943 & -1.151309 \\
\hline $\mathrm{H}$ & 3.663503 & 0.893197 & -1.016812 \\
\hline $\mathrm{H}$ & -4.673395 & 1.191399 & 1.099200 \\
\hline $\mathrm{H}$ & -5.027869 & -0.525143 & 1.456168 \\
\hline $\mathrm{H}$ & -4.285328 & 0.515947 & 2.714778 \\
\hline $\mathrm{H}$ & -2.872758 & -4.777226 & -0.206211 \\
\hline $\mathrm{H}$ & -1.780294 & -4.484937 & -1.597531 \\
\hline $\mathrm{H}$ & -1.098302 & -4.705018 & 0.041014 \\
\hline $\mathrm{H}$ & 1.242342 & -0.450179 & -1.556683 \\
\hline $\mathrm{H}$ & 0.859150 & -1.227450 & 1.173025 \\
\hline
\end{tabular}




\section{TS-9m}

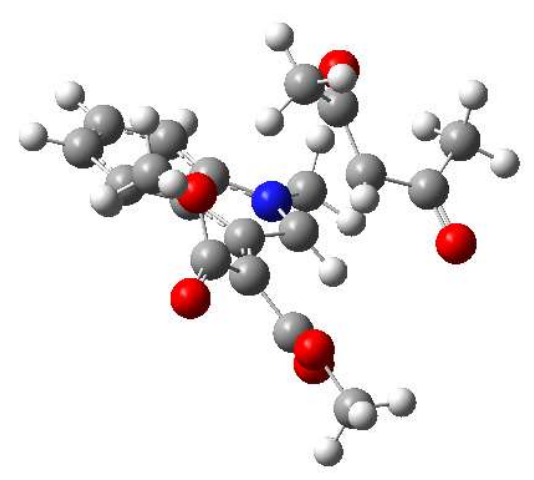

$\begin{array}{llll}\mathrm{C} & 0.817898 & 0.192293 & 2.723304 \\ \mathrm{C} & 1.643123 & -0.669704 & 1.793367 \\ \mathrm{C} & 0.900829 & -1.610354 & 0.935956 \\ \mathrm{C} & 1.430843 & -2.954096 & 0.628342 \\ \mathrm{C} & 2.863350 & -3.336795 & 0.938954 \\ \mathrm{O} & 2.874718 & -0.542704 & 1.737101 \\ \mathrm{O} & 0.676866 & -3.792282 & 0.104186 \\ \mathrm{C} & 2.396928 & 3.582380 & -1.025939 \\ \mathrm{C} & 1.070174 & 3.930900 & -0.709253 \\ \mathrm{C} & 0.090872 & 2.948925 & -0.529705 \\ \mathrm{C} & 0.434275 & 1.587738 & -0.659916 \\ \mathrm{C} & 1.781549 & 1.264634 & -1.019850 \\ \mathrm{C} & 2.766659 & 2.239177 & -1.190855 \\ \mathrm{C} & -0.308517 & 0.353560 & -0.606106 \\ \mathrm{C} & 0.688061 & -0.716477 & -0.802480 \\ \mathrm{~N} & 1.879694 & -0.099443 & -1.202656 \\ \mathrm{C} & 3.081389 & -0.790635 & -1.624645 \\ \mathrm{C} & -1.705310 & 0.116404 & -0.391046 \\ \mathrm{C} & -2.552427 & 1.035820 & 0.357224 \\ \mathrm{O} & -3.755809 & 1.253910 & 0.191475 \\ \mathrm{O} & -1.861891 & 1.707622 & 1.335132 \\ \mathrm{C} & -2.567403 & 2.756605 & 2.013323 \\ \mathrm{C} & -2.309825 & -1.069854 & -0.980491 \\ \mathrm{O} & -1.858411 & -1.714228 & -1.936576 \\ \mathrm{C} & -3.452001 & -1.483254 & -0.353864 \\ & -4.135359 & -2.595743 & -0.949070\end{array}$




$\begin{array}{llll}\mathrm{H} & -0.004277 & 0.670169 & 2.180774 \\ \mathrm{H} & 0.365159 & -0.436519 & 3.501340 \\ \mathrm{H} & 1.447347 & 0.952701 & 3.192842 \\ \mathrm{H} & 3.573508 & -2.649416 & 0.477529 \\ \mathrm{H} & 3.039746 & -3.280495 & 2.019809 \\ \mathrm{H} & 3.041519 & -4.357659 & 0.592125 \\ \mathrm{H} & 3.144278 & 4.361379 & -1.152069 \\ \mathrm{H} & 0.802119 & 4.978963 & -0.603261 \\ \mathrm{H} & -0.925468 & 3.239483 & -0.293944 \\ \mathrm{H} & 3.784652 & 1.965786 & -1.451884 \\ \mathrm{H} & 3.472665 & -0.336871 & -2.542361 \\ \mathrm{H} & 3.855854 & -0.753886 & -0.851157 \\ \mathrm{H} & 2.836151 & -1.834158 & -1.831248 \\ \mathrm{H} & -2.932318 & 3.508910 & 1.306229 \\ \mathrm{H} & -3.415102 & 2.357468 & 2.578737 \\ \mathrm{H} & -1.842756 & 3.205772 & 2.695128 \\ \mathrm{H} & -5.041916 & -2.733032 & -0.356114 \\ \mathrm{H} & -4.399741 & -2.385715 & -1.990700 \\ \mathrm{H} & -3.524044 & -3.503234 & -0.910769 \\ \mathrm{H} & 0.419617 & -1.629545 & -1.321506 \\ \mathrm{H} & -0.165294 & -1.665187 & 1.152784\end{array}$

9m

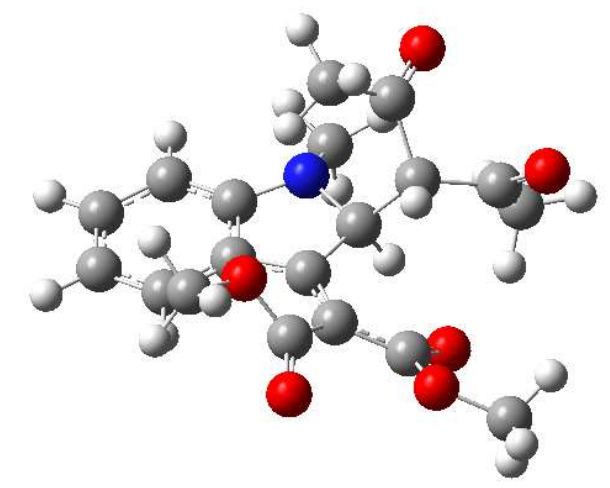

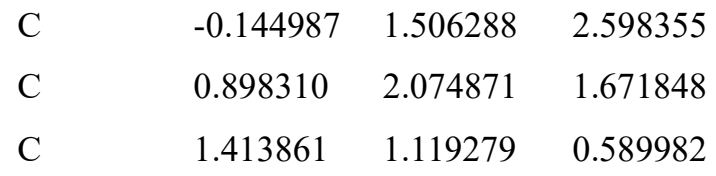




\begin{tabular}{|c|c|c|c|}
\hline $\mathrm{C}$ & 2.845041 & 1.479370 & 0.182596 \\
\hline $\mathrm{C}$ & 3.127117 & 2.183371 & -1.117344 \\
\hline $\mathrm{O}$ & 1.333414 & 3.213700 & 1.795920 \\
\hline $\mathrm{O}$ & 3.755494 & 1.171078 & 0.944978 \\
\hline $\mathrm{C}$ & -4.348332 & 1.277917 & -0.882163 \\
\hline $\mathrm{C}$ & -4.247086 & -0.121957 & -0.844764 \\
\hline $\mathrm{C}$ & -3.003701 & -0.747597 & -0.694401 \\
\hline $\mathrm{C}$ & -1.831685 & 0.038530 & -0.577039 \\
\hline $\mathrm{C}$ & -1.958086 & 1.466577 & -0.611743 \\
\hline $\mathrm{C}$ & -3.195491 & 2.086831 & -0.772598 \\
\hline $\mathrm{C}$ & -0.455100 & -0.297606 & -0.474247 \\
\hline $\mathrm{C}$ & 0.331912 & 1.004279 & -0.543865 \\
\hline $\mathrm{N}$ & -0.708753 & 2.056894 & -0.455268 \\
\hline $\mathrm{C}$ & -0.427325 & 3.359720 & -1.036454 \\
\hline $\mathrm{C}$ & 0.159190 & -1.594201 & -0.336473 \\
\hline $\mathrm{C}$ & -0.523540 & -2.675043 & 0.362927 \\
\hline $\mathrm{O}$ & -0.426377 & -3.891504 & 0.173790 \\
\hline $\mathrm{O}$ & -1.371171 & -2.200339 & 1.337500 \\
\hline $\mathrm{C}$ & -2.249640 & -3.158148 & 1.940541 \\
\hline $\mathrm{C}$ & 1.490911 & -1.783673 & -0.858857 \\
\hline $\mathrm{O}$ & 2.104800 & -0.964645 & -1.568943 \\
\hline $\mathrm{O}$ & 2.116357 & -2.940170 & -0.468448 \\
\hline $\mathrm{C}$ & 3.441314 & -3.137686 & -0.978736 \\
\hline $\mathrm{H}$ & -0.820119 & 0.825758 & 2.072504 \\
\hline $\mathrm{H}$ & 0.376883 & 0.915409 & 3.364046 \\
\hline $\mathrm{H}$ & -0.704234 & 2.308379 & 3.085778 \\
\hline $\mathrm{H}$ & 2.969573 & 1.475340 & -1.939243 \\
\hline $\mathrm{H}$ & 2.446438 & 3.024237 & -1.274950 \\
\hline $\mathrm{H}$ & 4.163402 & 2.527755 & -1.131681 \\
\hline $\mathrm{H}$ & -5.319837 & 1.749664 & -1.004687 \\
\hline $\mathrm{H}$ & -5.144141 & -0.728828 & -0.942758 \\
\hline $\mathrm{H}$ & -2.936267 & -1.830334 & -0.685795 \\
\hline $\mathrm{H}$ & -3.280440 & 3.168587 & -0.810544 \\
\hline $\mathrm{H}$ & -0.333722 & 3.310006 & -2.133598 \\
\hline $\mathrm{H}$ & -1.225543 & 4.061303 & -0.782953 \\
\hline $\mathrm{H}$ & 0.500630 & 3.754713 & -0.619089 \\
\hline
\end{tabular}




$\begin{array}{llll}\mathrm{H} & -2.839475 & -3.687181 & 1.184510 \\ \mathrm{H} & -1.692703 & -3.891581 & 2.533134 \\ \mathrm{H} & -2.911753 & -2.583170 & 2.591357 \\ \mathrm{H} & 3.763195 & -4.106534 & -0.590350 \\ \mathrm{H} & 3.448169 & -3.156240 & -2.073773 \\ \mathrm{H} & 4.123254 & -2.353787 & -0.632589 \\ \mathrm{H} & 0.843535 & 1.091847 & -1.509665 \\ \mathrm{H} & 1.498198 & 0.134716 & 1.063465\end{array}$


10. NMR spectra<smiles>Cc1ccc(C(=O)CC(=O)c2ccc(C)cc2)cc1</smiles>

2c

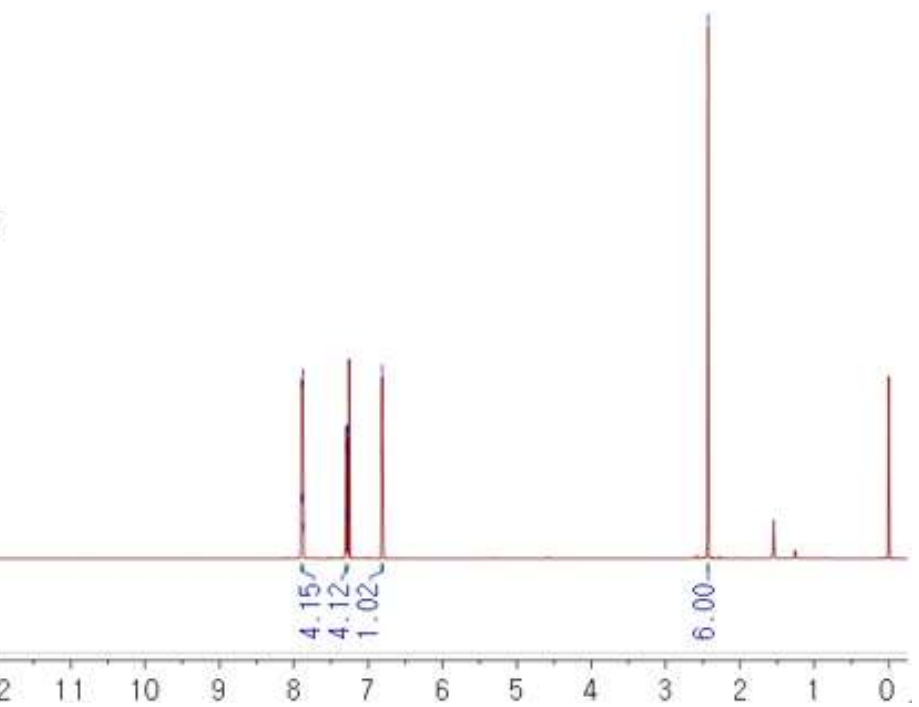

${ }^{1} \mathrm{H}$ NMR (400 MHz, $\mathrm{CDCl}_{3}$ ) spectra of 1,3-di-p-tolylpropane-1,3-dione (2c)

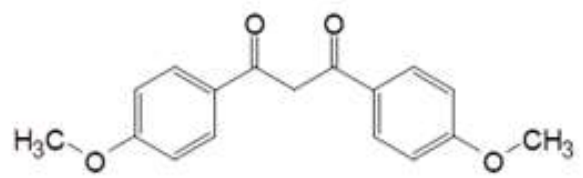

2d

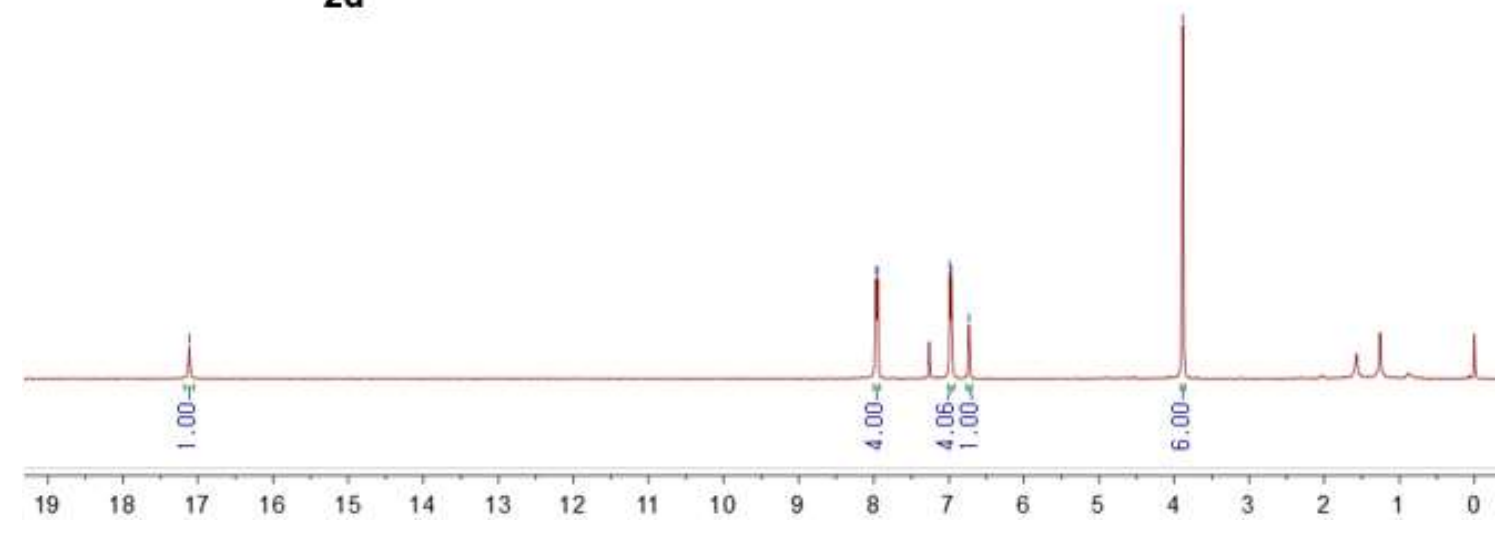

${ }^{1} \mathrm{H}$ NMR (400 MHz, $\mathrm{CDCl}_{3}$ ) spectra of 1,3-bis(4-methoxyphenyl)propane-1,3-dione (2d) 

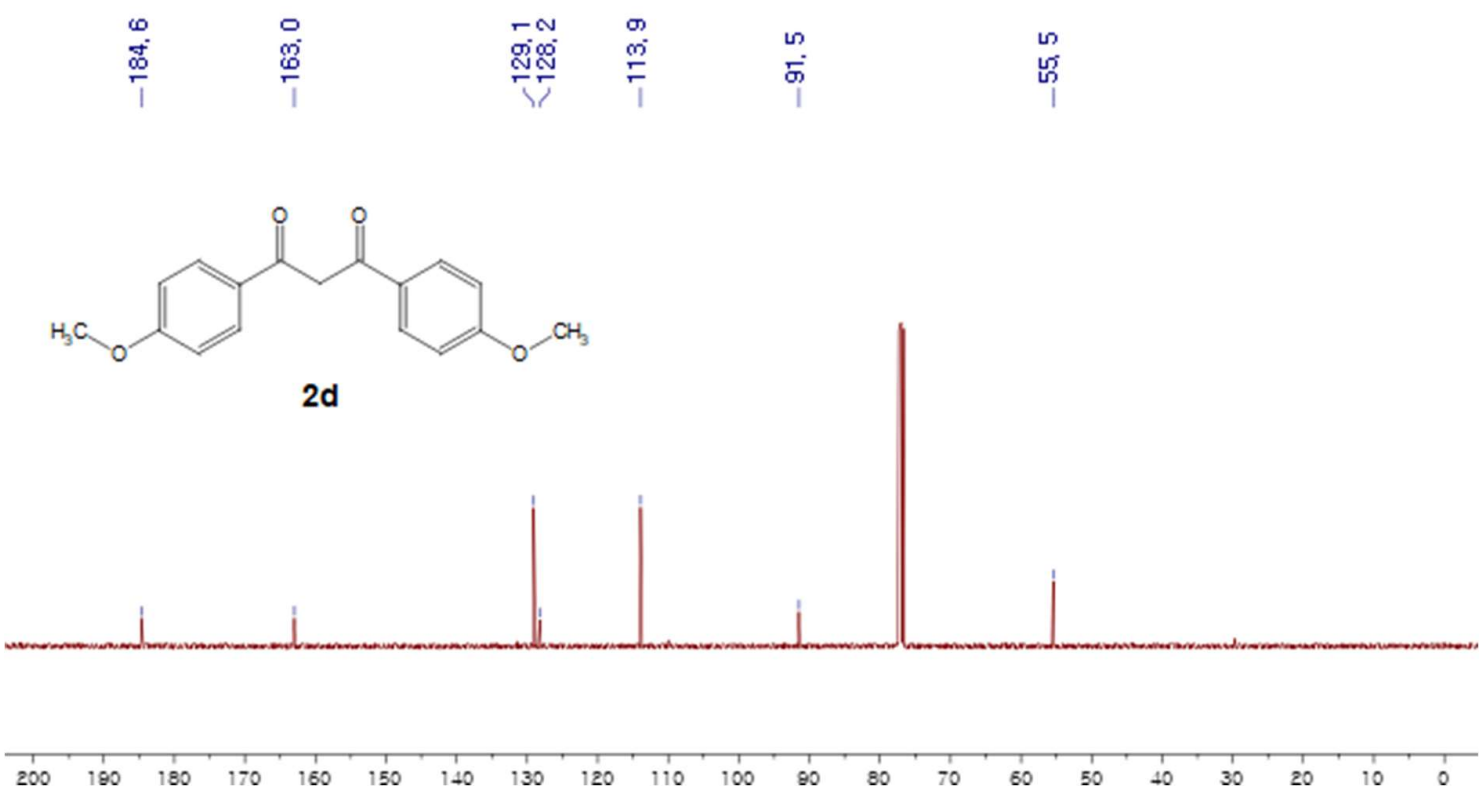

${ }^{13} \mathrm{C}$ NMR (100 MHz, $\mathrm{CDCl}_{3}$ ) spectra of 1,3-bis(4-methoxyphenyl)propane-1,3-dione (2d)
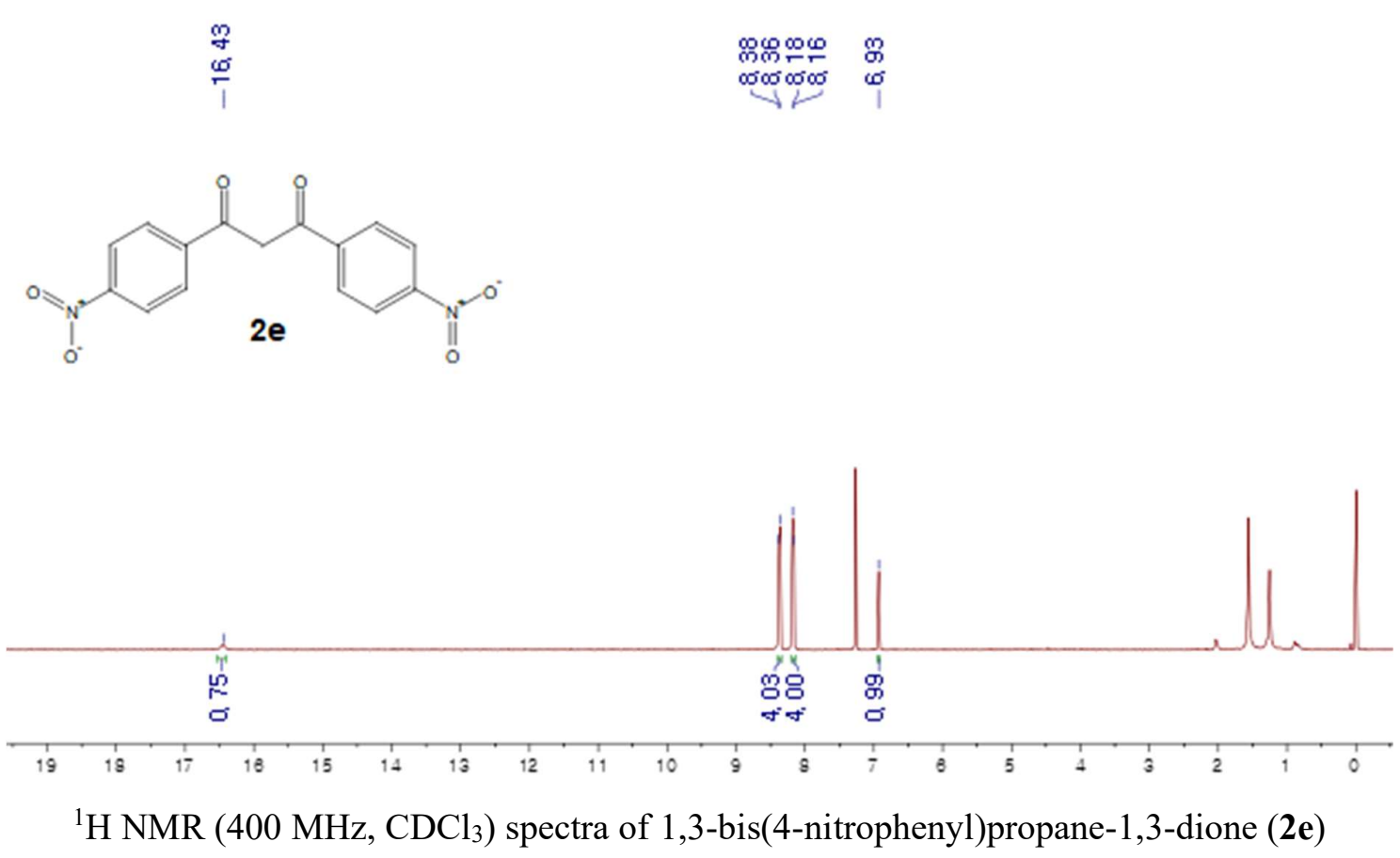


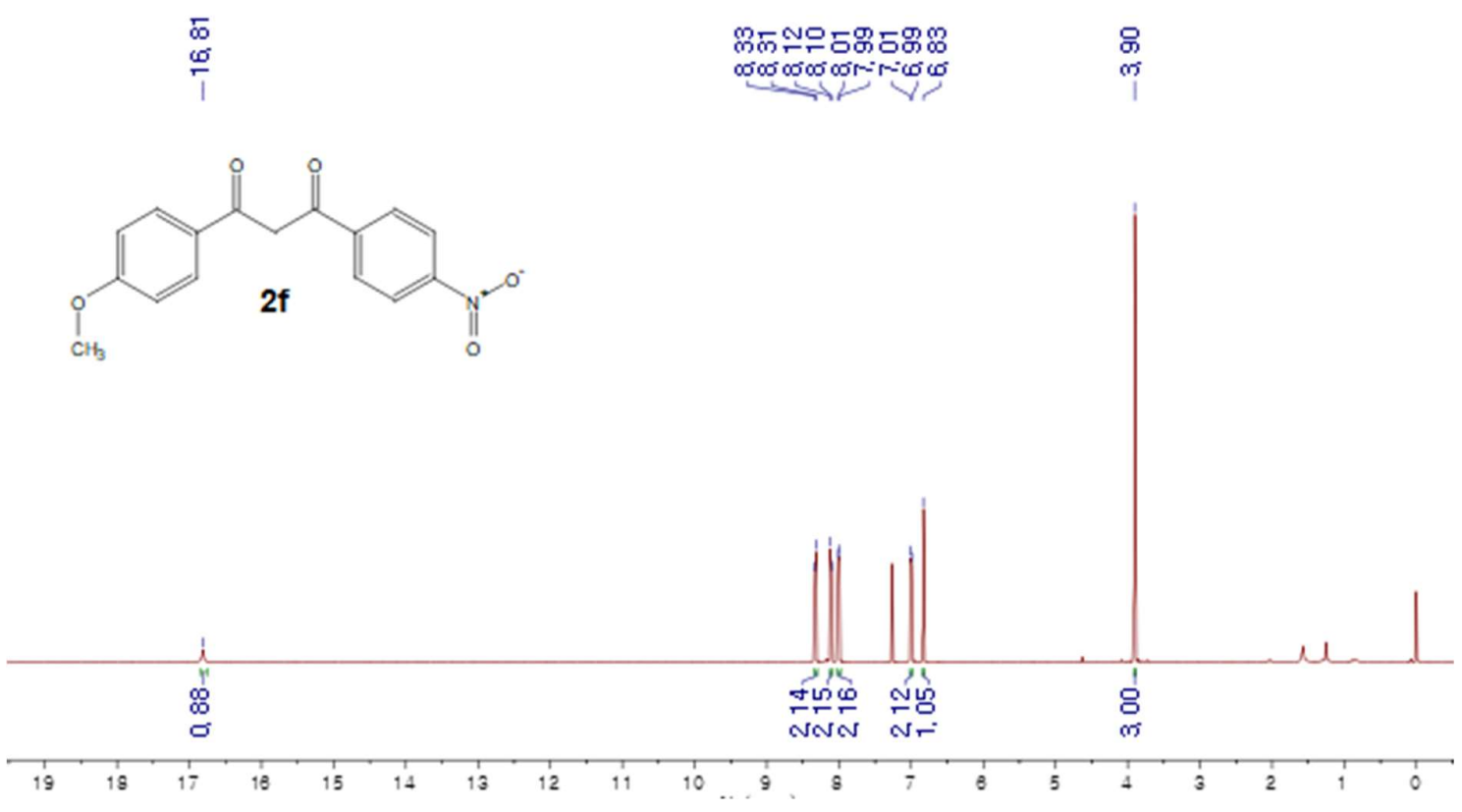

${ }^{1} \mathrm{H}$ NMR (400 MHz, $\mathrm{CDCl}_{3}$ ) spectra of 1-(4-methoxyphenyl)-3-(4-nitrophenyl)propane-1,3dione (2f)
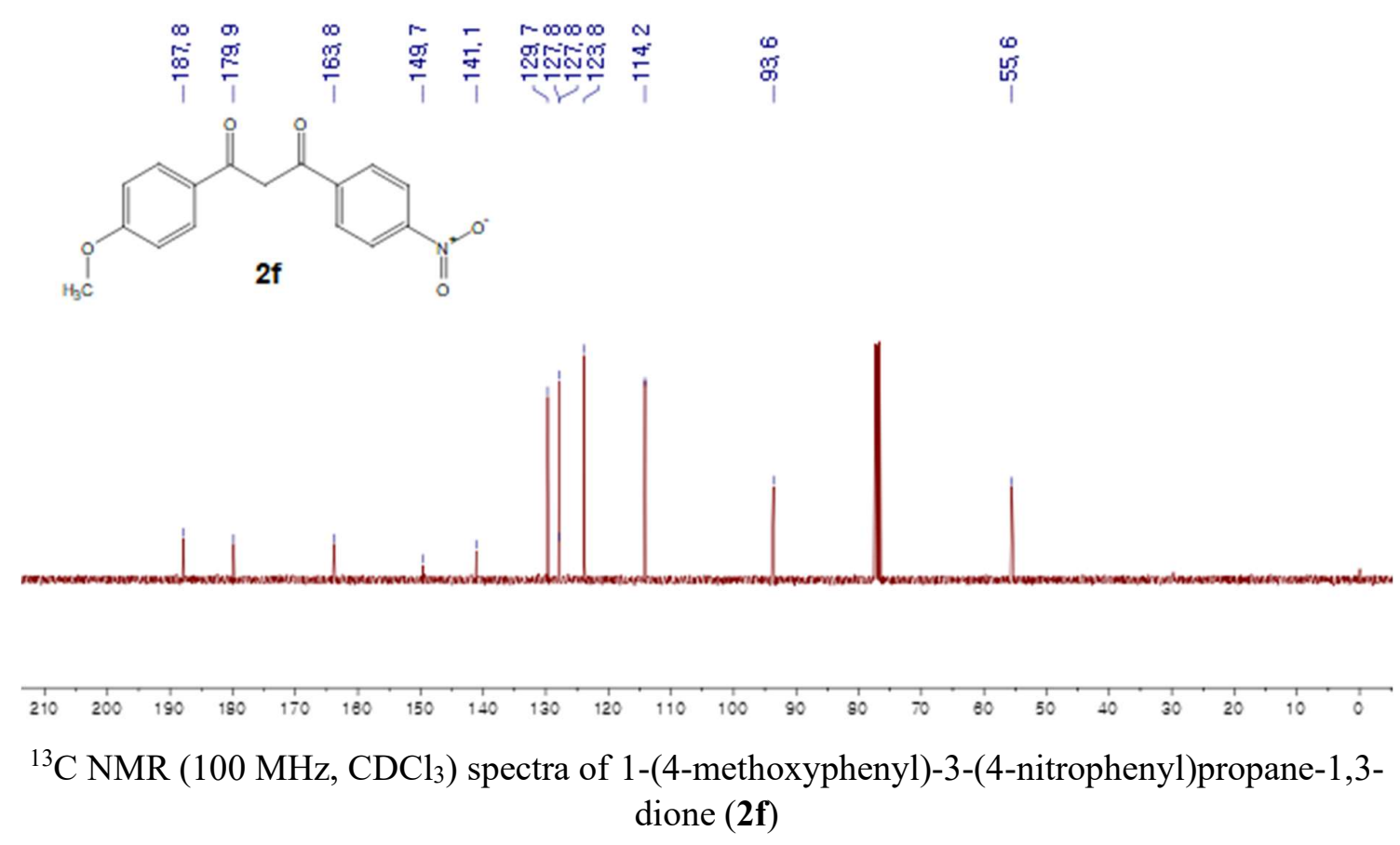


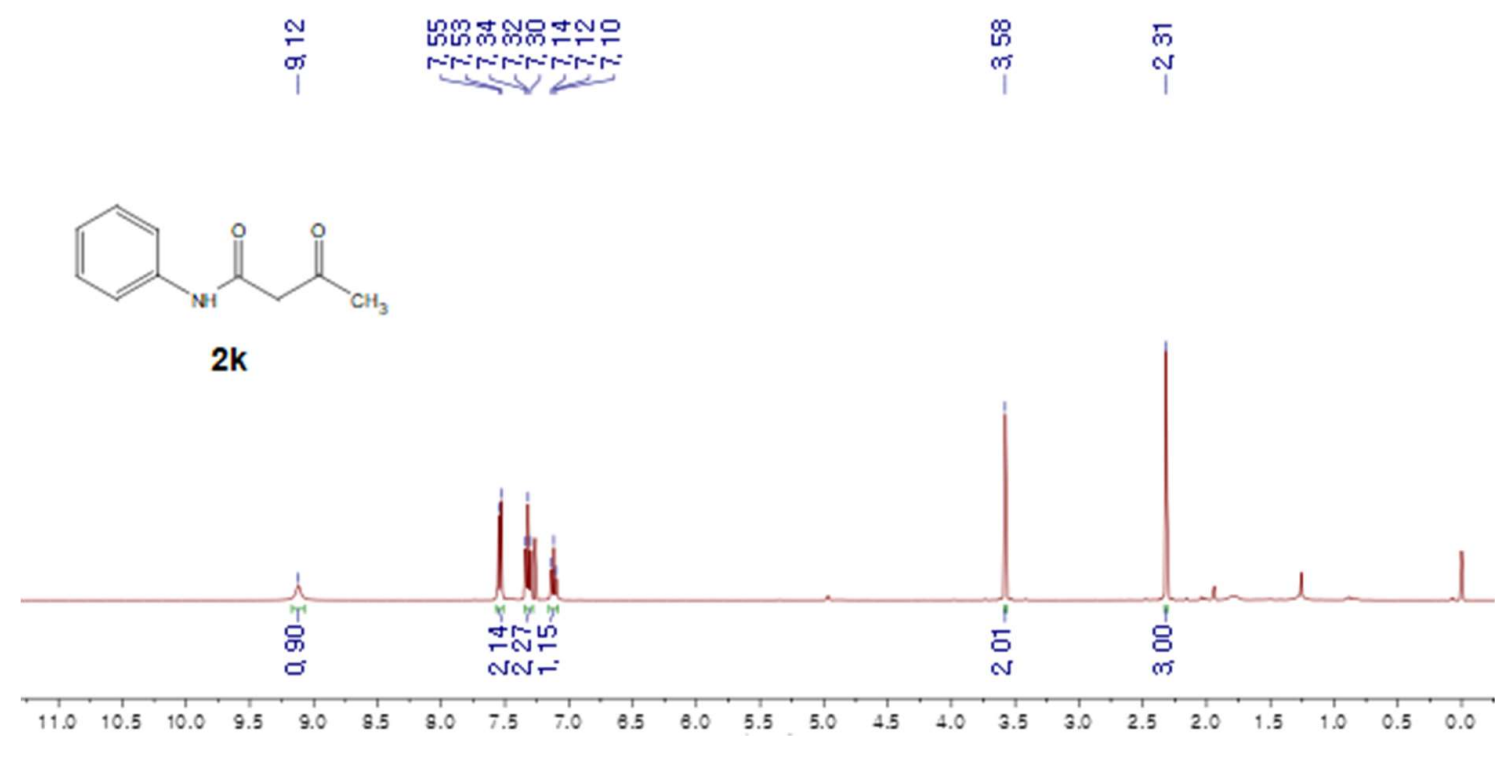

${ }^{1} \mathrm{H}$ NMR (400 MHz, $\mathrm{CDCl}_{3}$ ) spectra of 3-oxo-N-phenylbutanamide (2k)

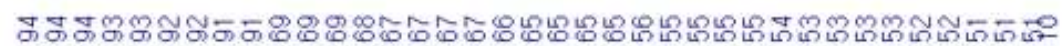

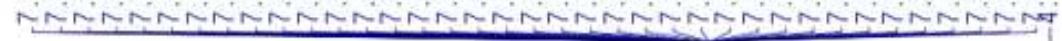<smiles>N#CCC(=O)c1ccccc1</smiles>

2I

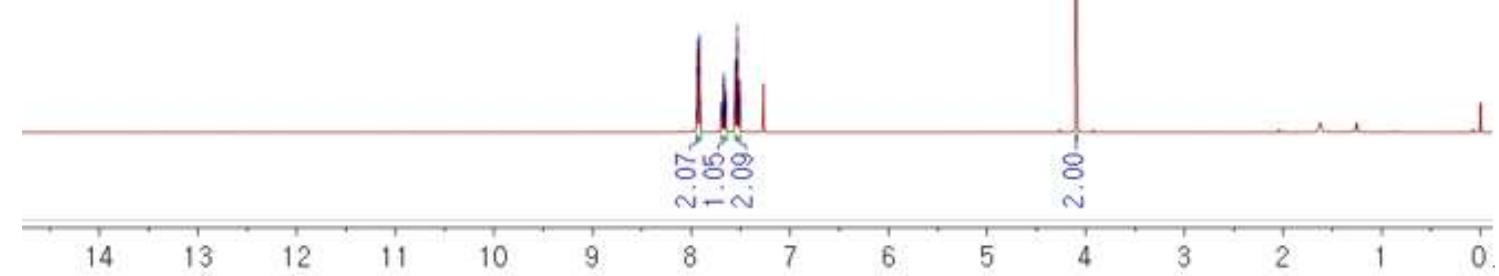

${ }^{1} \mathrm{H}$ NMR (400 MHz, $\mathrm{CDCl}_{3}$ ) spectra of 3-oxo-3-phenylpropanenitrile (2l) 


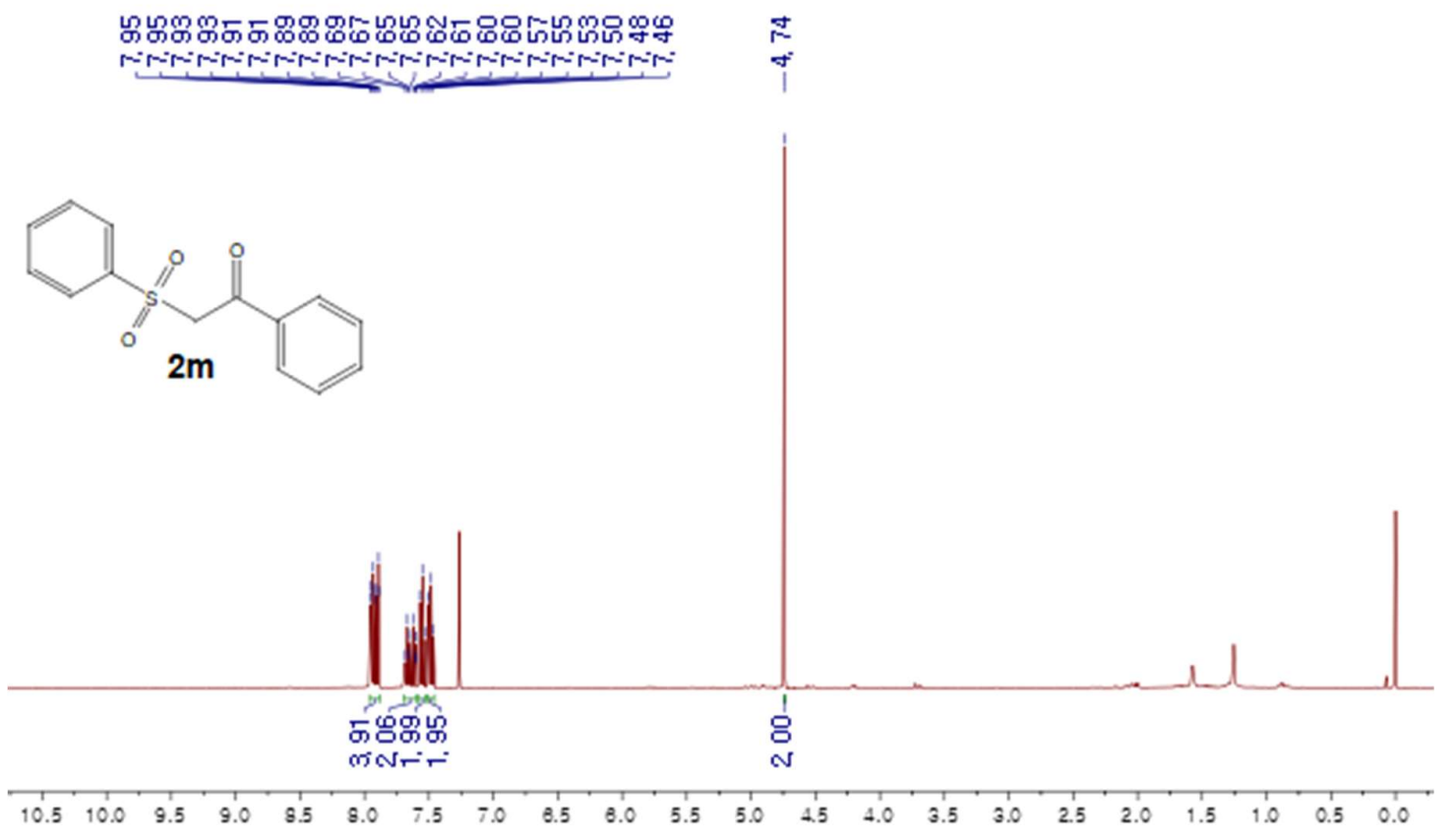

${ }^{1} \mathrm{H}$ NMR (400 MHz, $\mathrm{CDCl}_{3}$ ) spectra of 1-phenyl-2-(phenylsulfonyl)ethan-1-one (2m)
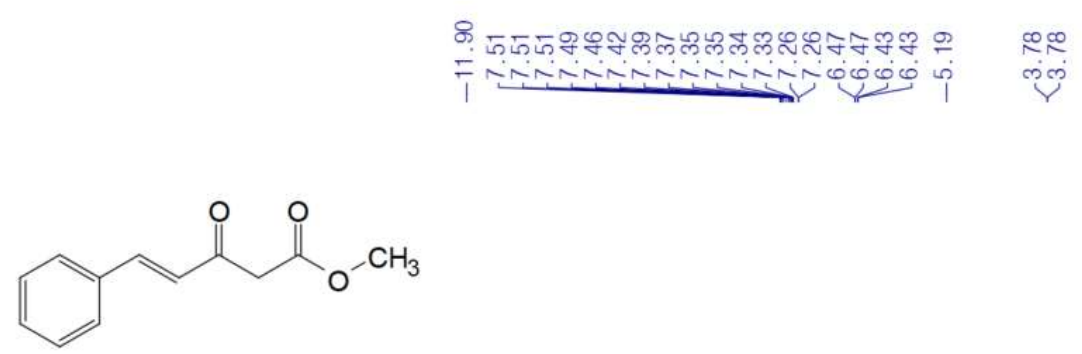

20

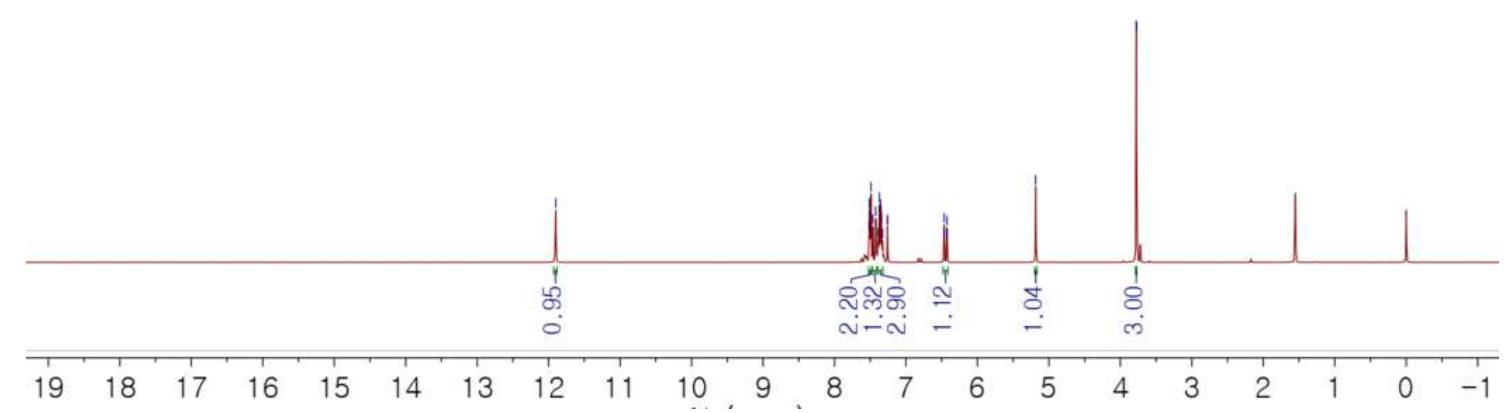

${ }^{1} \mathrm{H}$ NMR (400 MHz, $\mathrm{CDCl}_{3}$ ) spectra of methyl (E)-3-oxo-5-phenylpent-4-enoate (2o) 


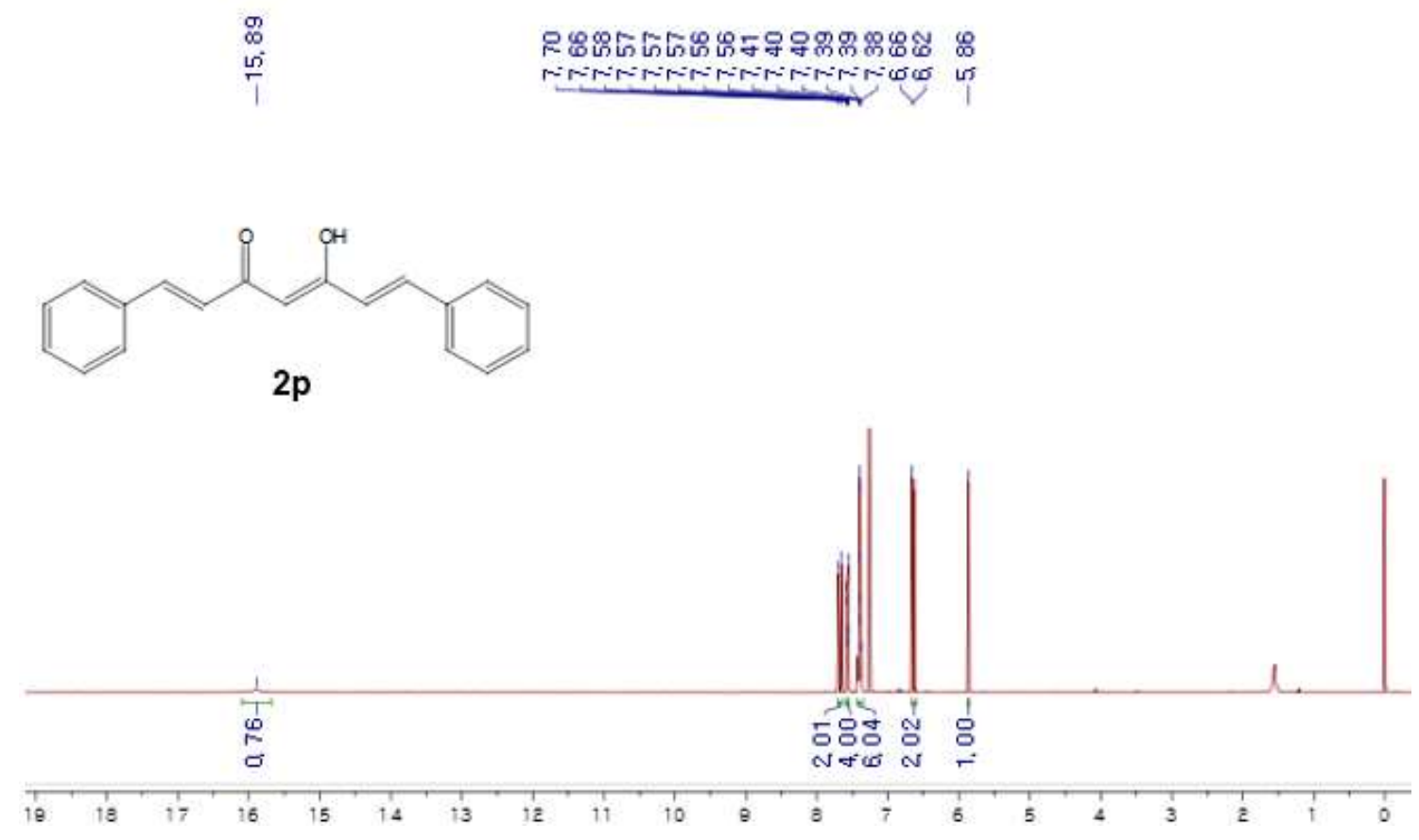

${ }^{1} \mathrm{H}$ NMR (400 MHz, $\mathrm{CDCl}_{3}$ ) spectra of (1E,6E)-1,7-diphenylhepta-1,6-diene-3,5-dione (2p)

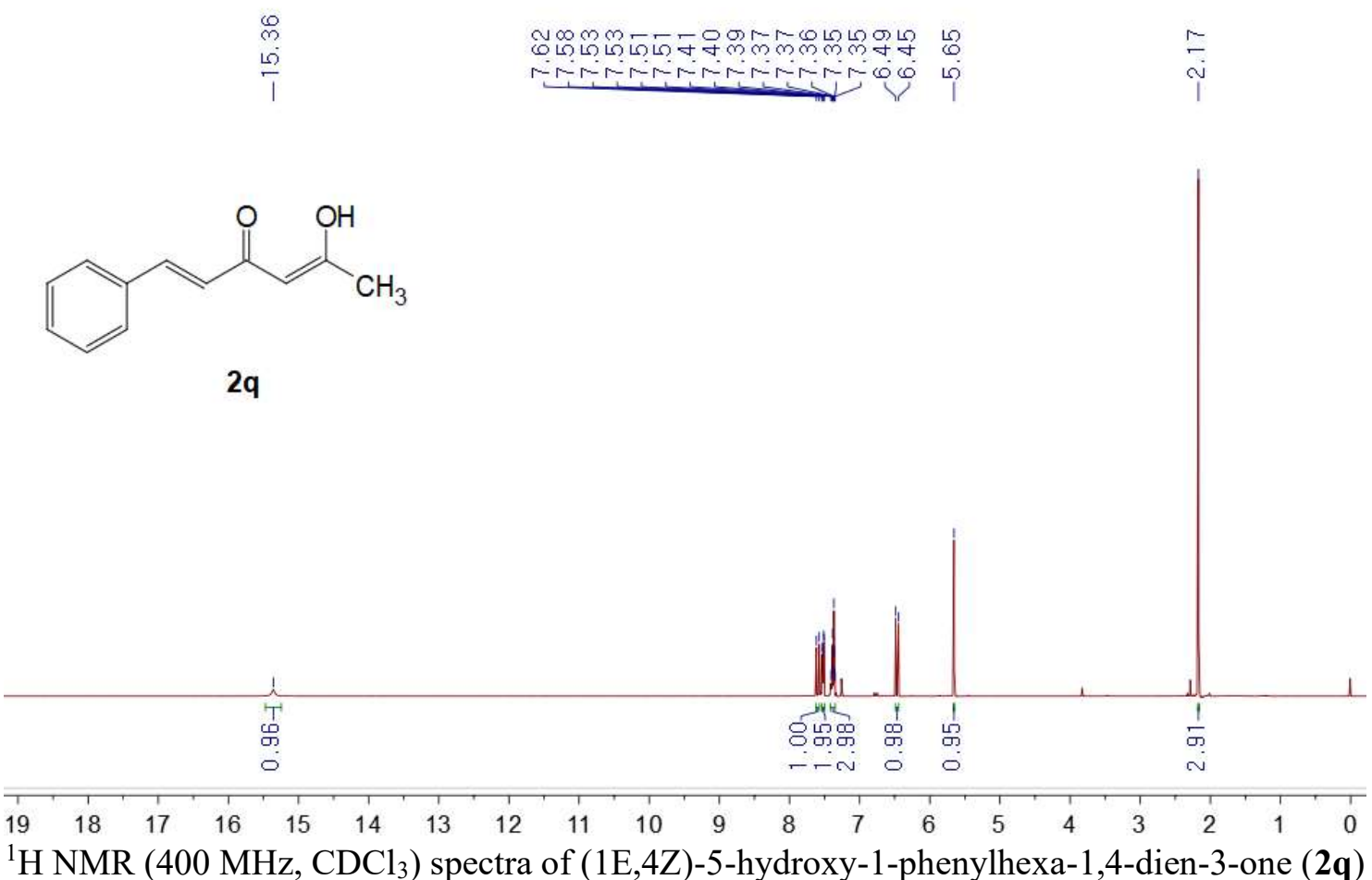



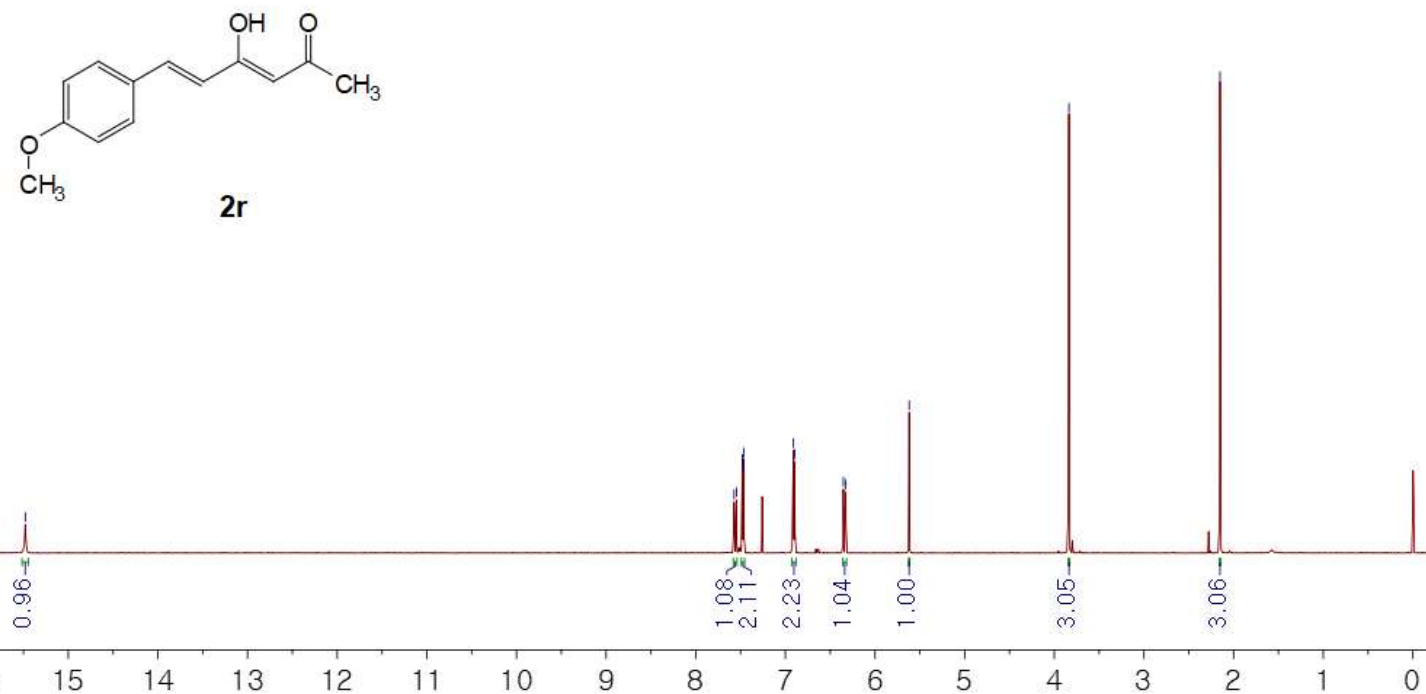

${ }^{1} \mathrm{H}$ NMR (600 MHz, $\mathrm{CDCl}_{3}$ ) spectra of (1E,4Z)-5-hydroxy-1-(4-methoxyphenyl)hexa-1,4dien-3-one (2r)
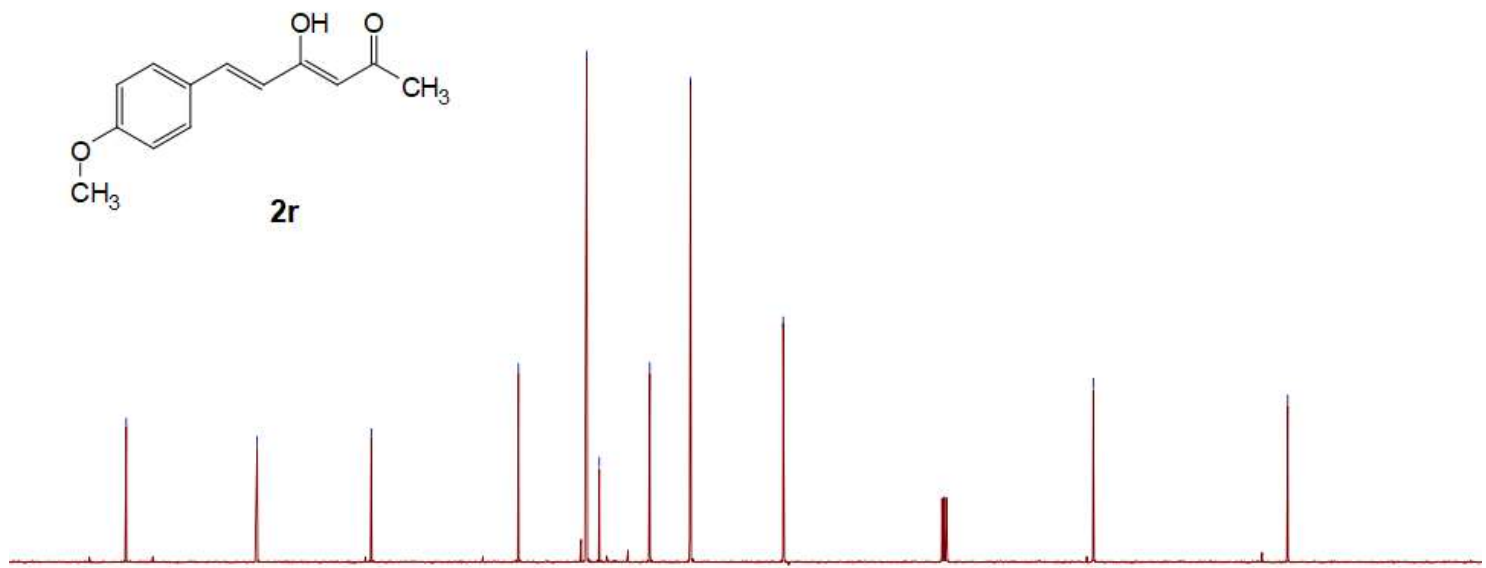

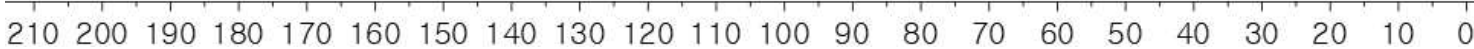

${ }^{13} \mathrm{C}$ NMR (100 MHz, $\left.\mathrm{CDCl}_{3}\right)$ spectra of (1E,4Z)-5-hydroxy-1-(4-methoxyphenyl)hexa-1,4dien-3-one (2r) 


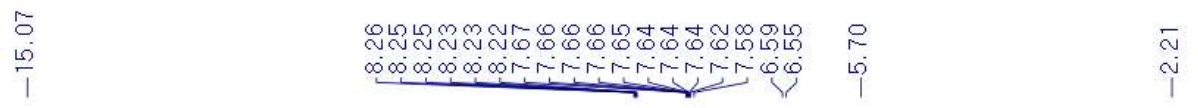<smiles>C/C(O)=C/C(=O)/C=C/c1ccc([N+](=O)[O-])cc1</smiles>

$2 s$

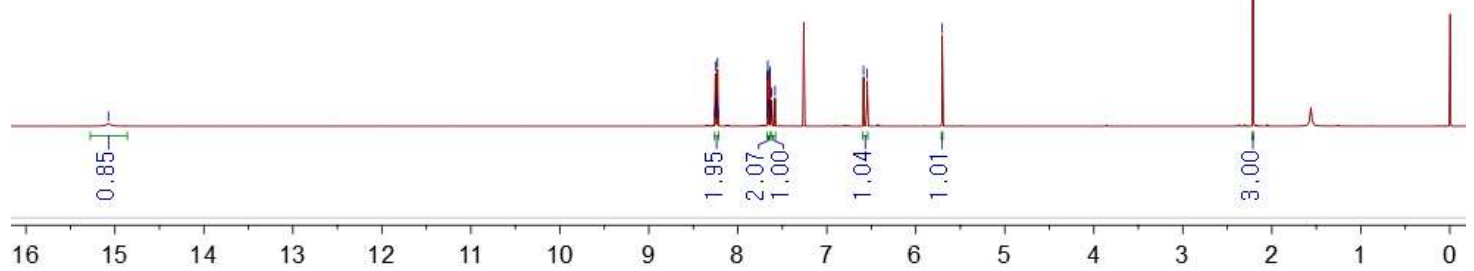

${ }^{1} \mathrm{H}$ NMR (400 MHz, $\mathrm{CDCl}_{3}$ ) spectra of (1E,4Z)-5-hydroxy-1-(4-nitrophenyl)hexa-1,4-dien-3one $(2 \mathrm{~s})$

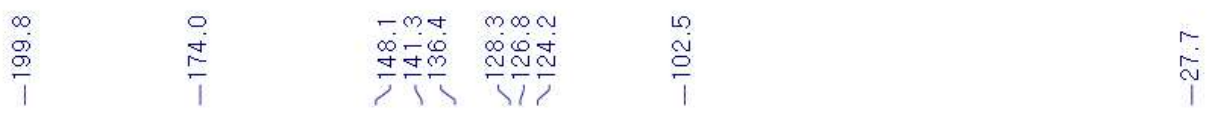<smiles>C/C(O)=C/C(=O)/C=C/c1ccc([N+](=O)[O-])cc1</smiles>

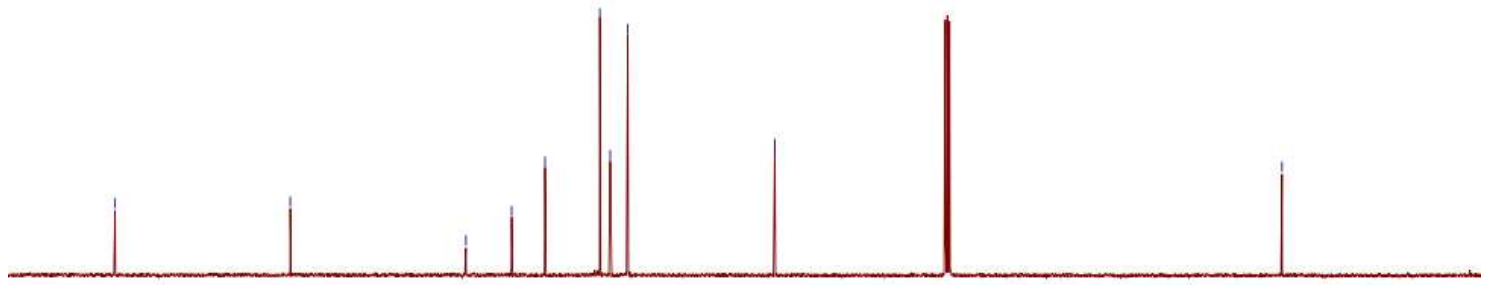

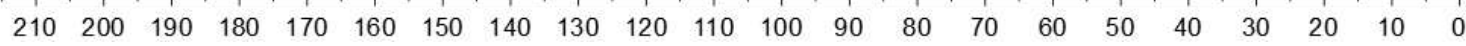
${ }^{13} \mathrm{C} \mathrm{NMR}\left(100 \mathrm{MHz}, \mathrm{CDCl}_{3}\right)$ spectra of (1E,4Z)-5-hydroxy-1-(4-nitrophenyl)hexa-1,4-dien3-one (2s) 
<smiles>[R1]C=C(O)C=Cc1ccc(Cl)cc1</smiles>

$2 t$

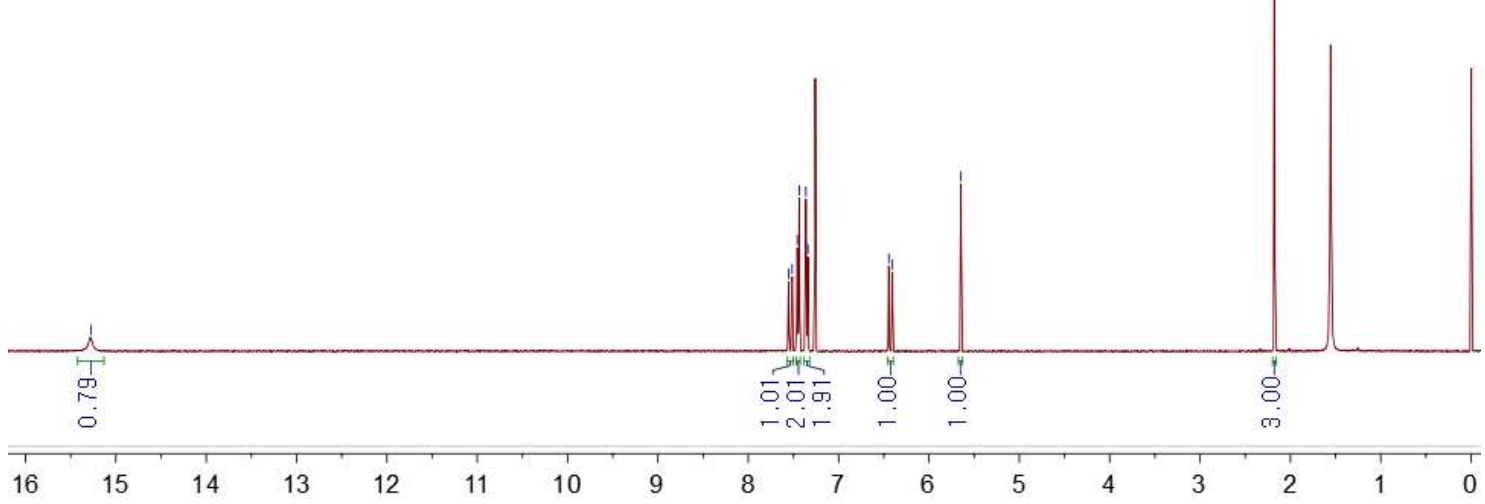

${ }^{1} \mathrm{H}$ NMR (400 MHz, $\mathrm{CDCl}_{3}$ ) spectra of (1E,4Z)-1-(4-chlorophenyl)-5-hydroxyhexa-1,4-dien3 -one $(\mathbf{2 t})$

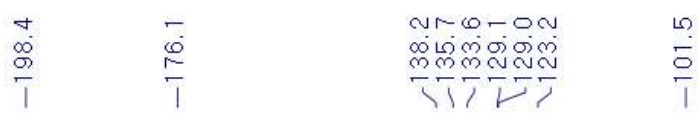
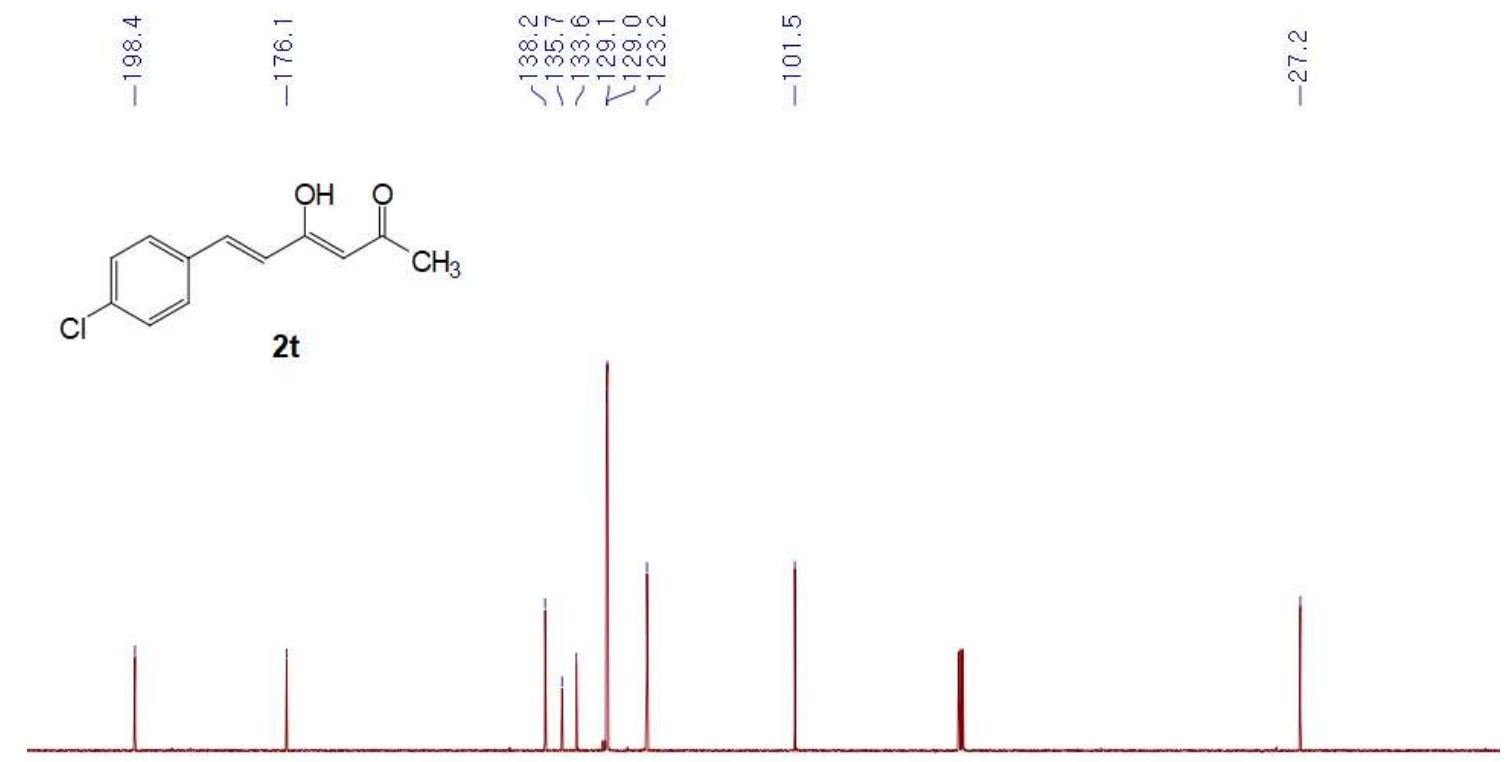

$\begin{array}{llllllllllllllllllllllllllllll}210 & 200 & 190 & 180 & 170 & 160 & 150 & 140 & 130 & 120 & 110 & 100 & 90 & 80 & 70 & 60 & 50 & 40 & 30 & 20 & 10 & 0\end{array}$ ${ }^{13} \mathrm{C}$ NMR (100 MHz, $\left.\mathrm{CDCl}_{3}\right)$ spectra of (1E,4Z)-1-(4-chlorophenyl)-5-hydroxyhexa-1,4-dien3-one (2t) 


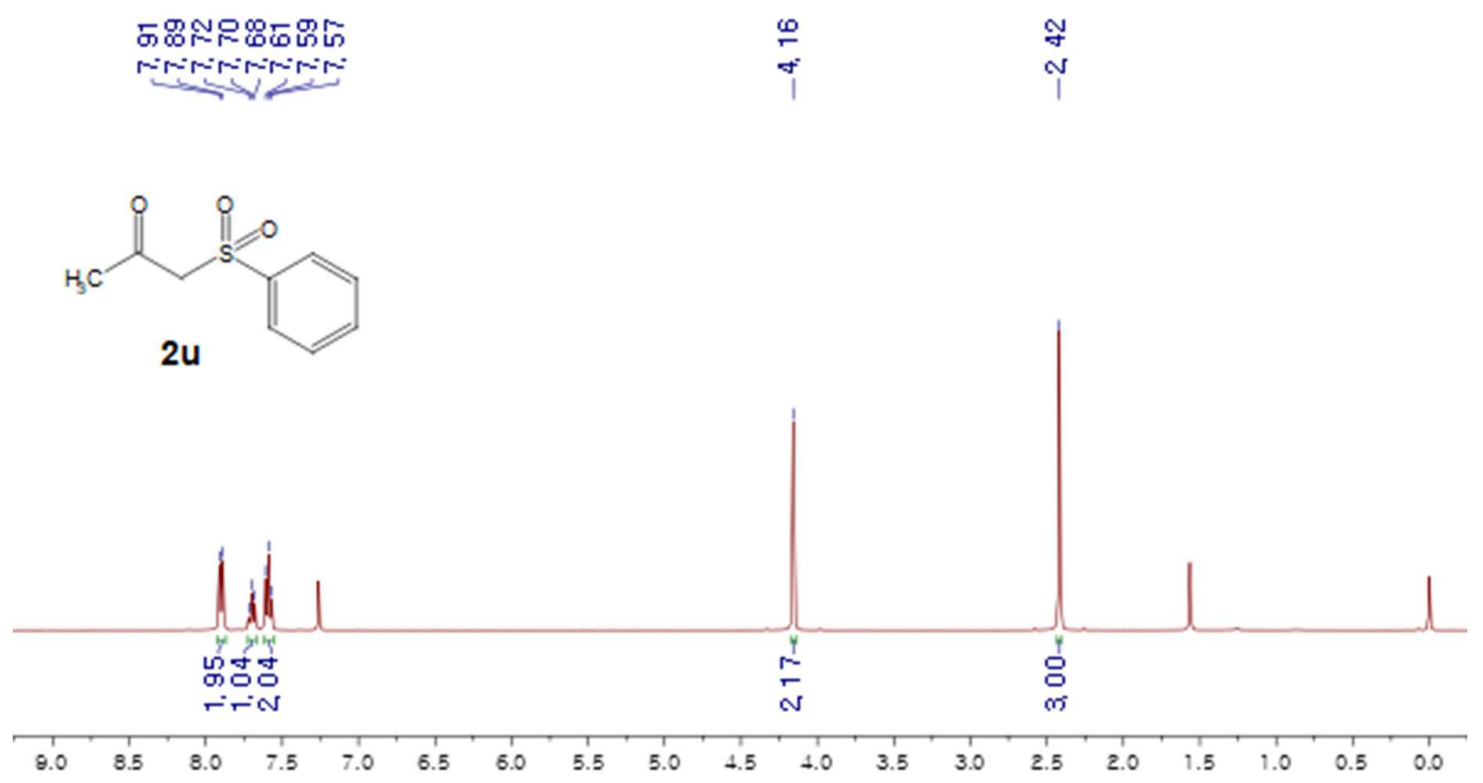

${ }^{1} \mathrm{H}$ NMR (400 MHz, $\mathrm{CDCl}_{3}$ ) spectra of 1-(phenylsulfonyl)propan-2-one (2u)

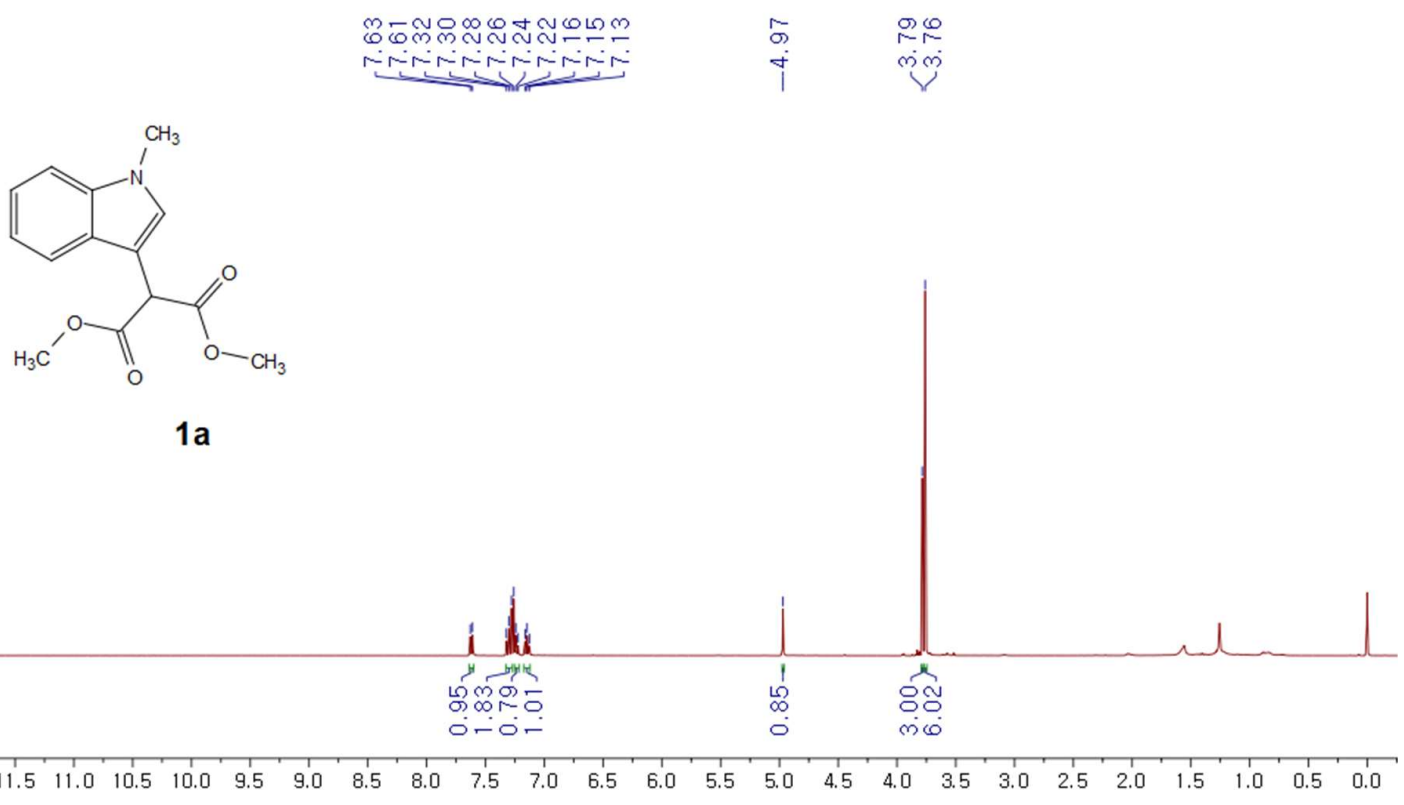

${ }^{1} \mathrm{H} \mathrm{NMR}\left(400 \mathrm{MHz}, \mathrm{CDCl}_{3}\right.$ ) spectra of dimethyl 2-(1-methyl-1H-indol-3-yl)malonate (1a) 


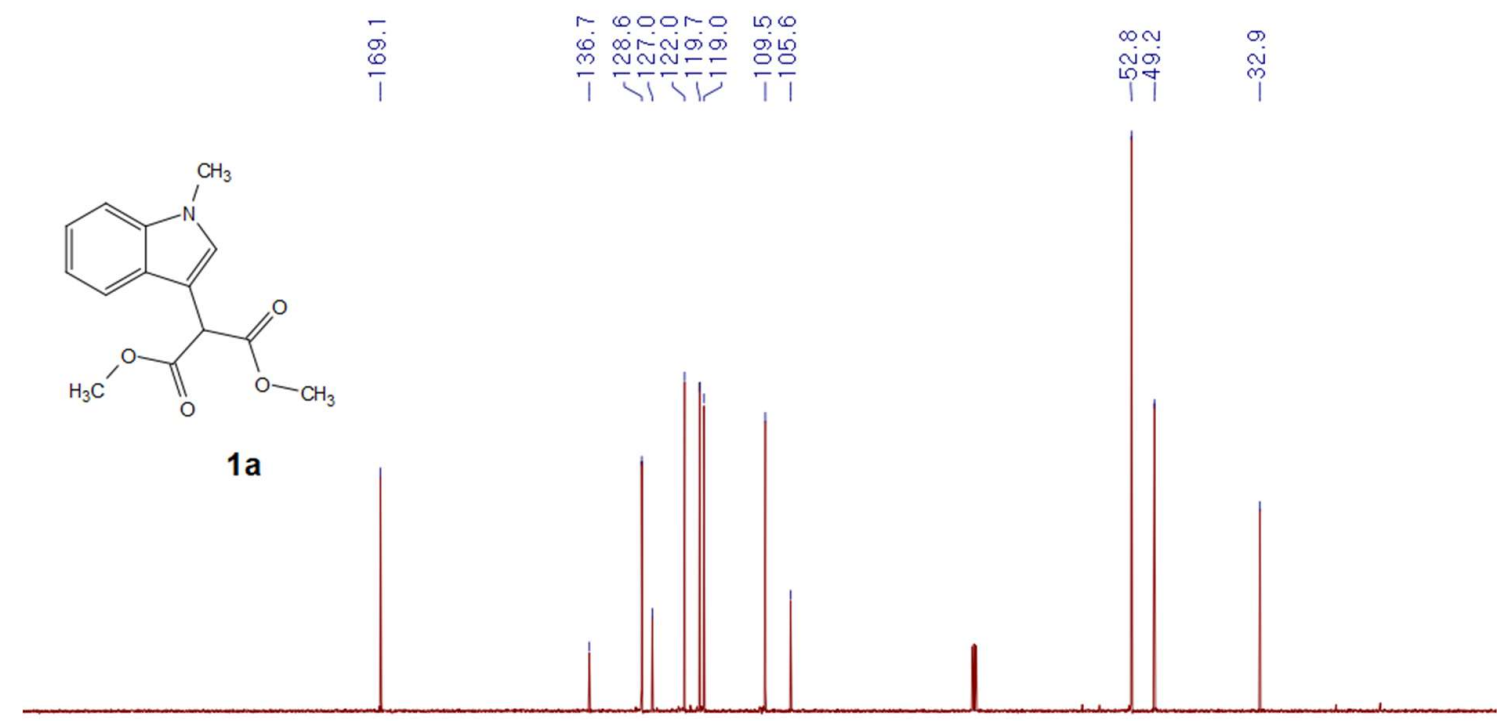

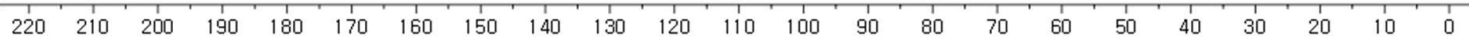

${ }^{13} \mathrm{C}$ NMR (100 MHz, $\mathrm{CDCl}_{3}$ ) spectra of dimethyl 2-(1-methyl-1H-indol-3-yl)malonate (1a)
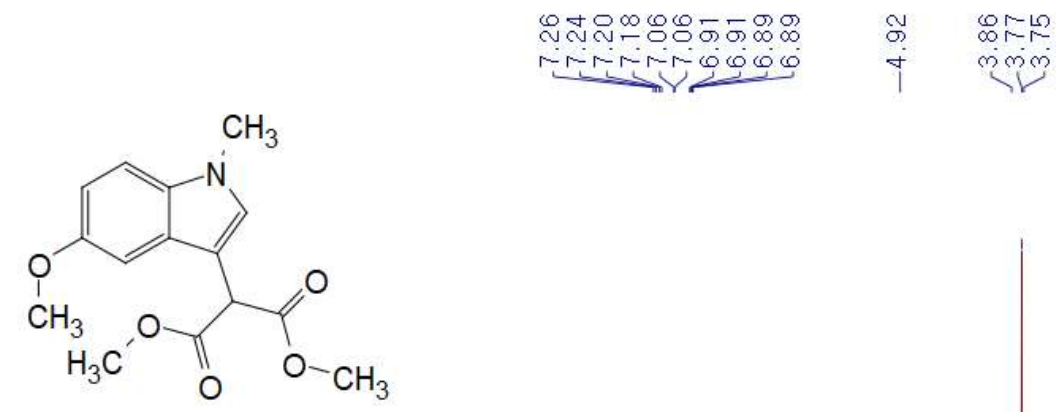

1b

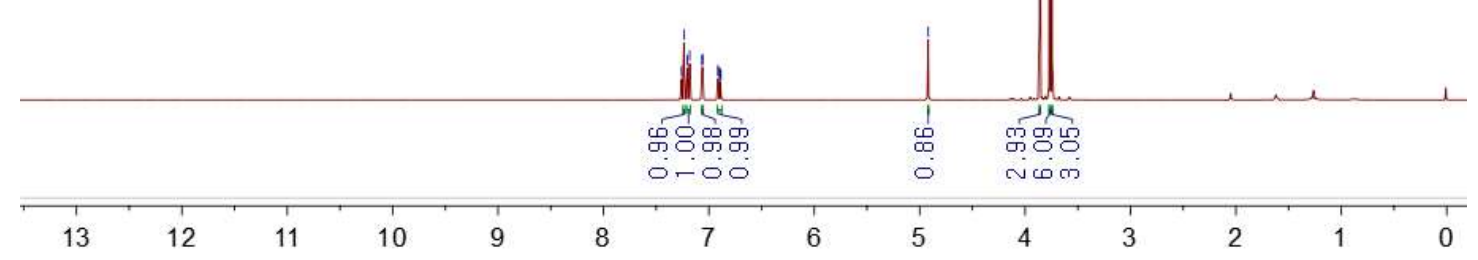

${ }^{1} \mathrm{H}$ NMR (400 MHz, $\mathrm{CDCl}_{3}$ ) spectra of Dimethyl 2-(5-methoxy-1-methyl-1H-indol-3-

yl)malonate (1b) 


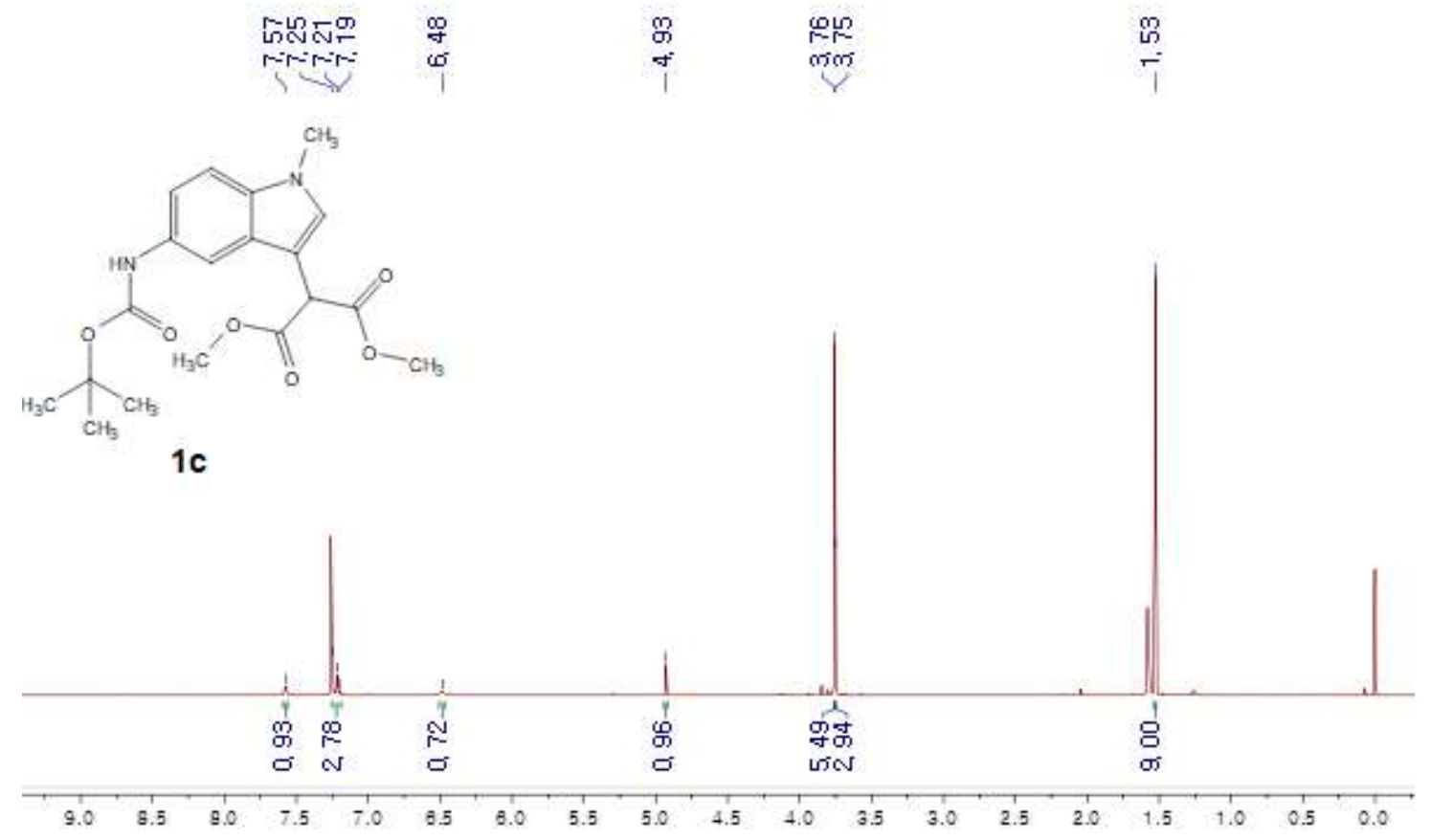

${ }^{1} \mathrm{H}$ NMR (400 MHz, $\left.\mathrm{CDCl}_{3}\right)$ spectra of dimethyl 2-(5-((tert-butoxycarbonyl)amino)-1methyl-1H-indol-3-yl)malonate (1c)
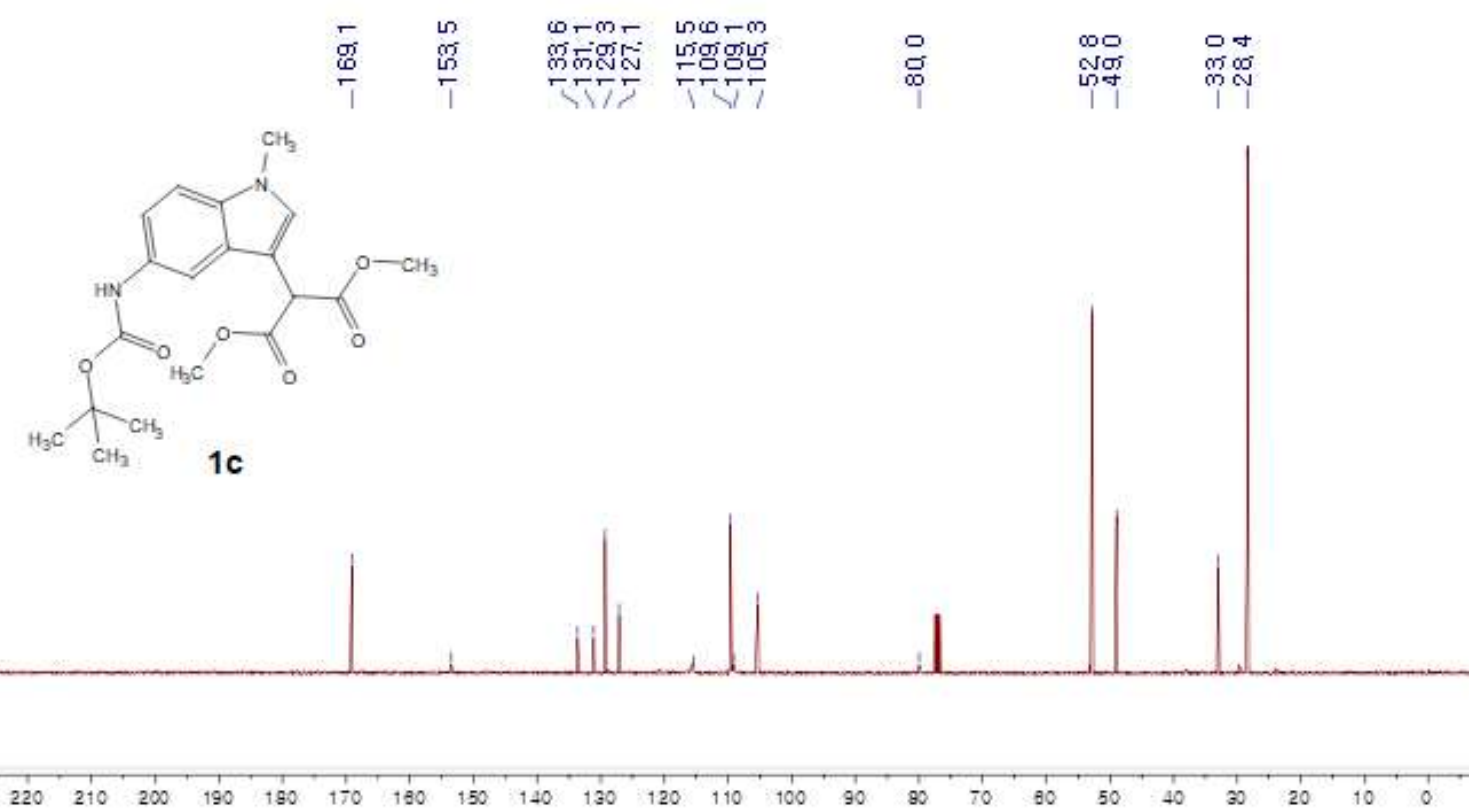

${ }^{13} \mathrm{C}$ NMR (100 MHz, $\mathrm{CDCl}_{3}$ ) spectra of dimethyl 2-(5-((tert-butoxycarbonyl)amino)-1methyl-1H-indol-3-yl)malonate (1c) 


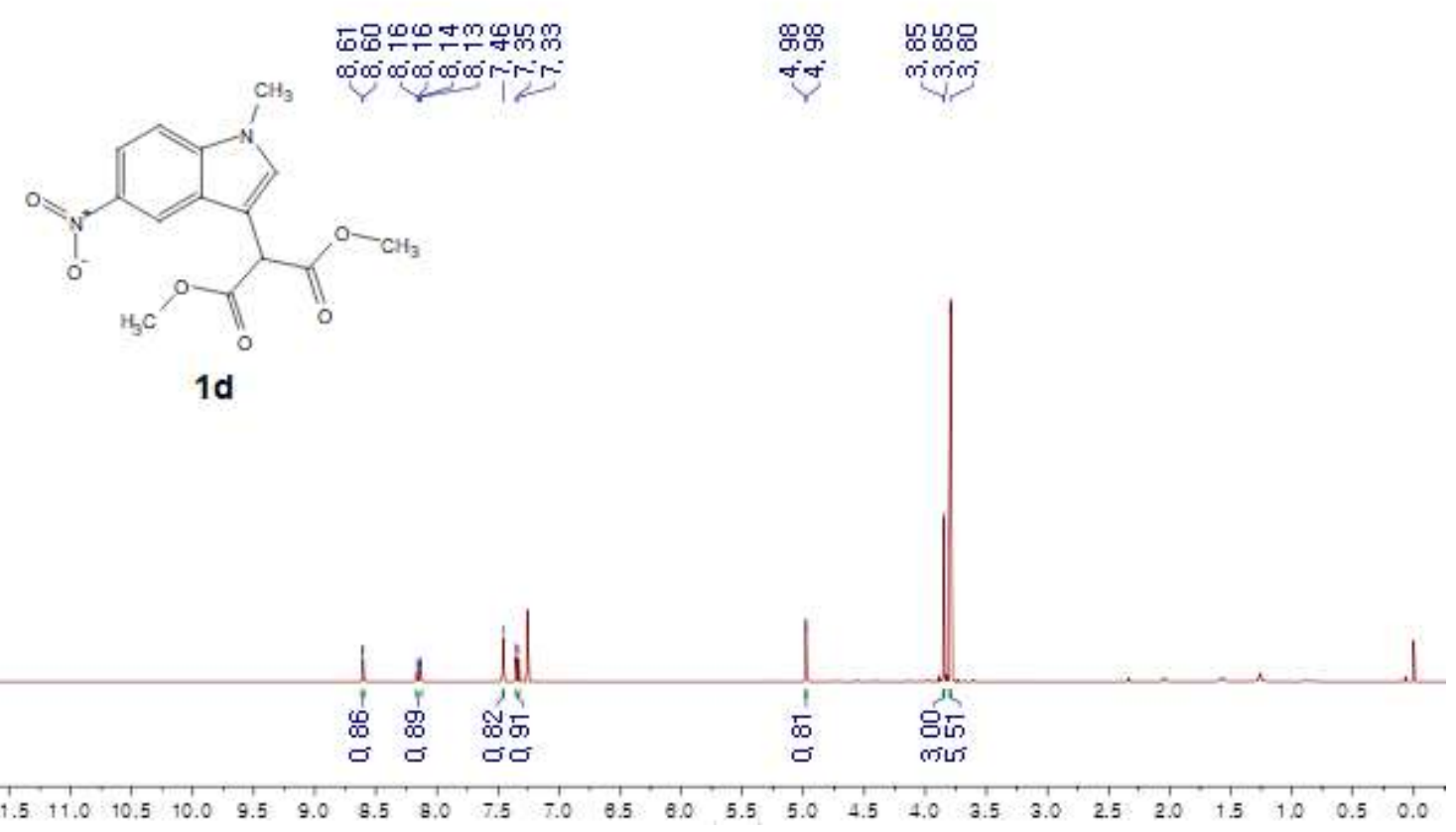

${ }^{1} \mathrm{H}$ NMR $\left(400 \mathrm{MHz}, \mathrm{CDCl}_{3}\right.$ ) spectra of dimethyl 2-(1-methyl-5-nitro-1H-indol-3-yl)malonate

(1d)

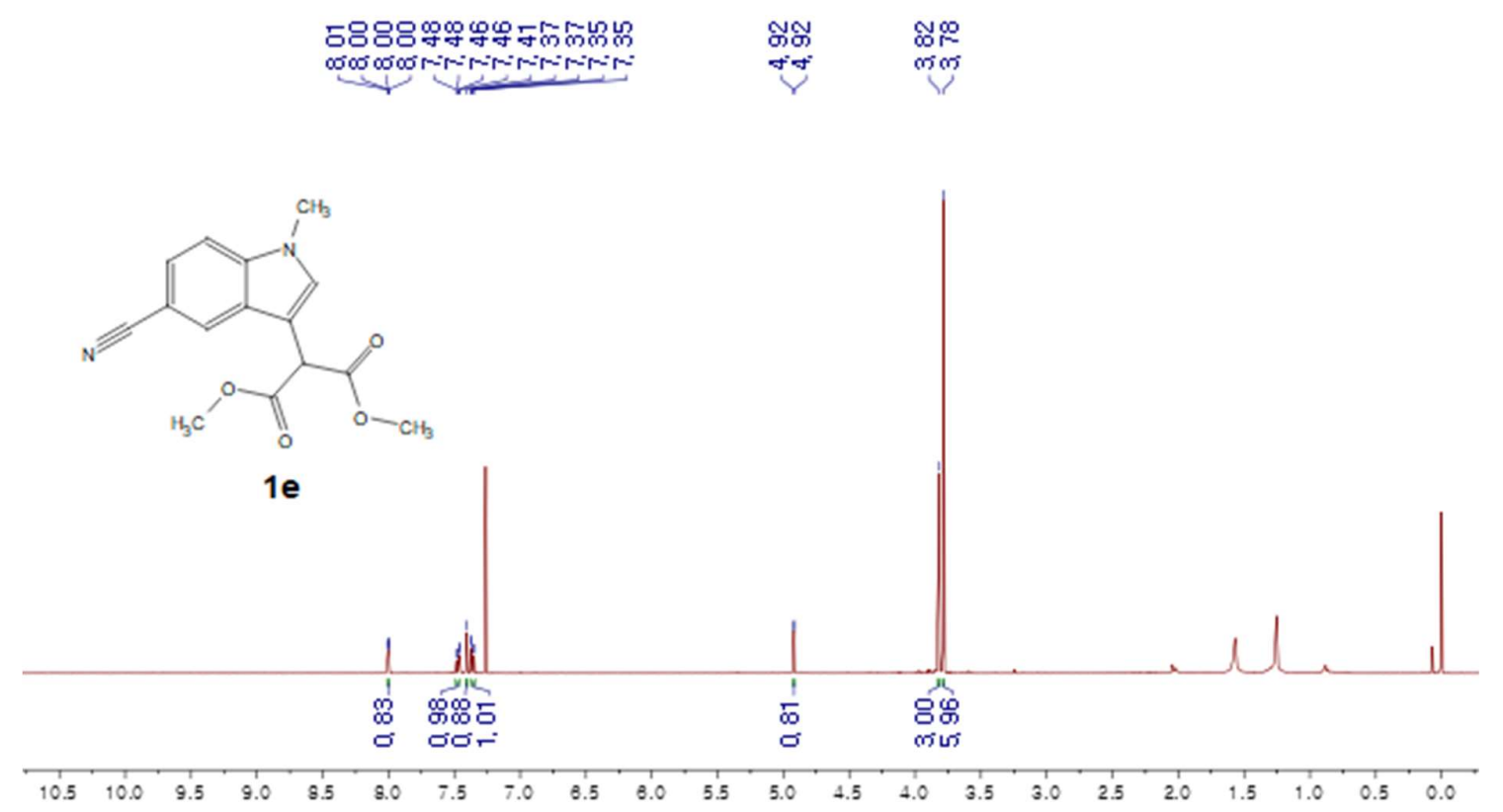

${ }^{1} \mathrm{H}$ NMR (400 MHz, $\mathrm{CDCl}_{3}$ ) spectra of dimethyl 2-(5-cyano-1-methyl-1H-indol-3yl)malonate (1e) 

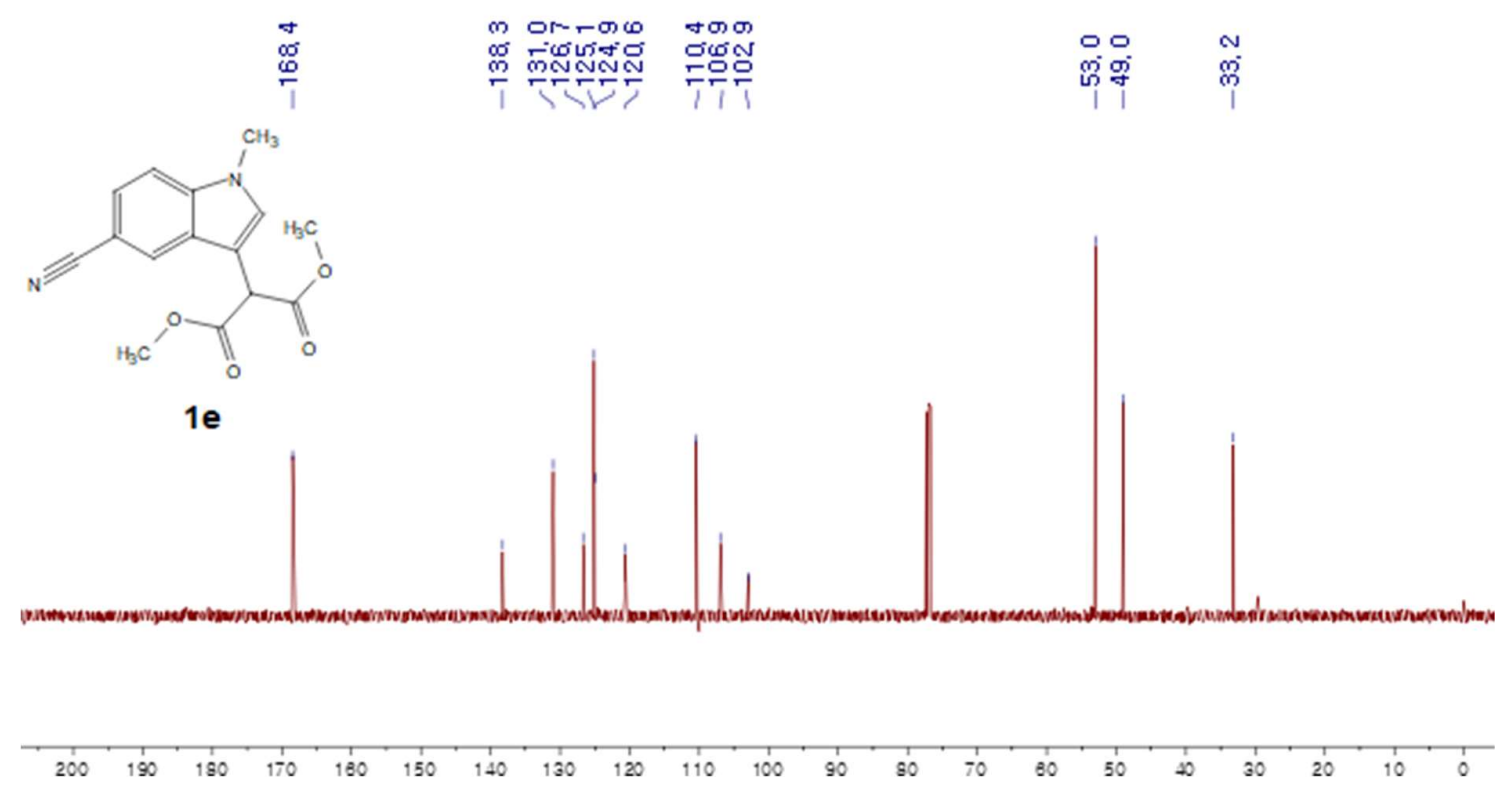

${ }^{13} \mathrm{C}$ NMR $\left(100 \mathrm{MHz}, \mathrm{CDCl}_{3}\right)$ spectra of dimethyl 2-(5-cyano-1-methyl-1H-indol-3yl)malonate (1e)

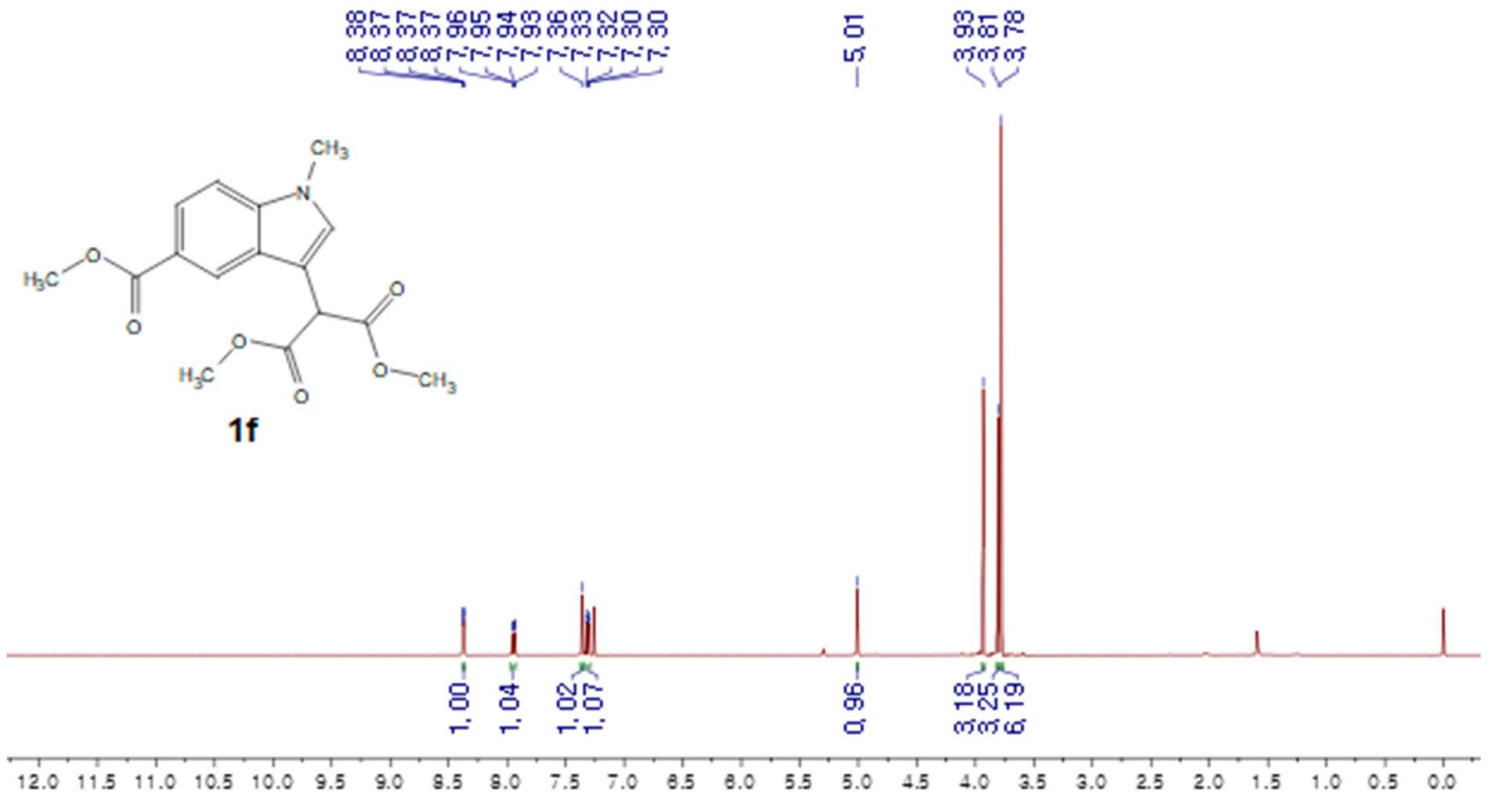

${ }^{1} \mathrm{H}$ NMR (400 MHz, $\mathrm{CDCl}_{3}$ ) spectra of dimethyl 2-(5-(methoxycarbonyl)-1-methyl-1Hindol-3-yl)malonate (1f) 

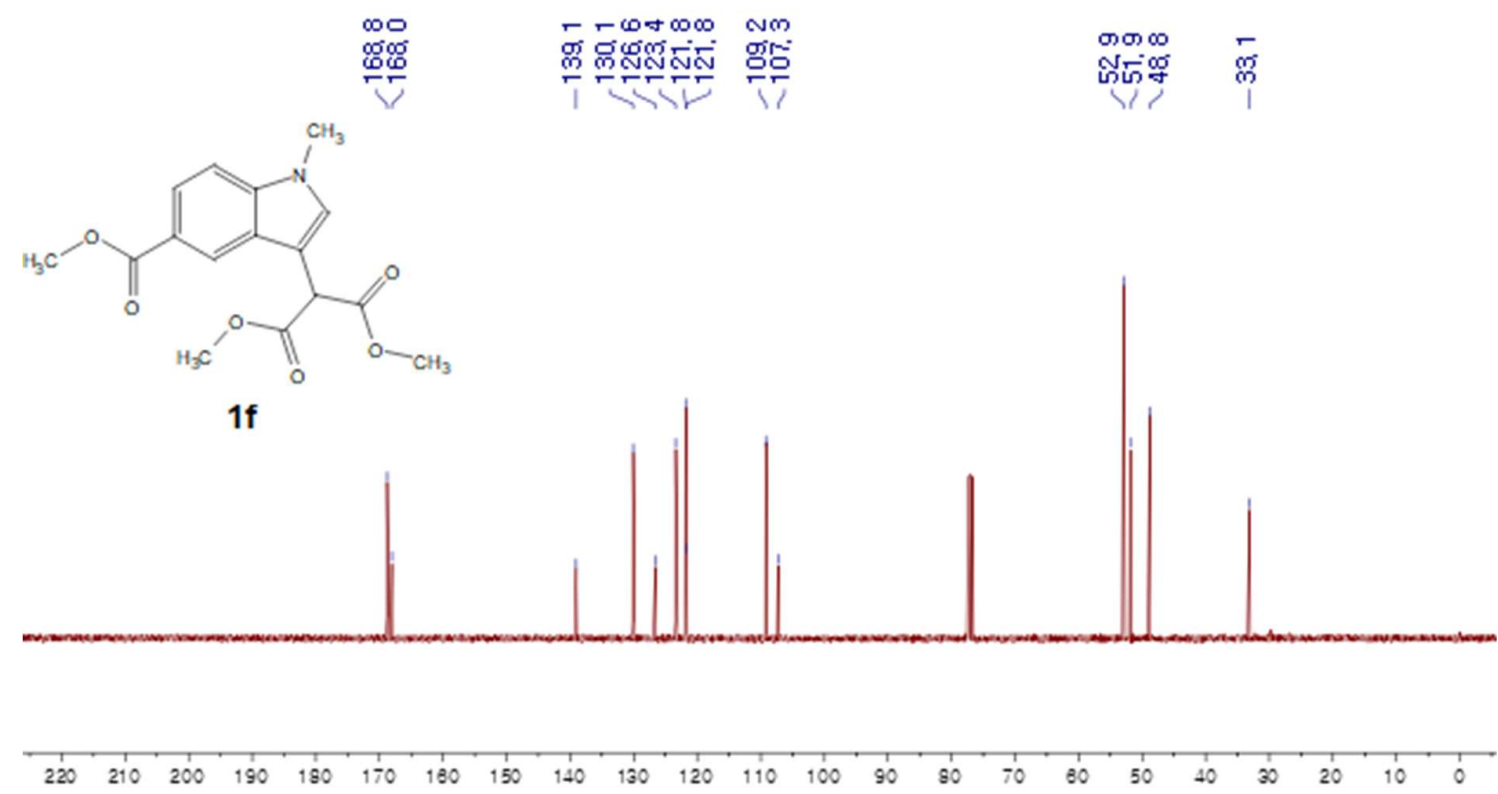

${ }^{13} \mathrm{C}$ NMR $\left(100 \mathrm{MHz}, \mathrm{CDCl}_{3}\right)$ spectra of dimethyl 2-(5-(methoxycarbonyl)-1-methyl-1Hindol-3-yl)malonate (1f)
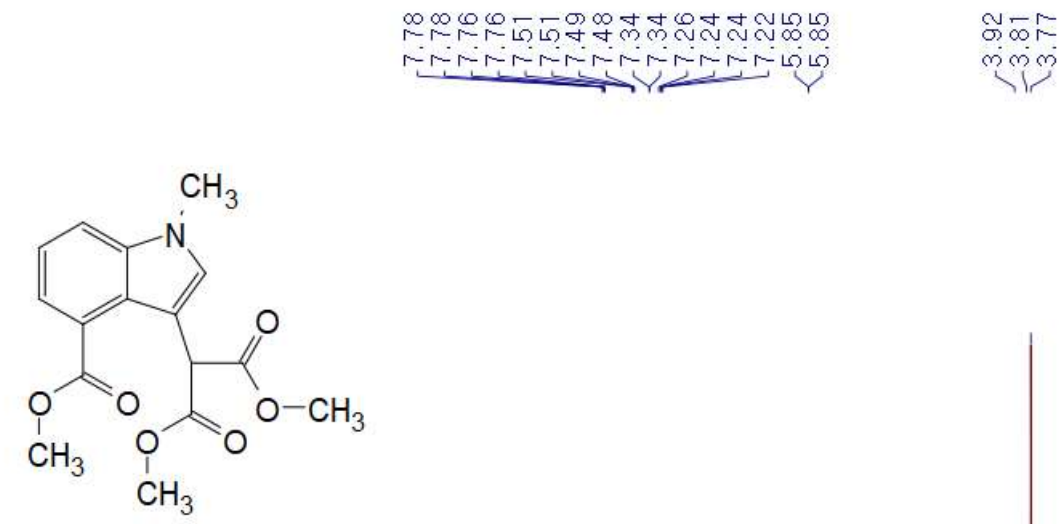

$1 \mathrm{~g}$

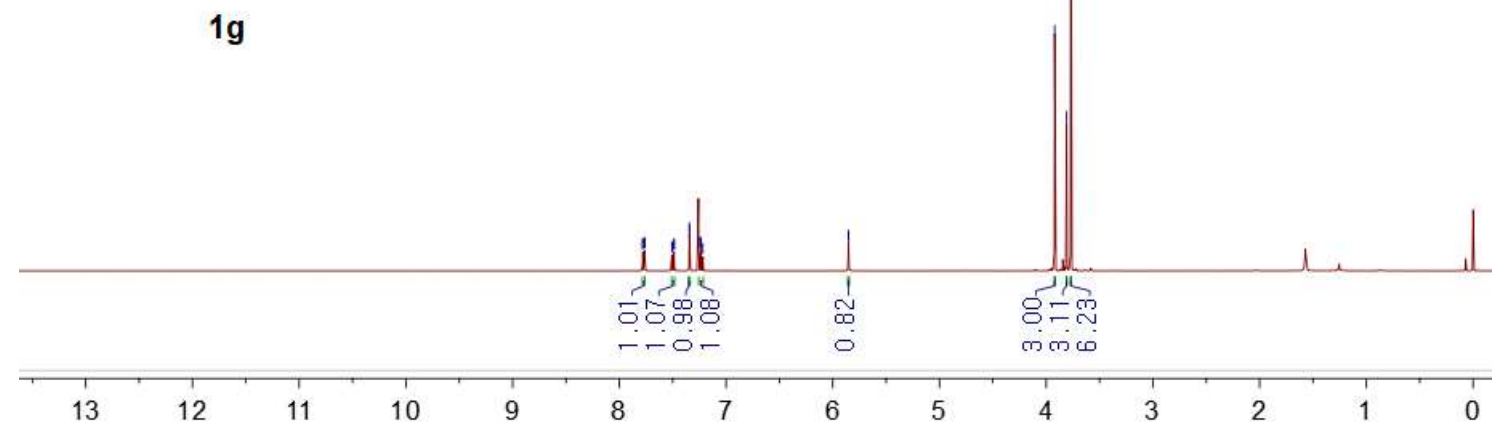

${ }^{1} \mathrm{H}$ NMR (400 MHz, $\mathrm{CDCl}_{3}$ ) spectra of Dimethyl 2-(4-(methoxycarbonyl)-1-methyl-1Hindol-3-yl)malonate (1g) 


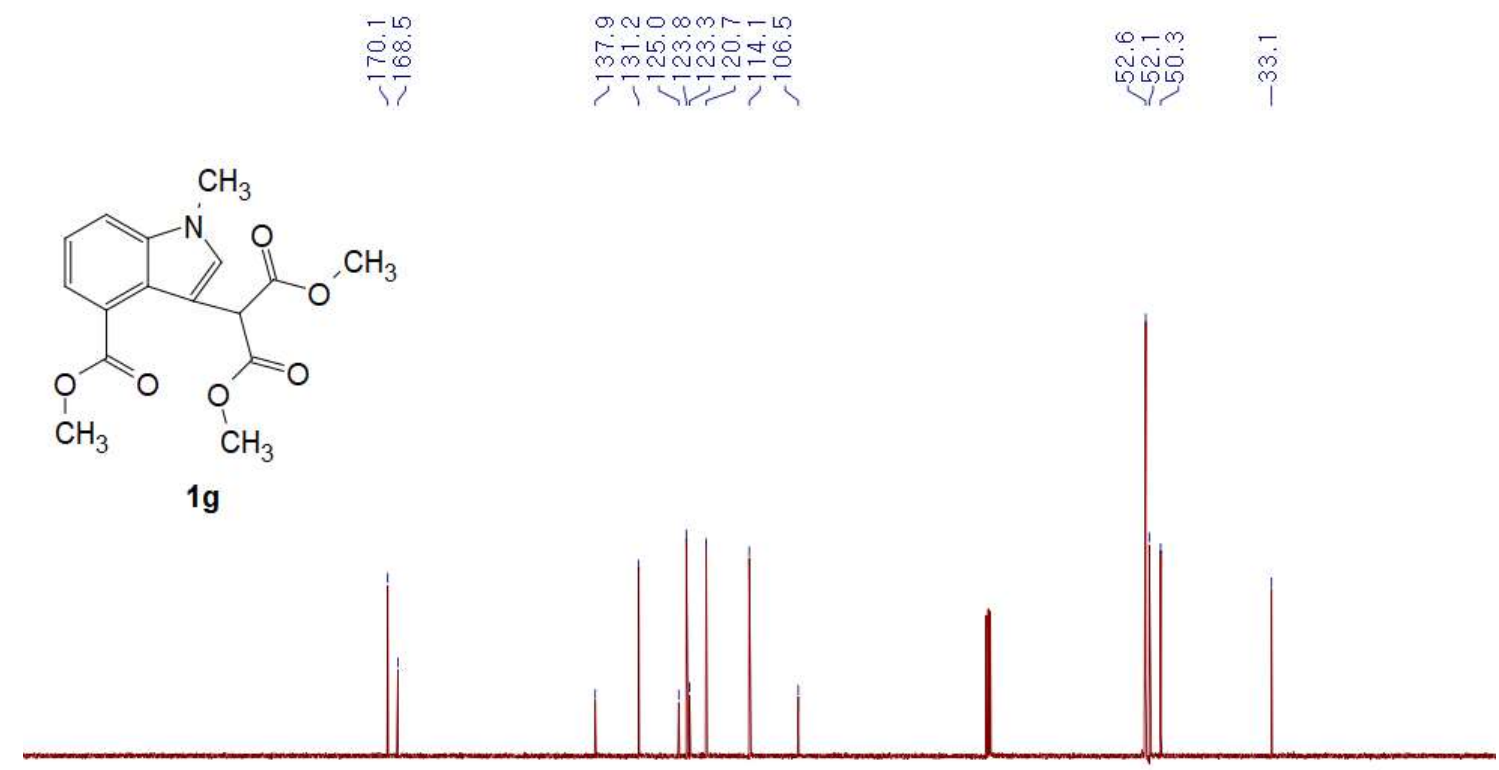

$\begin{array}{lllllllllllllllllllllll}220 & 210 & 200 & 190 & 180 & 170 & 160 & 150 & 140 & 130 & 120 & 110 & 100 & 90 & 80 & 70 & 60 & 50 & 40 & 30 & 20 & 10 & 0\end{array}$

${ }^{13} \mathrm{C}$ NMR (100 MHz, $\mathrm{CDCl}_{3}$ ) spectra of Dimethyl 2-(4-(methoxycarbonyl)-1-methyl-1Hindol-3-yl)malonate (1g)

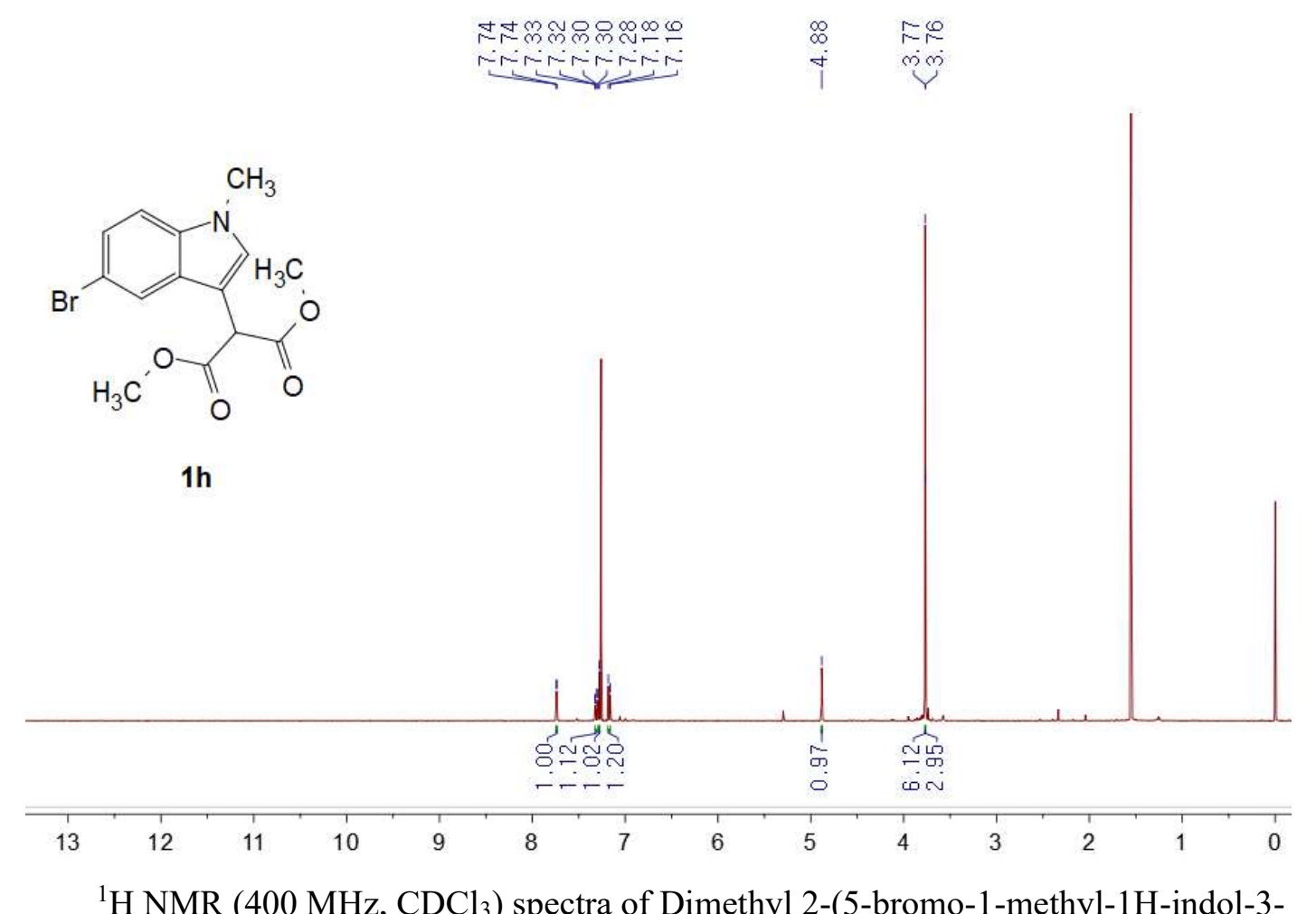

yl)malonate (1h) 


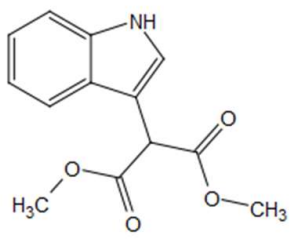

$1 \mathbf{i}$
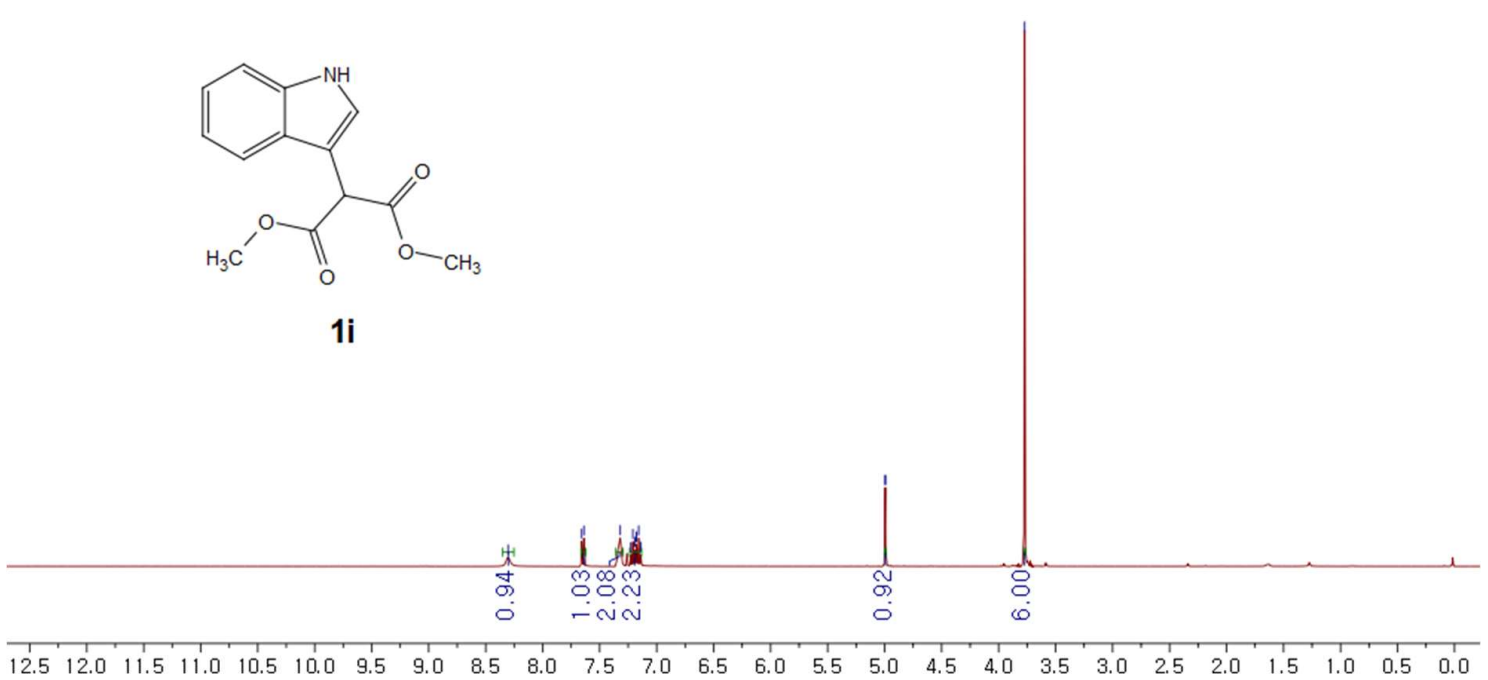

${ }^{1} \mathrm{H}$ NMR (400 MHz, $\left.\mathrm{CDCl}_{3}\right)$ spectra of dimethyl 2-(1H-indol-3-yl)malonate (1i)

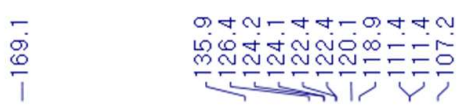

çm

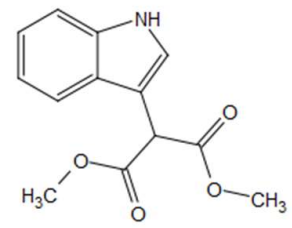

$1 \mathbf{i}$

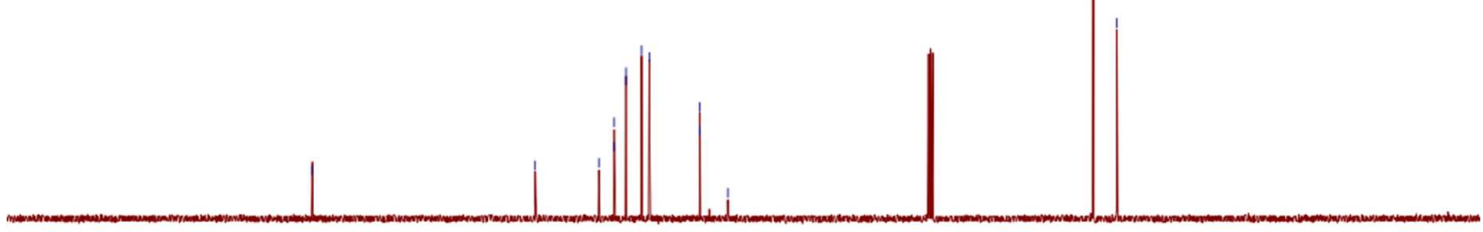

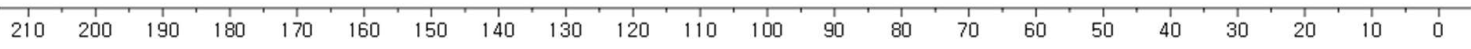

${ }^{13} \mathrm{C} \mathrm{NMR}\left(100 \mathrm{MHz}, \mathrm{CDCl}_{3}\right)$ spectra of dimethyl 2-(1H-indol-3-yl)malonate (1i) 


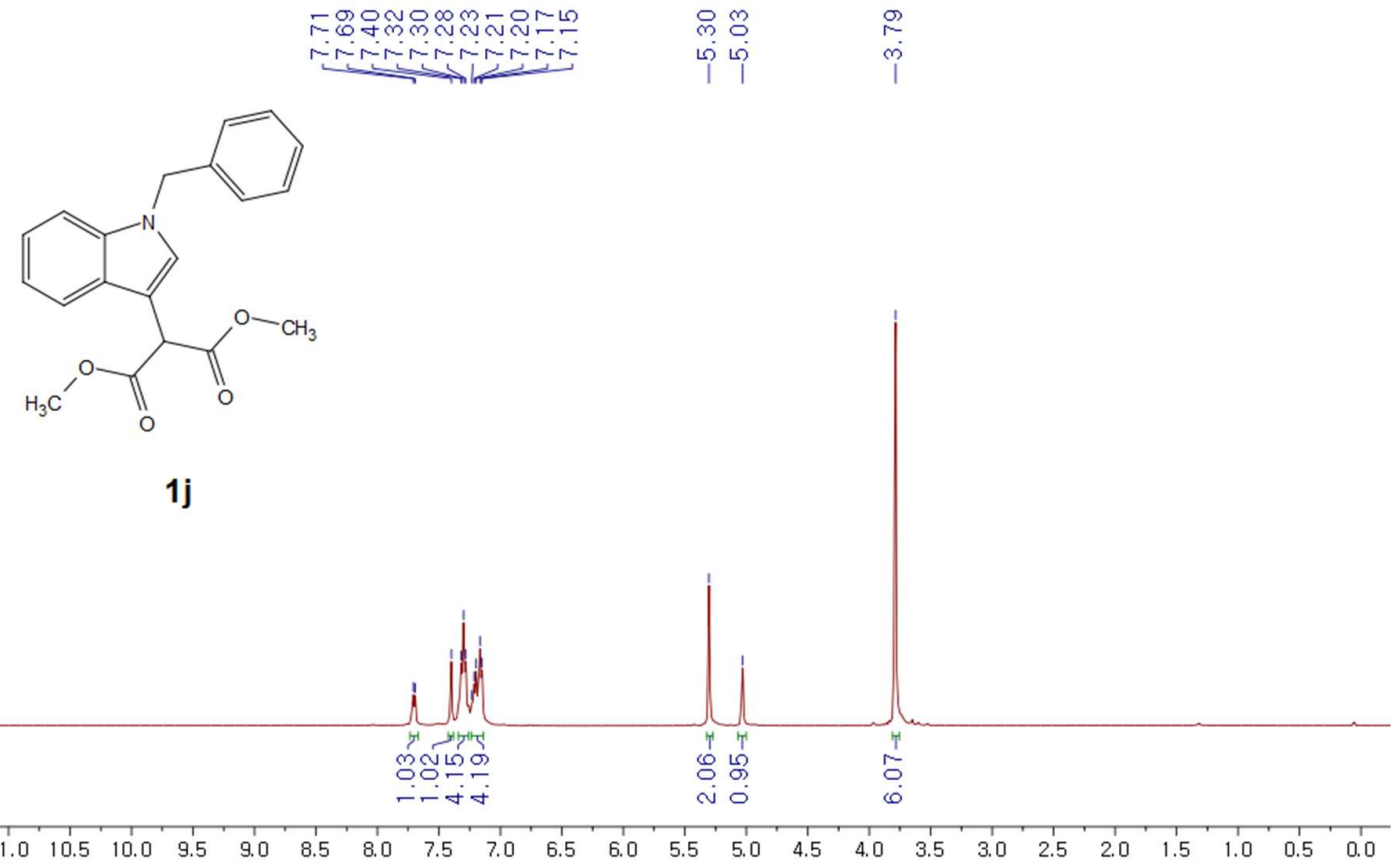

${ }^{1} \mathrm{H}$ NMR (400 MHz, $\mathrm{CDCl}_{3}$ ) spectra of dimethyl 2-(1-benzyl-1H-indol-3-yl)malonate (1j)

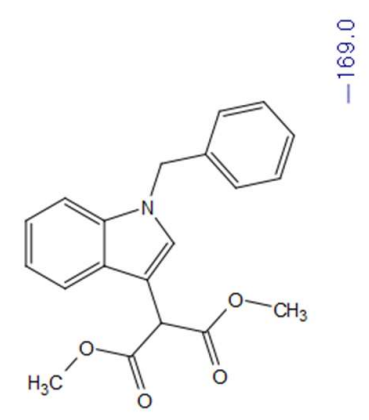

1j

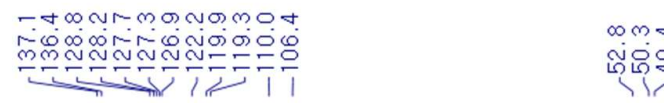

กิ่ง

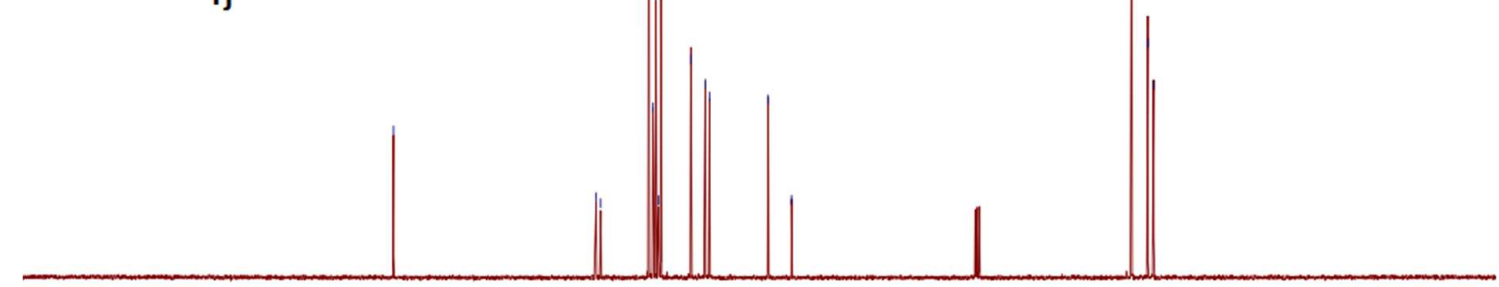

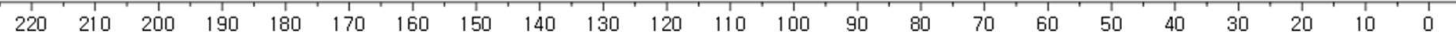

${ }^{13} \mathrm{C}$ NMR (100 MHz, $\left.\mathrm{CDCl}_{3}\right)$ spectra of dimethyl 2-(1-benzyl-1H-indol-3-yl)malonate (1j) 


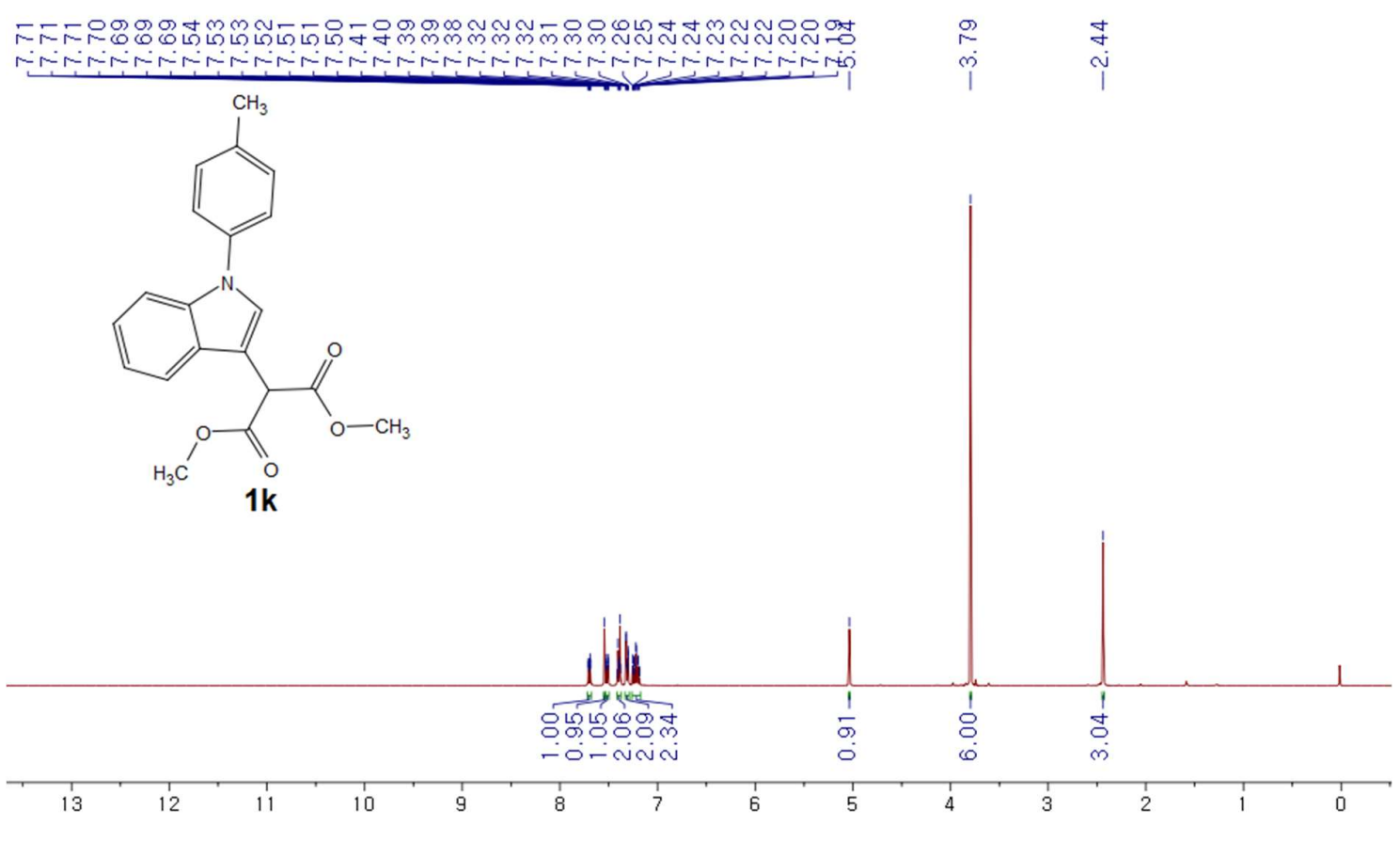

${ }^{1} \mathrm{H}$ NMR (400 MHz, $\mathrm{CDCl}_{3}$ ) spectra of dimethyl 2-(1-(p-tolyl)-1H-indol-3-yl)malonate (1k)

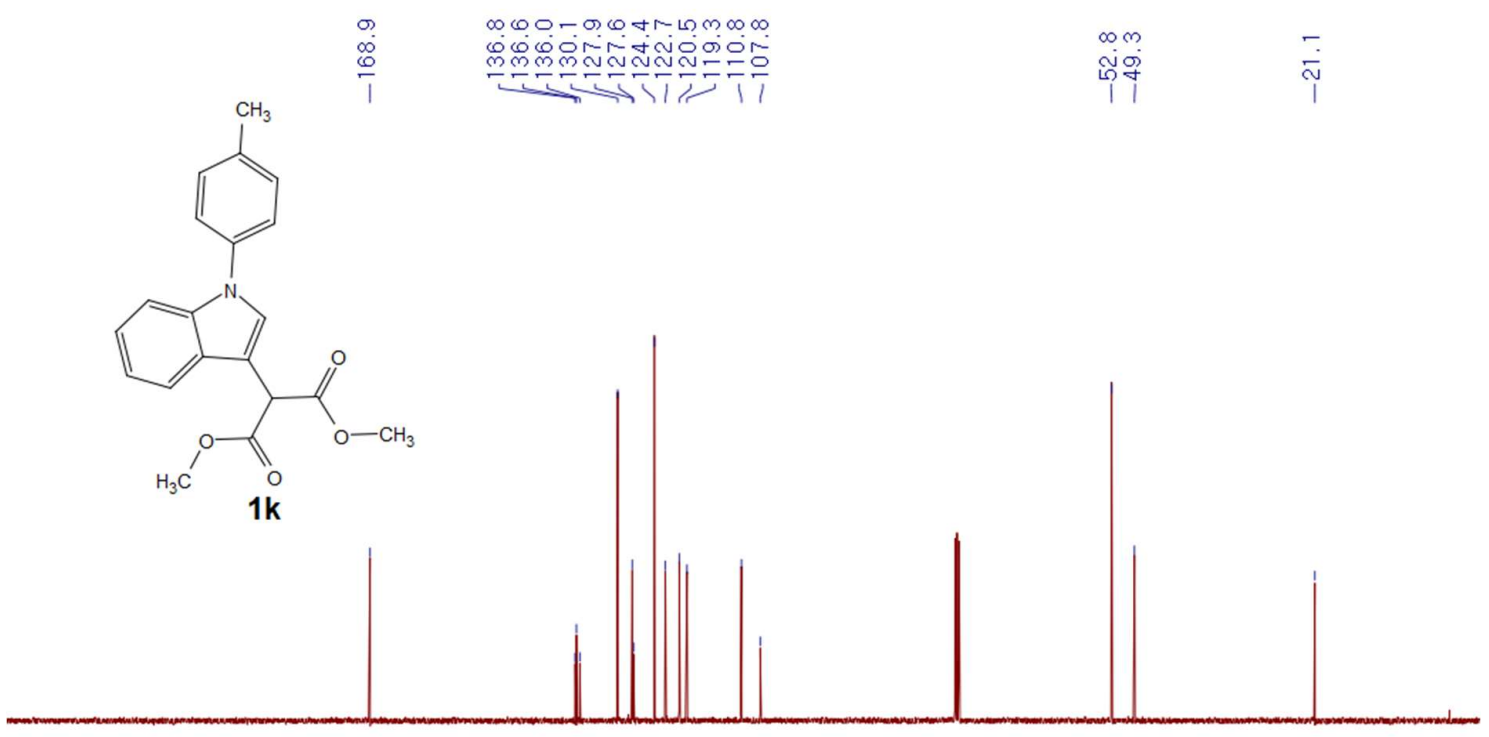

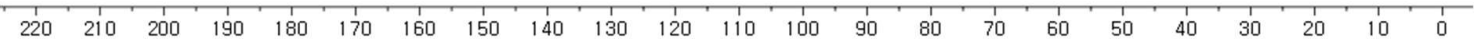

${ }^{13} \mathrm{C} \mathrm{NMR}\left(100 \mathrm{MHz}, \mathrm{CDCl}_{3}\right)$ spectra of dimethyl 2-(1-(p-tolyl)-1H-indol-3-yl)malonate (1k) 

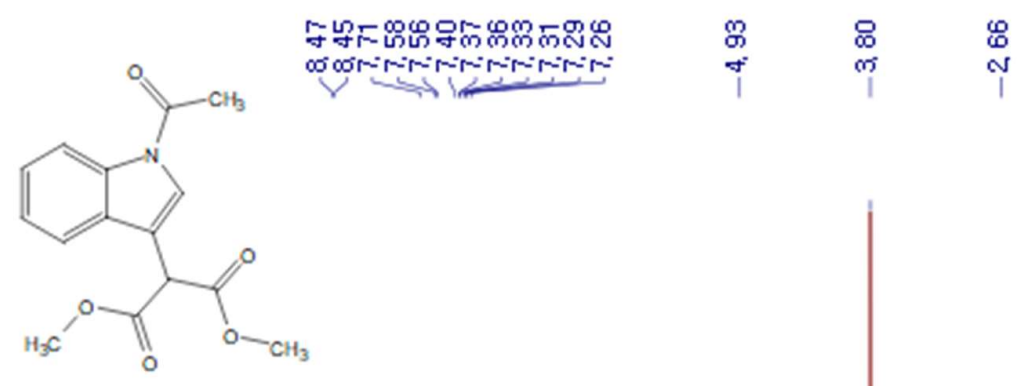

11

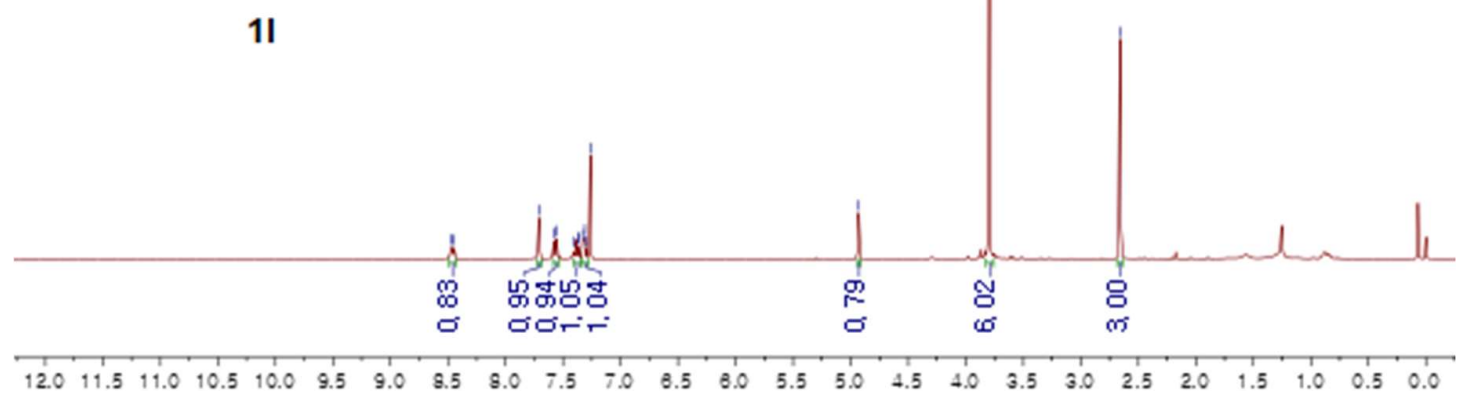

${ }^{1} \mathrm{H}$ NMR (400 MHz, $\mathrm{CDCl}_{3}$ ) spectra of dimethyl 2-(1-acetyl-1H-indol-3-yl)malonate (11)

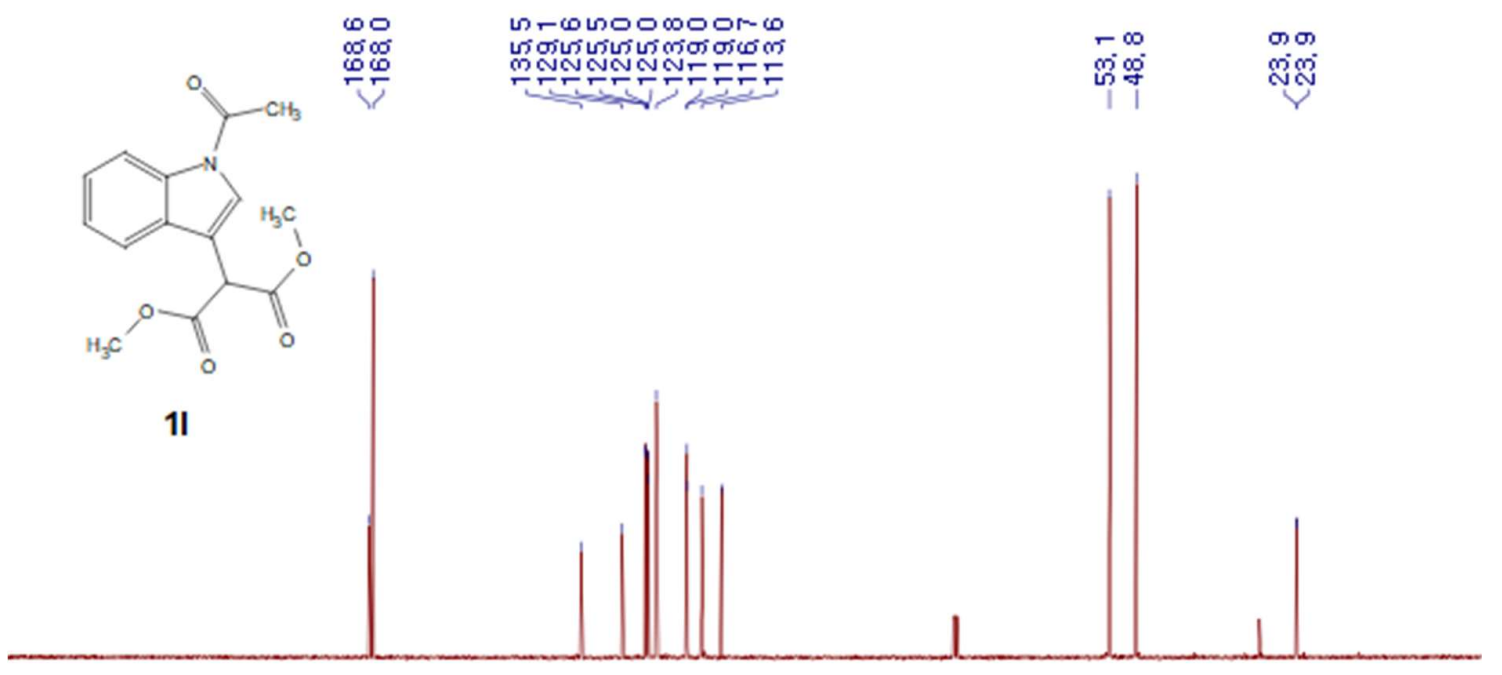

$\begin{array}{llllllllllllllllllllllllllllllllllllll}220 & 210 & 200 & 190 & 180 & 170 & 180 & 150 & 140 & 130 & 120 & 110 & 100 & 90 & 80 & 70 & 80 & 50 & 40 & 30 & 20 & 10 & 0\end{array}$

${ }^{13} \mathrm{C} \mathrm{NMR}\left(100 \mathrm{MHz}, \mathrm{CDCl}_{3}\right)$ spectra of dimethyl 2-(1-acetyl-1H-indol-3-yl)malonate (11) 


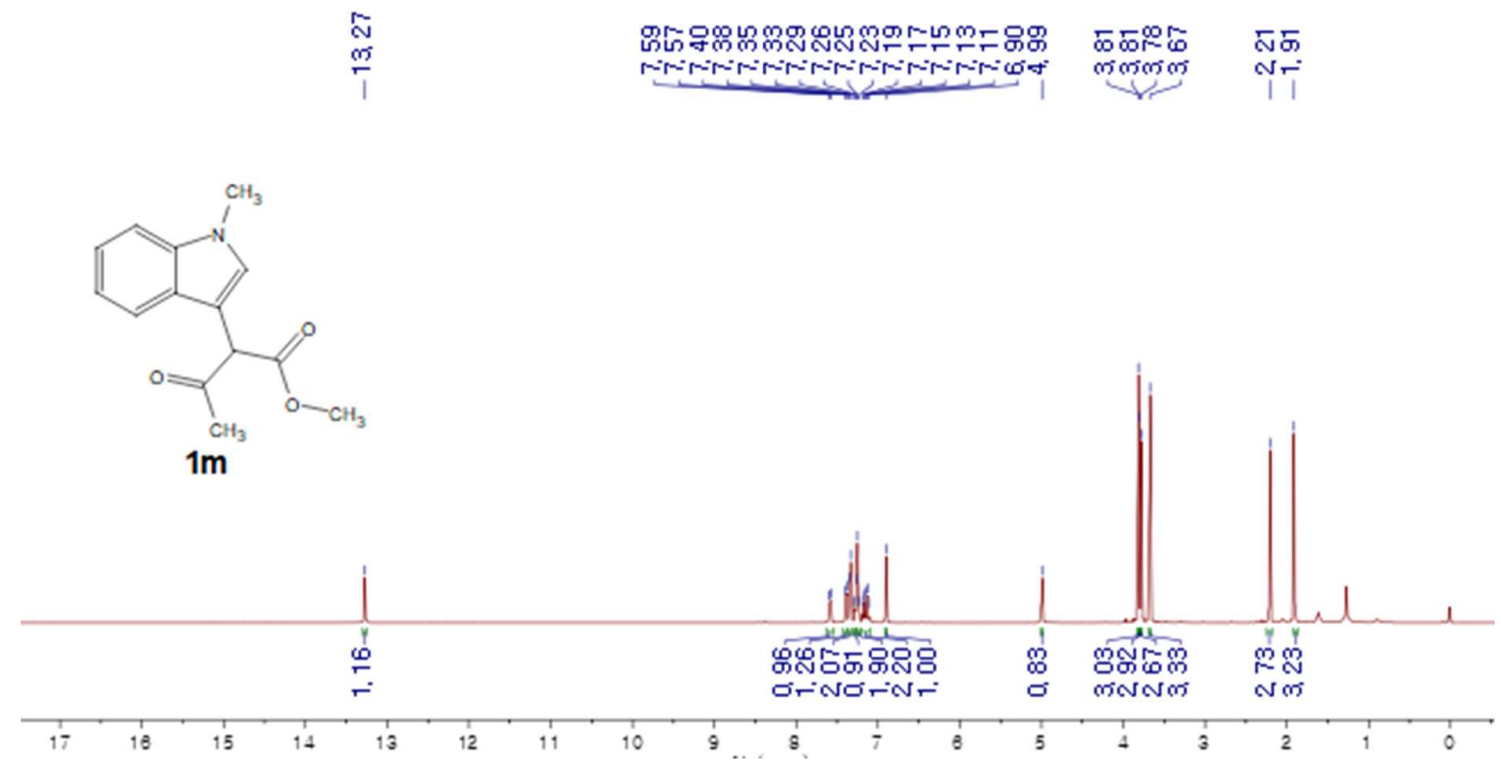

${ }^{1} \mathrm{H}$ NMR (400 MHz, $\mathrm{CDCl}_{3}$ ) spectra of methyl 2-(1-methyl-1H-indol-3-yl)-3-oxobutanoate (1m)

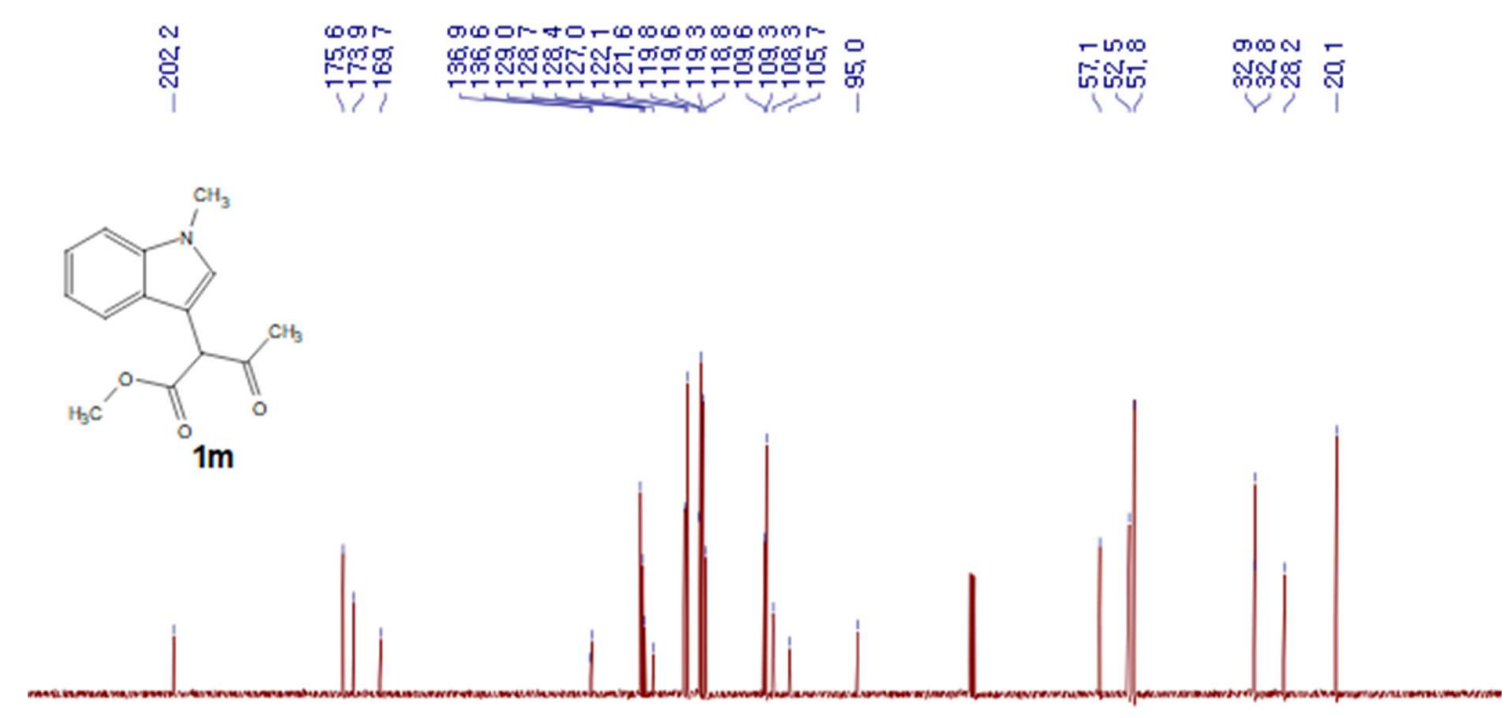

$\begin{array}{lllllllllllllllllllllllllllll}220 & 210 & 200 & 190 & 180 & 170 & 180 & 150 & 140 & 130 & 120 & 110 & 100 & 90 & 80 & 70 & 80 & 50 & 40 & 30 & 20 & 10 & 0\end{array}$

${ }^{13} \mathrm{C}$ NMR (100 MHz, $\left.\mathrm{CDCl}_{3}\right)$ spectra of methyl 2-(1-methyl-1H-indol-3-yl)-3-oxobutanoate

(1m) 

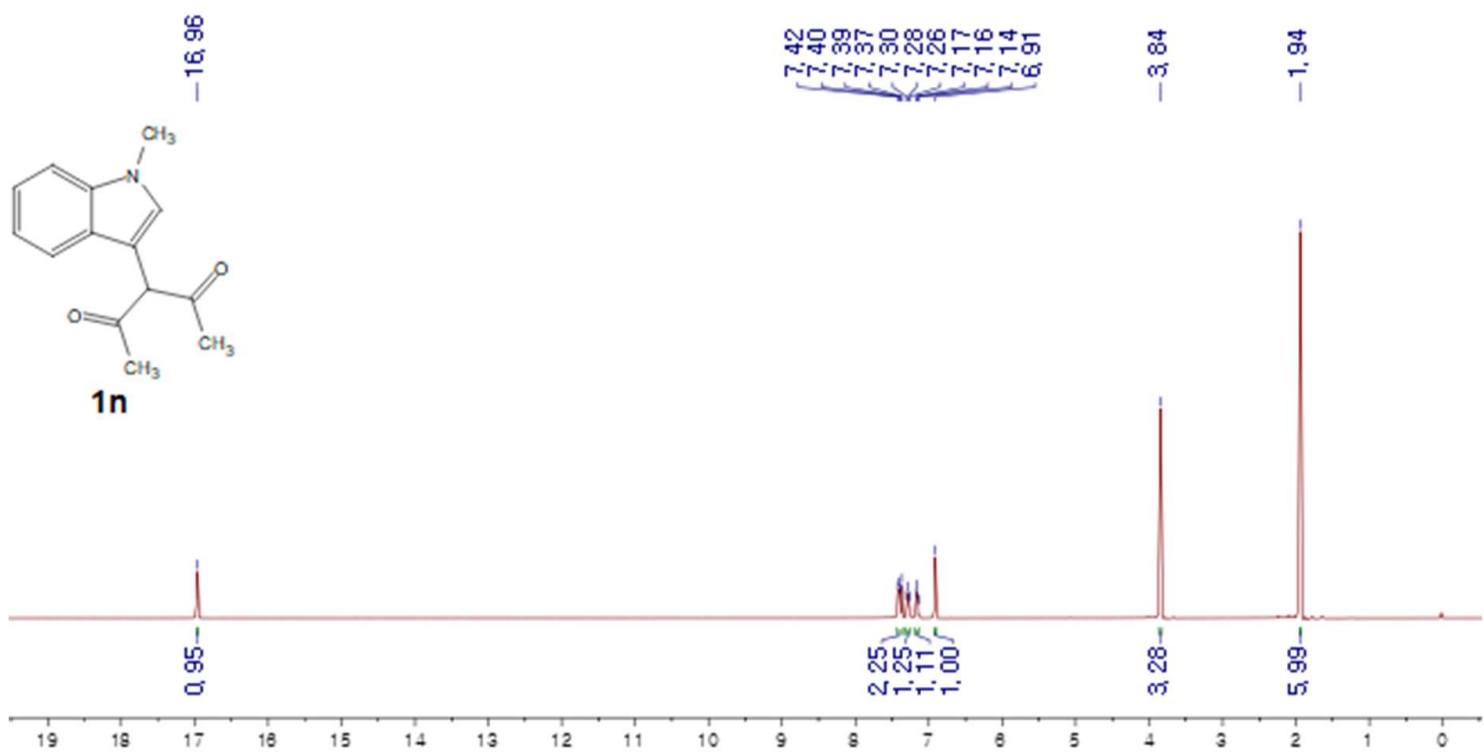

${ }^{1} \mathrm{H}$ NMR (400 MHz, $\mathrm{CDCl}_{3}$ ) spectra of 3-(1-methyl-1H-indol-3-yl)pentane-2,4-dione (1n)

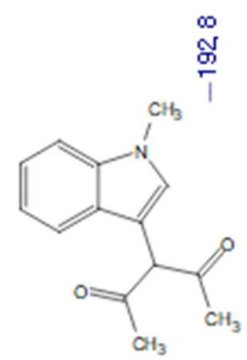

の $\infty \pi \omega m$ m

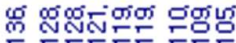

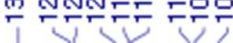

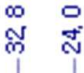

$1 \mathrm{n}$

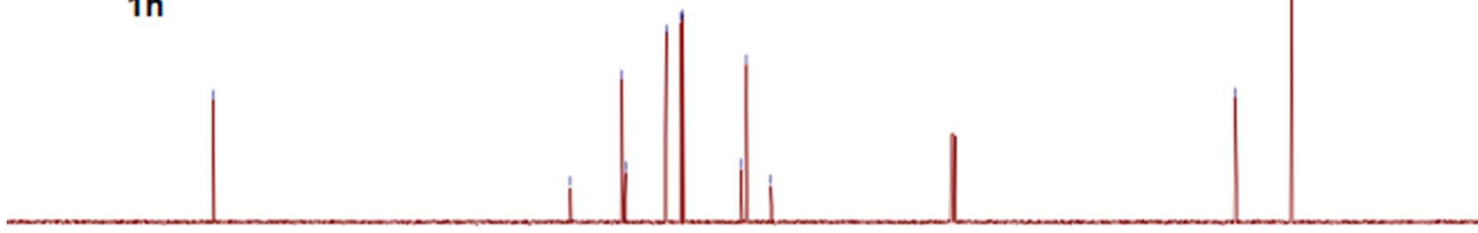

$\begin{array}{llllllllllllllllllllllllll}220 & 210 & 200 & 190 & 180 & 170 & 180 & 150 & 140 & 130 & 120 & 110 & 100 & 90 & 80 & 70 & 80 & 50 & 40 & 30 & 20 & 10 & 0\end{array}$ 13C NMR (100 MHz, CDCl3) spectra of 3-(1-methyl-1H-indol-3-yl)pentane-2,4-dione (1n) 
<smiles>COP(=O)(OC)C(C(C)=O)c1cn(C)c2ccccc12</smiles>

10

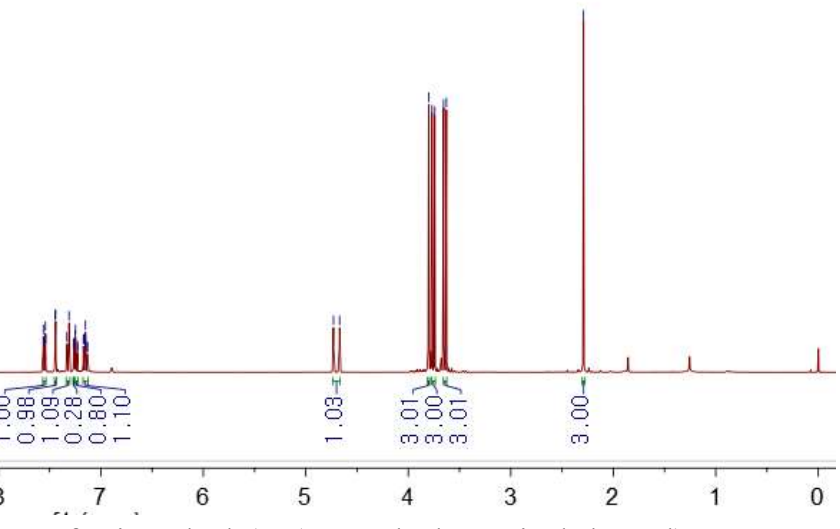

${ }^{1} \mathrm{H}$ NMR (400 MHz, $\mathrm{CDCl}_{3}$ ) spectra of Dimethyl (1-(1-methyl-1H-indol-3-yl)-2oxopropyl)phosphonate (10)

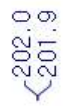

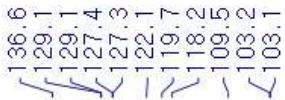

$\infty \infty m m 00000$

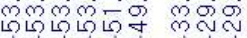
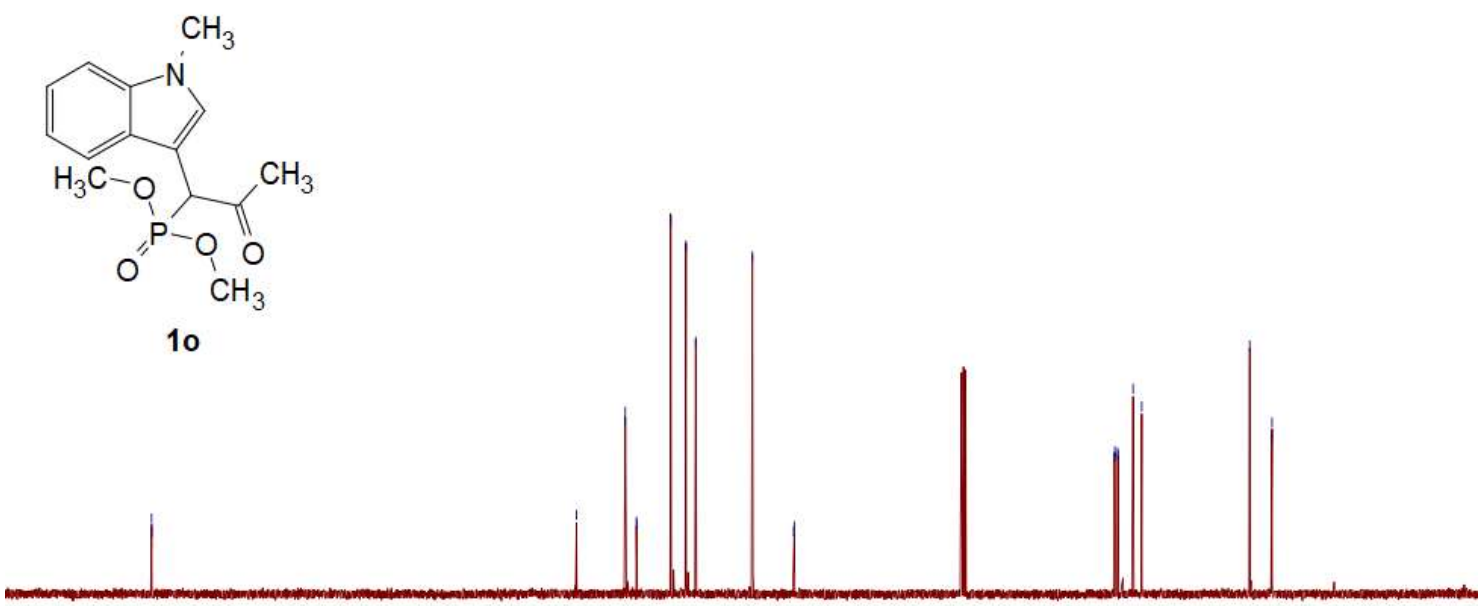

$\begin{array}{lllllllllllllllllllllll}220 & 210 & 200 & 190 & 180 & 170 & 160 & 150 & 140 & 130 & 120 & 110 & 100 & 90 & 80 & 70 & 60 & 50 & 40 & 30 & 20 & 10 & 0\end{array}$

${ }^{13} \mathrm{C}$ NMR (100 MHz, $\mathrm{CDCl}_{3}$ ) spectra of Dimethyl (1-(1-methyl-1H-indol-3-yl)-2oxopropyl)phosphonate (10) 


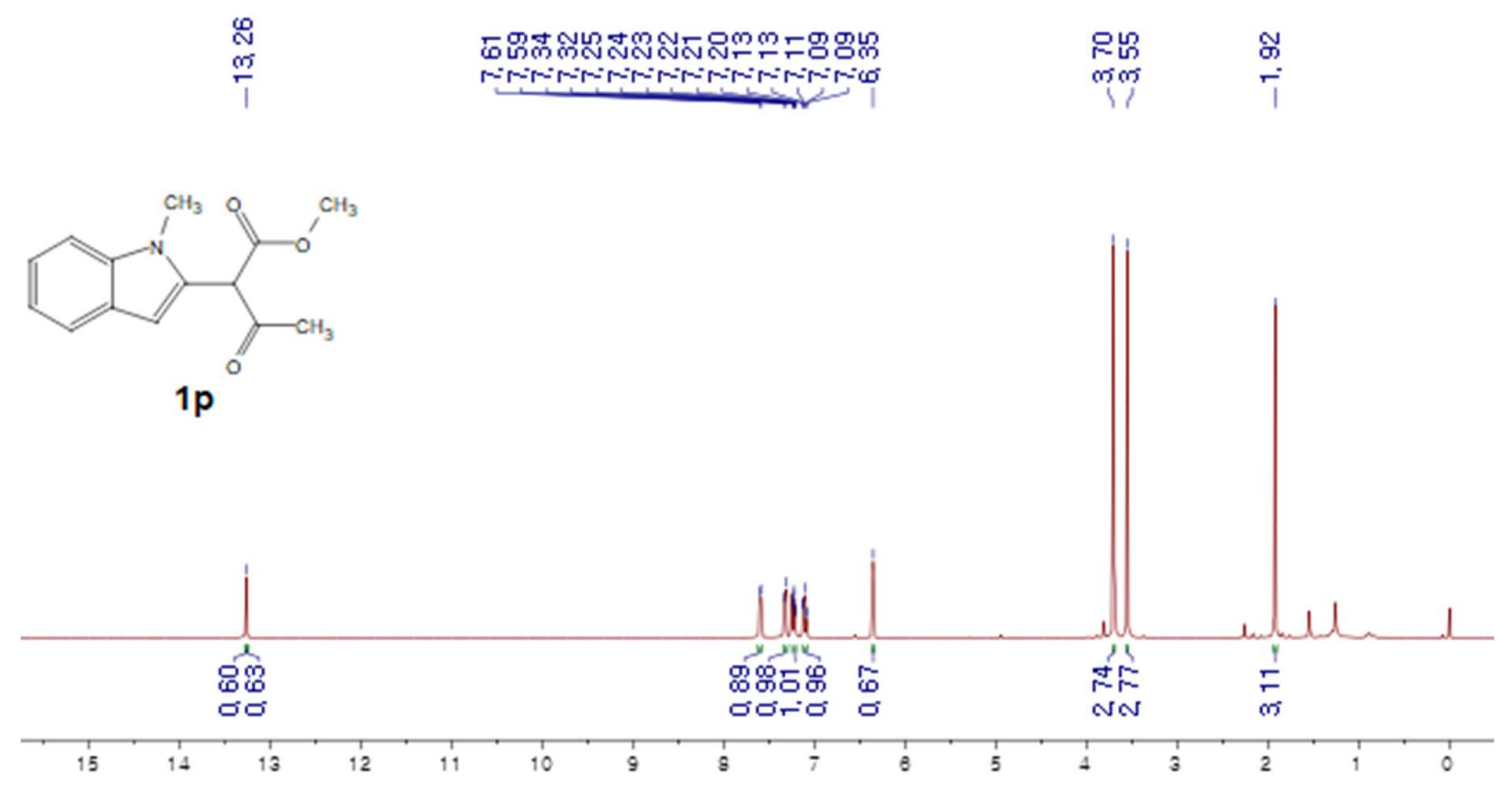

${ }^{1} \mathrm{H}$ NMR (400 MHz, $\mathrm{CDCl}_{3}$ ) spectra of methyl 2-(1-methyl-1H-indol-2-yl)-3-oxobutanoate (1p)
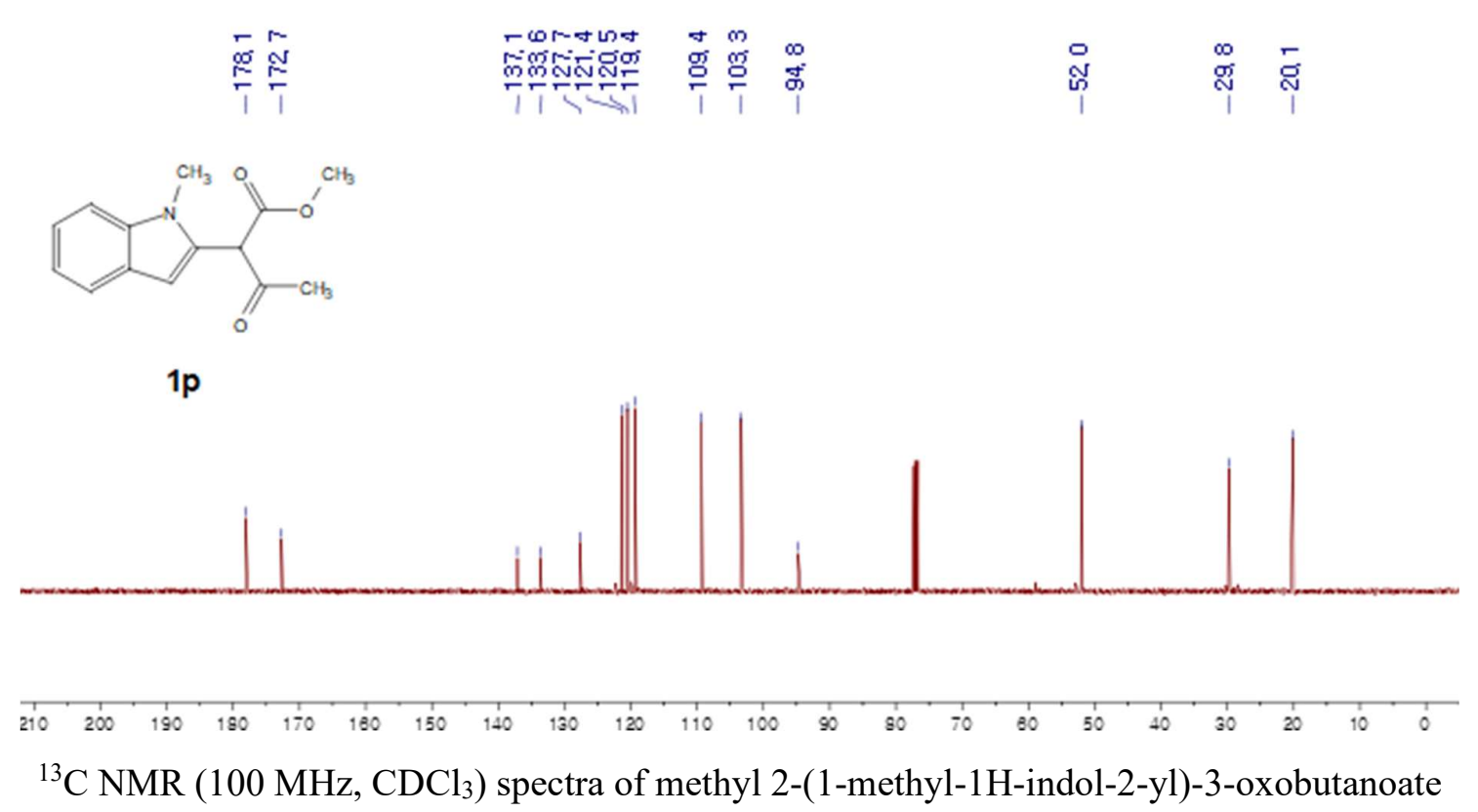

(1p) 


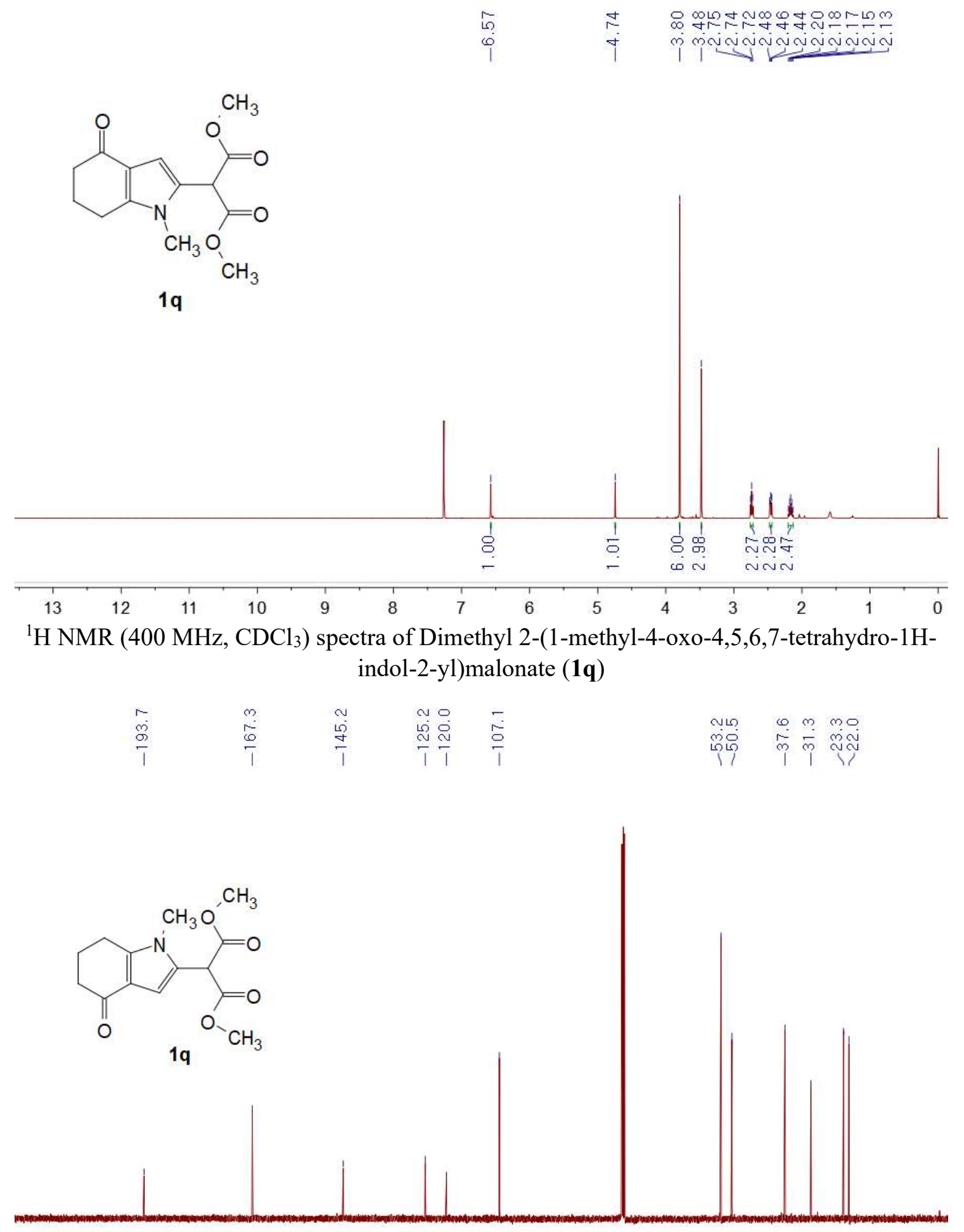

$\begin{array}{llllllllllllllllllllllllllllll}220 & 210 & 200 & 190 & 180 & 170 & 160 & 150 & 140 & 130 & 120 & 110 & 100 & 90 & 80 & 70 & 60 & 50 & 40 & 30 & 20 & 10 & 0\end{array}$

${ }^{13} \mathrm{C}$ NMR (100 MHz, $\mathrm{CDCl}_{3}$ ) spectra of Dimethyl 2-(1-methyl-4-oxo-4,5,6,7-tetrahydro-1Hindol-2-yl)malonate (1q) 

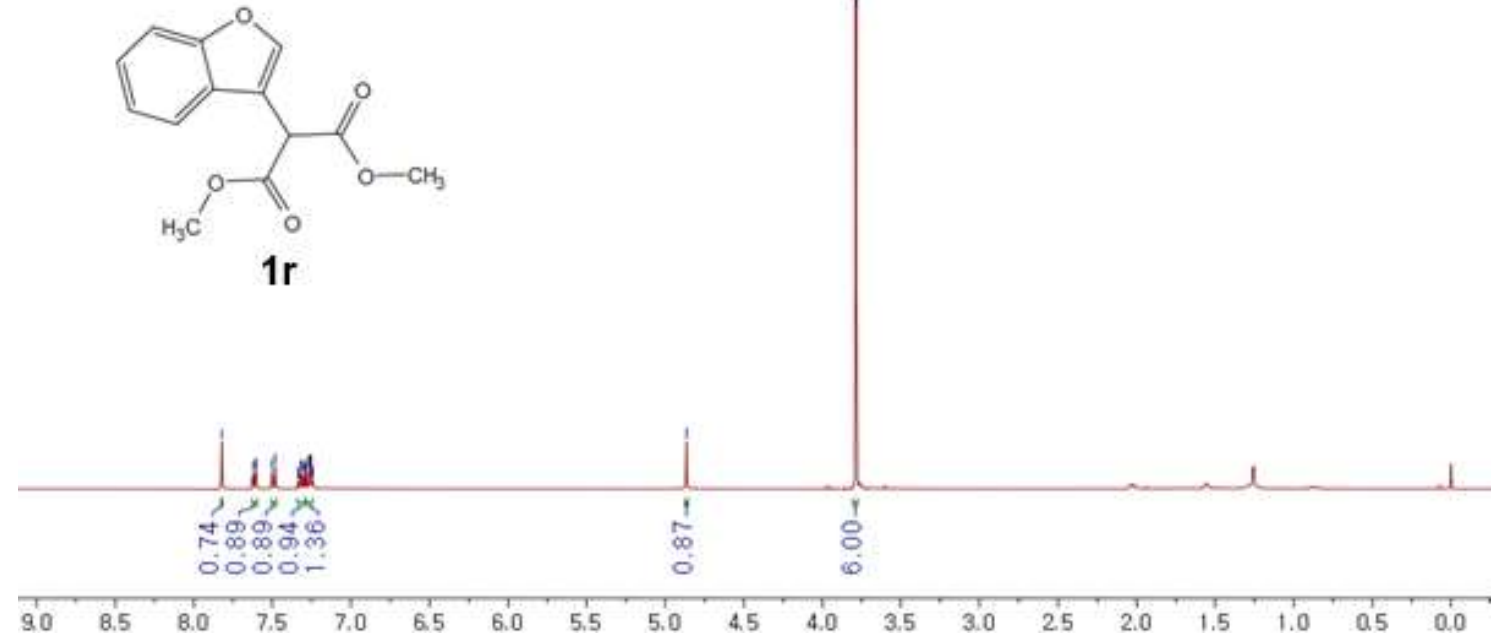

${ }^{1} \mathrm{H}$ NMR (400 MHz, $\mathrm{CDCl}_{3}$ ) spectra of dimethyl 2-(benzofuran-3-yl)malonate (1r)

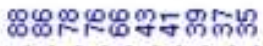

ririniniririnirim
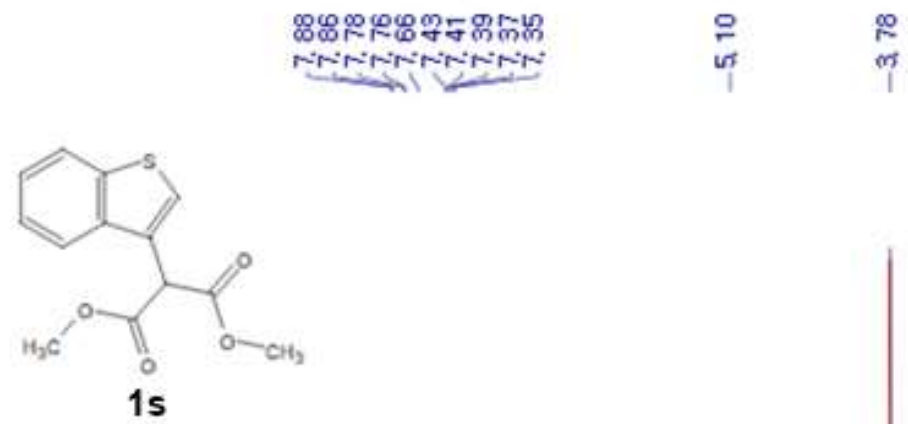

i

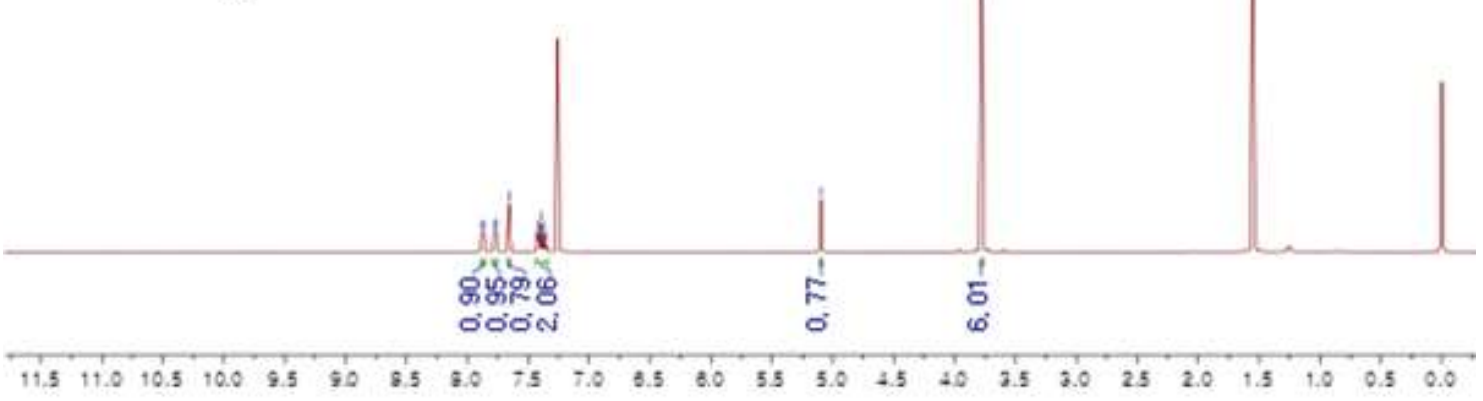

${ }^{1} \mathrm{H}$ NMR (400 MHz, $\mathrm{CDCl}_{3}$ ) spectra of dimethyl 2-(benzo[b]thiophen-3-yl)malonate (1s) 

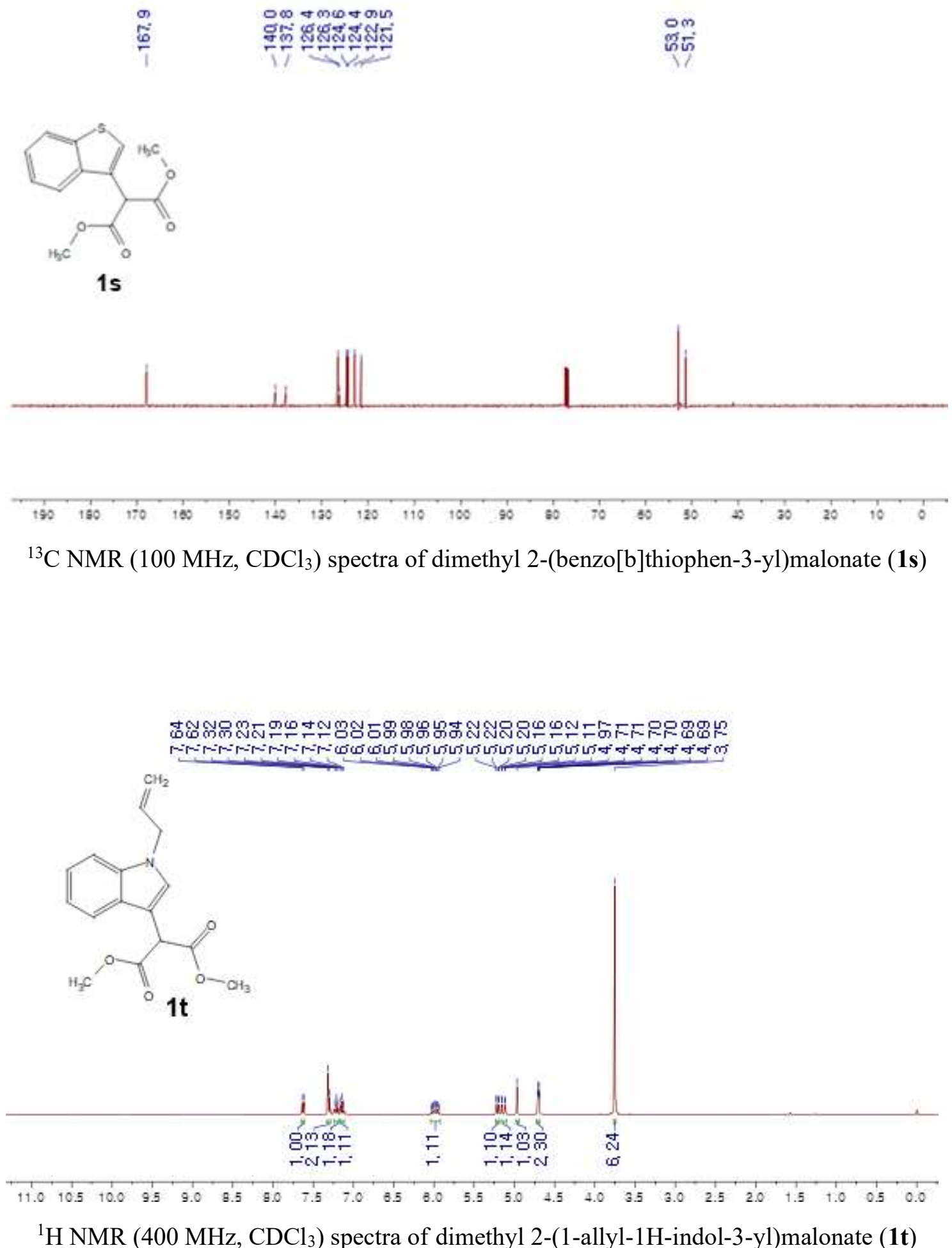

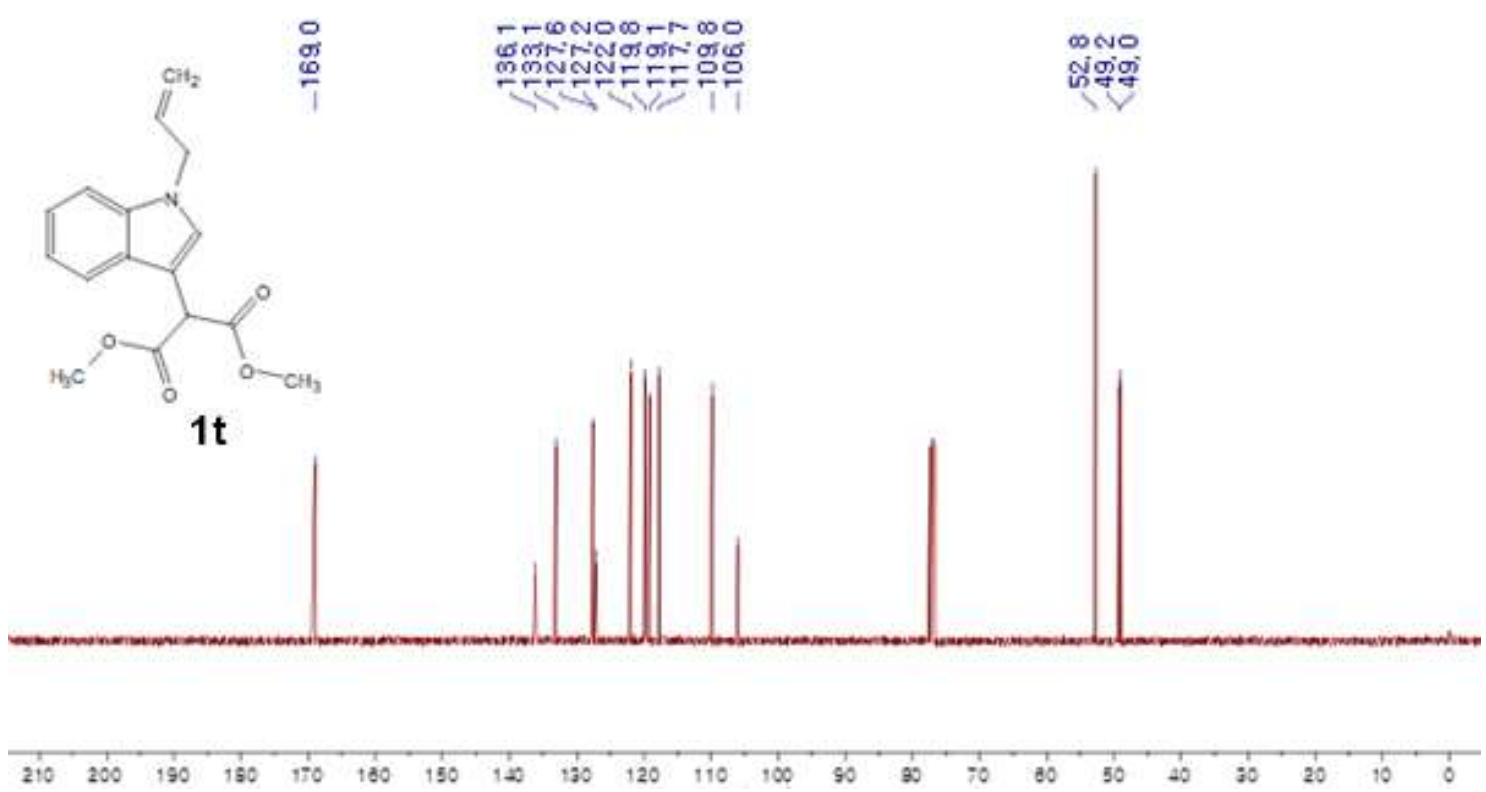

${ }^{13} \mathrm{C}$ NMR (100 MHz, $\left.\mathrm{CDCl}_{3}\right)$ spectra of dimethyl 2-(1-allyl-1H-indol-3-yl)malonate (1t)

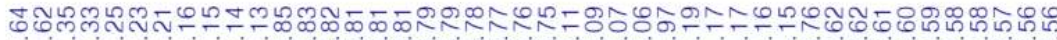

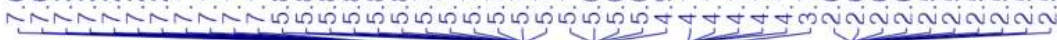

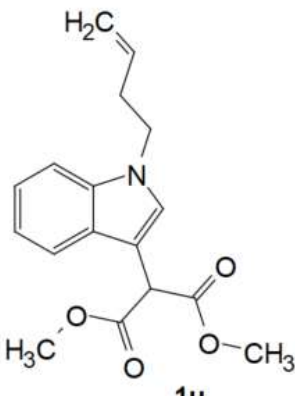

1u

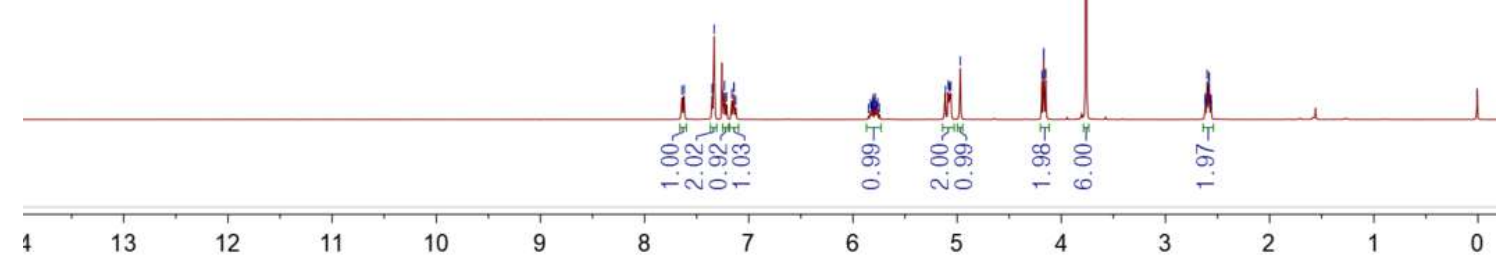

${ }^{1} \mathrm{H}$ NMR (400 MHz, $\mathrm{CDCl}_{3}$ ) spectra of dimethyl 2-(1-(but-3-en-1-yl)-1H-indol-3-

yl)malonate $(\mathbf{1 u})$ 


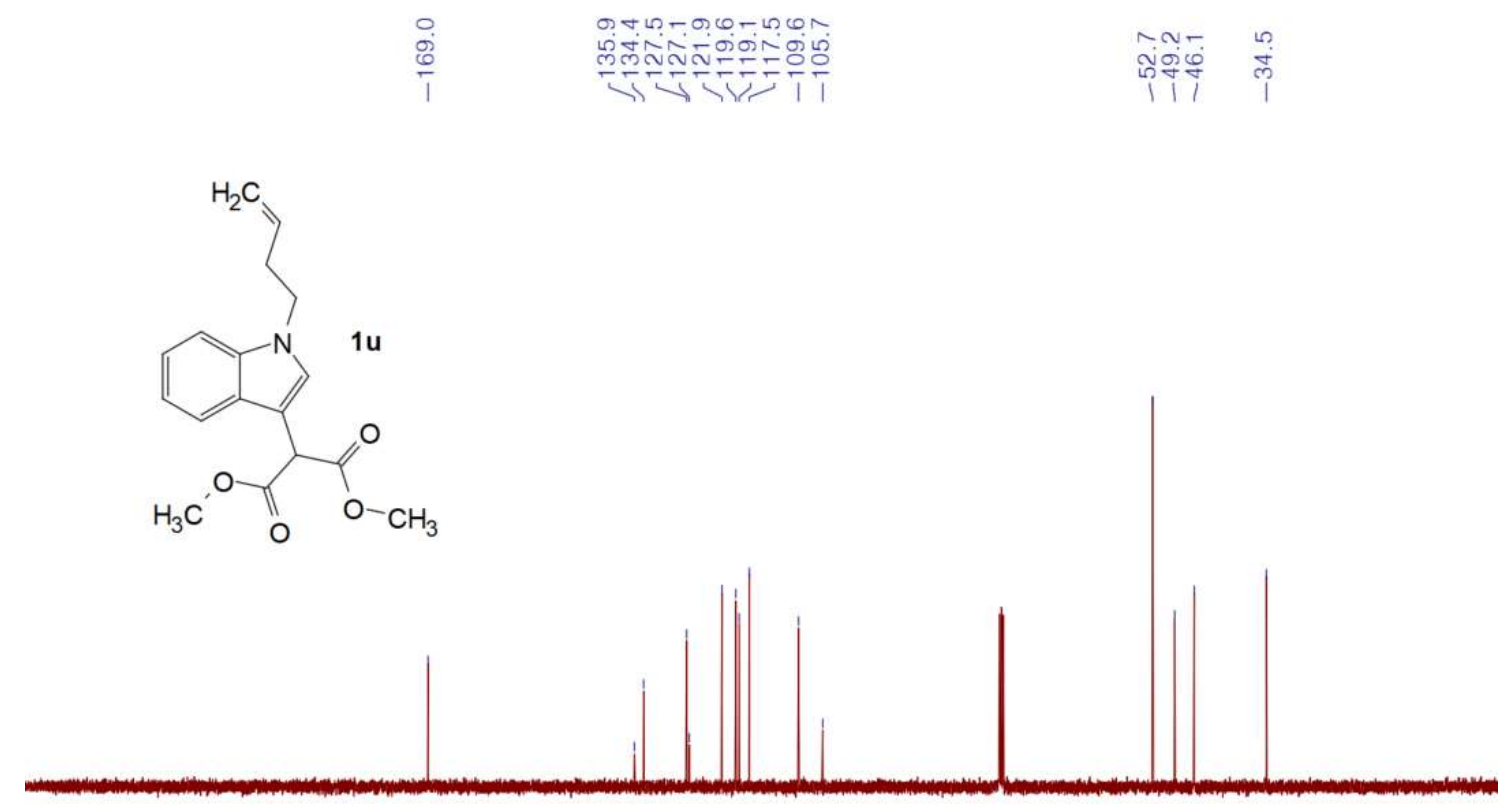

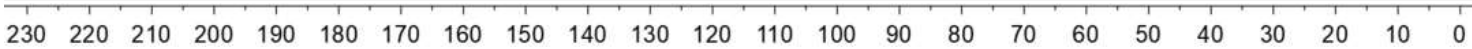

${ }^{13} \mathrm{C}$ NMR (100 MHz, $\mathrm{CDCl}_{3}$ ) spectra of dimethyl 2-(1-(but-3-en-1-yl)-1H-indol-3-

yl)malonate (1u)

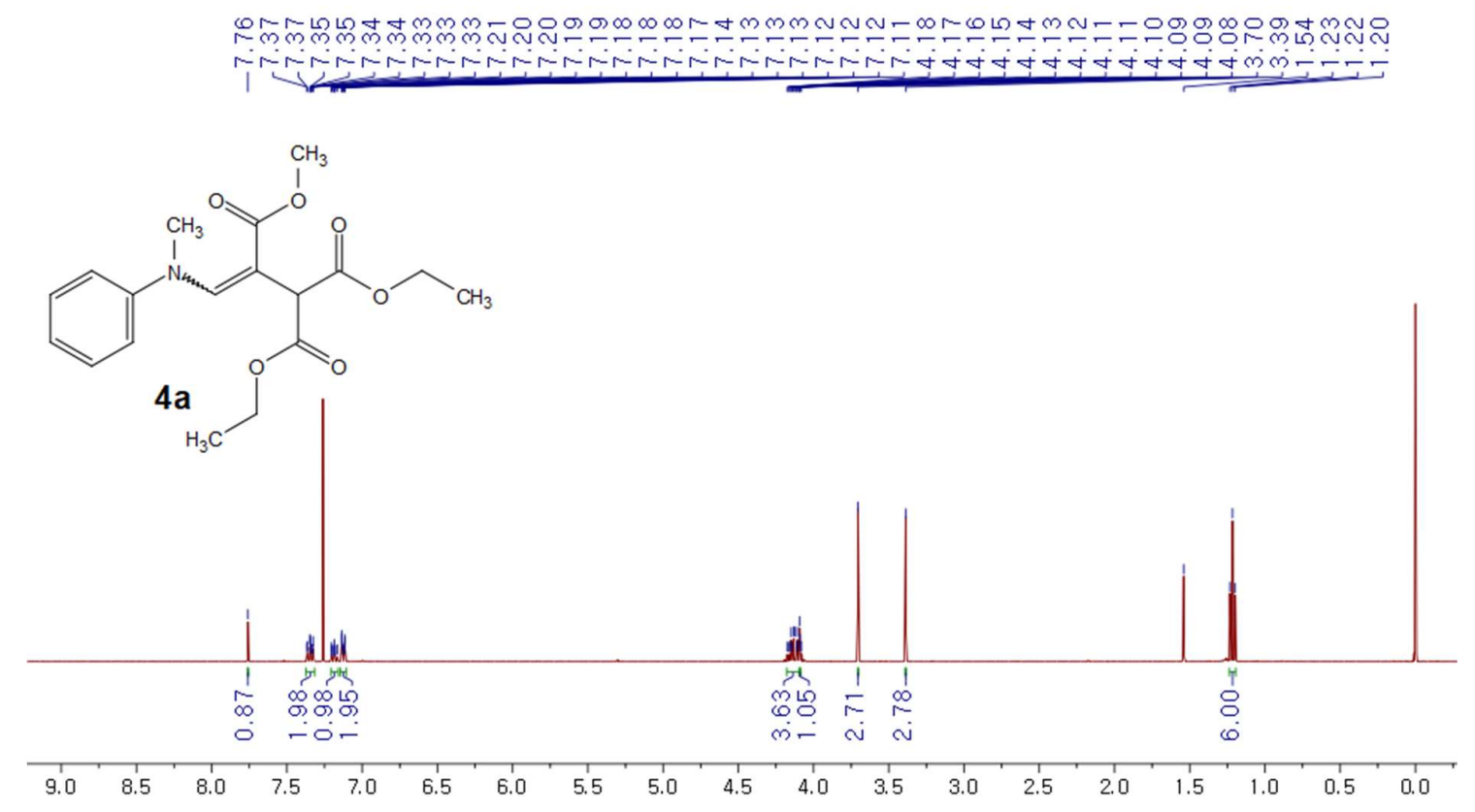

${ }^{1} \mathrm{H}$ NMR (400 MHz, $\mathrm{CDCl}_{3}$ ) spectra of 1,1-diethyl 2-methyl 3-(methyl(phenyl)amino)prop-2ene-1,1,2-tricarboxylate (4a) 

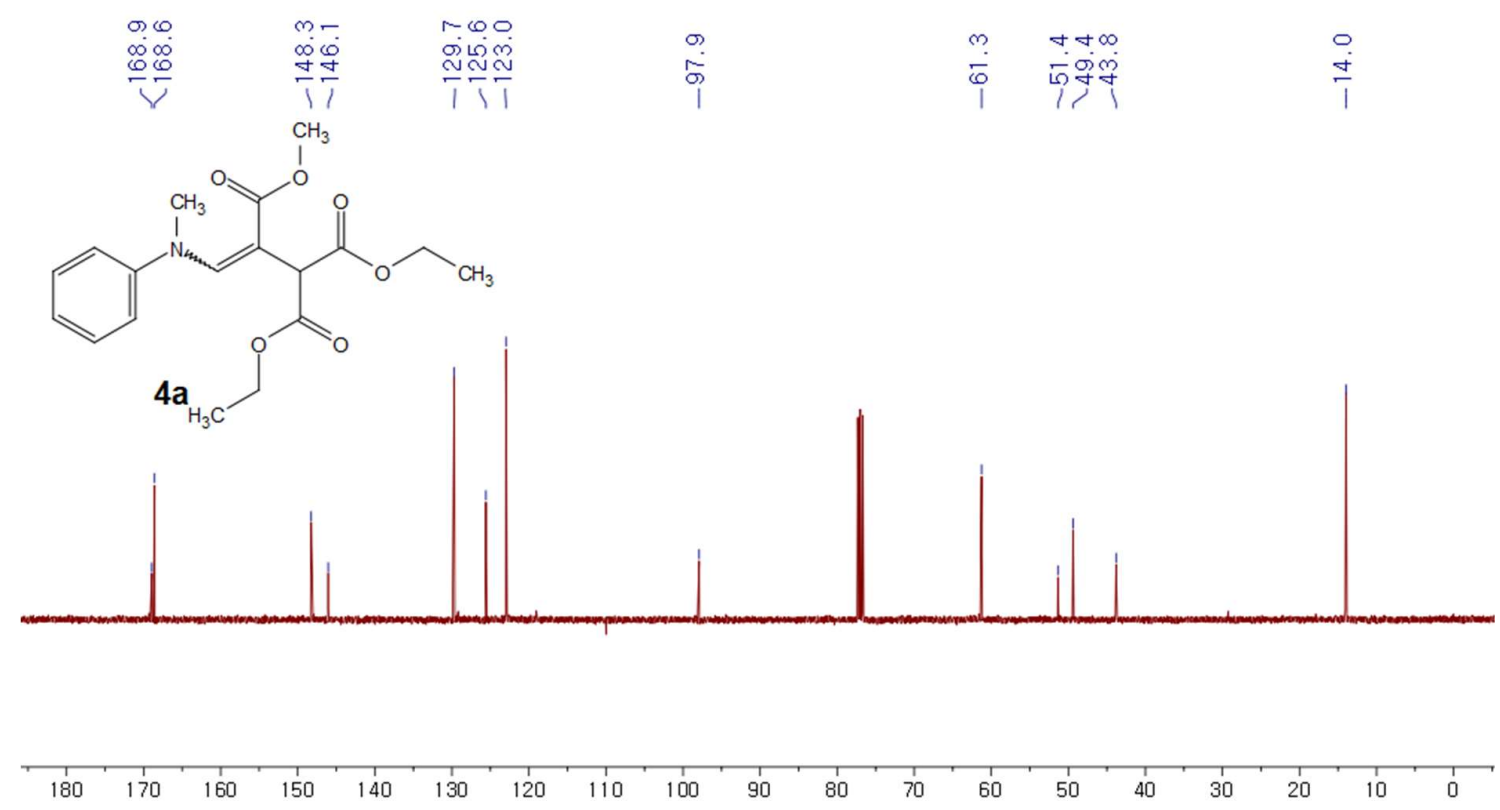

${ }^{13} \mathrm{C} \mathrm{NMR}\left(100 \mathrm{MHz}, \mathrm{CDCl}_{3}\right)$ spectra of 1,1-diethyl 2-methyl 3-(methyl(phenyl)amino)prop2-ene-1,1,2-tricarboxylate (4a)

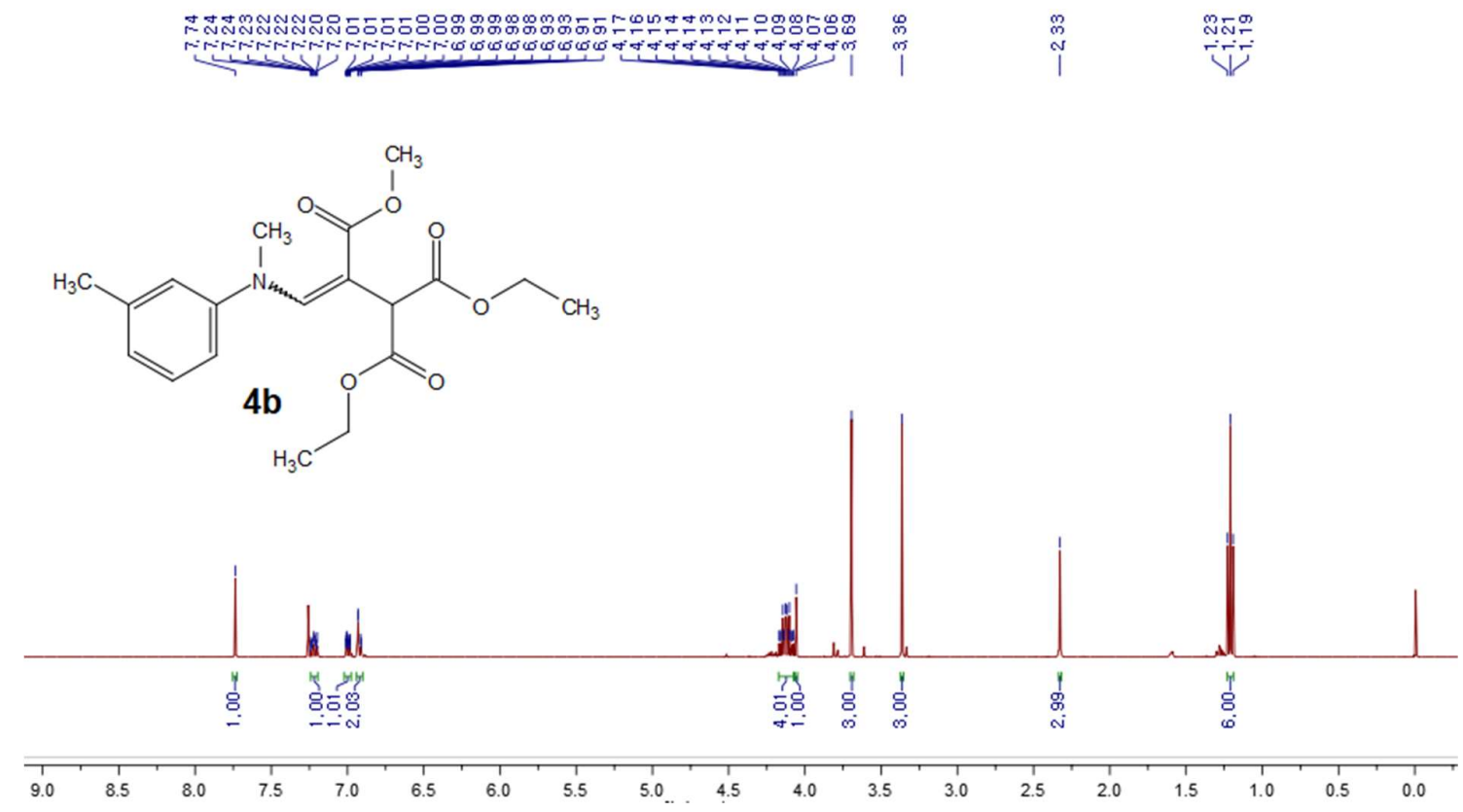

${ }^{1} \mathrm{H}$ NMR (400 MHz, $\mathrm{CDCl}_{3}$ ) spectra of 1,1-diethyl 2-methyl 3-(methyl(m-tolyl)amino)prop2-ene-1,1,2-tricarboxylate (4b) 

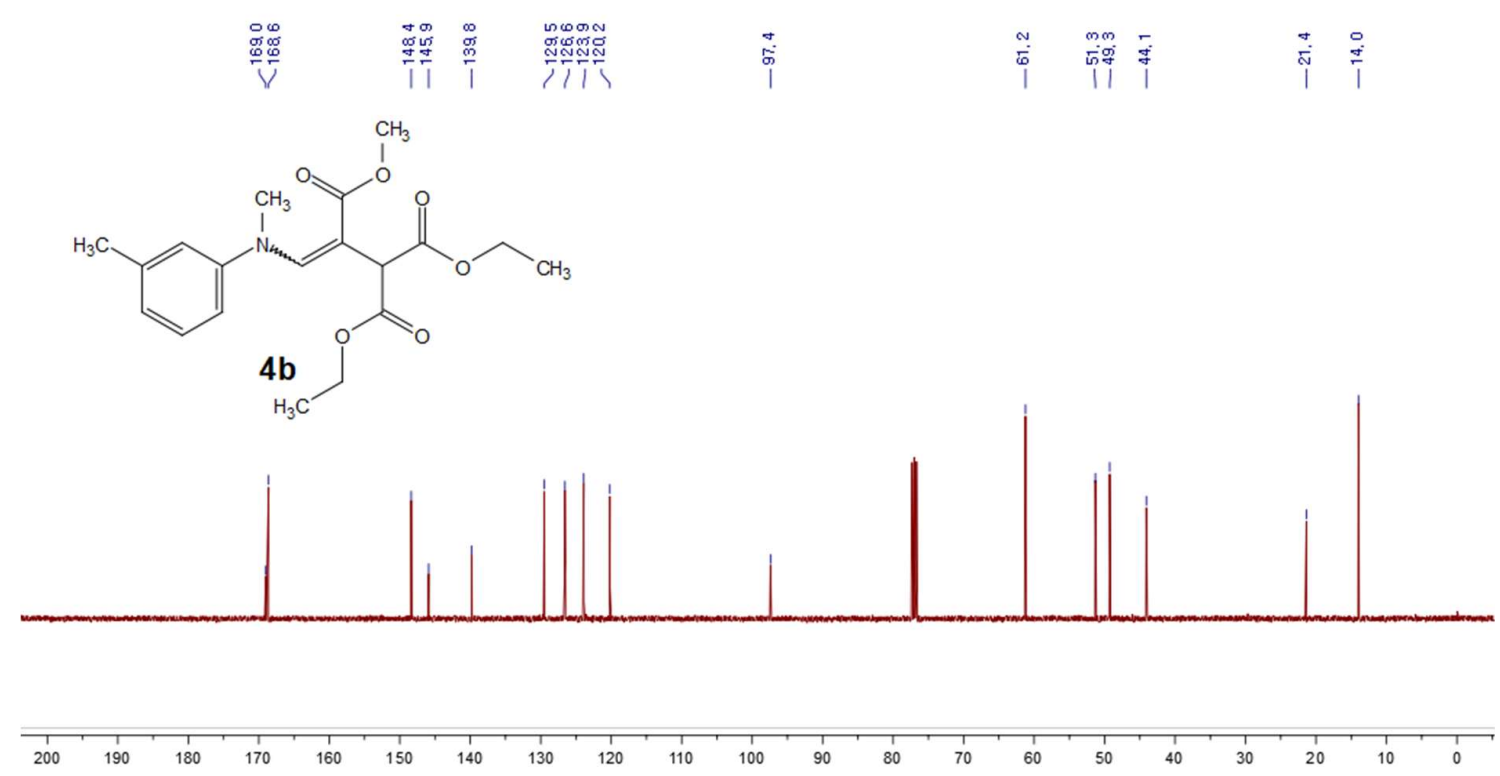

${ }^{13} \mathrm{C} \mathrm{NMR}\left(100 \mathrm{MHz}, \mathrm{CDCl}_{3}\right)$ spectra of 1,1-diethyl 2-methyl 3-(methyl(m-tolyl)amino)prop2-ene-1,1,2-tricarboxylate (4b)

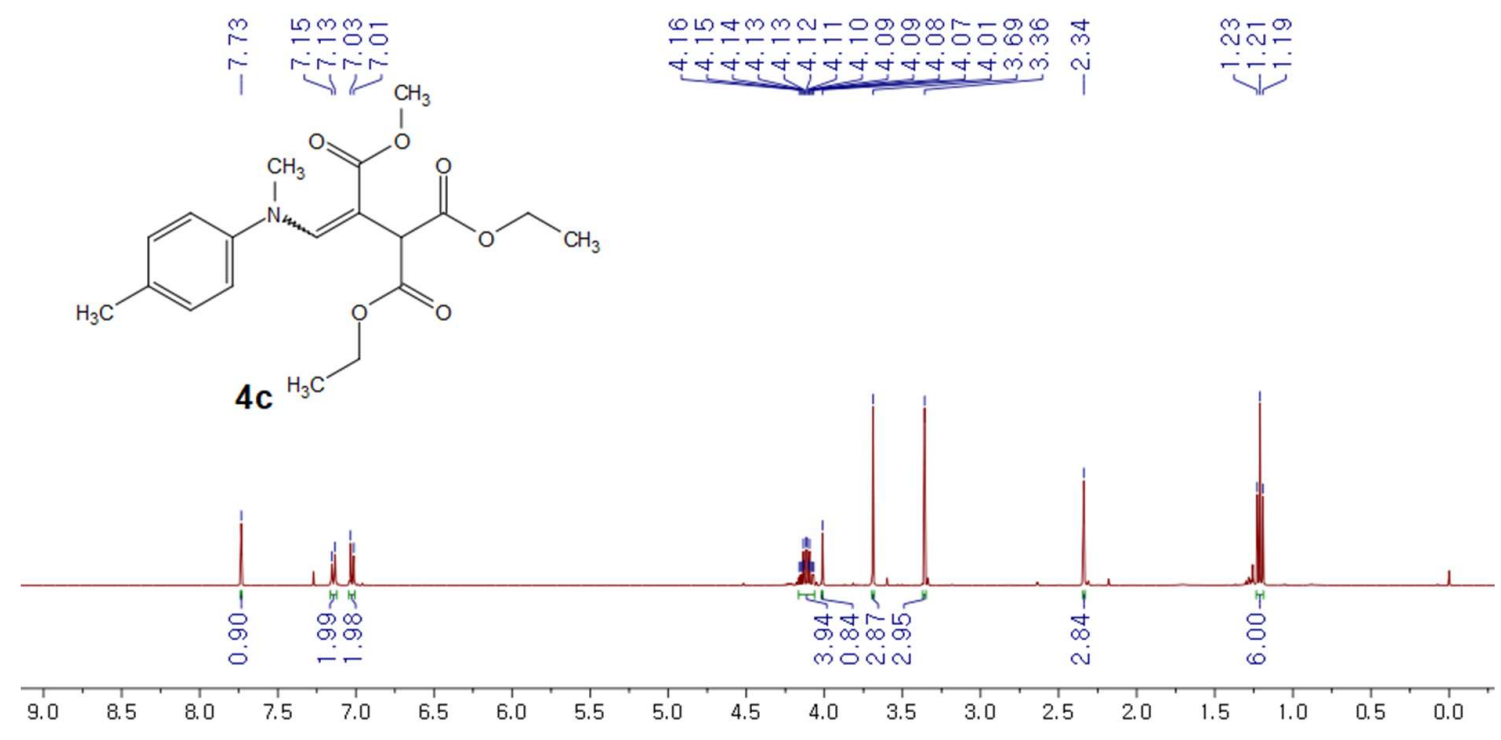

${ }^{1} \mathrm{H}$ NMR (400 MHz, $\mathrm{CDCl}_{3}$ ) spectra of 1,1-diethyl 2-methyl 3-(methyl(p-tolyl)amino)prop-2ene-1,1,2-tricarboxylate $(\mathbf{4 c})$ 


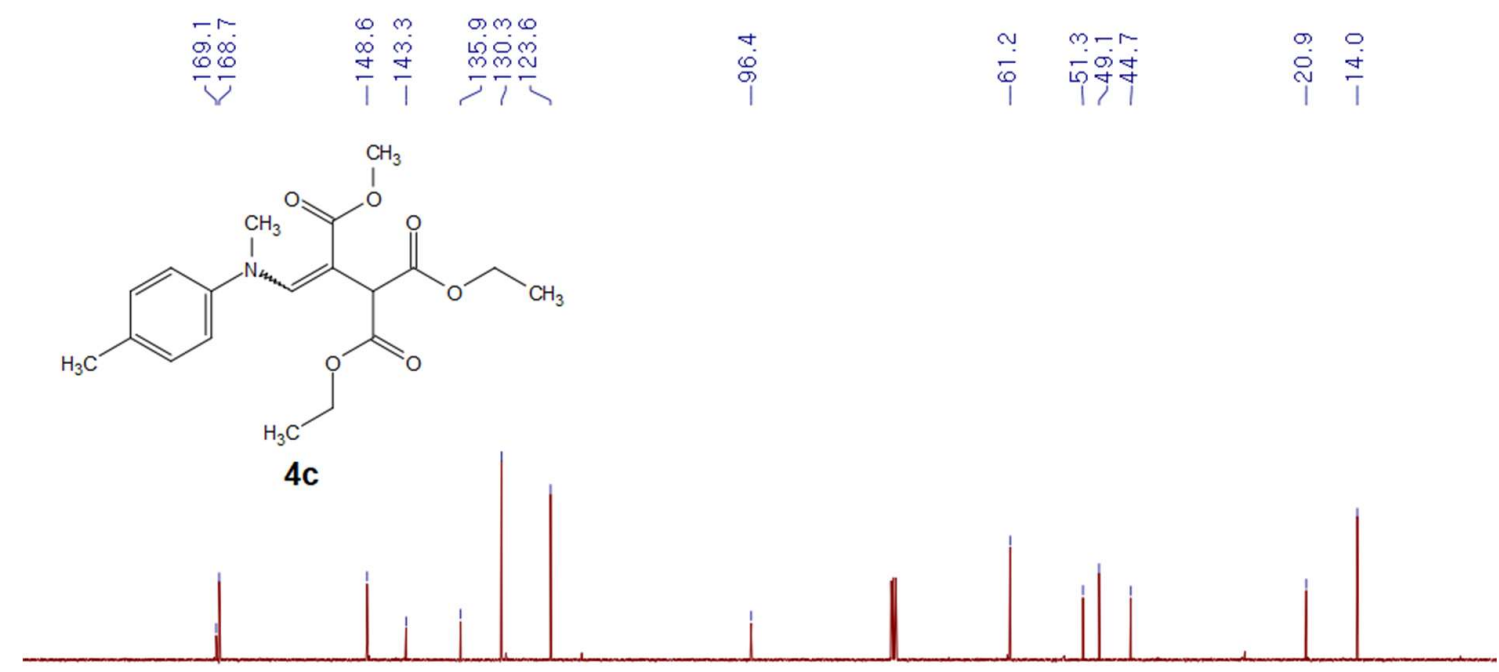

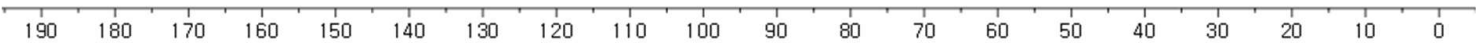

${ }^{13} \mathrm{C} \mathrm{NMR}\left(100 \mathrm{MHz}, \mathrm{CDCl}_{3}\right)$ spectra of 1,1-diethyl 2-methyl 3-(methyl(p-tolyl)amino)prop2-ene-1,1,2-tricarboxylate $(\mathbf{4 c})$

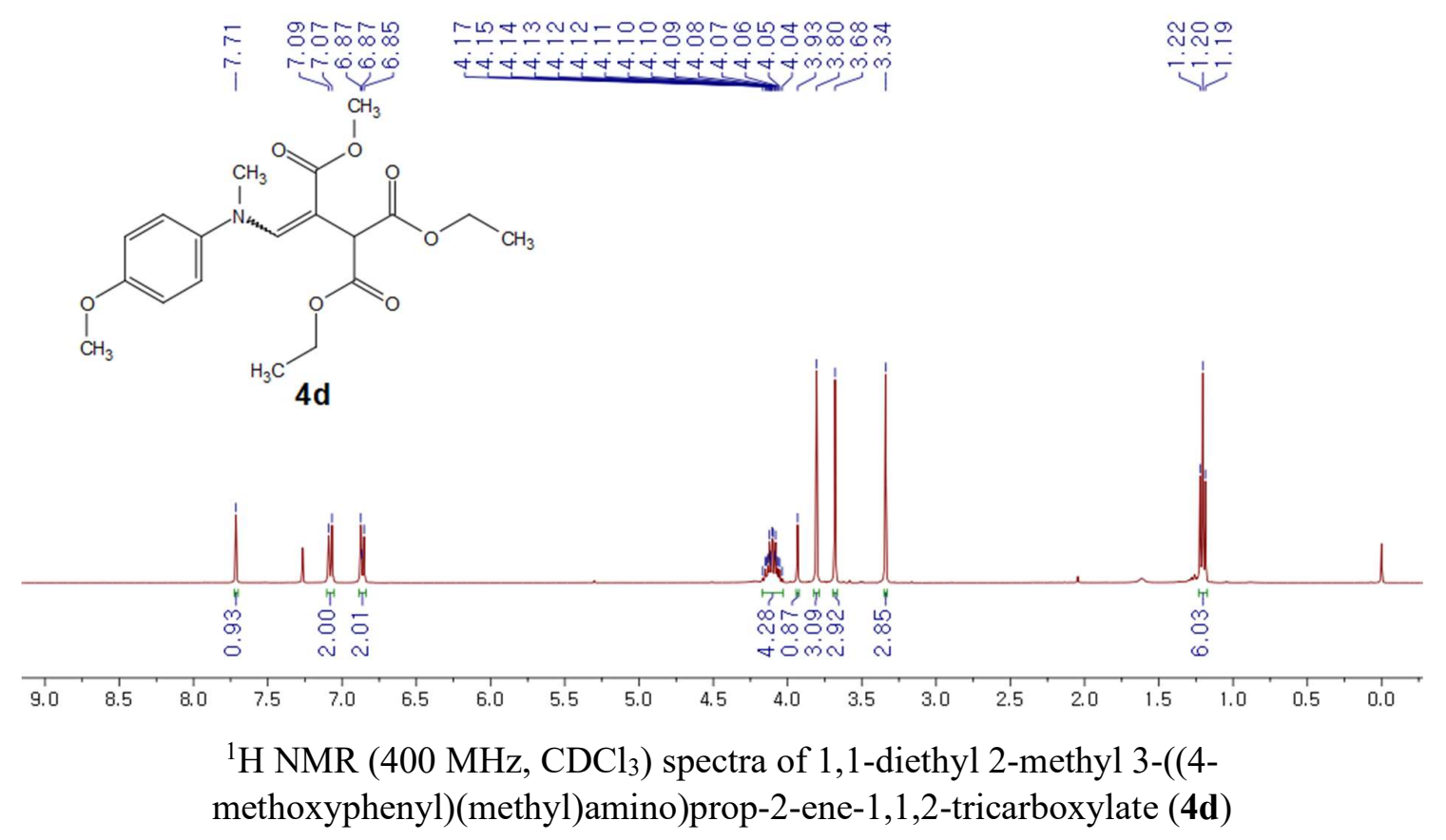



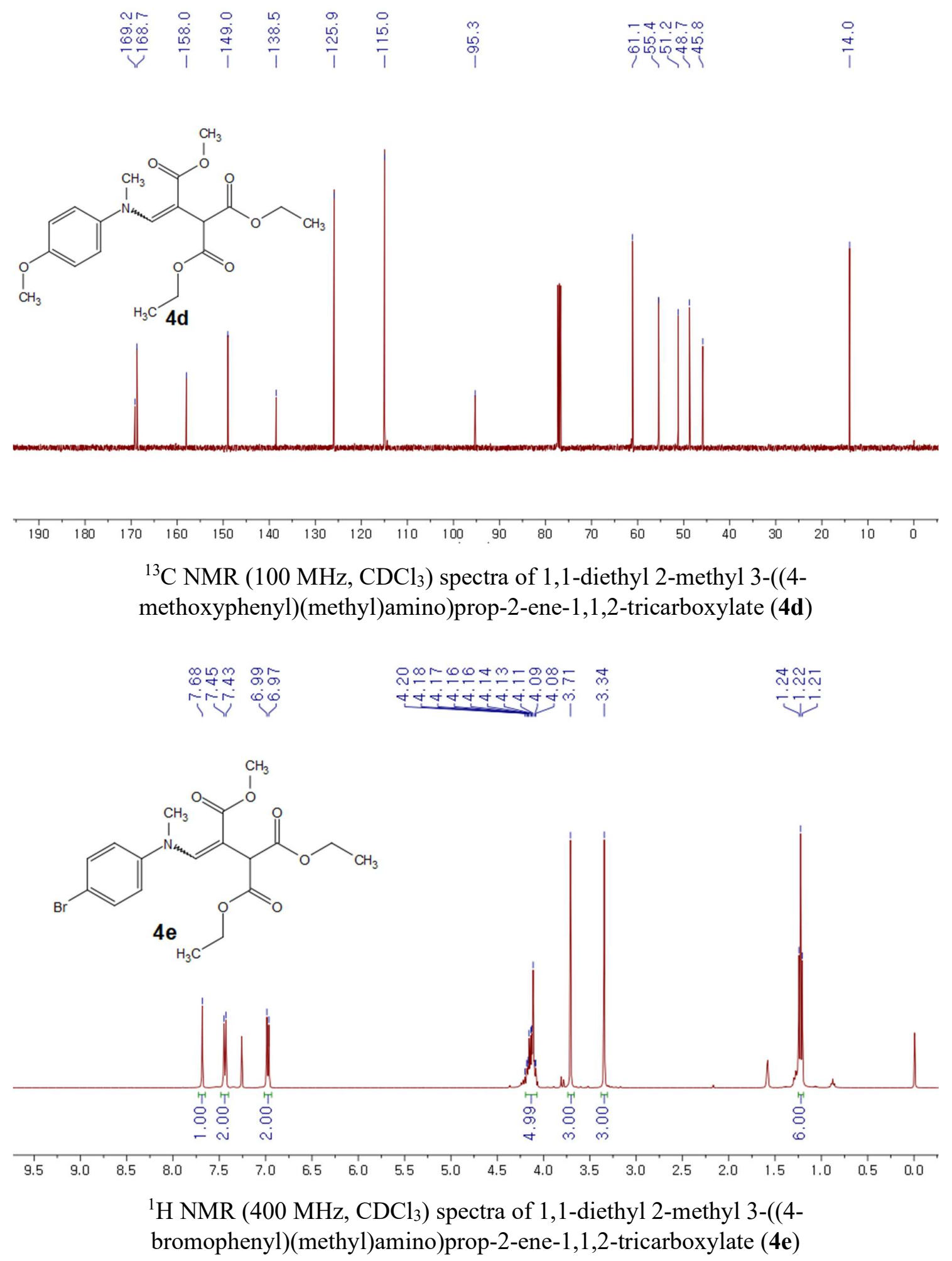

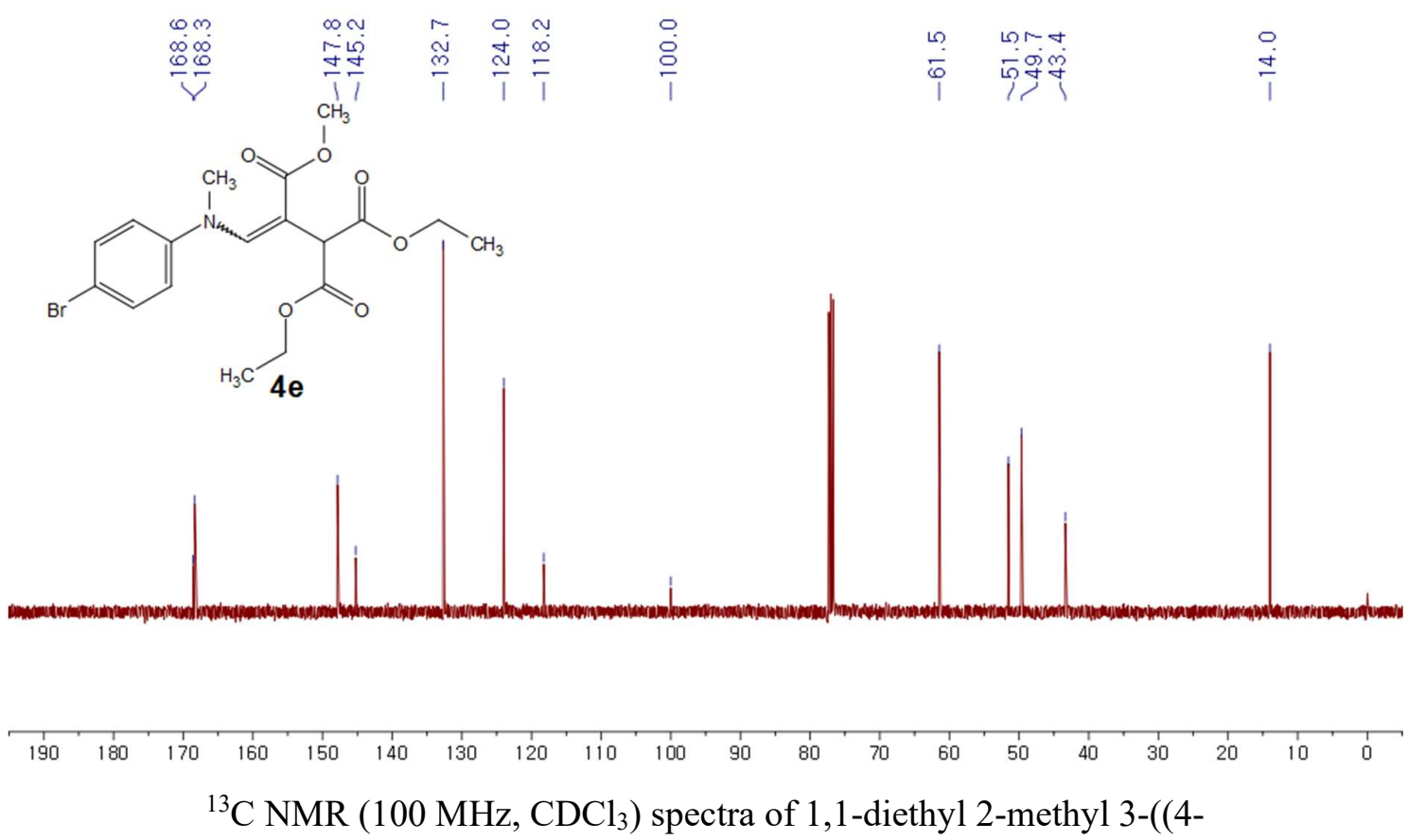
bromophenyl)(methyl)amino)prop-2-ene-1,1,2-tricarboxylate (4e)

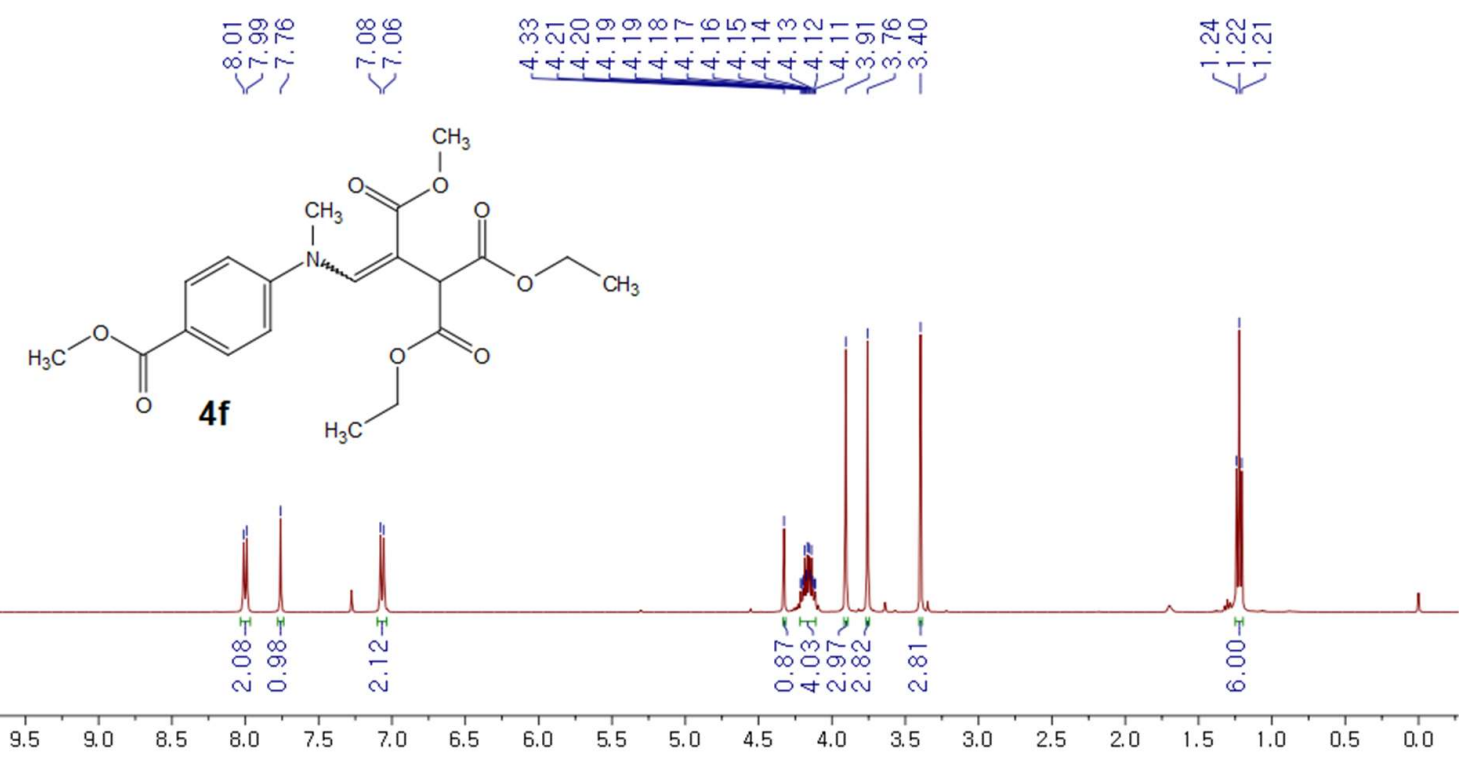

${ }^{1} \mathrm{H}$ NMR (400 MHz, $\left.\mathrm{CDCl}_{3}\right)$ spectra of 1,1-diethyl 2-methyl 3-((4(methoxycarbonyl)phenyl)(methyl)amino)prop-2-ene-1,1,2-tricarboxylate (4f) 

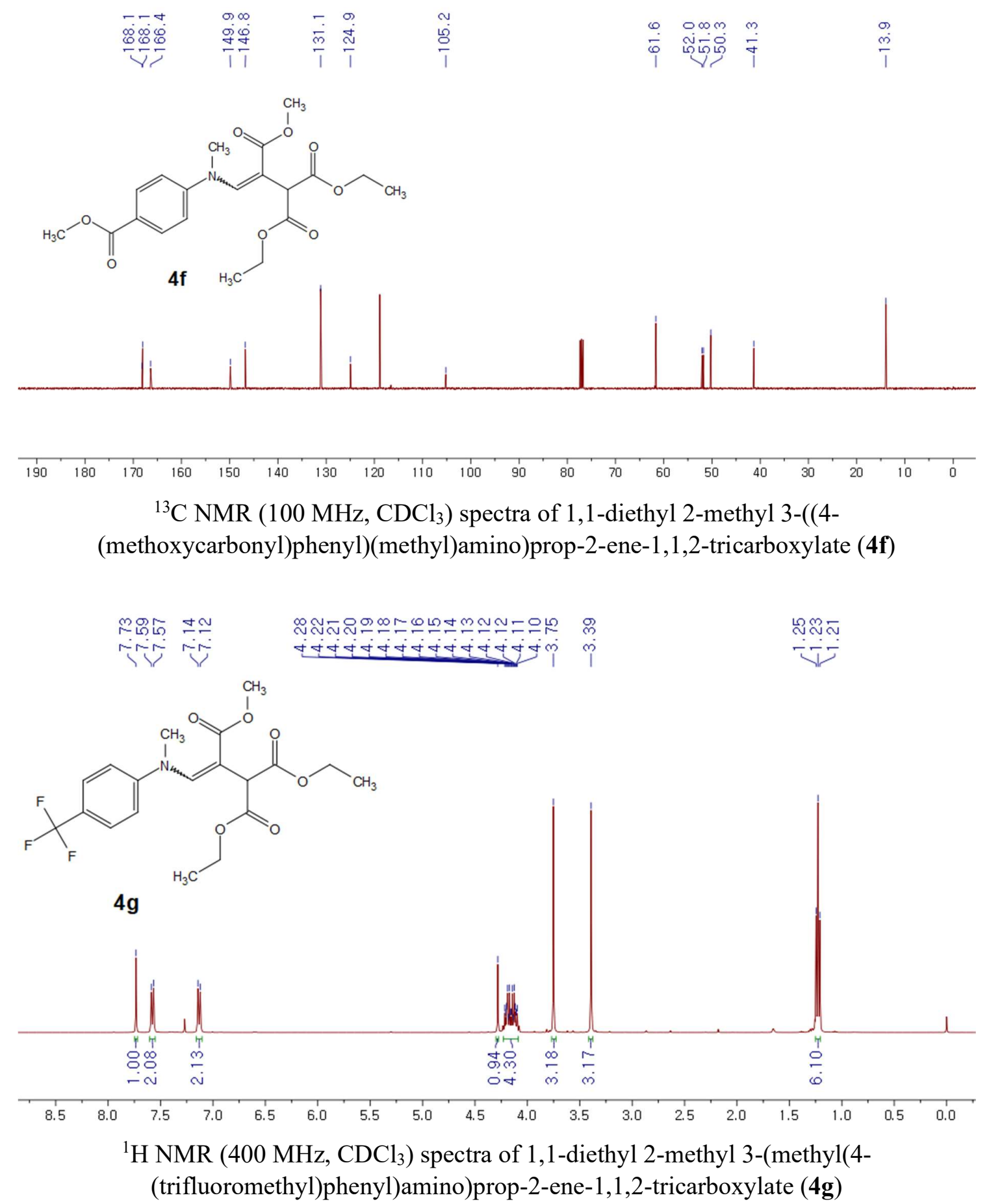


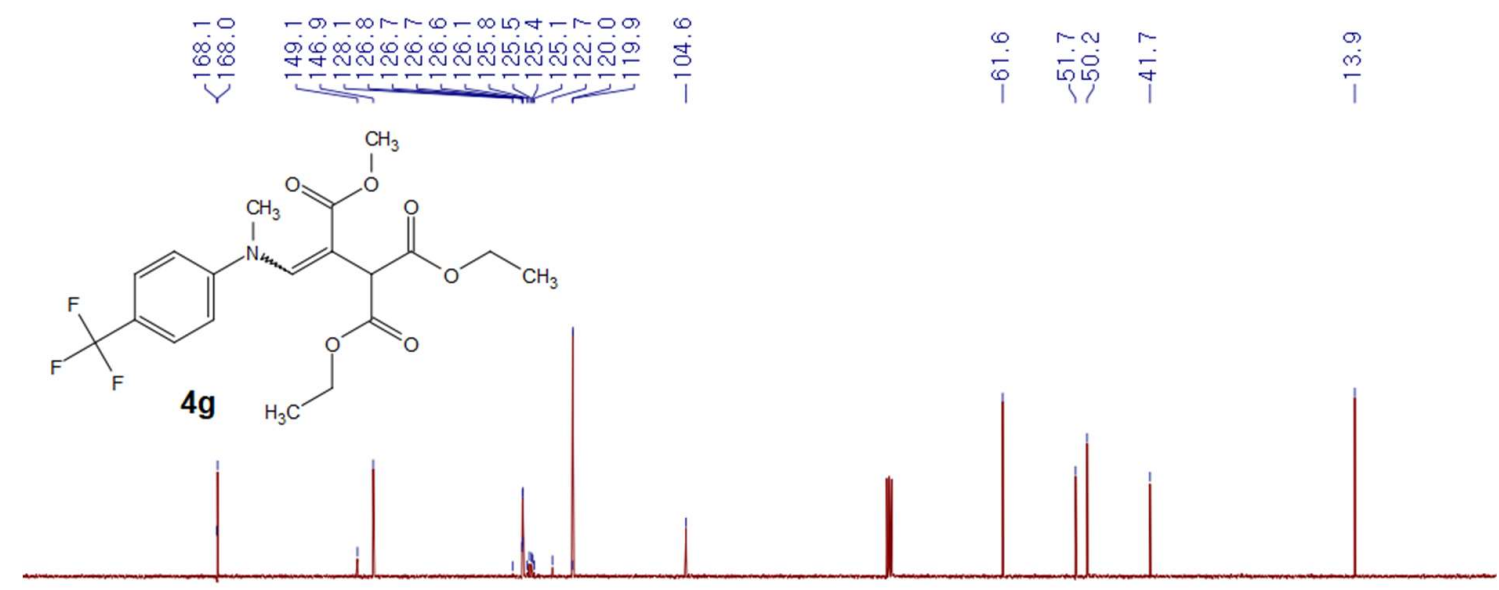

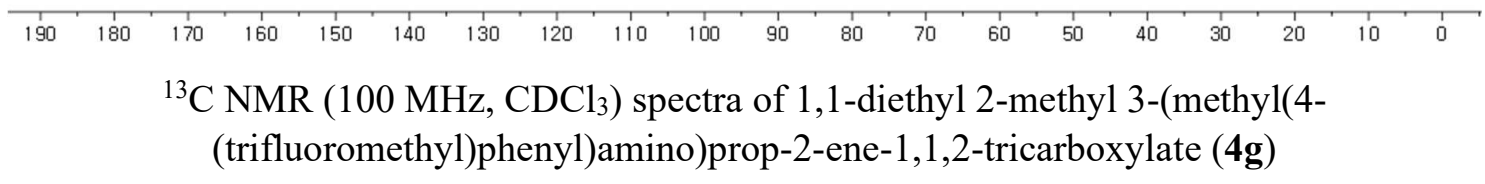




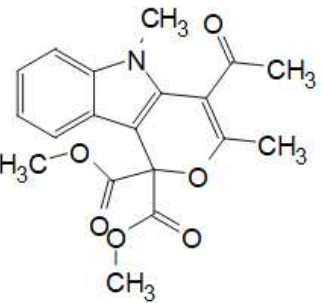

3aa

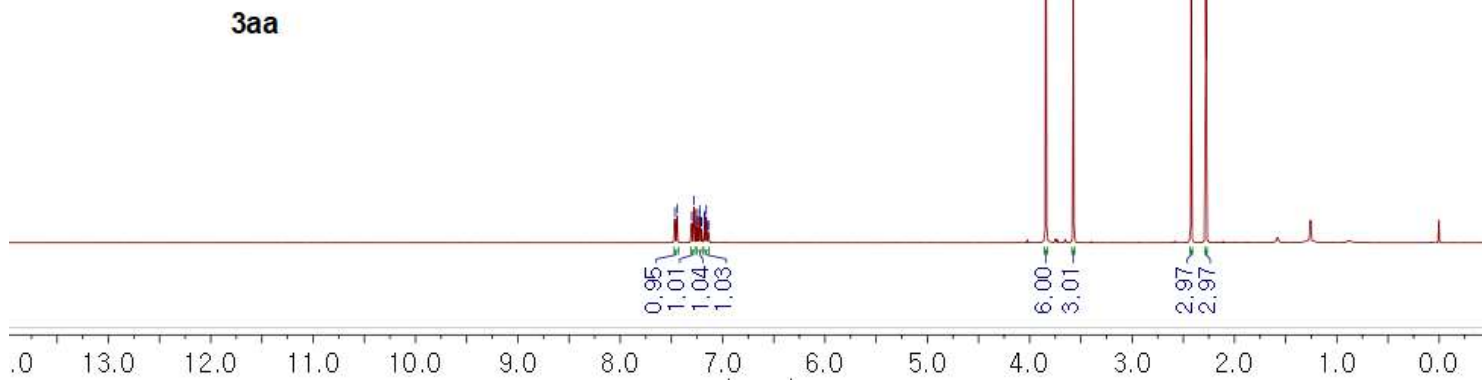

${ }^{1} \mathrm{H}$ NMR (400 MHz, $\mathrm{CDCl}_{3}$ ) spectra of dimethyl 4-acetyl-3,5-dimethylpyrano[4,3-b]indole$1,1(5 \mathrm{H})$-dicarboxylate (3aa)
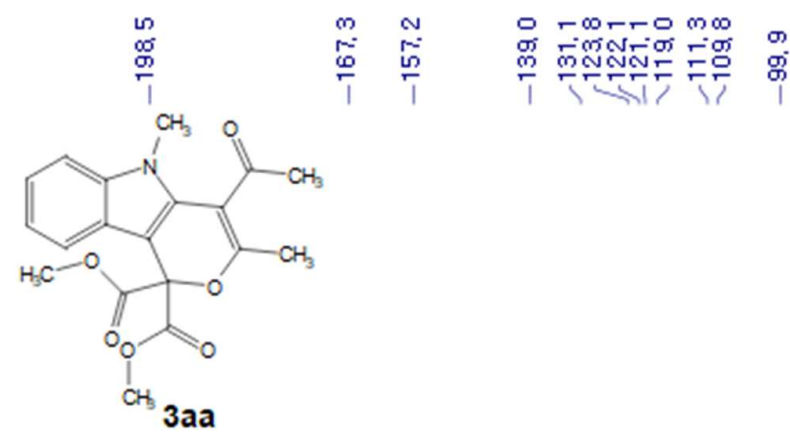

$\stackrel{\infty}{\infty}$

ஸे
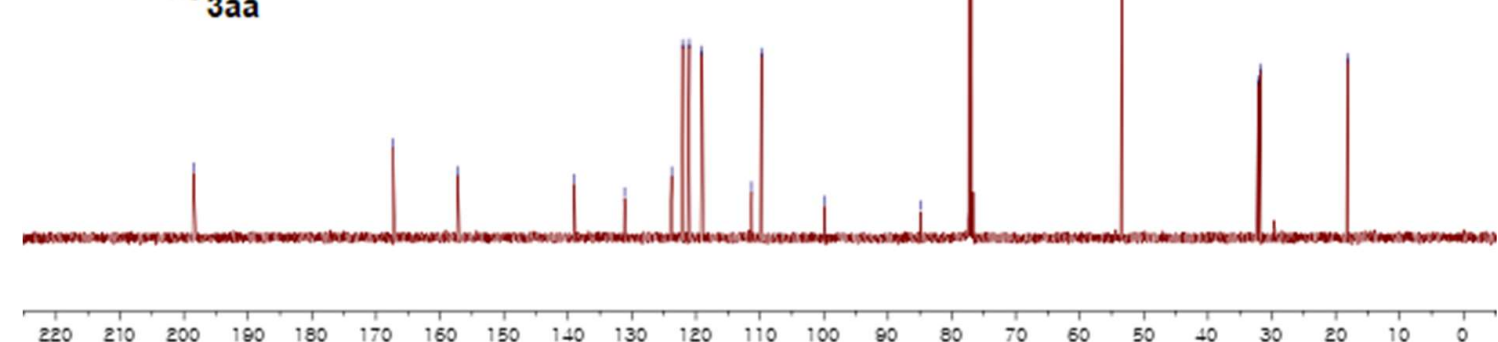

${ }^{13} \mathrm{C}$ NMR (100 MHz, $\left.\mathrm{CDCl}_{3}\right)$ spectra of dimethyl 4-acetyl-3,5-dimethylpyrano[4,3-b]indole1,1(5H)-dicarboxylate (3aa) 

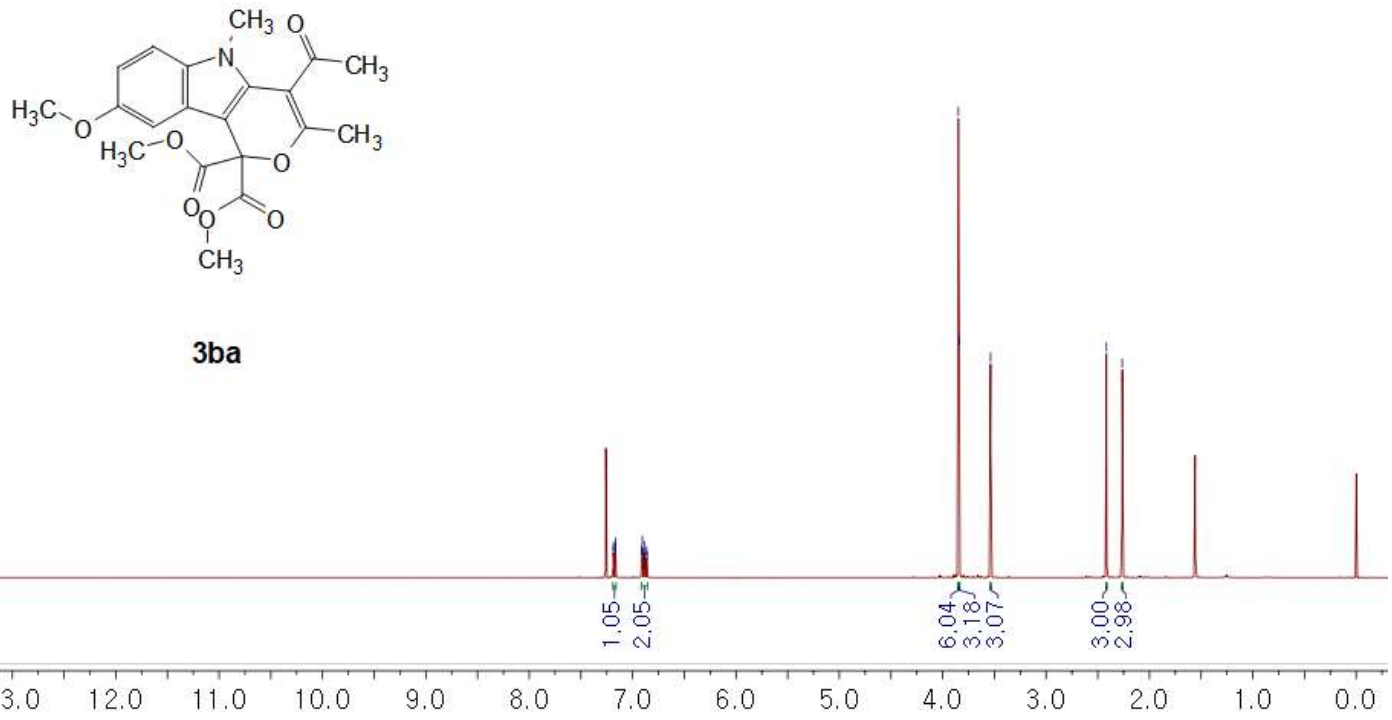

${ }^{1} \mathrm{H}$ NMR (400 MHz, $\mathrm{CDCl}_{3}$ ) spectra of dimethyl 4-acetyl-8-methoxy-3,5dimethylpyrano[4,3-b]indole-1,1(5H)-dicarboxylate (3ba)
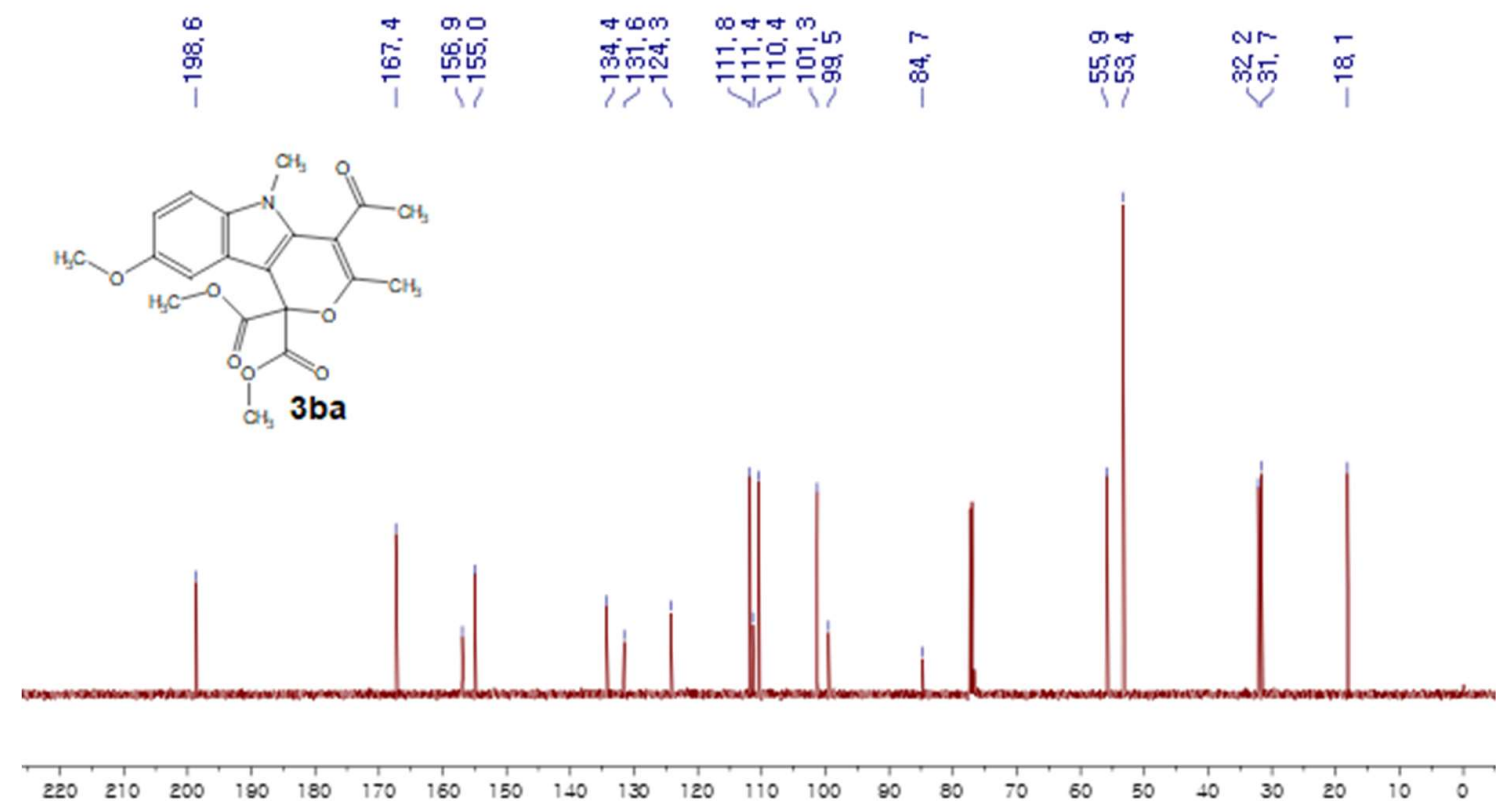

${ }^{13} \mathrm{C}$ NMR (100 MHz, $\mathrm{CDCl}_{3}$ ) spectra of dimethyl 4-acetyl-8-methoxy-3,5dimethylpyrano[4,3-b]indole-1,1(5H)-dicarboxylate (3ba) 


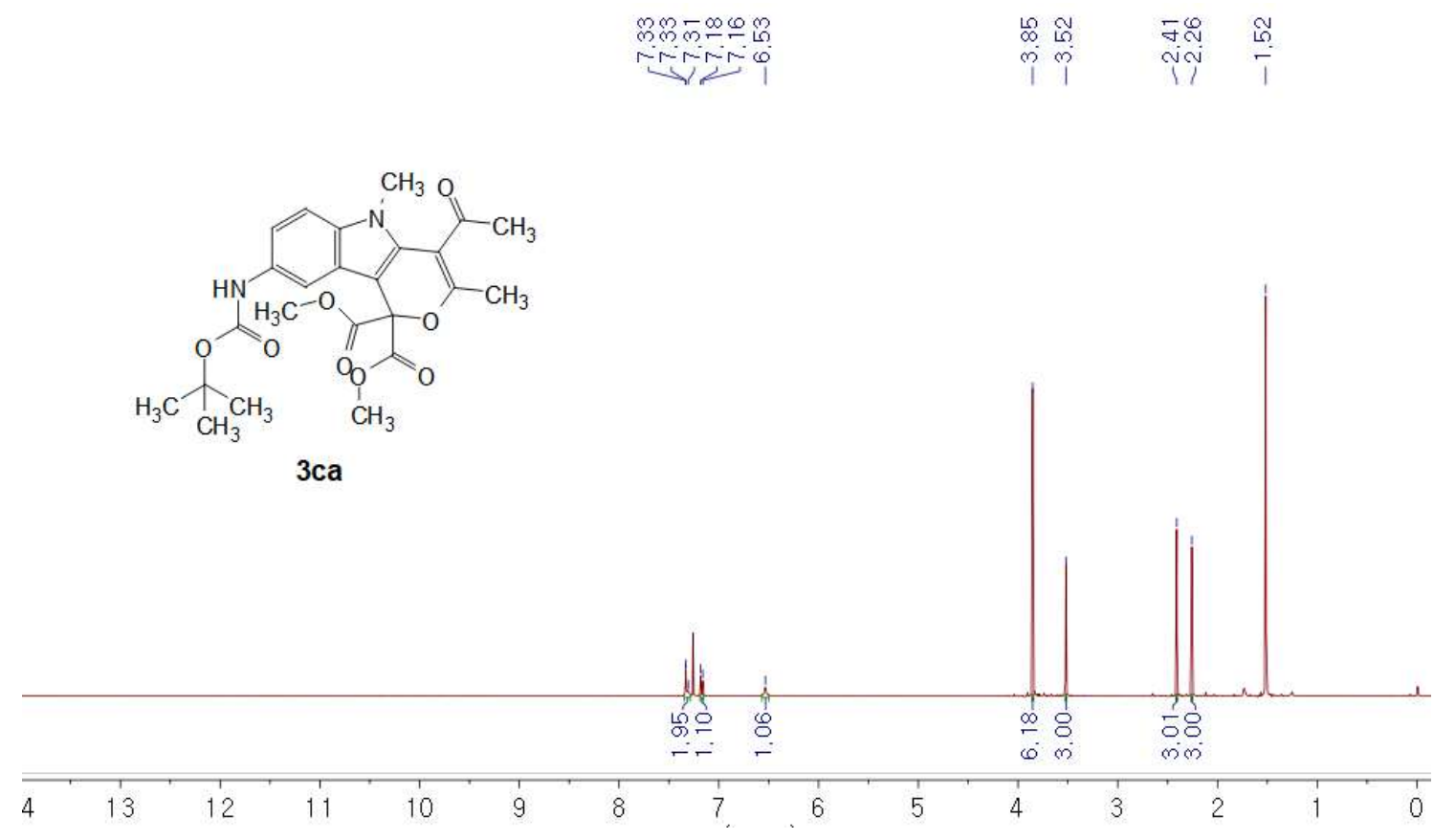

${ }^{1} \mathrm{H}$ NMR (400 MHz, $\mathrm{CDCl}_{3}$ ) spectra of dimethyl 4-acetyl-8-((tert-butoxycarbonyl)amino)3,5-dimethylpyrano[4,3-b]indole-1,1(5H)-dicarboxylate (3ca)
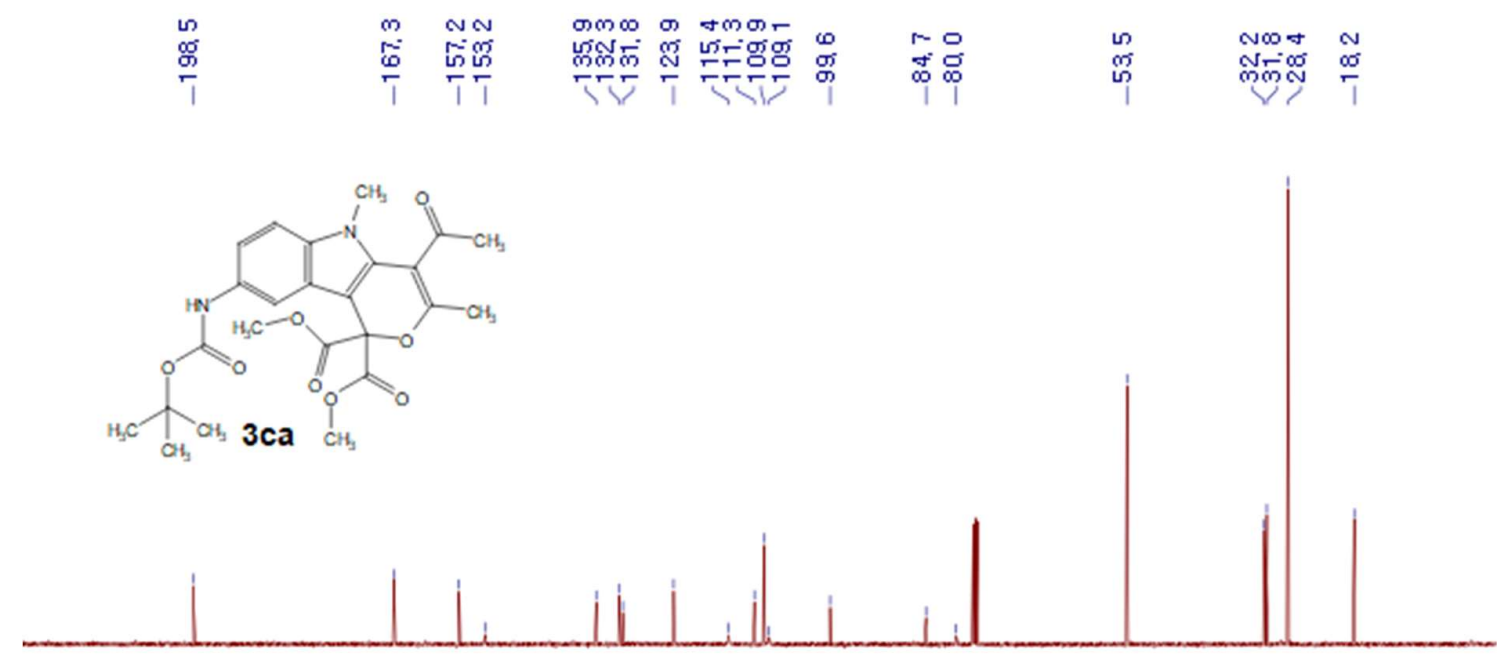

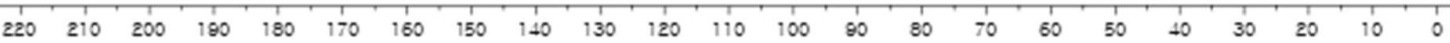

${ }^{13} \mathrm{C}$ NMR (100 MHz, $\left.\mathrm{CDCl}_{3}\right)$ spectra of dimethyl 4-acetyl-8-((tert-butoxycarbonyl)amino)3,5-dimethylpyrano[4,3-b]indole-1,1(5H)-dicarboxylate (3ca) 


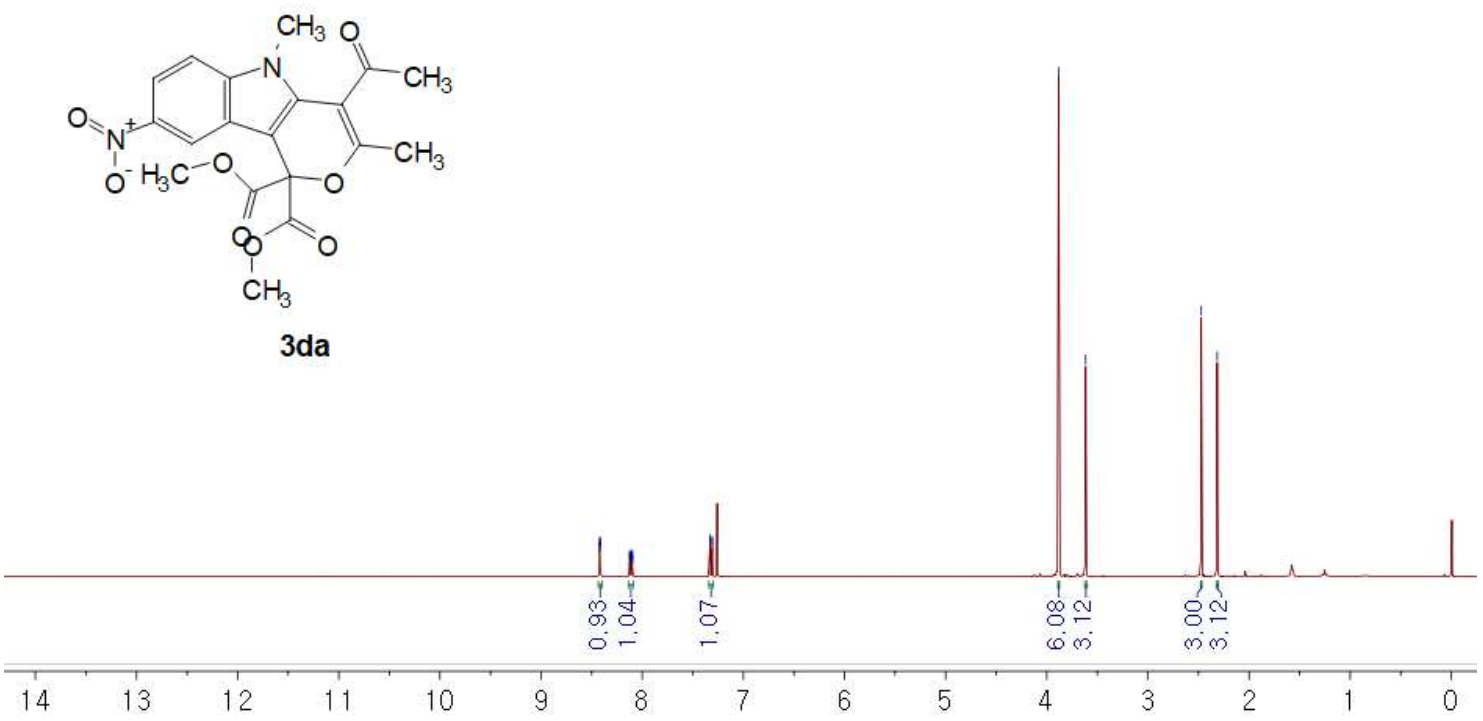

${ }^{1} \mathrm{H}$ NMR (400 MHz, $\mathrm{CDCl}_{3}$ ) spectra of dimethyl 4-acetyl-3,5-dimethyl-8-nitropyrano[4,3b]indole-1,1(5H)-dicarboxylate (3da)
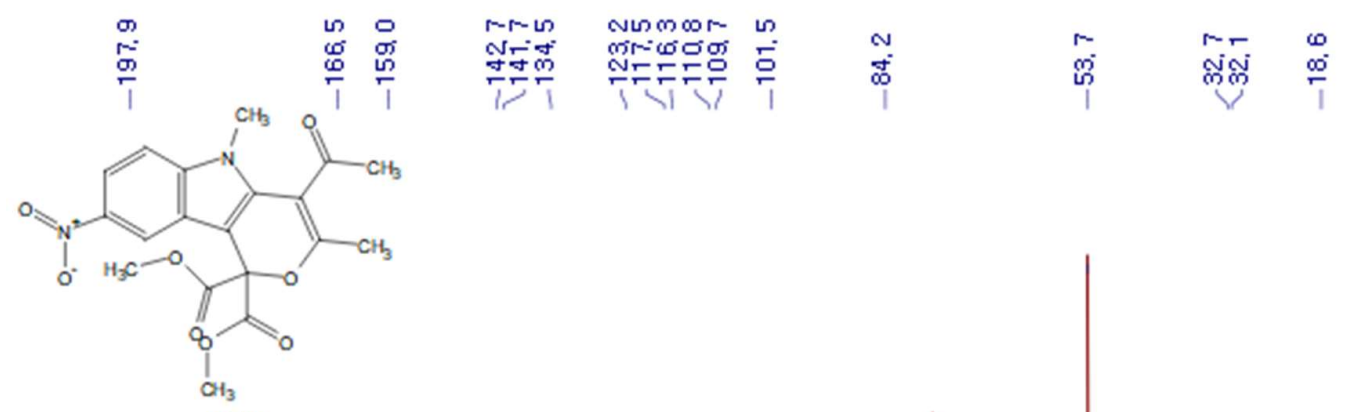

$3 d a$
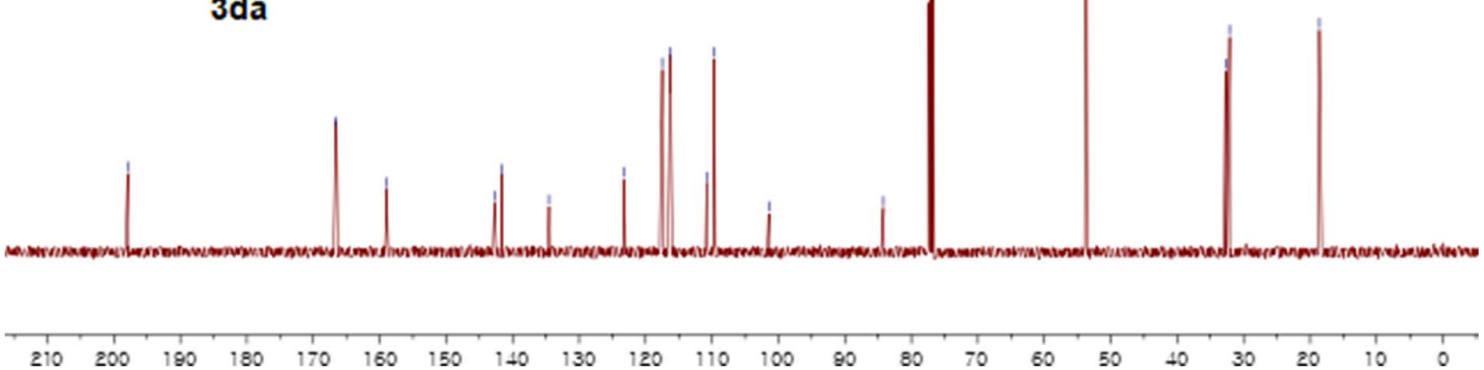

${ }^{13} \mathrm{C}$ NMR (100 MHz, $\mathrm{CDCl}_{3}$ ) spectra of dimethyl 4-acetyl-3,5-dimethyl-8-nitropyrano[4,3b]indole-1,1(5H)-dicarboxylate (3da) 


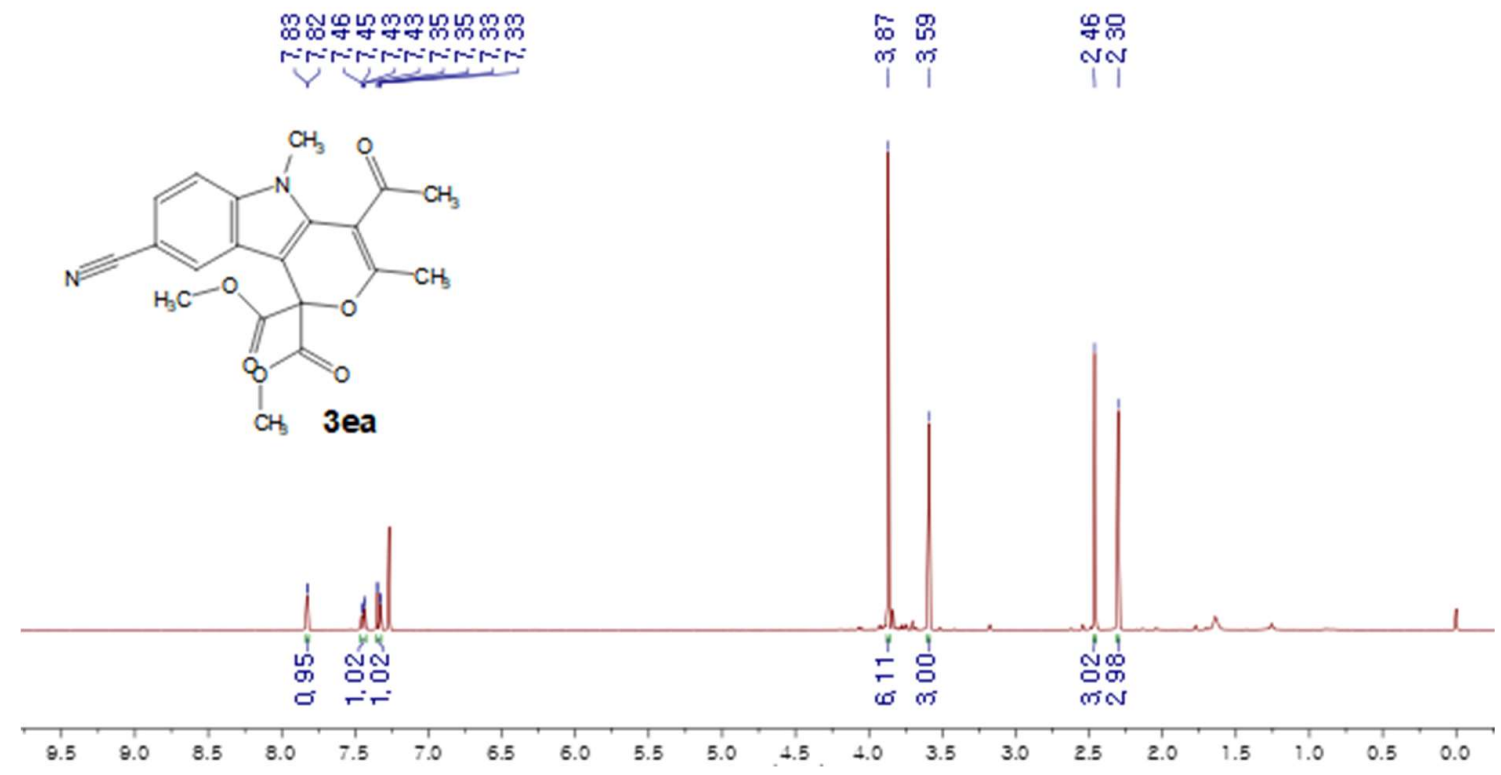

${ }^{1} \mathrm{H}$ NMR (400 MHz, $\mathrm{CDCl}_{3}$ ) spectra of dimethyl 4-acetyl-8-cyano-3,5-dimethylpyrano[4,3b]indole-1,1(5H)-dicarboxylate (3ea)
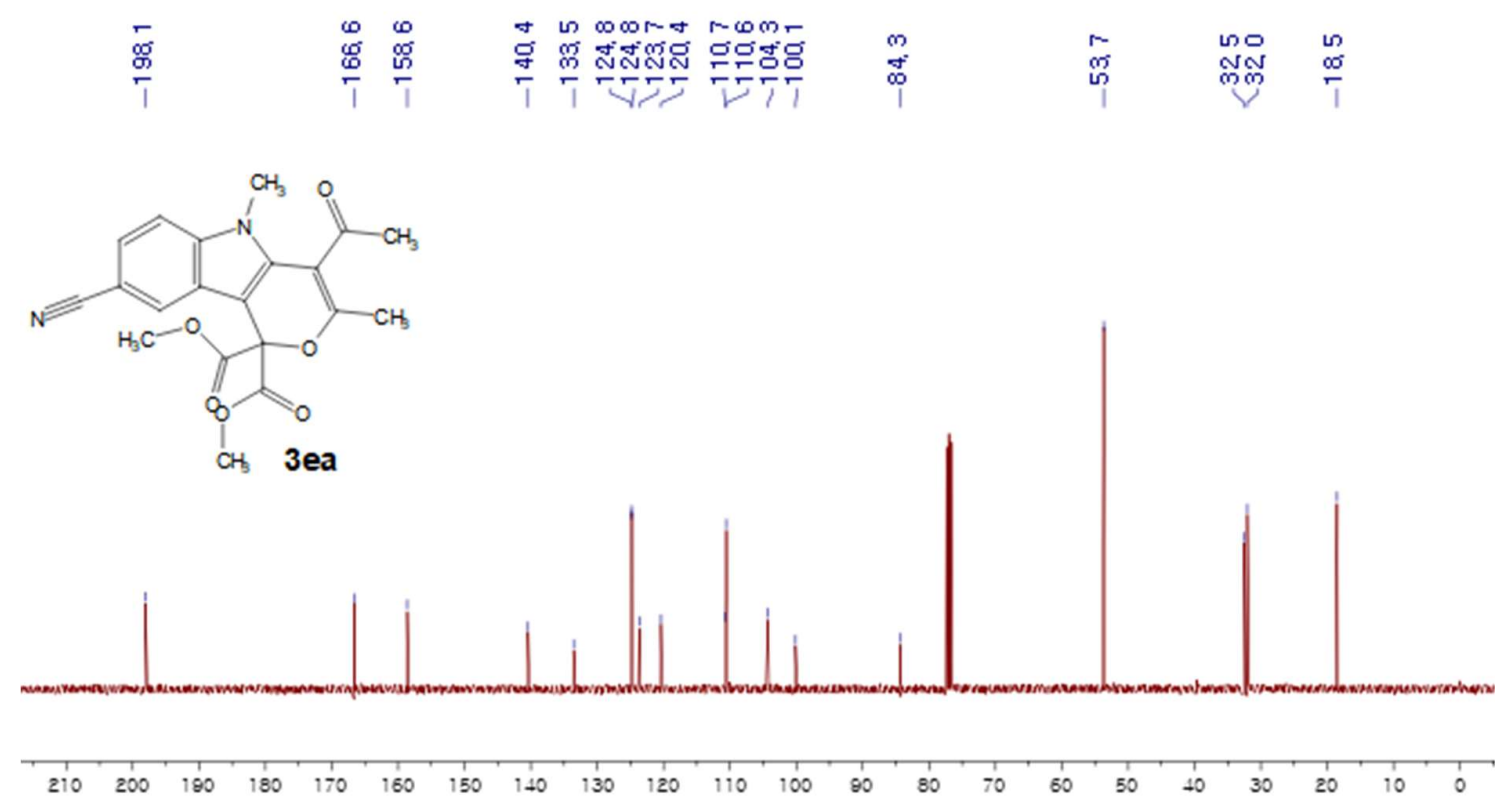

${ }^{13} \mathrm{C}$ NMR (100 MHz, $\mathrm{CDCl}_{3}$ ) spectra of dimethyl 4-acetyl-8-cyano-3,5-dimethylpyrano[4,3b]indole-1,1(5H)-dicarboxylate (3ea) 


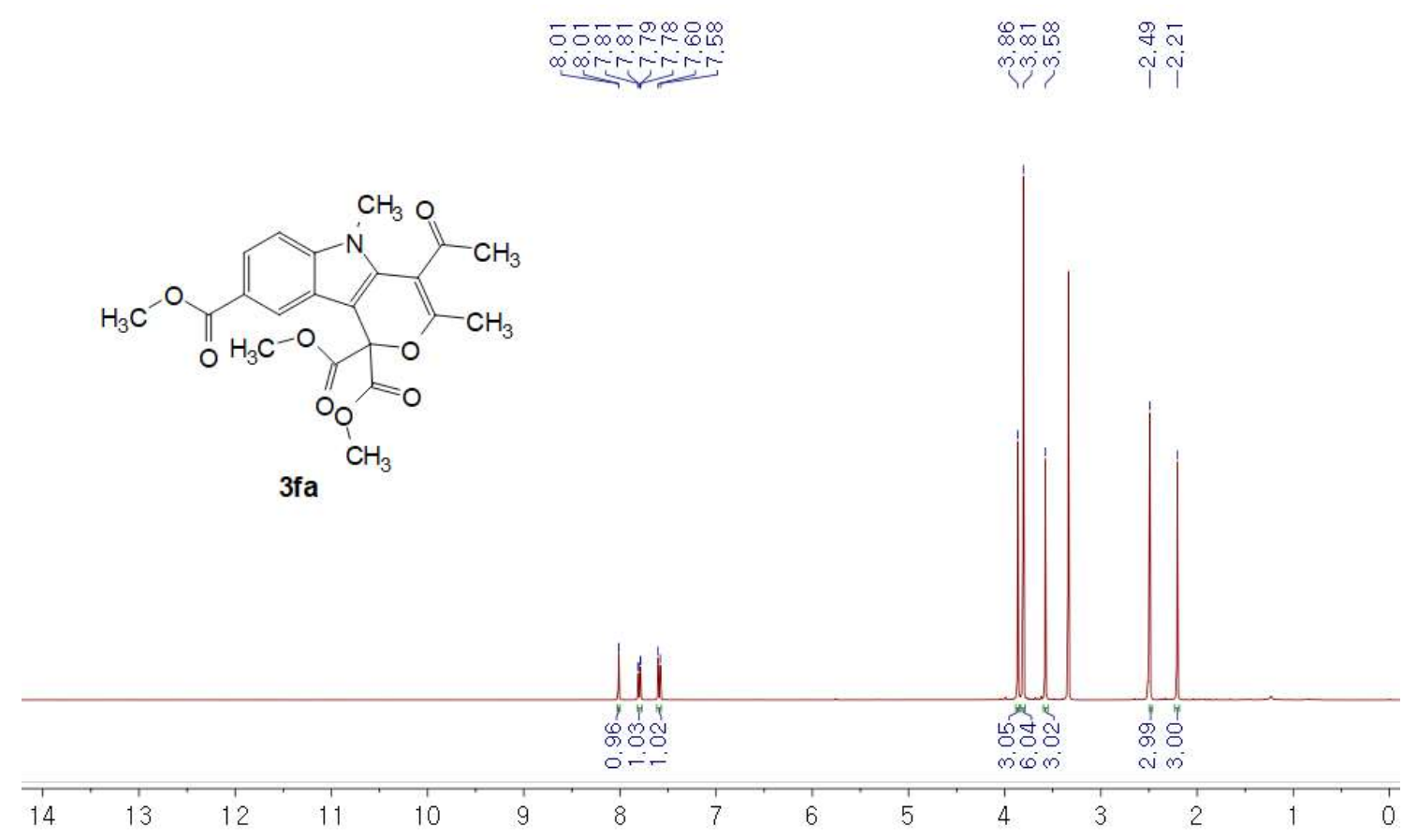

${ }^{1} \mathrm{H}$ NMR (400 MHz, DMSO) spectra of trimethyl 4-acetyl-3,5-dimethylpyrano[4,3-b]indole$1,1,8(5 \mathrm{H})$-tricarboxylate $(\mathbf{3 f a})$

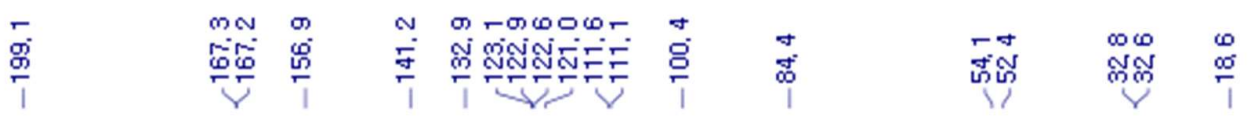
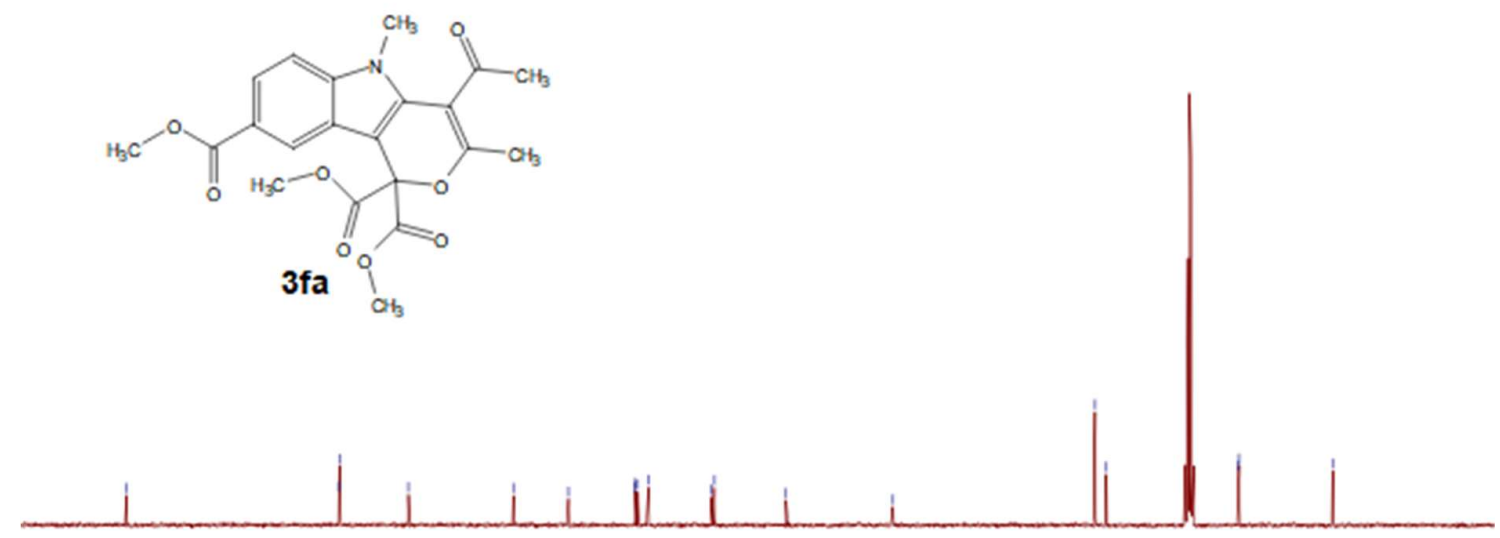

\begin{tabular}{llllllllllllllllllllllllll}
\hline 10 & 200 & 190 & 180 & 170 & 160 & 150 & 140 & 130 & 120 & 110 & 100 & 90 & 80 & 70 & 6 & 50 & 40 & 30 & 20 & 10 & 0
\end{tabular}

${ }^{13} \mathrm{C}$ NMR (100 MHz, DMSO) spectra of trimethyl 4-acetyl-3,5-dimethylpyrano[4,3-b]indole1,1,8(5H)-tricarboxylate (3fa) 


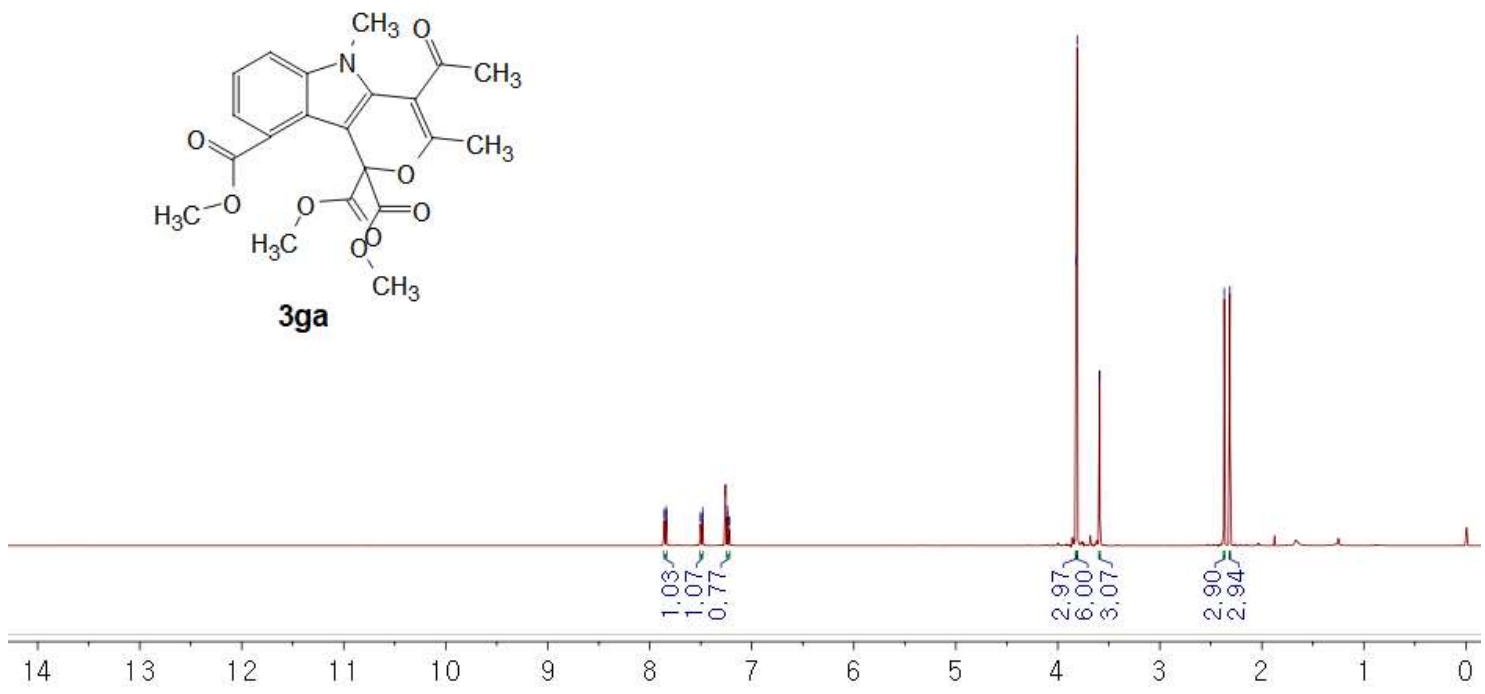

${ }^{1} \mathrm{H}$ NMR (400 MHz, $\mathrm{CDCl}_{3}$ ) spectra of trimethyl 4-acetyl-3,5-dimethylpyrano[4,3-b]indole$1,1,9(5 \mathrm{H})$-tricarboxylate $(\mathbf{3 g a})$
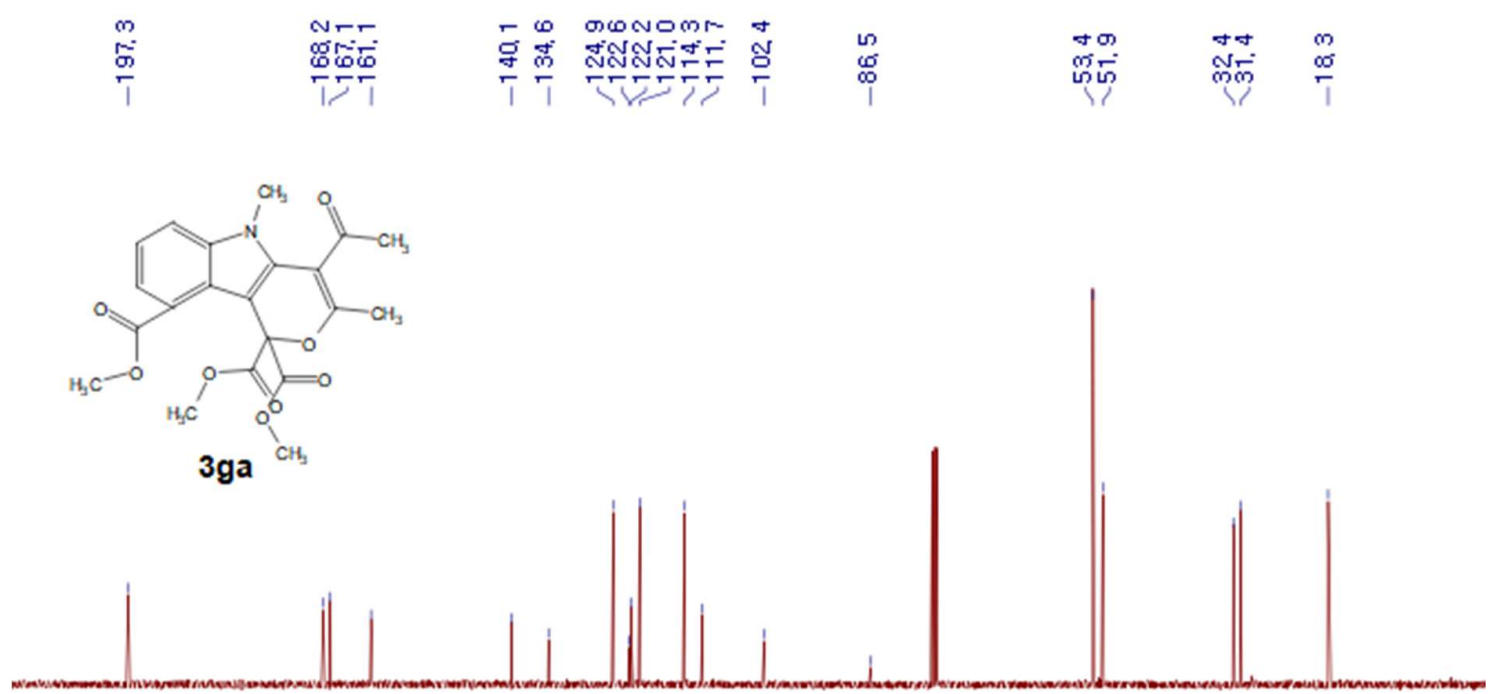

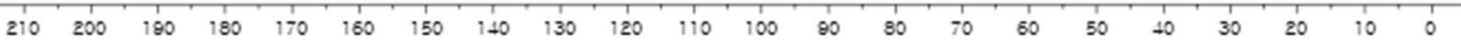

${ }^{13} \mathrm{C} \mathrm{NMR}\left(100 \mathrm{MHz}, \mathrm{CDCl}_{3}\right)$ spectra of trimethyl 4-acetyl-3,5-dimethylpyrano[4,3-b]indole1,1,9(5H)-tricarboxylate (3ga) 


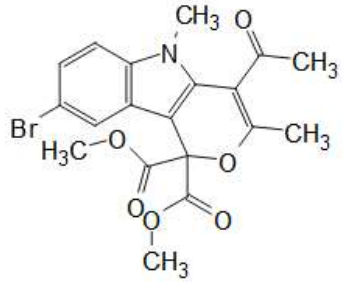

3ha

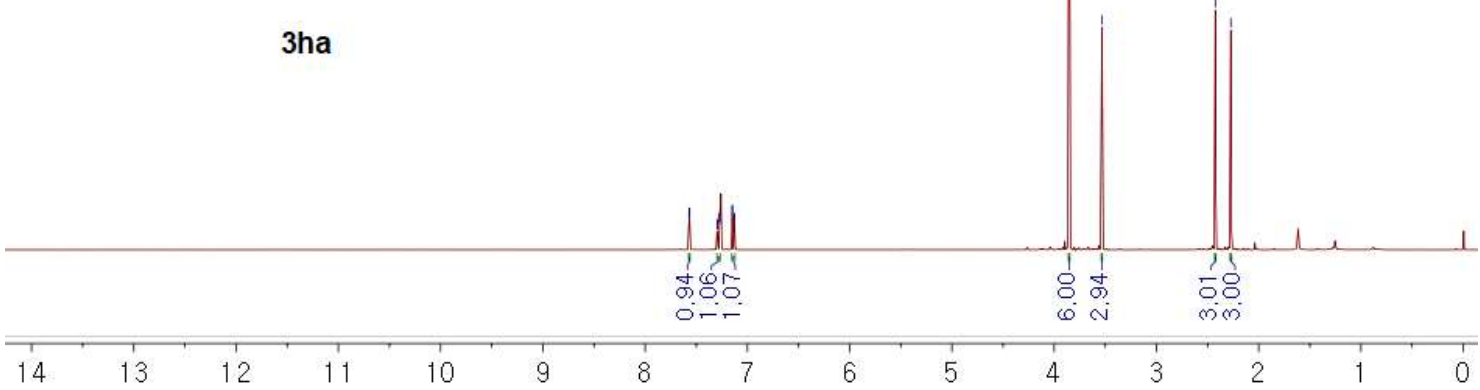

${ }^{1} \mathrm{H}$ NMR (400 MHz, $\mathrm{CDCl}_{3}$ ) spectra of dimethyl 4-acetyl-8-bromo-3,5-dimethylpyrano[4,3b]indole-1,1(5H)-dicarboxylate (3ha)
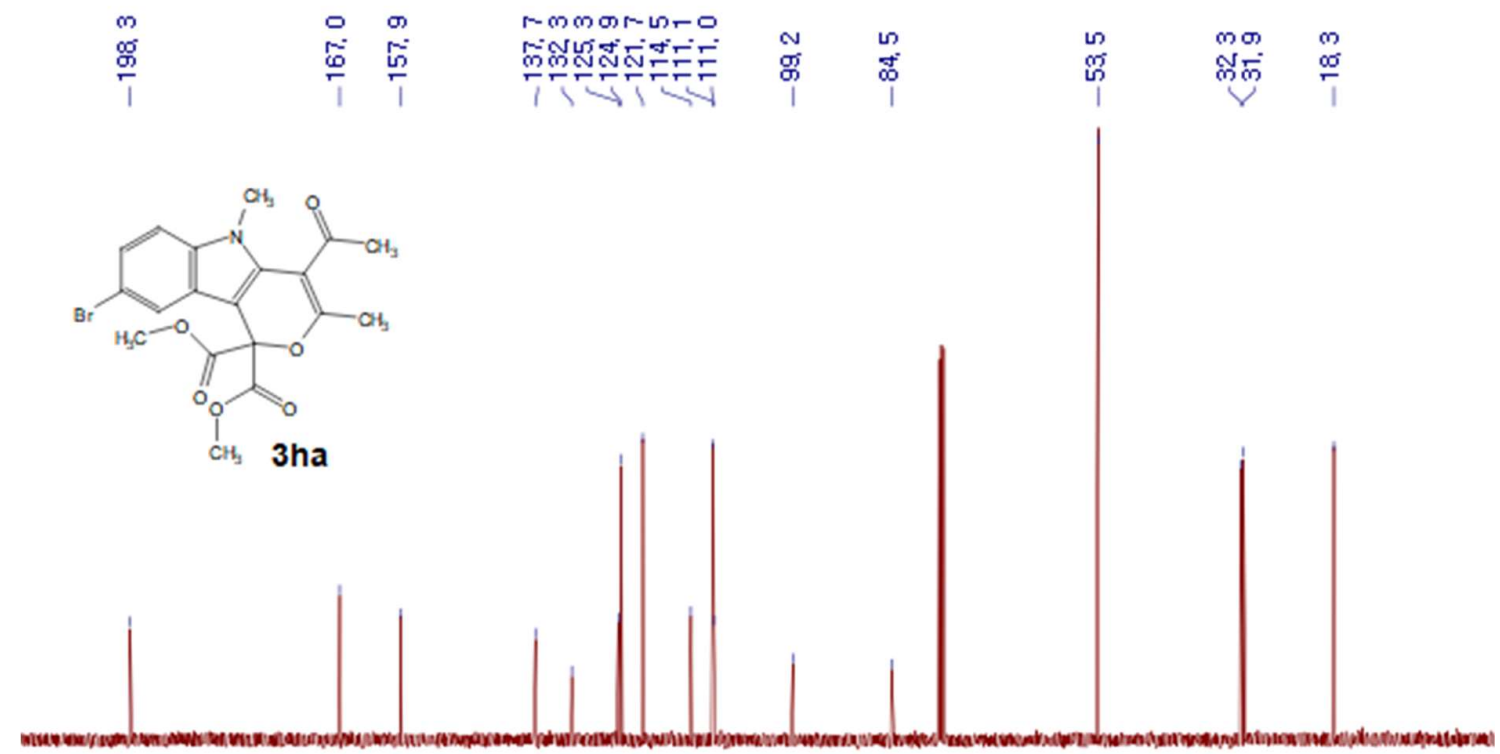

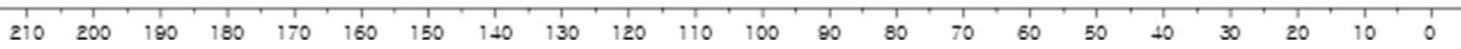

${ }^{13} \mathrm{C} \mathrm{NMR}\left(100 \mathrm{MHz}, \mathrm{CDCl}_{3}\right)$ spectra of dimethyl 4-acetyl-8-bromo-3,5-dimethylpyrano[4,3b]indole-1,1(5H)-dicarboxylate (3ha) 


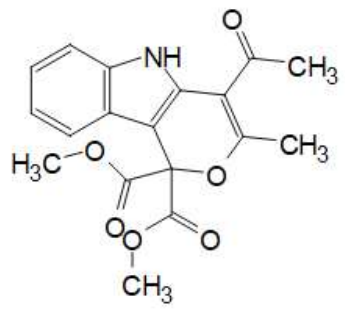

3ia

$\begin{array}{llllllllllllllll}1 & 1 & 1 & 1 & 1 & 1 & 1 & 1 & 1 & 1 & 1 & 1 & 1\end{array}$

${ }^{1} \mathrm{H}$ NMR (400 MHz, $\mathrm{CDCl}_{3}$ ) spectra of dimethyl 4-acetyl-3-methylpyrano[4,3-b]indole$1,1(5 \mathrm{H})$-dicarboxylate (3ia)

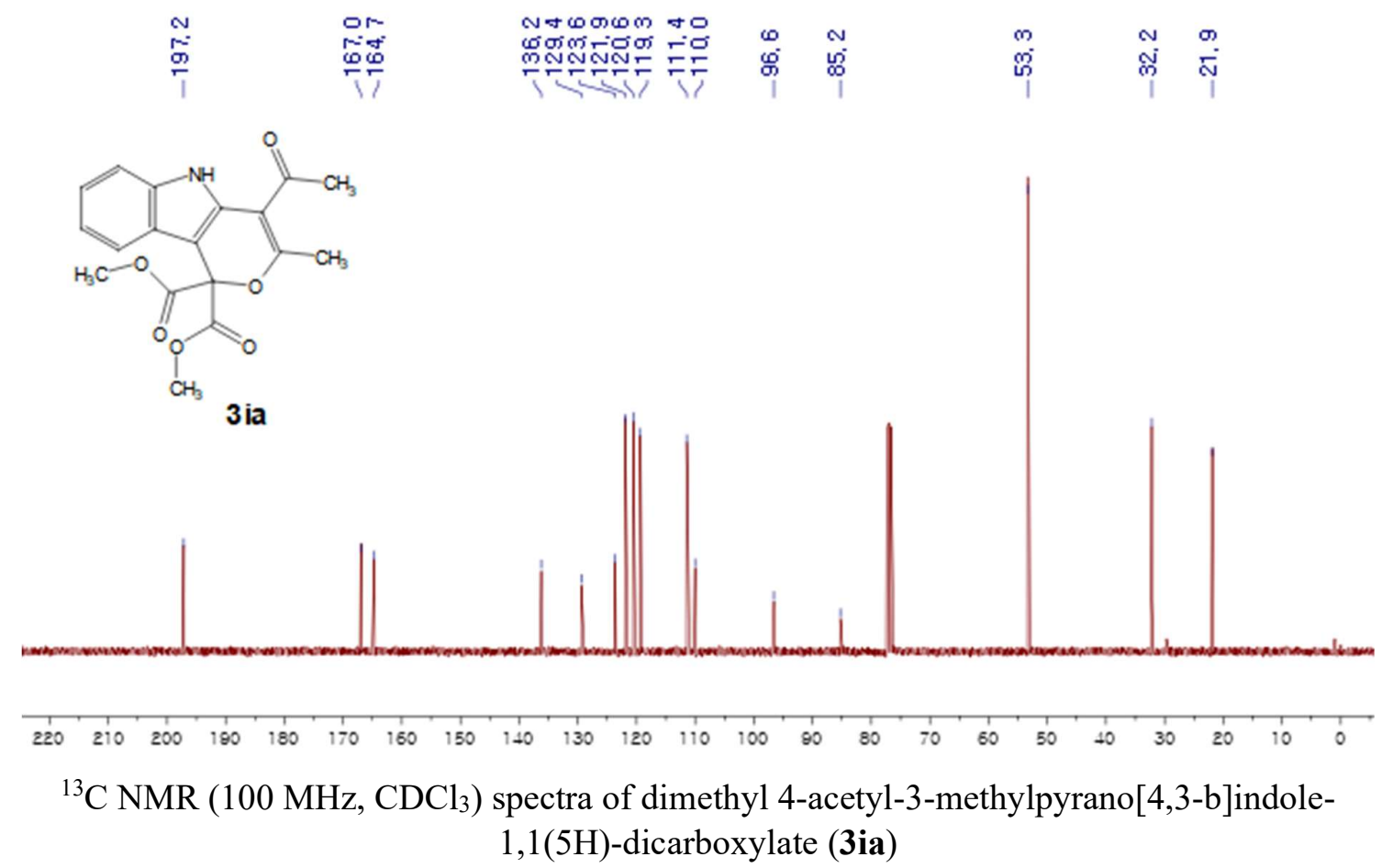




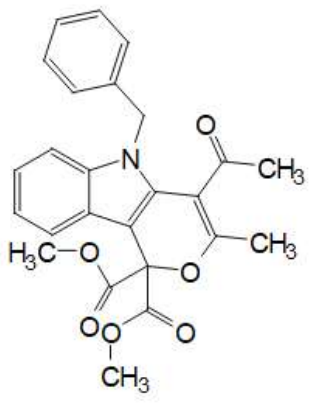

3ja

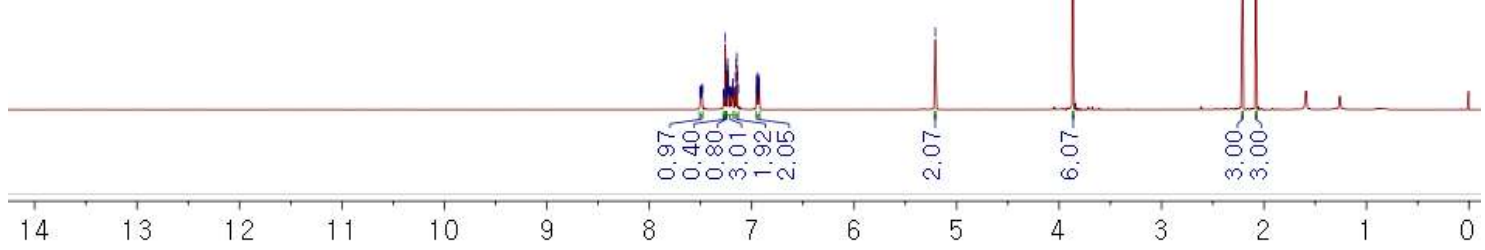

${ }^{1} \mathrm{H}$ NMR (400 MHz, $\mathrm{CDCl}_{3}$ ) spectra of dimethyl 4-acetyl-5-benzyl-3-methylpyrano[4,3b]indole-1,1(5H)-dicarboxylate $(\mathbf{3 j a})$
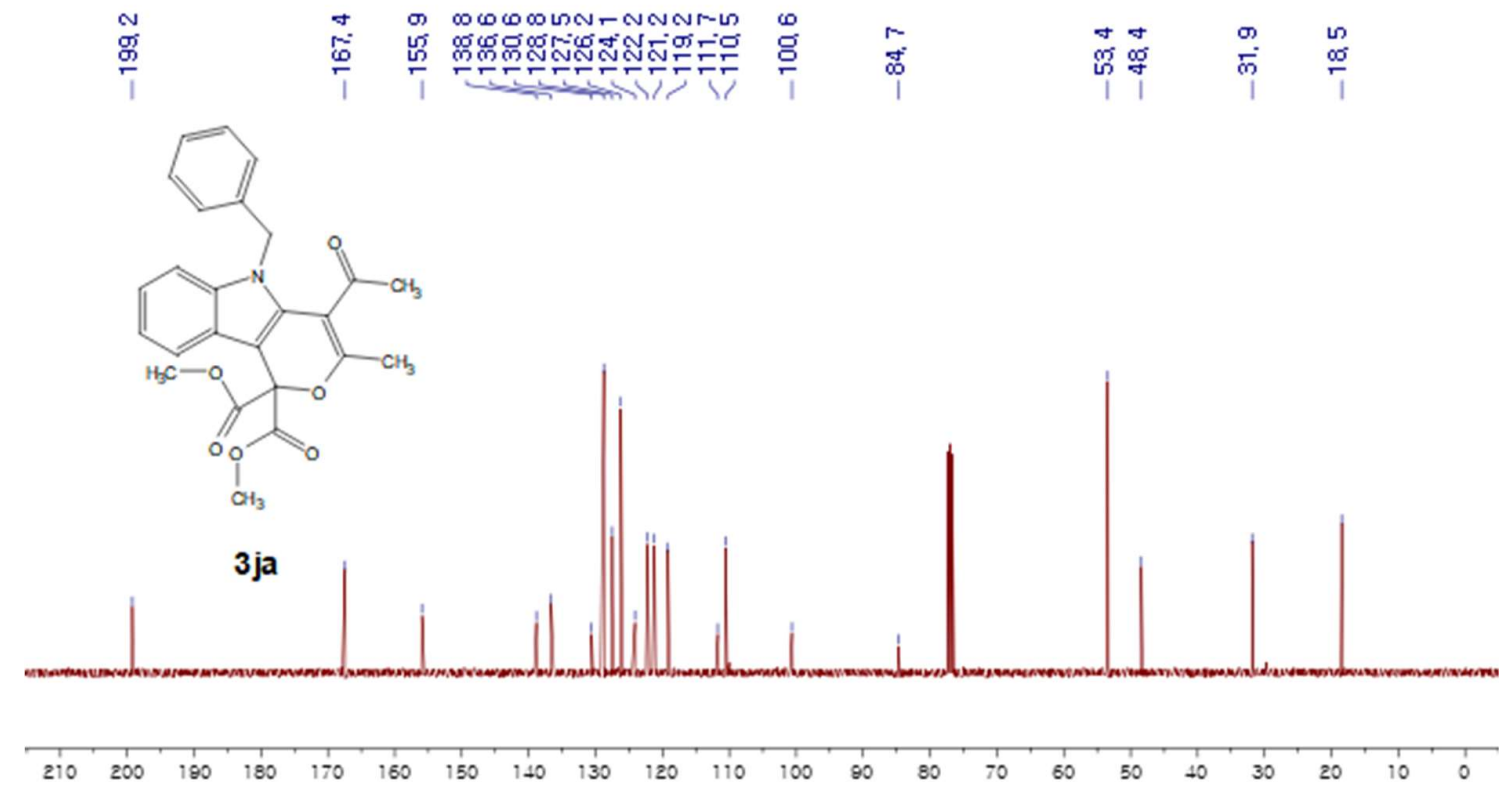

${ }^{13} \mathrm{C}$ NMR (100 MHz, $\left.\mathrm{CDCl}_{3}\right)$ spectra of dimethyl 4-acetyl-5-benzyl-3-methylpyrano[4,3b]indole-1,1(5H)-dicarboxylate (3ja) 


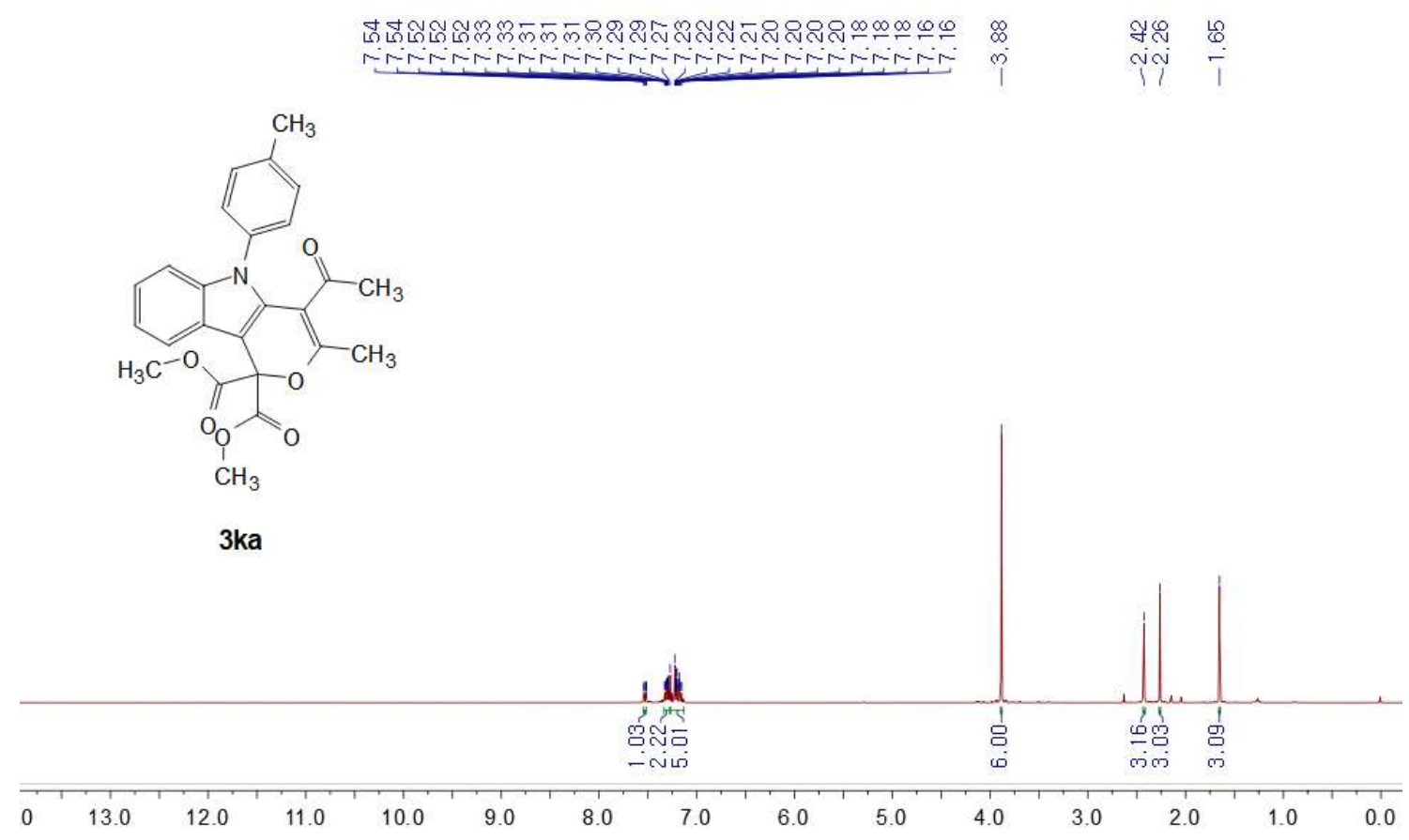

${ }^{1} \mathrm{H}$ NMR (400 MHz, $\mathrm{CDCl}_{3}$ ) spectra of dimethyl 4-acetyl-3-methyl-5-(p-tolyl)pyrano[4,3b]indole-1,1(5H)-dicarboxylate (3ka)

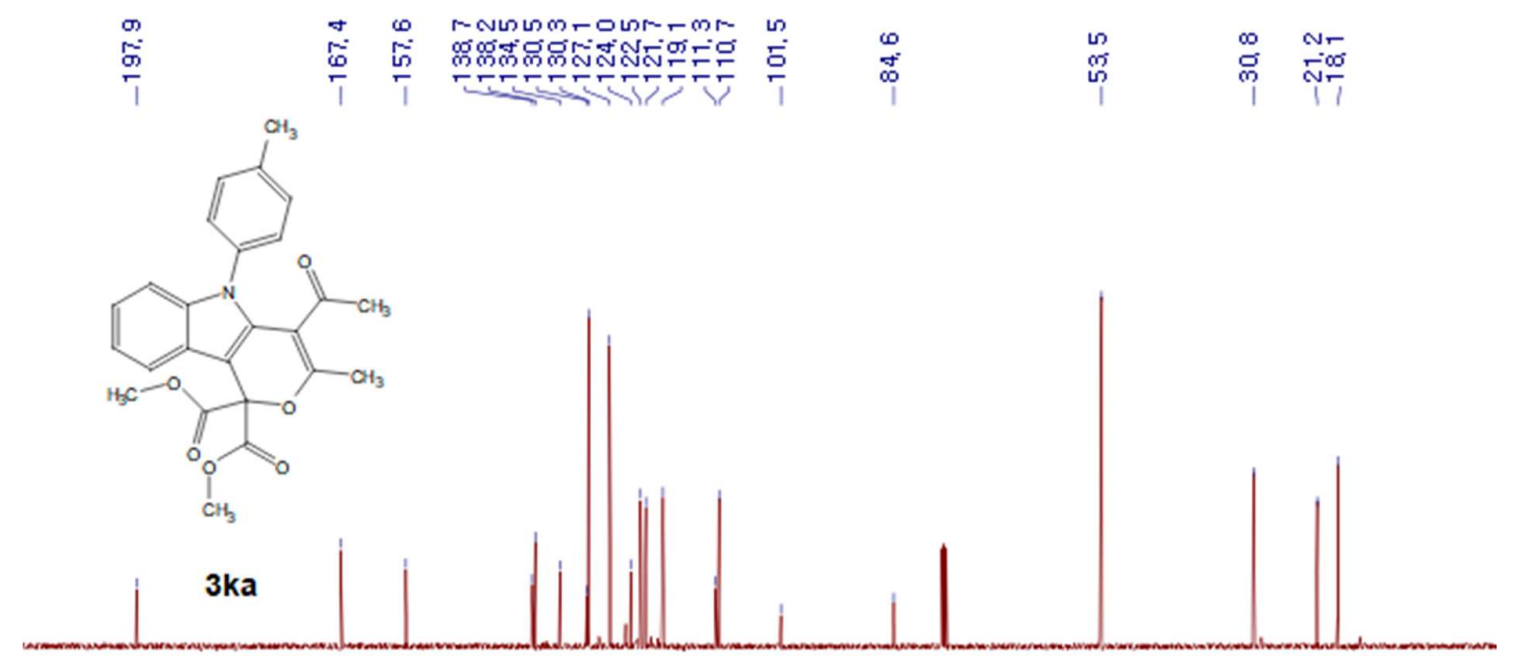

$\begin{array}{lllllllllllllllllllllllll}1 & 10 & 200 & 190 & 180 & 170 & 160 & 150 & 140 & 130 & 120 & 110 & 100 & 90 & 80 & 70 & 60 & 50 & 40 & 30 & 20 & 10 & 0\end{array}$

${ }^{13} \mathrm{C}$ NMR $\left(100 \mathrm{MHz}, \mathrm{CDCl}_{3}\right)$ spectra of dimethyl 4-acetyl-3-methyl-5-(p-tolyl)pyrano[4,3b]indole-1,1(5H)-dicarboxylate $(3 \mathbf{k a})$ 


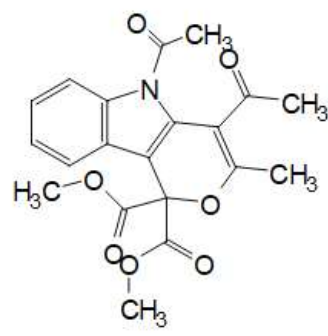

3la

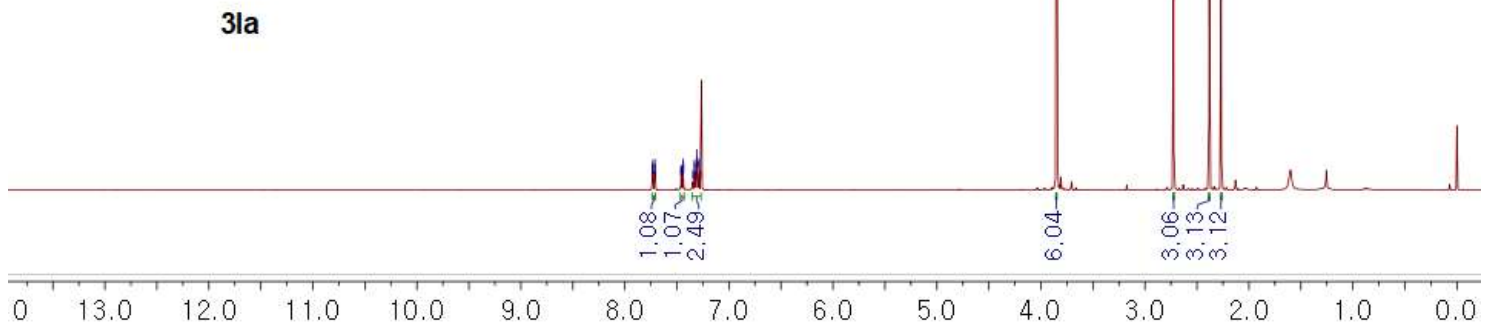

${ }^{1} \mathrm{H}$ NMR (400 MHz, $\mathrm{CDCl}_{3}$ ) spectra of dimethyl 4,5-diacetyl-3-methylpyrano[4,3-b]indole1,1(5H)-dicarboxylate (3la)
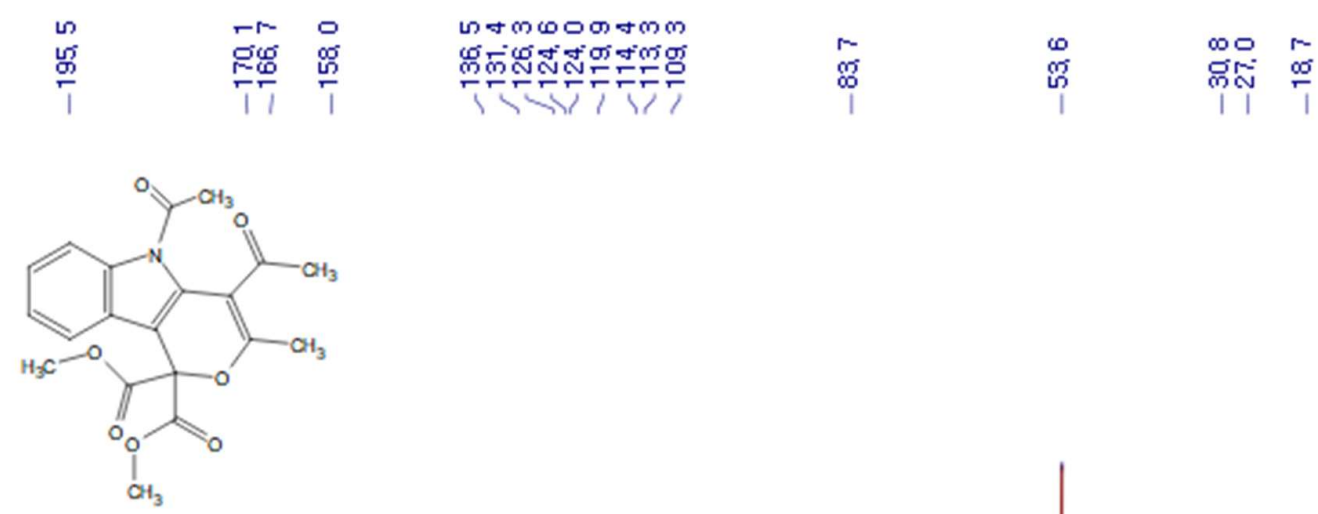

3la
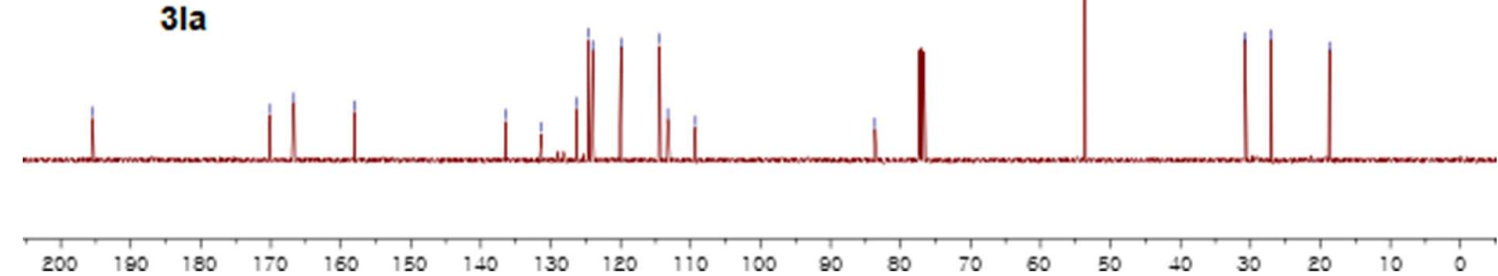

${ }^{13} \mathrm{C}$ NMR $\left(100 \mathrm{MHz}, \mathrm{CDCl}_{3}\right.$ ) spectra of dimethyl 4,5-diacetyl-3-methylpyrano[4,3-b]indole1,1(5H)-dicarboxylate (3la) 

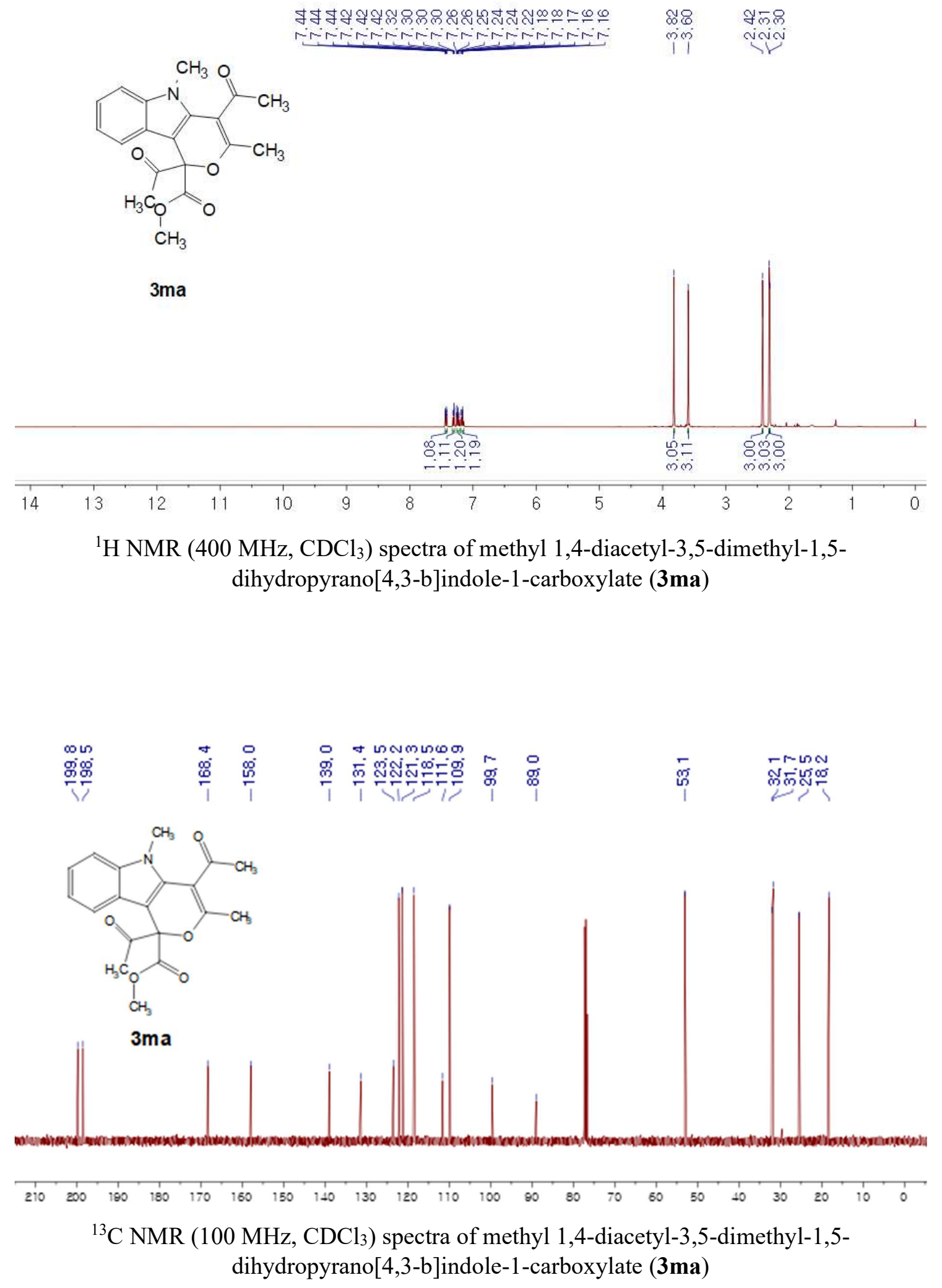


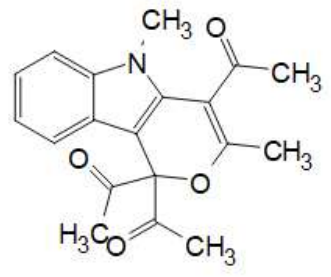

3na

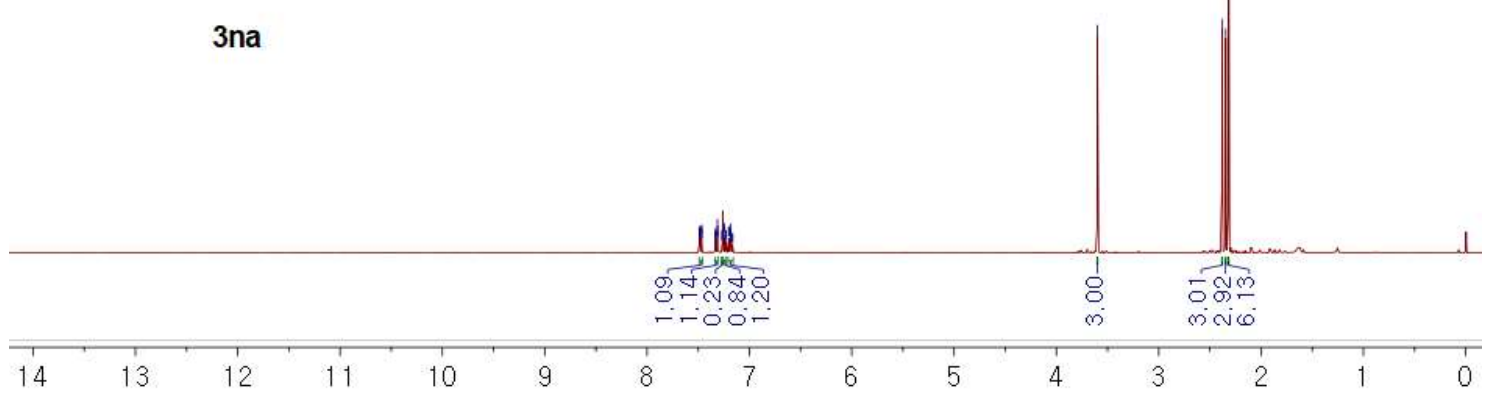

${ }^{1} \mathrm{H}$ NMR (400 MHz, $\mathrm{CDCl}_{3}$ ) spectra of 1,1',1"-(3,5-dimethyl-1,5-dihydropyrano[4,3b]indole-1,1,4-triyl)tris(ethan-1-one) (3na)

कू⿻

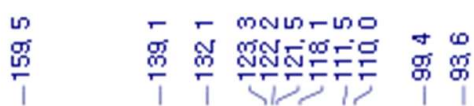

लํㅐㅇㅛ<smiles>CC(=O)C1=C(C)OC(C(C)=O)(C(C)=O)c2c1n(C)c1ccccc21</smiles>

3na
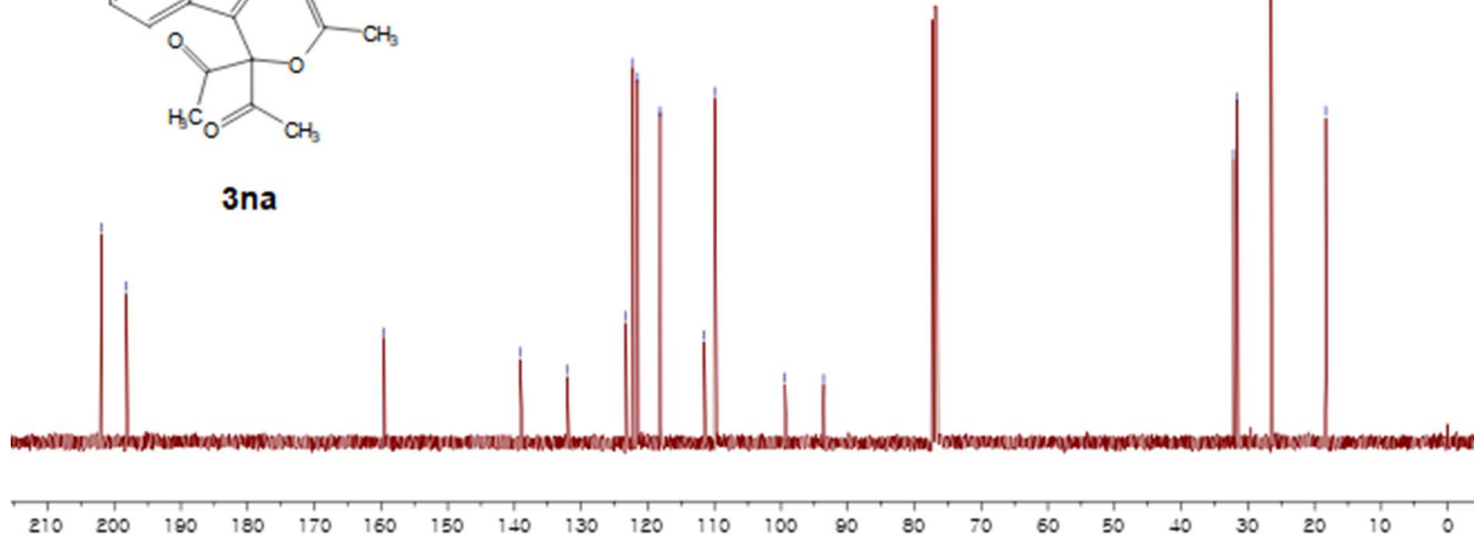

${ }^{13} \mathrm{C}$ NMR (100 MHz, $\left.\mathrm{CDCl}_{3}\right)$ spectra of 1,1',1'-(3,5-dimethyl-1,5-dihydropyrano[4,3b]indole-1,1,4-triyl)tris(ethan-1-one) (3na) 


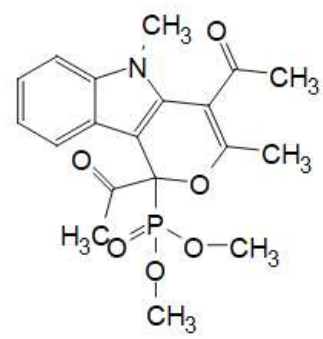

3oa

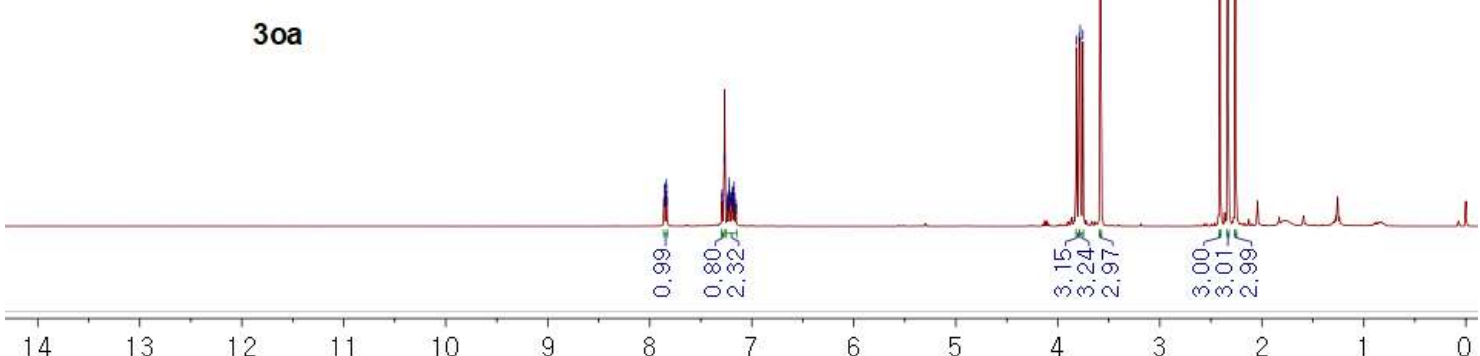

${ }^{1} \mathrm{H}$ NMR (400 MHz, $\left.\mathrm{CDCl}_{3}\right)$ spectra of dimethyl (1,4-diacetyl-3,5-dimethyl-1,5dihydropyrano[4,3-b]indol-1-yl)phosphonate (3oa)
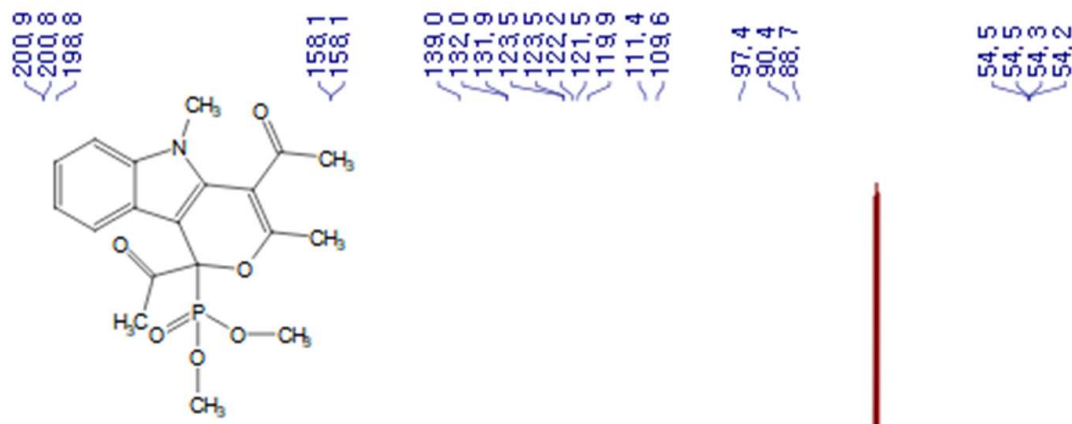

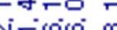

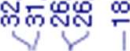

$30 a$

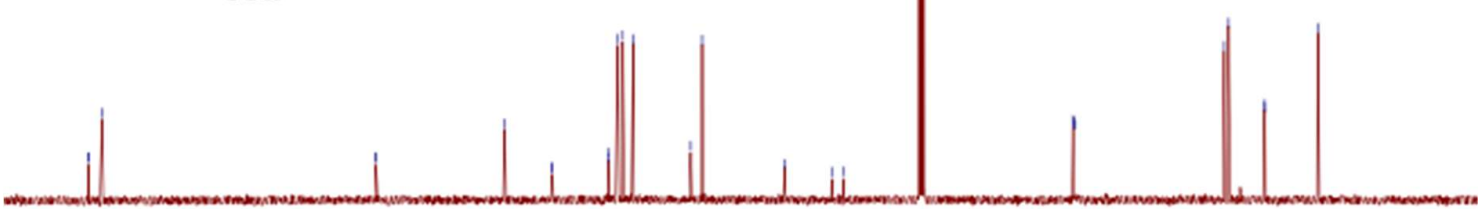

$\begin{array}{llllllllllllllllllllllllllll}210 & 200 & 190 & 180 & 170 & 160 & 150 & 140 & 130 & 120 & 110 & 100 & 90 & 80 & 70 & 60 & 50 & 40 & 30 & 20 & 10 & 0\end{array}$

${ }^{13} \mathrm{C}$ NMR (100 MHz, $\left.\mathrm{CDCl}_{3}\right)$ spectra of dimethyl (1,4-diacetyl-3,5-dimethyl-1,5dihydropyrano[4,3-b]indol-1-yl)phosphonate (3oa) 


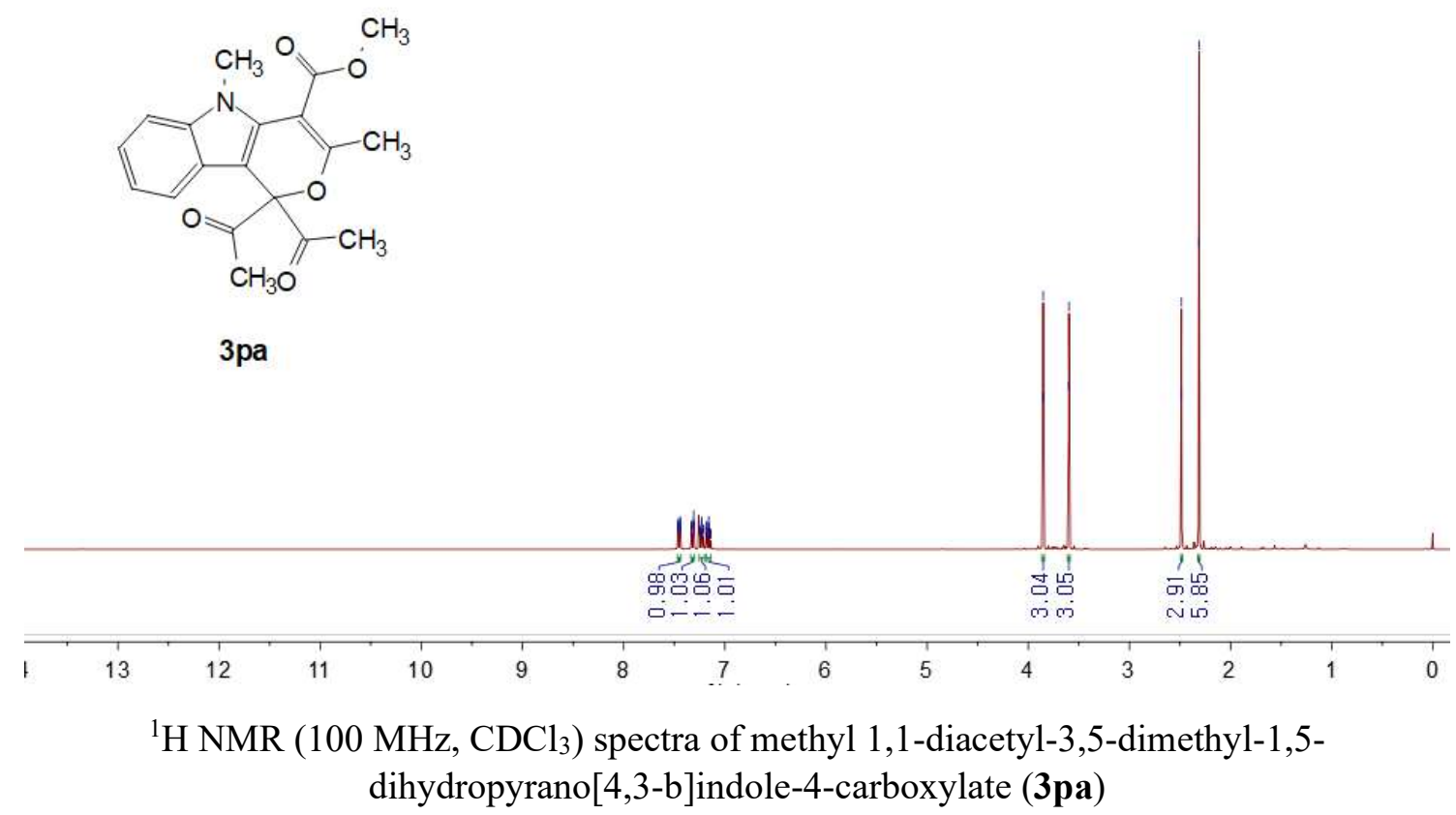

Б)

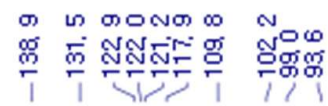

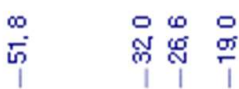
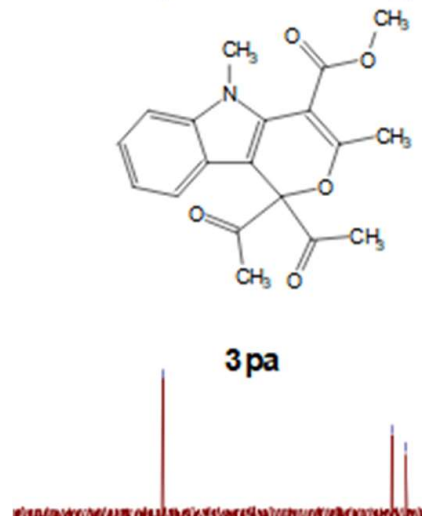

$3 \mathrm{pa}$

$220 \quad 210$

${ }^{13} \mathrm{C}$ NMR (100 MHz, $\mathrm{CDCl}_{3}$ ) spectra of methyl 1,1-diacetyl-3,5-dimethyl-1,5dihydropyrano[4,3-b]indole-4-carboxylate (3pa) 


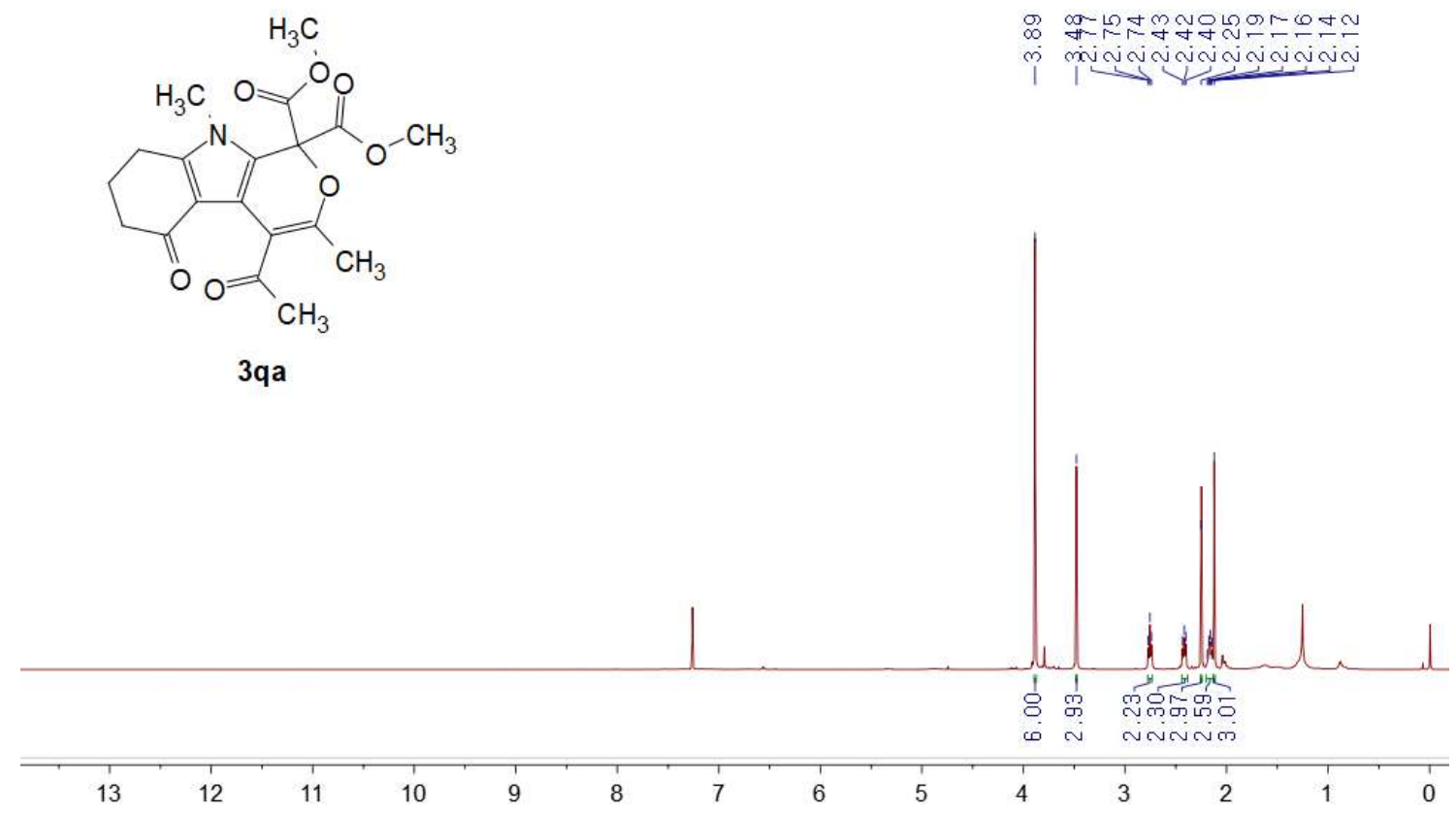

${ }^{1} \mathrm{H}$ NMR (400 MHz, $\mathrm{CDCl}_{3}$ ) spectra of Dimethyl 4-acetyl-3,9-dimethyl-5-oxo-6,7,8,9tetrahydropyrano[3,4-b]indole-1,1(5H)-dicarboxylate (3qa)
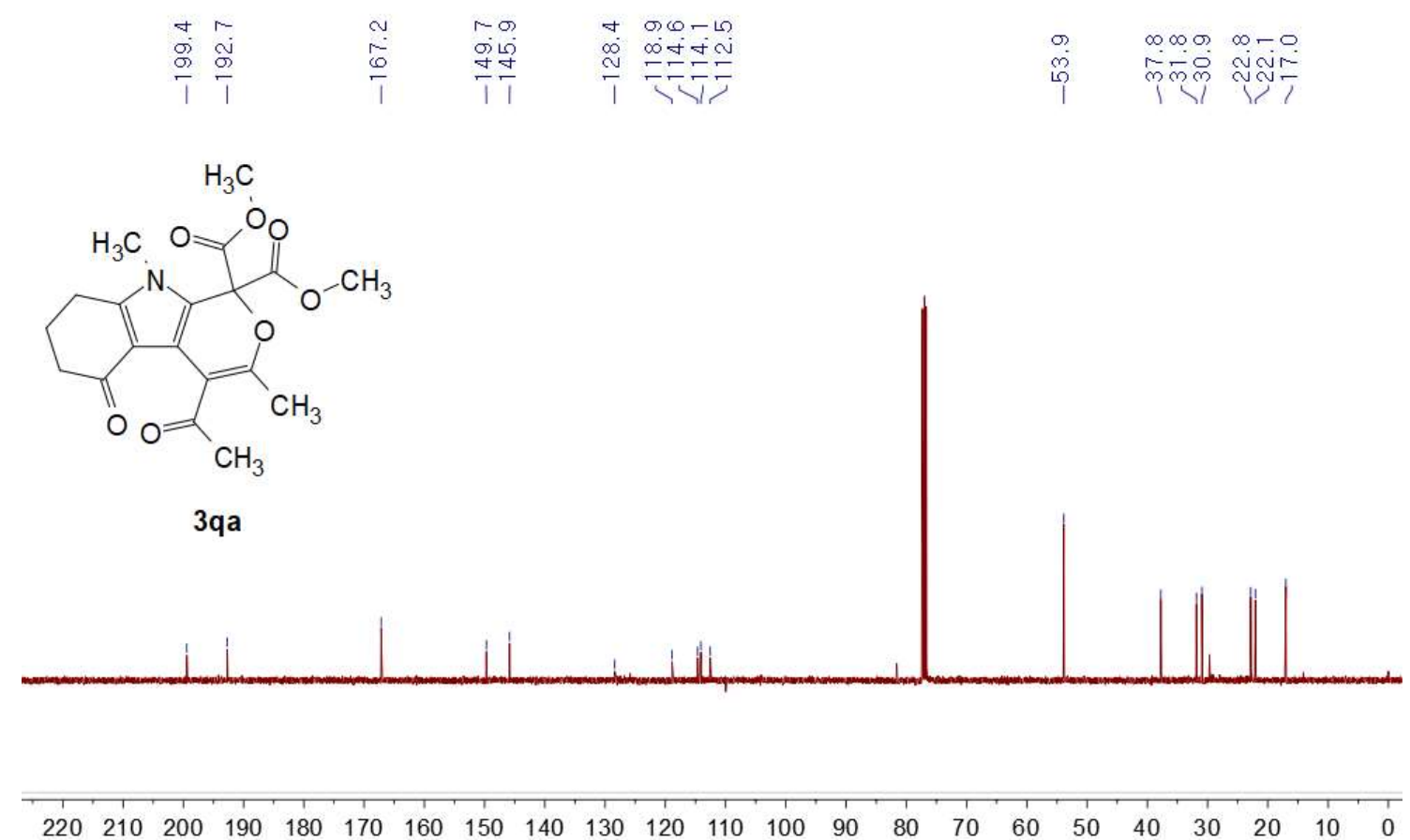

${ }^{13} \mathrm{C}$ NMR (100 MHz, $\mathrm{CDCl}_{3}$ ) spectra of Dimethyl 4-acetyl-3,9-dimethyl-5-oxo-6,7,8,9tetrahydropyrano[3,4-b]indole-1,1(5H)-dicarboxylate (3qa) 

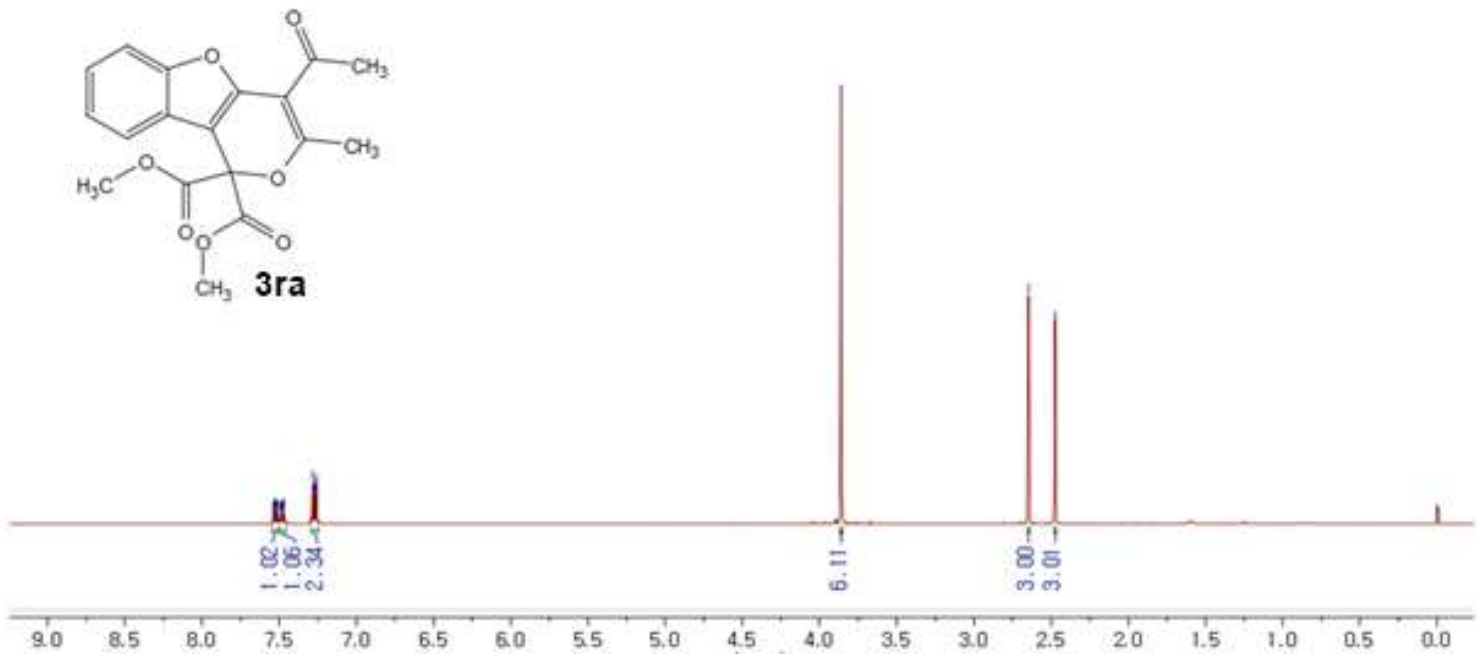

${ }^{1} \mathrm{H}$ NMR (400 MHz, $\mathrm{CDCl}_{3}$ ) spectra of dimethyl 4-acetyl-3-methyl-1H-pyrano[4,3b] benzofuran-1,1-dicarboxylate (3ra)
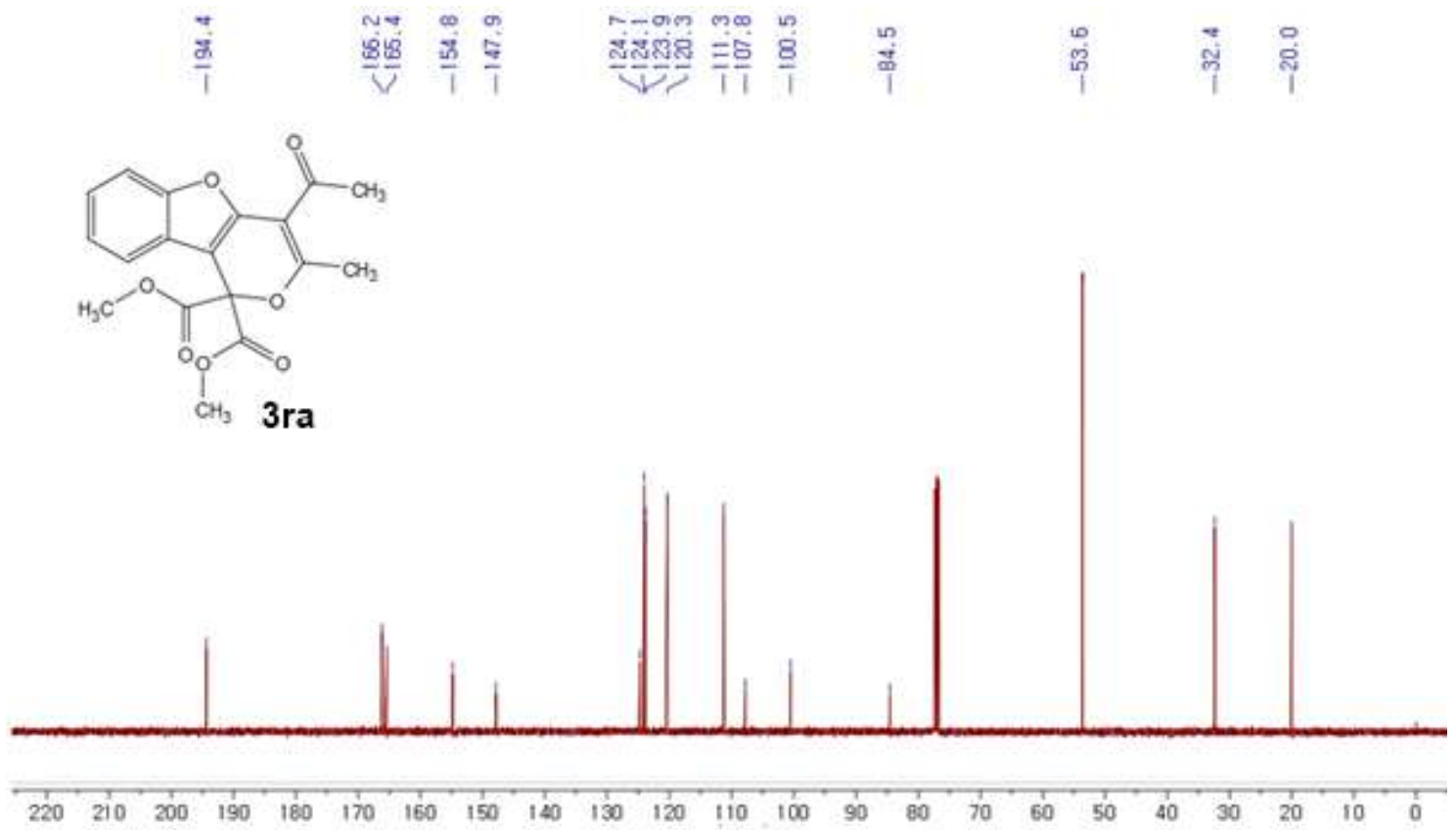

${ }^{13} \mathrm{C}$ NMR $\left(100 \mathrm{MHz}, \mathrm{CDCl}_{3}\right)$ spectra of dimethyl 4-acetyl-3-methyl-1H-pyrano[4,3b] benzofuran-1,1-dicarboxylate (3ra) 


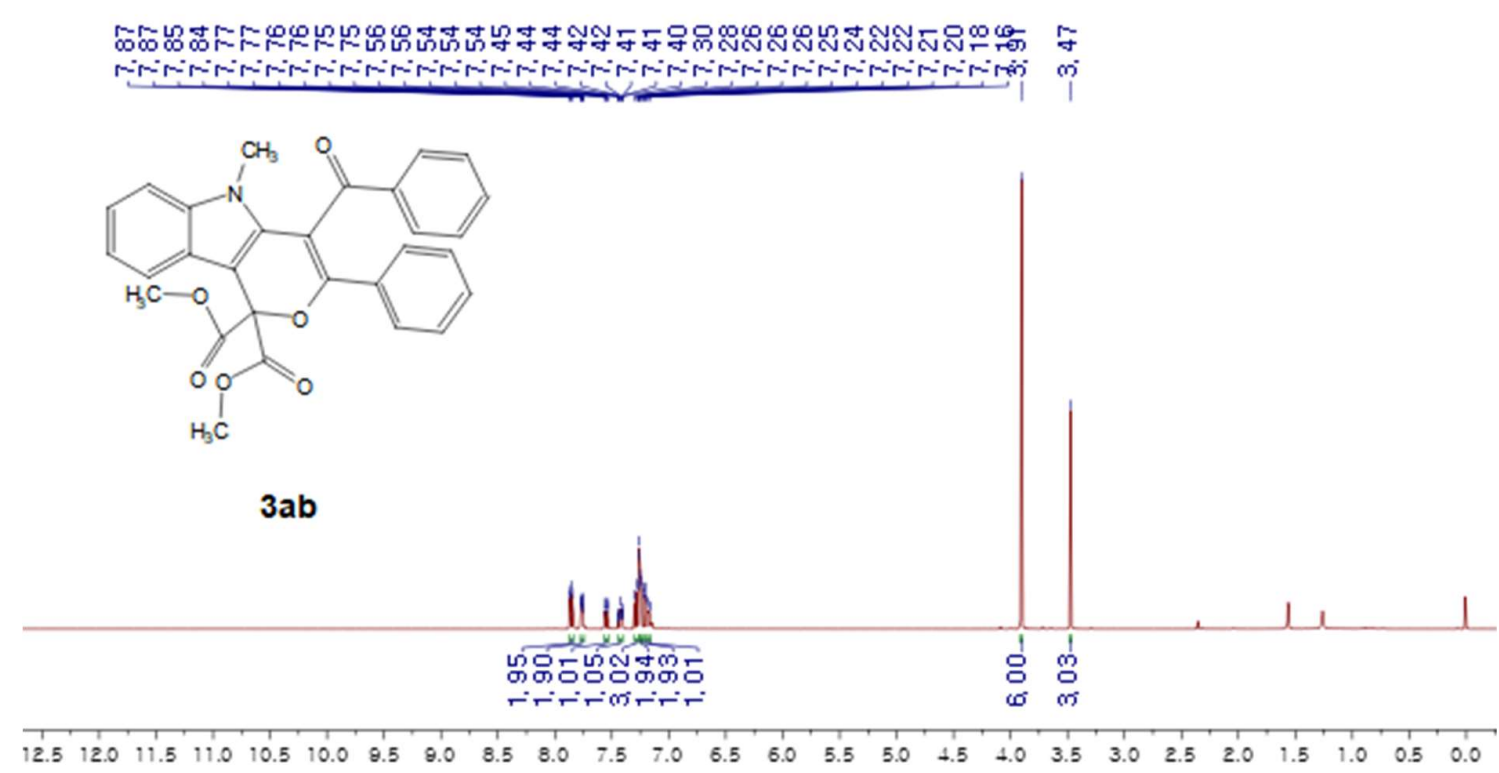

${ }^{1} \mathrm{H}$ NMR (400 MHz, $\mathrm{CDCl}_{3}$ ) spectra of dimethyl 4-benzoyl-5-methyl-3-phenylpyrano[4,3b]indole-1,1(5H)-dicarboxylate (3ab)
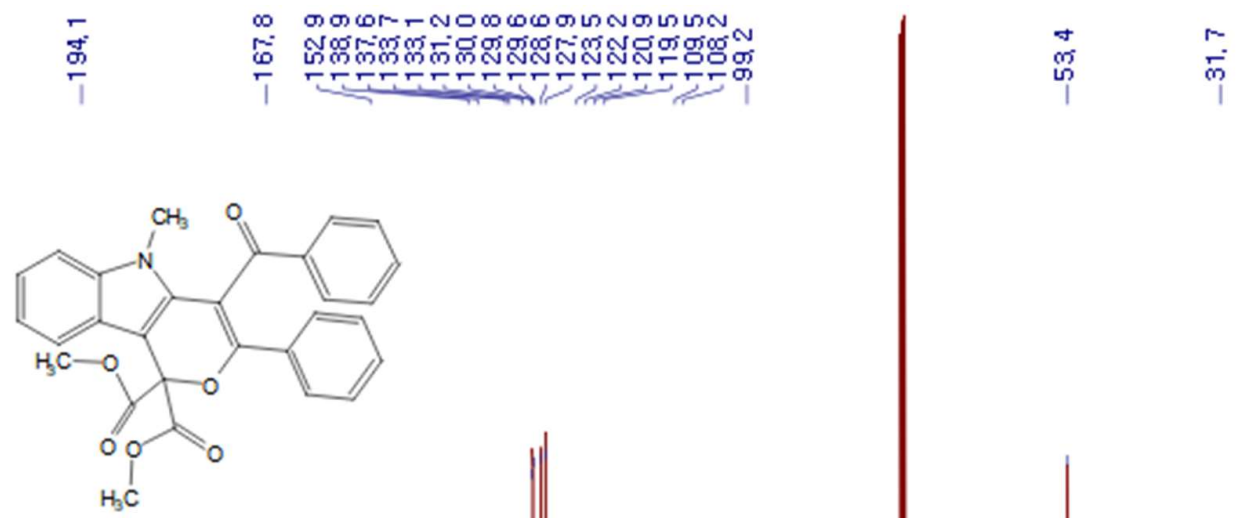

$3 a b$

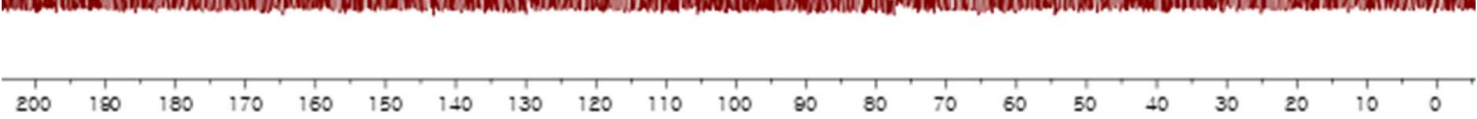

${ }^{13} \mathrm{C}$ NMR (100 MHz, $\mathrm{CDCl}_{3}$ ) spectra of dimethyl 4-benzoyl-5-methyl-3-phenylpyrano[4,3b]indole-1,1(5H)-dicarboxylate (3ab) 

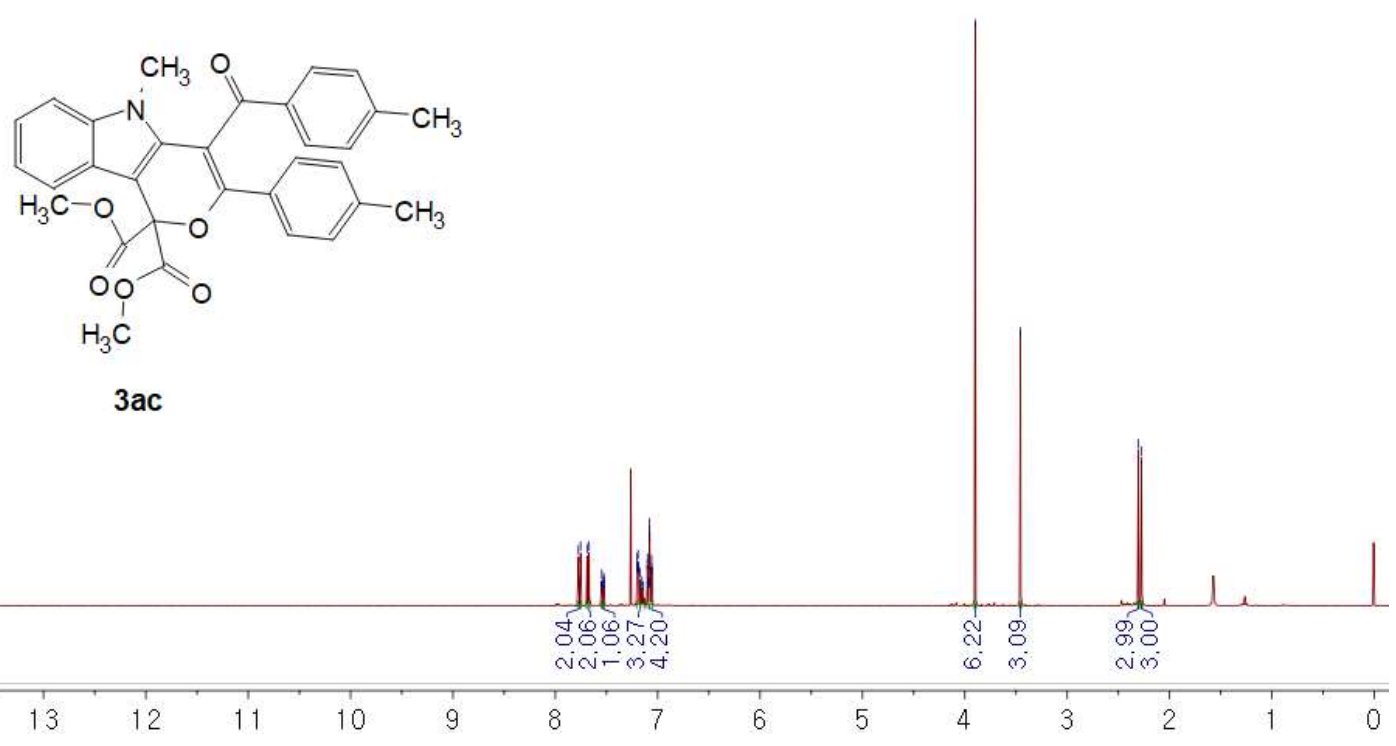

${ }^{1} \mathrm{H}$ NMR (400 MHz, $\mathrm{CDCl}_{3}$ ) spectra of dimethyl 5-methyl-4-(4-methylbenzoyl)-3-(ptolyl)pyrano[4,3-b]indole-1,1(5H)-dicarboxylate (3ac)

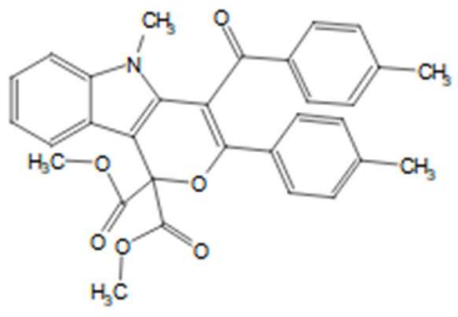

$3 a c$
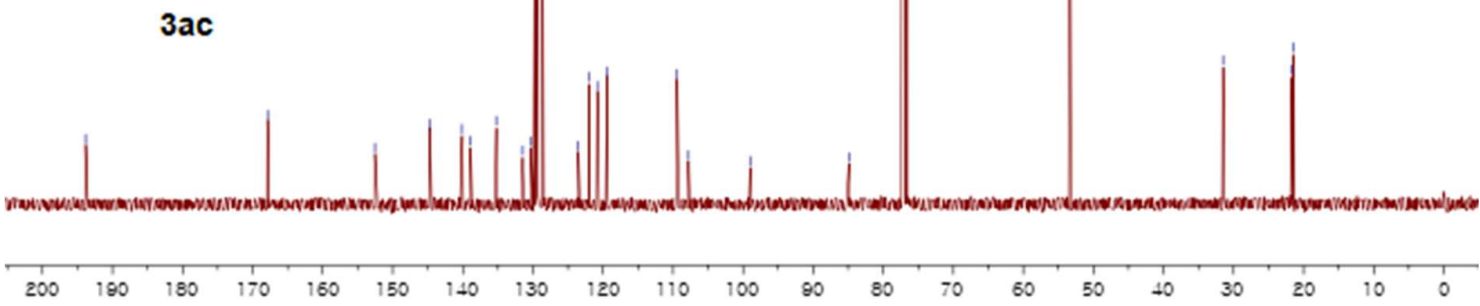

${ }^{13} \mathrm{C}$ NMR (100 MHz, $\mathrm{CDCl}_{3}$ ) spectra of dimethyl 5-methyl-4-(4-methylbenzoyl)-3-(ptolyl)pyrano[4,3-b]indole-1,1(5H)-dicarboxylate (3ac) 


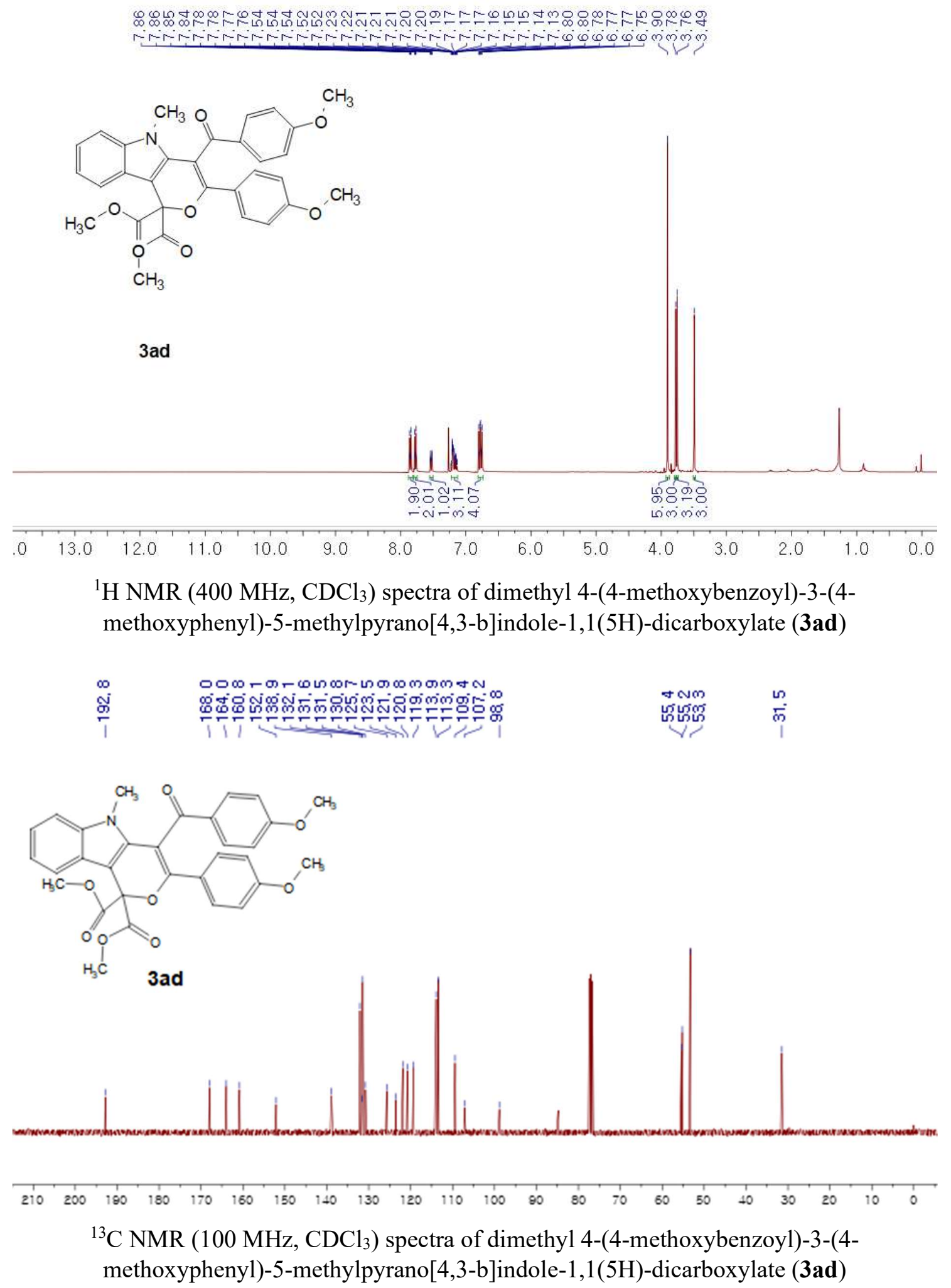




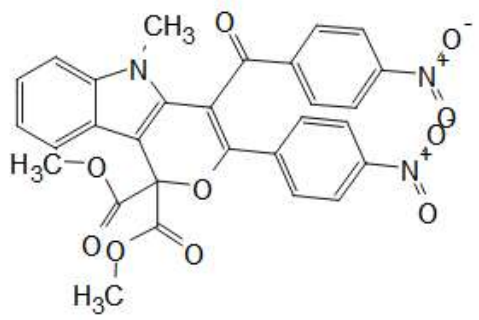

3ae

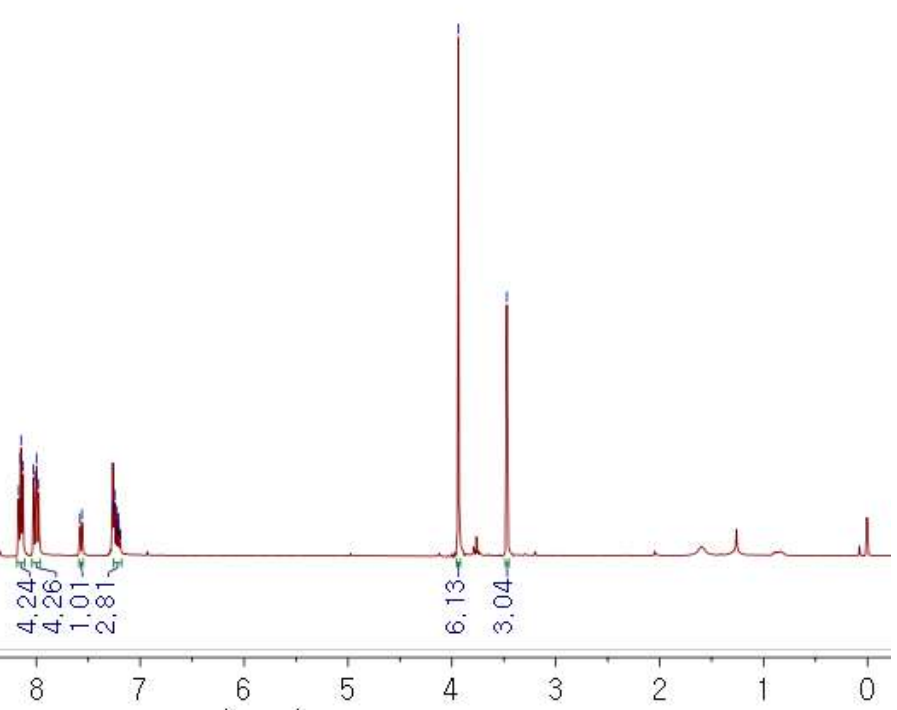

${ }^{1} \mathrm{H}$ NMR (400 MHz, $\mathrm{CDCl}_{3}$ ) spectra of dimethyl 5-methyl-4-(4-nitrobenzoyl)-3-(4nitrophenyl)pyrano[4,3-b]indole-1,1(5H)-dicarboxylate (3ae)
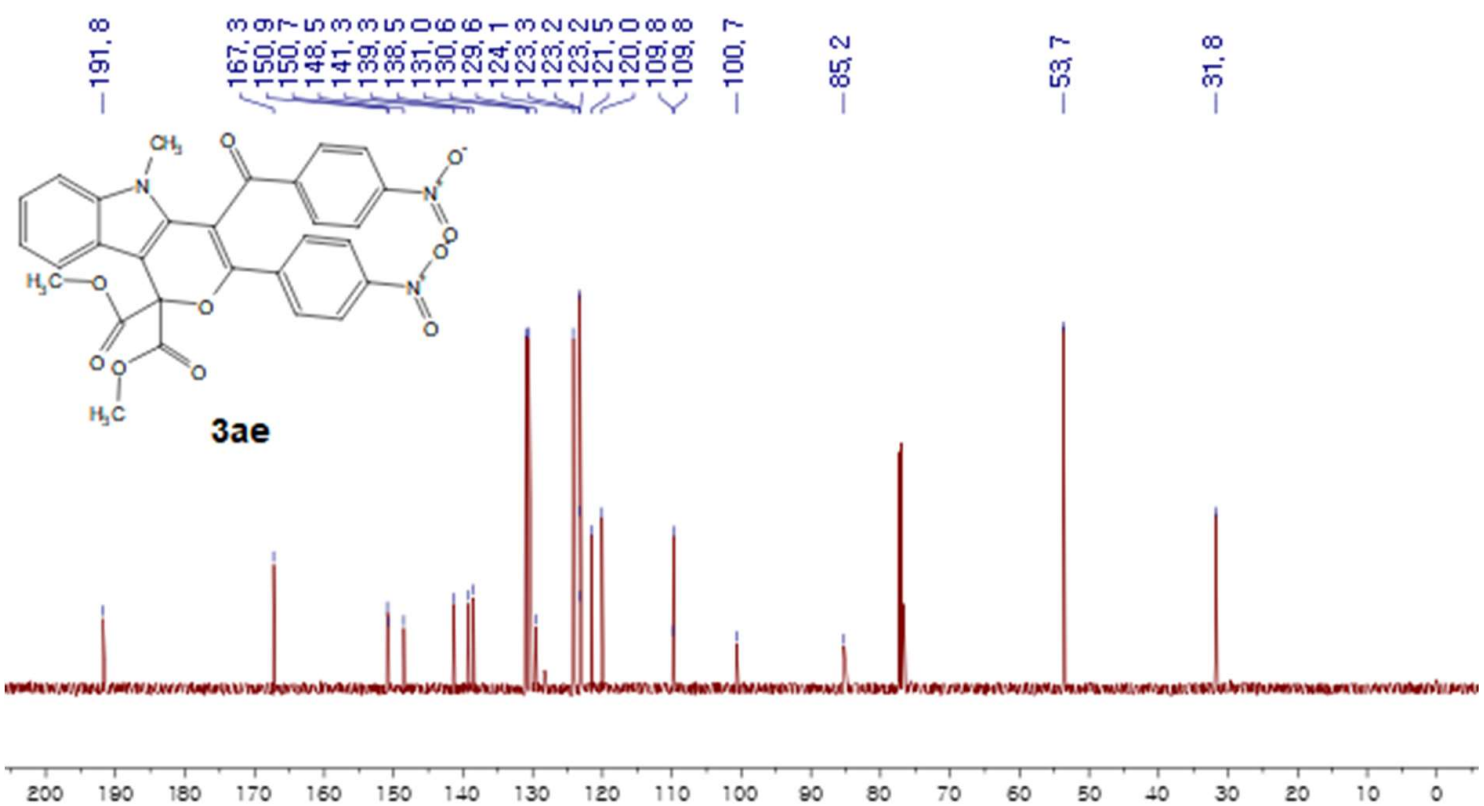

${ }^{13} \mathrm{C}$ NMR (100 MHz, $\mathrm{CDCl}_{3}$ ) spectra of dimethyl 5-methyl-4-(4-nitrobenzoyl)-3-(4nitrophenyl)pyrano[4,3-b]indole-1,1(5H)-dicarboxylate (3ae) 


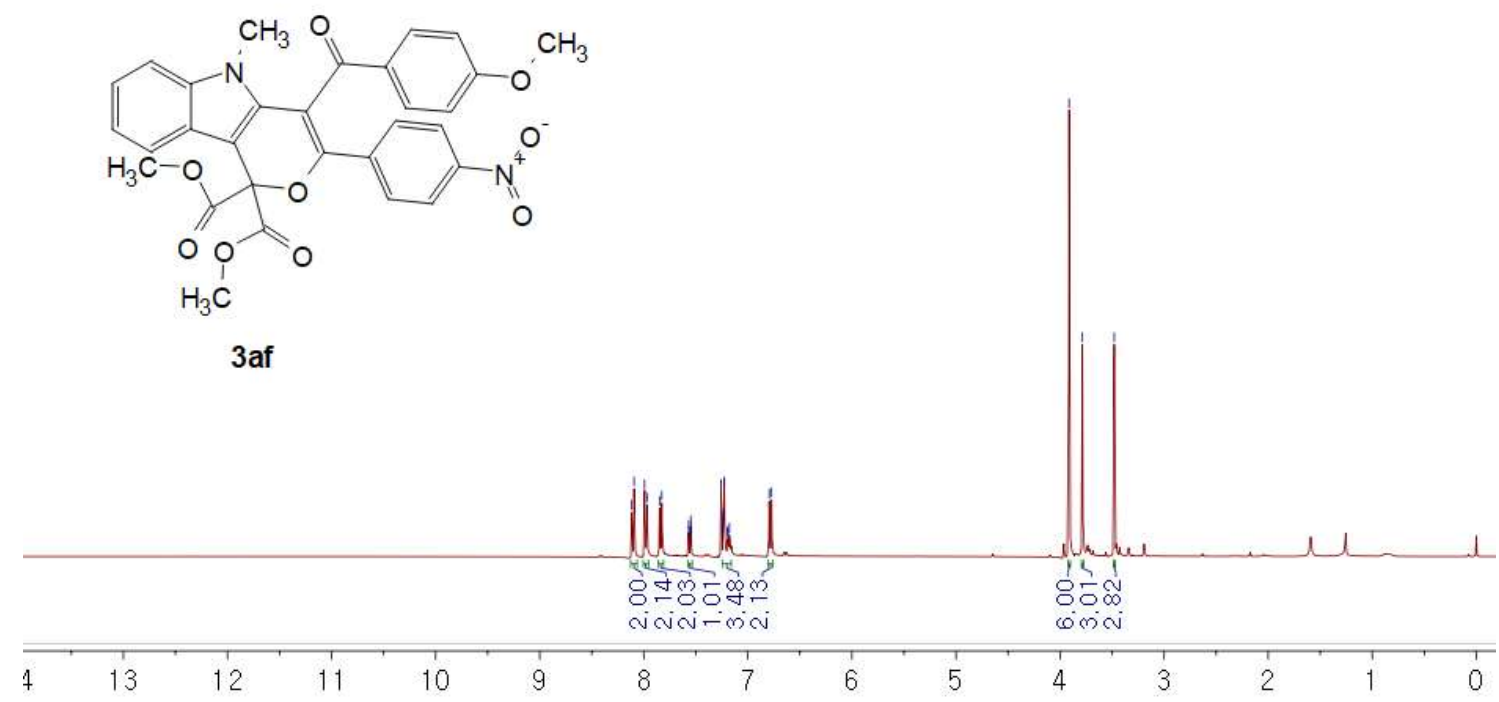

${ }^{1} \mathrm{H}$ NMR (400 MHz, $\mathrm{CDCl}_{3}$ ) spectra of dimethyl 4-(4-methoxybenzoyl)-5-methyl-3-(4nitrophenyl)pyrano[4,3-b]indole-1,1(5H)-dicarboxylate (3af)
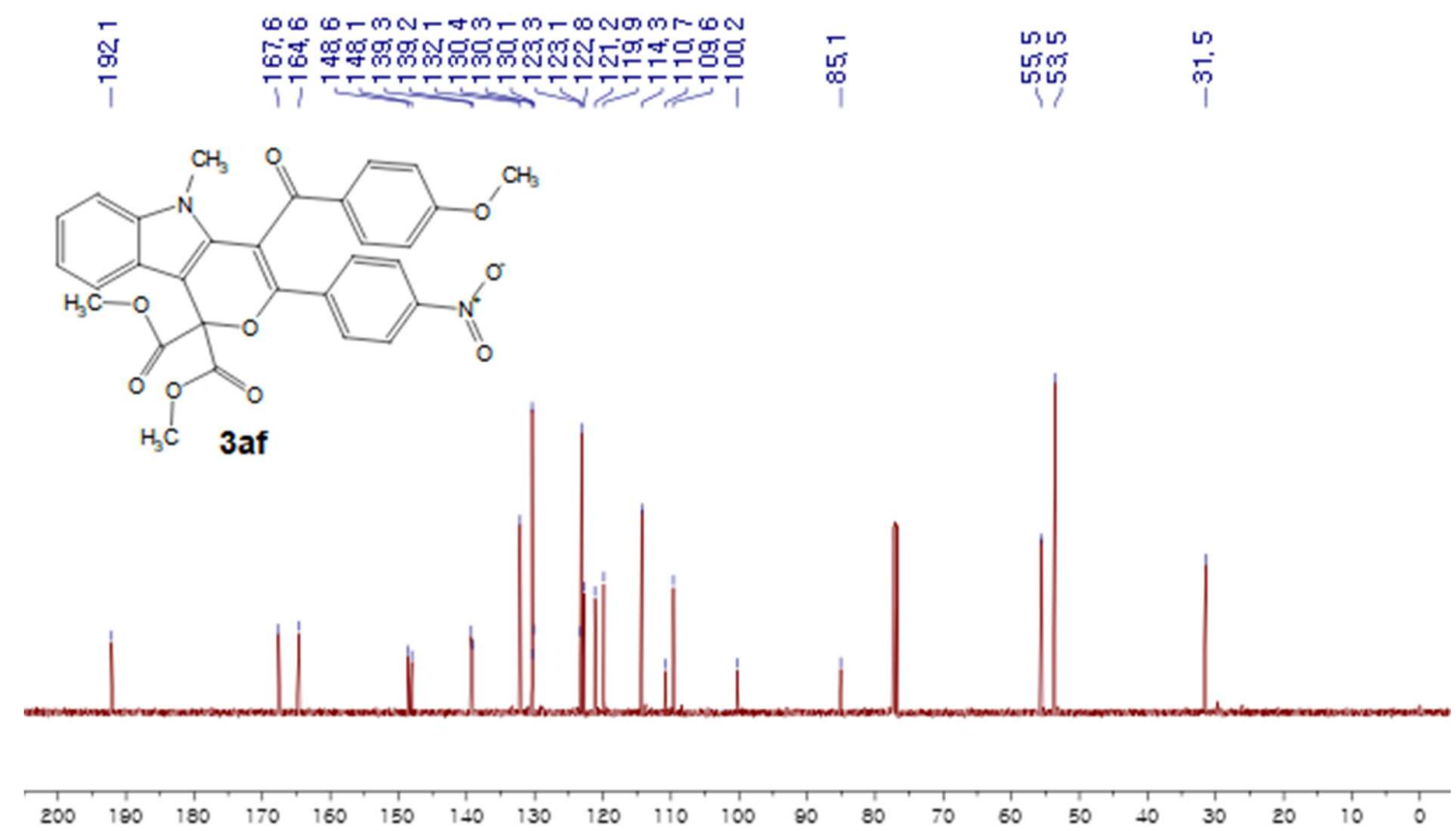

${ }^{13} \mathrm{C}$ NMR (100 MHz, $\mathrm{CDCl}_{3}$ ) spectra of dimethyl 4-(4-methoxybenzoyl)-5-methyl-3-(4nitrophenyl)pyrano[4,3-b]indole-1,1(5H)-dicarboxylate (3af) 


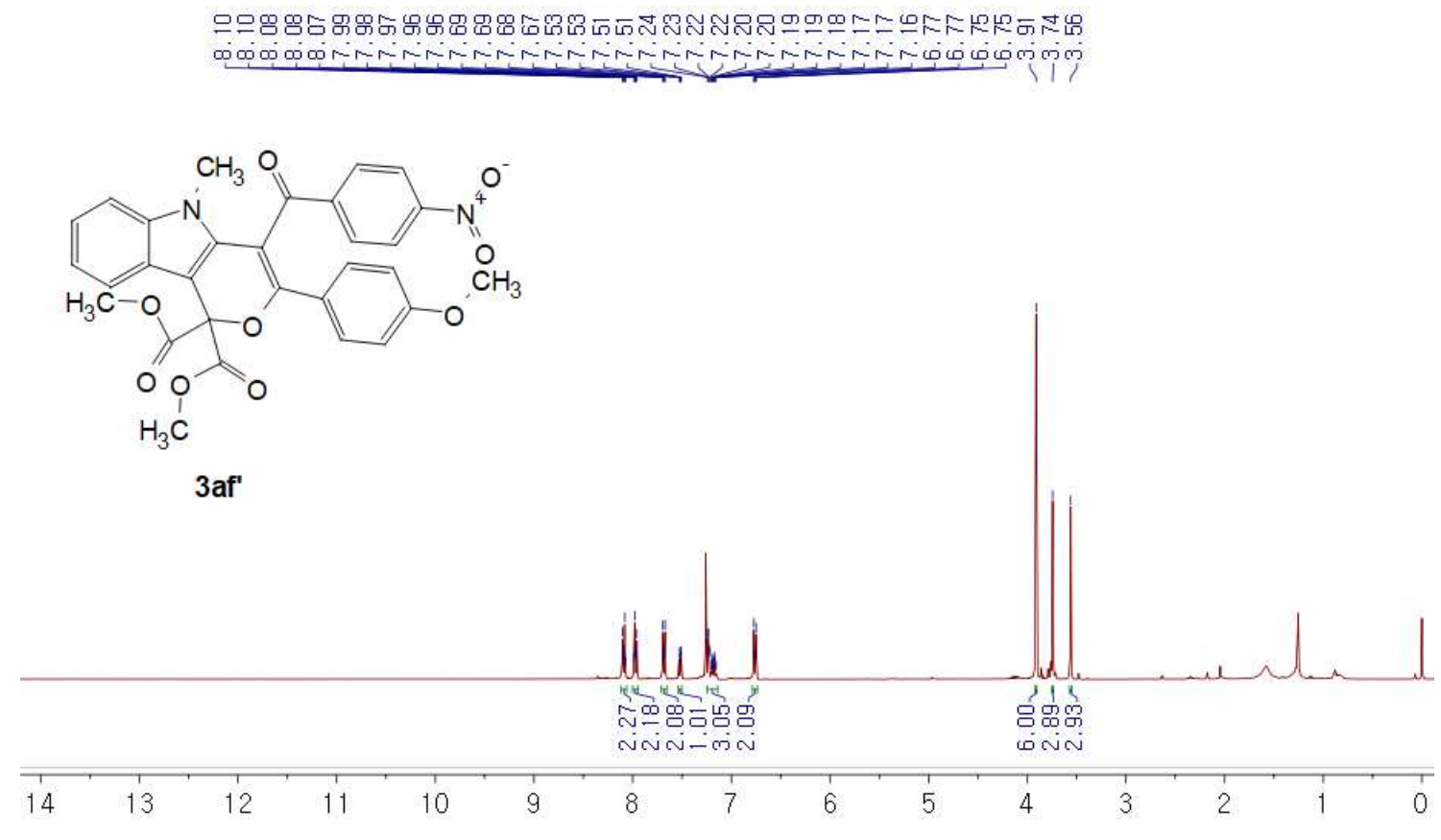

${ }^{1} \mathrm{H}$ NMR (400 MHz, $\mathrm{CDCl}_{3}$ ) spectra of dimethyl 3-(4-methoxyphenyl)-5-methyl-4-(4nitrobenzoyl)pyrano[4,3-b]indole-1,1(5H)-dicarboxylate (3af')
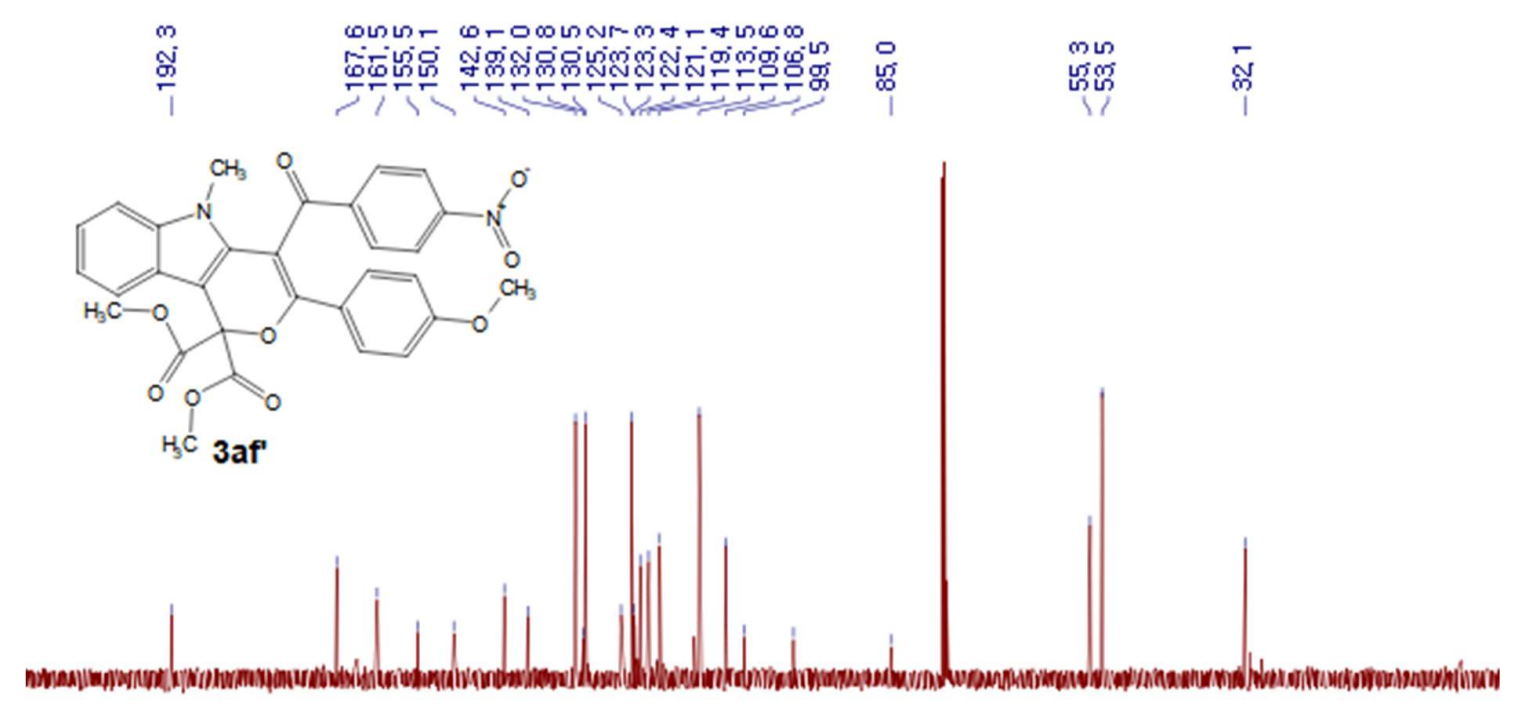

\begin{tabular}{lllllllllllllllllllllll}
\hline 10 & 200 & 190 & 180 & 170 & 160 & 150 & 140 & 130 & 120 & 110 & 100 & 90 & 80 & 70 & 60 & 50 & 40 & 30 & 20 & 10 & 0 & 1
\end{tabular}

${ }^{13} \mathrm{C}$ NMR (100 MHz, $\mathrm{CDCl}_{3}$ ) spectra of dimethyl 3-(4-methoxyphenyl)-5-methyl-4-(4nitrobenzoyl)pyrano[4,3-b]indole-1,1(5H)-dicarboxylate (3af') 


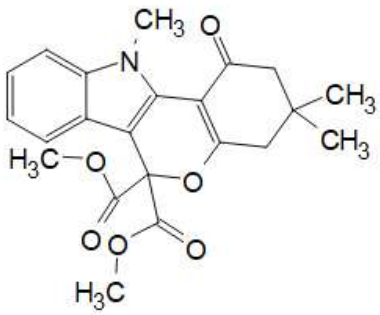

3ag

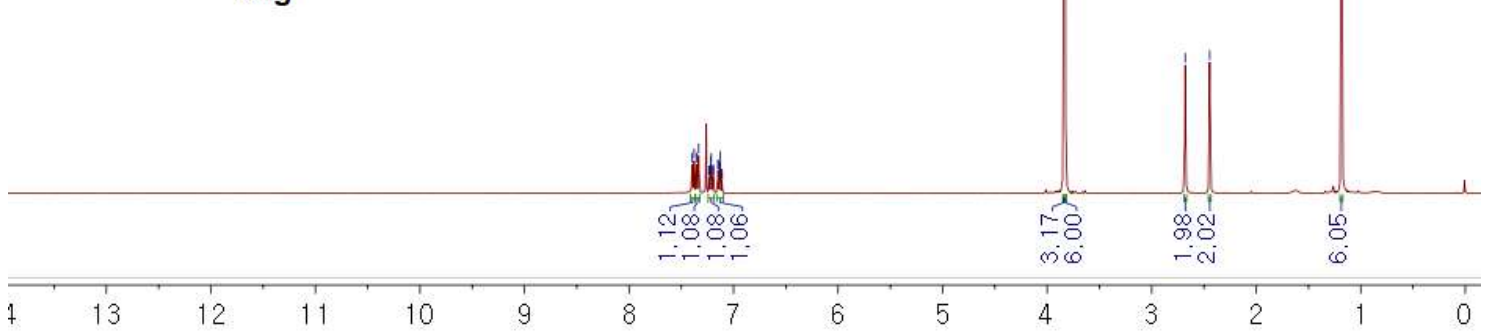

${ }^{1} \mathrm{H}$ NMR (400 MHz, $\mathrm{CDCl}_{3}$ ) spectra of dimethyl 3,3,11-trimethyl-1-oxo-2,3,4,11tetrahydrochromeno[4,3-b]indole-6,6(1H)-dicarboxylate (3ag)
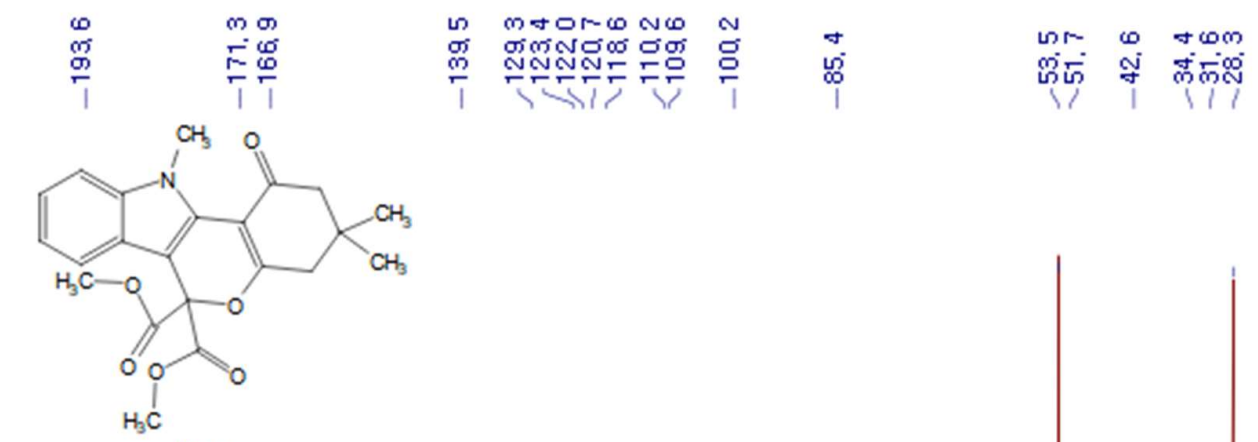

3ag

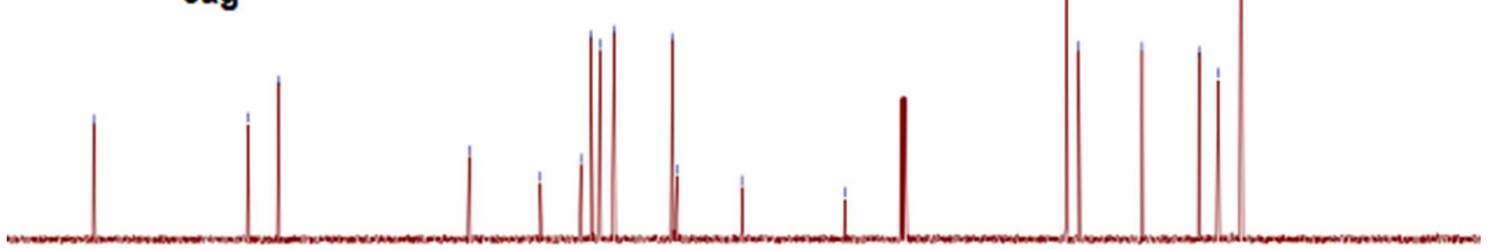

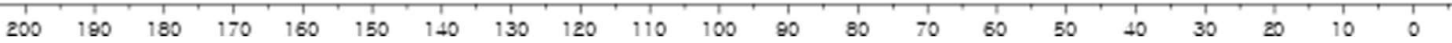

${ }^{13} \mathrm{C}$ NMR (100 MHz, $\mathrm{CDCl}_{3}$ ) spectra of dimethyl 3,3,11-trimethyl-1-oxo-2,3,4,11tetrahydrochromeno[4,3-b]indole-6,6(1H)-dicarboxylate (3ag) 


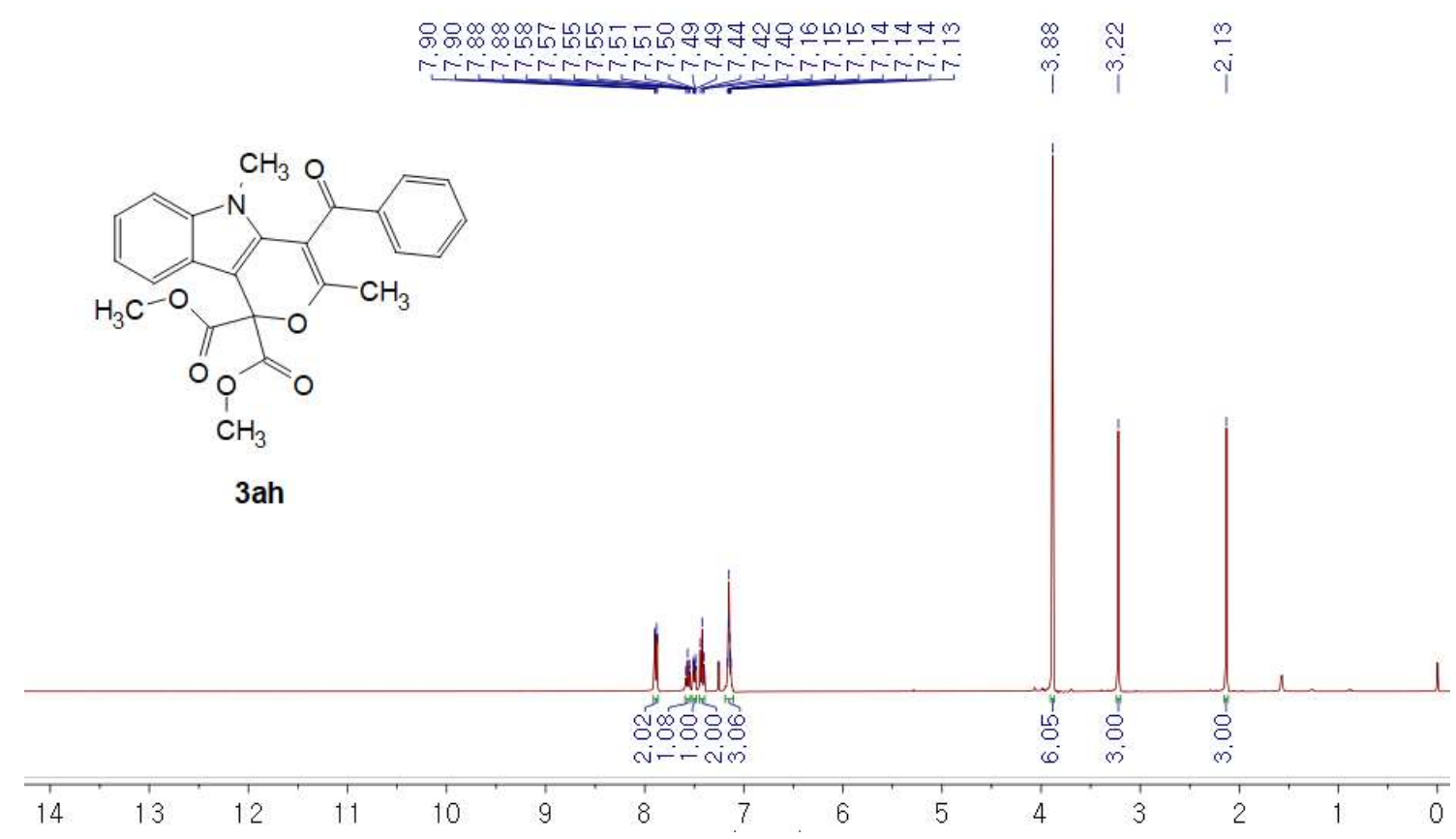

${ }^{1} \mathrm{H}$ NMR (400 MHz, $\mathrm{CDCl}_{3}$ ) spectra of dimethyl 4-benzoyl-3,5-dimethylpyrano[4,3-b]indole1,1(5H)-dicarboxylate (3ah)

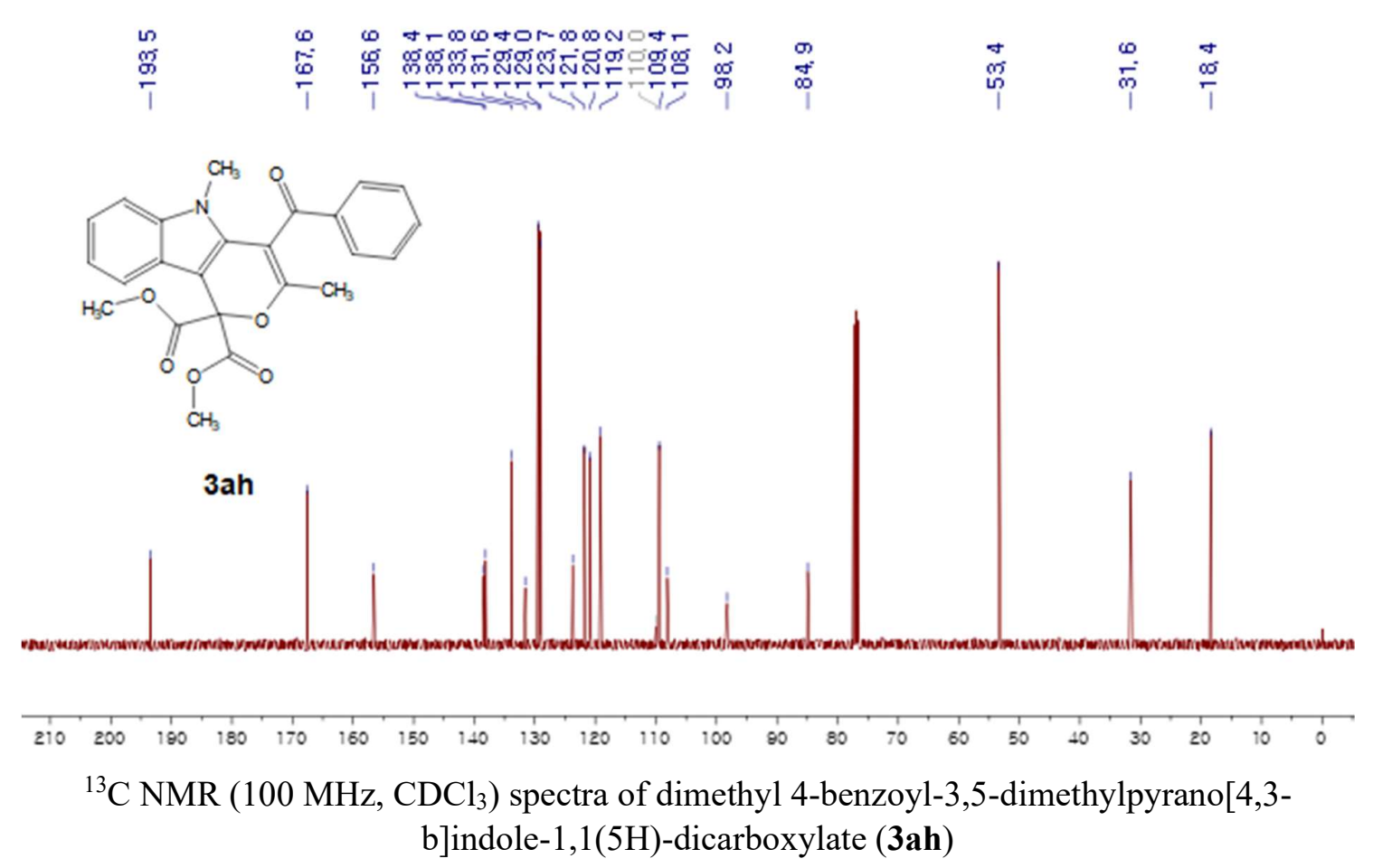




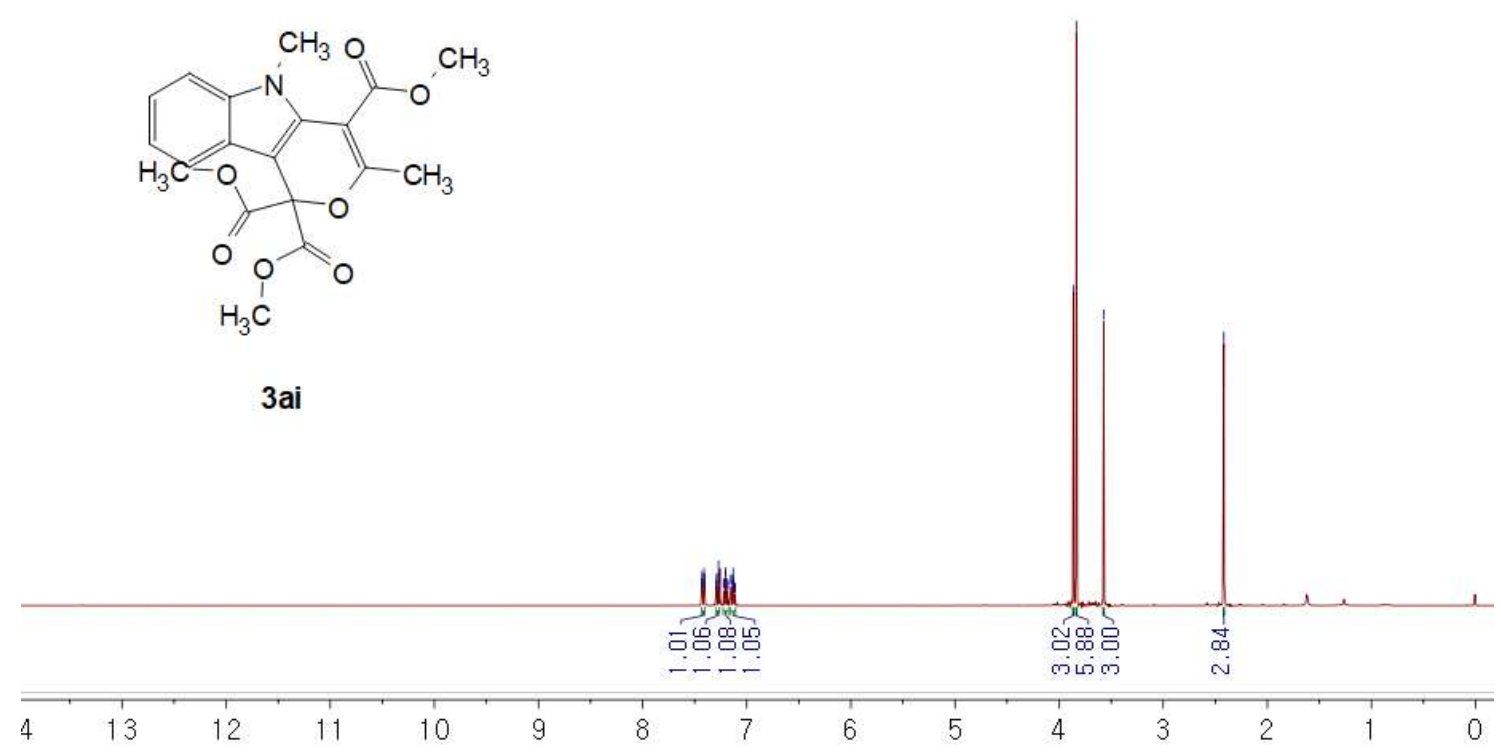

${ }^{1} \mathrm{H}$ NMR (400 MHz, $\mathrm{CDCl}_{3}$ ) spectra of trimethyl 3,5-dimethylpyrano[4,3-b]indole-1,1,4(5H)tricarboxylate (3ai)
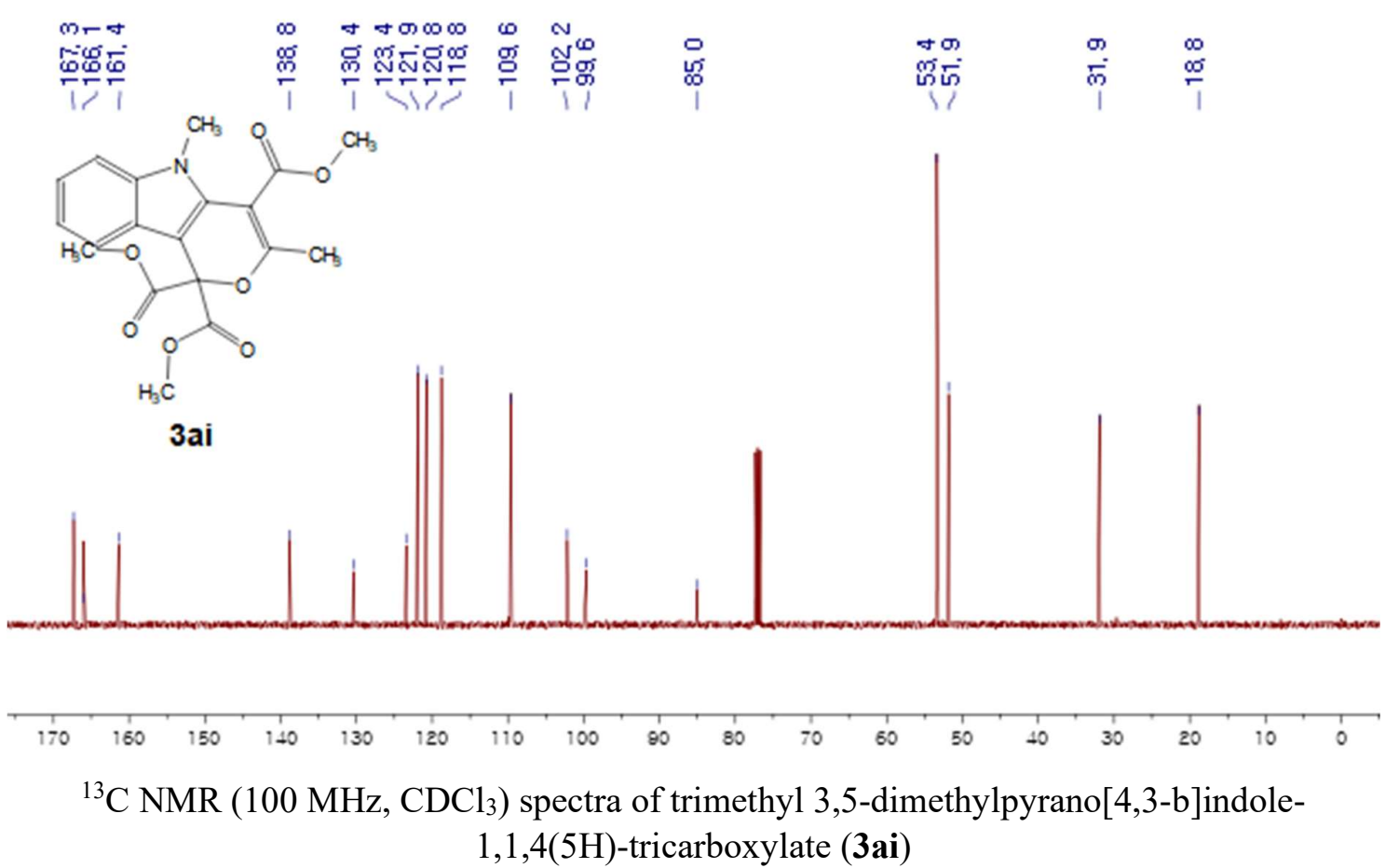


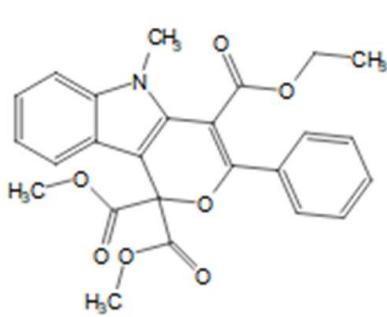

3aj

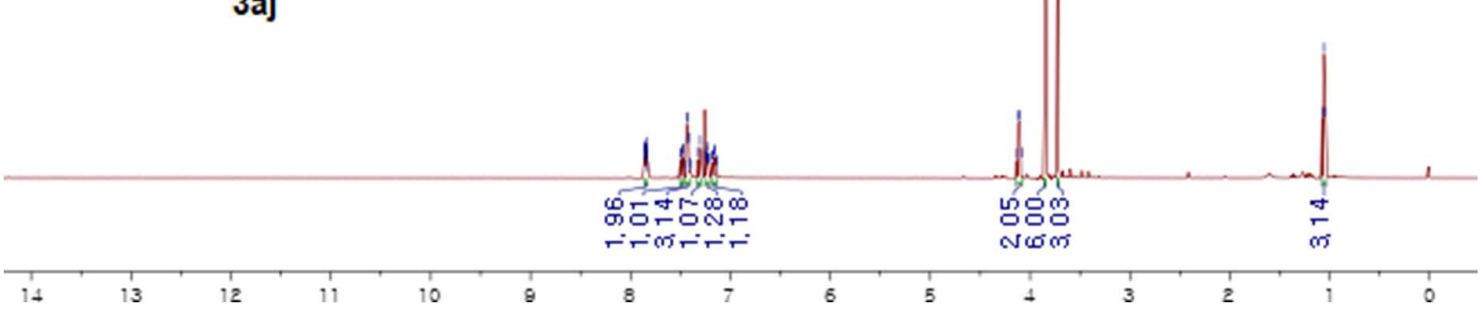

${ }^{1} \mathrm{H}$ NMR (400 MHz, $\mathrm{CDCl}_{3}$ ) spectra of 4-ethyl 1,1-dimethyl 5-methyl-3-phenylpyrano[4,3b]indole-1,1,4(5H)-tricarboxylate (3aj)

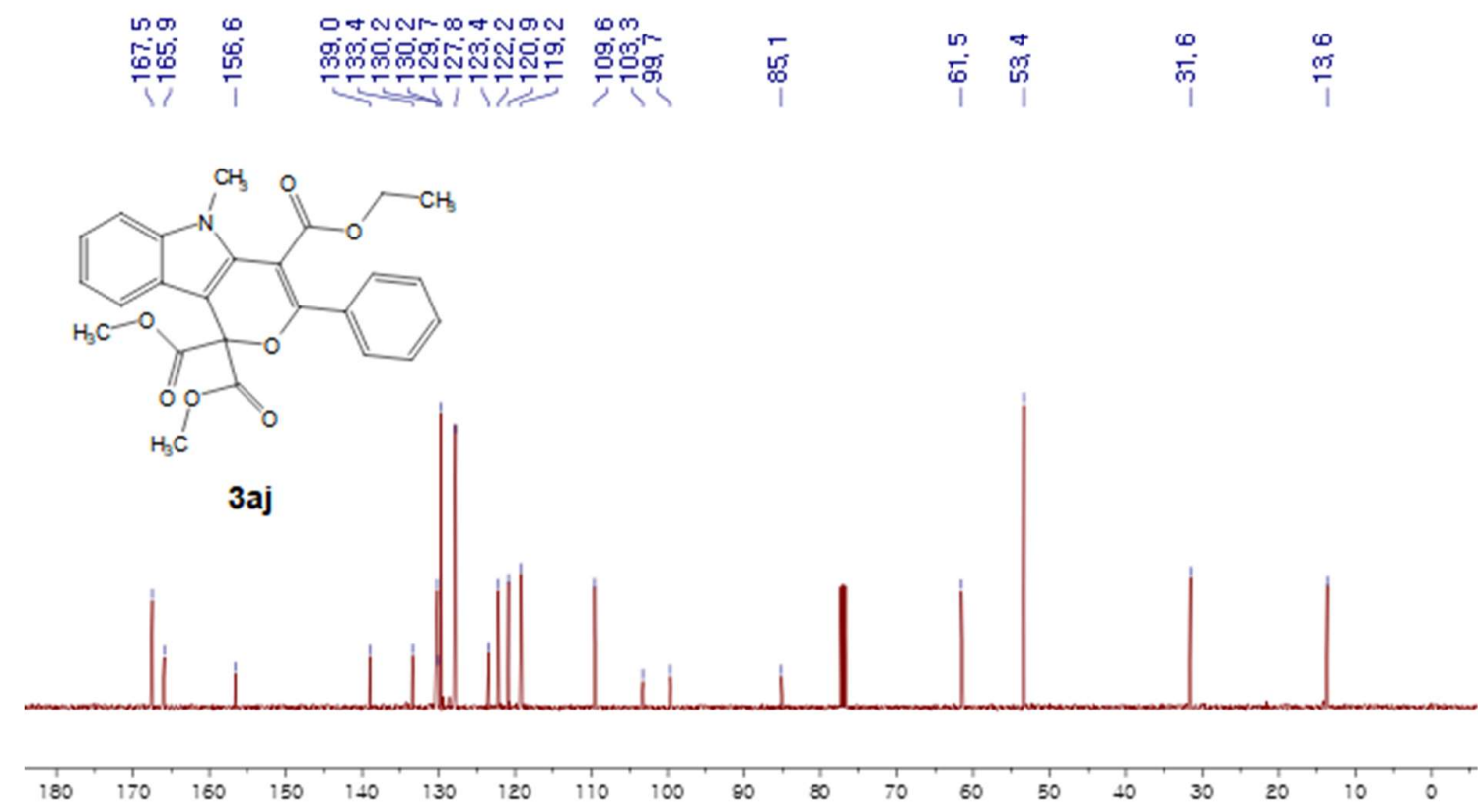

${ }^{13} \mathrm{C}$ NMR $\left(100 \mathrm{MHz}, \mathrm{CDCl}_{3}\right)$ spectra of 4-ethyl 1,1-dimethyl 5-methyl-3-phenylpyrano[4,3b]indole-1,1,4(5H)-tricarboxylate (3aj) 


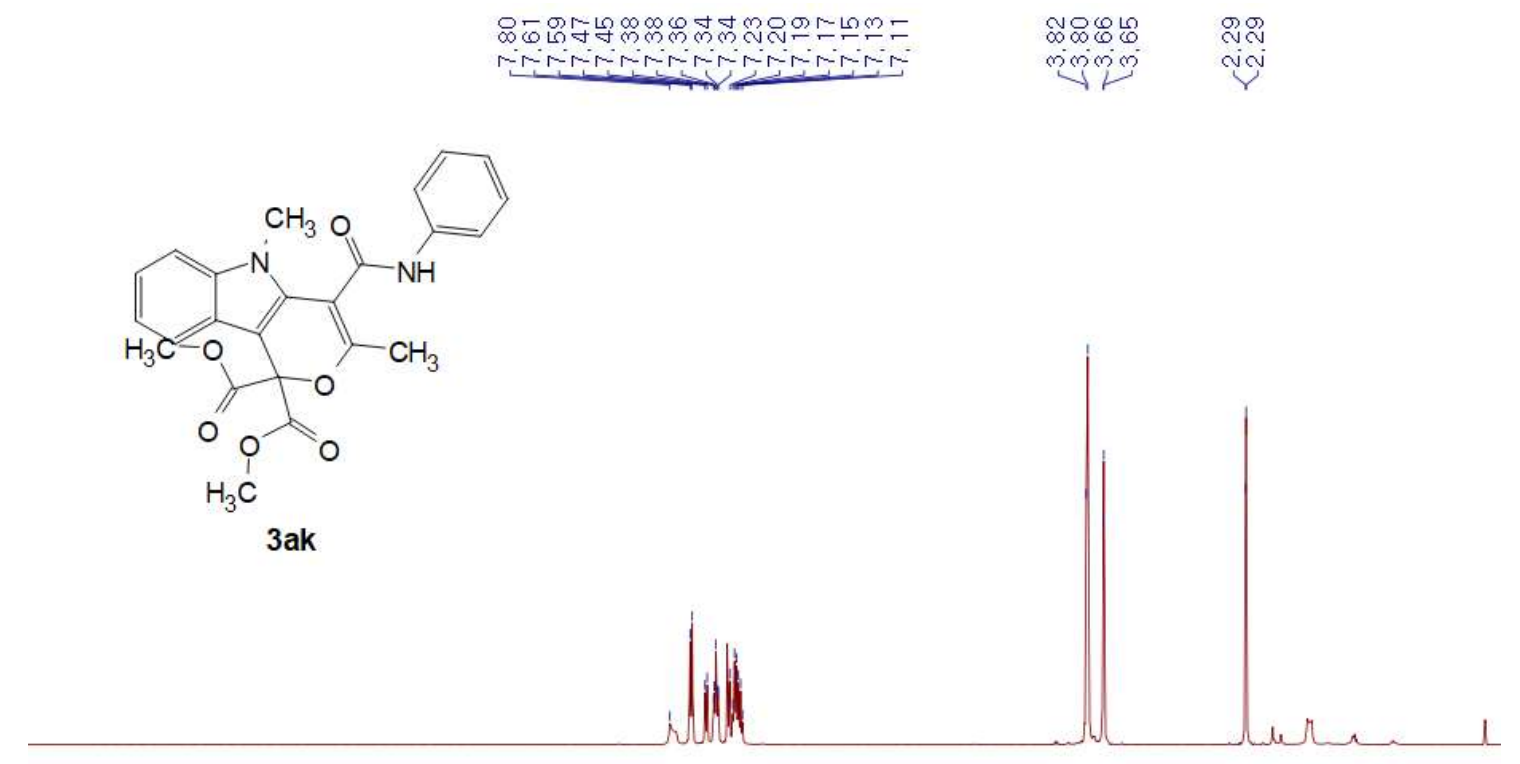

\begin{tabular}{llllllllllllllllll}
\hline \hline & 13 & 12 & 11 & 10 & 9 & 8 & 7 & 6 & 5 & 4 & 3 & 2 & 1 & 0
\end{tabular}

${ }^{1} \mathrm{H}$ NMR (400 MHz, $\mathrm{CDCl}_{3}$ ) spectra of dimethyl 3,5-dimethyl-4(phenylcarbamoyl)pyrano[4,3-b]indole-1,1(5H)-dicarboxylate (3ak)

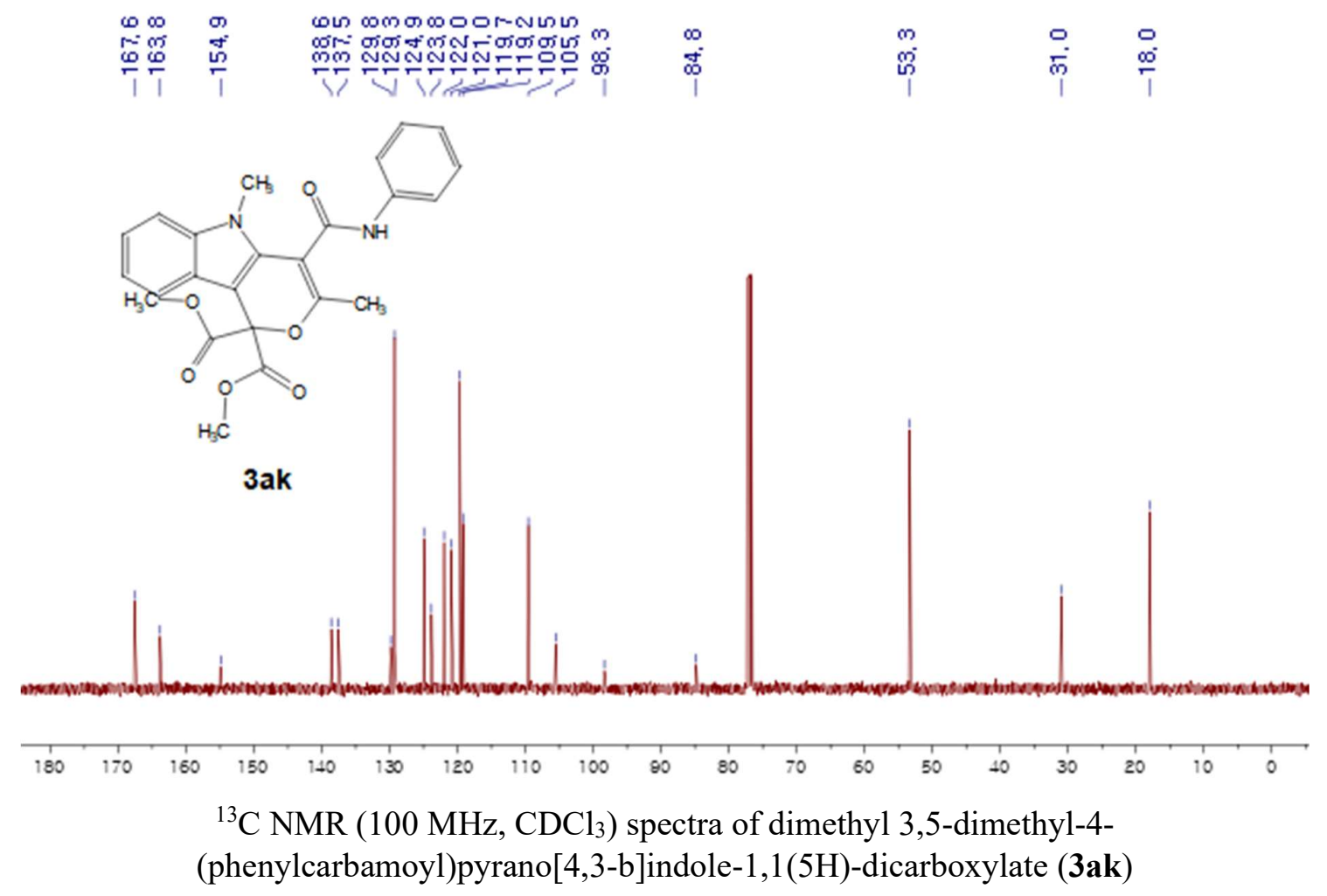




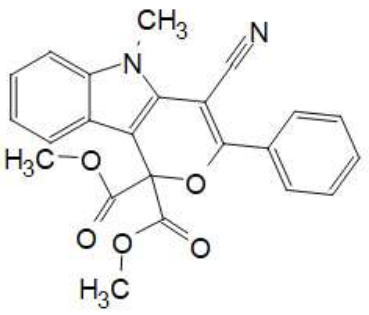

3al

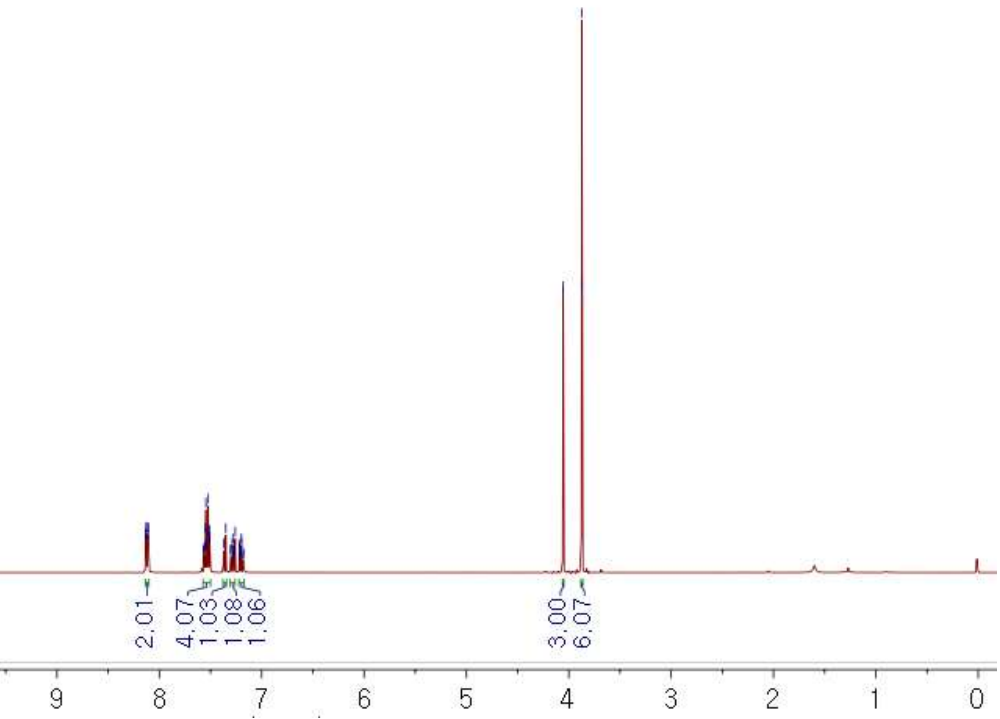

${ }^{1} \mathrm{H}$ NMR (400 MHz, $\mathrm{CDCl}_{3}$ ) spectra of dimethyl 4-cyano-5-methyl-3-phenylpyrano[4,3b]indole-1,1(5H)-dicarboxylate (3al)

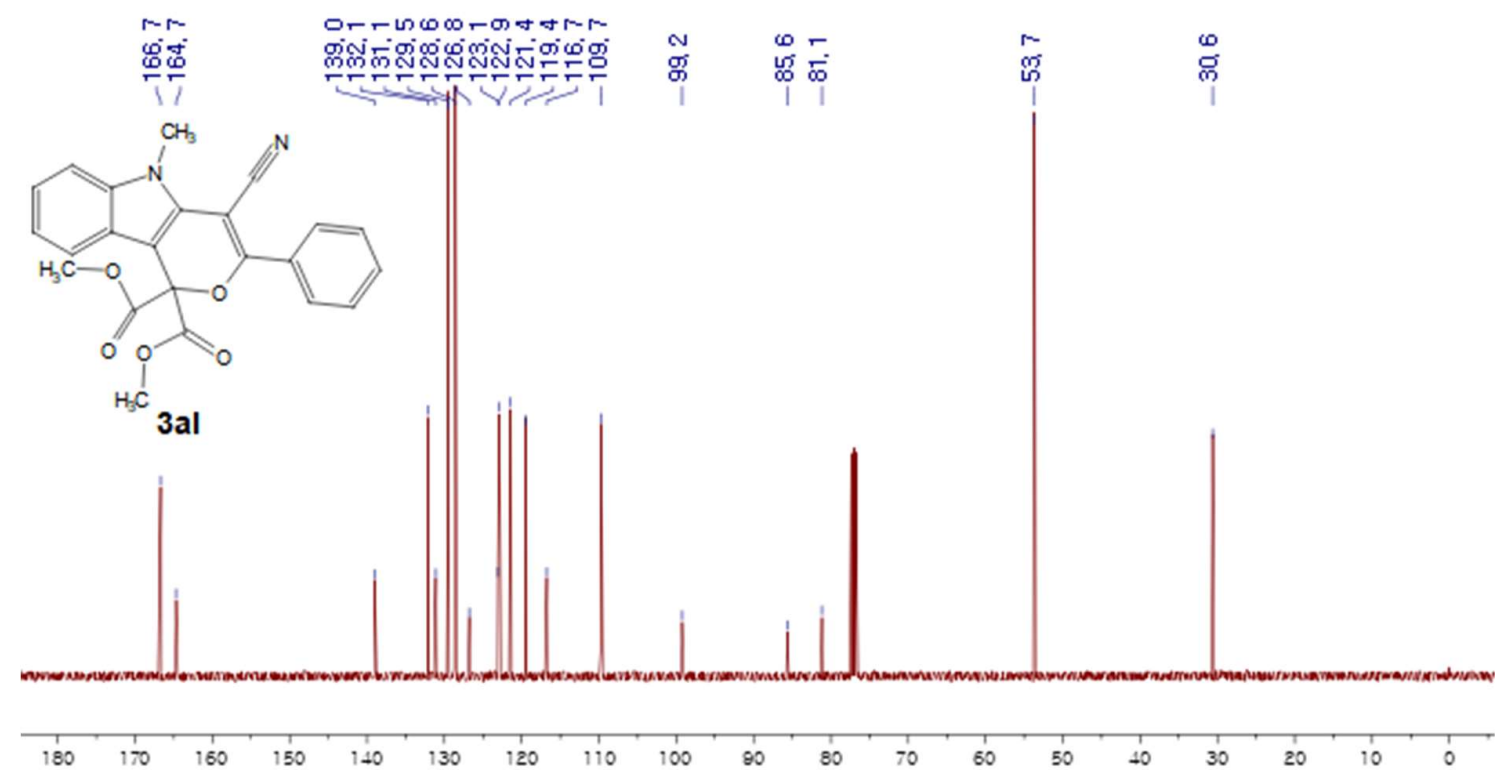

${ }^{13} \mathrm{C}$ NMR $\left(100 \mathrm{MHz}, \mathrm{CDCl}_{3}\right)$ spectra of dimethyl 4-cyano-5-methyl-3-phenylpyrano[4,3b]indole-1,1(5H)-dicarboxylate (3al) 


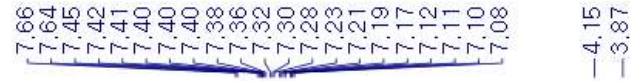

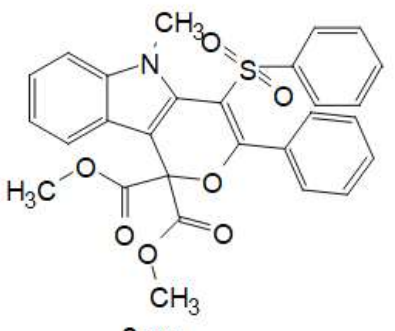

3am

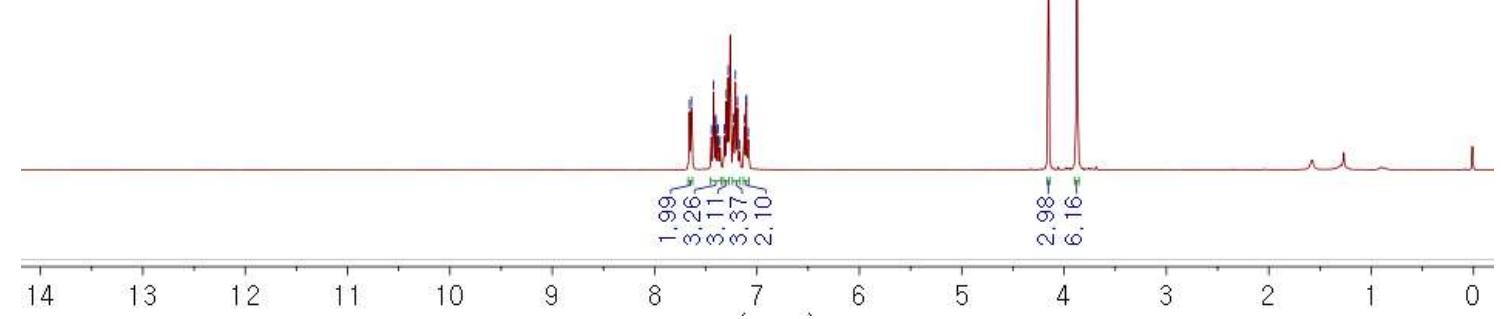

${ }^{1} \mathrm{H}$ NMR (400 MHz, $\mathrm{CDCl}_{3}$ ) spectra of dimethyl 5-methyl-3-phenyl-4-

(phenylsulfonyl)pyrano[4,3-b]indole-1,1(5H)-dicarboxylate (3am)
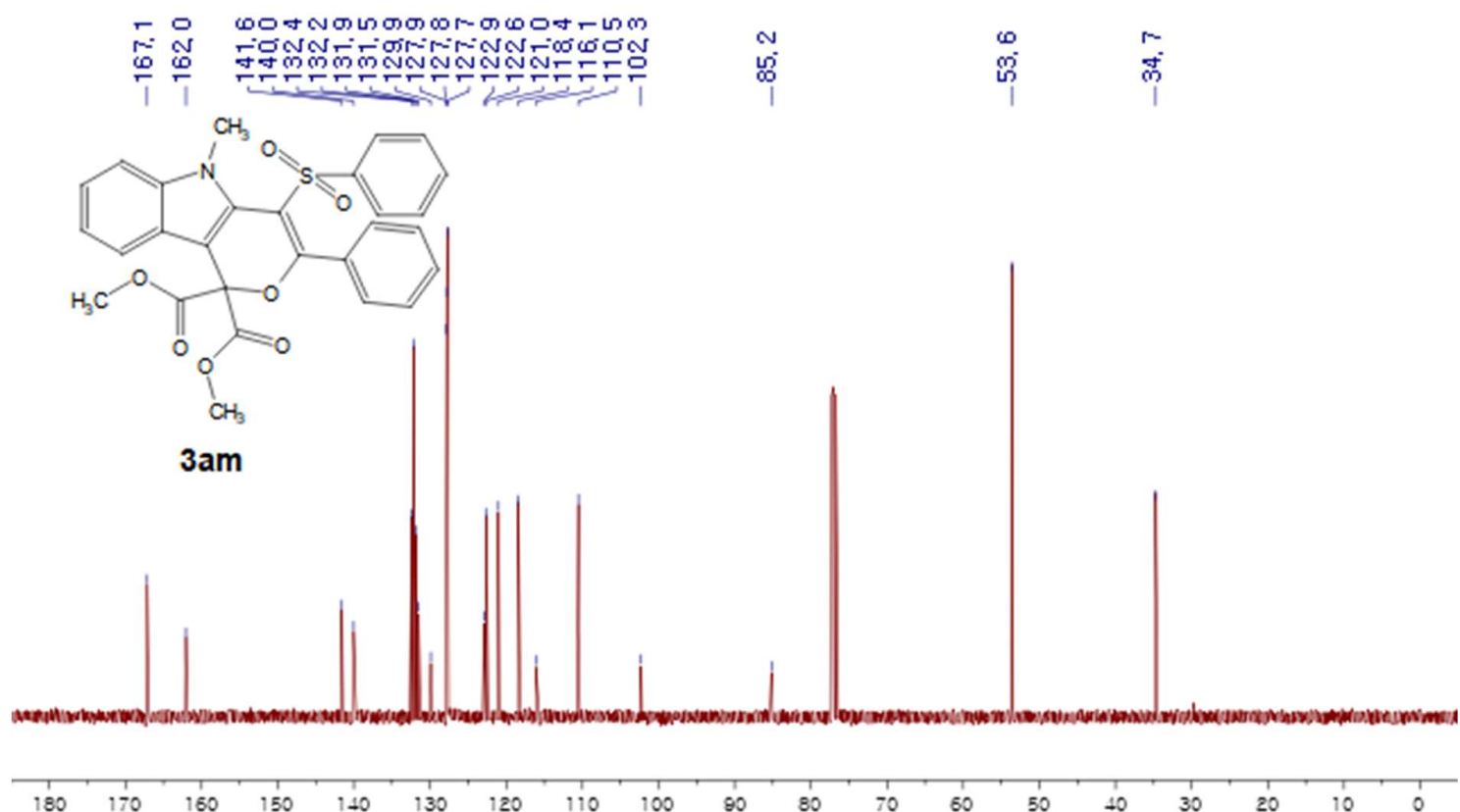

${ }^{13} \mathrm{C}$ NMR (100 MHz, $\left.\mathrm{CDCl}_{3}\right)$ spectra of dimethyl 5-methyl-3-phenyl-4(phenylsulfonyl)pyrano[4,3-b]indole-1,1(5H)-dicarboxylate (3am) 


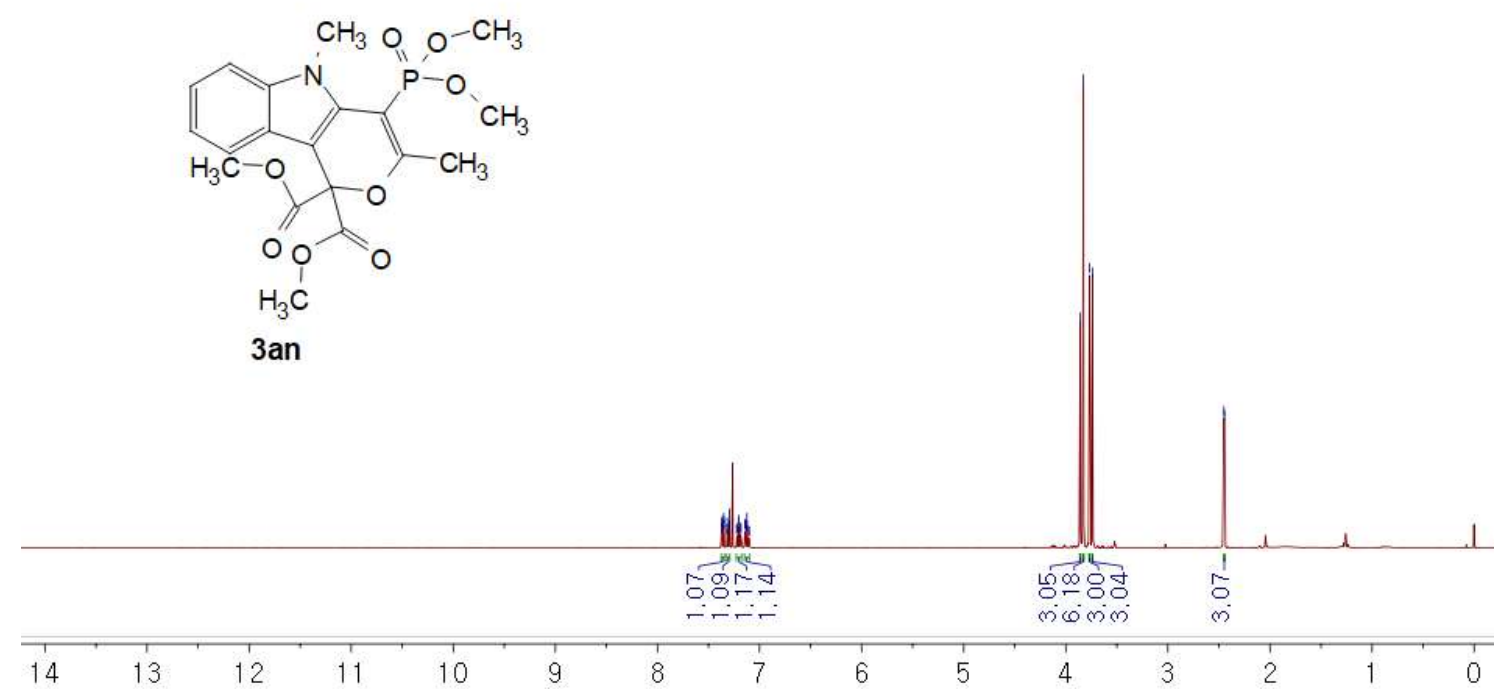

${ }^{1} \mathrm{H}$ NMR (400 MHz, $\mathrm{CDCl}_{3}$ ) spectra of dimethyl 4-(dimethoxyphosphoryl)-3,5dimethylpyrano[4,3-b]indole-1,1(5H)-dicarboxylate (3an)

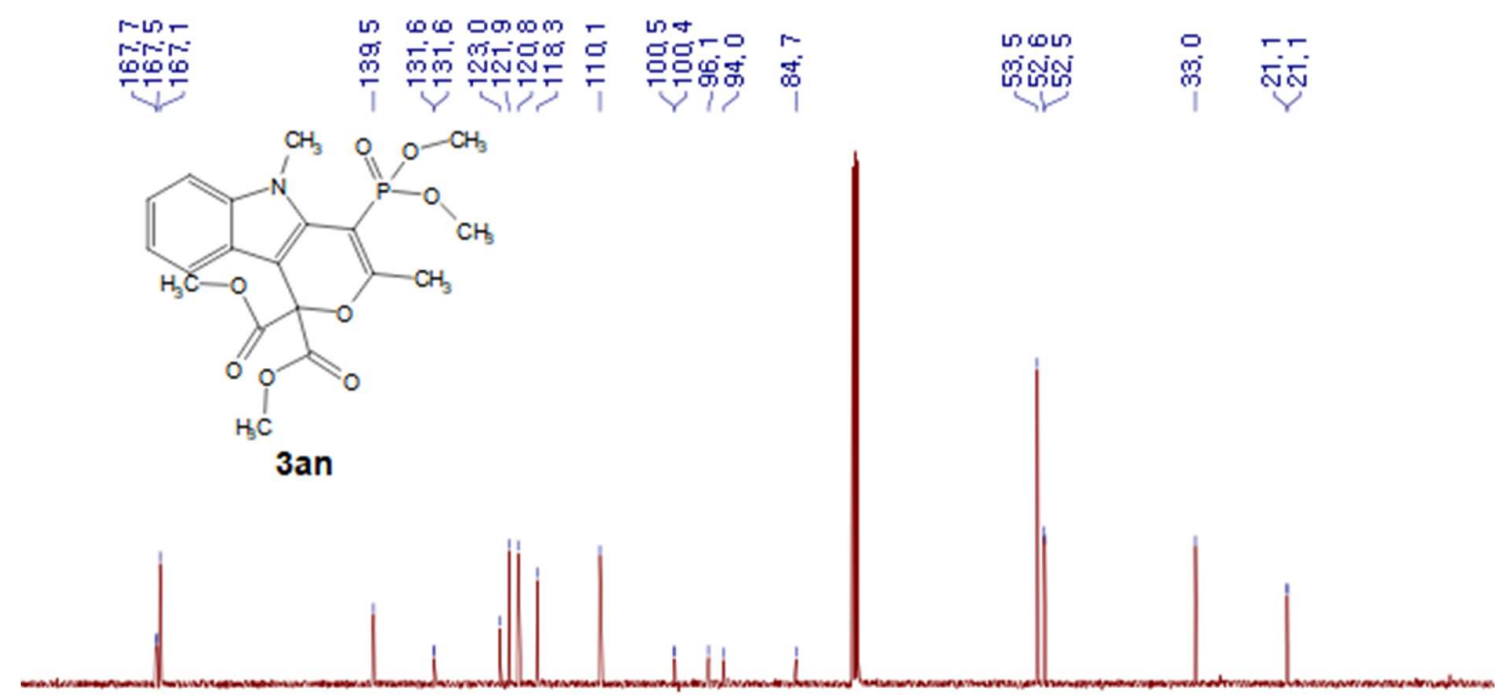

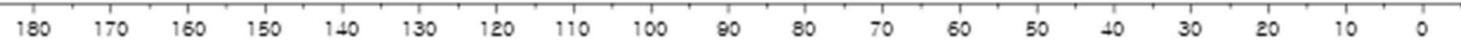

${ }^{13} \mathrm{C}$ NMR (100 MHz, $\mathrm{CDCl}_{3}$ ) spectra of dimethyl 4-(dimethoxyphosphoryl)-3,5dimethylpyrano[4,3-b]indole-1,1(5H)-dicarboxylate (3an) 


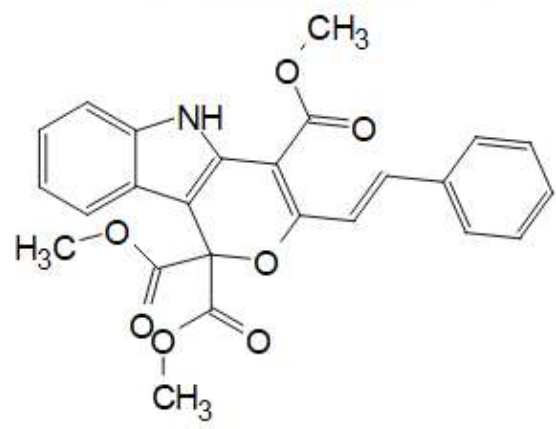

3io

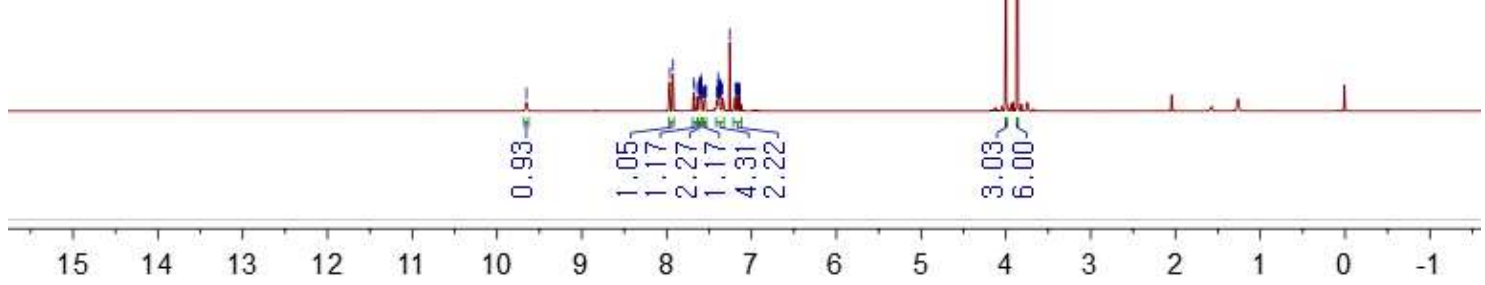

${ }^{1} \mathrm{H}$ NMR (400 MHz, $\mathrm{CDCl}_{3}$ ) spectra of trimethyl (E)-3-styrylpyrano[4,3-b]indole-1,1,4(5H)tricarboxylate (3io)
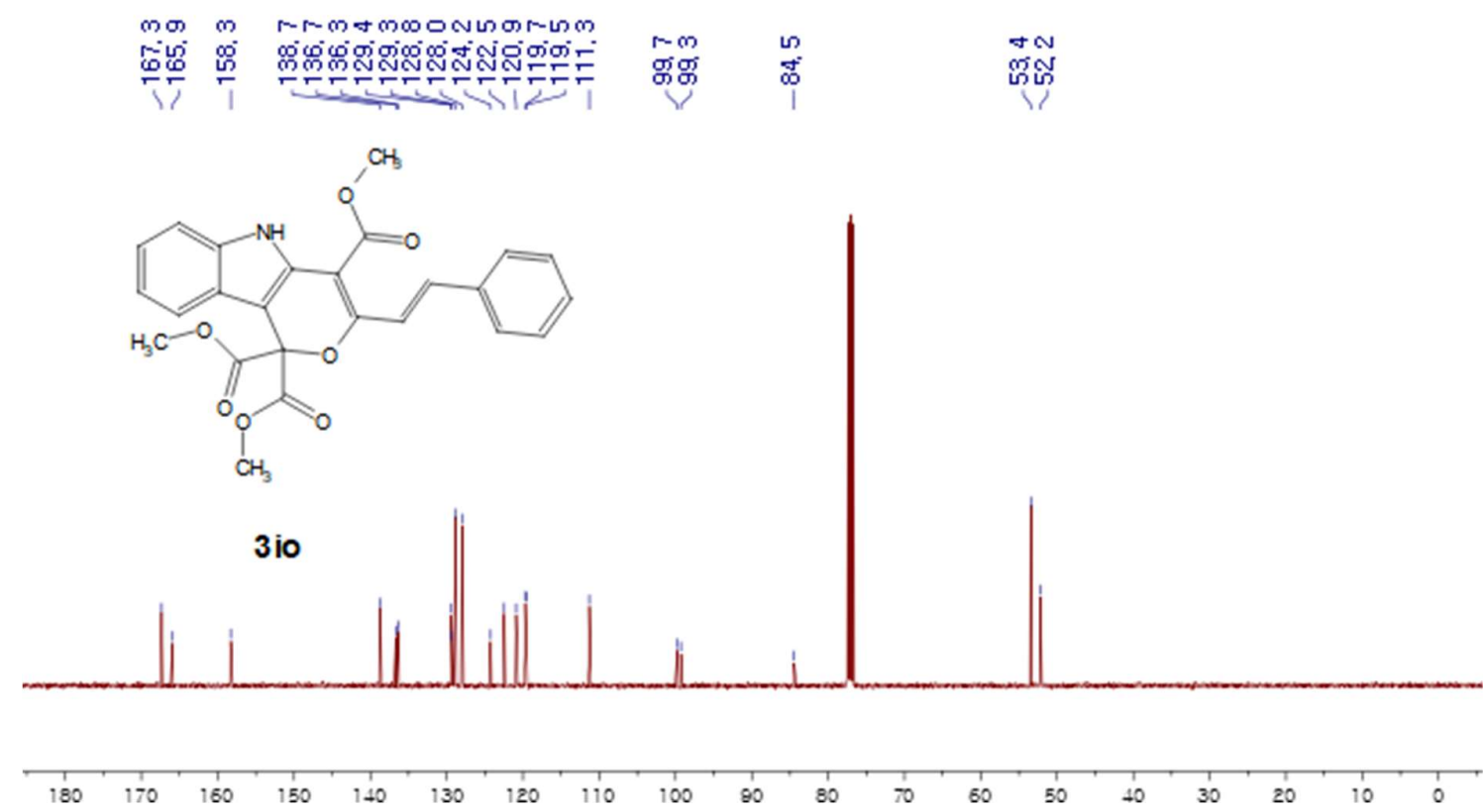

${ }^{13} \mathrm{C}$ NMR (100 MHz, $\mathrm{CDCl}_{3}$ ) spectra of trimethyl (E)-3-styrylpyrano[4,3-b]indole-1,1,4(5H)tricarboxylate (3io) 

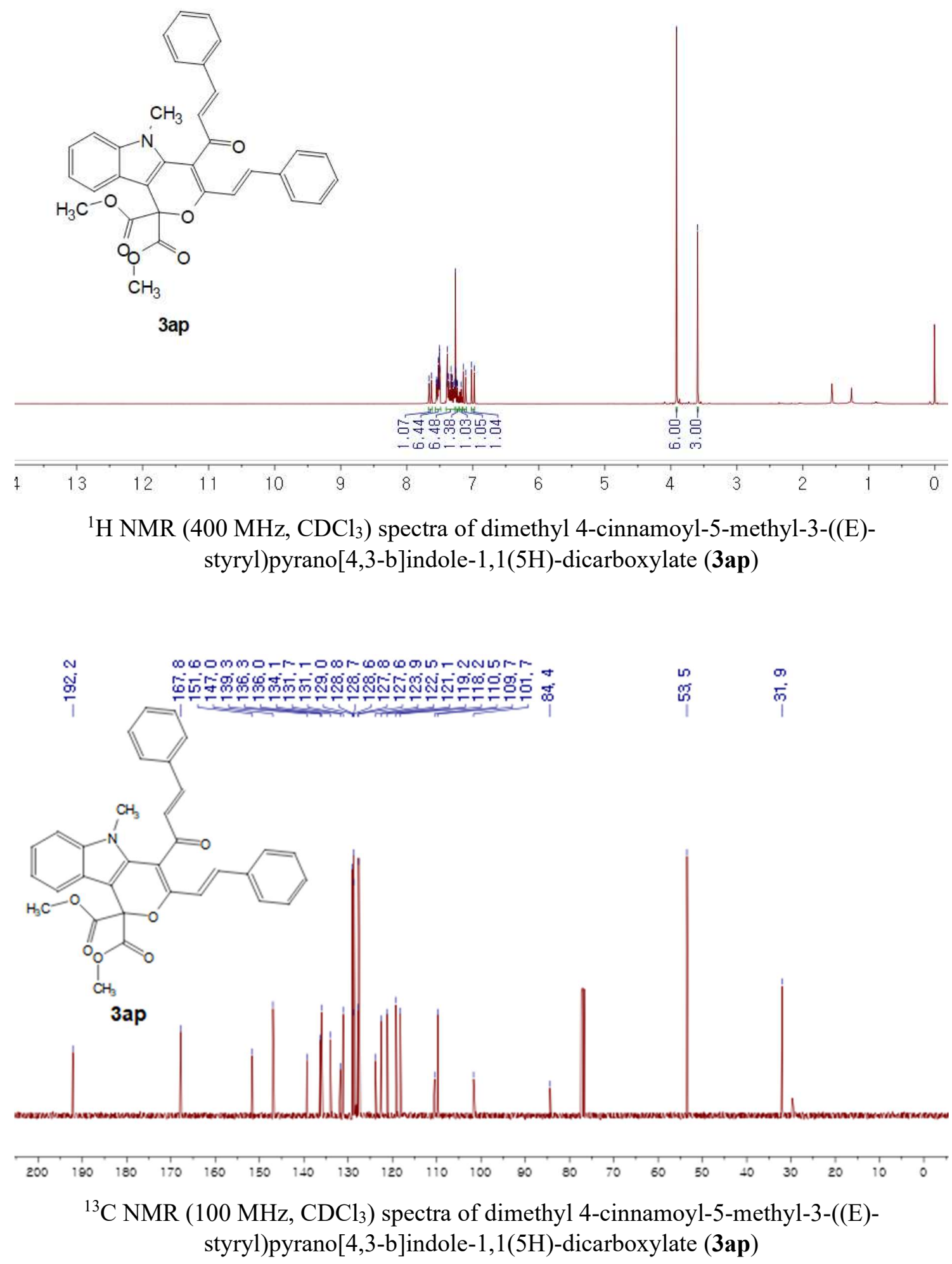


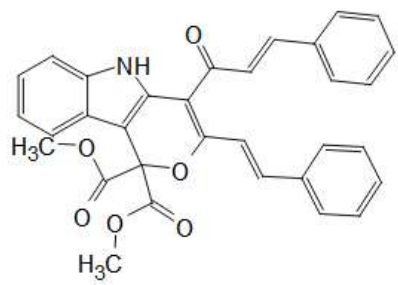

3ip

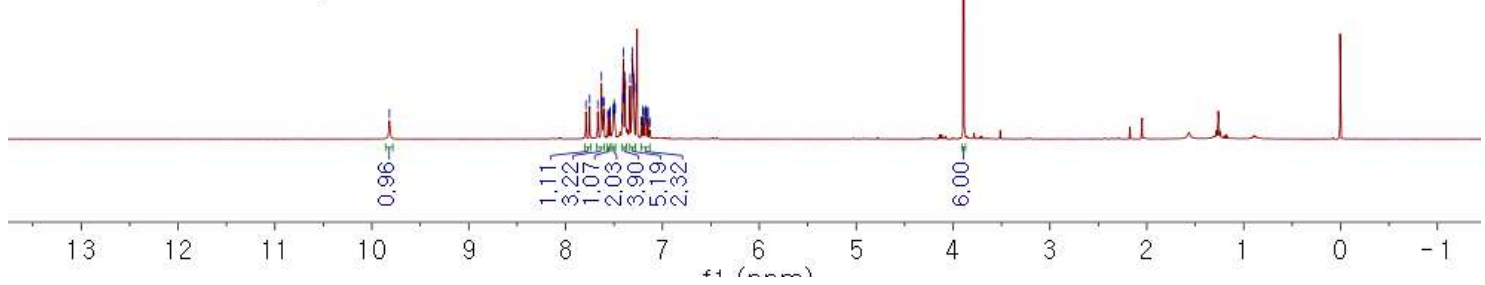

${ }^{1} \mathrm{H}$ NMR (400 MHz, $\mathrm{CDCl}_{3}$ ) spectra of dimethyl 4-cinnamoyl-3-((E)-styryl)pyrano[4,3b]indole-1,1(5H)-dicarboxylate (3ip)
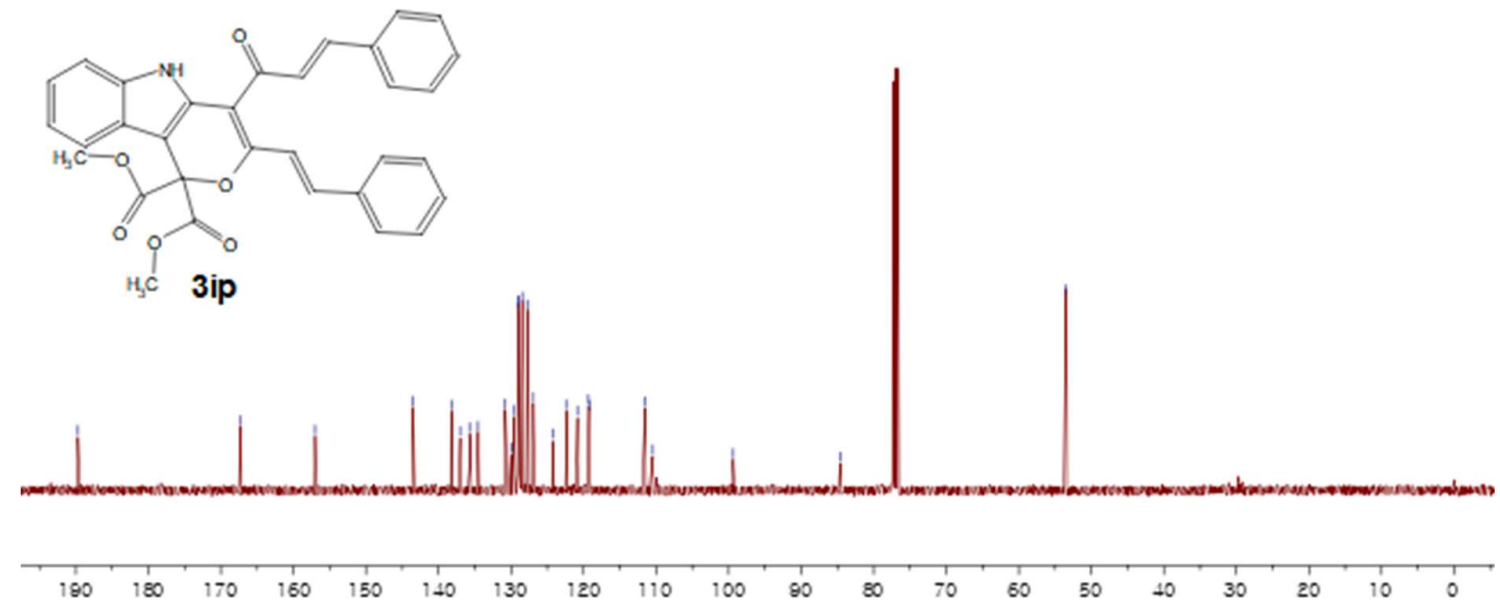

${ }^{13} \mathrm{C}$ NMR (100 MHz, $\left.\mathrm{CDCl}_{3}\right)$ spectra of dimethyl 4-cinnamoyl-3-((E)-styryl)pyrano[4,3b]indole-1,1(5H)-dicarboxylate (3ip) 


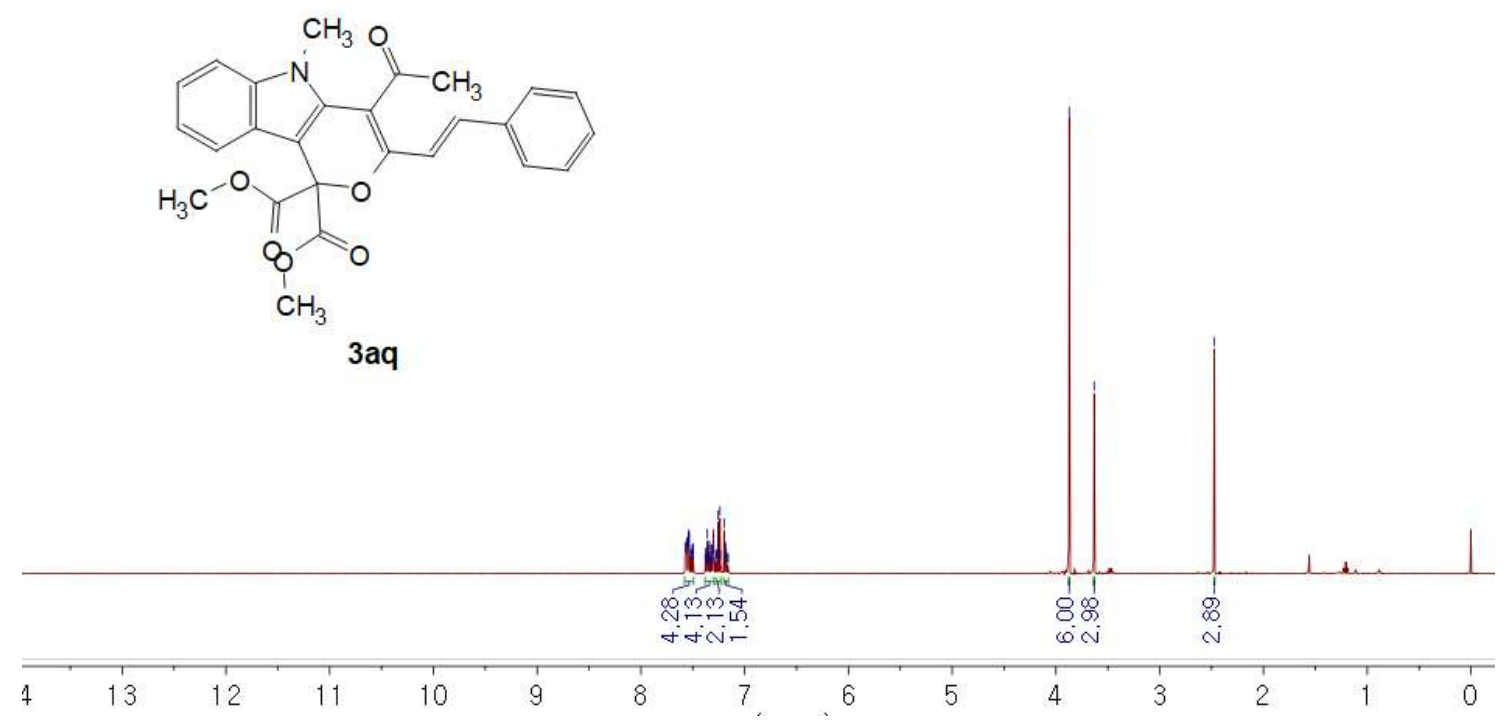

${ }^{1} \mathrm{H}$ NMR (400 MHz, $\mathrm{CDCl}_{3}$ ) spectra of dimethyl (E)-4-acetyl-5-methyl-3-styrylpyrano[4,3b]indole-1,1(5H)-dicarboxylate (3aq)

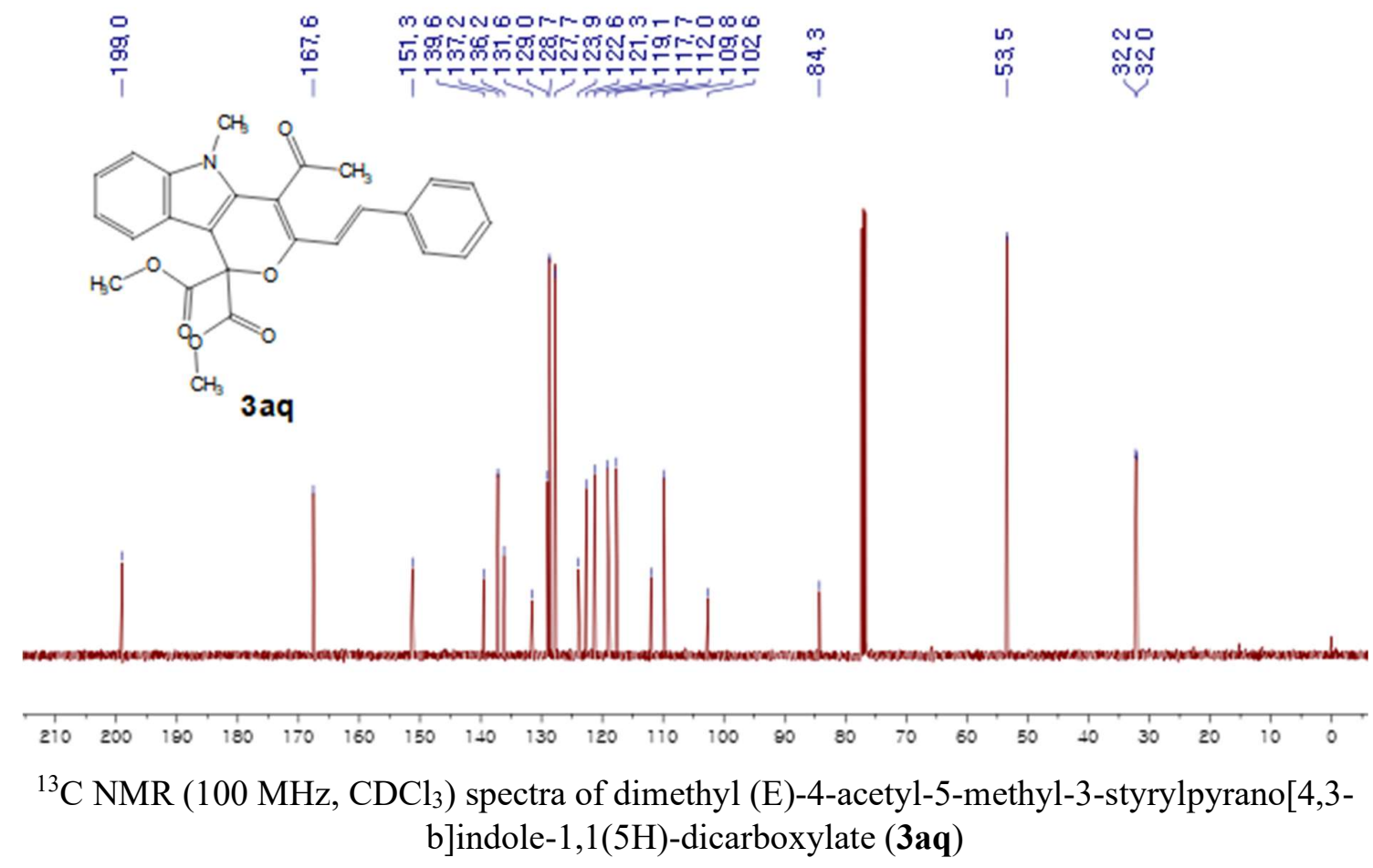




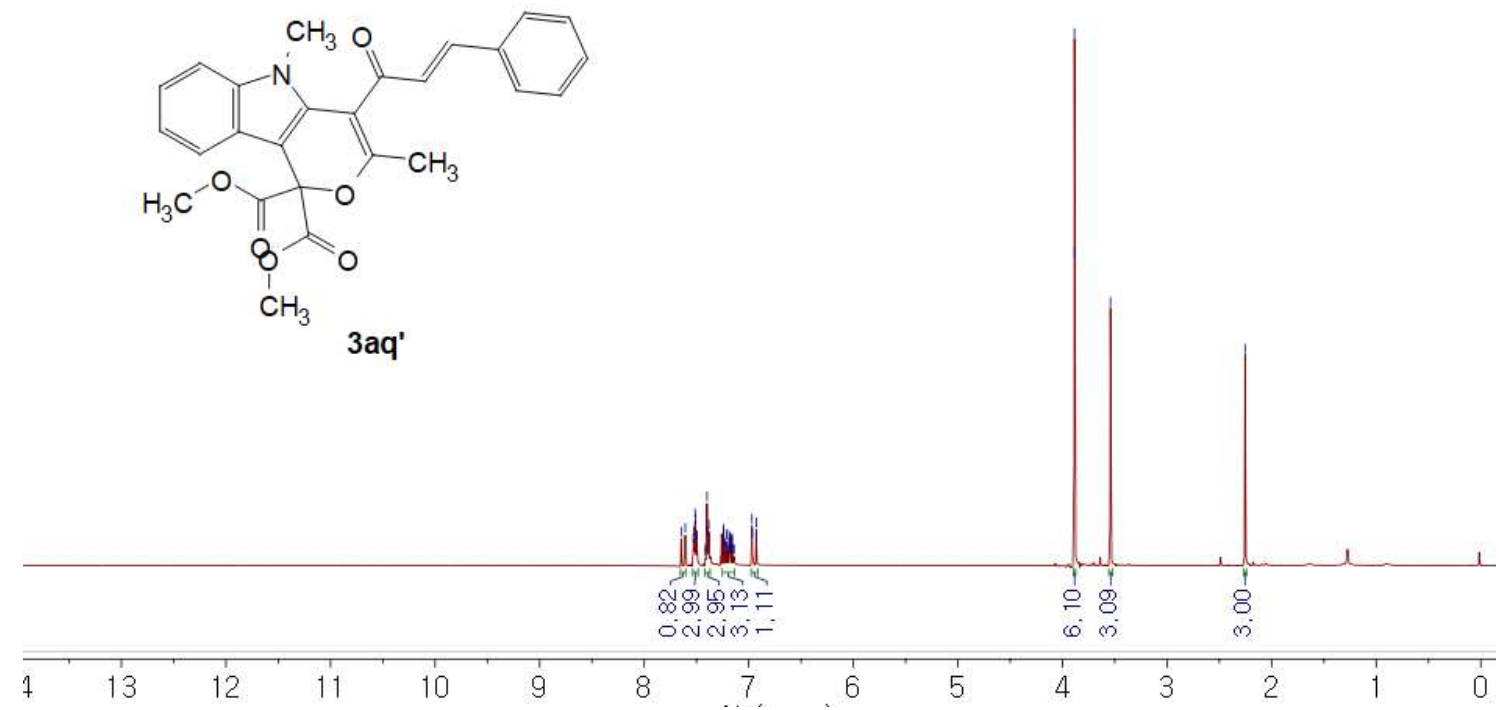

${ }^{1} \mathrm{H}$ NMR (400 MHz, $\mathrm{CDCl}_{3}$ ) spectra of dimethyl 4-cinnamoyl-3,5-dimethylpyrano[4,3b]indole-1,1(5H)-dicarboxylate (3aq')
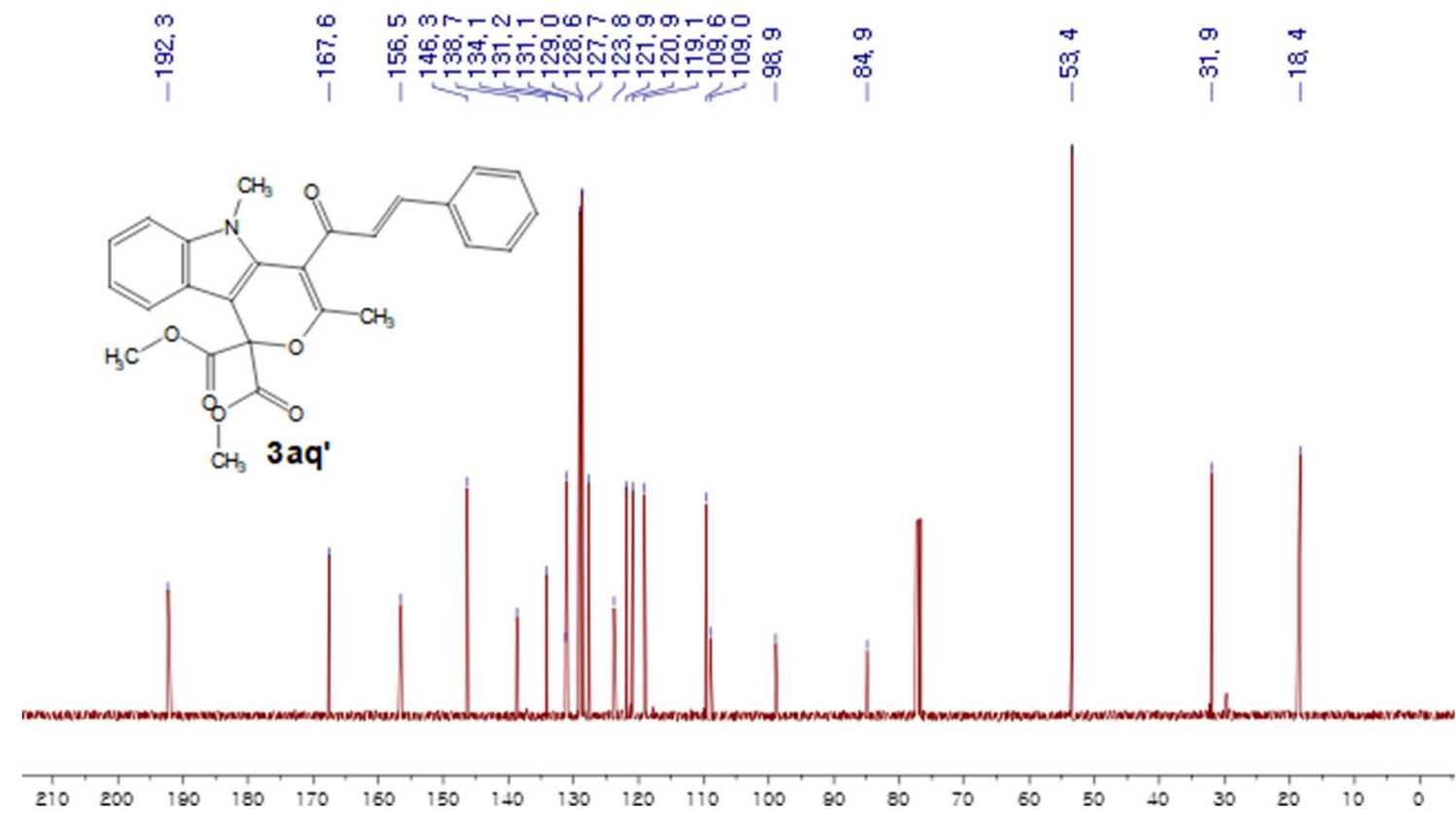

${ }^{13} \mathrm{C}$ NMR (100 MHz, $\left.\mathrm{CDCl}_{3}\right)$ spectra of dimethyl 4-cinnamoyl-3,5-dimethylpyrano[4,3b]indole-1,1(5H)-dicarboxylate (3aq') 
<smiles>COC(=O)C1(C(=O)OC)OC(/C=C/c2ccccc2)=C(C(C)=O)c2[nH]c3ccccc3c21</smiles>

3iq<smiles>COC(=O)C1=C2C(C(=O)/C=C/c3ccccc3)=C(C)OC1(C(=O)OC)Nc1ccccc12</smiles>

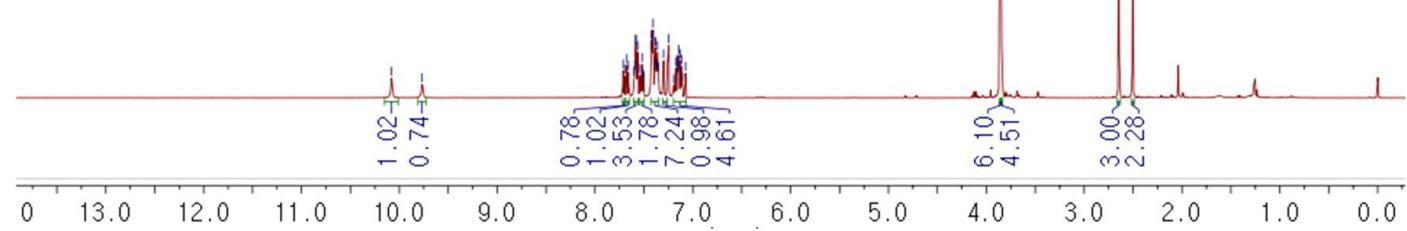

${ }^{1} \mathrm{H} \mathrm{NMR}\left(400 \mathrm{MHz} \mathrm{CDCl}_{3}\right.$ ) spectra of dimethyl (E)-4-acetyl-3-styrylpyrano[4,3-b]indole1,1(5H)-dicarboxylate and dimethyl 4-cinnamoyl-3-methylpyrano[4,3-b]indole-1,1(5H)dicarboxylate (3iq, 3iq')

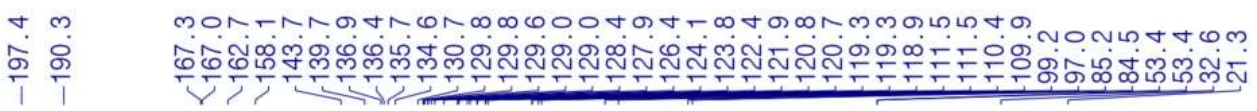
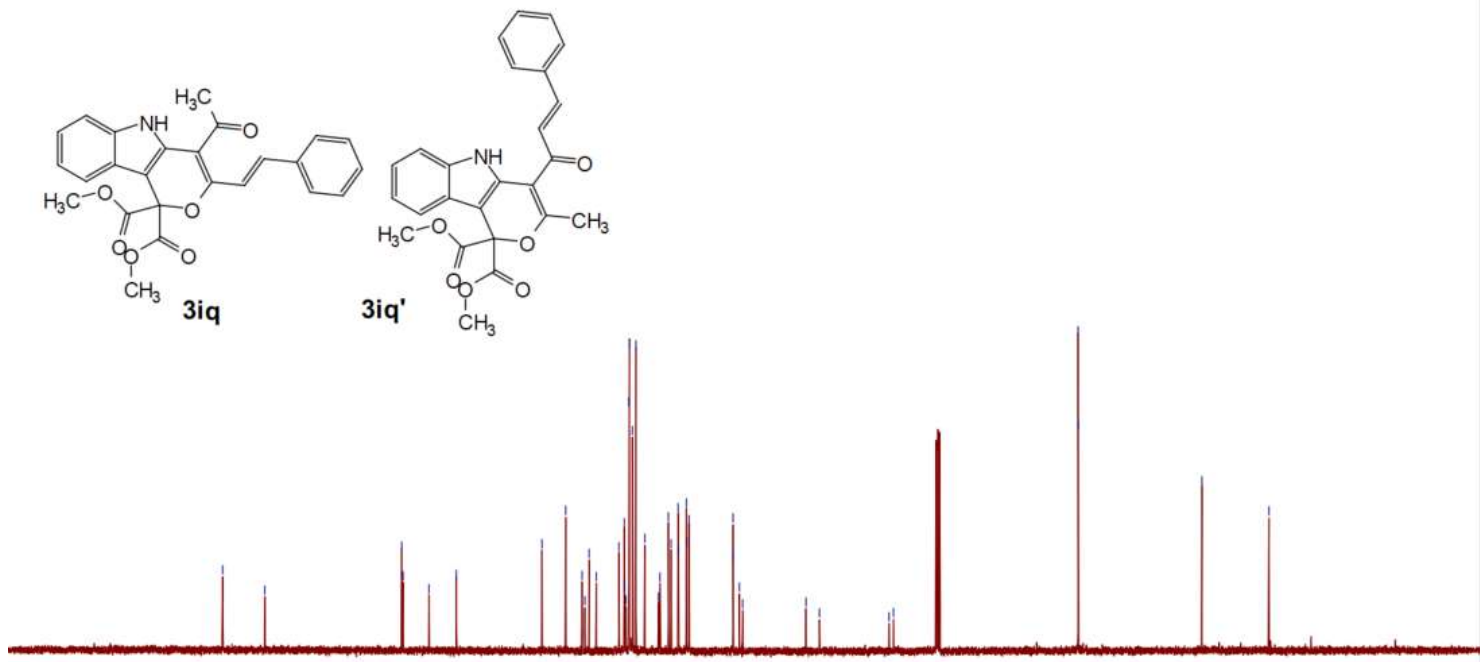

$\begin{array}{llllllllllllllllllllllllll}230 & 220 & 210 & 200 & 190 & 180 & 170 & 160 & 150 & 140 & 130 & 120 & 110 & 100 & 90 & 80 & 70 & 60 & 50 & 40 & 30 & 20 & 10 & 0 & -10\end{array}$

${ }^{13} \mathrm{C}$ NMR (100 MHz, CDCl 3 ) spectra of dimethyl (E)-4-acetyl-3-styrylpyrano[4,3-b]indole1,1(5H)-dicarboxylate and dimethyl 4-cinnamoyl-3-methylpyrano[4,3-b]indole-1,1(5H)dicarboxylate (3iq, 3iq') 

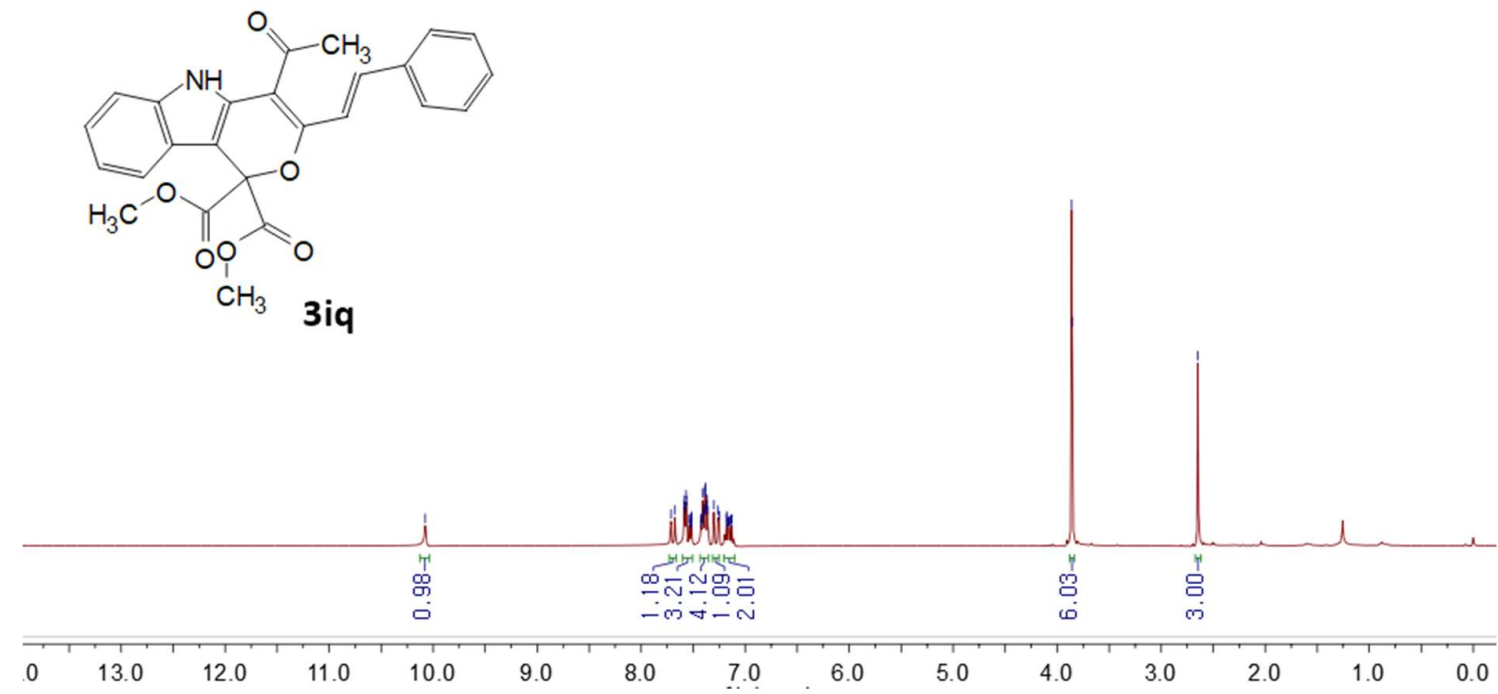

${ }^{1} \mathrm{H}$ NMR (400 MHz, $\mathrm{CDCl}_{3}$ ) spectra of dimethyl (E)-4-acetyl-3-styrylpyrano[4,3-b]indole$1,1(5 \mathrm{H})$-dicarboxylate (3iq)
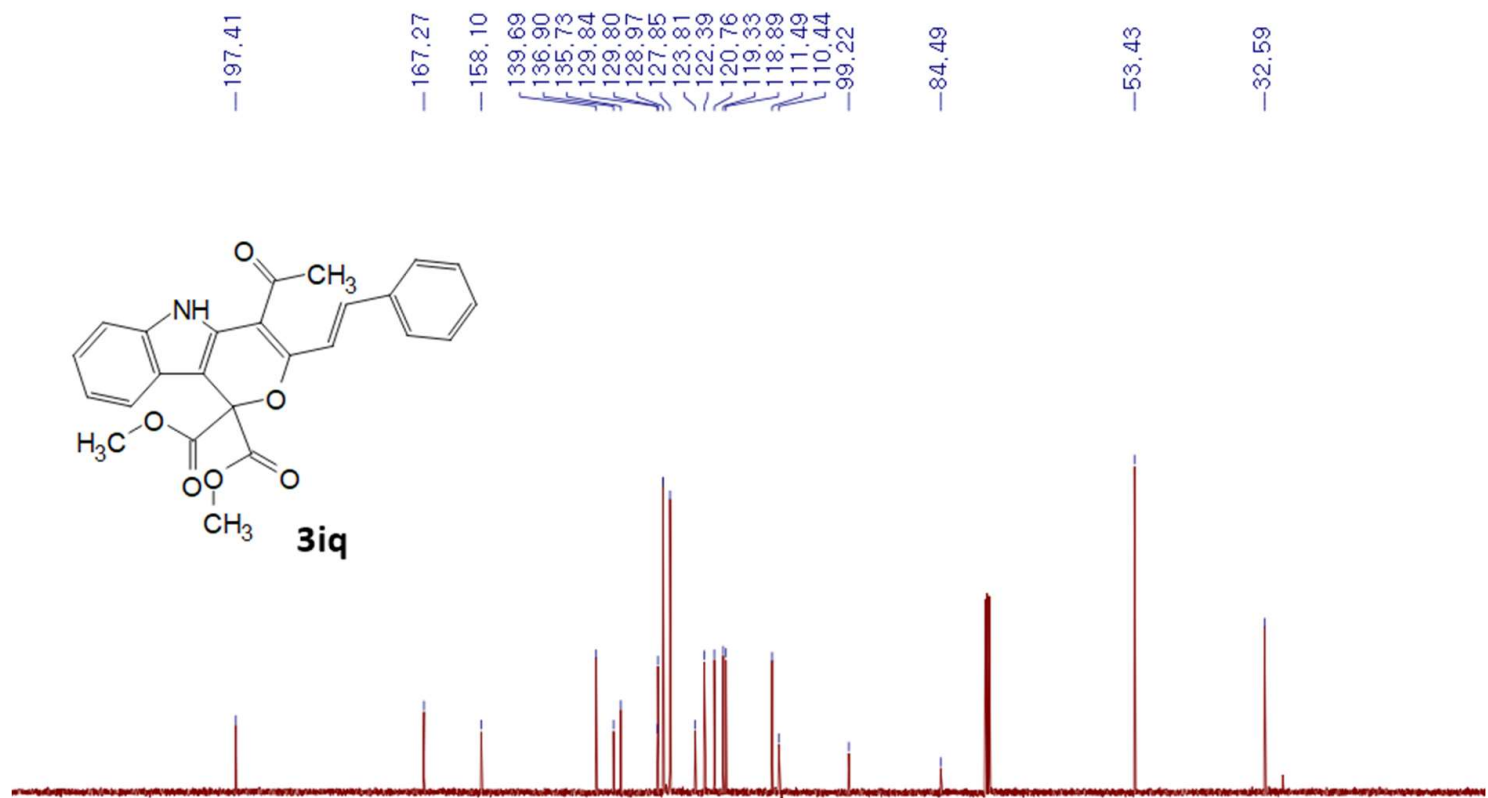

$\begin{array}{llllllllllllllllllllllll}230 & 220 & 210 & 200 & 190 & 180 & 170 & 160 & 150 & 140 & 130 & 120 & 110 & 100 & 90 & 80 & 70 & 60 & 50 & 40 & 30 & 20 & 10 & 0\end{array}$

${ }^{13} \mathrm{C}$ NMR $\left(100 \mathrm{MHz}, \mathrm{CDCl}_{3}\right.$ ) spectra of dimethyl (E)-4-acetyl-3-styrylpyrano[4,3-b]indole1,1(5H)-dicarboxylate (3iq) 

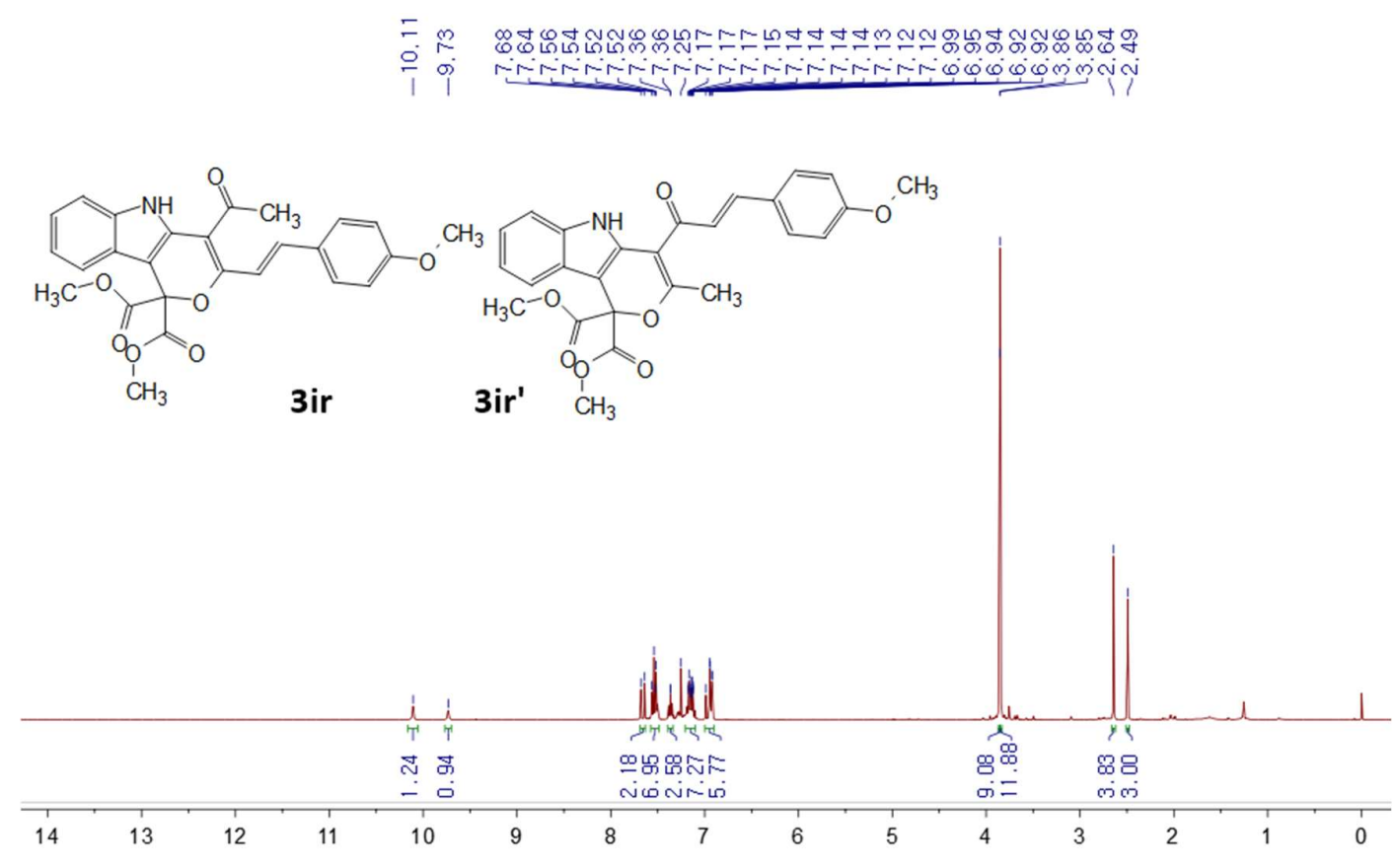

${ }^{1} \mathrm{H}$ NMR (400 MHz, $\mathrm{CDCl}_{3}$ ) spectra of dimethyl (E)-4-acetyl-3-(4methoxystyryl)pyrano[4,3-b]indole-1,1(5H)-dicarboxylate and dimethyl (E)-4-(3-(4methoxyphenyl)acryloyl)-3-methylpyrano[4,3-b]indole-1,1(5H)-dicarboxylate (3ir, 3ir')

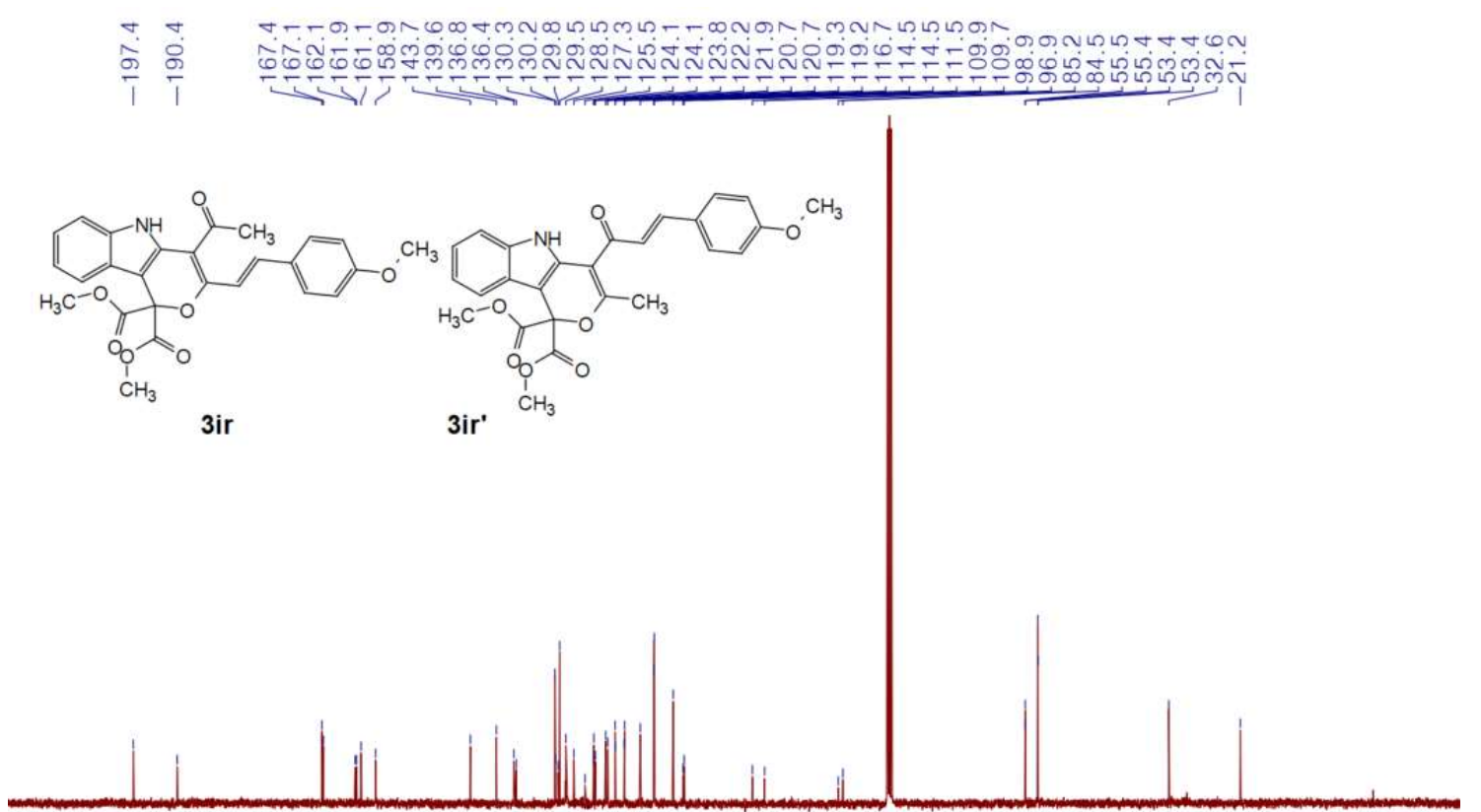

$\begin{array}{llllllllllllllllllllllllllll}210 & 200 & 190 & 180 & 170 & 160 & 150 & 140 & 130 & 120 & 110 & 100 & 90 & 80 & 70 & 60 & 50 & 40 & 30 & 20 & 10 & 0 & -10\end{array}$

${ }^{13} \mathrm{C}$ NMR (100 MHz, $\left.\mathrm{CDCl}_{3}\right)$ spectra of dimethyl (E)-4-acetyl-3-(4methoxystyryl)pyrano[4,3-b]indole-1,1(5H)-dicarboxylate and dimethyl (E)-4-(3-(4methoxyphenyl)acryloyl)-3-methylpyrano[4,3-b]indole-1,1(5H)-dicarboxylate (3ir, 3ir') 


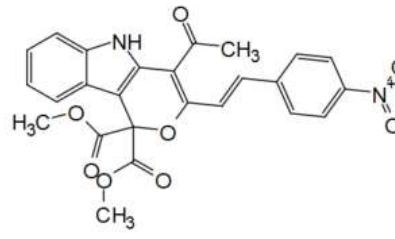

3is

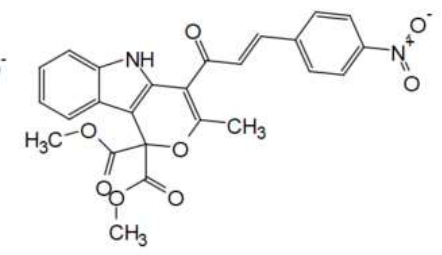

3is"

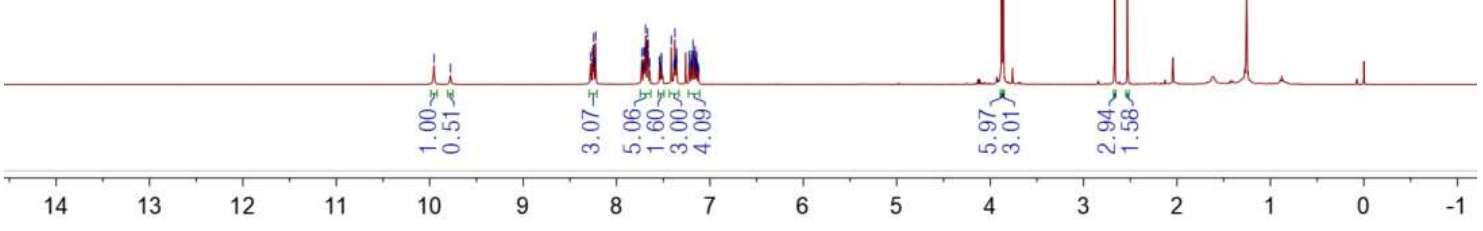

${ }^{1} \mathrm{H}$ NMR (400 MHz, $\mathrm{CDCl}_{3}$ ) spectra of dimethyl (E)-4-acetyl-3-(4-nitrostyryl)pyrano[4,3-

b]indole-1,1(5H)-dicarboxylate and dimethyl (E)-3-methyl-4-(3-(4nitrophenyl)acryloyl)pyrano[4,3-b]indole-1,1(5H)-dicarboxylate (3is, 3is')
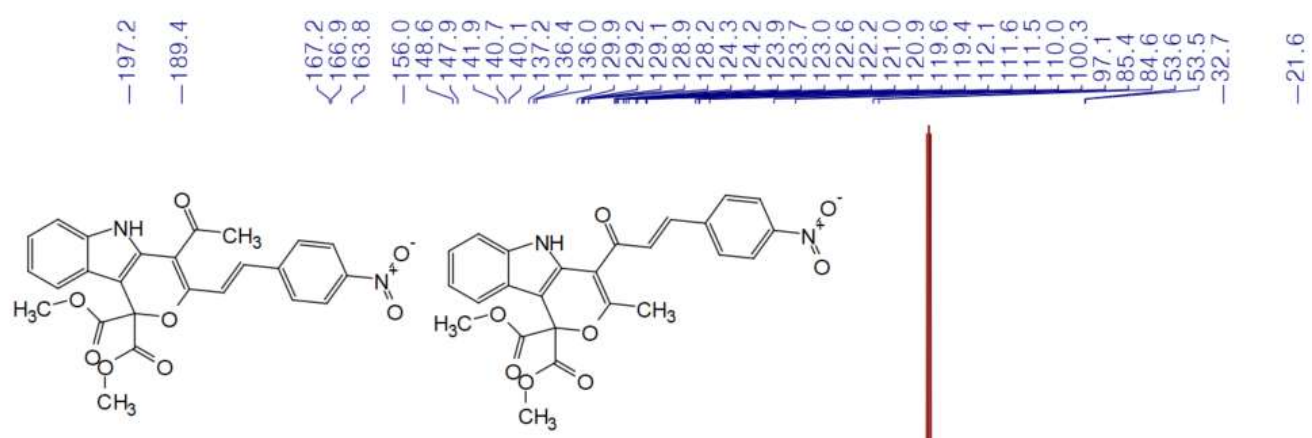

3 is

3is'

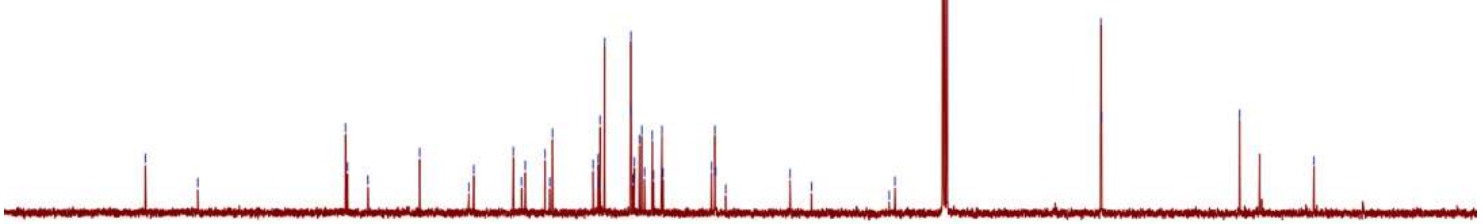

$\begin{array}{llllllllllllllllllllllll}210 & 200 & 190 & 180 & 170 & 160 & 150 & 140 & 130 & 120 & 110 & 100 & 90 & 80 & 70 & 60 & 50 & 40 & 30 & 20 & 10 & 0\end{array}$

${ }^{13} \mathrm{C}$ NMR (100 MHz, $\mathrm{CDCl}_{3}$ ) spectra of dimethyl (E)-4-acetyl-3-(4-nitrostyryl)pyrano[4,3-

b]indole-1,1(5H)-dicarboxylate and dimethyl (E)-3-methyl-4-(3-(4nitrophenyl)acryloyl)pyrano[4,3-b]indole-1,1(5H)-dicarboxylate (3is, 3is') 

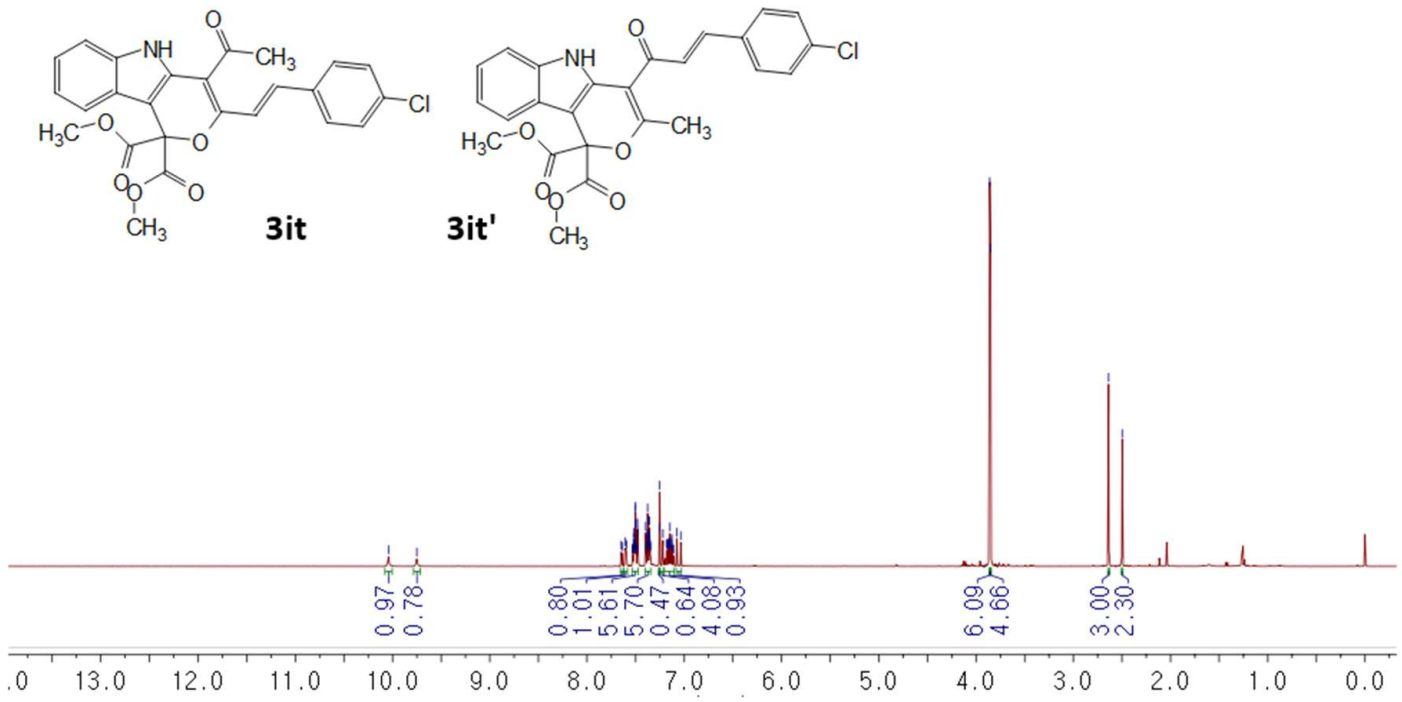

${ }^{1} \mathrm{H}$ NMR (400 MHz, $\mathrm{CDCl}_{3}$ ) spectra of dimethyl (E)-4-acetyl-3-(4-chlorostyryl)pyrano[4,3b]indole-1,1(5H)-dicarboxylate and dimethyl (E)-4-(3-(4-chlorophenyl)acryloyl)-3methylpyrano[4,3-b]indole-1,1(5H)-dicarboxylate (3it, 3it')

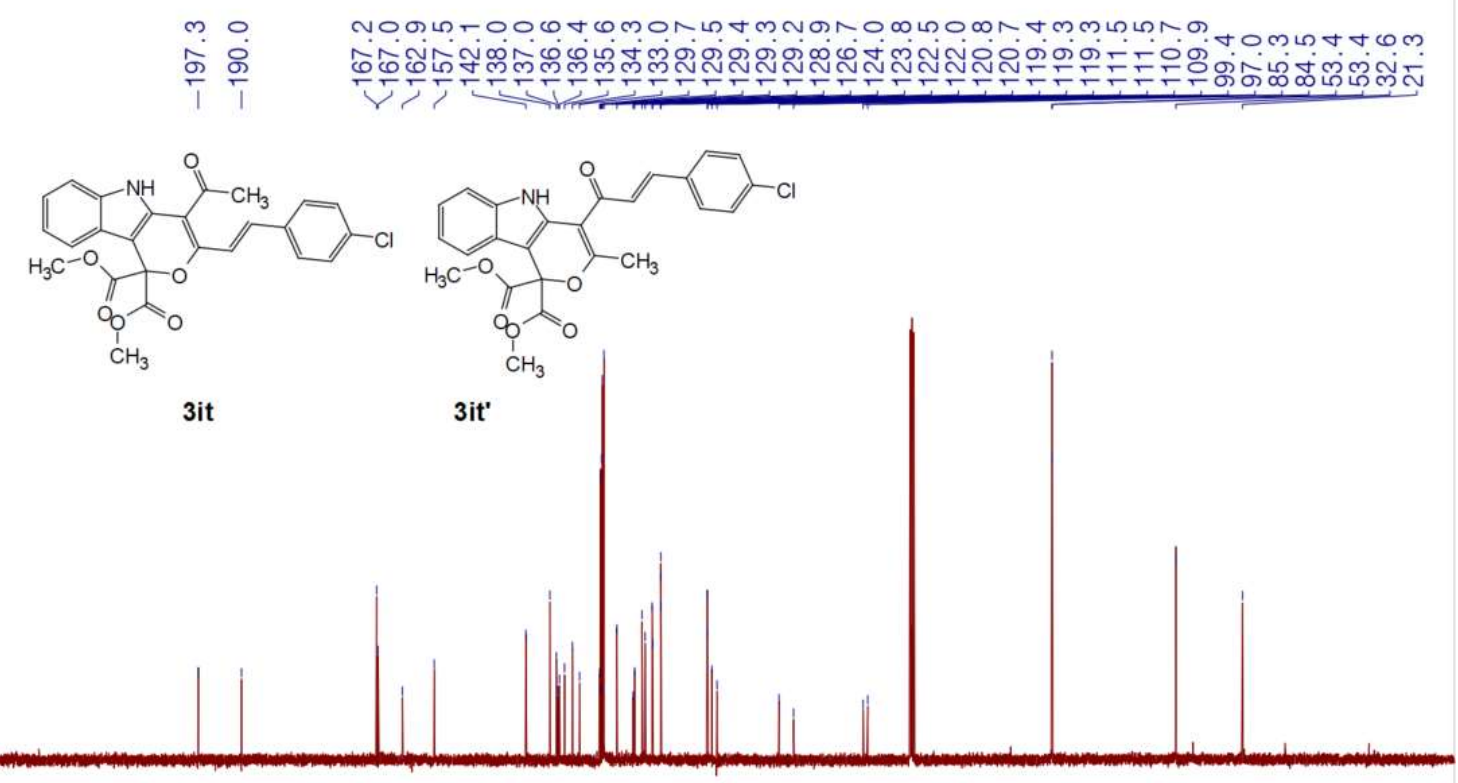

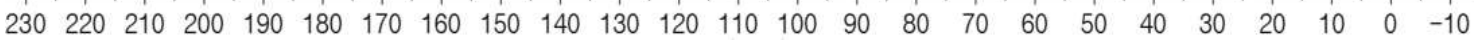

${ }^{13} \mathrm{C}$ NMR (100 MHz, $\mathrm{CDCl}_{3}$ ) spectra of dimethyl (E)-4-acetyl-3-(4-chlorostyryl)pyrano[4,3b]indole-1,1(5H)-dicarboxylate and dimethyl (E)-4-(3-(4-chlorophenyl)acryloyl)-3methylpyrano[4,3-b]indole-1,1(5H)-dicarboxylate (3it, 3it') 


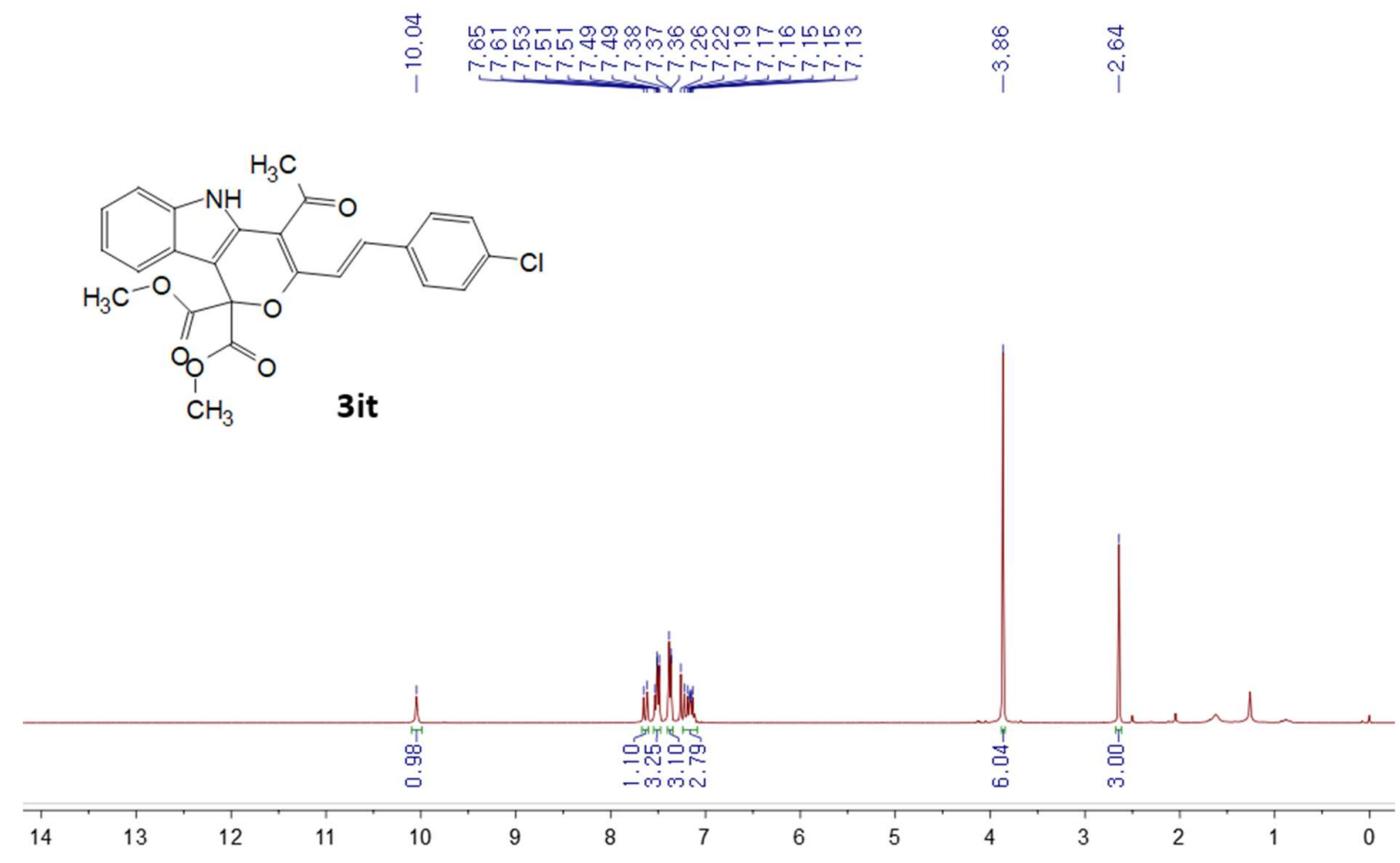

${ }^{1} \mathrm{H}$ NMR (400 MHz, $\mathrm{CDCl}_{3}$ ) spectra of dimethyl (E)-4-acetyl-3-(4-chlorostyryl)pyrano[4,3b]indole-1,1(5H)-dicarboxylate (3it)

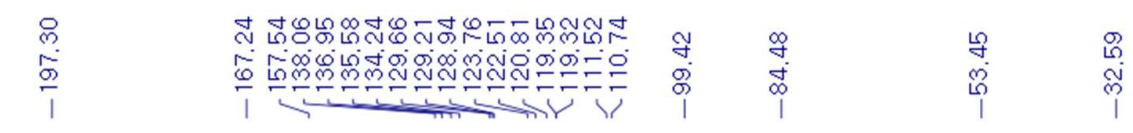<smiles>COC(=O)C1(C(=O)OC)OC(/C=C/c2ccc(Cl)cc2)=C(C(C)=O)c2[nH]c3ccccc3c21</smiles>

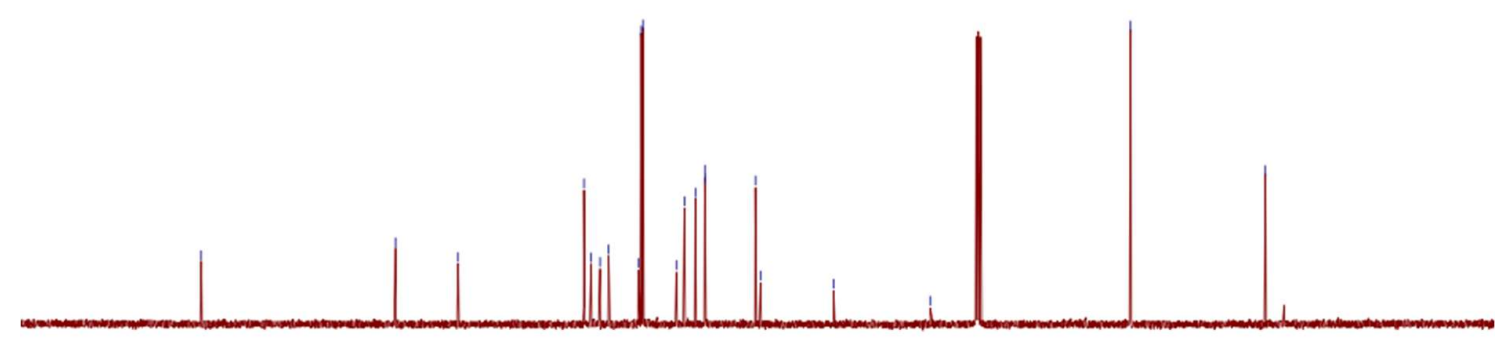

$\begin{array}{llllllllllllllllllllllllllll}220 & 210 & 200 & 190 & 180 & 170 & 160 & 150 & 140 & 130 & 120 & 110 & 100 & 90 & 80 & 70 & 60 & 50 & 40 & 30 & 20 & 10 & 0\end{array}$

${ }^{13} \mathrm{C} \mathrm{NMR}\left(100 \mathrm{MHz}, \mathrm{CDCl}_{3}\right)$ spectra of dimethyl (E)-4-acetyl-3-(4-chlorostyryl)pyrano[4,3b]indole-1,1(5H)-dicarboxylate (3it) 

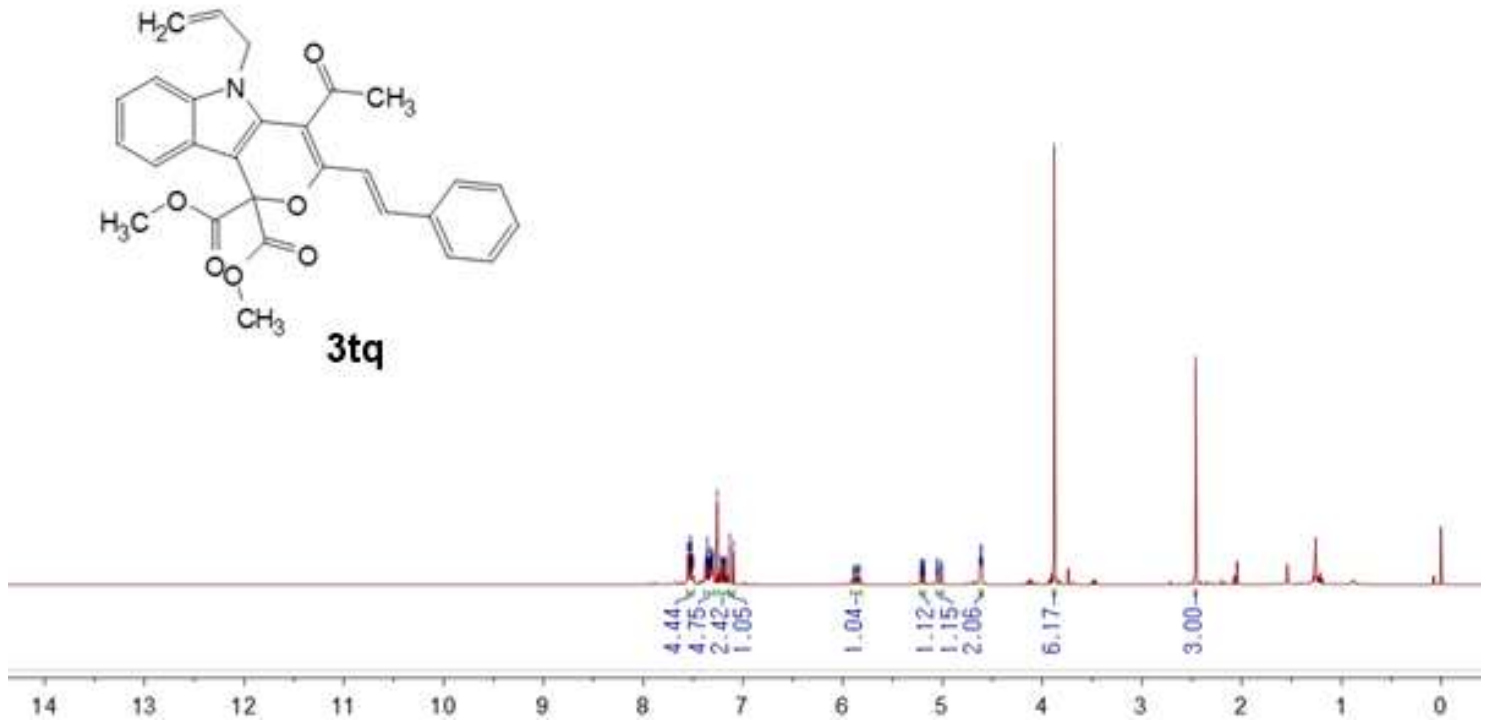

${ }^{1} \mathrm{H}$ NMR (400 MHz, $\mathrm{CDCl}_{3}$ ) spectra of dimethyl (E)-4-acetyl-5-allyl-3-styrylpyrano[4,3b]indole-1,1(5H)-dicarboxylate (3tq)

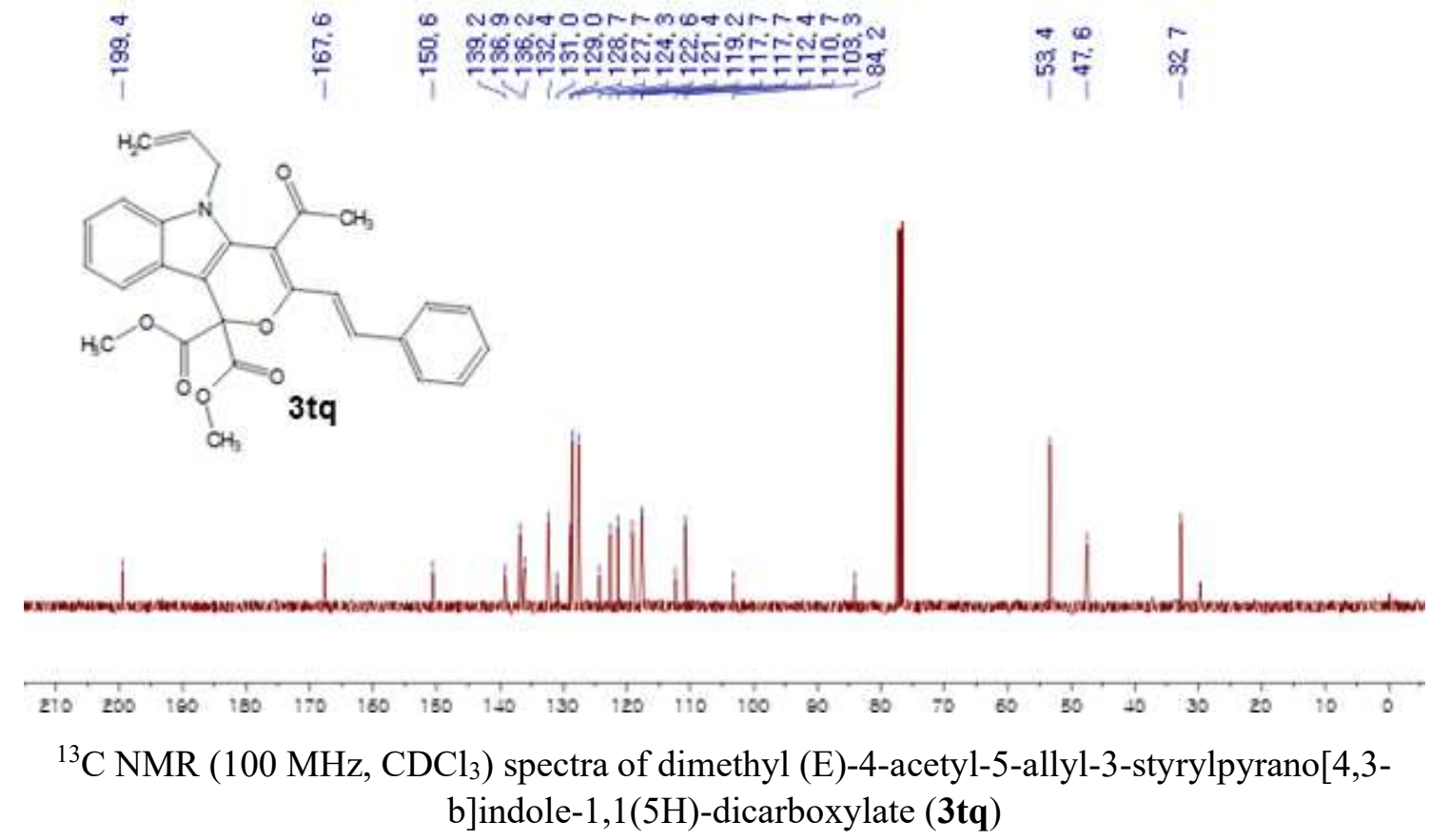



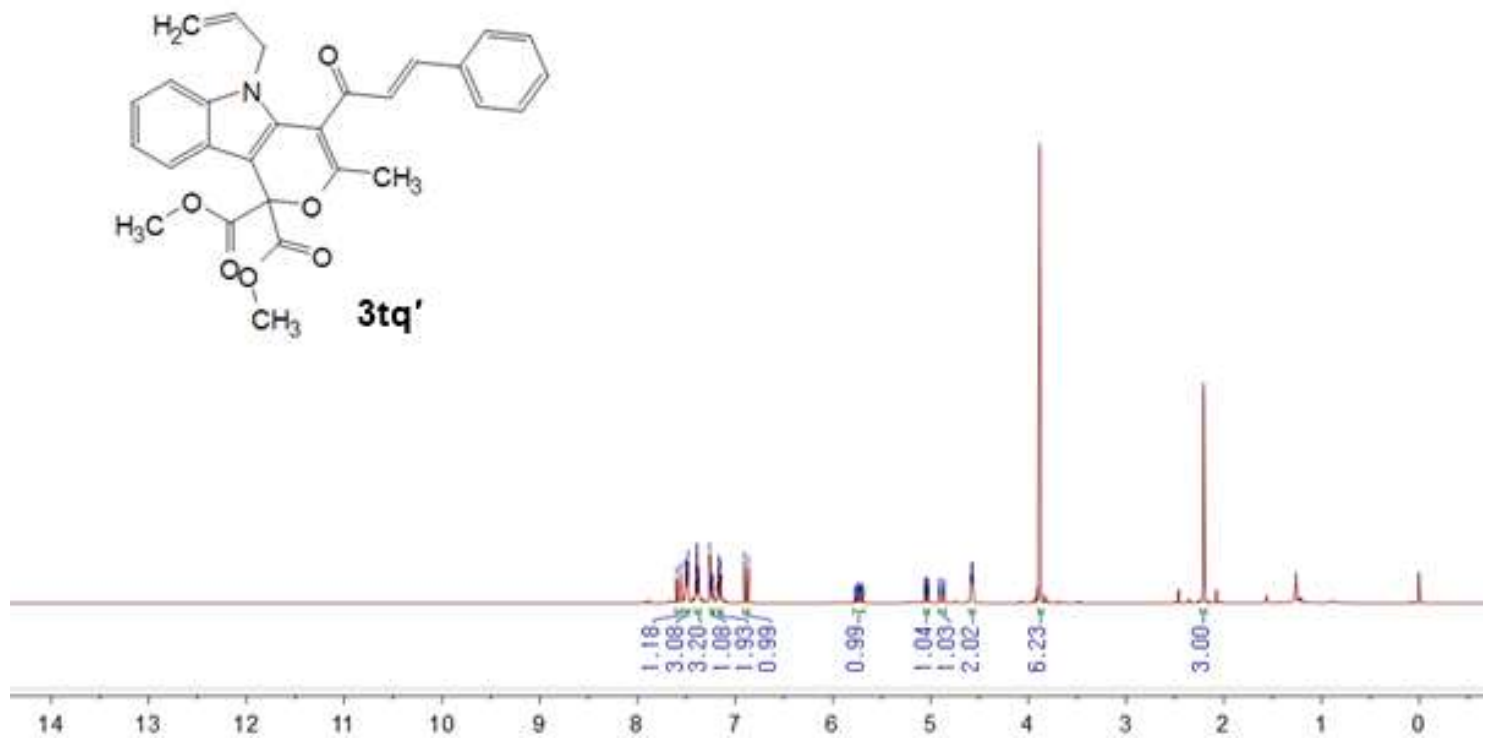

${ }^{1} \mathrm{H}$ NMR (400 MHz, $\mathrm{CDCl}_{3}$ ) spectra of dimethyl 5-allyl-4-cinnamoyl-3-methylpyrano[4,3b]indole-1,1(5H)-dicarboxylate (3tq')
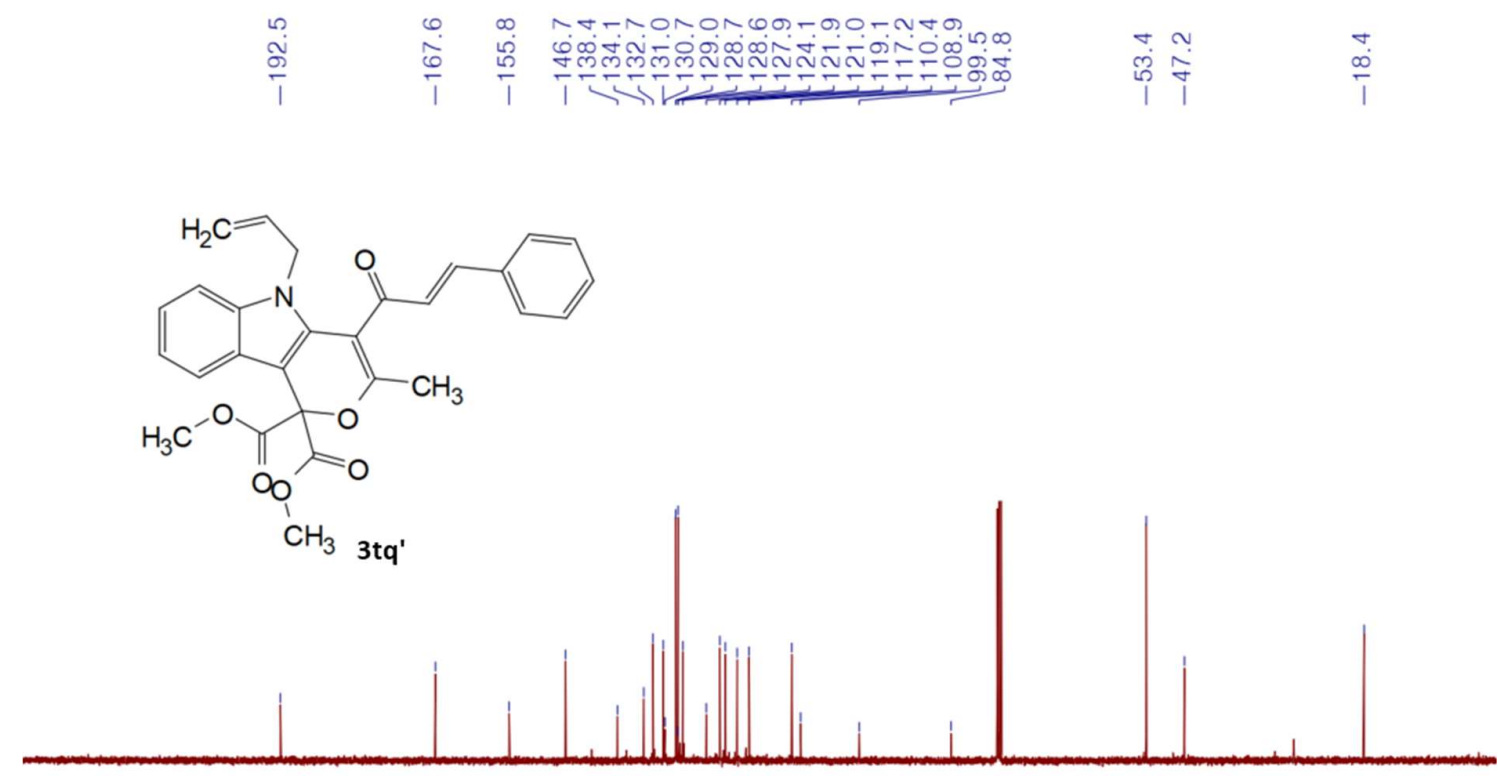

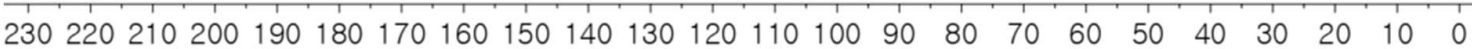

${ }^{13} \mathrm{C}$ NMR $\left(100 \mathrm{MHz}, \mathrm{CDCl}_{3}\right)$ spectra of dimethyl 5-allyl-4-cinnamoyl-3-methylpyrano[4,3b]indole-1,1(5H)-dicarboxylate (3tq') 

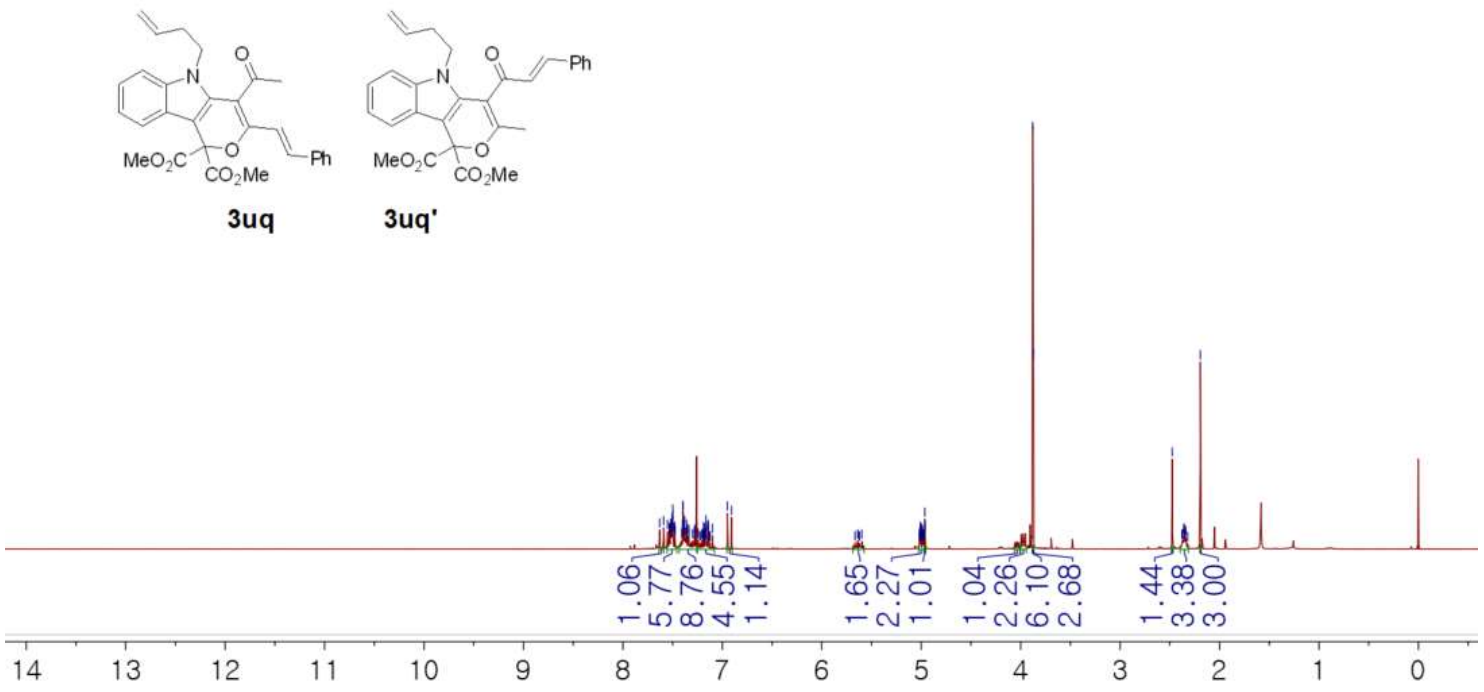

${ }^{1} \mathrm{H}$ NMR (400 MHz, $\mathrm{CDCl}_{3}$ ) spectra of Dimethyl (E)-4-acetyl-5-(but-3-en-1-yl)-3styrylpyrano[4,3-b]indole-1,1(5H)-dicarboxylate (3uq) and Dimethyl 5-(but-3-en-1-yl)-4-cinnamoyl3-methylpyrano[4,3-b]indole-1,1(5H)-dicarboxylate (3uq')

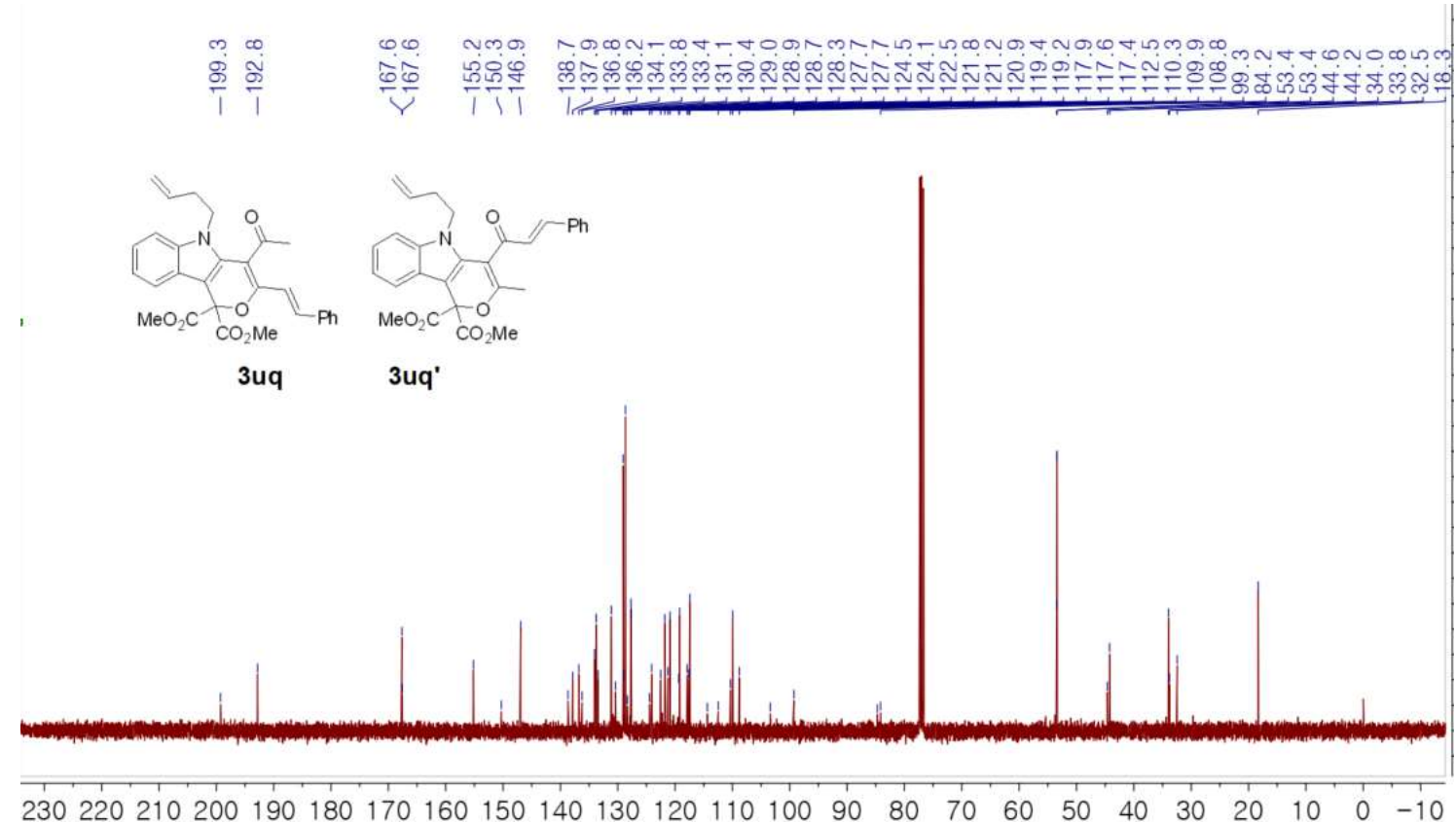

${ }^{13} \mathrm{C}$ NMR (100 MHz, $\left.\mathrm{CDCl}_{3}\right)$ spectra of Dimethyl (E)-4-acetyl-5-(but-3-en-1-yl)-3styrylpyrano[4,3-b]indole-1,1(5H)-dicarboxylate (3uq) and Dimethyl 5-(but-3-en-1-yl)-4-cinnamoyl3-methylpyrano[4,3-b]indole-1,1(5H)-dicarboxylate (3uq') 


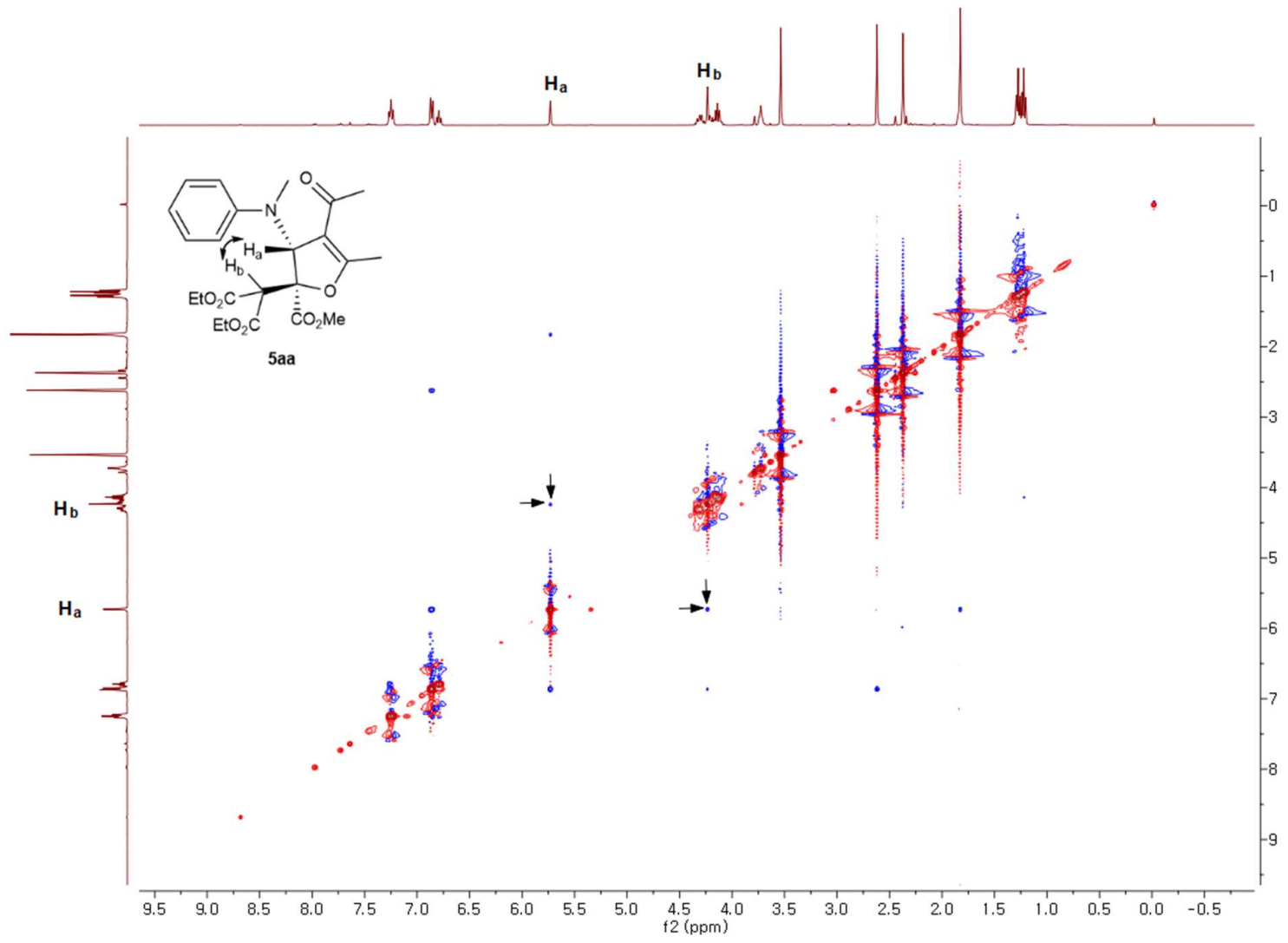

Supplementary Figure 4. NOESY of compound 5aa
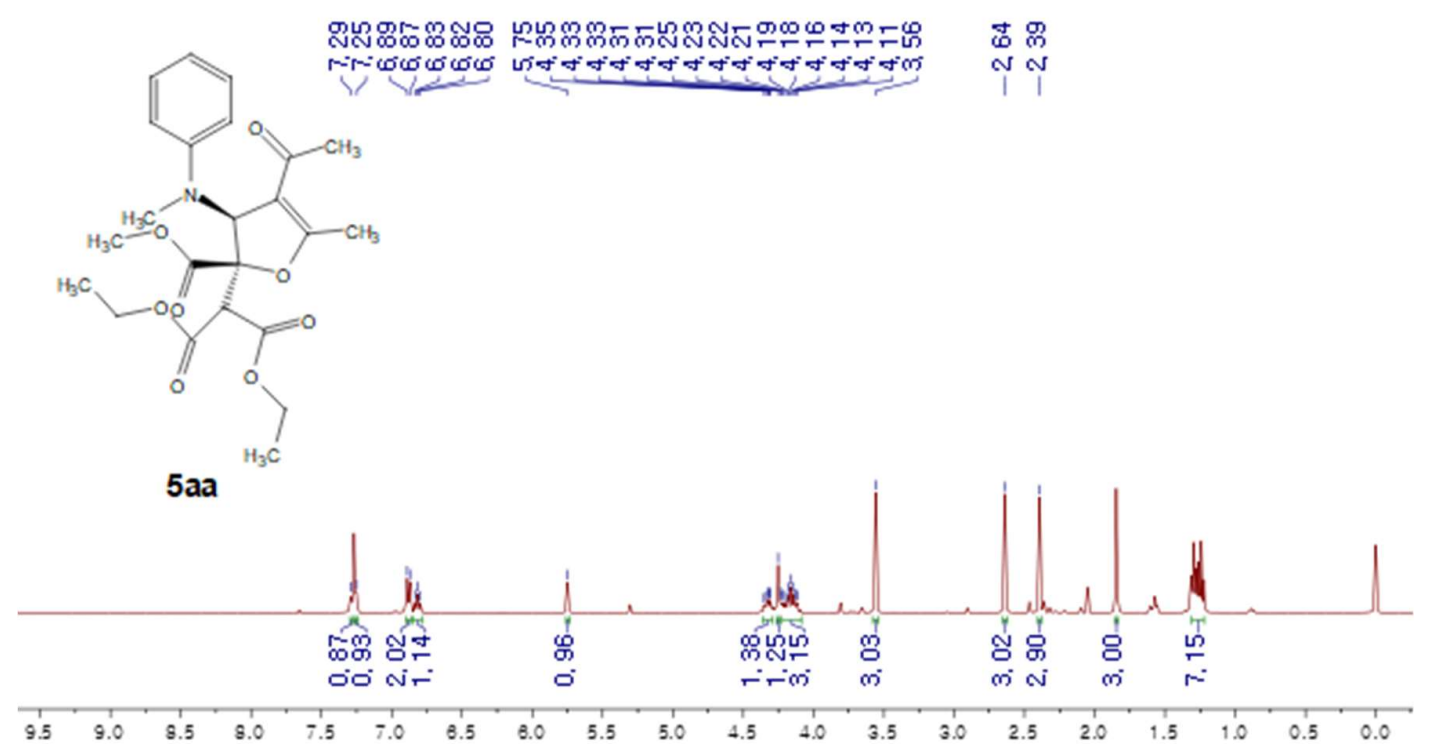

${ }^{1} \mathrm{H}$ NMR (400 MHz, $\mathrm{CDCl}_{3}$ ) spectra of diethyl 2-((2R,3S)-4-acetyl-2-(methoxycarbonyl)-5methyl-3-(methyl(phenyl)amino)-2,3-dihydrofuran-2-yl)malonate (5aa) 

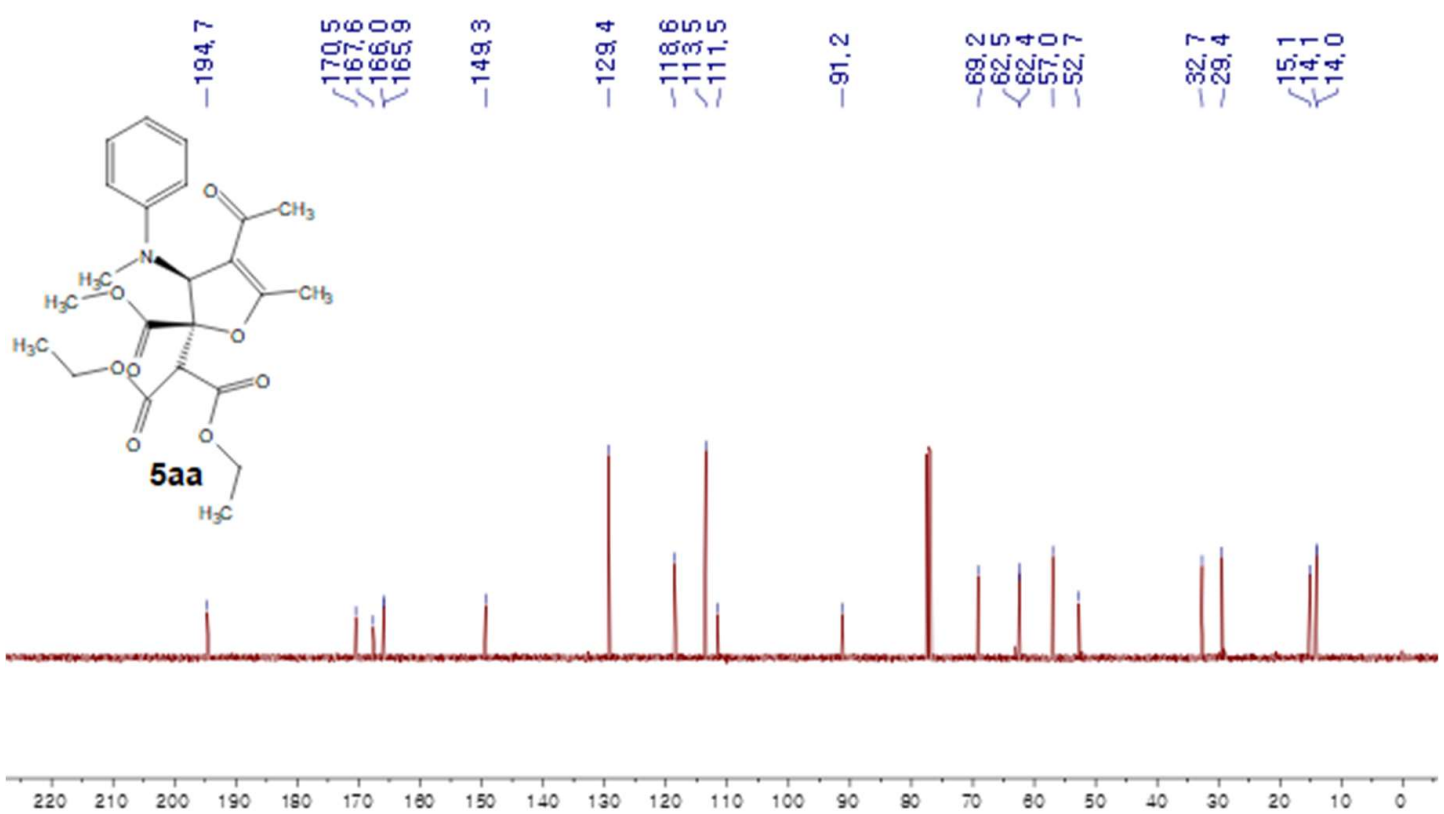

${ }^{13} \mathrm{C}$ NMR (100 MHz, $\left.\mathrm{CDCl}_{3}\right)$ spectra of diethyl 2-((2R,3S)-4-acetyl-2-(methoxycarbonyl)-5methyl-3-(methyl(phenyl)amino)-2,3-dihydrofuran-2-yl)malonate (5aa)

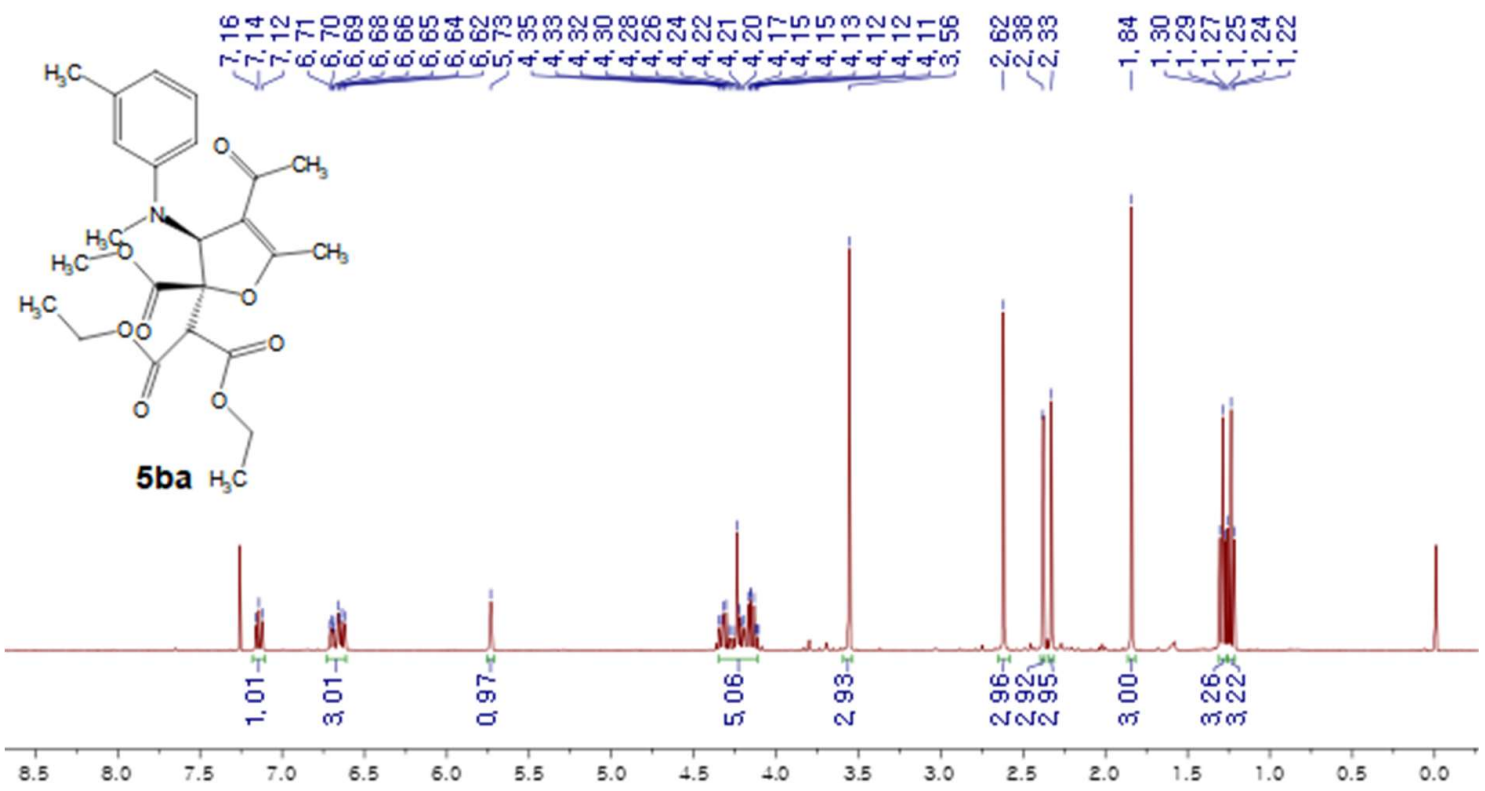

${ }^{1} \mathrm{H}$ NMR (400 MHz, $\left.\mathrm{CDCl}_{3}\right)$ spectra of diethyl 2-((2R,3S)-4-acetyl-2-(methoxycarbonyl)-5methyl-3-(methyl(m-tolyl)amino)-2,3-dihydrofuran-2-yl)malonate (5ba) 


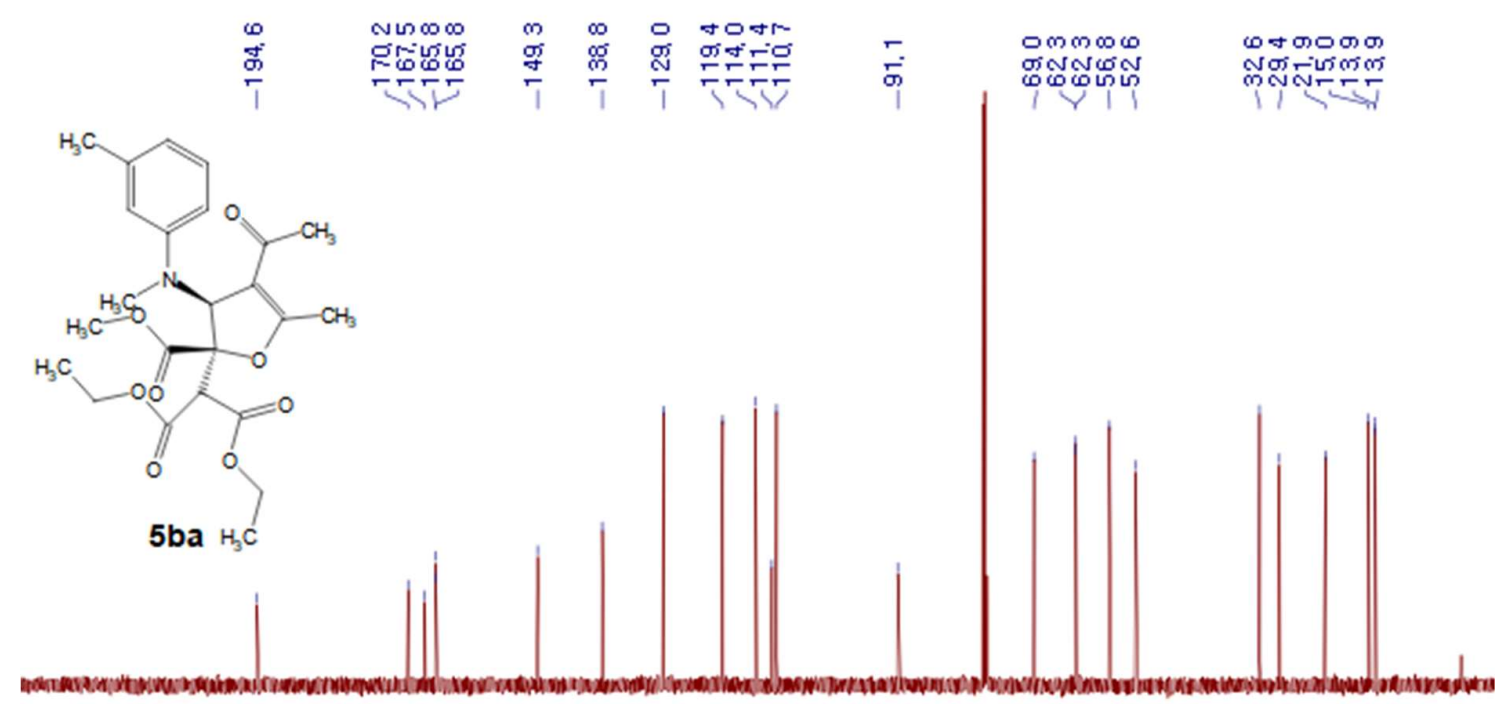

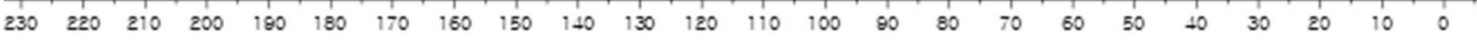

${ }^{13} \mathrm{C}$ NMR (100 MHz, $\left.\mathrm{CDCl}_{3}\right)$ spectra of diethyl 2-((2R,3S)-4-acetyl-2-(methoxycarbonyl)-5methyl-3-(methyl(m-tolyl)amino)-2,3-dihydrofuran-2-yl)malonate (5ba)

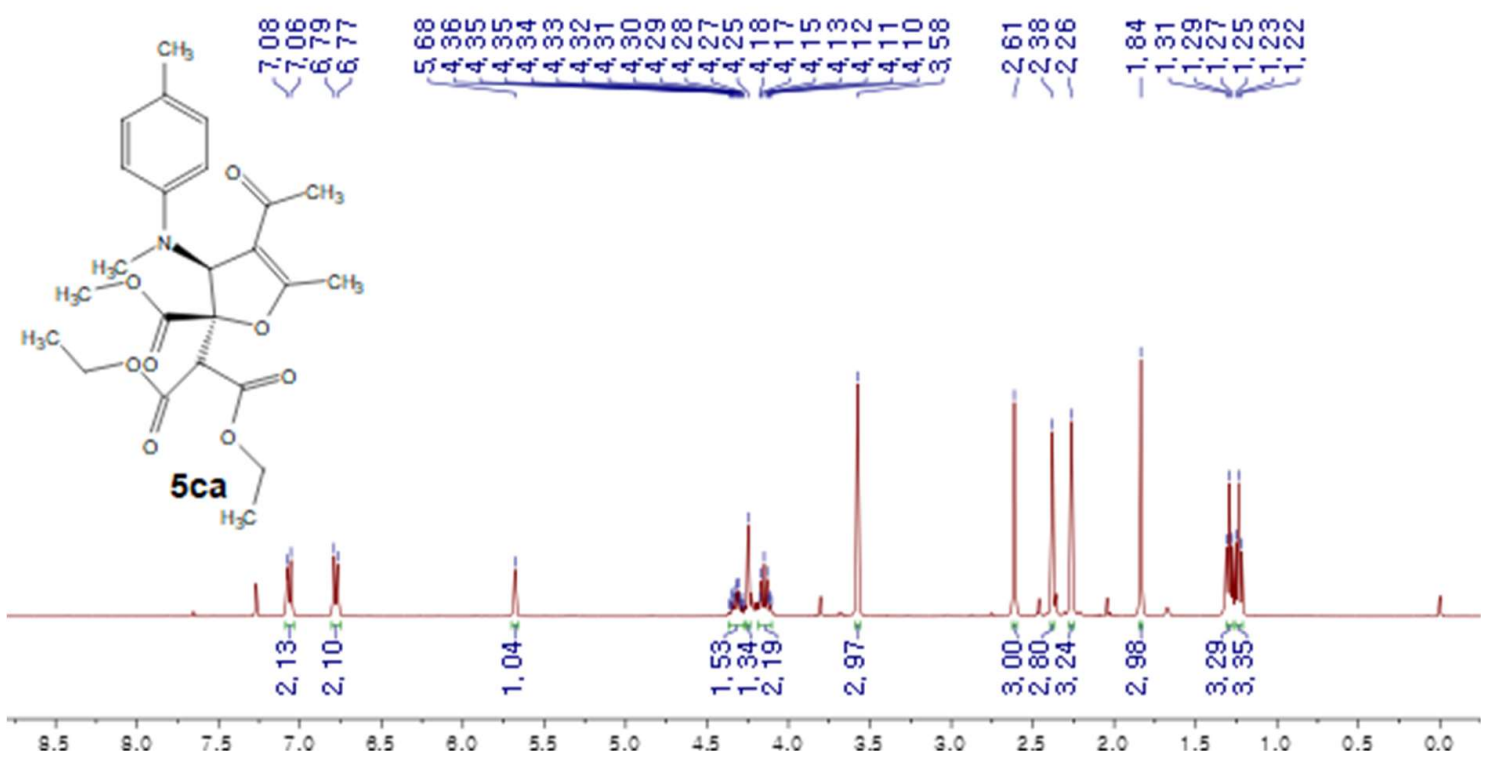

${ }^{1} \mathrm{H}$ NMR (400 MHz, $\left.\mathrm{CDCl}_{3}\right)$ spectra of diethyl 2-((2R,3S)-4-acetyl-2-(methoxycarbonyl)-5methyl-3-(methyl(p-tolyl)amino)-2,3-dihydrofuran-2-yl)malonate (5ca) 


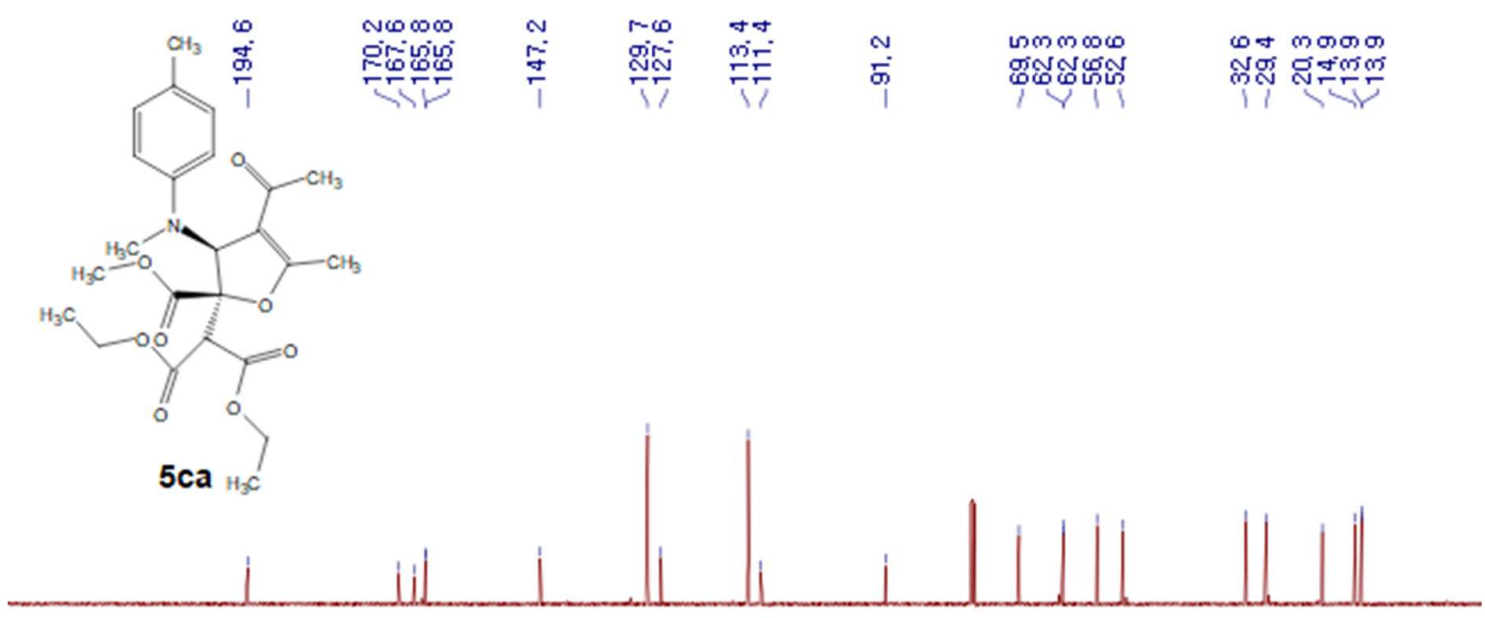

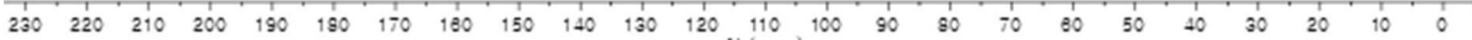

${ }^{13} \mathrm{C}$ NMR (100 MHz, $\left.\mathrm{CDCl}_{3}\right)$ spectra of diethyl 2-((2R,3S)-4-acetyl-2-(methoxycarbonyl)-5methyl-3-(methyl(p-tolyl)amino)-2,3-dihydrofuran-2-yl)malonate (5ca)

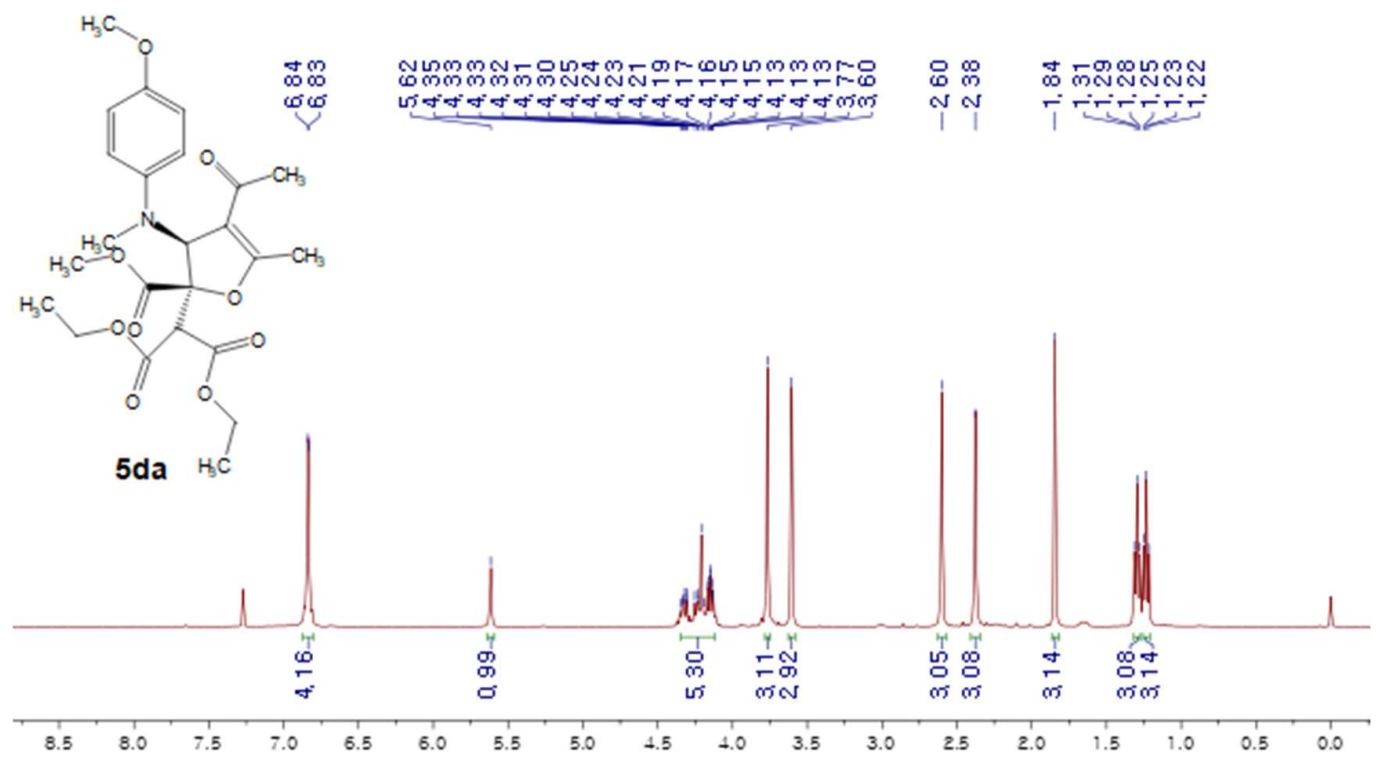

${ }^{1} \mathrm{H}$ NMR (400 MHz, $\left.\mathrm{CDCl}_{3}\right)$ spectra of diethyl 2-((2R,3S)-4-acetyl-2-(methoxycarbonyl)-3((4-methoxyphenyl)(methyl)amino)-5-methyl-2,3-dihydrofuran-2-yl)malonate (5da) 


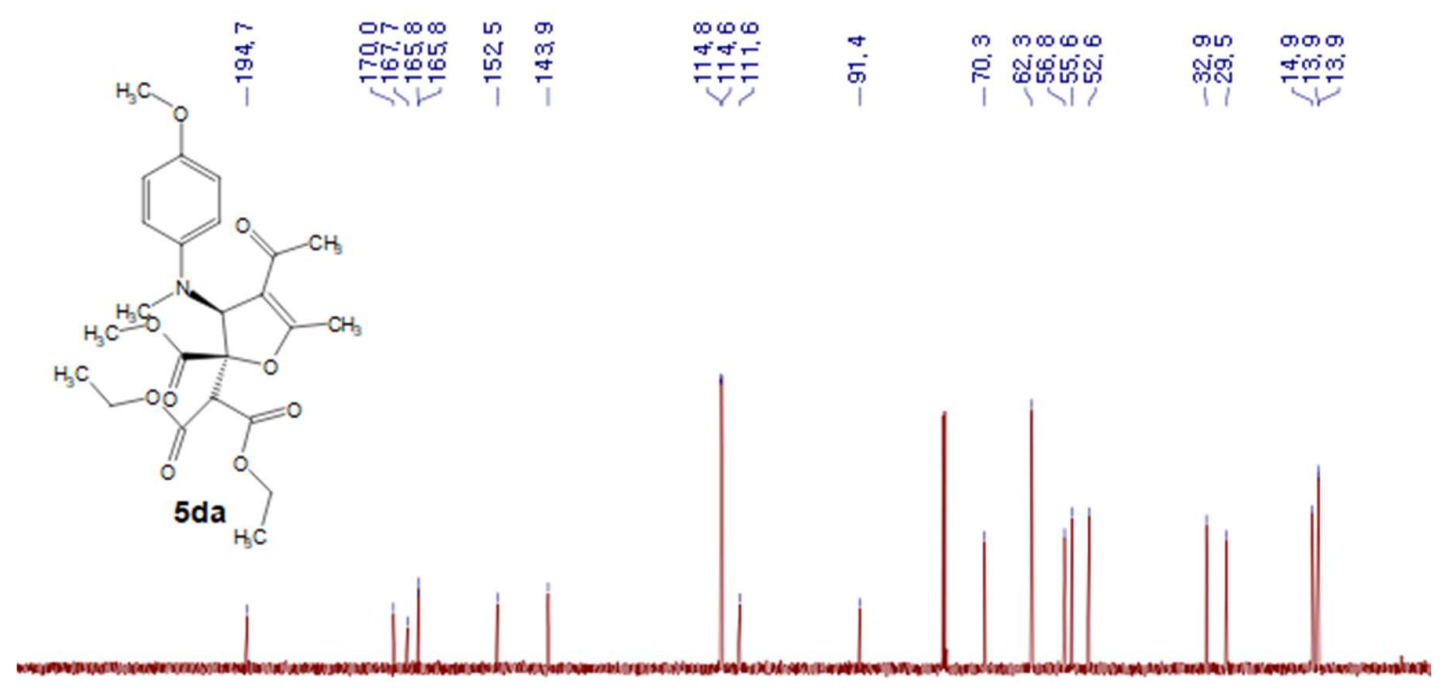

\begin{tabular}{llllllllllllllllllllllll}
\hline 230 & 220 & 210 & 200 & 190 & 180 & 170 & 160 & 150 & 140 & 130 & 120 & 110 & 100 & 90 & 80 & 70 & 60 & 50 & 40 & 30 & 20 & 10 & 0
\end{tabular}

${ }^{13} \mathrm{C}$ NMR (100 MHz, $\left.\mathrm{CDCl}_{3}\right)$ spectra of diethyl 2-((2R,3S)-4-acetyl-2-(methoxycarbonyl)-3((4-methoxyphenyl)(methyl)amino)-5-methyl-2,3-dihydrofuran-2-yl)malonate (5da)

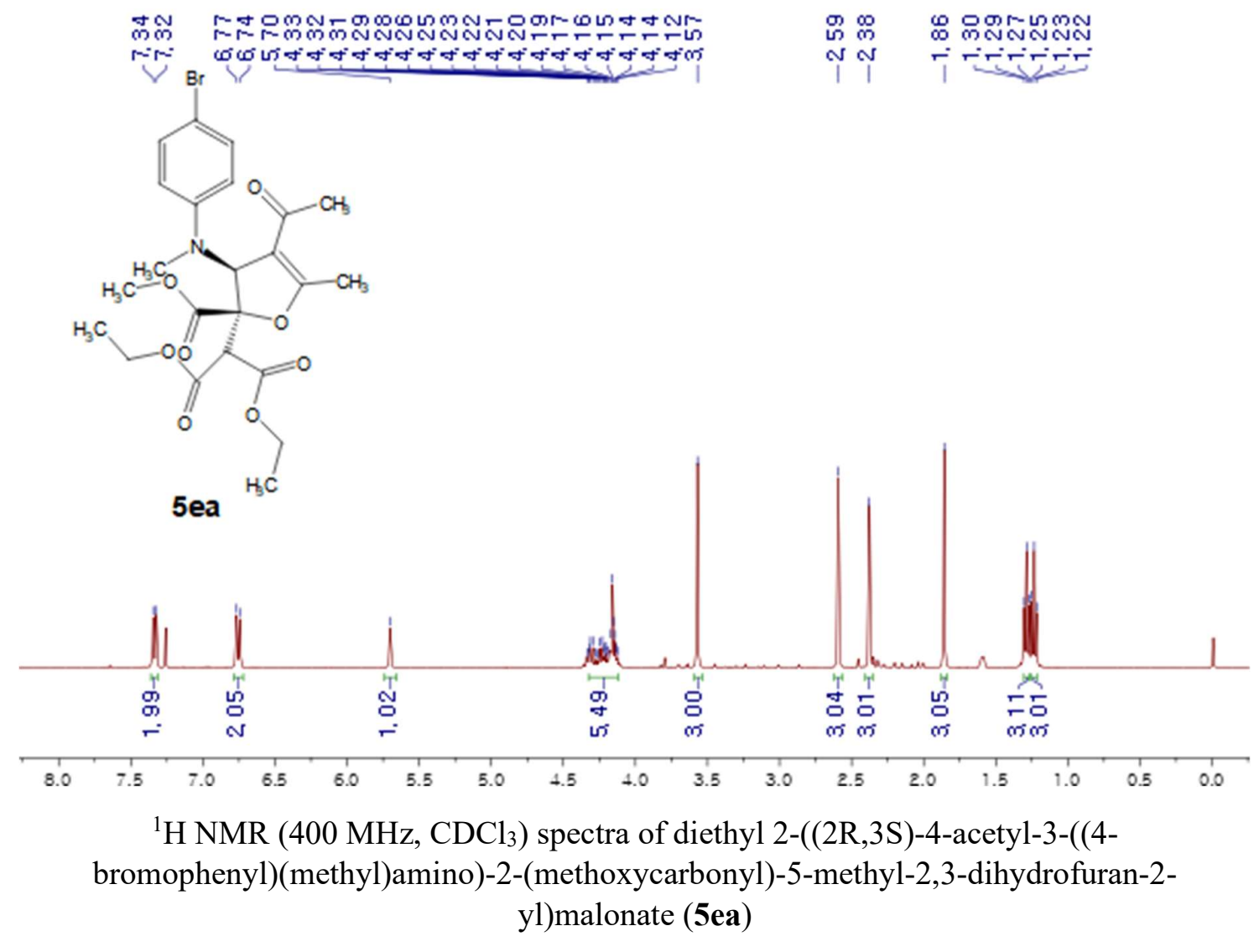




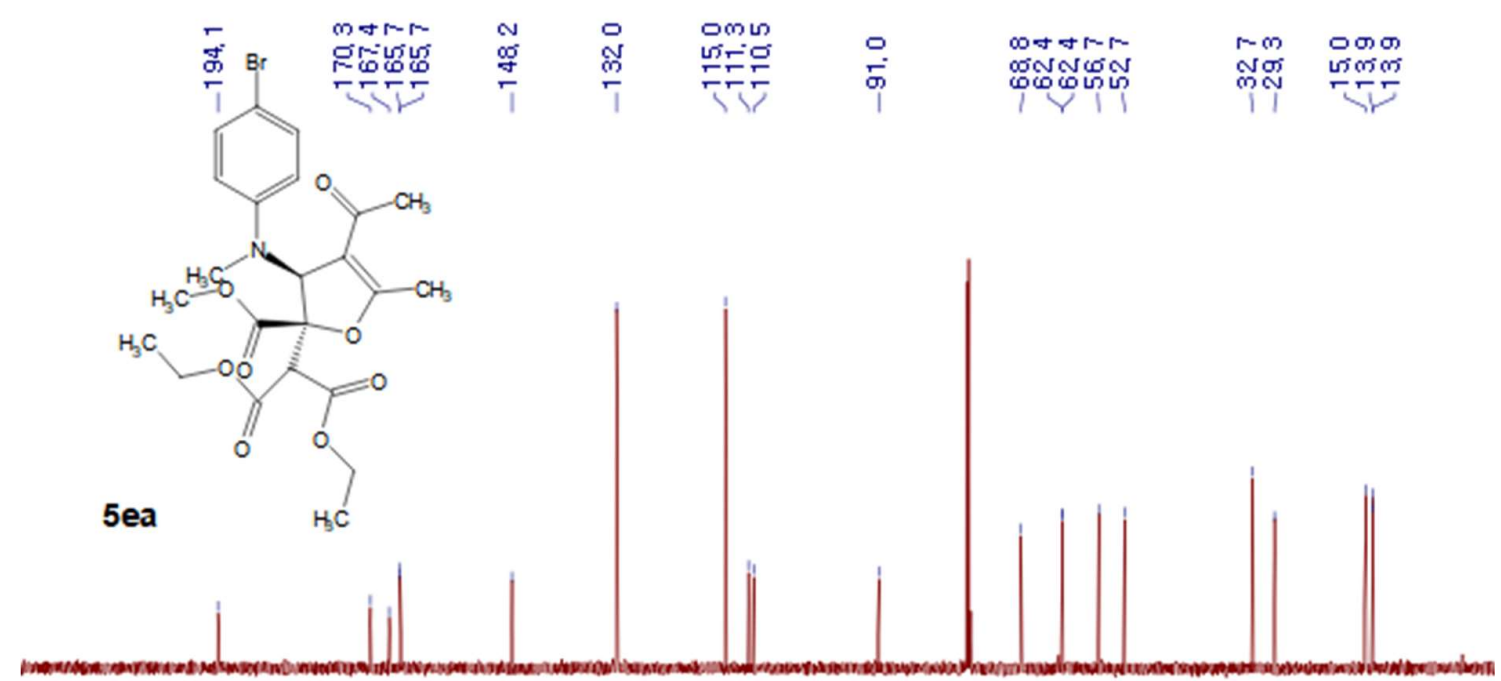

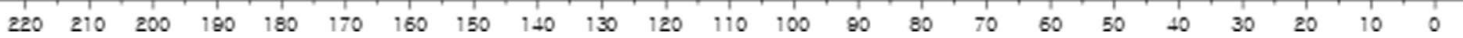

${ }^{13} \mathrm{C}$ NMR (100 MHz, $\left.\mathrm{CDCl}_{3}\right)$ spectra of diethyl 2-((2R,3S)-4-acetyl-3-((4-

bromophenyl)(methyl)amino)-2-(methoxycarbonyl)-5-methyl-2,3-dihydrofuran-2yl)malonate (5ea)

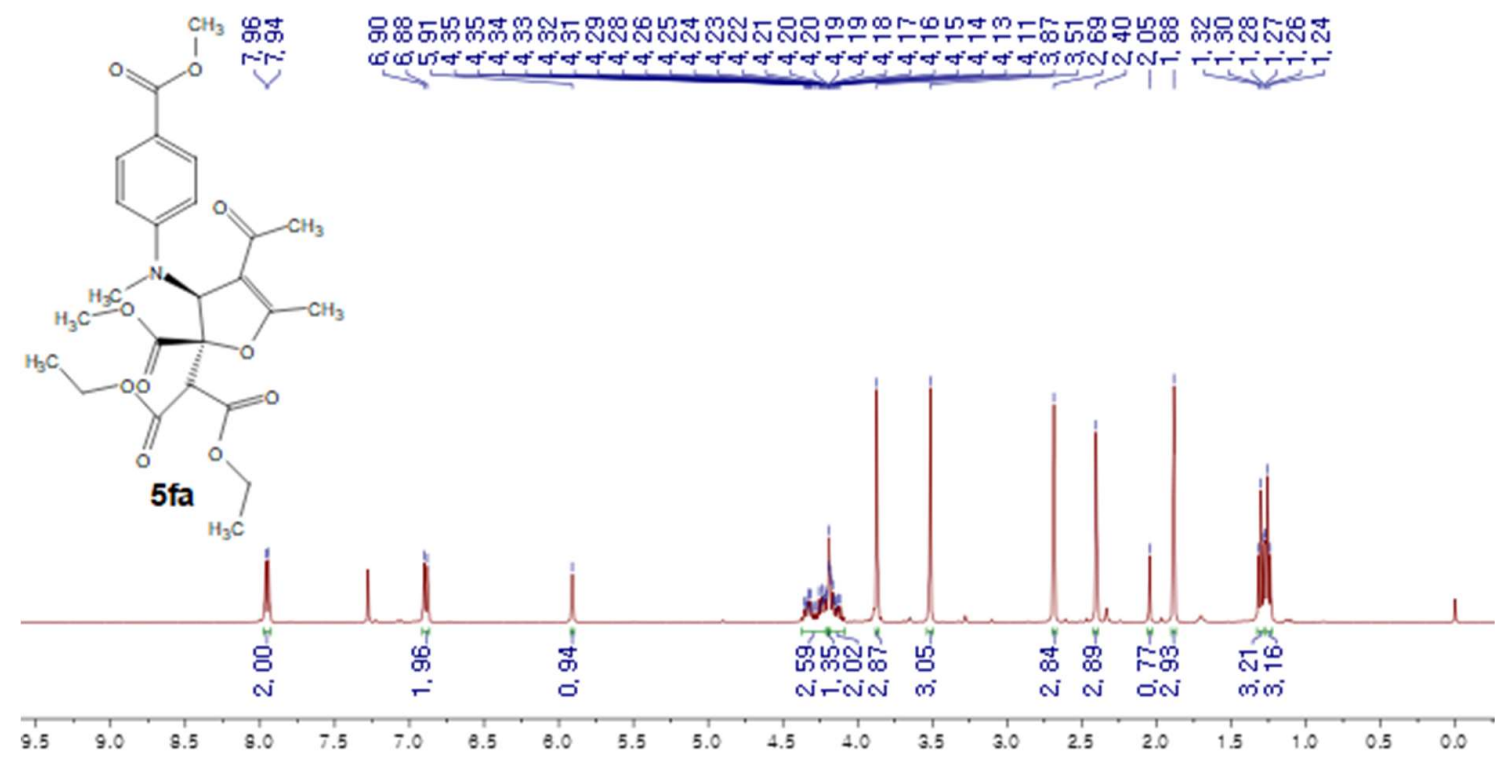

${ }^{1} \mathrm{H}$ NMR (400 MHz, $\left.\mathrm{CDCl}_{3}\right)$ spectra of diethyl 2-((2R,3S)-4-acetyl-2-(methoxycarbonyl)-3((4-(methoxycarbonyl)phenyl)(methyl)amino)-5-methyl-2,3-dihydrofuran-2-yl)malonate (5fa) 


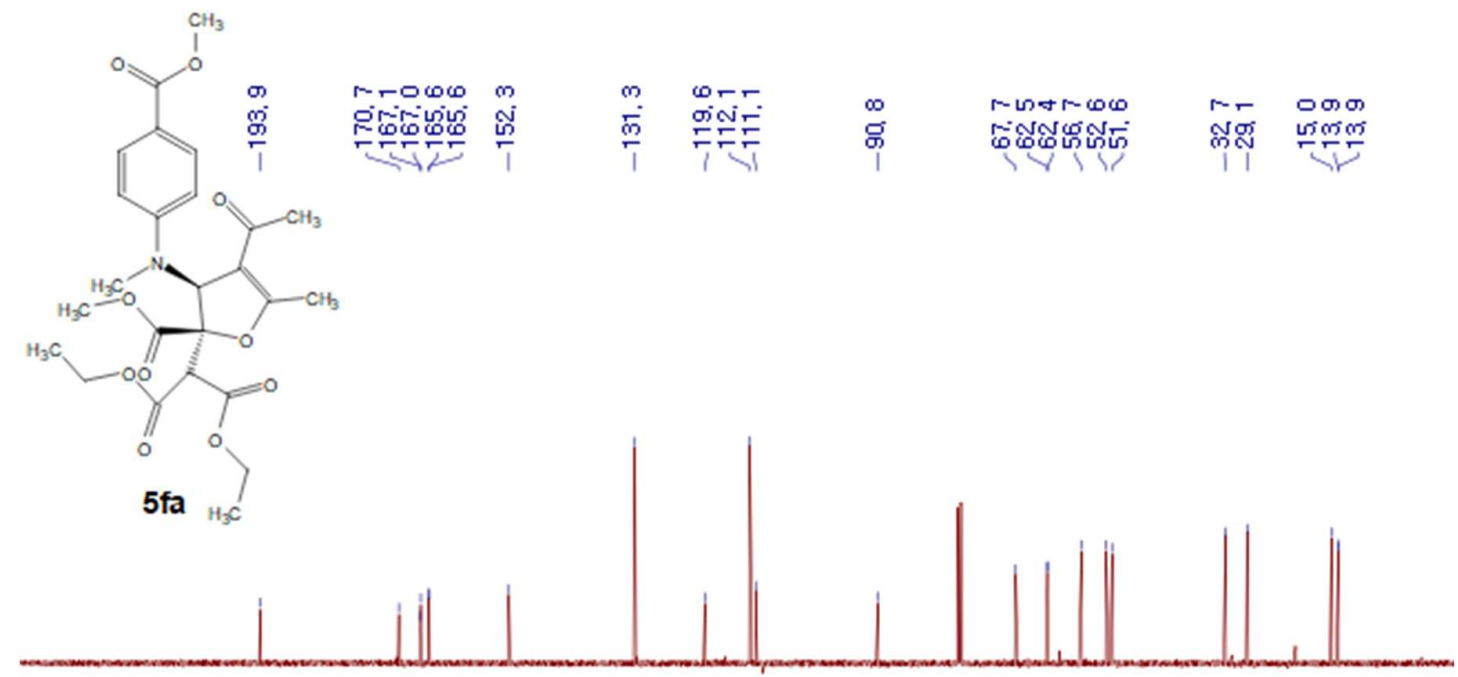

$\begin{array}{llllllllllllllllllllllllllllllllll}130 & 220 & 210 & 200 & 190 & 180 & 170 & 180 & 150 & 140 & 130 & 120 & 110 & 100 & 90 & 80 & 70 & 80 & 50 & 40 & 30 & 20 & 10 & 0\end{array}$

${ }^{13} \mathrm{C}$ NMR (100 MHz, $\left.\mathrm{CDCl}_{3}\right)$ spectra of diethyl 2-((2R,3S)-4-acetyl-2-(methoxycarbonyl)-3((4-(methoxycarbonyl)phenyl)(methyl)amino)-5-methyl-2,3-dihydrofuran-2-yl)malonate

(5fa)

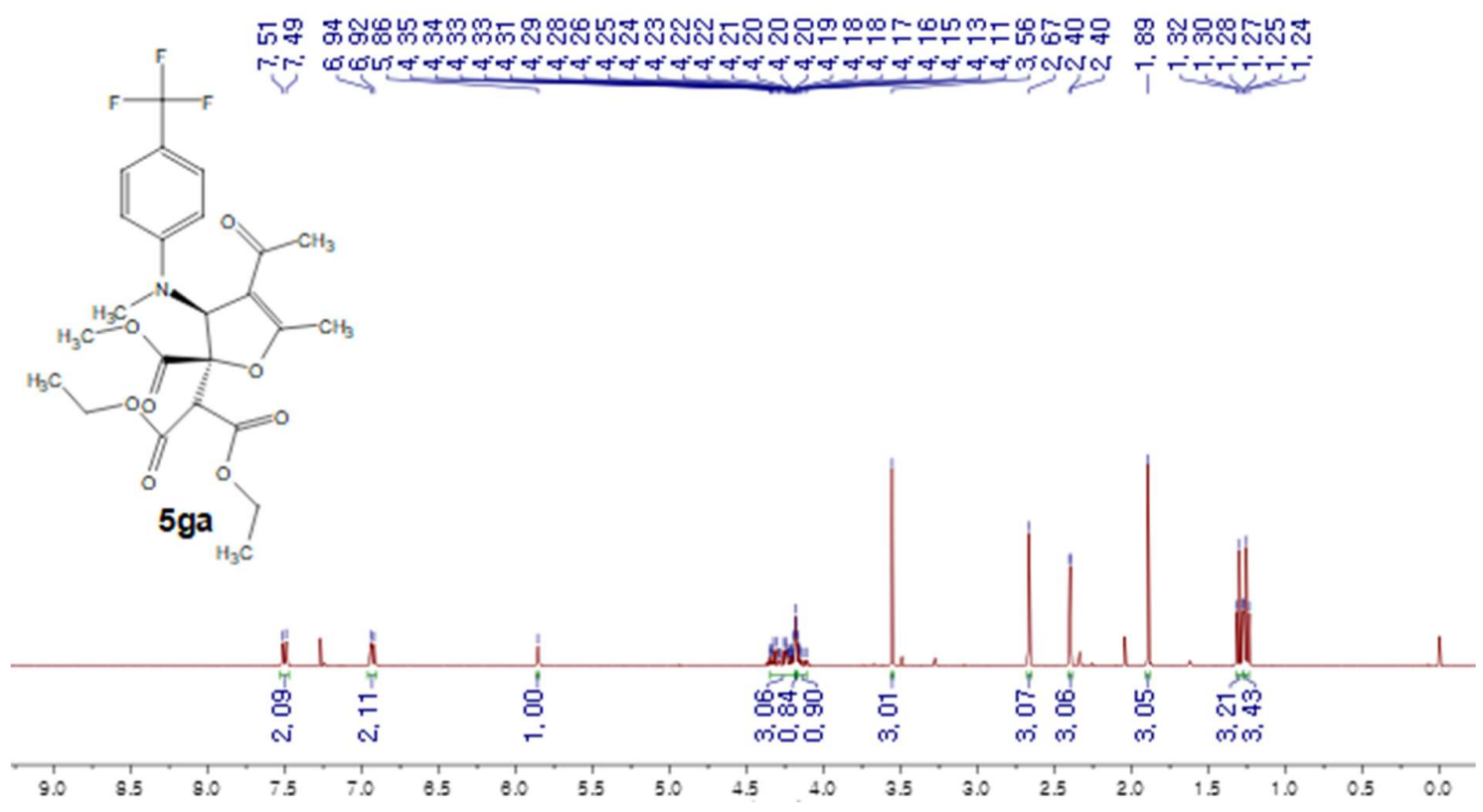

${ }^{1} \mathrm{H}$ NMR (400 MHz, $\left.\mathrm{CDCl}_{3}\right)$ spectra of diethyl 2-((2R,3S)-4-acetyl-2-(methoxycarbonyl)-5methyl-3-(methyl(4-(trifluoromethyl)phenyl)amino)-2,3-dihydrofuran-2-yl)malonate (5ga) 


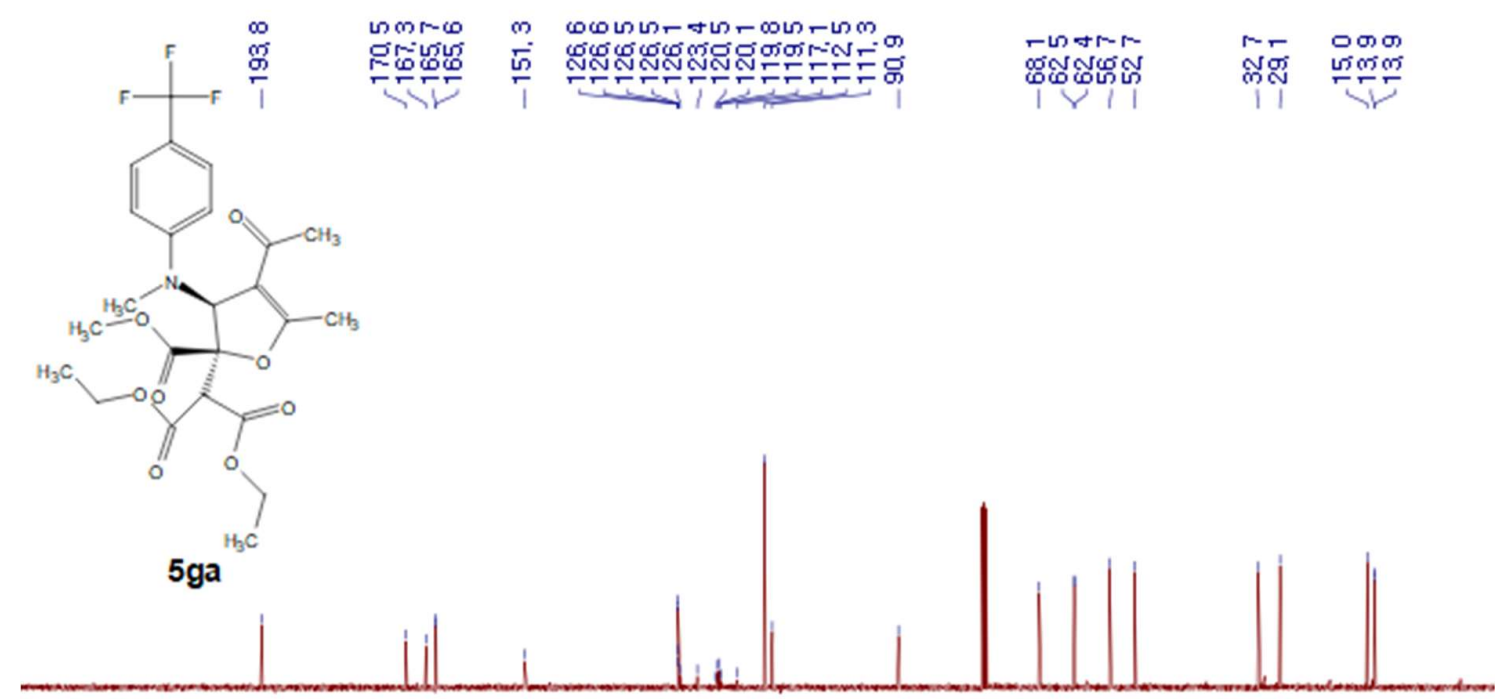

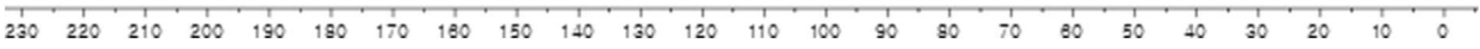

${ }^{13} \mathrm{C}$ NMR (100 MHz, $\left.\mathrm{CDCl}_{3}\right)$ spectra of diethyl 2-((2R,3S)-4-acetyl-2-(methoxycarbonyl)-5methyl-3-(methyl(4-(trifluoromethyl)phenyl)amino)-2,3-dihydrofuran-2-yl)malonate (5ga)

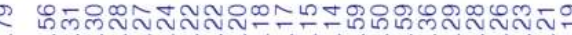

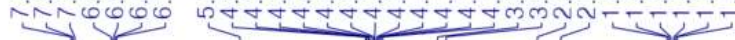

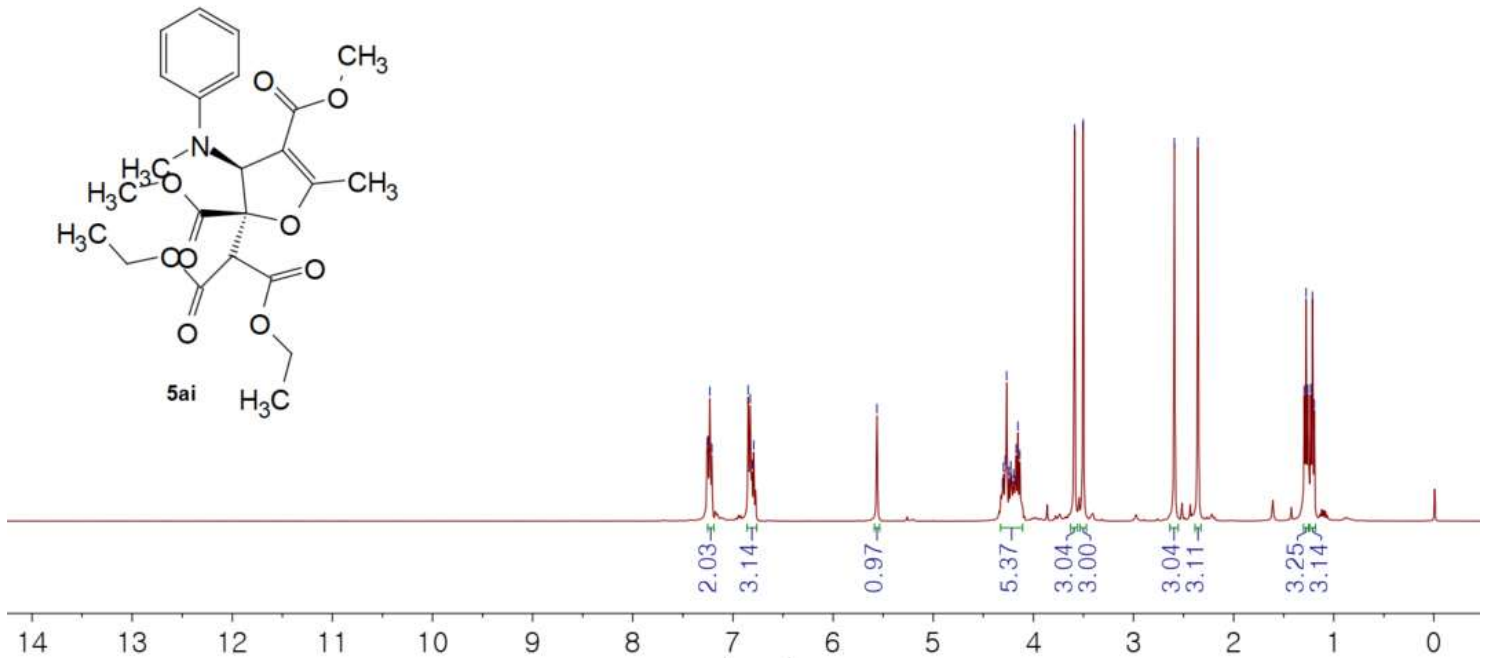

${ }^{1} \mathrm{H}$ NMR (400 MHz, $\mathrm{CDCl}_{3}$ ) spectra of dimethyl (2R,3S)-2-(1,3-diethoxy-1,3-dioxopropan-2yl)-5-methyl-3-(methyl(phenyl)amino)-2,3-dihydrofuran-2,4-dicarboxylate (5ai) 


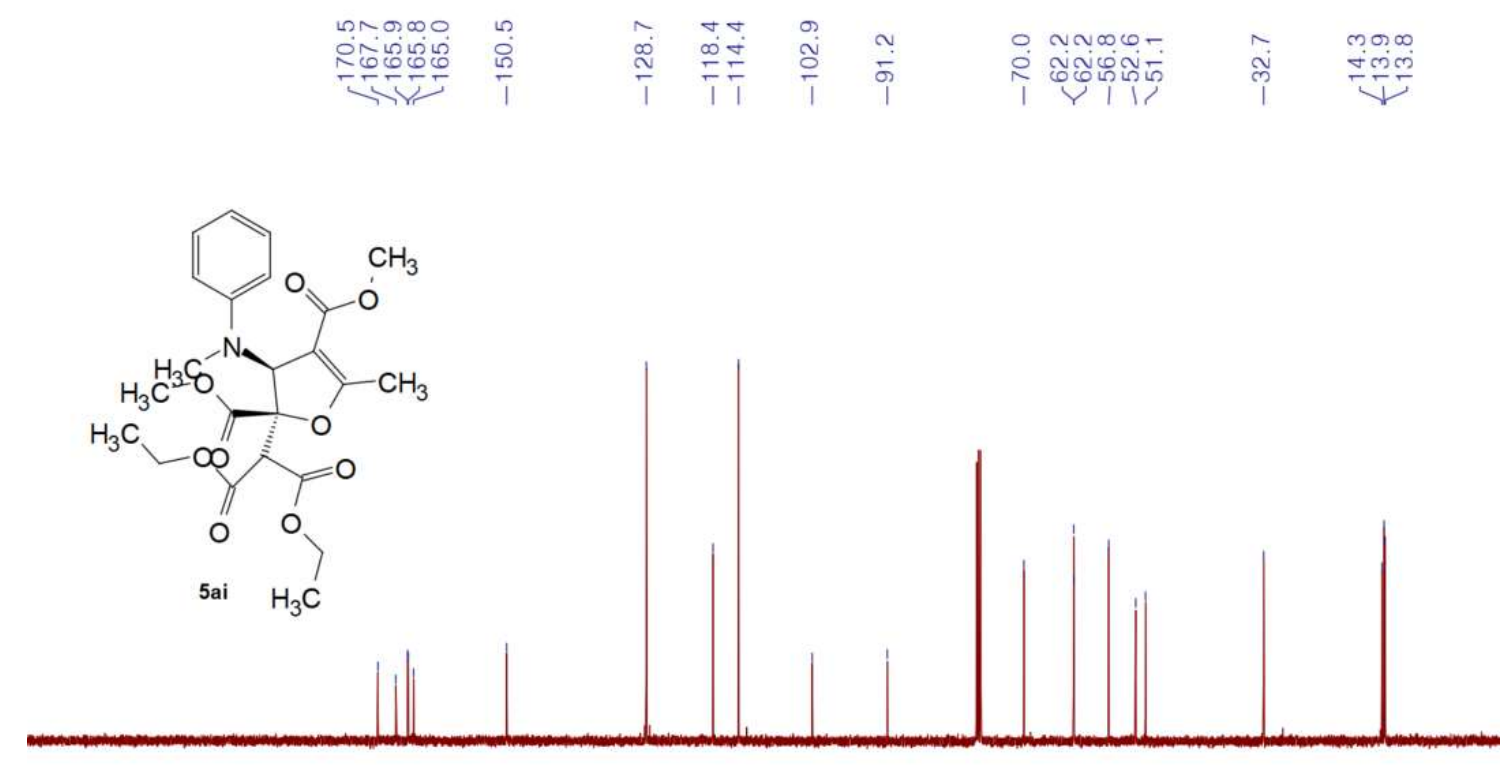

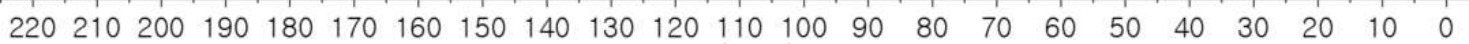

${ }^{13} \mathrm{C}$ NMR (100 MHz, $\left.\mathrm{CDCl}_{3}\right)$ spectra of dimethyl (2R,3S)-2-(1,3-diethoxy-1,3-dioxopropan2-yl)-5-methyl-3-(methyl(phenyl)amino)-2,3-dihydrofuran-2,4-dicarboxylate (5ai)

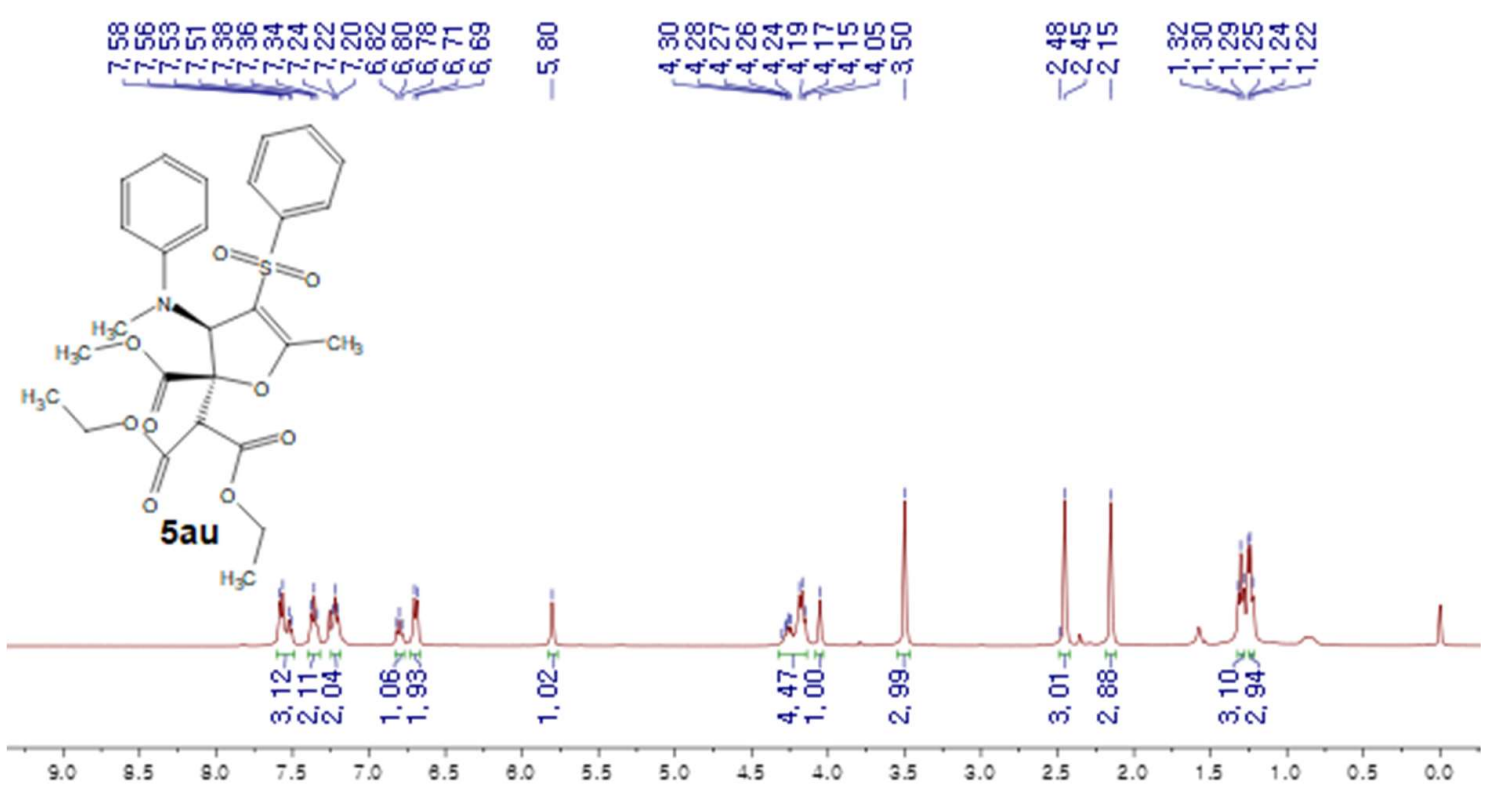

${ }^{1} \mathrm{H}$ NMR (400 MHz, $\left.\mathrm{CDCl}_{3}\right)$ spectra of diethyl 2-((2R,3R)-2-(methoxycarbonyl)-5-methyl-3(methyl(phenyl)amino)-4-(phenylsulfonyl)-2,3-dihydrofuran-2-yl)malonate (5au) 

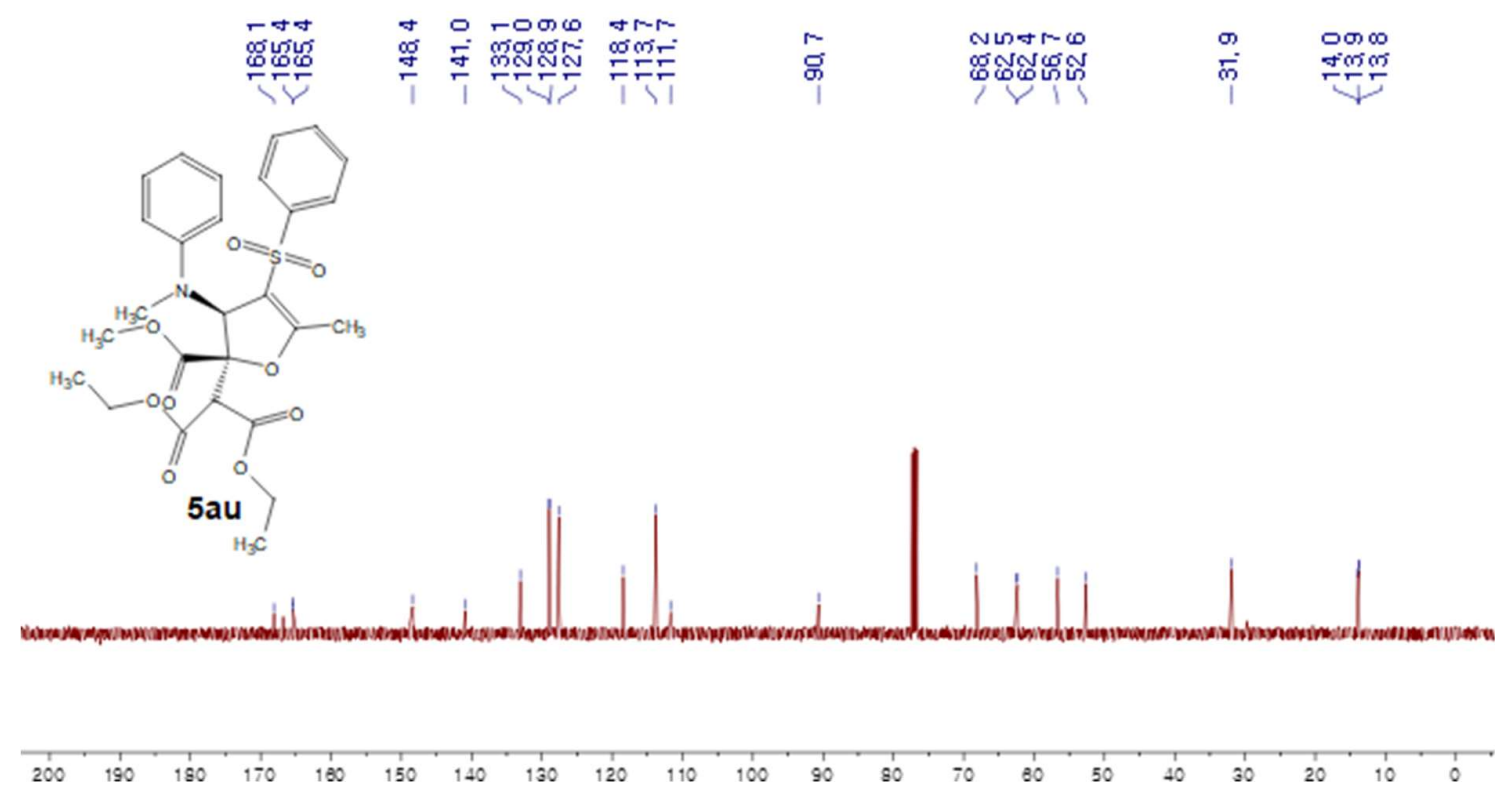

${ }^{13} \mathrm{C}$ NMR (100 MHz, $\left.\mathrm{CDCl}_{3}\right)$ spectra of diethyl 2-((2R,3R)-2-(methoxycarbonyl)-5-methyl-3(methyl(phenyl)amino)-4-(phenylsulfonyl)-2,3-dihydrofuran-2-yl)malonate (5au)

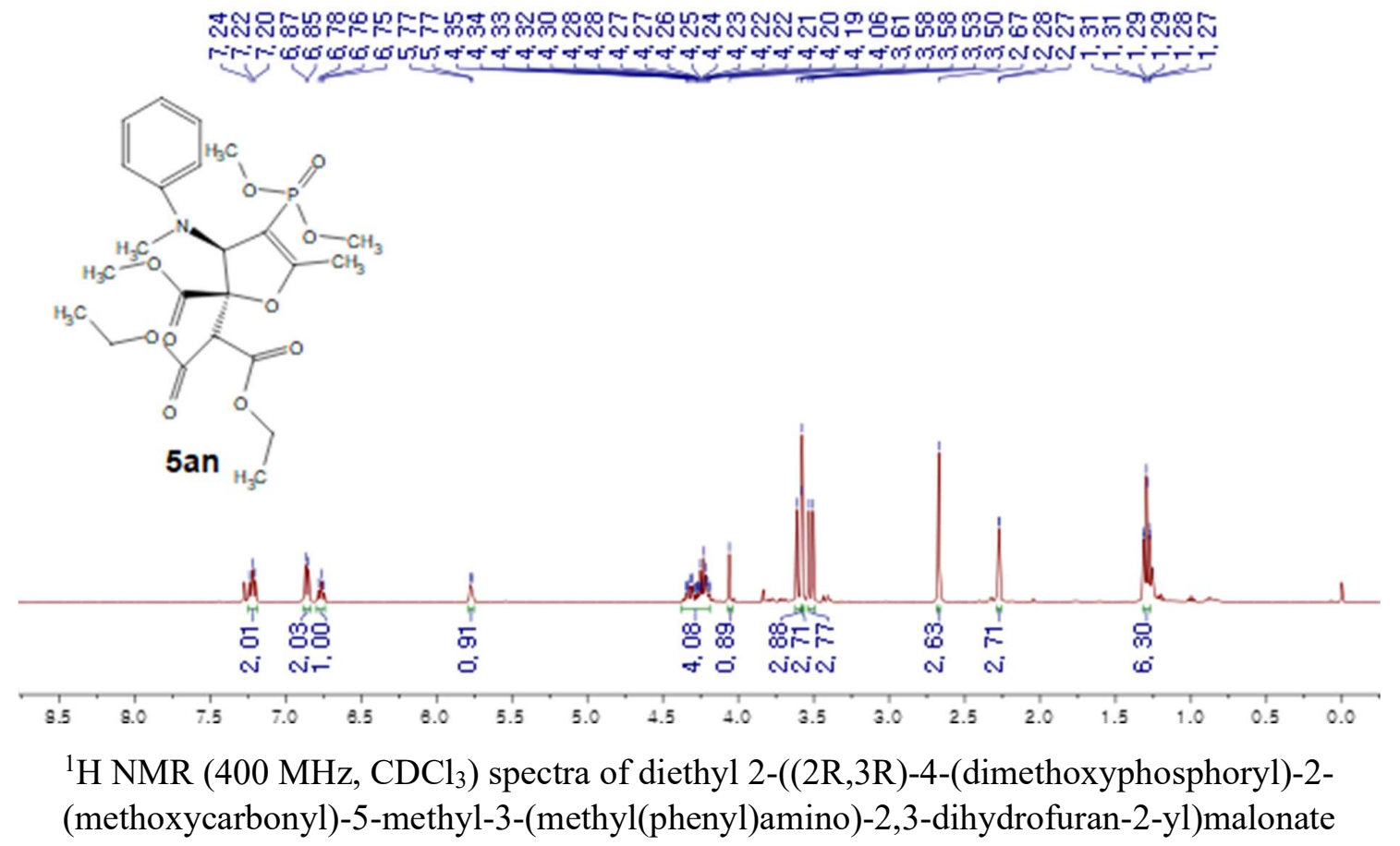
(5an) 


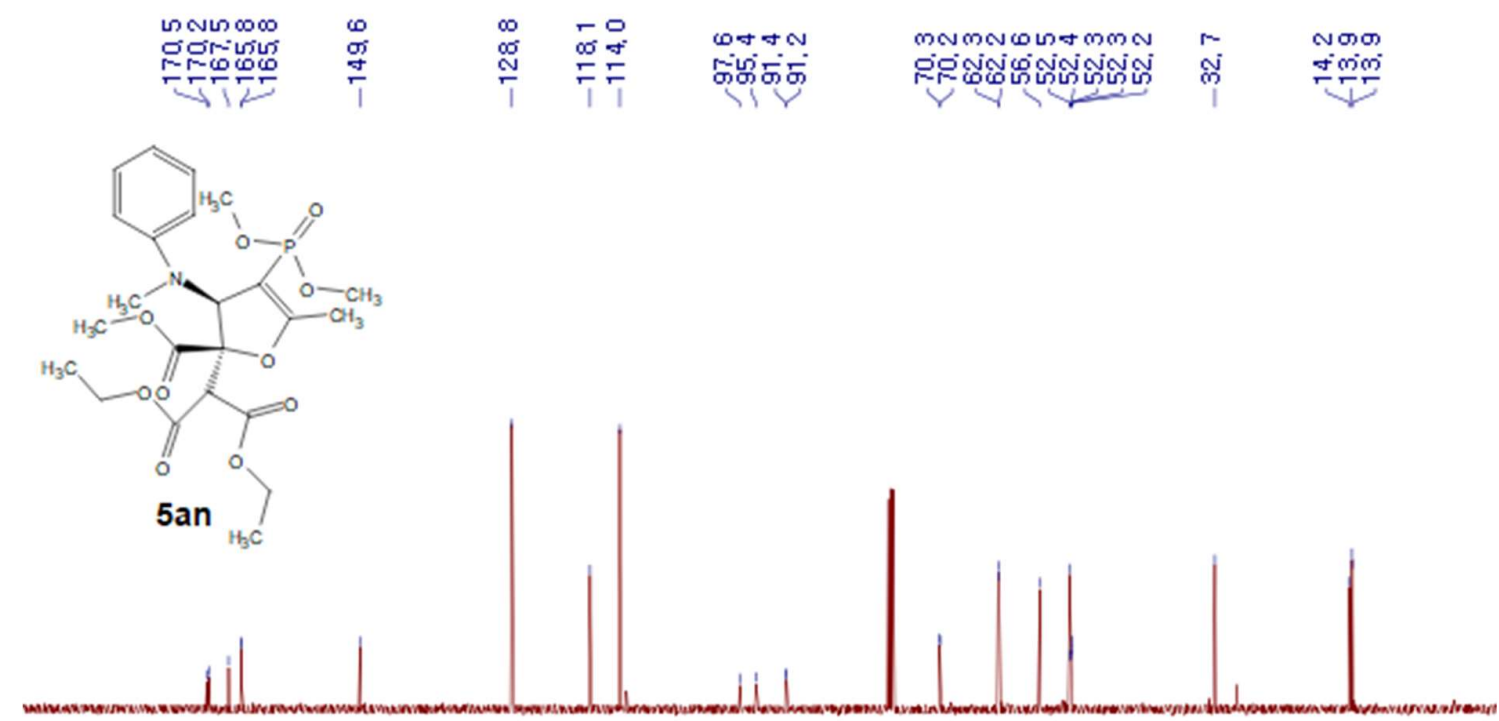

$\underset{\substack{6 \\ \stackrel{5}{1}}}{\stackrel{1}{1}}$
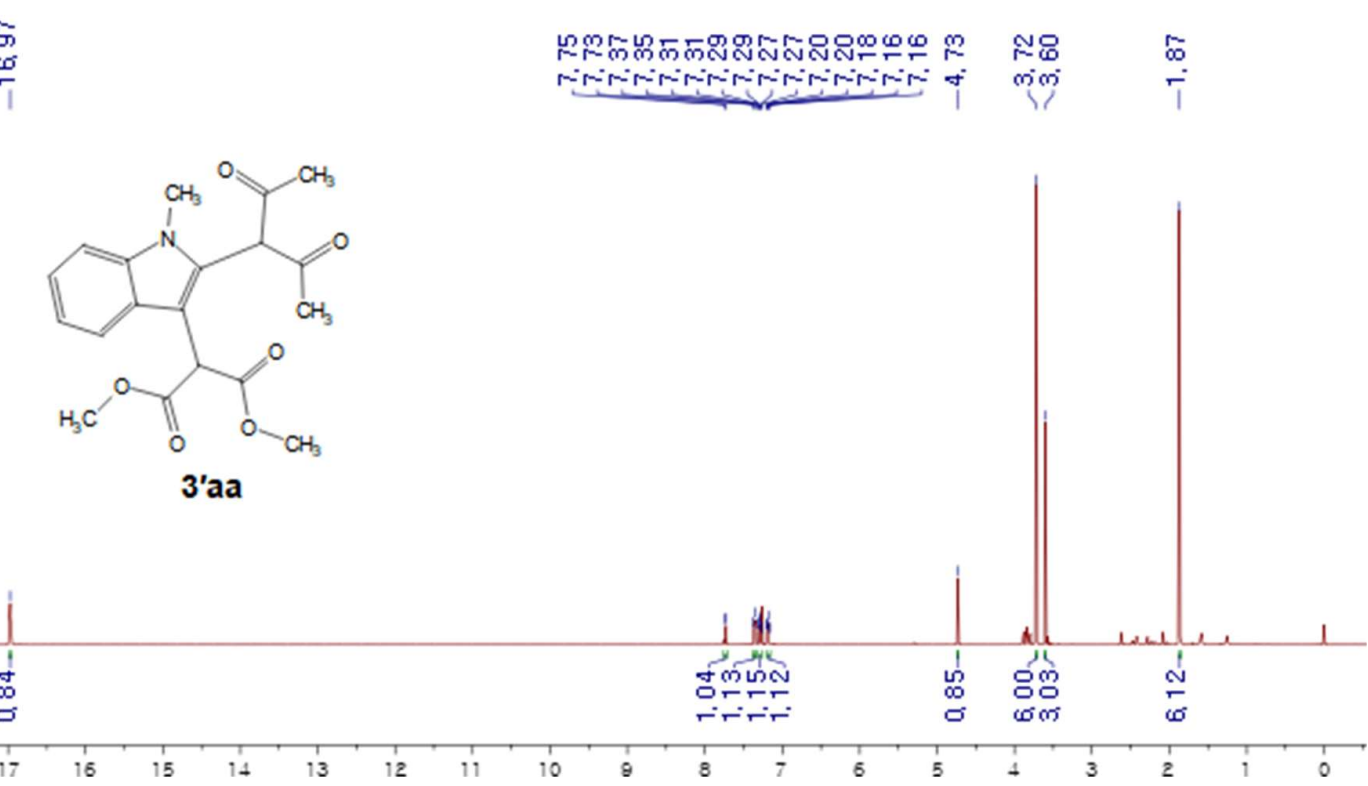

(5an)

${ }^{13} \mathrm{C}$ NMR (100 MHz, $\left.\mathrm{CDCl}_{3}\right)$ spectra of diethyl 2-((2R,3R)-4-(dimethoxyphosphoryl)-2(methoxycarbonyl)-5-methyl-3-(methyl(phenyl)amino)-2,3-dihydrofuran-2-yl)malonate

${ }^{1} \mathrm{H}$ NMR (400 MHz, CDCl3) spectra of dimethyl 2-(2-(2,4-dioxopentan-3-yl)-1-methyl-1Hindol-3-yl)malonate (3'aa) 

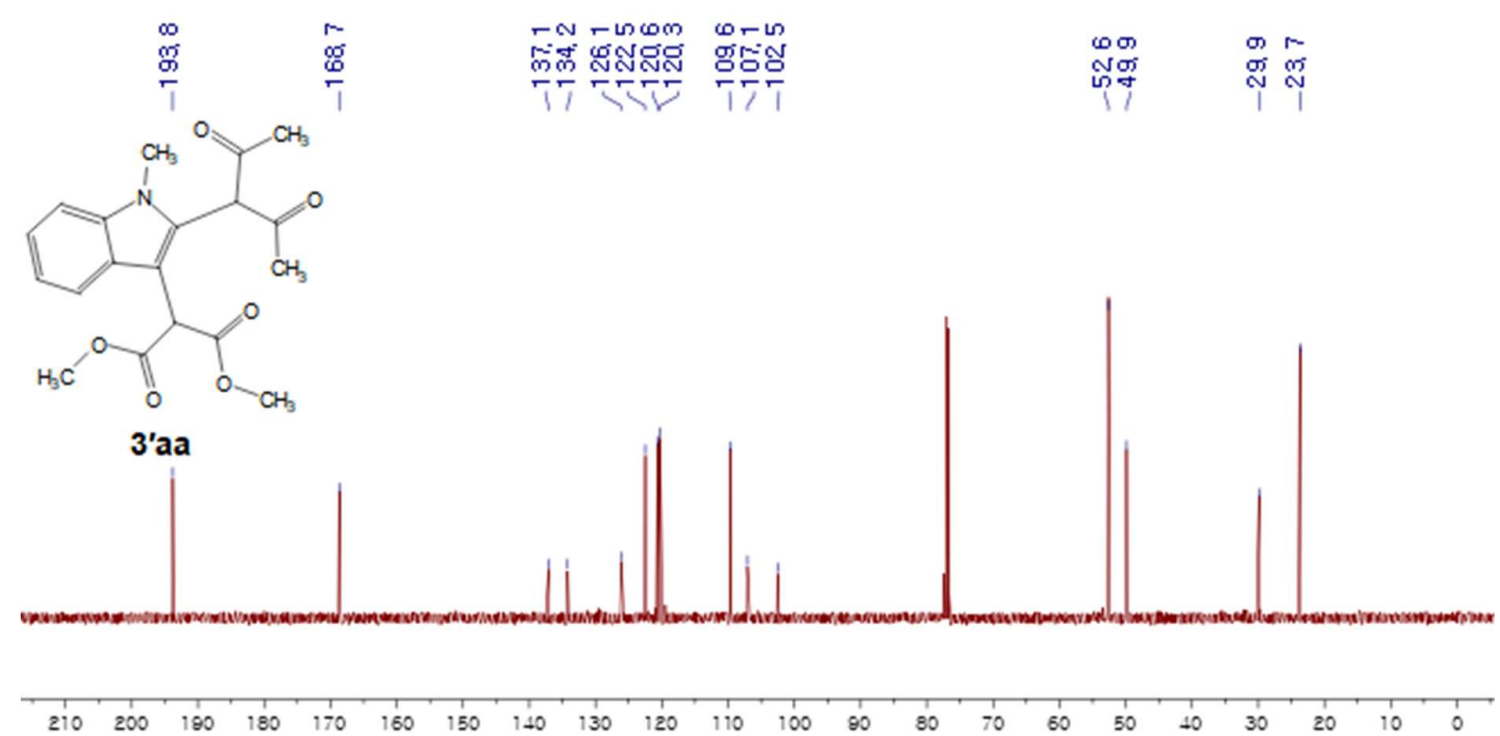

${ }^{13} \mathrm{C}$ NMR (100 MHz, $\left.\mathrm{CDCl}_{3}\right)$ spectra of dimethyl 2-(2-(2,4-dioxopentan-3-yl)-1-methyl-1Hindol-3-yl)malonate (3'aa)

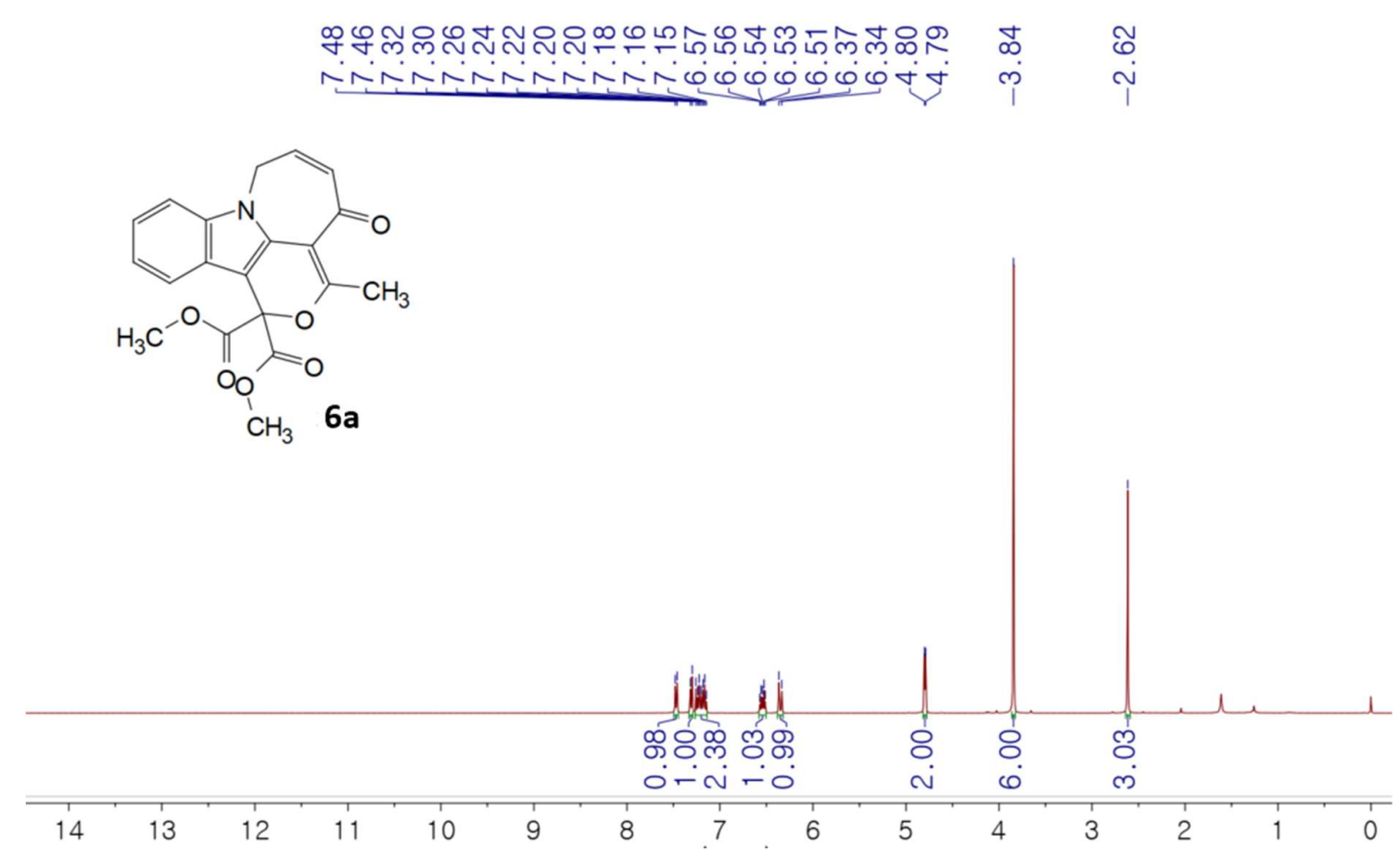

${ }^{1} \mathrm{H}$ NMR (400 MHz, $\mathrm{CDCl}_{3}$ ) spectra of dimethyl 3-methyl-4-oxo-4,7-dihydro-1H-2-oxa-7aazacyclohepta[jk]fluorene-1,1-dicarboxylate (6a) 


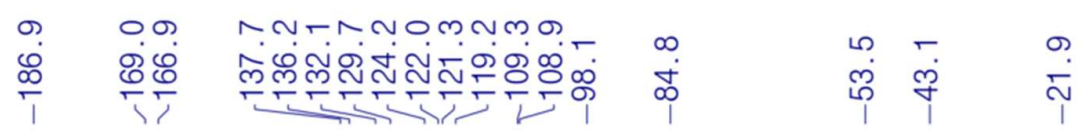<smiles>COC(=O)C1(C(=O)OC)OC(C)=C2C(=O)C=CCn3c2c1c1ccccc13</smiles>

$230220210200190180170160150140 \quad 130120110100 \quad 90 \quad 80 \quad 70 \quad 60 \quad 50 \quad 40 \quad 30 \quad 20 \quad 10 \quad 0$

${ }^{13} \mathrm{C}$ NMR (100 MHz, $\mathrm{CDCl}_{3}$ ) spectra of dimethyl 3-methyl-4-oxo-4,7-dihydro-1H-2-oxa-7aazacyclohepta[jk]fluorene-1,1-dicarboxylate (6a)

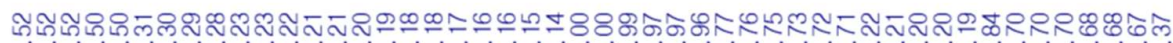

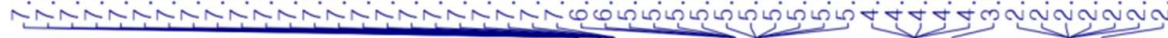<smiles>COC(=O)C1(C(=O)OC)OC(C)=C2C(=O)C=CCCn3c2c1c1ccccc13</smiles>

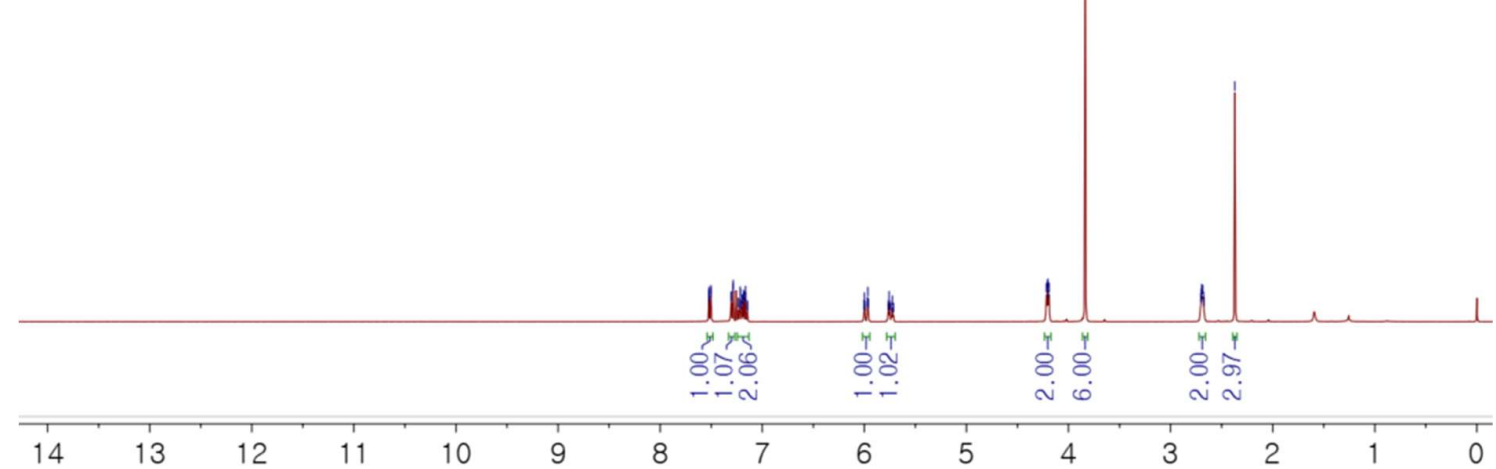

${ }^{1} \mathrm{H}$ NMR (400 MHz, $\mathrm{CDCl}_{3}$ ) spectra of dimethyl (Z)-3-methyl-4-oxo-7,8-dihydro-1H,4H-2oxa-8a-azacycloocta[jk]fluorene-1,1-dicarboxylate $(\mathbf{6 b})$ 

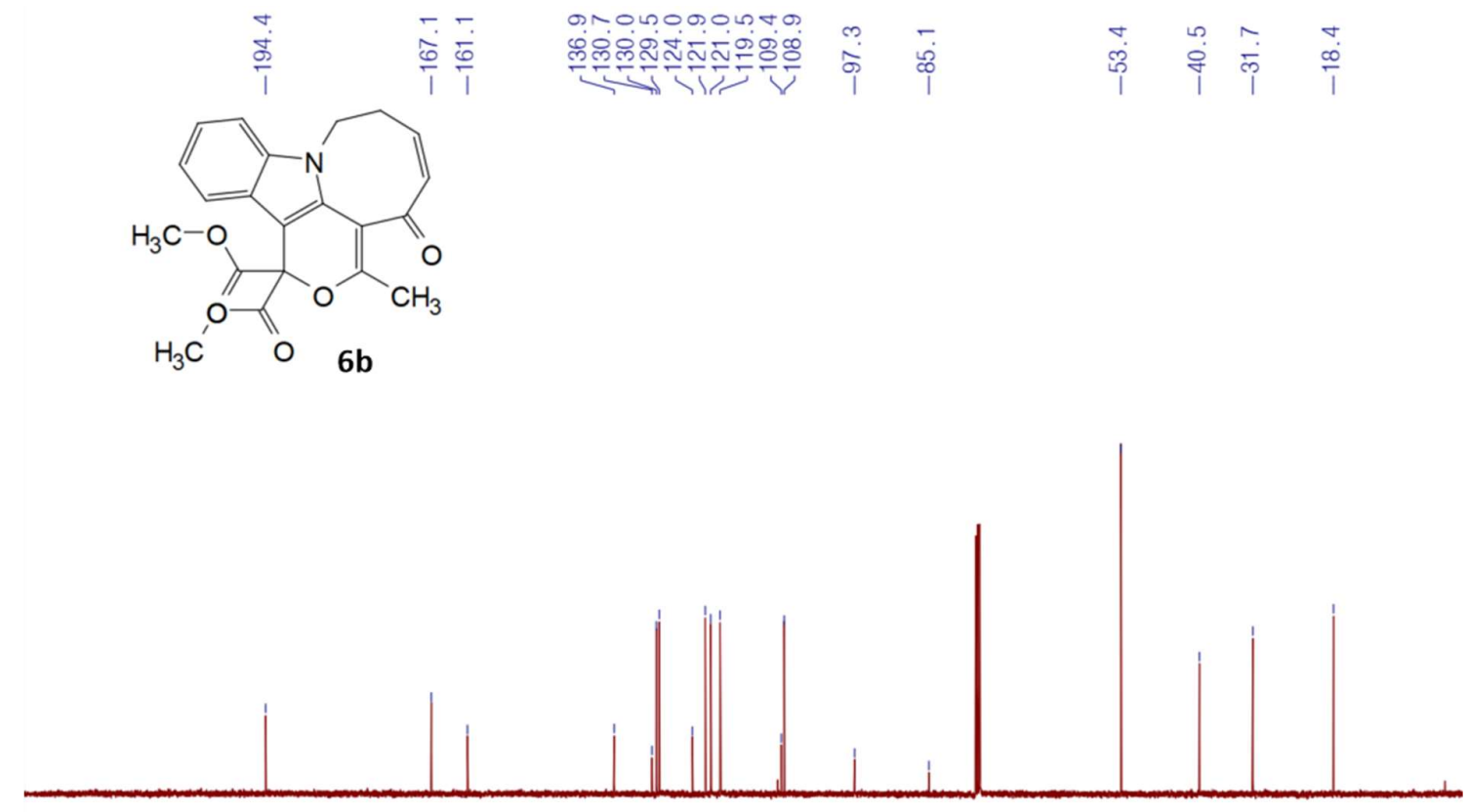
$230220210200190180170160150140130120110100 \quad 90 \quad 80 \quad 70 \quad 60 \quad 50 \quad 40 \quad 30 \quad 20 \quad 10 \quad 0$

${ }^{13} \mathrm{C}$ NMR (100 MHz, $\mathrm{CDCl}_{3}$ ) spectra of dimethyl (Z)-3-methyl-4-oxo-7,8-dihydro-1H,4H-2oxa-8a-azacycloocta[jk]fluorene-1,1-dicarboxylate $(\mathbf{6 b})$

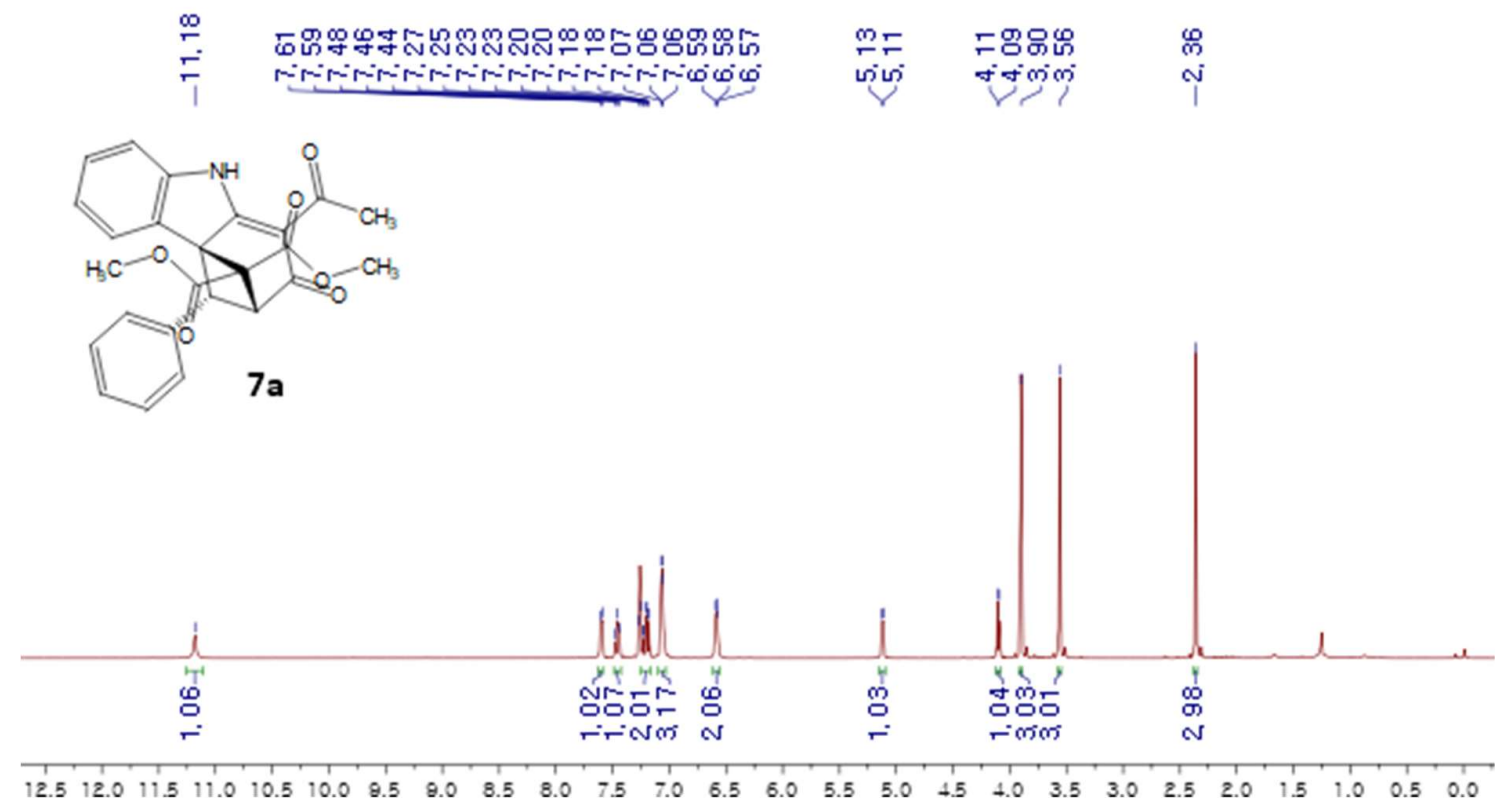

${ }^{1} \mathrm{H}$ NMR (400 MHz, $\mathrm{CDCl}_{3}$ ) spectra of dimethyl (3R,4aR,10R)-1-acetyl-2-oxo-10-phenyl2,9-dihydro-3,4a-methanocarbazole-4,4(3H)-dicarboxylate (7a) 


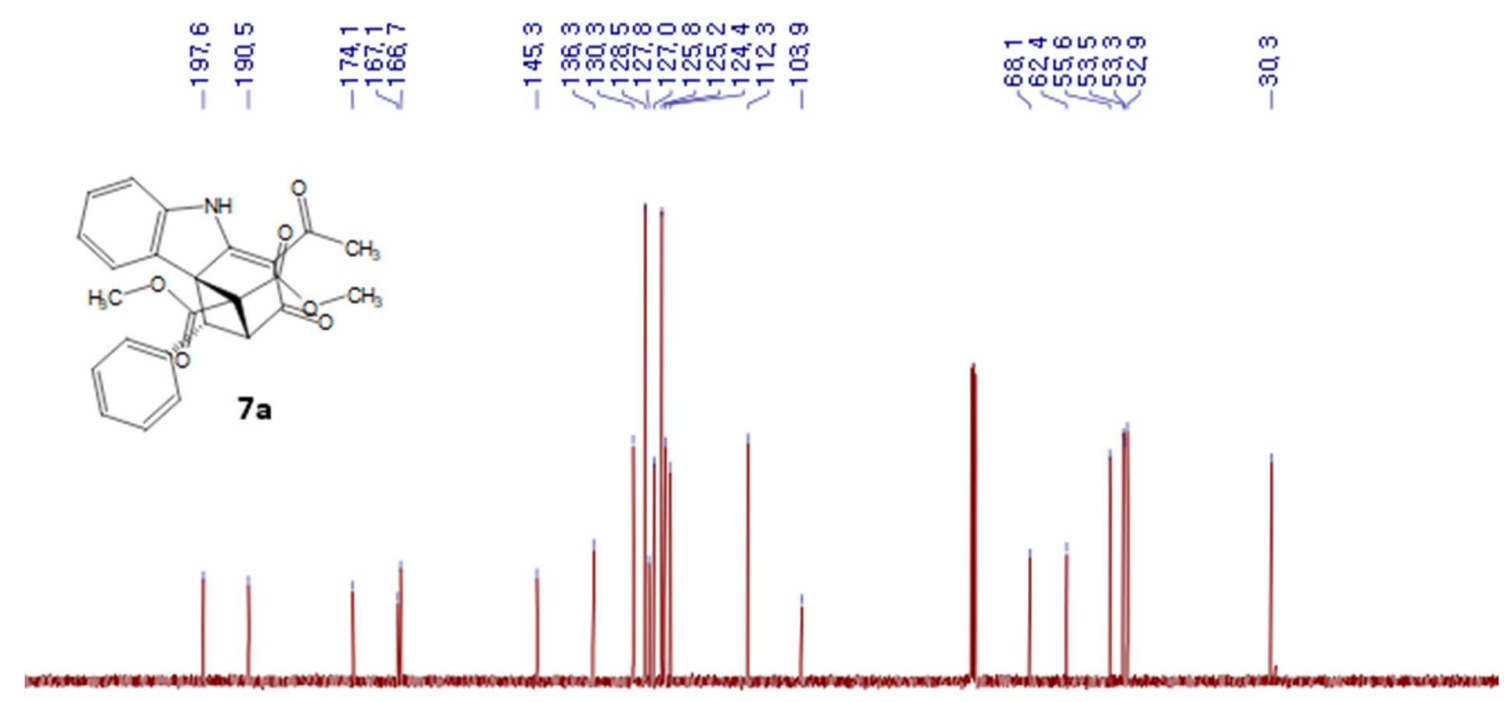

$\begin{array}{llllllllllllllllllllllllll}1 & 1 & 1 & 100 & 200 & 100 & 180 & 170 & 160 & 150 & 140 & 130 & 120 & 110 & 100 & 90 & 80 & 70 & 60 & 50 & 40 & 30 & 20 & 10 & 0\end{array}$

${ }^{13} \mathrm{C}$ NMR (100 MHz, $\mathrm{CDCl}_{3}$ ) spectra of dimethyl (3R,4aR,10R)-1-acetyl-2-oxo-10-phenyl2,9-dihydro-3,4a-methanocarbazole-4,4(3H)-dicarboxylate (7a)
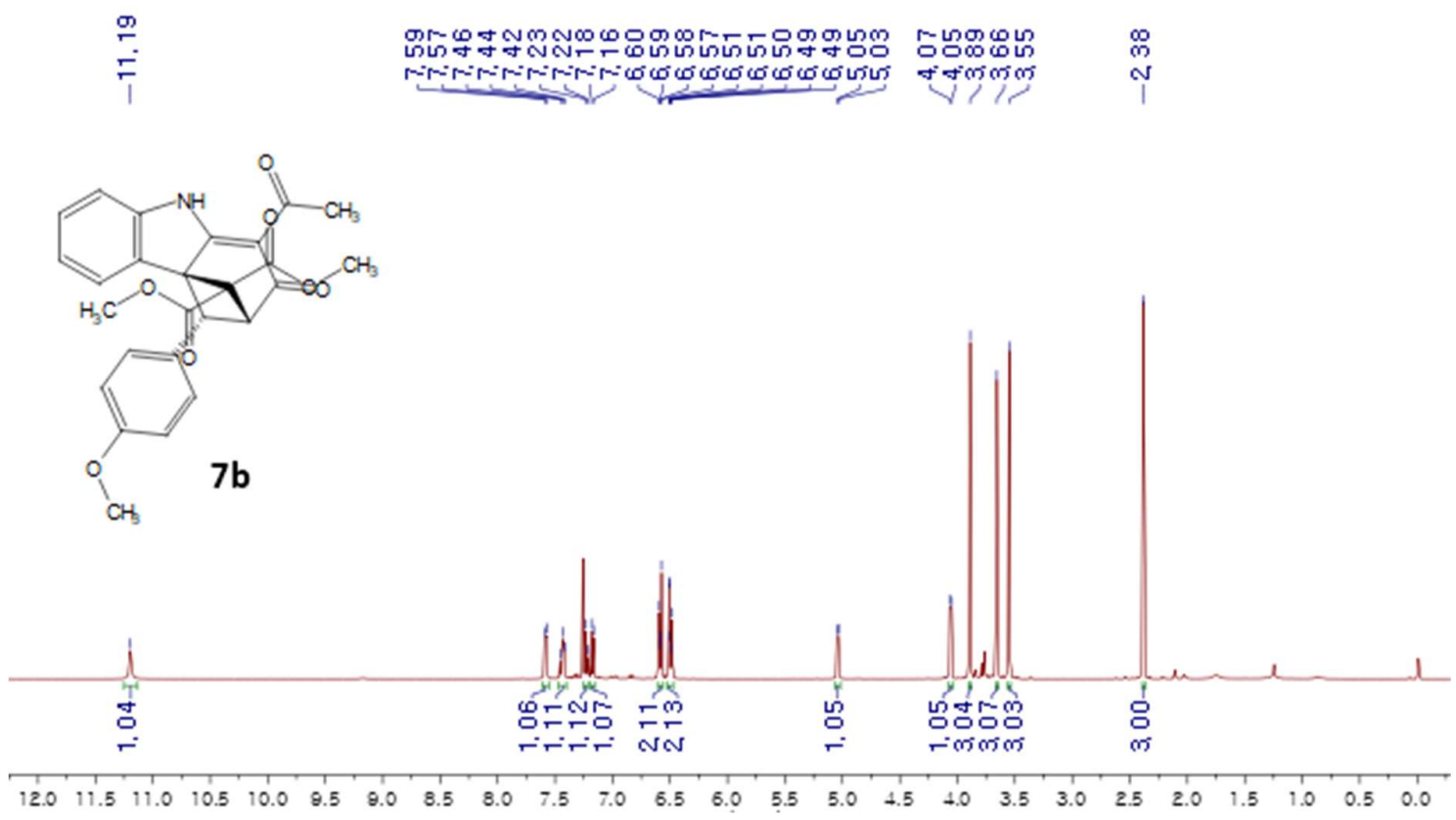

${ }^{1} \mathrm{H}$ NMR (400 MHz, $\mathrm{CDCl}_{3}$ ) spectra of dimethyl (3R,4aR,10R)-1-acetyl-10-(4methoxyphenyl)-2-oxo-2,9-dihydro-3,4a-methanocarbazole-4,4(3H)-dicarboxylate (7b) 


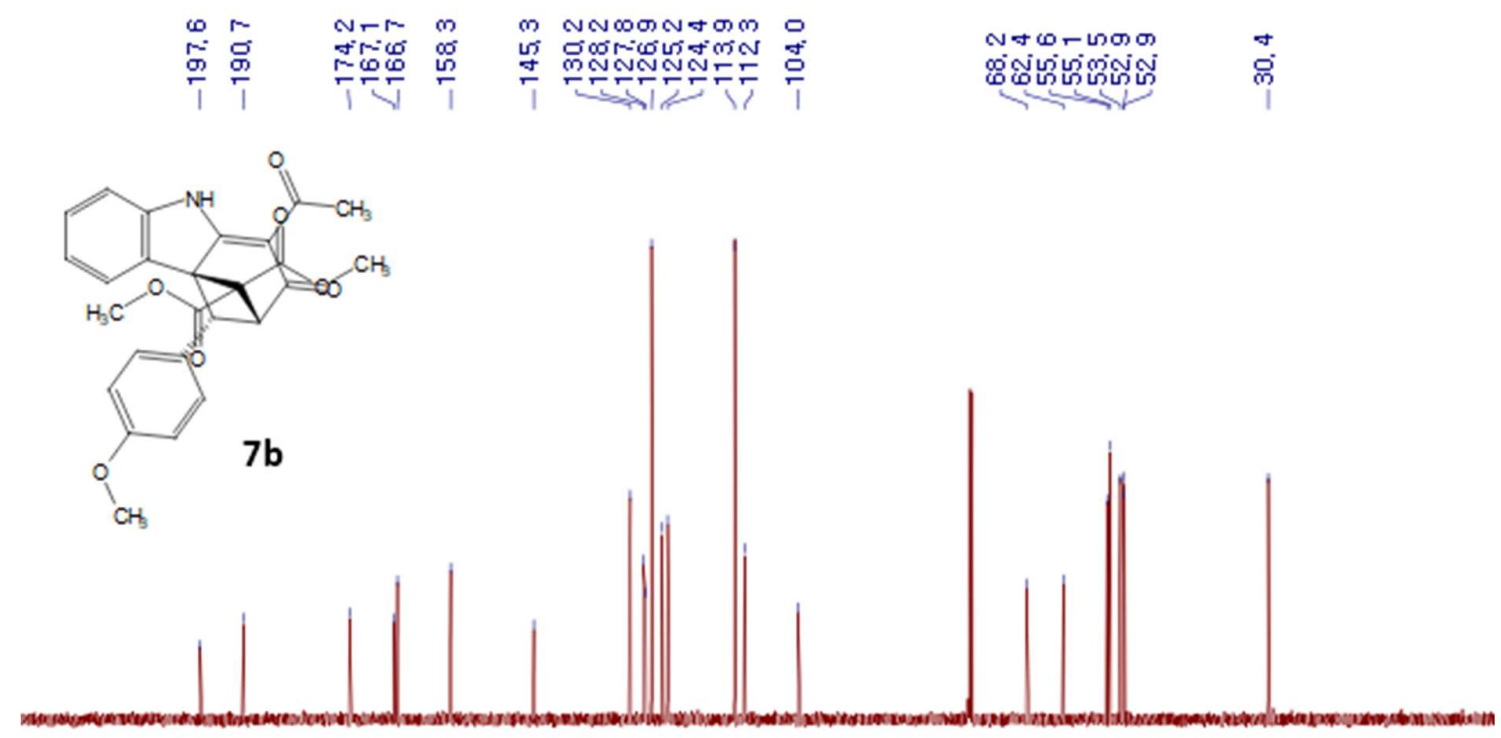

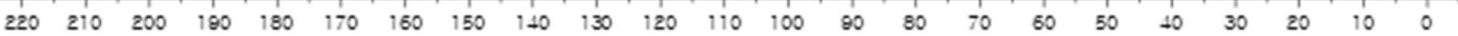

${ }^{13} \mathrm{C}$ NMR $\left(100 \mathrm{MHz}, \mathrm{CDCl}_{3}\right)$ spectra of dimethyl (3R,4aR,10R)-1-acetyl-10-(4methoxyphenyl)-2-oxo-2,9-dihydro-3,4a-methanocarbazole-4,4(3H)-dicarboxylate (7b)

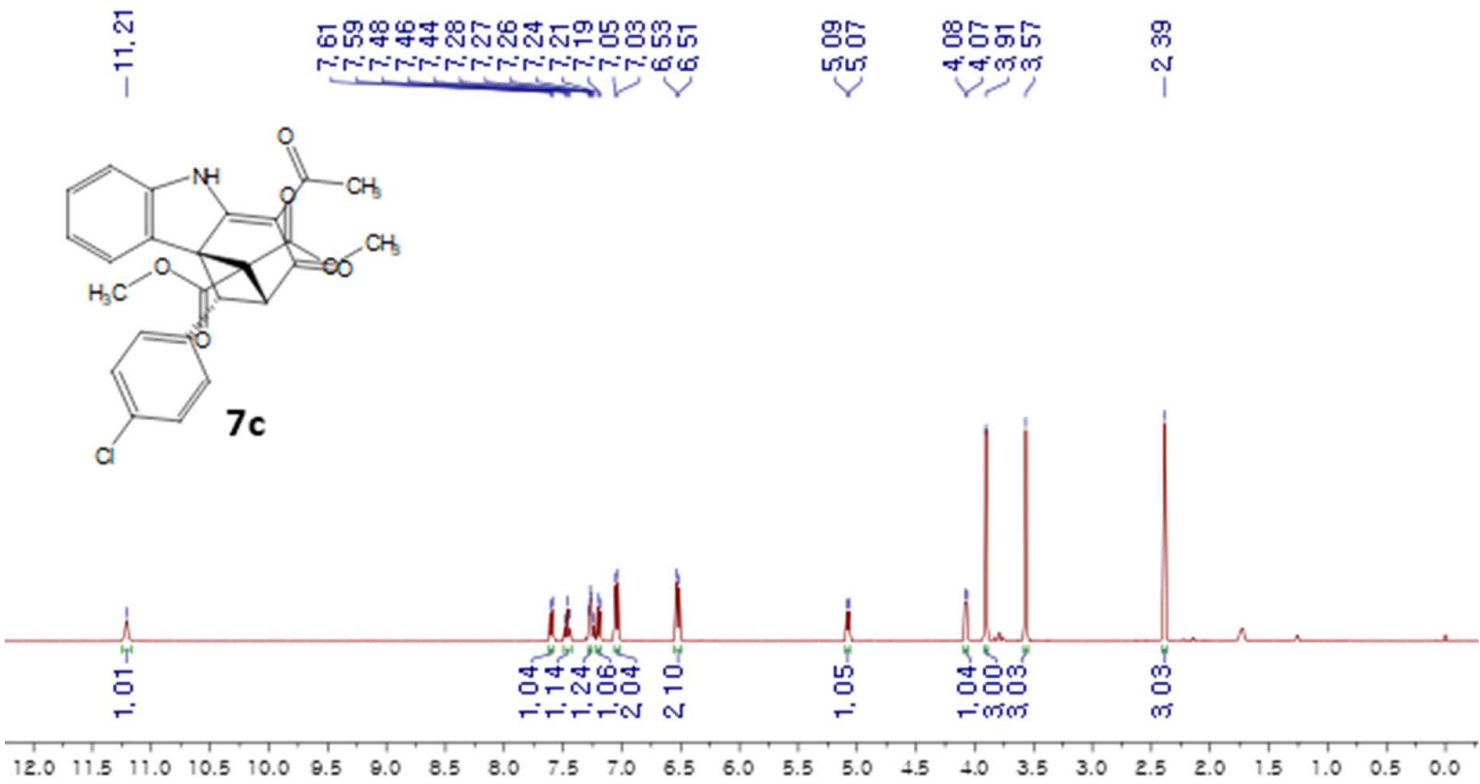

${ }^{1} \mathrm{H}$ NMR (400 MHz, $\mathrm{CDCl}_{3}$ ) spectra of dimethyl (3R,4aR,10R)-1-acetyl-10-(4chlorophenyl)-2-oxo-2,9-dihydro-3,4a-methanocarbazole-4,4(3H)-dicarboxylate (7c) 
๘

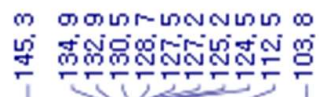

$-\cos c 0$

๓ำํํํำ

官
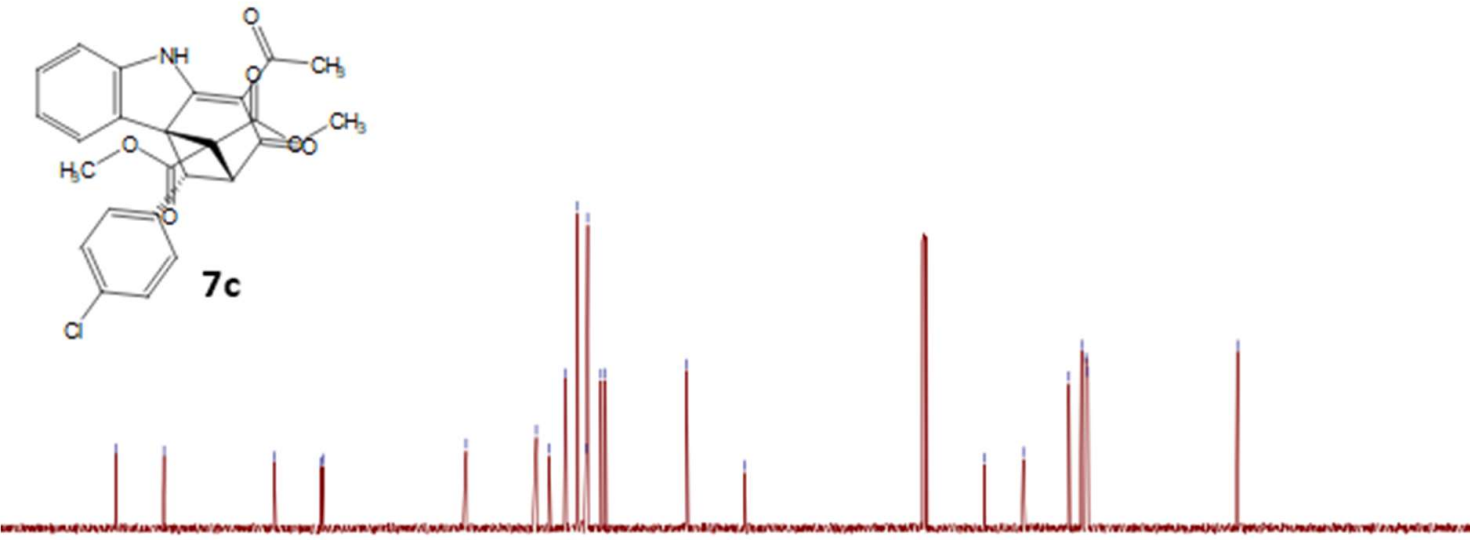

$\begin{array}{llllllllllllllllllllllllllll}1 & 10 & 200 & 180 & 180 & 170 & 160 & 150 & 140 & 130 & 120 & 110 & 100 & 90 & 80 & 70 & 60 & 50 & 40 & 30 & 20 & 10 & 0\end{array}$

${ }^{13} \mathrm{C}$ NMR (100 MHz, $\mathrm{CDCl}_{3}$ ) spectra of dimethyl (3R,4aR,10R)-1-acetyl-10-(4-

chlorophenyl)-2-oxo-2,9-dihydro-3,4a-methanocarbazole-4,4(3H)-dicarboxylate (7c)

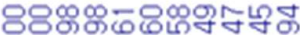

oonininininimo<smiles>O=C(c1ccccc1)C(I)C(=O)c1ccccc1</smiles>

8a

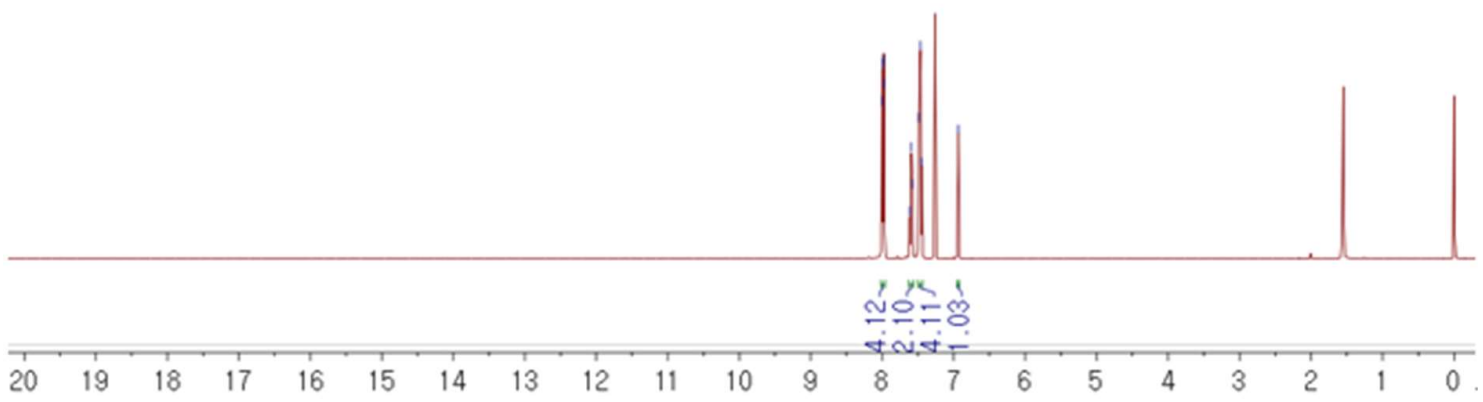

${ }^{1} \mathrm{H}$ NMR (400 MHz, CDCl3) spectra of 2-iodo-1,3-diphenylpropane-1,3-dione (8a) 


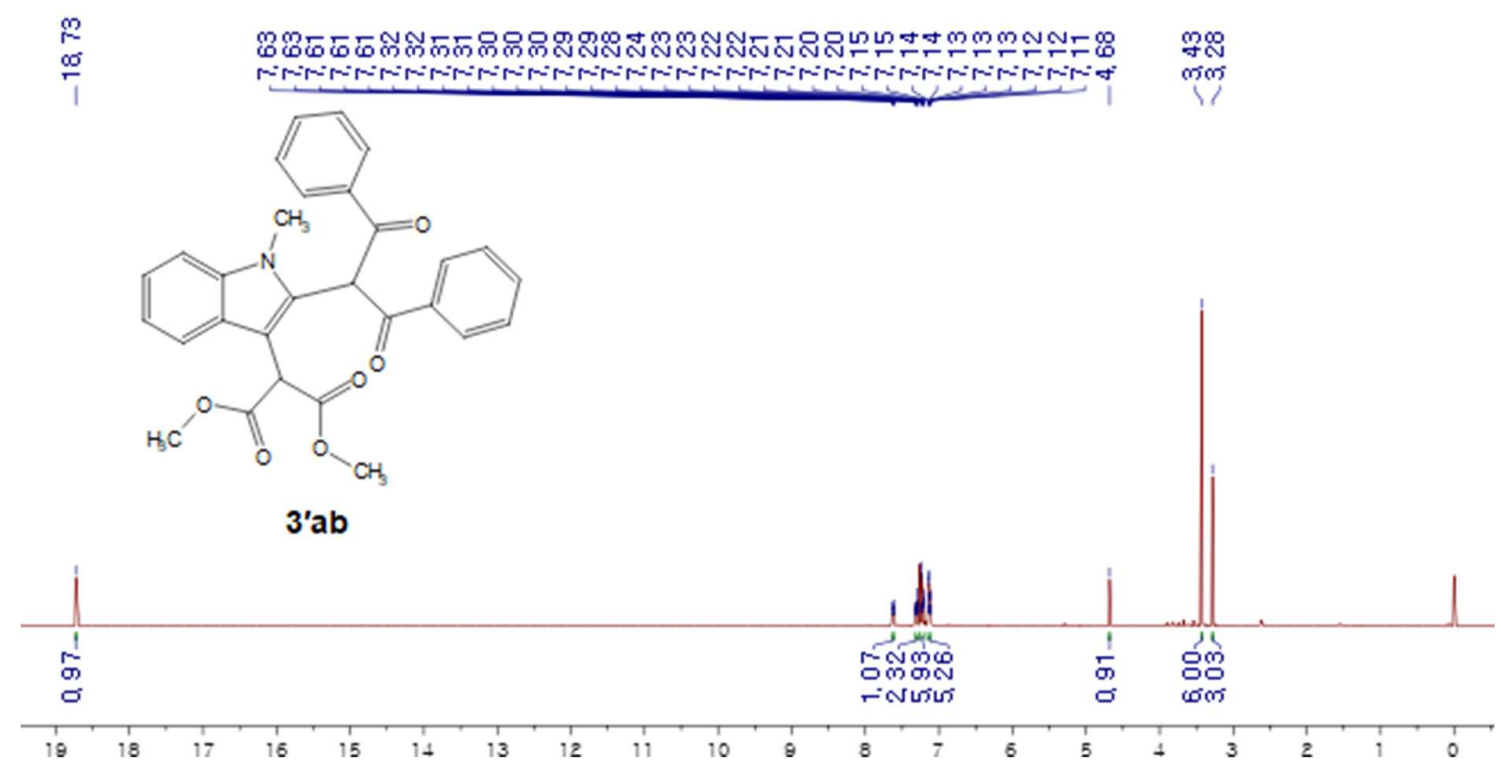

${ }^{1} \mathrm{H}$ NMR (400 MHz, $\mathrm{CDCl}_{3}$ ) spectra of dimethyl 2-(2-(1,3-dioxo-1,3-diphenylpropan-2-yl)-1methyl-1H-indol-3-yl)malonate (3'ab)

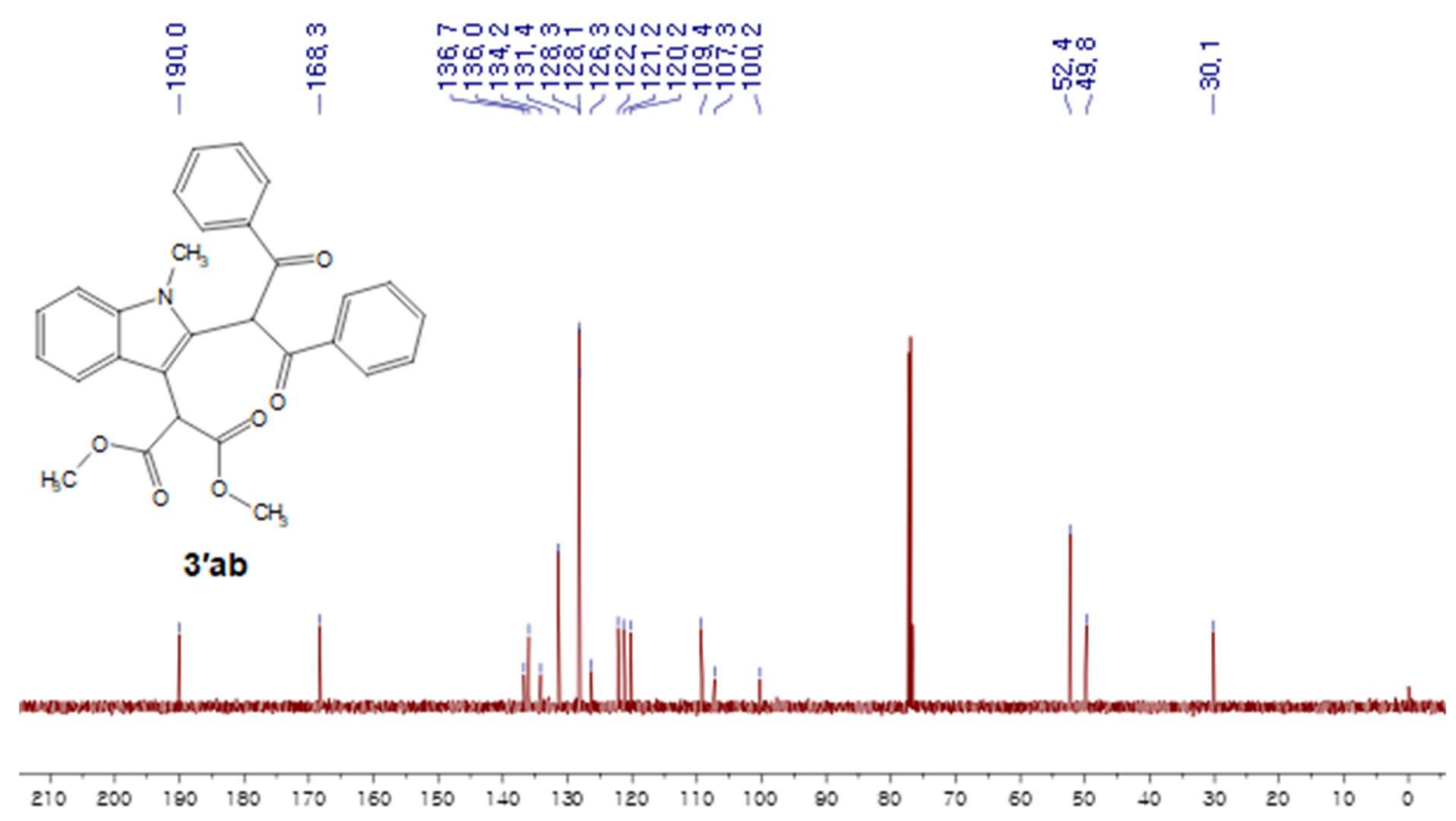

${ }^{13} \mathrm{C} \mathrm{NMR}\left(100 \mathrm{MHz}, \mathrm{CDCl}_{3}\right)$ spectra of dimethyl 2-(2-(1,3-dioxo-1,3-diphenylpropan-2-yl)1-methyl-1H-indol-3-yl)malonate (3'ab) 


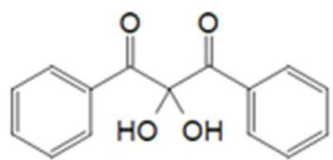

8b

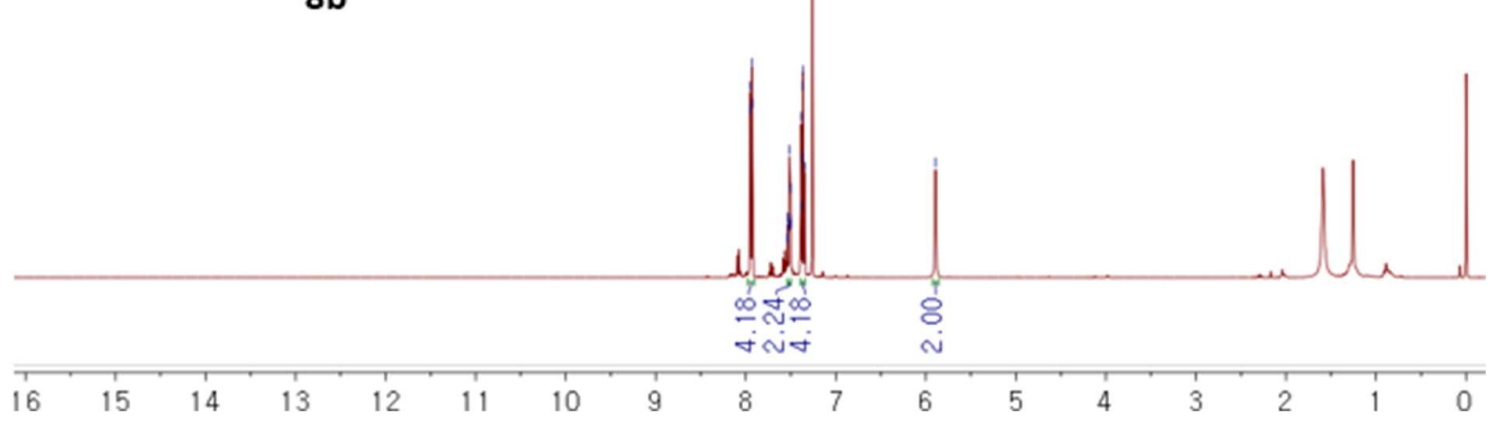

${ }^{1} \mathrm{H}$ NMR (400 MHz, $\mathrm{CDCl}_{3}$ ) spectra of 2,2-dihydroxy-1,3-diphenylpropane-1,3-dione (8b)

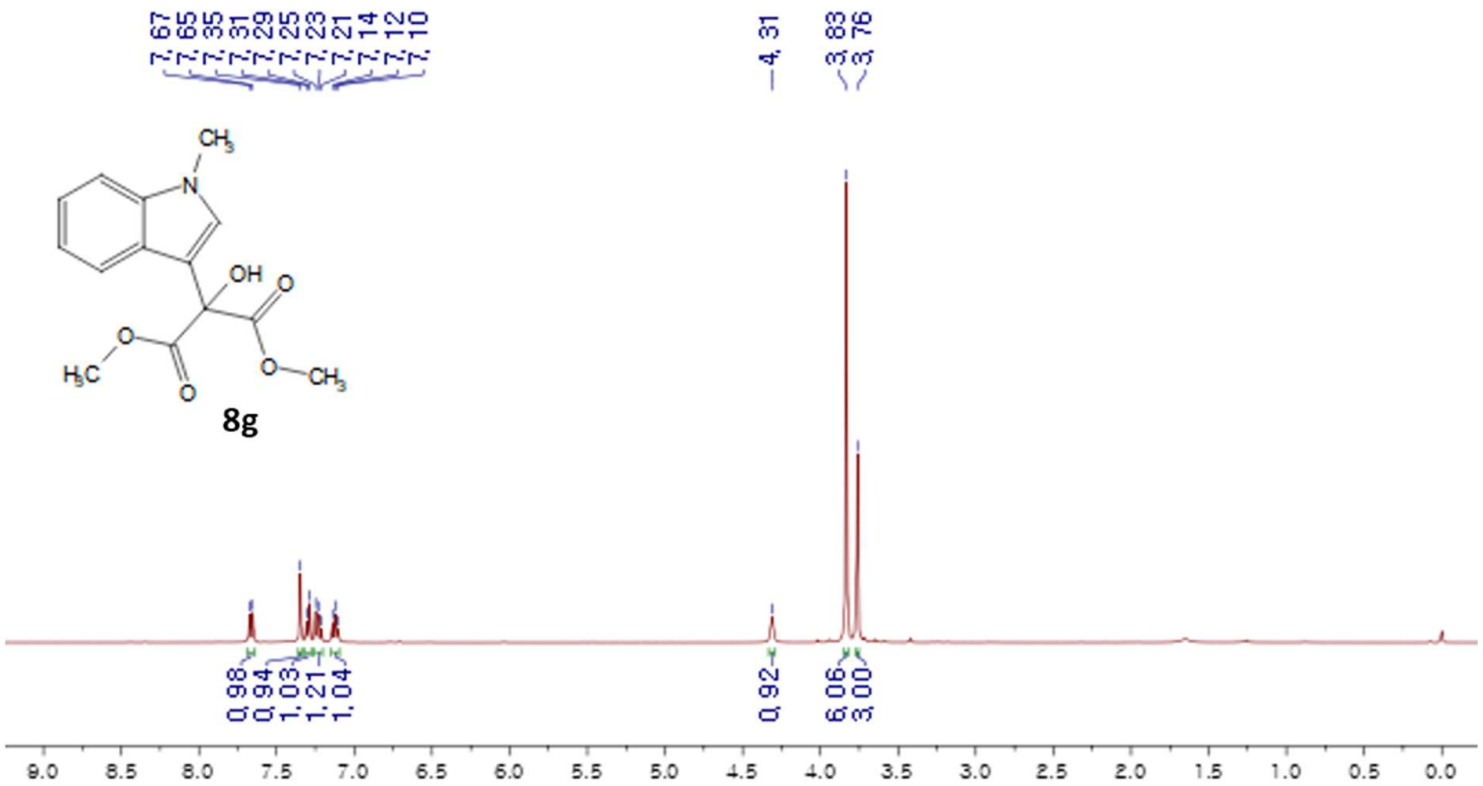

${ }^{1} \mathrm{H}$ NMR (400 MHz, $\mathrm{CDCl}_{3}$ ) spectra of dimethyl 2-hydroxy-2-(1-methyl-1H-indol-3yl)malonate $(\mathbf{8 g})$ 


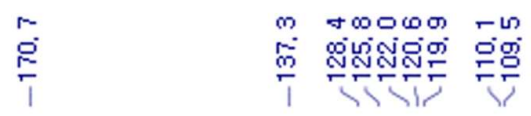

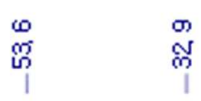

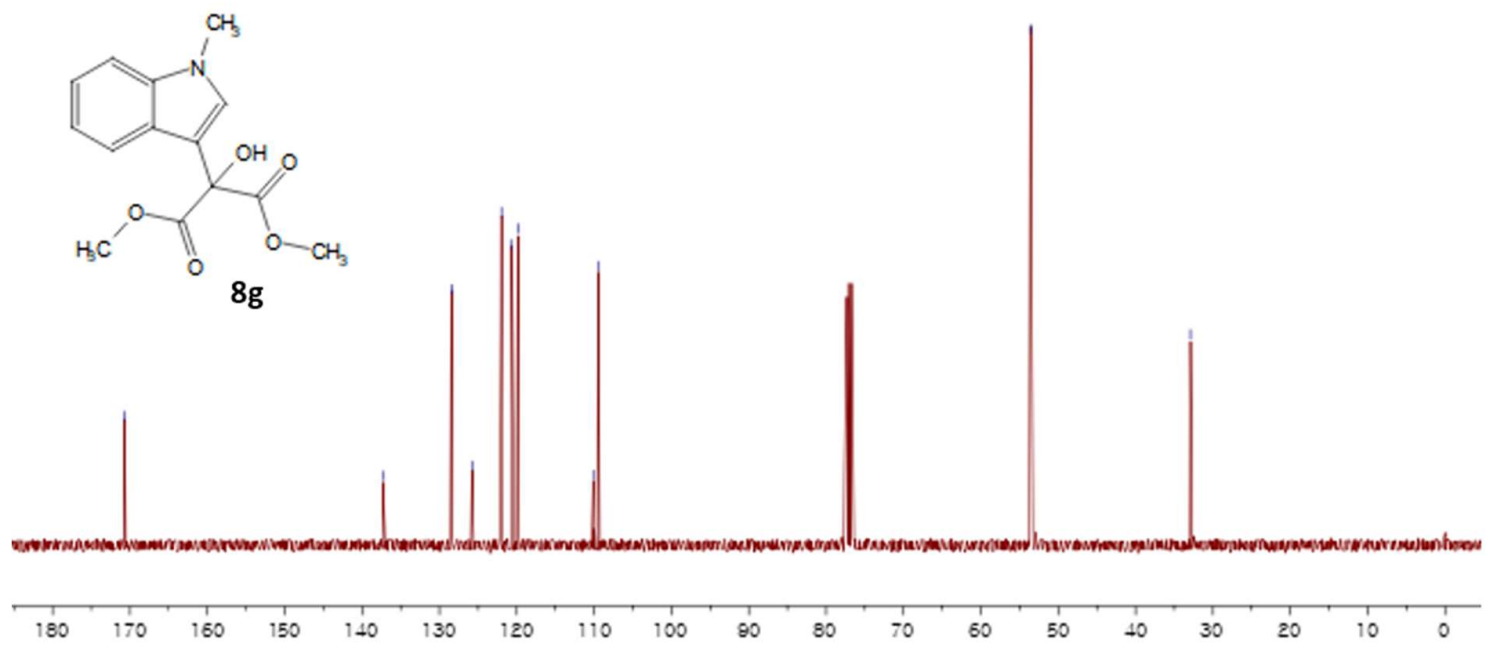

${ }^{13} \mathrm{C}$ NMR (100 MHz, $\mathrm{CDCl}_{3}$ ) spectra of dimethyl 2-hydroxy-2-(1-methyl-1H-indol-3yl)malonate (8g)
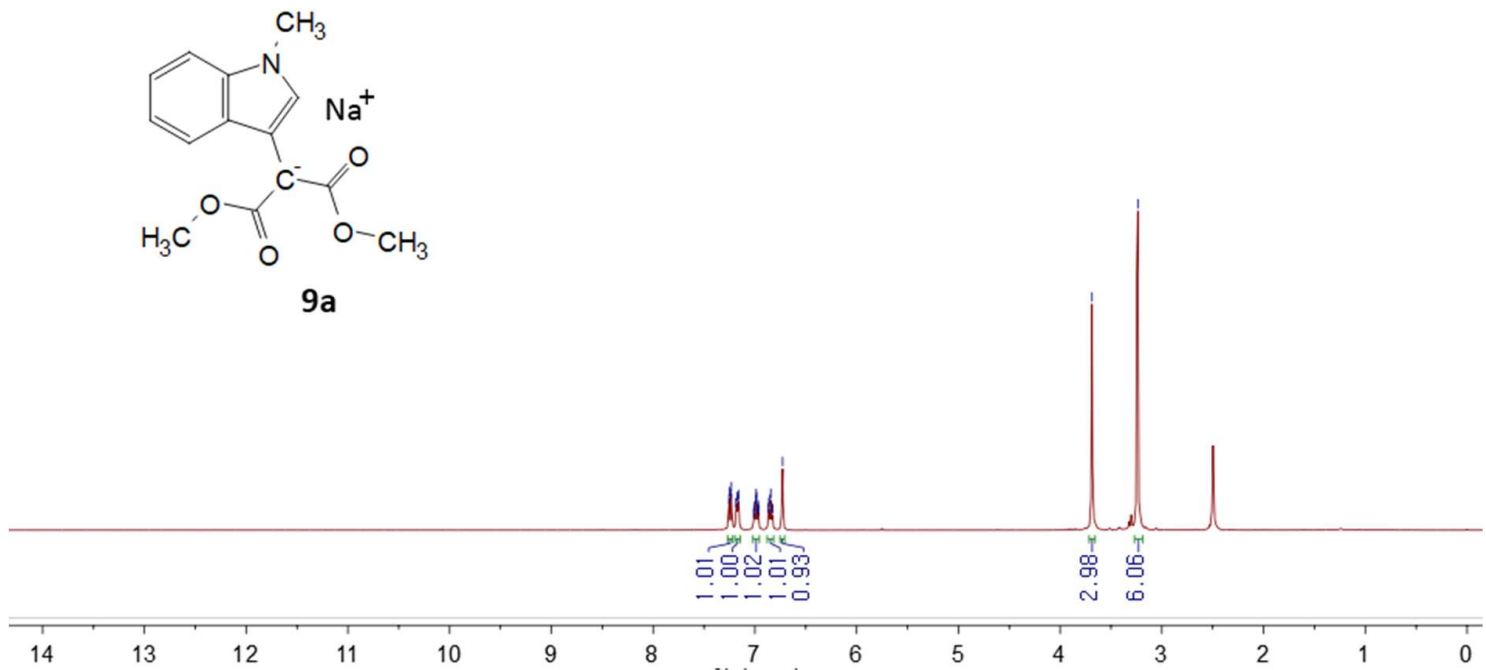

${ }^{1} \mathrm{H}$ NMR (400 MHz, $\mathrm{CDCl}_{3}$ ) spectra of 1,3-dimethoxy-2-(1-methyl-1H-indol-3-yl)-1,3dioxopropan-2-ide (9a) 


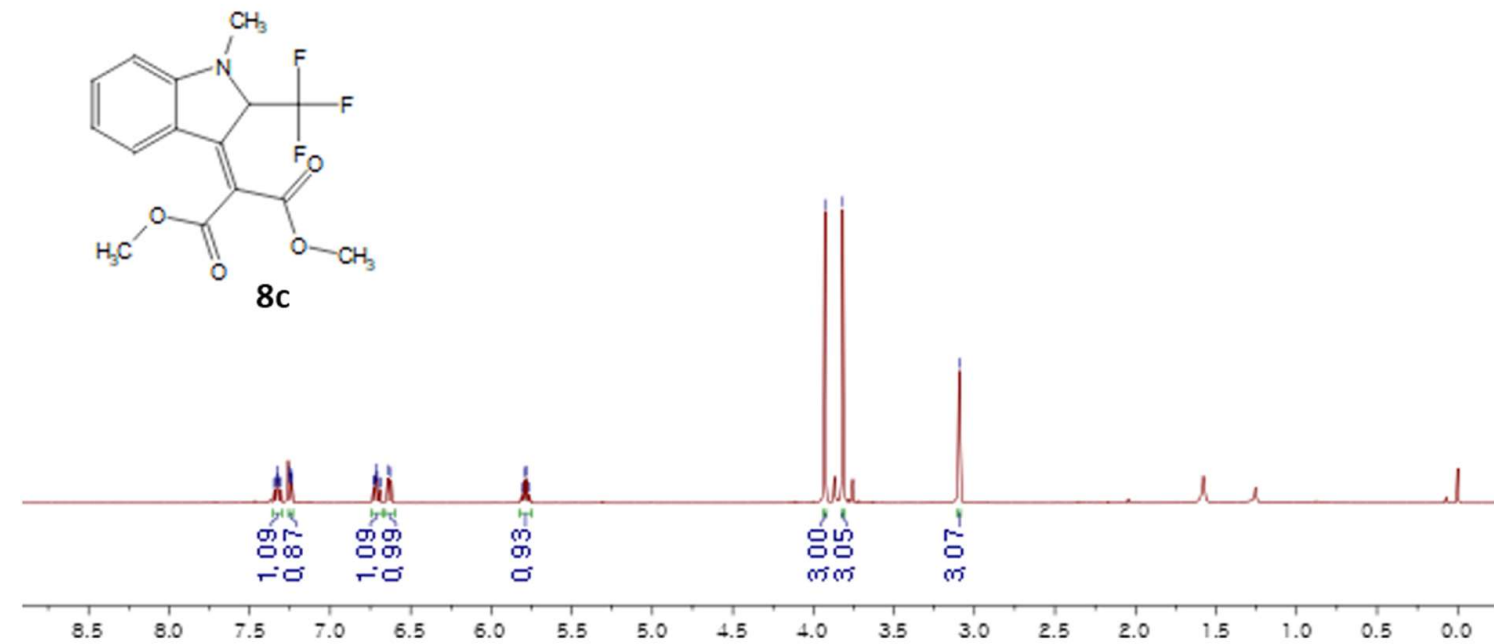

${ }^{1} \mathrm{H}$ NMR (400 MHz, $\mathrm{CDCl}_{3}$ ) spectra of dimethyl 2-(1-methyl-2-(trifluoromethyl)indolin-3ylidene)malonate $(\mathbf{8 c})$
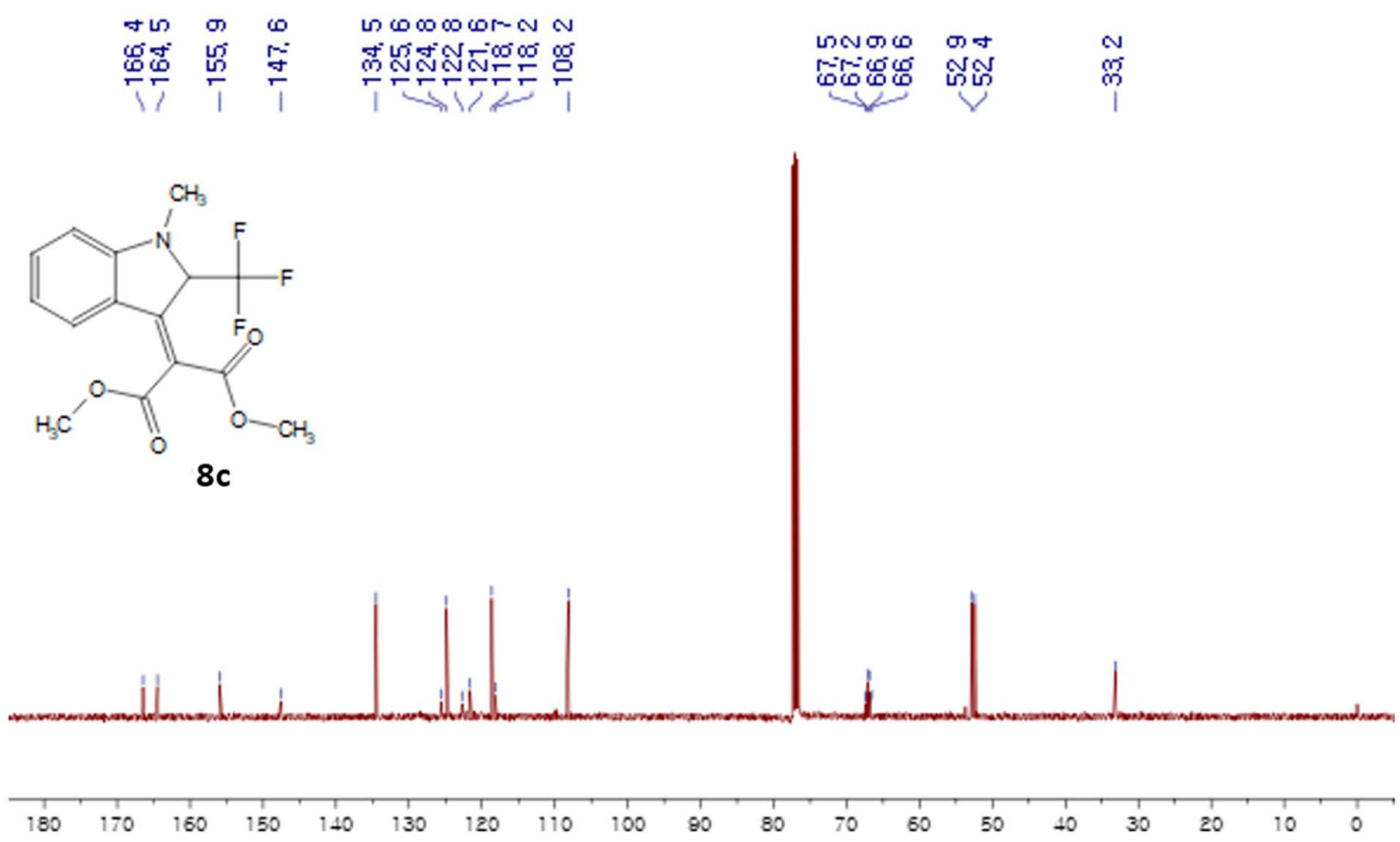

${ }^{13} \mathrm{C}$ NMR (100 MHz, $\mathrm{CDCl}_{3}$ ) spectra of dimethyl 2-(1-methyl-2-(trifluoromethyl)indolin-3ylidene)malonate (8c) 


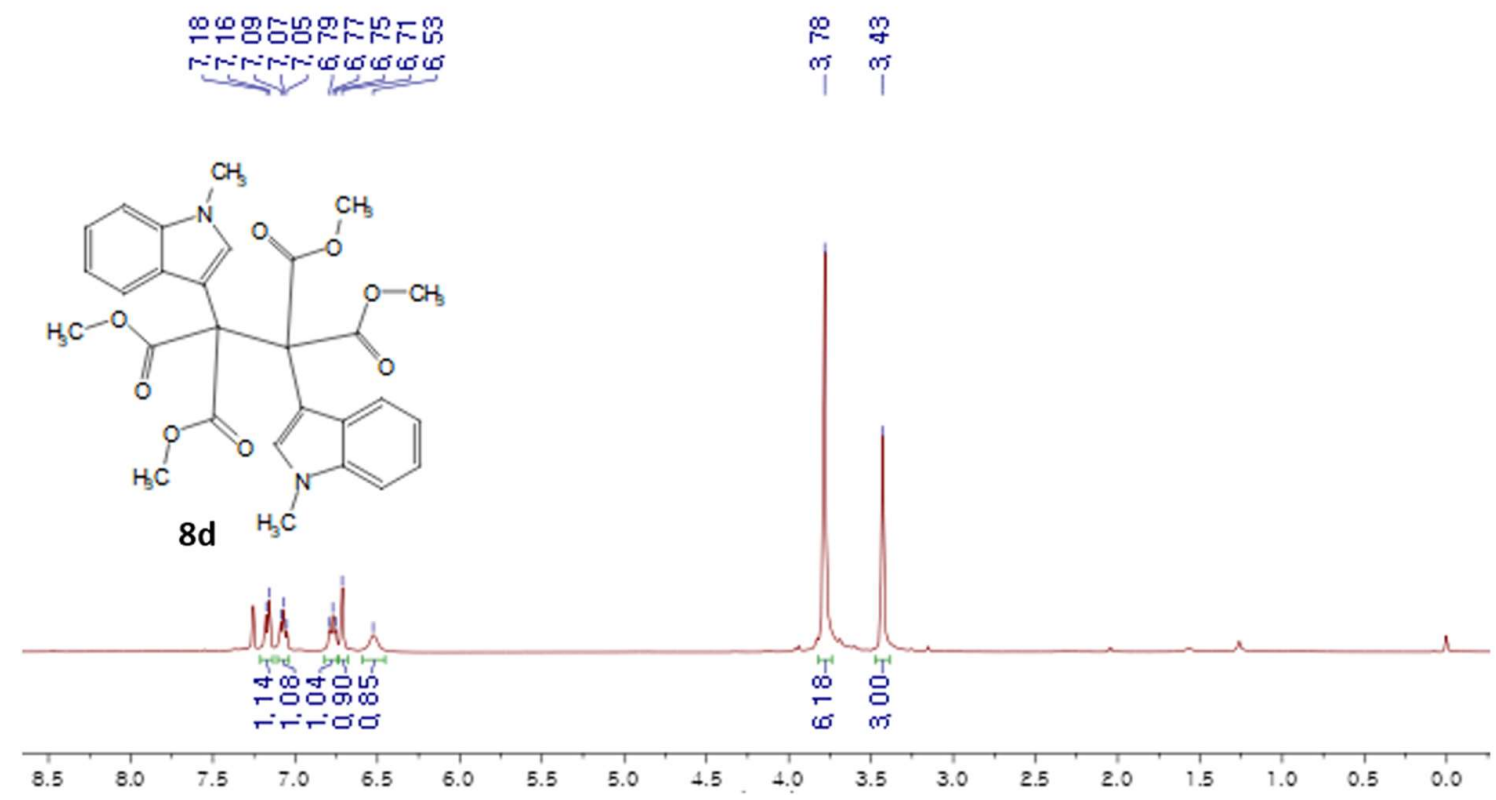

${ }^{1} \mathrm{H}$ NMR (400 MHz, $\mathrm{CDCl}_{3}$ ) spectra of tetramethyl 1,2-bis(1-methyl-1H-indol-3-yl)ethane1,1,2,2-tetracarboxylate (8d)
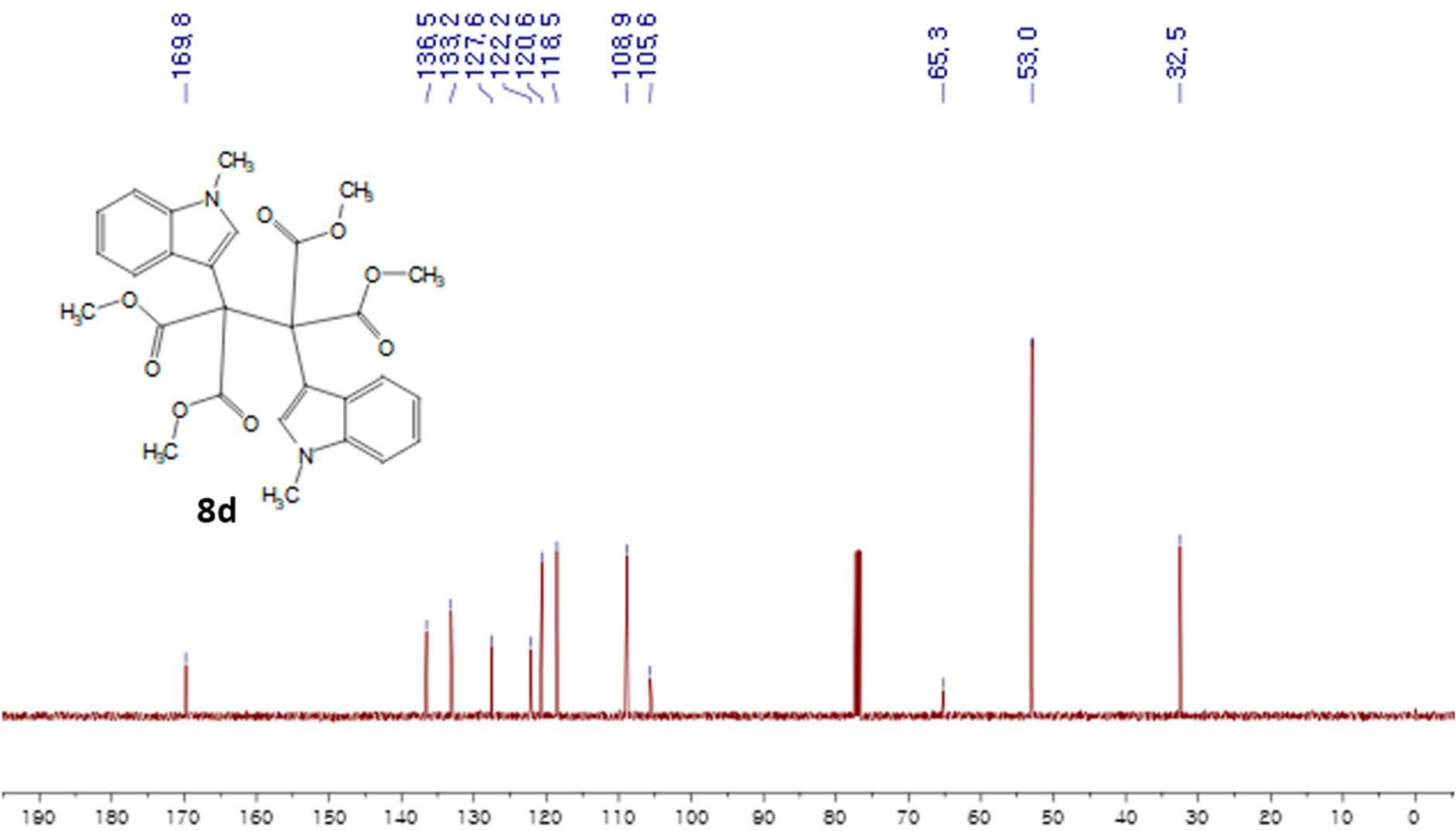

${ }^{13} \mathrm{C}$ NMR (100 MHz, $\mathrm{CDCl}_{3}$ ) spectra of tetramethyl 1,2-bis(1-methyl-1H-indol-3-yl)ethane1,1,2,2-tetracarboxylate (8d) 


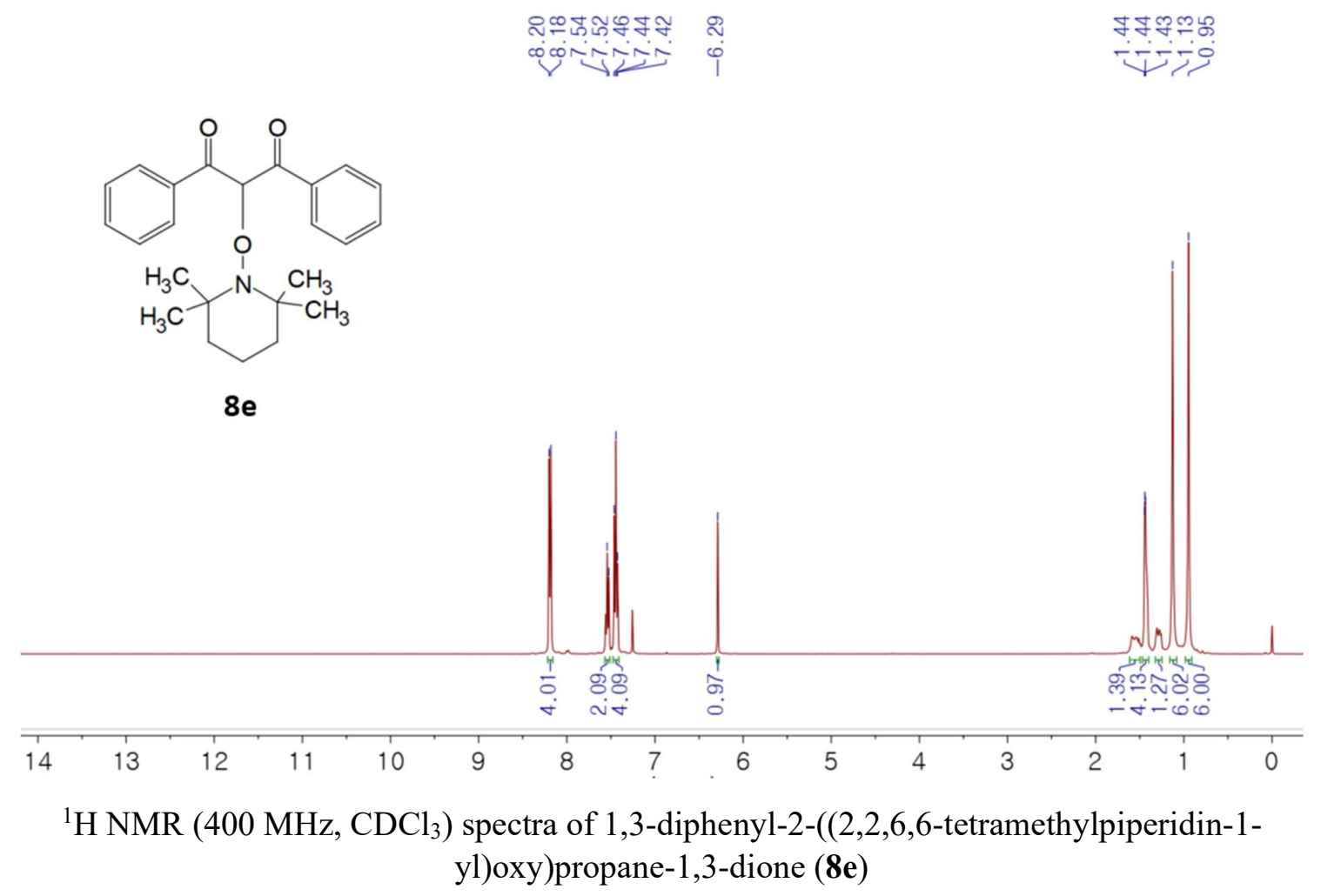




\section{References}

1. Zou, L.-H.; Priebbenow, D. L.; Wang, L.; Mottweiler, J.; Bolm, C., Copper-Catalyzed Synthesis of $\alpha$-Thioaryl Carbonyl Compounds Through S-S and C-C Bond Cleavage. Adv. Synth. Catal. 2013, 355, 2558-2563.

2. Dhondi, P. K.; Carberry, P.; Chisholm, J. D., Ligand effects in the rhodium-catalyzed addition of alkynes to aldehydes and diketones. Modification of the $\beta$-diketonate ligand. Tetrahedron Letters 2007, $48,8743-8746$.

3. Jin, C.-Y.; Du, J.-Y.; Zeng, C.; Zhao, X.-H.; Cao, Y.-X.; Zhang, X.-Z.; Lu, X.-Y.; Fan, C.-A., Hypervalent Iodine(III)-Mediated Oxidative Dearomatizing Cyclization of Arylamines. Adv. Syn. Catal. 2014, 356, 2437-2444.

4. Zawadiak, J.; Mrzyczek, M., Influence of substituent on UV absorption and keto-enol tautomerism equilibrium of dibenzoylmethane derivatives. Spectrochimica Acta Part A: Molecular and Biomolecular Spectroscopy 2012, 96, 815-819.

5. Suri, O. P.; Satti, N. K.; Suri, K. A., Microwave Induced Acetoacetylation of Hetaryl and Aryl Amines. Synth. Commun. 2000, 30, 3709-3718.

6. Sharma, P.; Liu, R.-S., Cu-Catalyzed Aerobic Oxidative Cyclizations of 3-N-Hydroxyamino-1,2propadienes with Alcohols, Thiols, and Amines To Form $\alpha-\mathrm{O}-$, S-, and N-Substituted 4-Methylquinoline Derivatives. Chem. Eur. J. 2015, 21, 4590-4594.

7. Sun, L.; Bera, H.; Chui, W. K., Synthesis of pyrazolo[1,5-a][1,3,5]triazine derivatives as inhibitors of thymidine phosphorylase. Eur. J. Med. Chem. 2013, 65, 1-11.

8. Li, X.; Golz, C.; Alcarazo, M., 5-(Cyano)dibenzothiophenium Triflate: A Sulfur-Based Reagent for Electrophilic Cyanation and Cyanocyclizations. Angew. Chem. Int. Ed. 2019, 58, 9496-9500.

9. Enders, D.; Grossmann, A.; Huang, H.; Raabe, G., Dual Secondary Amine/N-Heterocyclic Carbene Catalysis in the Asymmetric Michael/Cross-Benzoin Cascade Reaction of $\beta$-Oxo Sulfones with Enals. Eur. J. Med. Chem. 2011, 2011, 4298-4301.

10. Ma, X.; Li, W.; Li, X.; Tao, X.; Fan, W.; Xie, X.; Ayad, T.; Ratovelomanana-Vidal, V.; Zhang, Z., Ru-catalyzed highly chemo- and enantioselective hydrogenation of $\gamma$-halo- $\gamma, \delta$-unsaturated- $\beta$-keto esters under neutral conditions. Chem. Commun. 2012, 48, 5352-5354.

11. Martichonok, V. V.; Chiang, P. K.; Dornbush, P. J.; Land, K. M., On Regioselectivity of Aldol Condensation of Aromatic Aldehydes with Borate Complex of Acetylacetone. Synth. Commun. 2014, $44,1245-1250$.

12. Khan, M. A.; El-Khatib, R.; Rainsford, K. D.; Whitehouse, M. W., Synthesis and anti-inflammatory properties of some aromatic and heterocyclic aromatic curcuminoids. Bioorg. Chem. 2012, 40, 30-38. 13. Peterson, J. R.; Winter, T. J.; Miller, C. P., Efficacious Entry Into Substituted 5,6-Dihydro-4Hydroxy-2H-Pyran-2-Ones and 2,3-Dihydro-4H-Pyran-4-Ones Utilizing Ketonic Dianions. Synth. Commun. 1988, 18, 949-963. 
14. Freifeld, I.; Bose, G.; Eckardt, T.; Langer, P., Synthesis of $\gamma$-Alkylidenebutenolides by Formal [3+2] Cyclizations of 1,5- and 2,4-Bis(trimethylsilyloxy)-1,3,5-hexatrienes with Oxalyl Chloride. Eur. J. Org. Chem. 2007, 2007, 351-355.

15. Wei, X.; Yang, Y.; Ge, J.; Lin, X.; Liu, D.; Wang, S.; Zhang, J.; Zhou, G.; Li, S., Synthesis, characterization, DNA/BSA interactions and in vitro cytotoxicity study of palladium(II) complexes of hispolon derivatives. J. Inorg. Biochem. 2020, 202, 110857.

16. Pal, A.; Koduri, N. D.; Wang, Z.; Quiroz, E. L.; Chong, A.; Vuong, M.; Rajagopal, N.; Nguyen, M.; Roberts, K. P.; Hussaini, S. R., Copper-catalyzed chemoselective cross-coupling reaction of thioamides and $\alpha$-diazocarbonyl compounds: Synthesis of enaminones. Tetrahedron Letters 2017, 58, 586-589.

17. Xiong, Y.-S.; Weng, J.; Lu, G., Manganese(III)-Mediated and -Catalyzed Decarboxylative Hydroxysulfonylation of Arylpropiolic Acids with Sodium Sulfinates in Water. Adv. Synth. Catal. 2018, 360, 1611-1616.

18. Fraile, J. M.; Le Jeune, K.; Mayoral, J. A.; Ravasio, N.; Zaccheria, F., CuO/SiO2 as a simple, effective and recoverable catalyst for alkylation of indole derivatives with diazo compounds. Org. Biomol. Chem. 2013, 11, 4327-4332.

19. Johansen, M. B.; Kerr, M. A., Direct Functionalization of Indoles: Copper-Catalyzed Malonyl Carbenoid Insertions. Org. Lett. 2010, 12, 4956-4959.

20. Kato, S.-i.; Furuya, T.; Nitani, M.; Hasebe, N.; Ie, Y.; Aso, Y.; Yoshihara, T.; Tobita, S.; Nakamura, Y., A Series of $\pi$-Extended Thiadiazoles Fused with Electron-Donating Heteroaromatic Moieties: Synthesis, Properties, and Polymorphic Crystals. Chem. Eur. J. 2015, 21, 3115-3128.

21. Yip, S. F.; Cheung, H. Y.; Zhou, Z.; Kwong, F. Y., Room-Temperature Copper-Catalyzed $\alpha$-Arylation of Malonates. Org. Lett. 2007, 9, 3469-3472.

22. Liu, Y.; Ma, S., Benzofurans or Isochromenes via the Ring-Opening Cyclization of Cyclopropene Derivatives with Organolithiums. Org. Lett. 2012, 14, 720-723.

23. Zhao, M.-N.; Lian, X.-L.; Ren, Z.-H.; Wang, Y.-Y.; Guan, Z.-H., Palladium-catalyzed oxidative amination of activated olefins with $\mathrm{N}$-alkyl anilines for synthesis of tertiary (E)-enamines. RSC $A d v$. 2014, 4, 62042-62045.

24. Krasnokutskaya, E. A.; Lesina, Y. A.; Gorlushko, D. A.; Filimonov, V. D., Comparative Study of the Reactivity of Iodinating Agents in Solution and Solid Phase. Russ. J. Org. 2005, 41, 855-859.

25. Zhou, P.-J.; Li, C.-K.; Zhou, S.-F.; Shoberu, A.; Zou, J.-P., Copper-catalyzed TEMPO oxidative cleavage of 1,3-diketones and $\beta$-keto esters for the synthesis of 1,2-diketones and $\alpha$-keto esters. Org. Biomol. Chem. 2017, 15, 2629-2637.

26. (a) Grimme, S.; Antony, J.; Ehrlich, S.; Krieg, H., A consistent and accurate ab initio parametrization of density functional dispersion correction (DFT-D) for the 94 elements H-Pu. J. Chem. Phys. 2010, 132, 154104; (b) Becke, A. D., Density-functional thermochemistry. III. The role of exact exchange. $J$. Chem. Phys. 1993, 98, 5648-5652; (c) Lee, C.; Yang, W.; Parr, R. G., Development of the Colle-Salvetti 
correlation-energy formula into a functional of the electron density. Phys. Rev. B. 1988, 37, 785-789.

27. Hehre, W. J.; Ditchfield, R.; Pople, J. A., Self-Consistent Molecular Orbital Methods. XII. Further Extensions of Gaussian-Type Basis Sets for Use in Molecular Orbital Studies of Organic Molecules. J. Chem. Phys. 1972, 56, 2257-2261.

28. Hay, P. J.; Wadt, W. R., Ab initio effective core potentials for molecular calculations. Potentials for $\mathrm{K}$ to Au including the outermost core orbitals. J. Chem. Phys. 1985, 82, 299-310.

29. (a) Feller, D., The role of databases in support of computational chemistry calculations. J. Comput. Chem. 1996, 17, 1571-1586; (b) Schuchardt, K. L.; Didier, B. T.; Elsethagen, T.; Sun, L.; Gurumoorthi, V.; Chase, J.; Li, J.; Windus, T. L., Basis Set Exchange: A Community Database for Computational Sciences. J. Chem. Inf. Model. 2007, 47, 1045-1052.

30. Marenich, A. V.; Cramer, C. J.; Truhlar, D. G., Universal Solvation Model Based on Solute Electron Density and on a Continuum Model of the Solvent Defined by the Bulk Dielectric Constant and Atomic Surface Tensions. J. Phys. Chem. B. 2009, 113, 6378-6396.

31. (a) Fukui, K., Formulation of the reaction coordinate. J. Phys. Chem. 1970, 74, 4161-4163; (b) Fukui, K., The path of chemical reactions - the IRC approach. Acc. Chem. Res. 1981, 14, 363-368.

32. Gaussian 09 : Gaussian 09, Revision D.01, M. J. Frisch, G. W. Trucks, H. B. Schlegel, G. E. Scuseria, M. A. Robb, J. R. Cheeseman, G. Scalmani, V. Barone, B. Mennucci, G. A. Petersson, H. Nakatsuji, M. Caricato, X. Li, H. P. Hratchian, A. F. Izmaylov, J. Bloino, G. Zheng, J. L. Sonnenberg, M. Hada, M. Ehara, K. Toyota, R. Fukuda, J. Hasegawa, M. Ishida, T. Nakajima, Y. Honda, O. Kitao, H. Nakai, T. Vreven, J. A. Montgomery, Jr., J. E. Peralta, F. Ogliaro, M. Bearpark, J. J. Heyd, E. Brothers, K. N. Kudin, V. N. Staroverov, T. Keith, R. Kobayashi, J. Normand, K. Raghavachari, A. Rendell, J. C. Burant, S. S. Iyengar, J. Tomasi, M. Cossi, N. Rega, J. M. Millam, M. Klene, J. E. Knox, J. B. Cross, V. Bakken, C. Adamo, J. Jaramillo, R. Gomperts, R. E. Stratmann, O. Yazyev, A. J. Austin, R. Cammi, C. Pomelli, J. W. Ochterski, R. L. Martin, K. Morokuma, V. G. Zakrzewski, G. A. Voth, P. Salvador, J. J. Dannenberg, S. Dapprich, A. D. Daniels, O. Farkas, J. B. Foresman, J. V. Ortiz, J. Cioslowski, and D. J. Fox, Gaussian, Inc., Wallingford CT, 2013. (b) B3LYP : A.D. Becke, J. Chem. Phys. 1993, 98, 5648. C.T. Lee, W.T Yang, R.G. Parr, Phys. Rev. B 1988, 37, 785. (c) GD3 : S. Grimme, J. Antony, S. Ehrlich, H. Krieg, J. Chem. Phys. 2010, 132, 154104-154119. (d) 631+g(d,p) : W.J. Hehre, R. Ditchfield, J.A. Pople, J. Chem. Phys. 1972, 56, 2257. (e) LANL2DZdp : W. R. Wadt, P. J. Hay, J. Chem. Phys. 1985, 82, 284-298. (f) EMSL Basis Set Exchange : Feller, D., J. Comp. Chem., 17(13), 1571-1586, 1996; J. Chem. Inf. Model., 47(3), 1045-1052, 2007. (f) IRC : K. Fukui, J. Phys. Chem. 1970, 74, 4161; K. Fukui, Acc. Chem. Res. 1981, 14, 363. (g) SMD model : A.V. Marenich, C.J. Cramer, D.G. Truhlar, J. Phys. Chem. B 2009, 113, 6378. 